\title{
Algebraic polynomial system solving and applications
}

Citation for published version (APA):

Bleylevens, I. W. M. (2010). Algebraic polynomial system solving and applications. [Doctoral Thesis, Maastricht University]. https://doi.org/10.26481/dis.20101209ib

Document status and date:

Published: 01/01/2010

DOI:

10.26481/dis.20101209ib

Document Version:

Publisher's PDF, also known as Version of record

\section{Please check the document version of this publication:}

- A submitted manuscript is the version of the article upon submission and before peer-review. There can be important differences between the submitted version and the official published version of record.

People interested in the research are advised to contact the author for the final version of the publication, or visit the DOI to the publisher's website.

- The final author version and the galley proof are versions of the publication after peer review.

- The final published version features the final layout of the paper including the volume, issue and page numbers.

Link to publication

\footnotetext{
General rights rights.

- You may freely distribute the URL identifying the publication in the public portal. please follow below link for the End User Agreement:

www.umlib.nl/taverne-license

Take down policy

If you believe that this document breaches copyright please contact us at:

repository@maastrichtuniversity.nl

providing details and we will investigate your claim.
}

Copyright and moral rights for the publications made accessible in the public portal are retained by the authors and/or other copyright owners and it is a condition of accessing publications that users recognise and abide by the legal requirements associated with these

- Users may download and print one copy of any publication from the public portal for the purpose of private study or research.

- You may not further distribute the material or use it for any profit-making activity or commercial gain

If the publication is distributed under the terms of Article $25 \mathrm{fa}$ of the Dutch Copyright Act, indicated by the "Taverne" license above, 
ALGEBRAIC POLYNOMIAL SYSTEM SOLVING AND APPLICATIONS

Ivo Bleylevens 
๑) Copyright I.W.M. Bleylevens, Maastricht 2010

Cover design: D\&L Graphics, Kerkrade

Printing: $\quad$ Schrijen-Lippertz, Voerendaal

ISBN: $\quad$ 978-90-8590-046-7

All rights reserved. No part of this thesis may be reproduced, stored in a retrieval system of any nature, or transmitted in any form by any means, electronic, mechanical, photocopying, recording or otherwise, included a complete or partial transcription, without the permission of the author. 


\section{ALGEBRAIC POLYNOMIAL SYSTEM SOLVING AND APPLICATIONS}

\section{PROEFSCHRIFT}

ter verkrijging van de graad van doctor aan de Universiteit Maastricht, op gezag van de Rector Magnificus, Prof. mr. G.P.M.F. Mols, volgens het besluit van het College van Decanen, in het openbaar te verdedigen op donderdag 9 december 2010 om 12.00 uur

door

Ivo Werner Maria Bleylevens. 


\section{Promotores:}

Prof. dr. ir. R.L.M. Peeters

Prof. dr. B. Hanzon (University College Cork, lerland)

Beoordelingscommissie:

Prof. dr. ir. C.P.M. van Hoesel (voorzitter)

Dr. M.E. Hochstenbach (TU Eindhoven)

Dr. M. Petreczky

Prof. dr. ir. J.H. van Schuppen (CWI/VU Amsterdam, TU Delft) 


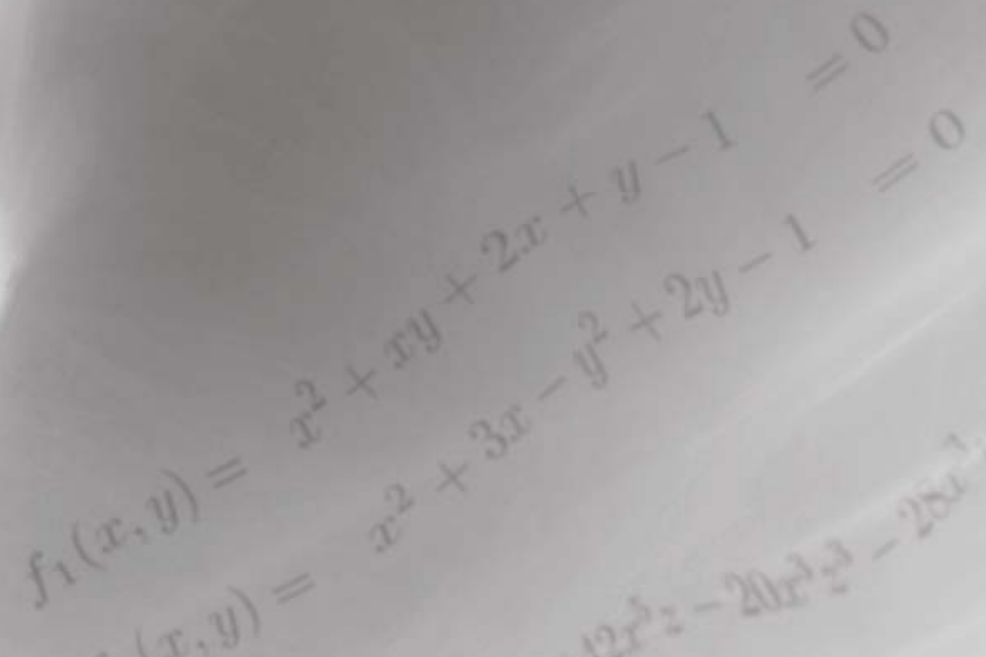

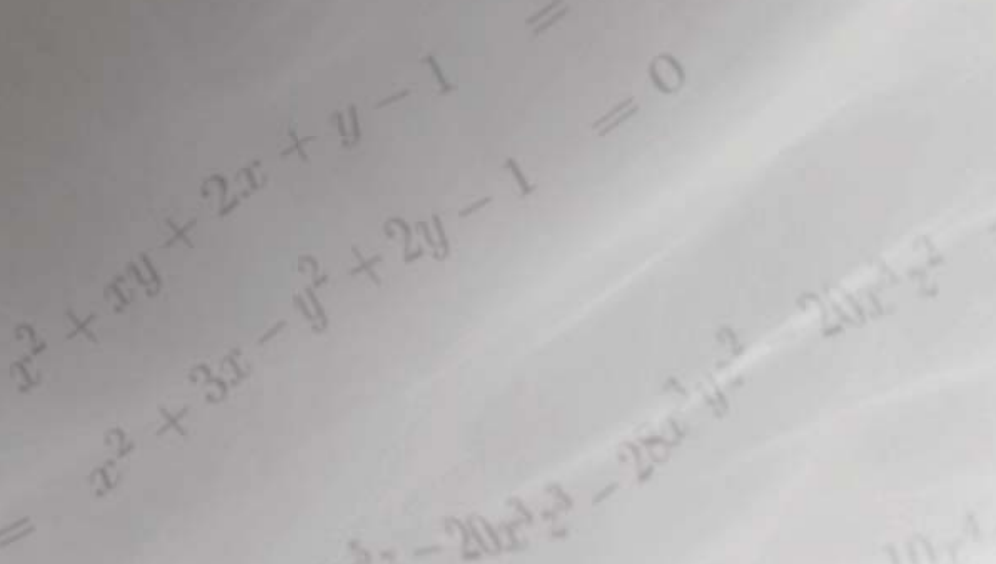

$+18 x^{3}+1 x^{2}+12^{3}$

25:-2015?

$\left(105 x^{3} 35-7 x^{2} z^{2}\right.$

$\int \log ^{2} x^{3} x^{3}+3 x^{3} y$

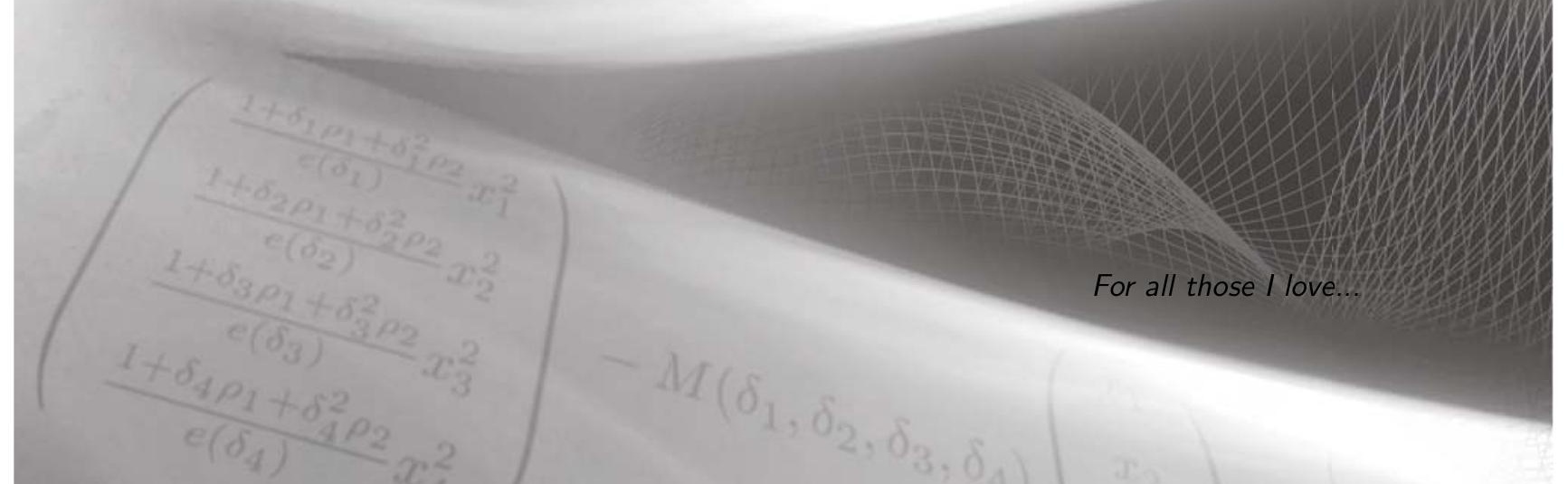





\section{Contents}

Contents

1 Introduction $\mathbf{3}$

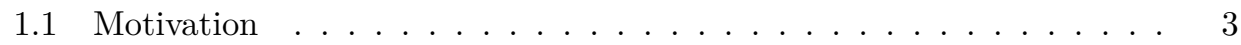

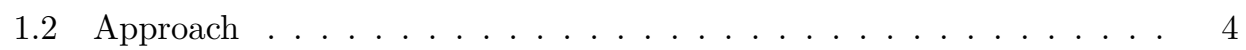

1.3 Research questions .................... 5

1.4 Thesis outline ..................... 6

I General introduction and background 9

2 Algebraic Background $\quad 11$

2.1 Rings and Ideals . . . . . . . . . . . . . . . . . . . . . 11

2.2 Varieties . . . . . . . . . . . . . . . . . 13

2.3 Monomial orderings . . . . . . . . . . . . . . . . . . 14

2.4 Gröbner Basis Theory . . . . . . . . . . . . . . . 16

2.5 The Buchberger Algorithm . . . . . . . . . . . . . . 19

3 Solving polynomial systems of equations 25

3.1 Solving polynomial equations in one variable . . . . . . . . . . 26

3.2 Methods for solving polynomial systems of equations . . . . . . . . . . 28

3.2.1 Resultant methods . . . . . . . . . . . . . . . . . 28

3.2 .2 Homotopy methods . . . . . . . . . . . . . . . 29

3.2.3 Subdivision methods . . . . . . . . . . . . . . . . . 32

3.2 .4 Algebraic methods . . . . . . . . . . . . . . . . . 32

3.3 The Stetter-Möller matrix method . . . . . . . . . . . . . . . . . . 35

3.4 Counting real solutions . . . . . . . . . . . . . . . . . . . . . . . . . . . . . . . . . .

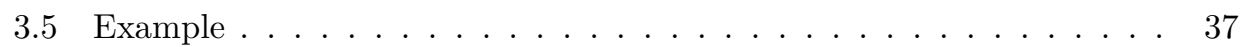


II Global Optimization

4 Global optimization of multivariate polynomials 43

4.1 The global minimum of a dominated polynomial . . . . . . . . . . . 44

4.2 The Stetter-Möller matrix method for global optimization . . . . . . . 46

4.3 An example . . . . . . . . . . . . . . . . 47

$5 \quad n$ D-systems approach in polynomial optimization $\quad 51$

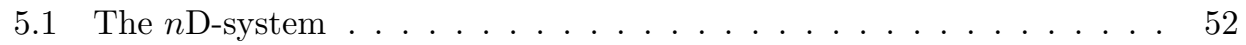

5.2 Usage of the $n \mathrm{D}$-system in polynomial optimization . . . . . . 58

5.3 Efficiency of the $n$ D-systems approach . . . . . . . . . . . 59

5.3.1 A linear complexity result for computing $y_{t_{1}, \ldots, t_{n}} \ldots \ldots \ldots$

5.3.2 Formulation as a shortest path problem . . . . . . . . . 60

5.3.3 Some heuristic procedures for the shortest path problem . . . . 69

5.3.4 Parallel computations in the $n$ D-systems approach . . . . . . 79

6 Iterative eigenvalue solvers $\quad 81$

6.1 Iterative eigenvalue solvers . . . . . . . . . . . . . . . . 82

6.2 The Jacobi-Davidson method . . . . . . . . . . . . . . . . . . 83

6.2 .1 Subspace expansion . . . . . . . . . . . . . . 83

6.2 .2 Subspace extraction $\ldots \ldots \ldots \ldots \ldots \ldots$

6.2 .3 Remaining important issues . . . . . . . . . . . . . . . 85

6.2.4 Pseudocode of the Jacobi-Davidson method . . . . . . . . . . 86

6.3 Target selection: the smallest real eigenvalue . . . . . . . . . 86

6.4 Projection to Stetter-structure . . . . . . . . . . . . . 87

6.4.1 Projecting an approximate eigenvector . . . . . . . . 87

6.4.2 Embedding projection in a Jacobi-Davidson method . . . . . 98

6.5 A Jacobi-Davidson method for commuting matrices . . . . . . . . . 102

6.5 .1 The method JDCOMM . . . . . . . . . . . . . . . 102

6.5.2 The JDCOMM expansion phase . . . . . . . . . . . . . 103

6.5.3 Pseudocode of the JDCOMM method . . . . . . . . . . . . 104

$\begin{array}{lll}7 & \text { Numerical experiments } & 107\end{array}$

7.1 Computing the minimum of a polynomial of order $8 \ldots \ldots \ldots$

7.2 Computing the global minimum using target selection . . . . . . 112

7.3 Projection to Stetter structure _. . . . . . . . . . . . . . 115

7.4 Parallel computations in the $n$ D-systems approach . . . . . . 117

7.5 Numerical experiments with JDCOMM software . . . . . . . . 117

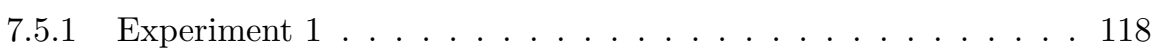

7.5 .2 Experiment $2 \ldots \ldots \ldots \ldots \ldots \ldots$

7.5 .3 Experiment $3 \ldots \ldots \ldots \ldots \ldots$

7.5 .4 Experiment $4 \ldots \ldots \ldots \ldots \ldots \ldots \ldots$ 
III $H_{2}$ Model-order Reduction

$8 H_{2}$ model-order reduction $\quad \mathbf{1 3 5}$

8.1 Introduction . . . . . . . . . . . . . . . . . 135

8.2 The $H_{2}$ model-order reduction problem . . . . . . . . . . . . . 137

8.3 A reparameterization of the model reduction problem . . . . . . . . . 141

8.4 The $H_{2}$-approximation criterion . . . . . . . . . . . . . . . . . . 146

8.5 Stetter-Möller for $H_{2}$ model-order reduction . . . . . . . . . . . . . . . 147

8.6 Computing the approximation $G(s) \ldots \ldots$. . . . . . . . . 149

$9 H_{2}$ Model-order reduction from order $\mathbf{N}$ to $\mathrm{N}-1 \quad 151$

9.1 Solving the system of quadratic equations . . . . . . . . . . . . . 151

9.2 Computing the approximation $G(s) \ldots \ldots \ldots \ldots$

9.3 Example ............................. 154

$10 \mathrm{H}_{2}$ Model-order reduction from order $\mathbf{N}$ to $\mathbf{N}-2 \quad 161$

10.1 Solving the system of quadratic equations . . . . . . . . . . . . 163

10.2 Linearizing a polynomial eigenvalue problem . . . . . . . . . . . 166

10.3 The Kronecker canonical form of a matrix pencil . . . . . . . . . . . . 168

10.3.1 The singular part of a pencil . . . . . . . . . . . . . . . . 172

10.3.2 The regular part of a pencil . . . . . . . . . . . . . . . 177

10.3.3 Deflation of a singular pencil . . . . . . . . . . . . . . . 179

10.4 Computing the approximation $G(s) \ldots \ldots$. . . . . . . 179

10.5 Algorithm for $H_{2}$ model-order reduction for the co-order $k=2$ case . . 180

10.6 Examples . . . . . . . . . . . . . . . . . . 181

10.6.1 Example 1. . . . . . . . . . . . . . . . 182

10.6 .2 Example $2 \ldots \ldots \ldots . \ldots \ldots 18 \ldots$

10.6.3 Example 3..................... 190

$11 \mathrm{H}_{2}$ Model-order reduction from order $\mathbf{N}$ to $\mathrm{N}-3 \quad 195$

11.1 Solving the system of quadratic equations . . . . . . . . . . . . . 196

11.2 Linearizing the two-parameter eigenvalue problem . . . . . . . . . . 197

11.3 Kronecker canonical form of a two-parameter pencil . . . . . . . . . . 198

11.3.1 Transformation into the Kronecker canonical form . . . . . . . 199

11.3.2 Transformation matrices for linear 2-parameter pencil . . . . . 203

11.3.3 Transformation matrices for non-linear 2-parameter pencil . . . 209

11.3.4 A second approach for a non-linear 2-parameter pencil . . . . . 215

11.4 Computing the approximation $G(s) \ldots \ldots$. . . . . . . . . . 221

11.5 Example . . . . . . . . . . . . . . . . . . 222

11.5.1 Model-order reduction from 4 to 1 . . . . . . . . . . . 222

11.5.2 Comparing co-order 3 technique with co-order 1 and 2 . . . . 228

12 Conclusions \& directions for further research $\quad 229$

12.1 Conclusions . . . . . . . . . . . . . . . . . . . . . 229 
12.2 Directions for further research . . . . . . . . . . . . . . . . . 234

$\begin{array}{lr}\text { Bibliography } & 237\end{array}$

A Linearizing a 2-parameter eigenvalue problem $\quad 247$

A.1 Linearization with respect to $\mu \ldots \ldots . . . . . . . . .247$

A.2 Linearization with respect to $\lambda \ldots \ldots \ldots . \ldots . \ldots . \ldots 248$

A.3 Example . . . . . . . . . . . . . . . . . 250

B Polynomial test set $\quad 255$

$\begin{array}{lr}\text { Summary } & 257\end{array}$

$\begin{array}{lr}\text { Samenvatting } & 261\end{array}$

$\begin{array}{ll}\text { Curriculum Vitae } & 265\end{array}$

$\begin{array}{ll}\text { List of Publications } & 267\end{array}$

$\begin{array}{ll}\text { List of Symbols and Abbreviations } & 269\end{array}$

$\begin{array}{ll}\text { List of Figures } & 270\end{array}$

List of Tables $\quad 272$

$\begin{array}{ll}\text { Index } & 273\end{array}$ 


\section{Acknowledgements}

It is a pleasure to finally thank those who made this thesis possible. Without the support of the people mentioned here it would have been impossible to complete this project.

First of all, I want to thank my promotores and supervisors professor Ralf Peeters and professor Bernard Hanzon. Ralf supervised and supported me from the beginning, when he introduced me to computer algebra during my master thesis research, all the way to the final level of this thesis. The same holds for Bernard. He was always very helpful in our research no matter if he worked at the CWI in Amsterdam or at the UCC in Cork (Ireland). He always created a very welcoming atmosphere.

Another word of thanks goes to my colleagues of the Department of Knowledge Engineering and especially to the people I shared a room with in the years 2004-2009, to the students I co-supervised, and of course to Jan Paredis, regarding the e-SAPAS project. It was always fun to work with all of you.

Another word of thanks for another colleague, Dr. Michiel Hochstenbach, from the Technical University Eindhoven. You were always very enthusiastic about our research, however I wish we had spent more time together.

A very special word of thanks goes to my friends, without giving any particular names. You know I feel endless love for all of you and this helped me through the good times, but also through the bad times I experienced during my $\mathrm{PhD}$ period.

Last but not least, I offer my regards and blessings to Jennifer, the love of my life and my best friend ever, and also to my parents, parents-in-law, grand-parents, and family. I am proud of you all.

Ivo Bleylevens

Maastricht, December 2010

(ivo.bleylevens@gmail.com) 



\section{Chapter 1}

\section{Introduction}

\subsection{Motivation}

Solving systems of polynomial equations in several unknowns is a classical problem in mathematics and it has many applications in various sciences. Often one needs a particular solution of the system of equations, e.g., the solution that optimizes a criterion of interest. The problem of computing the solutions of a system of polynomial equations can be approached in various ways, such as for example algebraic techniques based on Gröbner basis computations. When the system of equations admits a finite number of solutions, the problem can be cast into the form of a large eigenvalue problem. This can be done by using the algebraic approach called the Stetter-Möller matrix method [48][50][81].

The problem of solving systems of polynomial equations together with the algebraic methods to solve them, are of great interest here because of the fact that in systems and control theory and in mathematical modeling there are plenty of optimization, approximation, estimation and identifiability questions involving problems of this kind. Moreover, they have our attention due to cross fertilization of the involved ideas because the techniques used to solve the polynomial systems of equations have again a system-theoretic interpretation.

The Stetter-Möller matrix method forms the starting point for the development of computational procedures for the two main applications addressed in this thesis:

- The global optimization of a multivariate polynomial, and

- the $H_{2}$ model-order reduction problem. 


\subsection{Approach}

Finding the global minimum of a real-valued multivariate polynomial is a problem which has many useful applications. To guarantee a finite number of solutions to the system of first-order conditions of the polynomial under consideration, we focus on Minkowski dominated polynomials. The system of first-order conditions of a Minkowski dominated polynomial is directly in Gröbner basis form with a known basis for the quotient space. However, in [63] and [64] it is shown that we could also start from general polynomials or rational functions.

The Stetter-Möller matrix method reformulates the problem of finding the global minimum of a Minkowski dominated polynomial as an eigenvalue problem. Actually, it yields a set of large and sparse commuting matrices. To study and improve the efficiency of the Stetter-Möller matrix method, it is worked out how the matrices that show up in this method admit an interpretation in terms of $n \mathrm{D}$-systems. Such an $n \mathrm{D}$-system is used to compute the action of a matrix more efficiently using recursions. In this way it is possible to avoid the explicit construction of these matrices.

The eigenvalues of these matrices determine the solutions of the system of firstorder conditions. To compute the global minimum, not all these eigenvalues and eigenvectors are required, but we focus on a few selected solutions of the system of first-order conditions. To achieve the computation of only a selected set of eigenvalues, we use iterative eigenvalue solvers. This yields many possibilities to improve the efficiency of the approach, and some of them have been investigated. First, the $n \mathrm{D}$ systems approach is incorporated in the Jacobi-Davidson eigenvalue solver, yielding a matrix-free solver, and the efficient computation within the $n \mathrm{D}$-system setting is investigated. Second, the Jacobi-Davidson method is tuned in various ways, such as changing the parameter settings, implementing a projection method that projects an approximate eigenvector to a vector with Stetter structure and changing the operators during the iteration process. In particular this last modification has lead to the development of the JDCOMM iterative eigenvalue solver which has the following properties: (i) it computes the eigenvalues of a matrix while iterating with a much sparser matrix which results in a speed up in computation time and a decrease in required floating point operations, and (ii) it targets on the smallest real eigenvalue of the matrix first. Moreover, this solver is compatible with the matrix-free $n \mathrm{D}$-system approach. Finally, all the methods mentioned here have been tested on a set of polynomials, varying in degree and number of variables. It turns out that in some cases the approach described here has a superior performance in terms of time and accuracy over other more conventional methods.

Preliminary and partial results described in Part II of this thesis have been communicated in [13], [14], [15], [16], [17], [18], [20], [53], and [88].

The same techniques are applied to address the $\mathrm{H}_{2}$ model-order reduction problem of a system of order $N$ to a reduced system of order $N-k$. The case where the order of the given system of order $N$ is reduced to order $N-k$ is called the co-order $k$ case. 
The goal is to find the globally optimal approximation of order $N-k$. This problem is approached as a rational optimization problem and can therefore be reformulated as a problem of finding solutions of a quadratic system of polynomial equations. This approach is introduced in [50] for the co-order $k=1$ case but is generalized and extended here which results in a joint framework in which to study the $\mathrm{H}_{2}$ modelorder reduction problem for various co-orders $k \geq 1$. In the more general case of $k \geq 1$ the system of quadratic equations contains $k-1$ additional parameters and its solutions should satisfy $k-1$ additional linear constraints.

In the co-order $k=1$ case, the system of equations is in Gröbner basis form and admits a finite number of solutions. This allows the direct application of the previous techniques such that the Stetter-Möller matrix method can directly be used to solve the system of equations. In this way the efficiency of the method in [50] is improved because we do not compute all the eigenvalues of the involved matrix but we use an iterative eigenvalue solver in a matrix-free fashion to focus on the smallest real eigenvalue only. This makes it possible to solve larger problem instances for the co-order $k=1$ case.

For $k=2$, the reformulation of the problem yields a system of quadratic equations in one additional parameter. Applying the Stetter-Möller matrix method to the linear constraint using this system of equations yields a rational matrix in the one parameter. A result of our research is that this rational problem can be made polynomial with a total degree of $N-1$ of the parameter. Thus, this yields a polynomial eigenvalue problem. By linearizing this polynomial eigenvalue problem it becomes equivalent with a larger and singular generalized eigenvalue problem. To reliably compute the eigenvalues of this singular eigenvalue problem, its singular parts and the infinite eigenvalues are removed by using the transformations of the Kronecker canonical form computation.

For the co-order $k=3$ case, the same approach yields a structured polynomial eigenvalue problem in two parameters involving two matrices and one common eigenvector. By using a generalization of the Kronecker canonical form techniques, used in the co-order $k=2$ case, this problem is cast by an algebraic transformation into a one-parameter eigenvalue problem. The solutions of this one-parameter eigenvalue problem determine the approximations of order $N-3$.

For all the co-order $k=1, k=2$, and $k=3$ cases worked examples are presented and their performances or compared with each other.

Preliminary and partial results described in Part III of this thesis have been communicated in [15], [18], [19], [21] and [22].

\subsection{Research questions}

Studying the two main applications of this thesis corresponds to the following research questions which represent the main objectives of this thesis.

1. How to improve the efficiency of the Stetter-Möller matrix method, applied to the global optimization of a multivariate polynomial, when a special basis for 
the quotient space is available, by means of an $n \mathrm{D}$-systems approach?

To answer this question we need to address the following subquestions:

a) How to perform efficient computations for $n \mathrm{D}$-systems?

b) How to exploit the Stetter structure of the involved eigenvectors?

c) How to tune the iterative eigenvalue solvers to gain efficiency?

2. How to find the global optimum to the $H_{2}$ model-order reduction problem for a reduction of order $N$ to order $N-k$, using the techniques of the Stetter-Möller matrix method in combination with an $n \mathrm{D}$-system and an iterative eigenvalue solver?

The corresponding subquestions are:

a) How to improve the performance for co-order $k=1$ reduction?

b) How to develop new techniques or extensions for co-order $k=2$ reduction and how to deal with the singular matrix pencils that occurs in this case?

c) How to develop new techniques or extensions for co-order $k=3$ reduction and how to deal with the structured and singular two-parameter polynomial eigenvalue problem that occurs in this case?

\subsection{Thesis outline}

For readers unfamiliar with the algebraic background a brief introduction into the most important concepts and definitions of algebraic geometry is given in Part I, Chapter 2 of this thesis. Chapter 3 provides an overview of various methods which are used to solve univariate and multivariate systems of polynomial equations.

The development and the efficiency improvements of the global polynomial optimization method based on the Stetter-Möller matrix method are described in Part II of this thesis.

In Chapter 4 we introduce the global optimization of a multivariate polynomial and, in particular, the optimization of a Minkowski dominated polynomial. Subsequently, we explain the techniques behind the Stetter-Möller matrix method.

In Chapter 5 the efficiency of the Stetter-Möller matrix method is improved by associating the system of first-order conditions with an $n \mathrm{D}$-system. The usage of the $n \mathrm{D}$-system is improved by setting up a corresponding shortest path problem as in Section 5.3.2 and by implementing some heuristic procedures as in Section 5.3.3. In Section 5.3.4 we try to improve the efficiency of the method by applying parallel computing techniques.

The first section of Chapter 6 describes the main implementation aspects of Jacobi-Davidson eigenvalue solvers including the pseudocode. Such a Jacobi-Davidson method is able to focus on some specific eigenvalues of the involved matrix and, therefore, in Section 6.3, a selection criterion is developed and embedded into the 
Jacobi-Davidson method such that only the smallest real eigenvalues are computed. In Section 6.4 we try to increase the speed of convergence of the Jacobi-Davidson methods by projecting approximate eigenvectors to close-by Stetter vectors. The development of a new Jacobi-Davidson eigenvalue solver for commuting matrices, the JDCOMM method, is described in Section 6.5.

Chapter 7 provides several worked examples of the techniques mentioned in Part II of this thesis.

In Part III of this thesis the techniques developed in Part II are applied to the problem of computing the $H_{2}$ globally optimal approximation along similar lines as in $[50]$.

In Chapter 8 the generalization of the approach of [50] is shown which results in the joint framework for various co-orders $k \geq 1$. The system of quadratic equations containing $k-1$ additional parameters, as a result of the reparametrization of the $H_{2}$ optimization problem, is described in Section 8.3. In Section 8.4 the $H_{2}$ criterion function for the general co-order $k$ case is worked out. In Section 8.5 the Stetter-Möller matrix method is applied using the system of quadratic equations which rewrites the $H_{2}$ problem into eigenvalue problems of different kind.

In Chapter 9 we show for the co-order $k=1$ case how the conventional eigenvalue problem can be solved efficiently in a matrix-free fashion by applying the $n \mathrm{D}$-systems approach, described in Chapter 5, in combination with an iterative solver as described in Chapter 6. In Section 9.2 we compute the globally optimal approximation of order $N-1$.

In the first section of Chapter 10 we describe how the Stetter-Möller matrix method, in the co-order $k=2$ case, transforms the problem into an eigenvalue problem involving a rational matrix in one unknown which can be made polynomial. To compute its eigenvalues a linearization method is used in Section 10.2 which yields a singular generalized eigenvalue problem. To reliably compute the eigenvalues of this singular matrix pencil, the Kronecker canonical form is computed in Section 10.3 to split off the parts of the matrix which cause the singularity. In this way all the solutions of the system of equations are obtained, and thus, the globally optimal approximation of order $N-2$ can be obtained as described in the Sections 10.4 and 10.5.

In Chapter 11 we describe the $H_{2}$ model-order reduction technique for the coorder $k=3$ case. Applying the Stetter-Möller matrix method to the two linear constraints using the system of equations parameterized by two parameters, yields a two-parameter polynomial eigenvalue problem involving two matrices and one common eigenvector, as described in Section 11.1. In Section 11.2 both these matrices are joined together in one rectangular and singular matrix in two parameters. In Section 11.3 three methods are given, based on the Kronecker canonical form techniques of Chapter 10, to split off singular parts of the involved singular matrix pencil, which make it possible to compute the solutions of the system of equations. Finally in Section 11.4, the globally optimal approximation of order $N-3$ is computed as the 
approximation that yields the smallest real value of the $H_{2}$ criterion.

Chapter 12 provides concluding remarks and directions for further research regarding the results described in the Parts II and III of this thesis. 


\section{Part I}

\section{General introduction and}

background 



\section{Chapter 2}

\section{Algebraic Background}

This chapter provides the reader with a basic introduction to rings, ideals, monomial orderings, varieties, and Gröbner bases; it is based on the first chapters of [28] and [51]. The purpose is to provide the required background for the next chapters of this thesis. The reader interested in a complete presentation of the theory is referred to [1], [10], [23], and [28].

Polynomial ideals are very useful for the expression and solution of many mathematical problems: ideals can be used to decide whether a system of equations is satisfiable, to eliminate variables, to solve equations, and so on. In solving these problems varieties play an important role. Varieties are sets consisting of the common zeros of polynomial ideals. This shows the intimate relation between ideals and varieties. In the 1960's Buchberger developed an algorithm to compute the Gröbner basis for an ideal of polynomials with respect to a given monomial ordering. It turned out to be a very useful tool for various mathematical questions. Gröbner bases are, among many other applications, used to prove that a polynomial is a member of an ideal, that a set of polynomials in an ideal have common zeros, that variables can be eliminated from an ideal and that there is a specified number of common zeros in an ideal (if the ideal has finitely many solutions).

\subsection{Rings and Ideals}

A set $\mathbb{K}$ with two binary operations ' + ' and ' ' is called a ring if the following conditions are satisfied:

(i) $\mathbb{K}$ is a commutative group with respect to addition. This means that for all $u, v, w \in \mathbb{K}$ it holds that: $u+(v+w)=(u+v)+w$ (the associative law), $u+v=v+u$ (the commutative law), there exists a neutral element $0 \in \mathbb{K}$ such that for all $u \in \mathbb{K}$ holds: $u+0=u$ and $0+u=u$ (the additive identity) and for all $u \in \mathbb{K}$ there exists an inverse with respect to addition, denoted by $-u$, such that $u+(-u)=0$ and $(-u)+u=0$ (the additive inverse). 
(ii) Over $\mathbb{K}$ multiplication is associative and there exists a neutral element $1 \in \mathbb{K}$ such that for all $u, v, w \in \mathbb{K}$ it holds that $u(v w)=(u v) w$ and, moreover, that $1 \cdot u=u$ and $u \cdot 1=u$ hold.

(iii) Over $\mathbb{K}$ the distributive laws, $u(v+w)=(u v)+(u w)$ and $(u+v) w=(u w)+(v w)$, apply.

A ring is called a field if the additional property holds that multiplication is a commutative group on $\mathbb{K} \backslash\{0\}$. Then the following two conditions should hold:

(i) $u v=v u$ (the commutative law)

(ii) for all $u \in \mathbb{K}, u \neq 0$, there exists an inverse with respect to multiplication, denoted by $u^{-1} \in \mathbb{K}$, such that: $u u^{-1}=1$ and $u^{-1} u=1$.

Example 2.1. An example of a field is the class $\mathbb{R}$ or $\mathbb{C}$ but not $\mathbb{Z}$ since division fails. The class of square matrices of a given size is a ring and not a field because not every non-zero matrix has an inverse.

In this thesis only polynomial rings over $\mathbb{R}$ and $\mathbb{C}$ are considered $(\mathbb{K}=\mathbb{R}, \mathbb{K}=\mathbb{C}$ or $\mathbb{K}=\mathbb{C}\left(p_{1}, \ldots, p_{r}\right)$, which is the polynomial ring over the complex rational functions in $\left.p_{1}, \ldots, p_{r}\right)$. The polynomial ring in the variables $X=\left\{x_{1}, \ldots, x_{n}\right\}$ over a ring $\mathbb{K}$ is denoted by $\mathbb{K}[X]$ or $\mathbb{K}\left[x_{1}, \ldots, x_{n}\right]$. This ring contains all the polynomials, including the zero and one polynomial, with coefficients in $\mathbb{K}$ and whose terms are power products of $x_{1}, \ldots, x_{n}$.

Definition 2.1. (Ideal). Let $\mathbb{K}[X]$ be a polynomial ring in the variables $X=x_{1}, \ldots$, $x_{n}$. A subring $I$ of $\mathbb{K}[X]$ is called an ideal of $\mathbb{K}[X]$ if

(i) $0 \in I$,

(ii) if $f, g \in I$, then $f+g \in I$,

(iii) if $f \in I$, then $\forall h \in \mathbb{K}[X]: h f \in I$.

Definition 2.2. (Generating system of ideal). Let I be an ideal of a polynomial ring $\mathbb{K}[X]$. A subset $F=\left\{f_{i}\right\}$ of $I$ is called a generating system of $I$ if every member $i \in I$ can be written as a sum of the form:

$$
i=\sum_{f_{i} \in F} h_{i} f_{i},
$$

where $h_{i} \in \mathbb{K}[X]$.

An ideal is said to be generated by $F \subseteq \mathbb{K}[X]$ if $F$ is a generating system of $I$. The ideal generated by $F$ will be denoted by $\langle F\rangle$. The ideal generated by $f_{1}, \ldots, f_{m}$ will be denoted by $\left\langle f_{1}, \ldots, f_{m}\right\rangle$. The elements of the generating system, the polynomials $f_{i}$, are called generators. An ideal generated by only one element is called a principal ideal.

Definition 2.3. If an ideal I has finitely many generators, $f_{1}, \ldots, f_{m}$, it is said to be finitely generated and $F=\left\{f_{1}, \ldots, f_{m}\right\}$ is called a basis of $I$.

Actually, the Hilbert Basis Theorem states that: 
Theorem 2.4. (Hilbert Basis Theorem). Every ideal in $\mathbb{K}\left[x_{1}, \ldots, x_{n}\right]$ is finitely generated.

Note that a given ideal may have many different bases. A particular kind of basis is a Gröbner bases, which has a number of useful additional properties as is shown in Section 2.4.

Definition 2.5. (Minimal basis). A basis $F$ of an ideal $I$ is said to be minimal if there exists no proper subset of $F$ which also is a basis of $I$.

Example 2.2. $\left\{x^{2}, x^{4}\right\}$ is not a minimal basis, since $x^{2}$ generates $x^{4}$.

A property of an ideal is that it can be added to another ideal. This results, again, in an ideal: the sum of ideal $I$ and $J$ is the set that contains all the sums of elements of the ideals $I$ and $J$. It is denoted by $I+J$ and is given by:

$$
I+J=\{i+j:(i, j) \in I \times J\}
$$

where $\times$ is the Cartesian product.

\subsection{Varieties}

An affine algebraic variety is a set which has the property that it is the smallest set containing all the common zeros of a subset of polynomials from a polynomial ring. Let $X=\left\{x_{1}, \ldots, x_{n}\right\}$ be a non-empty set of variables, and let $S \subseteq \mathbb{K}[X]$. The variety of $S$ is the set where each of the polynomials in $S$ becomes zero.

Definition 2.6. (Affine algebraic variety). Let $S=\left\{f_{1}, \ldots f_{m}\right\}$ be polynomials in $\mathbb{K}[X]$. The affine variety of $S$ in $\mathbb{K}^{n}$ is denoted by $V(S)$ and is defined as:

$$
V(S)=\left\{\left(a_{1}, \ldots, a_{n}\right) \in \mathbb{K}^{n}: f_{i}\left(a_{1}, \ldots, a_{n}\right)=0 \text { for all } 1 \leq i \leq m\right\}
$$

Example 2.3. Let $\mathbb{K}=\mathbb{R}$ and $X=\left\{x_{1}, x_{2}\right\}$, let $S=\left\{f_{1}, f_{2}\right\}$ with $f_{1}=3 x_{1}+x_{2}+5$, and $f_{2}=x_{1}+x_{2}-1$. The intersection of the lines $f_{1}=0$ and $f_{2}=0$ in the two-dimensional real space is in one-to-one correspondence with the set of common zeros: the variety in $\mathbb{R}^{2}$ of the ideal $I$ of $\mathbb{R}\left[x_{1}, x_{2}\right]$ which is generated by $\left\langle f_{1}, f_{2}\right\rangle$. The following demonstrates how to find this intersection by manipulating with the 
polynomials $f_{1}$ and $f_{2}$ :

$$
\begin{aligned}
V(I) & =V\left(\left\langle f_{1}, f_{2}\right\rangle\right) \\
& =V\left(\left\langle 3 x_{1}+x_{2}+5, x_{1}+x_{2}-1\right\rangle\right) \\
& =V\left(\left\langle 3 x_{1}+x_{2}+5-\left(x_{1}+x_{2}-1\right), x_{1}+x_{2}-1\right\rangle\right) \\
& =V\left(\left\langle 2 x_{1}+6, x_{1}+x_{2}-1\right\rangle\right) \\
& =V\left(\left\langle x_{1}+3, x_{1}+x_{2}-1\right\rangle\right) \\
& =V\left(\left\langle x_{1}+3, x_{1}+x_{2}-1-\left(x_{1}+3\right)\right\rangle\right) \\
& =V\left(\left\langle x_{1}+3, x_{2}-4\right\rangle\right) \\
& =\{(-3,4)\}
\end{aligned}
$$

Of course, finding the variety of an ideal generated by polynomials $f_{1}, \ldots, f_{m}$, which are all linear in $x_{1}, \ldots, x_{n}$, is achieved by Gaussian elimination. When the polynomials are non-linear in $x_{1}, \ldots, x_{n}$, Gröbner basis theory provides a systematic approach to deal with such (much more complicated) situations.

Conversely, starting from a variety we can also introduce a vanishing ideal. A vanishing ideal is the ideal of a variety $V$ that contains all the polynomials that vanish on $V$. The vanishing ideal of the variety $V$ is denoted by $I(V)$.

Definition 2.7. (Vanishing ideal). Let $V$ be an affine variety in $\mathbb{K}^{n}$. Then:

$$
I(V)=\left\{f \in \mathbb{K}[X]: f\left(a_{1}, \ldots, a_{n}\right)=0 \text { for all }\left(a_{1}, \ldots, a_{n}\right) \in V\right\}
$$

is the vanishing ideal of $V$.

Example 2.4. Suppose that in $\mathbb{K}^{2}$ the variety is $V=\{(0,0)\}$, then $I(V)$ is the ideal generated by $x_{1}, x_{2}:\left\langle x_{1}, x_{2}\right\rangle$. In fact, any polynomial of the form $p\left(x_{1}, x_{2}\right) x_{1}+$ $q\left(x_{1}, x_{2}\right) x_{2}$ vanishes at $(0,0)$.

A zero-dimensional ideal is an ideal of which the cardinality of the corresponding variety is finite. In other words: an ideal $I$ generated by some polynomials $\left\{f_{1}, \ldots, f_{m}\right\}$ with a finite number of solutions is called a zero-dimensional ideal $I$.

\subsection{Monomial orderings}

In the next sections we will define what a Gröbner basis is and what its precise relation with the previous sections is. The notion of monomial orderings is important in this theory and will be treated in this section.

A monomial ordering is to a Gröbner basis what a variable ordering is to Gaussian Elimination. A monomial ordering is a generalization in such a way that it not only orders variables (linear terms) but also orders non-linear terms. 
Let $X=\left\{x_{1}, \ldots, x_{n}\right\}$ be a set of variables. The set containing all power products of $x_{1}, \ldots, x_{n}$ is denoted by $\mathbb{T}_{X}$ :

$$
\mathbb{T}_{X}=\left\{x^{\alpha}\right\}=\left\{x_{1}^{\alpha_{1}} \cdots x_{n}^{\alpha_{n}}:\left(\alpha_{1}, \ldots, \alpha_{n}\right) \in \mathbb{N}_{0}^{n}\right\}
$$

Since a polynomial is a linear combination of monomials, we would often like to arrange the terms in a polynomial in an unambiguous way in descending (or ascending) order. So we must be able to compare monomials to each other to establish their proper relative positions by use of monomial orderings.

Every $x^{\alpha} \in \mathbb{T}_{X}$ is called a monomial and the total degree of a monomial is defined as $|\alpha|=\alpha_{1}+\ldots+\alpha_{n}$. The most significant term of a non-zero polynomial $p$ with respect to a monomial ordering $\succ$ is called the leading term $L T_{\succ}(p)$ of that polynomial with respect to the chosen ordering. A leading term $L T_{\succ}(p)$ of some polynomial $p$ consists of a leading coefficient $L C_{\succ}(p)$ and the corresponding leading monomial $L M_{\succ}(p)$ such that $L T_{\succ}(p)=L C_{\succ}(p) L M_{\succ}(p)$.

Definition 2.8. (Monomial ordering). Let $X$ be a non-empty set of variables. A monomial ordering $\succ$ on $\mathbb{T}_{X}$ is a total ordering with the additional property that $u \succ 1$ for all $u \in \mathbb{T}_{X}$ and that if $u \succ v$ then $t u \succ t v$ for all $u, v, t \in \mathbb{T}_{X}$.

There are several monomial orderings of which we will explicitly review three: the lexicographic ordering, the graded lex ordering and the graded reverse lex ordering.

Definition 2.9. (Lexicographic Ordering). Let $X$ be a set of $n$ variables $x_{1}, \ldots, x_{n}$ and let $\alpha=\left(\alpha_{1}, \ldots, \alpha_{n}\right)$ and $\beta=\left(\beta_{1}, \ldots, \beta_{n}\right) \in \mathbb{N}_{0}^{n}$. Then $\alpha \succ_{\text {lex }} \beta$ is a monomial ordering on $\mathbb{T}_{X}$ if in the vector difference $\alpha-\beta$ the left-most non-zero entry is positive. We write $x^{\alpha} \succ_{\text {lex }} x^{\beta}$ if $\alpha \succ_{\text {lex }} \beta$. The lexicographic ordering is often abbreviated as lex ordering.

Example 2.5. $(3,2,4) \succ_{\text {lex }}(2,5,3)$ since the left-most entry in $(3,2,4)-(2,5,3)=$ $(1,-3,1)$ is positive. Hence, it holds that $x_{1}^{3} x_{2}^{2} x_{3}^{4} \succ_{\text {lex }} x_{1}^{2} x_{2}^{5} x_{3}^{3}$.

Definition 2.10. (Graded Lex Ordering). Let $X$ be a set of $n$ variables $x_{1}, \ldots, x_{n}$ and let $\alpha=\left(\alpha_{1}, \ldots, \alpha_{n}\right)$ and $\beta=\left(\beta_{1}, \ldots, \beta_{n}\right) \in \mathbb{N}_{0}^{n}$. Then $\alpha \succ_{\text {grlex }} \beta$ is a monomial ordering on $\mathbb{T}_{X}$ if $|\alpha|=\sum_{i=1}^{n} \alpha_{i}>|\beta|=\sum_{i=1}^{n} \beta_{i}$, or $|\alpha|=|\beta|$ and $\alpha \succ_{\text {lex }} \beta$. The graded lex ordering is often abbreviated as grlex ordering.

Example 2.6. $(4,3,4) \succ_{\text {grlex }}(3,5,3)$ since $|(4,3,4)|=|(3,5,3)|=11$ and $(4,3,4)$ $\succ_{\text {lex }}(3,5,3)$. This holds since the left-most entry in $(4,3,4)-(3,5,3)=(1,-2,1)$ is positive. Hence, it holds that $x_{1}^{4} x_{2}^{3} x_{3}^{4} \succ_{\text {grlex }} x_{1}^{3} x_{2}^{5} x_{3}^{3}$.

In the literature the graded lex ordering is also called the total degree ordering.

Definition 2.11. (Graded Reverse Lex Ordering). Let $X$ be a set of $n$ variables $x_{1}, \ldots, x_{n}$ and let $\alpha=\left(\alpha_{1}, \ldots, \alpha_{n}\right)$ and $\beta=\left(\beta_{1}, \ldots, \beta_{n}\right) \in \mathbb{N}_{0}^{n}$. Then $\alpha \succ_{\text {grevlex }} \beta$ is a monomial ordering on $\mathbb{T}_{X}$ if $|\alpha|=\sum_{i=1}^{n} \alpha_{i}>|\beta|=\sum_{i=1}^{n} \beta_{i}$, or $|\alpha|=|\beta|$ and the right-most non-zero entry in $\alpha-\beta \in \mathbb{Z}^{n}$ is negative. The graded reverse lex ordering is often abbreviated as grevlex ordering. 
Table 2.1: Monomial orderings of the terms of $f(x, y, z)$

\begin{tabular}{l|ccc} 
& $\begin{array}{c}\text { Lexicographic } \\
\text { Ordering }\end{array}$ & $\begin{array}{c}\text { Graded Lex } \\
\text { Ordering }\end{array}$ & $\begin{array}{c}\text { Graded Reverse Lex } \\
\text { Ordering }\end{array}$ \\
\hline Ordering & $4 x^{4} \succ 3 x^{3} y^{4} z^{4} \succ 2 x^{2} y^{6} z^{3}$ & $3 x^{3} y^{4} z^{4} \succ 2 x^{2} y^{6} z^{3} \succ 4 x^{4}$ & $2 x^{2} y^{6} z^{3} \succ 3 x^{3} y^{4} z^{4} \succ 4 x^{4}$ \\
$L T_{\succ}(p)$ & $4 x^{4}$ & $3 x^{3} y^{4} z^{4}$ & $2 x^{2} y^{6} z^{3}$ \\
$L C_{\succ}(p)$ & 4 & 3 & 2 \\
$L M_{\succ}(p)$ & $x^{4}$ & $x^{3} y^{4} z^{4}$ & $x^{2} y^{6} z^{3}$ \\
\hline
\end{tabular}

Example 2.7. $(3,5,3) \succ_{\text {grevlex }}(3,4,4)$ since $|(3,5,3)|=|(3,4,4)|=11$ and the rightmost non-zero entry in $(3,5,3)-(3,4,4)=(0,1,-1)$ is negative. Hence, it holds that $x_{1}^{3} x_{2}^{5} x_{3}^{3} \succ_{\text {grevlex }} x_{1}^{3} x_{2}^{4} x_{3}^{4}$.

Note that in the previous three examples one could also have used $x, y, z, \ldots$ instead of $x_{1}, x_{2}, x_{3}, \ldots$. We will use this in the next example.

Example 2.8. Let the polynomial $f$ be defined as $f(x, y, z)=2 x^{2} y^{6} z^{3}+3 x^{3} y^{4} z^{4}+4 x^{4}$. The monomial ordering, the leading terms and the corresponding leading monomials and coefficients of polynomial $f$ are shown in Table 2.1 for each of the monomial orderings.

\subsection{Gröbner Basis Theory}

Gröbner Basis Theory, which originates from the 1960's, provides theory and algorithms which generalize the theory of Gaussian Elimination and Euclidean division to the multivariate and non-linear polynomial case. A Gröbner basis is a finite generating system of a polynomial ideal. It has the additional property that the leading terms of the members of this generating system (with respect to a chosen monomial ordering) can be used to characterize the leading terms of the members of the ideal (with respect to the same monomial ordering). This property can be used when one tries to solve a system of polynomial equations.

In order to prepare for the definition of a Gröbner basis given shortly, we first introduce the generalized division algorithm for multivariate polynomials. This algorithm uses the notion of polynomial reduction as stated in the next theorem:

Definition 2.12. (Polynomial reduction). Fix a monomial ordering $\succ$ and let $F$ be a finite generating system of polynomials $\left\{f_{1}, \ldots, f_{m}\right\}$ in $\mathbb{K}[X]$. A polynomial $g \in \mathbb{K}[X]$ is reduced with respect to (or modulo) $F$ if no $L M\left(f_{i}\right)$ divides $L M(g)$ for all $i=1, \ldots, m$.

If some polynomial $g$ is not reduced with respect to $F$, one can subtract a multiple of a member of $F$ from it, to remove its leading monomial. This yields a new polynomial $h$ which again is a member of the ideal $I$ which is generated by the set $\left\{f_{1}, \ldots, f_{m}\right\}$ and whose leading monomial is less than the leading monomial of $g$. This process is called reduction of $g$ with respect to $f$ and is denoted by: $g \longrightarrow_{F} h$.

The definition above only concerns the leading monomials. But one can imagine that there exists a polynomial whose leading monomial is reduced with respect to $F$, 
but which contains (non-leading) terms that can also be reduced with respect to $F$. Therefore we introduce the concept of the complete reduction of a polynomial:

Definition 2.13. (Complete polynomial reduction). Fix a monomial ordering $\succ$ and let $F$ be a finite generating system of polynomials $\left\{f_{1}, \ldots, f_{m}\right\}$ in $\mathbb{K}[X]$. A polynomial $g \in \mathbb{K}[X]$ is completely reduced with respect to (or modulo) $F$ if no monomial of $g$ is divisible by $\operatorname{LM}\left(f_{i}\right)$ for all $i=1, \ldots, m$.

Example 2.9. Let $g=x+y+z^{2}$ and $F=\{x y-1, x z+y\}$ with monomial ordering $\succ_{l e x}$. Then $g$ is completely reduced with respect to $F$ because $x, y$ and $z^{2}$ are not divisible by $L M\left(f_{1}\right)=x y$ or $L M\left(f_{2}\right)=x z$.

Example 2.10. Let $g=x+y^{2}+y$ and $F=\left\{f_{1}\right\}=\{y-1\}$ with monomial ordering $\succ_{l e x}$. Then $g$ can be reduced by subtracting $y$ times $f_{1}$ which gives $x+2 y$. This is still not completely reduced because $2 y$ is still divisible by $L M\left(f_{1}\right)=y$. Subtracting again 2 times $f_{1}$ from this result, gives $x+2$ which is completely reduced with respect to $F$.

This reduction process is finite and the completely reduced polynomial $h$ is called a Normal form of $g$ with respect to $F$. This is denoted by: $g \longrightarrow_{F}^{*} h$.

This reduction process is combined into an algorithm which is called the Generalized Division Algorithm in $\mathbb{K}[X]$ formulated in the next Theorem (based on Theorem 3 of $\S 2.3$ of [28]:

Definition 2.14. (Generalized Division algorithm). Fix a monomial ordering $\succ$ and let $F$ be a finite generating system of polynomials $\left\{f_{1}, \ldots, f_{m}\right\}$ in $\mathbb{K}[X]$. Then every $f \in \mathbb{K}[X]$ can be written as:

$$
f=a_{1} f_{1}+a_{2} f_{2}+\ldots+a_{m} f_{m}+r
$$

where $a_{i}, r \in \mathbb{K}[X]$ and either the remainder of division $r=0$ or $r$ is completely reduced with respect to $F$.

Note that the remainder $r$ is generally not uniquely determined for division with respect to $F$.

Suppose some polynomial $f$ is reduced by $F=\left\{f_{1}, \ldots, f_{m}\right\}$ and the normal form of $f$ turns out to be zero, then: $f=a_{1} f_{1}+\ldots+a_{m} f_{m}$ and therefore $f \in\left\langle f_{1}, \ldots, f_{m}\right\rangle$. But $r=0$ is a sufficient condition for $f$ being a member of $\langle I\rangle$ and not a necessary condition. One can question what might be a good basis $\left\{g_{1}, \ldots, g_{t}\right\}$ of the ideal $\left\langle f_{1}, \ldots, f_{m}\right\rangle$, for which the remainder $r$ on division by the generators $g_{i}$ is uniquely determined and the condition $r=0$ is equivalent to membership in the ideal. It will turn out that a Gröbner basis will be such a basis.

Definition 2.15. Let $I=\left\langle f_{1}, \ldots, f_{m}\right\rangle$ be an ideal in $\mathbb{K}[X]$, then we define for a given ordering $L T(I)=\{L T(f): f \in I\}$. The ideal generated by the elements of $L T(I)$ is denoted by $\langle L T(I)\rangle$.

Note that if $I=\left\langle f_{1}, \ldots, f_{m}\right\rangle$ then $\left\langle L T\left(f_{1}\right), \ldots, L T\left(f_{m}\right)\right\rangle$ and $\langle L T(I)\rangle$ may be different ideals but $\left\langle L T\left(f_{1}\right), \ldots, L T\left(f_{m}\right)\right\rangle \subset\langle L T(I)\rangle$ is true. 
Definition 2.16. Fix a monomial ordering $\succ$. A finite subset $G=\left\{g_{1}, \ldots, g_{m}\right\}$ of an ideal I is said to be a Gröbner basis (or standard basis) if:

$$
\langle L T(I)\rangle=\left\langle L T\left(g_{1}\right), \ldots, L T\left(g_{m}\right)\right\rangle .
$$

This means that a subset $G=\left\{g_{1}, \ldots, g_{m}\right\}$ of an ideal $I$ is a Gröbner basis if and only if the leading term of any element of $I$ is divisible by one of $\operatorname{LT}\left(g_{i}\right)$ for $i=1, \ldots, n$.

Theorem 2.17. Fix a monomial ordering $\succ$. Then every ideal $I=\left\langle f_{1}, \ldots, f_{s}\right\rangle$ in $\mathbb{K}[X]$ other than 0 has a Gröbner basis $G=\left\{g_{1}, \ldots, g_{t}\right\}$. Furthermore, any Gröbner basis of $I$ is a basis of $I: I=\left\langle f_{1}, \ldots, f_{s}\right\rangle=\left\langle g_{1}, \ldots, g_{t}\right\rangle$.

For a proof of this theorem see $\S 2.5$ and Corollary 6 and its proof in [28].

Example 2.11. Let $I=\left\langle f_{1}, f_{2}\right\rangle$ with $f_{1}=x^{2}+2 x y^{2}$ and $f_{2}=x y+2 y^{3}-1$ with a lexicographic ordering $\succ_{l e x}$. The set $\left\{f_{1}, f_{2}\right\}$ is not a Gröbner basis because: $y f_{1}-$ $x f_{2}=x$ and therefore $x \in I$ and $x \in L T(I)$. But $x \notin\left\langle L T\left(f_{1}\right), L T\left(f_{2}\right)\right\rangle$ which is $\left\langle x^{2}, x y\right\rangle$.

Example 2.12. Let $I_{1}=\left\langle f_{1}, f_{2}\right\rangle$ with $f_{1}=x^{2}-2 y^{2}$ and $f_{2}=x y-3$. The monomials are ordered with respect to the lexicographic ordering $\succ_{\text {lex }}$. Then $\left\{g_{1}, g_{2}\right\}=$ $\left\{2 y^{4}-9,3 x-2 y^{3}\right\}$ is a Gröbner basis for $I_{1}$. To demonstrate this we show that (i) $\left\{f_{1}, f_{2}\right\}$ is not a Gröbner Basis for $I_{1}$, (ii) $\left\{g_{1}, g_{2}\right\}$ does satisfy the condition in Definition 2.16 for being a Gröbner Basis for the ideal $\left\langle g_{1}, g_{2}\right\rangle$, and (iii) the ideal $I_{1}=\left\langle f_{1}, f_{2}\right\rangle$ is the same ideal as $I_{2}=\left\langle g_{1}, g_{2}\right\rangle$ generated by $g_{1}$ and $g_{2}$.

(i) The ideal $\left\langle L T\left(f_{1}\right), L T\left(f_{2}\right)\right\rangle$ is equal to $\left\langle x^{2}, x y\right\rangle$. If $f_{1}$ and $f_{2}$ are combined as $y f_{1}-x f_{2}=3 x-2 y^{3}$ then the leading term $L T\left(y f_{1}-x f_{2}\right)$ is $3 x$. Because it holds that $3 x \in\left\langle I_{1}\right\rangle$ and $3 x \notin\left\langle L T\left(f_{1}\right), L T\left(f_{2}\right)\right\rangle$, we can conclude according to Definition 2.16 that $\left\langle f_{1}, f_{2}\right\rangle$ is not a Gröbner Basis.

(ii) The leading terms of $g_{1}$ and $g_{2}$ are $L T\left(g_{1}\right)=2 y^{4}$ and $L T\left(g_{2}\right)=3 x$. The ideal $I_{2}=\left\langle g_{1}, g_{2}\right\rangle$ is given by $I_{2}=\left\{p_{1}\left(2 y^{4}-9\right)+p_{2}\left(3 x-2 y^{3}\right) \mid p_{1}, p_{2} \in \mathbb{R}[x, y]\right\}$. To show that $\left\{g_{1}, g_{2}\right\}$ is a Gröbner basis, we need to make clear that the leading term $\operatorname{LT}(g)$ of every polynomial $g \in I_{2}$ is contained in $\left\langle L T\left(g_{1}\right), L T\left(g_{2}\right)\right\rangle=\left\langle 2 y^{4}, 3 x\right\rangle$.

If $g \in I_{2}$ has any term involving $x$, then $L T(g)$ also involves $x$ and clearly $L T(g) \in$ $\left\langle 2 y^{4}, 3 x\right\rangle$. If terms exist $g \in I_{2}$ not involving $x$, we may search for any pair of polynomials $\left(p_{1}, p_{2}\right)$ for which $g=p_{1}\left(2 y^{4}-9\right)+p_{2}\left(3 x-2 y^{3}\right)$ does not involve $x$ and such that the degree of $p_{1}$ with respect to $x$ is as small as possible.

We may write $p_{1}(x, y)=x r_{1}(x, y)+s_{1}(y)$ and $p_{2}(x, y)=x r_{2}(x, y)+s_{2}(y)$. Then:

$$
\begin{aligned}
g & =\left(x r_{1}+s_{1}\right)\left(2 y^{4}-9\right)+\left(x r_{2}+s_{2}\right)\left(3 x-2 y^{3}\right) \\
& =x\left(r_{1}\left(2 y^{4}-9\right)+r_{2}\left(3 x-2 y^{3}\right)+3 s_{2}\right)+s_{1}\left(2 y^{4}-9\right)-2 s_{2} y^{3}
\end{aligned}
$$


Since $g$ does not involve $x$, it holds that $r_{1}\left(2 y^{4}-9\right)+r_{2}\left(3 x-2 y^{3}\right)=-3 s_{2}$. Thus, $\left(r_{1}, r_{2}\right)$ is a pair of polynomials for which $r_{1}\left(2 y^{4}-9\right)+r_{2}\left(3 x-2 y^{3}\right)$ does not involve $x$, but the degree of $r_{1}$ is strictly less than the degree of $p_{1}$ with respect to $x$, yielding a contradiction unless $r_{1} \equiv 0$. Then it follows that $r_{2} \equiv 0$ and $s_{2} \equiv 0$, so that $p_{2} \equiv 0$ and we have that $g=s_{1}(y)\left(2 y^{4}-9\right)$. Clearly, for $s_{1} \neq \equiv 0$, it follows that $L T(g)=2 y^{4} L T\left(s_{1}\right)$ and $\operatorname{LT}(g) \in L T\left(2 y^{4}, 3 x\right)$.

(iii) To prove that $I_{1}=I_{2}$, consider the polynomial $y f_{1}-x f_{2}=3 x-2 y^{3}=g_{2}$. It holds that $g_{2} \in I_{1}$. Furthermore, $-y^{2} f_{1}+(x y+3) f_{2}=-y g_{2}+3 f_{2}=2 y^{4}-9=g_{1}$ and it holds that $g_{1} \in I_{1}$. Therefore we conclude that $I_{2} \subset I_{1}$.

Conversely, it holds that: $\frac{1}{3} g_{1}+\frac{1}{3} y g_{2}=f_{2}$ and $f_{2} \in I_{2}$, and moreover that $\frac{2}{9} y^{2} g_{1}+\left(\frac{2}{9} y^{3}+\frac{1}{3} x\right) g_{2}=\frac{1}{3} x g_{2}+\frac{2}{3} y^{2} f_{2}=f_{1}$ and $f_{1} \in I_{2}$. From this we conclude that $I_{1} \subset I_{2}$.

Combining $I_{2} \subset I_{1}$ and $I_{1} \subset I_{2}$, yields $I_{1}=I_{2}$ and $\left\{g_{1}, g_{2}\right\}$ is indeed a Gröbner basis for $I_{1}$.

When dividing by the elements of a Gröbner basis, the remainder will always be unique, this in contrast to what holds for an arbitrary basis.

Proposition 2.18. Let $G$ be a Gröbner basis for an ideal $I$ in $\mathbb{K}[X]$ and let $f$ be a polynomial in $\mathbb{K}[X]$. Then there is a unique $r \in \mathbb{K}[X]$ with the following two properties:

(i) $r$ is completely reduced with respect to $G$

(ii) there is $h \in I$ such that $f=h+r$.

In particular, $r$ is the remainder on division of $f$ by $G$ no matter how the elements of $G$ are listed when using the division algorithm.

For a proof see Proposition 1 of $\S 2.6$ of [28].

Proposition 2.18 can be used to determine if a polynomial belongs to an ideal or not; also called the 'ideal membership problem': let $G$ be a Gröbner basis for an ideal $I$ and $f$ some polynomial in $\mathbb{K}[X]$. Polynomial $f$ belongs to ideal $I$ if and only if the remainder $r$ on division of $f$ by $G$ is zero.

\subsection{The Buchberger Algorithm}

The Buchberger algorithm is a method of transforming a given set of generators for a polynomial ideal into a Gröbner basis with respect to some monomial ordering. It was developed by the Austrian mathematician Bruno Buchberger (see [23]). One can view it as a generalization of the Euclidean algorithm for univariate GCD computation and of Gaussian elimination for linear systems.

To transform a set of generators into a Gröbner basis, the S-polynomial is introduced. 
Definition 2.19. Let $f$ and $g$ be two non-zero polynomials in $\mathbb{K}[X]$, then the $S$ polynomial of $f$ and $g$ is defined as:

$$
S(f, g)=\frac{J}{L T(f)} f-\frac{J}{L T(g)} g
$$

where $J=L C M(L M(f), L M(g))$ and where $L C M$ denotes the Least Common Multiple of two monomials. The LCM is defined as follows: if $x^{\alpha}=L M(f)$ and $x^{\beta}=L M(g)$, then $J=L C M\left(x^{\alpha}, x^{\beta}\right)=x^{\gamma}$, with $\gamma_{i}=\max \left(\alpha_{i}, \beta_{i}\right)$ for $i=1, \ldots, n$.

Note that $S(f, g)$ belongs also to any ideal which contains $f$ and $g$.

Example 2.13. Let $f=3 x^{2} y^{3}+x y^{2}-3$ and $g=y^{2}+y-1$ with a lexicographic ordering $\succ_{\text {lex }}$. Then $J=\operatorname{LCM}\left(x^{2} y^{3}, y^{2}\right)=x^{2} y^{3}$ and $S(f, g)=\frac{x^{2} y^{3}}{3 x^{2} y^{3}} f-\frac{x^{2} y^{3}}{y^{2}} g=$ $\left(x^{2} y^{3}+\frac{1}{3} x y^{2}-1\right)-\left(x^{2} y^{3}+x^{2} y^{2}-x^{2} y\right)=-x^{2} y^{2}+x^{2} y+\frac{1}{3} x y^{2}-1$.

A constructive way to test if a set of polynomials is a Gröbner basis for the ideal it generates, is given by Theorem 2.20.

Theorem 2.20. A set $G=\left\{g_{1}, \ldots, g_{m}\right\}$ is a Gröbner basis for the ideal I it generates if and only if $S\left(g_{i}, g_{j}\right) \longrightarrow{ }_{G}^{*} 0$ for all $g_{i}, g_{j} \in G, g_{i} \neq g_{j}$.

For a proof see Theorem 6 in $\S 2.6$ of [28].

Theorem 2.20 motivates a method to transform an arbitrary set of polynomials into a Gröbner basis, which is the main idea behind the Buchberger algorithm: given a set F of polynomials in $\mathbb{K}[X]$ one can test for all $p, q \in F$ if $S(p, q) \longrightarrow_{F}^{*} 0, p \neq q$ holds. If a pair $(p, q)$ with a remainder $r \neq 0$ is found, it is valid to add $r$ to $F$ because it is an element of the same ideal. It is proven that repetition of this operation on $F$ is a finite process and yields a Gröbner basis $G$ for $F$. See again the proof of Theorem 6 in $\$ 2.6$ of [28].

Using this knowledge the Buchberger Algorithm can be defined in pseudocode as follows:

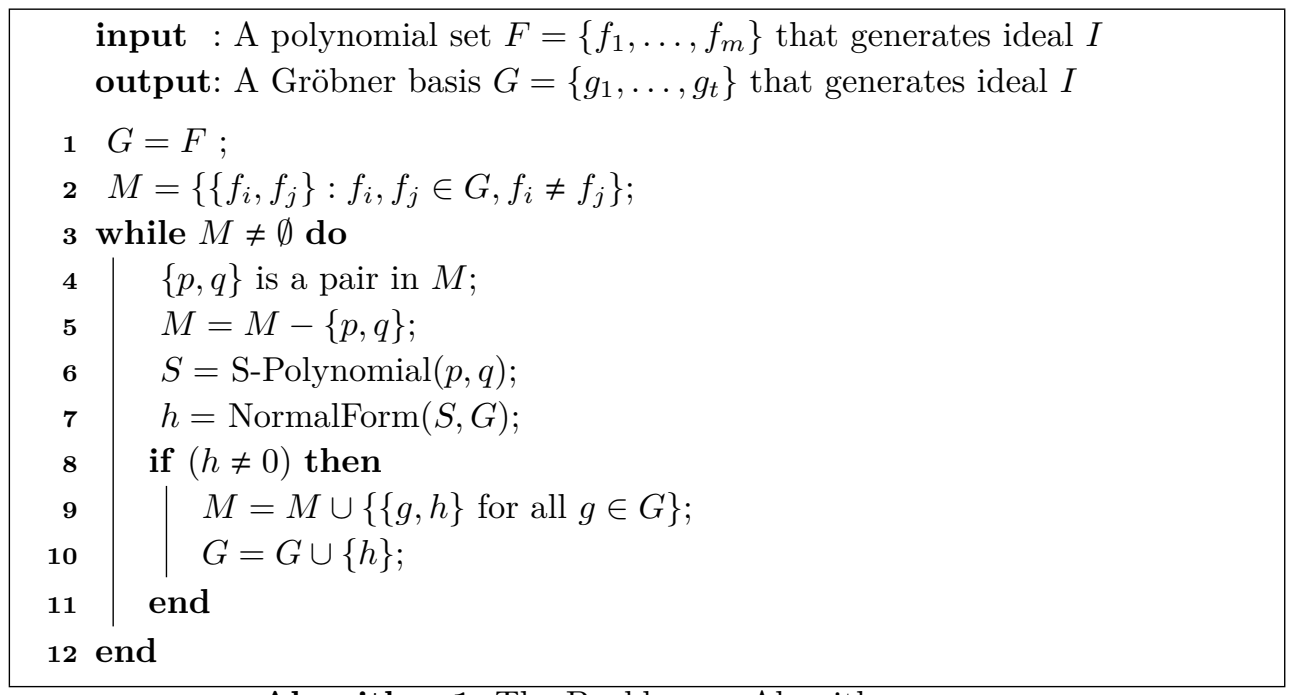

Algorithm 1: The Buchberger Algorithm 
The algorithm above gives a set of generators $G=\left\{g_{1}, \ldots, g_{t}\right\}$ which is a Gröbner basis for the ideal $I$. If there is any member of $G$ whose leading term can be divided by the leading term of any other member of $G$, one can eliminate this member from the basis to get a minimal size of the basis.

Example 2.12 (continued). Suppose we start with the set of polynomials $\left\{f_{1}, f_{2}\right\}=$ $\left\{x^{2}-2 y^{2}, x y-3\right\}$ of Example 2.12 where the monomials are ordered with respect to a lexicographic ordering. In Example 2.12 the Gröbner basis for $I=\left\langle f_{1}, f_{2}\right\rangle$ is given by $\left\{g_{1}, g_{2}\right\}=\left\{2 y^{4}-9,3 x-2 y^{3}\right\}$. We now show step-by-step how to obtain this Gröbner basis for the ideal $I$ using the Buchberger algorithm as shown in Algorithm 1 (each line starts with a number denoting the line of pseudocode in Algorithm 1).

1 The set $G$ is initialized as $G=F=\left\{f_{1}, f_{2}\right\}$.

2 The set $M$ is initialized as $M=\left\{\left\{f_{i}, f_{j}\right\}: f_{i}, f_{j} \in G, f_{i} \neq f_{j}\right\}=\left\{\left\{f_{1}, f_{2}\right\}\right\}$. Note that $\left\{f_{2}, f_{1}\right\}$ need not be included.

3 The set $M$ is not empty: new iteration starts.

4 The pair $\{p, q\}$ can only be chosen as $\left\{f_{1}, f_{2}\right\}$.

5 The set $M$ becomes: $M=M-\left\{f_{1}, f_{2}\right\}=\emptyset$.

6 The S-polynomial of $\left\{f_{1}, f_{2}\right\}$ is computed using $\operatorname{LT}\left(f_{1}\right)=x^{2}, \operatorname{LT}\left(f_{2}\right)=x y$ and $J=L C M\left(L M\left(f_{1}\right), L M\left(f_{2}\right)\right)=L C M\left(x^{2}, x y\right)=x^{2} y$ as: $S=S-\operatorname{polynomial}\left(f_{1}\right.$, $\left.f_{2}\right)=\frac{J}{L T\left(f_{1}\right)} f_{1}-\frac{J}{L T\left(f_{2}\right)} f_{2}=\frac{x^{2} y}{x^{2}} f_{1}-\frac{x^{2} y}{x y} f_{2}=y f_{1}-x f_{2}=3 x-2 y^{3}=g_{2}$.

7 The NormalForm of $g_{2}$ is computed as $h=\operatorname{NormalForm}\left(g_{2}, G\right)=g_{2}=3 x-2 y^{3}$ because:

$3 x$ is not divisible by $\operatorname{LM}\left(f_{1}\right)=x^{2}$ nor divisible by $L M\left(f_{2}\right)=x y$.

$-2 y^{3}$ is not divisible by $L M\left(f_{1}\right)=x^{2}$ nor divisible by $L M\left(f_{2}\right)=x y$.

Therefore the polynomial $g_{2}$ is already in normal form.

8 Because $h=g_{2} \neq 0$, the sets $M$ and $G$ are extended.

9 The set $M$ is extended to $M=\left\{\left\{f_{1}, g_{2}\right\},\left\{f_{2}, g_{2}\right\}\right\}$.

10 The set $G$ becomes $G=\left\{f_{1}, f_{2}, g_{2}\right\}=\left\{x^{2}-2 y^{2}, x y-3,3 x-2 y^{3}\right\}$.

3 The set $M$ is not empty: new iteration starts.

4 The pair $\{p, q\}$ is chosen as $\left\{f_{1}, g_{2}\right\}$.

5 The set $M$ becomes: $M=M-\left\{f_{1}, g_{2}\right\}=\left\{\left\{f_{2}, g_{2}\right\}\right\}$.

6 The S-polynomial of $\left\{f_{1}, g_{2}\right\}$ is computed using $\operatorname{LT}\left(f_{1}\right)=x^{2}, \operatorname{LT}\left(g_{2}\right)=3 x$ and $J=L C M\left(L M\left(f_{1}\right), L M\left(g_{2}\right)\right)=L C M\left(x^{2}, x\right)=x^{2}$ as: $S=S-\operatorname{polynomial}\left(f_{1}\right.$, $\left.g_{2}\right)=\frac{J}{L T\left(f_{1}\right)} f_{1}-\frac{J}{L T\left(g_{2}\right)} g_{2}=\frac{x^{2}}{x^{2}} f_{1}-\frac{x^{2}}{3 x}\left(3 x-2 y^{3}\right)=x^{2}-2 y^{2}-x^{2}+\frac{2}{3} x y^{3}=$ $\frac{2}{3} x y^{3}-2 y^{2}$.

7 The NormalForm of $\frac{2}{3} x y^{3}-2 y^{2}$ is computed as $h=\operatorname{NormalForm}\left(\frac{2}{3} x y^{3}-\right.$ $\left.2 y^{2}, G\right)=\frac{2}{3} x y^{3}-2 y^{2}-\frac{2}{3}(x y-3) y^{2}=0$.

$8 h=0$.

9 Because $h=0$ the set $M$ is not extended.

10 Because $h=0$ the set $G$ is not extended.

3 The set $M$ is not empty: new iteration starts.

4 The pair $\{p, q\}$ is chosen as $\left\{f_{2}, g_{2}\right\}$.

5 The set $M$ becomes: $M=M-\left\{f_{2}, g_{2}\right\}=\emptyset$. 
6 The S-polynomial of $\left\{f_{2}, g_{2}\right\}$ is computed using $\operatorname{LT}\left(f_{2}\right)=x y, \operatorname{LT}\left(g_{2}\right)=3 x$ and $J=L C M\left(L M\left(f_{2}\right), L M\left(g_{2}\right)\right)=L C M(x y, x)=x y$ as: $S=S$-polynomial $\left(f_{2}\right.$, $\left.g_{2}\right)=\frac{J}{L T\left(f_{2}\right)} f_{2}-\frac{J}{L T\left(g_{2}\right)} g_{2}=\frac{x y}{x y} f_{2}-\frac{x y}{3 x} g_{2}=x y-3-\frac{x y}{3 x}\left(3 x-2 y^{3}\right)=\frac{2}{3} y^{4}-3=\frac{1}{3} g_{1}$.

7 The NormalForm of $\frac{1}{3} g_{1}$ is computed as $h=\operatorname{NormalForm}\left(\frac{1}{3} g_{1}, G\right)=\frac{1}{3} g_{1}$ because $\frac{1}{3} g_{1}$ is already in normal form.

8 Because $h=\frac{1}{3} g_{1} \neq 0$, the sets $M$ and $G$ are extended.

9 The set $M$ is extended to $\left\{\left\{f_{1}, \frac{1}{3} g_{1}\right\},\left\{f_{2}, \frac{1}{3} g_{1}\right\},\left\{g_{2}, \frac{1}{3} g_{1}\right\}\right\}$

10 The set $G$ becomes $G=\left\{f_{1}, f_{2}, g_{2}, \frac{1}{3} g_{1}\right\}=\left\{x^{2}-2 y^{2}, x y-3,3 x-2 y^{3}, \frac{2}{3} y^{4}-3\right\}$.

3 The set $M$ is not empty: new iteration starts.

4 Etcetera...

The computation of the Gröbner basis using the Buchberger algorithm is now quickly finished since all the remaining pairs of the set $M=\left\{\left\{f_{1}, \frac{1}{3} g_{1}\right\},\left\{f_{2}, \frac{1}{3} g_{1}\right\}\right.$, $\left.\left\{g_{2}, \frac{1}{3} g_{1}\right\}\right\}$ yield a NormalForm $h$ equal to zero. Therefore the sets $M$ and $G$ are not extended anymore:

- the pair $\left\{f_{1}, \frac{1}{3} g_{1}\right\}$ yields an S-polynomial of $S=y^{4} f_{1}-x^{2} \frac{1}{3} g_{1}=\frac{1}{3} x^{2} y^{4}+3 x^{2}-$ $2 y^{6}$, using $\operatorname{LT}\left(f_{1}\right)=x^{2}, \operatorname{LT}\left(\frac{1}{3} g_{1}\right)=\frac{2}{3} y^{4}$ and $J=L C M\left(x^{2}, y^{4}\right)=x^{2} y^{4}$, and $\mathrm{h}=\operatorname{NormalForm}(S, G)=0$.

- the pair $\left\{f_{2}, \frac{1}{3} g_{1}\right\}$ yields an S-polynomial of $S=y^{3} f_{2}-\frac{1}{2} x g_{1}=4 \frac{1}{2} x-3 y^{3}$, using $\operatorname{LT}\left(f_{2}\right)=x y, \operatorname{LT}\left(\frac{1}{3} g_{1}\right)=\frac{2}{3} y^{4}$ and $J=\operatorname{LCM}\left(x y, y^{4}\right)=x y^{4}$, and $\mathrm{h}=$ $\operatorname{NormalForm}(S, G)=0$.

- the pair $\left\{g_{2}, \frac{1}{3} g_{1}\right\}$ yields an S-polynomial of $S=\frac{1}{3} y^{4} g_{2}-\frac{1}{2} x g_{1}=4 \frac{1}{2} x-\frac{2}{3} y^{7}$, using $\operatorname{LT}\left(g_{2}\right)=3 x, \operatorname{LT}\left(\frac{1}{3} g_{1}\right)=\frac{2}{3} y^{4}$ and $J=\operatorname{LCM}\left(x, y^{4}\right)=x y^{4}$, and $\mathrm{h}=$ $\operatorname{NormalForm}(S, G)=0$.

Thus, we conclude that the set $G=\left\{f_{1}, f_{2}, g_{2}, \frac{1}{3} g_{1}\right\}=\left\{x^{2}-2 y^{2}, x y-3,3 x-2 y^{3}, \frac{2}{3} y^{4}-\right.$ $3\}$ is a Gröbner basis for the ideal $I=\left\langle f_{1}, f_{2}\right\rangle$. Using the algorithm above, the resulting Gröbner Basis $G$ is often larger than necessary. The generators $f_{1}$ and $f_{2}$ can be eliminated from the Gröbner basis $G$ because the leading terms $L T\left(f_{1}\right)=x^{2}$ and $L T\left(f_{2}\right)=x y$ are divisible by the leading term $L T\left(g_{2}\right)=3 x$ of the generator $g_{2}$. Then the set $G^{\prime}=\left\{g_{2}, \frac{1}{3} g_{1}\right\}=\left\{3 x-2 y^{3}, \frac{2}{3} y^{4}-3\right\}$ is still a Gröbner basis for the ideal $I=\left\langle f_{1}, f_{2}\right\rangle$.

If we furthermore require that all the leading coefficients of a Gröbner basis $G=$ $\left\{g_{1}, \ldots, g_{m}\right\}$ for an ideal $I$ are equal to one and all the generators are completely reduced modulo the other generators in $G$, then we get a unique basis which is called a reduced Gröbner basis.

Definition 2.21. (Reduced Gröbner basis). A reduced Gröbner basis of a polynomial ideal $I$ is a Gröbner basis $G$ for the ideal I such that

(i) $L C(p)=1$ for all $p \in G$, 
(ii) each $p \in G$ is completely reduced modulo $G-\{p\}$.

Theorem 2.22. Every polynomial ideal I (not equal to $\{0\}$ ) has a unique reduced Gröbner basis with respect to a monomial ordering.

For a proof see Proposition 6 of $\S 2.7$ of [28].

Example 2.12 (continued). A reduced Gröbner basis for the ideal $I=\left\langle f_{1}, f_{2}\right\rangle=$ $\left\langle x^{2}-2 y^{2}, x y-3\right\rangle$ is $G^{\prime \prime}=\left\{x-\frac{2}{3} y^{3}, y^{4}-\frac{9}{2}\right\}$.

The Buchberger algorithm yields a method to solve the 'ideal equality problem': two ideals $I_{1}$ and $I_{2}$ have the same reduced Gröbner basis if and only if they are equal.

There are many improvements which have been made to this basic Buchberger algorithm in the last decades, i.e., yielding the Refined Buchberger Algorithm or the Faugère F4 Algorithm. Improvements are mostly aimed at avoiding redundancy in the set of generators of the Gröbner basis or involving variations in efficiently selecting a pair $\{p, q\} \in M$. Other improvements are based on predicting a-priori reductions of the S-polynomial which yield a zero solution. These reductions can then be discarded. Despite all the improvements made to the basic Buchberger Algorithm it is still computational very demanding because a Gröbner basis can be very large. In fact, it can be much larger than the initial set of polynomials. For state-of-the-art implementations of the Buchberger algorithm the reader may wish to consult Chapter 5.5 of $[10]$ and [44]. 



\section{Chapter 3}

\section{Solving polynomial systems of equations}

Solving systems of polynomial equations in several unknowns is a classical problem in mathematics. This problem arises across various sciences like for example computer science, engineering, economics, robotics, and also several subfields in mathematics like coding theory, optimization, control theory, machine learning, and many more. The set of solutions of a system of polynomial equations, the variety, is one of the basic objects of geometry. In the last years the computation of varieties of systems of equations has witnessed huge improvements caused by developments in numerical mathematics and symbolic algebra. Next to these developments, also the vast improvements in available powerful computer hardware has made previously untractable problems solvable and has made further developments possible.

Some commercial and non-commercial software packages which can deal with the problem of solving system of polynomial equations and which contain state of the art software for this are Mathematica, Matlab, Maple, Singular [45], Macauly [35], SOSTOOLS [90], and COCOA [71].

In this chapter we first discuss how to solve a polynomial equation in a single variable. The next section describes some families of methods to solve zero-dimensional systems of polynomial equations in more than one variable. Section 3.3 discusses in detail the Stetter-Möller matrix method, an exact algebraic method to solve systems of polynomial equations in Gröbner basis form. This method is used in the next chapters to serve as the basis for the two main applications addressed in this thesis: (i) the global optimization of a multivariate polynomial, and (ii) the $H_{2}$ model-order reduction problem. Section 3.4 describes a method to count real solutions of a system of polynomial equations using the Stetter-Möller matrix method. Finally, Section 3.5 shows an illustrative example of some techniques discussed in this chapter. Note that only zero-dimensional systems are studied here. 
This chapter is largely based on [47], [48], [50], [81], and [98] and own work [13], $[16]$, and [20].

\subsection{Solving polynomial equations in one variable}

For a good understanding of solving systems of polynomial equations, knowledge about solving univariate polynomial equations is required. The basic subject in this section is the computation of the zeros of a polynomial of degree $d$ in the variable $x$ :

$$
f(x)=a_{d} x^{d}+a_{d-1} x^{d-1}+\ldots+a_{1} x+a_{0}
$$

with coefficients $a_{i}$ in the field of real numbers $\mathbb{R}$ and $a_{d} \neq 0$. The variable $x$ ranges over the field of complex numbers $\mathbb{C}$.

The fundamental theorem of algebra [31] states that the polynomial $f(x)$ has $d$ roots in the field $\mathbb{C}$ of complex numbers counting multiplicities. If the degree $d$ of $f(x)$ is smaller than or equal to 4 , there exist explicit formulas for the roots of $f(x)$ in terms of the coefficients $a_{0}, \ldots, a_{d}$, involving square roots and standard arithmetic operations. One of the main results from Galois Theory states that for univariate polynomials with a degree larger than 4 such explicit formulas for the roots in terms of the coefficients do not exist [31].

Numerical root finding can be a challenging task even for the univariate case, especially when the degree $d$ in (3.1) is high, or when the coefficients $a_{i}$ are given in floating point expressions or approximations of these.

Numerical methods for solving the univariate polynomial equation $f(x)=0$ can be based on Newton methods, bisection techniques or sum of squares reformulations but also on reducing the problem to an eigenvalue problem involving the companion matrix $T_{x}$ of the polynomial $f(x)$. In this case the companion matrix $T_{x}$ attains the form:

$$
T_{x}=\left(\begin{array}{ccccc}
0 & 0 & \ldots & 0 & \frac{-a_{0}}{a_{d}} \\
1 & 0 & \ldots & 0 & \frac{-a_{1}}{a_{d}} \\
0 & 1 & \ldots & 0 & \vdots \\
\vdots & \vdots & \ddots & \vdots & \vdots \\
0 & 0 & \ldots & 1 & \frac{-a_{d-1}}{a_{d}}
\end{array}\right) .
$$

Note that there are different versions of companion matrices with the coefficients showing up in the last or first column or the last or first row. The matrix $T_{x}$ is just one possible companion form which applies to this situation, allowing for an interesting algebraic interpretation as will be discussed shortly.

Proposition 3.1. The zeros of $f(x)$, including multiplicities, are the eigenvalues of the matrix $T_{x}$.

Proposition 3.1 is a standard result in linear algebra. For a proof, see e.g., [98]. 
Thus, the zeros of $f(x)$ can be computed by performing a numerical eigenvalue computation on the companion matrix $T_{x}$. A generalized version of this is also used by the Stetter- Möller method in the multivariate case, as will be shown in Section 3.3. Therefore it is important to understand what the matrix $T_{x}$ exactly represents.

Let $V$ denote the quotient of the polynomial ring modulo the ideal $\langle f(x)\rangle$ generated by the univariate polynomial $f(x)$. This quotient ring $V=\mathbb{R}[x] /\langle f(x)\rangle$ is a $d$-dimensional $\mathbb{R}$-vector space. The operation 'multiplication by $x$ ' on the elements of $V$ defines a linear mapping from this vector space to itself:

$$
\mathcal{T}_{x}: V \mapsto V: g \quad \mapsto \quad x \cdot g
$$

The companion matrix $T_{x}$ is the $d \times d$ matrix which represents this linear operator $\mathcal{T}_{x}$ with respect to the monomial basis $\left\{1, x, \ldots, x^{d-1}\right\}$ of $V$.

Example 3.1. The zeros of the polynomial $f(x)=x^{4}+3 x^{3}+2 x^{2}+4 x-1$ are $-2.8360,0.21793$, and $-0.19098 \pm 1.2576 i$. These values coincide with the eigenvalues of the matrix:

$$
T_{x}=\left(\begin{array}{rrrr}
0 & 0 & 0 & 1 \\
1 & 0 & 0 & -4 \\
0 & 1 & 0 & -2 \\
0 & 0 & 1 & -3
\end{array}\right)
$$

To explain the particular structure of the matrix $T_{x}$, consider the following:

Take a polynomial $g(x)=g_{3} x^{3}+g_{2} x^{2}+g_{1} x+g_{0}$. Then $h(x)=x \cdot g(x) /\langle f(x)\rangle=$ $g_{3} x^{4}+g_{2} x^{3}+g_{1} x^{2}+g_{0} x /\langle f(x)\rangle$. In the quotient space $V=\mathbb{R}[x] /\langle f(x)\rangle$, this is equivalent to $h(x)=g_{3} x^{4}+g_{2} x^{3}+g_{1} x^{2}+g_{0} x-\frac{g_{3}}{a_{4}}\left(a_{4} x^{4}+a_{3} x^{3}+a_{2} x^{2}+a_{1} x+a_{0}\right)$ where $a_{0}=-1, a_{1}=4, a_{2}=2, a_{3}=3$, and $a_{4}=1$. This is rewritten as $h_{3} x^{3}+h_{2} x^{2}+h_{1} x+h_{0}$ with

$$
\begin{aligned}
& h_{3}=g_{2}-g_{3} \frac{a_{3}}{a_{4}} \\
& h_{2}=g_{1}-g_{3} \frac{a_{2}}{a_{4}} \\
& h_{1}=g_{0}-g_{3} \frac{a_{1}}{a_{4}} \\
& h_{0}=-g_{3} \frac{a_{0}}{a_{4}}
\end{aligned}
$$

which can also be expressed in matrix-vector form as:

$$
\left(\begin{array}{l}
h_{0} \\
h_{1} \\
h_{2} \\
h_{3}
\end{array}\right)=\left(\begin{array}{cccc}
0 & 0 & 0 & -\frac{a_{0}}{a_{4}} \\
1 & 0 & 0 & -\frac{a_{1}}{a_{4}} \\
0 & 1 & 0 & -\frac{a_{2}}{a_{4}} \\
0 & 0 & 1 & -\frac{a_{3}}{a_{4}}
\end{array}\right)\left(\begin{array}{l}
g_{0} \\
g_{1} \\
g_{2} \\
g_{3}
\end{array}\right) .
$$

The $4 \times 4$ matrix in Equation (3.6) equals the matrix $T_{x}$ in Equation (3.4) for the associated specific choice of values for the coefficients. 
This demonstrates the fact that $T_{x}$ represents the action $V \mapsto V$ given by $g(x) \mapsto$ $x \cdot g(x) /\langle f(x)\rangle$ and that this is indeed a linear operator on a finite dimensional space. Note that this procedure is also valid for $\mathbb{C}[x] /\langle f(x)\rangle$.

\subsection{Methods for solving polynomial systems of equations}

In the previous section a matrix approach to solve one univariate polynomial equation is discussed. In this section the computation of the solutions of a system of real polynomial equations in the multivariate case is addressed.

Suppose a system of multivariate polynomial equations

$$
\left\{\begin{array}{ccc}
f_{1}\left(x_{1}, \ldots, x_{n}\right) & = & 0 \\
\vdots & \vdots & \vdots \\
\vdots & \vdots & \vdots \\
f_{m}\left(x_{1}, \ldots, x_{n}\right) & = & 0
\end{array}\right.
$$

is given, where each $f_{i}\left(x_{1}, \ldots, x_{n}\right), i=1, \ldots, m$, is a possibly real non-linear polynomial in $\mathbb{R}\left[x_{1}, \ldots, x_{n}\right]$. Because we only study zero-dimensional ideals in this thesis, the ideal $I=\left\langle f_{1}, \ldots, f_{m}\right\rangle$, generated by the polynomials $f_{i}\left(x_{1}, \ldots, x_{n}\right), i=1, \ldots, m$, is assumed to have only finitely many zeros in $\mathbb{C}^{n}$. The n-tuple of complex numbers $\left(s_{1}, \ldots, s_{n}\right)$ is a solution of the system if $f_{i}\left(s_{1}, \ldots, s_{n}\right)=0$ for all $i=1, \ldots, m$. In this section some families of methods for solving zero-dimensional polynomial systems of non-linear equations over the field $\mathbb{R}$ are explored [84].

\subsubsection{Resultant methods}

Resultant methods are often called geometric methods because they use the geometric properties of the solutions. Computing resultants is a tool for solving systems of equations and is aimed at eliminating all but one variable from a system with finitely many solutions. The Sylvester resultant method is often used to determine the zeros of two polynomials in one or two variables. It is not advised to use this approach in the multivariate case because repeatedly applying the Sylvester resultant method proves to be inefficient as the elimination of one variable doubles the complexity of the remaining problem [65]. Multivariate resultant methods are much more efficient in this case because they are designed to eliminate any number of variables jointly from a set of polynomials. Some common multivariate resultant methods are the Dixon [67], the Macauly [79], and the sparse resultant method [24].

To eliminate variables in two polynomials with the Sylvester resultant method the Sylvester matrix of both polynomials has to be constructed. When the polynomials are of degree $d_{1}$ and $d_{2}$, the Sylvester matrix is of size $\left(d_{1}+d_{2}\right) \times\left(d_{1}+d_{2}\right)$. The points at which the determinant of this matrix (the Sylvester resultant) vanishes yield the common zeros of both polynomials [70]. 
Example 3.2. Consider the example involving a system of two polynomial equations $f_{1}=0$ and $f_{2}=0$ provided by Lazard in [76]:

$$
\left\{\begin{array}{l}
f_{1}(x, y)=x^{2}+x y+2 x+y-1=0 \\
f_{2}(x, y)=x^{2}+3 x-y^{2}+2 y-1=0
\end{array}\right.
$$

The roots of the Sylvester resultant of $f_{1}(x, y)$ and $f_{2}(x, y)$, treated as polynomials in $y$ with coefficients in $x$, are the $x$-coordinates of the common zeros of $f_{1}(x, y)$ and $f_{2}(x, y)$. The Sylvester matrix in this example has the dimension $\left(d_{1}+d_{2}\right) \times\left(d_{1}+d_{2}\right)=$ $3 \times 3$ and the Sylvester resultant of this system with respect to $y$ is given by the determinant:

$$
\left|\begin{array}{ccc}
x+1 & x^{2}+2 x-1 & 0 \\
0 & x+1 & x^{2}+2 x-1 \\
-1 & 2 & x^{2}+3 x-1
\end{array}\right|=-x^{3}-2 x^{2}+3 x .
$$

The roots of this univariate determinant are $\{-3,0,1\}$. These are the $x$-coordinates of the zeros of $f_{1}(x, y)$ and $f_{2}(x, y)$. The $y$-coordinates can be obtained as $\{1,1,-1\}$, respectively, by substituting the $x$-coordinates into $f_{1}(x, y)$ or $f_{2}(x, y)$ and addressing the resulting univariate expression.

This method can be extended recursively to a larger set of multivariate polynomials using polynomial GCD computations. Unfortunately, eliminating many variables using this method proves to be very inefficient due to the enormous increase in degrees of the involved polynomials in every elimination step (also called the 'expression swell').

The vanishing of a generalization of the Sylvester resultant of two polynomials in a single variable, is used for the multivariate case in Dixon's resultant method. This method controls the intermediate expression swell and makes use of the internal structure of the Sylvester matrix. In this case the dimension of the Sylvester matrix is $\max \left(d_{1}, d_{2}\right) \times \max \left(d_{1}, d_{2}\right)$ where $d_{1}$ and $d_{2}$ are the degrees of the involved polynomials [26], [33].

The Macauly resultant method [79] constructs a resultant for $n$ homogeneous polynomials in $n$ variables, which simultaneously generalizes the Sylvester resultant and the determinant of a system of linear equations [25], [65].

As an improvement on this, the sparse resultant method was developed which acts on sparse polynomial systems where only certain monomials in each of the polynomials have non-zero coefficients [36], [43], [97], [99]. For a detailed overview of all resultant methods one may refer to [66].

\subsubsection{Homotopy methods}

A homotopy method solves a system of polynomial equations by tracking the solutions of a deformed and nearby system of equations. The deformed system is transformed 
iteratively in a finite number of steps into the original system of equations. Each deformed system is solved numerically with a certain accuracy. This procedure provides an insight into the solutions of the original system [3], [83].

Suppose the original system of equations is denoted by $F\left(x_{1}, \ldots, x_{n}\right)$ and a parameter $t$ is introduced where $0 \leq t \leq 1$. Now define a homotopy map as follows:

$$
H\left(x_{1}, \ldots, x_{n}, t\right)=t F\left(x_{1}, \ldots, x_{n}\right)+(1-t) G\left(x_{1}, \ldots, x_{n}\right)
$$

where $G\left(x_{1}, \ldots, x_{n}\right)$ is an easily solvable system of polynomial equations free to choose. By following the paths of the solutions $\left(s_{1}, \ldots, s_{n}\right)$ of $H\left(s_{1}, \ldots, s_{n}, t\right)$ when the parameter $t$ varies from 0 to 1 , the solutions of the original system of equations can be obtained. Note that in the literature there are various ways to define the homotopy map. These are called the global homotopy map or the general homotopy map and they differ in the way how $t$ combines $F$ and $G$ to form $H$. Homotopy methods in use are numerically stable methods and the deformed systems $H$ are commonly solved by iterative numerical methods like Newton methods.

Example 3.3. To illustrate the homotopy approach, an example is presented involving two multivariate polynomials $f_{1}(x, y)$ and $f_{2}(x, y)$. Suppose the original system of equations $F(x, y)$ is defined as:

$$
\begin{cases}f_{1}(x, y)=3 x^{3}-4 x^{2} y-x+y^{2}+2 y^{3} & =0 \\ f_{2}(x, y)=-6 x^{3}+2 x y-5 y^{3}-y & =0\end{cases}
$$

and let $G(x, y)$ be:

$$
\left\{\begin{array}{l}
g_{1}(x, y)=x^{3}-1-y=0 \\
g_{2}(x, y)=y^{3}-8-x=0
\end{array}\right.
$$

Let us define the homotopy map as in (3.10). Then $H(x, y, t)$ is given by:

$$
H(x, y, t)=\left\{\begin{array}{l}
h_{1}(x, y)=t f_{1}(x, y)+(1-t) g_{1}(x, y)=0 \\
h_{2}(x, y)=t f_{2}(x, y)+(1-t) g_{2}(x, y)=0
\end{array}\right.
$$

Note that $H(x, y, 0)=G(x, y)$ holds and that $H(x, y, 1)=F(x, y)$ which is the original system of equations. Now $H(x, y, t)=0$ is solved while $t$ ranges from 0 to 1 in a finite number of steps; i.e., steps of size 0.001 . Solving $H(x, y, t)=0$ is easy for $t=0$ by construction: $y=x^{3}-1$ can be substituted into $g_{2}(x, y)$ yielding a univariate polynomial of order 9 . Bezout's Theorem states that there are at most $3 \times 3$ roots of the system $F(x, y)=0$. Therefore in this homotopy procedure only 9 solution paths have to be followed, which constitute continuous curves in the complex plane. The 9 solutions of system $G(x, y)=0$ are $(-0.2267+1.0743 i, 0.2267-1.0743 i)$, $(-0.2267-1.0743 i-0.2267+1.0743 i),(-0.7052+1.0387 i 0.9319+0.4290 i),(-0.7052-$ $1.0387 i 0.9319-0.4290 i),(0.9319-0.4290 i-0.7052-1.0387 i),(0.9319+0.4290 i$ $-0.7052+1.0387 i),(1.32471 .3247),(-0.6624+0.5623 i-0.6624+0.5623 i)$, and $(-0.6624-0.5623 i-0.6624-0.5623 i)$ and the solutions of system $F(x, y)=0$ are 
$(-0.48620 .2886),(0.4757+0.6118 i-0.2171+0.7286 i),(0.4757-0.6118 i-0.2171-$ $0.7286 i),(0.3650-0.3418),(-0.07632+0.1989 i-0.05853-0.4920 i),(-0.07632-$ $0.1989 i-0.05853+0.4920 i),(0.04140+0.4376 i 0.3593+0.3717 i),(0.04140-0.4376 i$ $0.3593-0.3717 i)$, and $(0,0)$. Figures $3.1 \mathrm{a}$ and $3.1 \mathrm{~b}$ show, respectively, the $x-$ and $y$-values of the 9 solution paths of $H(x, y, t)=0$ where the value of $t$ increases from 0 to 1 with steps of 0.001 . The 9 solutions of system $G(x, y)=0$ are denoted by green squares and the 9 solutions of system $F(x, y)=0$ by red squares.

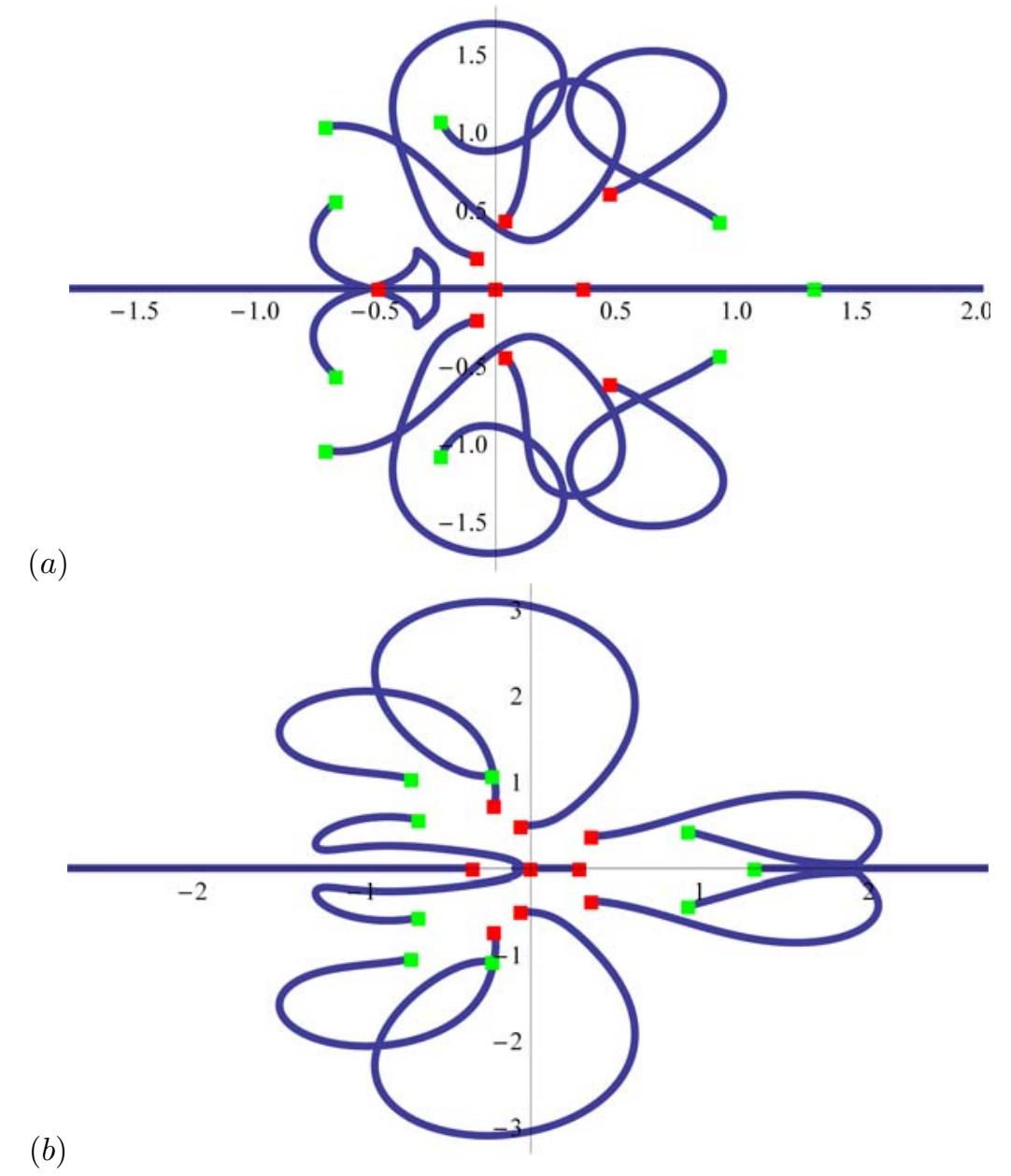

Figure 3.1: (a) $x$-values and (b) $y$-values of the solution paths of $H(x, y, t)=0$ for $0 \leq t \leq 1$ (green squares: solutions of $G(x, y)$, red squares: solutions of $F(x, y)$ ) 


\subsubsection{Subdivision methods}

A subdivision method is used to find the roots of a system of polynomial equations which lie in a specific domain or to isolate real roots. They use an exclusion criterion to remove a domain if no roots are found to lie inside this domain [85].

By rewriting the set of polynomials as polynomials in the Bernstein form, which yields a set of linear combinations of Bernstein polynomials $B_{i, j}(x)=\left(\begin{array}{c}j \\ i\end{array}\right) x^{i}(1-x)^{j-i}$ [37], the problem of searching for roots can be reduced to univariate root finding. The main ingredients of a subdivision solver are a preconditioner to transform the system of equations into a system which is more numerically stable, a reduction strategy to reduce the initial domain into a (smaller) search domain and a subdivision strategy, which is applied iteratively, to subdivide the search domain. The output is a set of small enough boxes which contain the roots of the original system of polynomial equations [91].

\subsubsection{Algebraic methods}

Algebraic methods to solve a system of equations exploit the algebraic relationships between the unknowns in the system of equations. These methods are mostly based on Gröbner Basis computations or normal form computations in a quotient algebra.

Gröbner basis computations can be used to solve systems of polynomial equations by eliminating variables from the system. In this situation the system in Gröbner basis form has the following upper triangular structure:

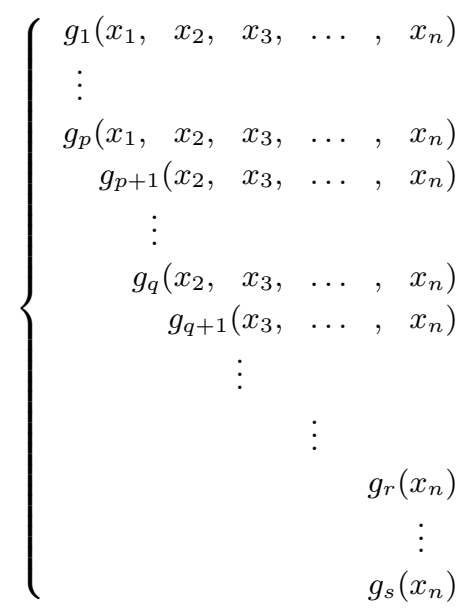

This structure can be achieved by using a proper monomial ordering like the lexicographic ordering. A system with such a triangular structure can be solved in a step wise order starting with the last (univariate) polynomial: first solve for the variable $x_{n}$, then for the variables $x_{n}, x_{n-1}$, then for $x_{n}, x_{n-1}, x_{n-2}$ and so on, until all the solutions $x_{1}, \ldots, x_{n}$ of the original system are found. This procedure is also called a 'back-solve strategy'. The transformation into such a Gröbner basis form is convenient when using such a procedure for solving a system of equations: one can always work 
backwards provided that a lexicographic ordering is used. However, this Gröbner basis computation can be computationally hard.

Example 3.4. Consider the system of polynomial equations:

$$
\begin{cases}f_{1}(x, y, z)=x^{2}-4 x y+4 x-y+3 z & =0 \\ f_{2}(x, y, z)=y^{2}-x-z & =0 \\ f_{3}(x, y, z)=z^{2}-x+y & \end{cases}
$$

To solve the system $\left\{f_{1}(x, y, z)=0, f_{2}(x, y, z)=0, f_{3}(x, y, z)=0\right\}$, the ideal $I=\left\langle f_{1}, f_{2}, f_{3}\right\rangle$ is considered. A Gröbner basis with a lexicographic monomial ordering for this ideal $I$ is given by $\left\{g_{1}(x, y, z), g_{2}(y, z), g_{3}(y, z), g_{4}(z)\right\}$ :

$$
\left\{\begin{array}{lll}
g_{1}(x, y, z) & =x-y-z^{2} & =0 \\
g_{2}(y, z) & =y^{2}-y-z^{2}-z & =0 \\
g_{3}(y, z) & =2 y z^{2}-z^{4}-z^{2} & =0 \\
g_{4}(z) & =z^{6}-4 z^{4}-4 z^{3}-z^{2} & =0
\end{array}\right.
$$

This system may seem more complicated on first inspection, because it contains 4 instead of 3 polynomials. However, among these 4 polynomials there is one univariate polynomial, $g_{4}(z)$, which is easily solved. Solving the univariate polynomial $g_{4}(z)=0$ yields the solutions $z=-1, z=0$, and $z=1 \pm \sqrt{2}$. The solutions for $z$ from the polynomial equation $g_{4}(z)=0$ are substituted into the polynomial $g_{3}(y, z)$. Solving the polynomial equation $g_{3}(y, z)=0$ for $y$, after substituting the values for $z$, yields the solution sets $\{y=1, z=-1\},\{y=2+\sqrt{2}, z=1+\sqrt{2}\}$ and $\{y=2-\sqrt{2}, z=$ $1-\sqrt{2}\}$. The solutions for $z$ from the equation $g_{4}(z)=0$ are also substituted into the polynomial $g_{2}(y, z)$. When the resulting expressions in the only variable $y$ are solved, this yields, next to the already known solutions, $\{y=0, z=-1\},\{y=0, z=0\},\{y=$ $1, z=0\},\{y=-1-\sqrt{2}, z=1+\sqrt{2}\}$, and $\{y=-1+\sqrt{2}, z=1-\sqrt{2}\}$. However, these new solutions should also still satisfy $g_{3}(y, z)=0$ and therefore the solutions $\{y=0, z=-1\},\{y=-1-\sqrt{2}, z=1+\sqrt{2}\}$, and $\{y=-1+\sqrt{2}, z=1-\sqrt{2}\}$ have to be discarded.

To summarize, we end up with five solutions: $\{y=1, z=-1\},\{y=0, z=0\}$, $\{y=1, z=0\},\{y=2+\sqrt{2}, z=1+\sqrt{2}\}$ and $\{y=2-\sqrt{2}, z=1-\sqrt{2}\}$ from the equations $g_{2}(y, z)=0, g_{3}(y, z)=0$ and $g_{4}(z)=0$. Subsequently, substituting these values for $y$ and for $z$ into $g_{1}(x, y, z)=0$ yields a univariate polynomial in the 
variable $x$. Solving for $x$ yields the solutions:

$$
\begin{aligned}
& x=2, y=1, z=-1 \\
& x=0, y=0, z=0 \\
& x=1, y=1, z=0 \\
& x=5+3 \sqrt{2}, y=2+\sqrt{2}, z=1+\sqrt{2} \\
& x=5-3 \sqrt{2}, y=2-\sqrt{2}, z=1-\sqrt{2}
\end{aligned}
$$

An immediate application of a Gröbner basis to solve polynomial equations in $\mathbb{C}$ is derived from the Weak Hilbert Nullstellensatz (Theorem 1 in Chapter 4 of [28]) and can be formulated as follows:

Theorem 3.2. (Consistency) If $I=\left\langle f_{1}, \ldots, f_{m}\right\rangle$ is an ideal in $\mathbb{C}\left[x_{1}, \ldots, x_{n}\right]$, then the system of polynomial equations $f_{1}\left(x_{1}, \ldots, x_{n}\right)=\ldots=f_{m}\left(x_{1}, \ldots, x_{n}\right)=0$ is unsolvable if and only if the reduced Gröbner basis of $I$ is $\{1\}$. Thus the variety $V(I)=\emptyset$.

Proof. The proof of this theorem is given in [23].

Example 3.5. Consider the following system of polynomial equations:

$$
\begin{cases}f_{1}=x^{2} y-z^{3} & =0 \\ f_{2}=2 x y-4 z-1 & =0 \\ f_{3}=y^{2}-z & =0 \\ f_{4}=x^{3}-4 y & =0\end{cases}
$$

The reduced Gröbner basis for $I=\left\langle f_{1}, f_{2}, f_{3}, f_{4}\right\rangle$ with respect to the lex ordering is $\{1\}$, hence the system is unsolvable.

A similar theorem in terms of the Gröbner basis of the corresponding ideal exists for a system with finitely many solutions. This theorem is based on Theorem 6 of Chapter 5 of $[28]$ :

Theorem 3.3. (Finiteness) If $I=\left\langle f_{1}, \ldots, f_{m}\right\rangle$ is an ideal in $\mathbb{C}\left[x_{1}, \ldots, x_{n}\right]$, then the system of polynomial equations $f_{1}\left(x_{1}, \ldots, x_{n}\right)=\ldots=f_{m}\left(x_{1}, \ldots, x_{n}\right)=0$ has finitely many solutions if and only if for all $i, 1 \leq i \leq n$, there exists a power of $x_{i}$ which belongs to the ideal generated by the leading terms of $I:\langle L T(I)\rangle$

Proof. The proof of this theorem is given in [23] and in [28].

An algebraic method which uses normal form computations in a quotient algebra to solve zero-dimensional ideals of multivariate polynomials, is presented in the next section. 


\subsection{The Stetter-Möller matrix method}

Suppose a system of the form (3.19) is given which is in Gröbner basis form with respect to a total degree monomial ordering, where each $f_{i}$ (for $i=1, \ldots, m$ ) is a (possibly) complex polynomial in the variables $x_{1}, \ldots, x_{n}$.

$$
\left\{\begin{array}{ccc}
f_{1}\left(x_{1}, \ldots, x_{n}\right) & = & 0 \\
\vdots & \vdots & \vdots \\
\vdots & \vdots & \vdots \\
f_{m}\left(x_{1}, \ldots, x_{n}\right) & = & 0
\end{array}\right.
$$

Because we only study zero-dimensional ideals in this chapter, we start from the assumption that the number of solutions of (3.19) in $\mathbb{C}^{n}$ is finite (for further details see [29] or Proposition 3.1 and Theorem 2.1 in [48] and the references given there).

To find all the solutions $x_{1}, \ldots, x_{n}$ in $\mathbb{C}^{n}$ of this system, we consider the quotient space $\mathbb{C}\left[x_{1}, \ldots, x_{n}\right] / I$, as in $[20]$, where $I$ is the ideal generated by the polynomials $f_{i}: I=\left\langle f_{1}, \ldots, f_{m}\right\rangle$. This quotient space is a finite-dimensional linear vector space of dimension $N$, and we denote the monomial basis for $\mathbb{C}\left[x_{1}, \ldots, x_{n}\right] / I$ as the set $B$ containing $N$ monomials $x^{u}=x_{1}^{u_{1}} x_{2}^{u_{2}} \cdots x_{n}^{u_{n}}$. Every polynomial can now be reduced modulo the polynomials $f_{1}, \ldots, f_{m}$ to a linear combination of the monomials in the basis $B$. Note that any total degree monomial ordering can be chosen.

For $i=1, \ldots, n$ we introduce the linear multiplication operators $\mathcal{A}_{x_{i}}$ on $\mathbb{C}\left[x_{1}, \ldots, x_{n}\right] / I$ by:

$$
\mathcal{A}_{x_{i}}: \mathbb{C}\left[x_{1}, \ldots, x_{n}\right] / I \quad \mapsto \mathbb{C}\left[x_{1}, \ldots, x_{n}\right] / I \quad: \quad g \quad \mapsto x_{i} \cdot g
$$

Each operator represents multiplication by $x_{i}$ modulo the ideal $I$. With respect to the monomial basis $B$, each (linear) operator $\mathcal{A}_{x_{i}}$ is represented by an $N \times N$ (possibly) complex non-symmetric matrix denoted by $A_{x_{i}}^{T}$ (where $T$ denotes the transpose of the matrix). In such a matrix $A_{x_{i}}^{T}$ the entry in position $(k, l)$ denotes the coefficient of the $l^{t h}$ monomial of $B$ in the normal form of the product of $x_{i}$ and the $k^{t h}$ monomial of $B$. In the literature the matrix $A_{x_{i}}^{T}$ is often called the $i^{t h}$ companion matrix or a multiplication table for the variable $x_{i}$ [81], [98] and the transposed matrix $A_{x_{i}}$ is called a representation matrix [82].

The matrices $A_{x_{1}}^{T}, \ldots, A_{x_{n}}^{T}$ commute pairwise with each other, which means that $A_{x_{i}}^{T} A_{x_{j}}^{T}=A_{x_{j}}^{T} A_{x_{i}}^{T}$ for all $i, j \in\{1, \ldots, n\}$. In fact, the set $A_{x_{1}}^{T}, \ldots, A_{x_{n}}^{T}$ is a matrix solution for system (3.19): $f_{i}\left(A_{x_{1}}^{T}, \ldots, A_{x_{n}}^{T}\right)=0$ for all $i=1, \ldots, m$ [48]. Note that all of this also holds without transposing the matrices.

Because of the commutative property of the set $A_{x_{1}}^{T}, \ldots, A_{x_{n}}^{T}$, these matrices share common eigenvectors $v$. Let $v \in \mathbb{C}^{n}$ denote such a common eigenvector of the matrices $A_{x_{1}}^{T}, \ldots, A_{x_{n}}^{T}$ with corresponding eigenvalues $\lambda_{1}, \ldots, \lambda_{n}$, respectively. Then $\left(x_{1}, \ldots, x_{n}\right)=\left(\lambda_{1}, \ldots, \lambda_{n}\right)$ is an element of the variety $V(I)$, the solution set of (complex) zeros of $I$. The $N$ eigenvalues $\lambda_{i}(1), \ldots, \lambda_{i}(N)$ of the matrix $A_{x_{i}}^{T}$ can be 
interpreted as the $x_{i}$-values of the zeros of the ideal $I$. A point $\left(\lambda_{1}, \ldots, \lambda_{n}\right) \in \mathbb{C}^{n}$ of eigenvalues of $A_{x_{1}}^{T}, \ldots, A_{x_{n}}^{T}$, respectively, corresponding to a common (non-zero) eigenvector $v \in \mathbb{C}^{n}$ is also called a multi-eigenvalue of the $n$-tuple of commuting matrices $\left(A_{x_{1}}^{T}, \ldots, A_{x_{n}}^{T}\right)$.

The following theorem characterizes the variety $V(I)$ :

Theorem 3.4. $V(I)=\left\{\left(\lambda_{1}, \ldots, \lambda_{n}\right) \in \mathbb{C}^{n}: \exists v \in \mathbb{C}^{n} \backslash\{0\} \forall i: A_{x_{i}}^{T} \cdot v=\lambda_{i} \cdot v\right\}$.

Proof. See [48] for the proof of this theorem.

The eigenvectors $v$ of the matrices $A_{x_{1}}^{T}, \ldots, A_{x_{n}}^{T}$ are highly structured. If all the eigenvalues of these matrices have algebraic multiplicity one, then it is wellknown that $A_{x_{i}}^{T}=U \Lambda L^{T}$, where $L^{T} U=I$ and $U$ is a generalized Vandermonde matrix. The rows of $U^{T}$ consist of the ordered basis $B$ evaluated at the points $x=v(k) \in \mathbb{C}^{n}, k=1, \ldots, N$, where $v(1), v(2), \ldots, v(N)$ are the complex solutions to the system of equations (3.19). Furthermore $\Lambda=\operatorname{diag}\left(\lambda_{1}, \ldots, \lambda_{N}\right)$ is a diagonal matrix with $\lambda_{k}=r(v(k)), k=1,2, \ldots, N$. The matrix $L$ has the property that its $k$-th column consists of the coefficients $l_{j k}$ of the Lagrange interpolation polynomials $l_{k}\left(x_{1}, \ldots, x_{n}\right)=\sum_{j=1}^{N} l_{j, k} b(j)$, where $b(j)$ denotes the $j$-th basis element in $B$, with the basic interpolation property that $l_{k}(v(k))=1$ and $l_{k}(v(j))=0$ for all $j \neq k$. In linear algebra terms this says that $L^{T}=U^{-1}$. The fact that the eigenvectors of $A_{x_{i}}^{T}$ are columns of the generalized Vandermonde matrix $U$ was noted by Stetter, which is the reason that eigenvectors of this form are sometimes called Stetter vectors [81]. The structure of these vectors can be used to read off the point $v(k)$ from an eigenvector of a single matrix $A_{x_{i}}^{T}$ directly, without having to apply the eigenvectors to all the operators $A_{x_{1}}^{T}, \ldots, A_{x_{n}}^{T}$, to obtain the corresponding eigenvalues. This is because the monomials $x_{i}, i=1, \ldots, n$, are all in the basis $B$, hence their values appear in the Stetter vector (see also [20]).

The method of rewriting the problem of finding solutions of a set of polynomial equations into an eigenvalue problem of a set of commuting matrices, is called the Stetter-Möller matrix method and can only be applied to systems of polynomial equations which generate a zero-dimensional ideal. This method is described in [81] and a similar approach can be found in [50] and [48] in which the matrix method was derived independently of the work of Stetter and Möller. For a further background on the constructive algebra and systems theory aspects of this approach, see also [47].

\subsection{Counting real solutions}

For some specific applications only real solutions are of importance. Therefore it can be convenient to have information about the number of real and complex solutions of an ideal $I$ generated by polynomials in $\mathbb{R}\left[x_{1}, \ldots, x_{n}\right]$ of a system of equations in Gröbner basis form. The numbers of real and complex solutions can be computed by 
applying the Stetter-Möller matrix method introduced in the previous section. Counting real solutions of a system of polynomial equations in $\mathbb{R}\left[x_{1}, \ldots, x_{n}\right]$ is facilitated by the following theorem (based on [84] and [98]):

Theorem 3.5. Construct the real symmetric matrix $M$ where the entries are the traces of the matrices which represent multiplication by the products of each possible pair of monomials in the set $B$, modulo the ideal $I$ :

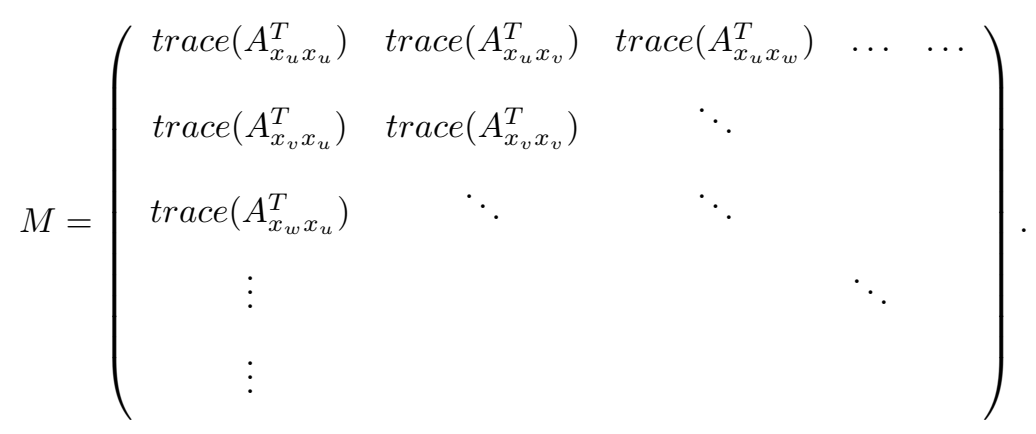

The number of real roots of the zero-dimensional ideal $I$ (in $\mathbb{R}\left[x_{1}, \ldots, x_{n}\right]$ ) in Gröbner basis form equals the number of positive eigenvalues of the matrix $M$ minus the number of negative eigenvalues of the matrix $M$.

Note that the matrix $M$ is real and symmetric and that therefore also all its eigenvalues are real. Because of the commuting properties of the matrices involved and the symmetry of the matrix $M$, i.e., $A_{x_{u} x_{v}}^{T}=A_{x_{u}}^{T} A_{x_{v}}^{T}=A_{x_{v} x_{u}}^{T}$, it is sufficient to compute only the upper-triangular part of the matrix $M$.

\subsection{Example: The Stetter-Möller matrix method to solve a system of equations and to count the number of real solutions}

Suppose that the following system of three (real) polynomial equations in the variables $x_{1}$ and $x_{2}$ is given:

$$
\begin{cases}f_{1}\left(x_{1}, x_{2}\right)=3 x_{2}^{2}+2 x_{1} x_{2}-4 x_{2} & =0 \\ f_{2}\left(x_{1}, x_{2}\right)=2 x_{1}^{3}-3 x_{2}^{3}+4 x_{2}^{2}-2 x_{1} & =0 \\ f_{3}\left(x_{1}, x_{2}\right)=39 x_{2}^{4}-124 x_{2}^{3}+132 x_{2}^{2}-48 x_{2} & =0\end{cases}
$$

This system is in Gröbner basis form and is zero-dimensional (in fact, it is the Gröbner basis with the lexicographic monomial ordering of the two polynomials $x_{1}^{3}+x_{1} x_{2}^{2}-x_{1}$ and $\left.3 x_{2}^{2}+2 x_{1} x_{2}-4 x_{2}\right)$. Let us now consider the quotient space $\mathbb{R}\left[x_{1}, x_{2}\right] / I$ where $I=\left\langle f_{1}, f_{2}, f_{3}\right\rangle$. This quotient space is a finite-dimensional vector space of dimension 6 and the monomial basis $B$ for this quotient space contains the 
6 monomials $1, x_{1}, x_{1}^{2}, x_{2}, x_{2}^{2}$, and $x_{2}^{3}$. The matrices $A_{x_{1}}^{T}$ and $A_{x_{2}}^{T}$ are given below:

$$
A_{x_{1}}^{T}=\left(\begin{array}{rrrrrr}
0 & 1 & 0 & 0 & 0 & 0 \\
0 & 0 & 1 & 0 & 0 & 0 \\
0 & 1 & 0 & -2 & 0 & \frac{3}{2} \\
0 & 0 & 0 & 2 & -\frac{3}{2} & 0 \\
0 & 0 & 0 & 0 & 2 & -\frac{3}{2} \\
0 & 0 & 0 & -\frac{24}{13} & \frac{66}{13} & -\frac{36}{13}
\end{array}\right),
$$

Let us, for example, have a look at the entry $(6,4)$ of the matrix $A_{x_{1}}^{T}$. This entry denotes the coefficient of the $4^{\text {th }}$ monomial of the basis $B$ in the normal form of the product of $x_{1}$ and the $6^{t h}$ monomial of $B$. The product of $x_{1}$ and the $6^{\text {th }}$ monomial of $B$ is $x_{1} x_{2}^{3}$ and its normal form takes the form $-\frac{36}{13} x_{2}^{3}+\frac{66}{13} x_{2}^{2}-\frac{24}{13} x_{2}$. The coefficient of the $4^{\text {th }}$ monomial of $B$ in this normal form is $-\frac{24}{13}$ which is exactly the element at position $(6,4)$ in the matrix $A_{x_{1}}^{T}$. It is easy to verify that the matrices commute and, thus, it holds that $A_{x_{1}}^{T} A_{x_{2}}^{T}=A_{x_{2}}^{T} A_{x_{1}}^{T}$.

The eigenvalue pairs $\left(\lambda_{1}, \lambda_{2}\right)$ of the matrices $A_{x_{1}}^{T}$ and $A_{x_{2}}^{T}$, respectively, are the solutions $\left(x_{1}, x_{2}\right)$ of the system $(3.22):(-1,0),(1,0),(0,1.3333),(0,0),(0.61538+$ $0.39970 i, 0.92308-0.26647 i)$ and $(0.61538-0.39970 i, 0.92308+0.26647 i)$.

However, once the eigenvalues and eigenvectors of the matrix $A_{x_{1}}^{T}$ are computed, the eigenvalues of $A_{x_{2}}^{T}$, which are the $x_{2}$-coordinates of system $(3.22)$, can also be read off from the corresponding eigenvectors $v$. The eigenvectors are structured conformably with the basis $B$, which means that the fourth element of $v$ denotes the value for the corresponding eigenvalue $\lambda_{2}$ (after normalization of $v$ to make its first element equal to 1) without having to construct the matrix $A_{x_{2}}^{T}$ explicitly and without having to perform additional eigenvalue computations.

Sometimes it can be helpful to split off unwanted solutions of a system of polynomial equations. This can be done by a so-called primary decomposition of the ideal $I=\left\langle f_{1}, f_{2}, f_{3}\right\rangle$ (see [98]). In this case however it would not be helpful because of the small dimensions of the matrices involved. Moreover, it goes beyond the scope of this chapter to go into all details of this. A primary decomposition of the ideal $I$ could 
be:

$$
\begin{gathered}
I=\left\langle 13 x_{2}^{2}-24 x_{2}+12,2 x_{1}+3 x_{2}-4\right\rangle+ \\
\left\langle x_{2},\left(x_{1}-1\right)\right\rangle+\left\langle x_{2},\left(x_{1}+1\right)\right\rangle+\left\langle 3 x_{2}-4, x_{1}\right\rangle+\left\langle x_{1}, x_{2}\right\rangle
\end{gathered}
$$

of which the last four ideals are responsible for the four solutions which contain a zero in one of its coordinates. In a situation where these solutions are meaningless, they can be split off from the solution set by removing the corresponding four ideals and one may continue with computing the solutions of $\left\{13 x_{2}^{2}-24 x_{2}+12,2 x_{1}+3 x_{2}-4\right\}$.

When counting real and complex solutions of the system of polynomial equations (3.22), one can construct the matrix $M$ mentioned in Theorem 3.5, which has the following structure:

$$
M=\left(\begin{array}{rrrrrr}
6.000 & 1.231 & 2.438 & 3.180 & 3.340 & 3.550 \\
1.231 & 2.438 & -0.124 & 1.349 & 1.355 & 1.255 \\
2.438 & -0.124 & 1.612 & 0.666 & 0.826 & 0.910 \\
3.180 & 1.349 & 0.666 & 3.340 & 3.550 & 3.897 \\
3.340 & 1.355 & 0.826 & 3.550 & 3.897 & 4.484 \\
3.550 & 1.255 & 0.910 & 3.897 & 4.484 & 5.438
\end{array}\right) .
$$

The eigenvalues of $M$ are: $16.632,3.5083,2.0467,0.39722,0.15356$, and -0.013531 . The difference between the number of positive and negative eigenvalues of the matrix $M$ indicates that there are four real solutions and two non-real complex solutions of the system of polynomial equations (3.22), which is consistent with our earlier findings. 



\section{Part II}

\section{Global Optimization}





\section{Chapter 4}

\section{Global optimization of multivariate polynomials}

Finding the global minimum of a real-valued multivariate polynomial is a problem which has several useful applications in systems and control theory as well as in many other quantitative sciences including statistics, mathematical finance, economics, systems biology, etc. Multivariate global polynomial optimization is a hard problem because of the non-convexity of the problem and the existence of local optima. In this chapter we focus on computing the global optimum of a special class of polynomials.

The class of polynomials discussed in this chapter is the class of so-called Minkowski dominated polynomials in several variables. When working with a polynomial $p_{\lambda}\left(x_{1}\right.$, $\left.\ldots, x_{n}\right)$ from this class, the problem of finding its global minimum can be reformulated as an eigenvalue problem by applying the Stetter-Möller matrix method introduced in the previous chapter. This is possible because the system of first-order conditions of such a polynomial is always in Gröbner basis form with a special structure, showing that the number of solutions is finite. Applying the Stetter-Möller matrix method yields a set of real non-symmetric large and sparse commuting matrices $A_{x_{1}}^{T}, \ldots, A_{x_{n}}^{T}$. Using the same approach, it is also possible to construct a matrix $A_{p_{\lambda}\left(x_{1}, \ldots, x_{n}\right)}^{T}$ which has some useful properties: it turns out that the smallest real eigenvalue of the matrix $A_{\left.p_{\lambda} x_{1}, \ldots, x_{n}\right)}^{T}$ is of interest for computing the global optimum of the polynomial $p_{\lambda}\left(x_{1}, \ldots, x_{n}\right)$.

Various extensions and generalizations of these techniques exist including the optimization of rational or logarithmic functions [63] and the use of systems that are not in Gröbner basis form [12]. However, these generalizations do not satisfy all the properties described below and therefore require adjustments to the techniques used here.

Numerical experiments using the approach described in this chapter are discussed in Chapter 7. 


\subsection{The global minimum of a Minkowski dominated polynomial}

Let $q\left(x_{1}, \ldots, x_{n}\right) \in \mathbb{R}\left[x_{1}, \ldots, x_{n}\right]$ be a real polynomial. We are interested to compute its infimum over $\mathbb{R}^{n}$. Let $d$ be a positive integer such that $2 d$ (strictly) exceeds the total degree of $q\left(x_{1}, \ldots, x_{n}\right)$ and consider the one-parameter family of what will be called (Minkowski) dominated polynomials:

$$
p_{\lambda}\left(x_{1}, \ldots, x_{n}\right):=\lambda\left(x_{1}^{2 d}+\ldots+x_{n}^{2 d}\right)+q\left(x_{1}, \ldots, x_{n}\right), \quad \lambda \in \mathbb{R}^{+} .
$$

Note that the nomenclature for this family derives from the property that the value of $p_{\lambda}$ is dominated by the term $\lambda\left(x_{1}^{2 d}+\ldots+x_{n}^{2 d}\right)$ when the Minkowski $2 d$-norm $\left\|\left(x_{1}, \ldots, x_{n}\right)\right\|_{2 d}=\left(x_{1}^{2 d}+x_{2}^{2 d}+\ldots+x_{n}^{2 d}\right)^{1 /(2 d)}$ becomes large. Consequently, the polynomial $p_{\lambda}$ has a global minimum over $\mathbb{R}^{n}$ for each $\lambda \in \mathbb{R}^{+}$: the presence of the dominating term $\lambda\left(x_{1}^{2 d}+\ldots+x_{n}^{2 d}\right)$ ensures that $p_{\lambda}$ has a global minimum. In fact information about the infimum (and its 'location') of an arbitrary real polynomial $q\left(x_{1}, \ldots, x_{n}\right)$ can be obtained by studying what happens to the global minima and the corresponding minimizing points of $p_{\lambda}\left(x_{1}, \ldots, x_{n}\right)$ for $\lambda \downarrow 0$, see [48], [64].

Note that there may be more than one point at which the value of the global minimum is obtained.

Example 4.1. The function: $p_{\lambda}\left(x_{1}, x_{2}\right)=\lambda\left(x_{1}^{6}+x_{2}^{6}\right)+10 x_{1}^{2} x_{2}^{2}-10 x_{1}^{2}-10 x_{2}^{2}+5$ with $\lambda=$ 0.5 has multiple points at which the global minimum is obtained, as shown in Figure 4.1. The global minimum value of -12.2133 is attained at 4 positions $(( \pm 1.6096,0)$ and $(0, \pm 1.6069))$. The method proposed in this chapter finds at least one point in every connected component of the set of points where this global minimum is attained.

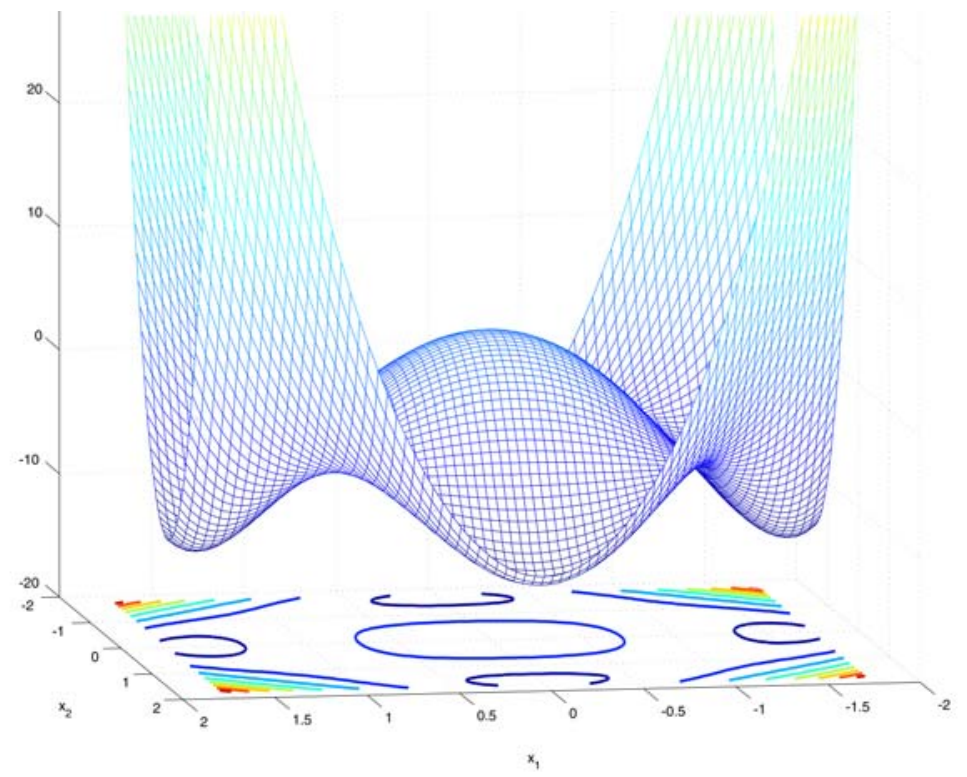

Figure 4.1: Example of a polynomial with multiple locations of the global optimum 
In certain applications it may be necessary to restrict the values of $x_{1}, \ldots, x_{n}$ to, for example, the positive orthant. However, in this research no attention is paid to this form of constrained polynomial optimization. The extension of the techniques described here to this more general setting is recommended for further research.

The global minimizers of $p_{\lambda}\left(x_{1}, \ldots, x_{n}\right)$ are of course among the stationary points of the polynomial $p_{\lambda}$, which are the real solutions to the corresponding system of firstorder conditions. This leads to a system of $n$ polynomial equations in $n$ variables of the form:

$$
d^{(i)}\left(x_{1}, \ldots, x_{n}\right)=0, \quad(i=1, \ldots, n)
$$

where

$$
d^{(i)}\left(x_{1}, \ldots, x_{n}\right)=x_{i}^{2 d-1}+\frac{1}{2 d \lambda} \frac{\partial}{\partial x_{i}} q\left(x_{1}, \ldots, x_{n}\right) .
$$

It will be convenient to write $d^{(i)}\left(x_{1}, \ldots, x_{n}\right)$ in the form:

$$
d^{(i)}\left(x_{1}, \ldots, x_{n}\right)=x_{i}^{m}-f^{(i)}\left(x_{1}, \ldots, x_{n}\right), \quad(i=1, \ldots, n),
$$

with $m=2 d-1$ and $f^{(i)}=-\frac{1}{2 d \lambda} \frac{\partial}{\partial x_{i}} q\left(x_{1}, \ldots, x_{n}\right) \in \mathbb{R}\left[x_{1}, \ldots, x_{n}\right]$ of total degree strictly less than $m$. Because of the special structure of the polynomial system, (i) the set of polynomials $\left\{d^{(i)} \mid i=1, \ldots, n\right\}$ is in Gröbner basis form with respect to any total degree monomial ordering and (ii) the associated variety $V$, the solution set to the system of equations (4.2), has dimension zero which means that the system of first-order conditions only admits a finite number of solutions in $\mathbb{C}^{n}$. The result that there exists a finite number of solutions in this situation is based on Theorem 3.3 of Section 3.2.4: for every $i, 1 \leq i \leq n$, there exists a power of $x_{i}$ which belongs to the ideal $\langle I\rangle$, where $I=\left\{d_{1}, \ldots, d_{n}\right\}$. (For more details see Proposition 3.1 and Theorem 2.1 in [48] and the references given there.)

Note that because of property (i) it is not necessary to run an algorithm in order to obtain a Gröbner basis for the ideal generated by the first-order conditions, because the first-order conditions of a Minkowski dominated polynomial are in Gröbner basis form already.

Because of these properties the Stetter-Möller matrix method, introduced in Section 3.3, can be applied to the system of equations (4.2). The associated ideal $I=\left\langle d^{(i)} \mid i=1, \ldots, n\right\rangle$, yields the quotient space $\mathbb{R}\left[x_{1}, \ldots, x_{n}\right] / I$ which is a finitedimensional vector space of dimension $N:=m^{n}$. A monomial basis for this quotient space is defined by the set:

$$
B=\left\{x_{1}^{\alpha_{1}} x_{2}^{\alpha_{2}} \cdots x_{n}^{\alpha_{n}} \mid \alpha_{1}, \alpha_{2}, \ldots, \alpha_{n} \in\{0,1, \ldots, m-1\}\right\} .
$$

For definiteness we will choose a permutation of the total degree reversed lexicographical monomial ordering throughout this thesis, unless stated otherwise. For the 2-dimensional situation this ordering corresponds to:

$$
\begin{gathered}
1 \prec x_{1} \prec x_{1}^{2} \prec \ldots \prec x_{1}^{2 d-2} \prec x_{2} \prec x_{1} x_{2} \prec x_{1}^{2} x_{2} \prec \ldots \\
\prec x_{1}^{2 d-2} x_{2} \prec x_{2}^{2} \prec \ldots \prec x_{1}^{2 d-2} x_{2}^{2 d-2}
\end{gathered}
$$


which is readily expanded to $n$ dimensions. Note that any other total degree monomial ordering could be chosen instead.

By applying the Stetter-Möller matrix method, an $n$-tuple of commuting $N \times N=$ $m^{n} \times m^{n}$ matrices $\left(A_{x_{1}}^{T}, A_{x_{2}}^{T}, \ldots, A_{x_{n}}^{T}\right)$ can be constructed. These matrices yield a matrix solution of the system of polynomial equations (4.2). Any common eigenvector $v$ of these matrices $A_{x_{1}}^{T}, A_{x_{2}}^{T}, \ldots, A_{x_{n}}^{T}$ leads to a scalar solution, constituted by the $n$-tuple of eigenvalues of the matrices $A_{x_{1}}^{T}, A_{x_{2}}^{T}, \ldots, A_{x_{n}}^{T}$ corresponding to $v$.

A straightforward method to compute the global minimum of a dominated real polynomial $p_{\lambda}\left(x_{1}, \ldots, x_{n}\right)$ over $\mathbb{R}^{n}$, is to first compute all the real stationary points of $p_{\lambda}\left(x_{1}, \ldots, x_{n}\right)$ by solving the system of first-order conditions $d^{(i)}\left(x_{1}, \ldots, x_{n}\right)=0$, $(i=1, \ldots, n)$ using the real eigenvalues of the matrices $A_{x_{1}}^{T}, A_{x_{2}}^{T}, \ldots, A_{x_{n}}^{T}$. The stationary points $\left(x_{1}, \ldots, x_{n}\right)$ can then be plugged into the polynomial $p_{\lambda}\left(x_{1}, \ldots\right.$, $x_{n}$ ) and selecting the real stationary point at which the minimal value is attained yields the global optimum of the polynomial $p_{\lambda}\left(x_{1}, \ldots, x_{n}\right)$.

However, there is another more efficient way of selecting the global optimum of the polynomial under consideration, by slightly extending the Stetter-Möller matrix method as discussed in the next section.

\subsection{The Stetter-Möller matrix method for global optimization}

In Section 3.3, the linear multiplication operators $\mathcal{A}_{x_{i}}$ on $\mathbb{R}\left[x_{1}, \ldots, x_{n}\right] / I$ are introduced, which represent multiplication by $x_{i}$ modulo the ideal $I$, generated by the first-order conditions $d^{(i)}$. More generally, a similar set-up of the Stetter-Möller matrix method for a polynomial $r\left(x_{1}, \ldots, x_{n}\right)$ can be used to introduce a linear operator $\mathcal{A}_{r\left(x_{1}, \ldots, x_{n}\right)}$. It turns out that this operator is of importance for the global optimization of a Minkowski dominated polynomial. The operator $\mathcal{A}_{r\left(x_{1}, \ldots, x_{n}\right)}$ is defined as:

$$
\mathcal{A}_{r\left(x_{1}, \ldots, x_{n}\right)}: \mathbb{R}\left[x_{1}, \ldots, x_{n}\right] / I \mapsto \mathbb{R}\left[x_{1}, \ldots, x_{n}\right] / I: g \mapsto r\left(x_{1}, \ldots, x_{n}\right) \cdot g
$$

A crucial observation now is that also in this case polynomial multiplication within $\mathbb{R}\left[x_{1}, \ldots, x_{n}\right] / I$ is a linear operation. Therefore, given any basis for $\mathbb{R}\left[x_{1}, \ldots, x_{n}\right] / I$, for instance the basis $B$ introduced in (4.5), it is possible to represent this operator $\mathcal{A}_{r}$ by a matrix: the matrix $A_{r\left(x_{1}, \ldots, x_{n}\right)}^{T}$ of dimension $N \times N=m^{n} \times m^{n}$. This matrix is then associated with the linear operation of multiplication by a polynomial $r\left(x_{1}, \ldots, x_{n}\right)$ within $\mathbb{R}\left[x_{1}, \ldots, x_{n}\right] / I$. It turns out that $A_{r\left(x_{1}, \ldots, x_{n}\right)}^{T}=r\left(A_{x_{1}}^{T}, \ldots, A_{x_{n}}^{T}\right)$ and that the matrix $A_{r}^{T}$ also commutes with the matrices $A_{x_{i}}^{T}$ (see also [20]). Depending on the choice of $r$, such a matrix $A_{r}$ is usually highly sparse and structured.

If $v$ is a common eigenvector of the matrices $A_{x_{1}}^{T}, \ldots, A_{x_{n}}^{T}$ for $\left(\lambda_{1}, \ldots, \lambda_{n}\right)$, then $v$ is also an eigenvector of $A_{r\left(x_{1}, \ldots, x_{n}\right)}^{T}$ with corresponding eigenvalue $r\left(\lambda_{1}, \ldots, \lambda_{n}\right)$ : $A_{r}^{T} v=r\left(A_{x_{1}}^{T}, \ldots, A_{x_{n}}^{T}\right) v=r\left(\lambda_{1}, \ldots, \lambda_{n}\right) v$. 
Using the matrices $A_{x_{i}}^{T}$, one can compute the coordinates of the stationary points of the polynomial $p_{\lambda}$ : the eigenvalues of each matrix $A_{x_{i}}^{T}$ are equal to the values of $x_{i}$ evaluated at the points of $V(I)$, which are equal to the $i^{t h}$ coordinates of the stationary points of the polynomial $p_{\lambda}$. As a consequence, one may also choose the polynomial $r$ equal to the polynomial $p_{\lambda}\left(x_{1}, \ldots, x_{n}\right)$, which yields the matrix $A_{p_{\lambda}}^{T}$. The matrix $A_{p_{\lambda}}^{T}$ makes it possible to evaluate $p_{\lambda}$ at its stationary points: the eigenvalues of $A_{p_{\lambda}}^{T}$ are the function values of $p_{\lambda}$ at the points of $V(I)$, i.e., at the (complex) solutions of the system of equations $d^{(i)}\left(x_{1}, \ldots, x_{n}\right)=0$ for $i=1, \ldots, n$.

A straightforward deployment of the Stetter-Möller matrix method for computing the global minimum and an associated global minimizer (over $\mathbb{R}^{n}$ ) for the real dominated polynomial $p_{\lambda}$ now proceeds as follows. As a suitable choice for the polynomial $r$, the polynomial $p_{\lambda}$ is chosen and the corresponding matrix $A_{p_{\lambda}}^{T}$ is constructed. Then its eigenvalues and corresponding eigenvectors are computed. Note that $p_{\lambda}$ is defined as a real polynomial and that therefore only real eigenvalues of the aforementioned matrix are of interest. The smallest real eigenvalue obtained in this way yields the global minimum and the corresponding minimizer(s) can be read off from the eigenvector which is structured in the same way as the basis $B$ in Equation (4.5) is structured (because of the same reason mentioned in Section 3.3). Note that the eigenvector is structured if and only if the geometric multiplicity of the smallest real eigenvalue is one. For higher multiplicities the corresponding eigenspace may be larger and the eigenvector is not structured (see again Section 3.3).

Remark 4.1. Another approach to retrieve the global minimizer is to apply the eigenvector $v$, corresponding to the smallest real eigenvalue of $A_{p_{\lambda}}^{T}$, to the matrices $A_{x_{1}}^{T}, \ldots, A_{x_{n}}^{T}$. The eigenvalues $\xi_{1}, \ldots, \xi_{n}$ are obtained by solving $\xi_{1}$ from the equation $A_{x_{i}}^{T} v=\xi_{i} v$ for $i=1, \ldots, n$. The point $\left(\xi_{1}, \ldots, \xi_{n}\right)$ found, yields the corresponding global minimizer. Using this approach the matrices $A_{x_{1}}^{T}, \ldots, A_{x_{n}}^{T}$ have to be constructed explicitly, which is not necessary and not efficient as the minimizers can be read off from the eigenvector at hand, as shown previously. But if the eigenvalue under consideration has a multiplicity larger than one, the corresponding eigenvectors may not necessarily contain the Stetter structure and this could then be an approach to compute the minimizers.

Remark 4.2. It is possible that there exists a real eigenvalue of the matrix $A_{p_{\lambda}}^{T}$ which has a non-real global minimizer. Non-real global minimizers which yield a real criterion value, when plugged into the polynomial $p_{\lambda}$, are discarded because they are of no interest in this application.

\subsection{An example}

Let the dominated polynomial to be minimized be chosen as:

$$
p_{1}\left(x_{1}, x_{2}\right)=\left(x_{1}^{4}+x_{2}^{4}\right)+x_{1}^{3}+2 x_{1}^{2}+3 x_{1} x_{2}
$$

for which $n=2, d=2, \lambda=1$, and $q\left(x_{1}, x_{2}\right)=x_{1}^{3}+2 x_{1}^{2}+3 x_{1} x_{2}$. This dominated polynomial is visualized in Figure 4.2. From this figure it is clear that there are 
two local minima near $(0.4,-0.6)^{T}$ and $(-0.6,0.8)^{T}$, however it is unclear what their exact location is and if one minimum has a lower value than the other.

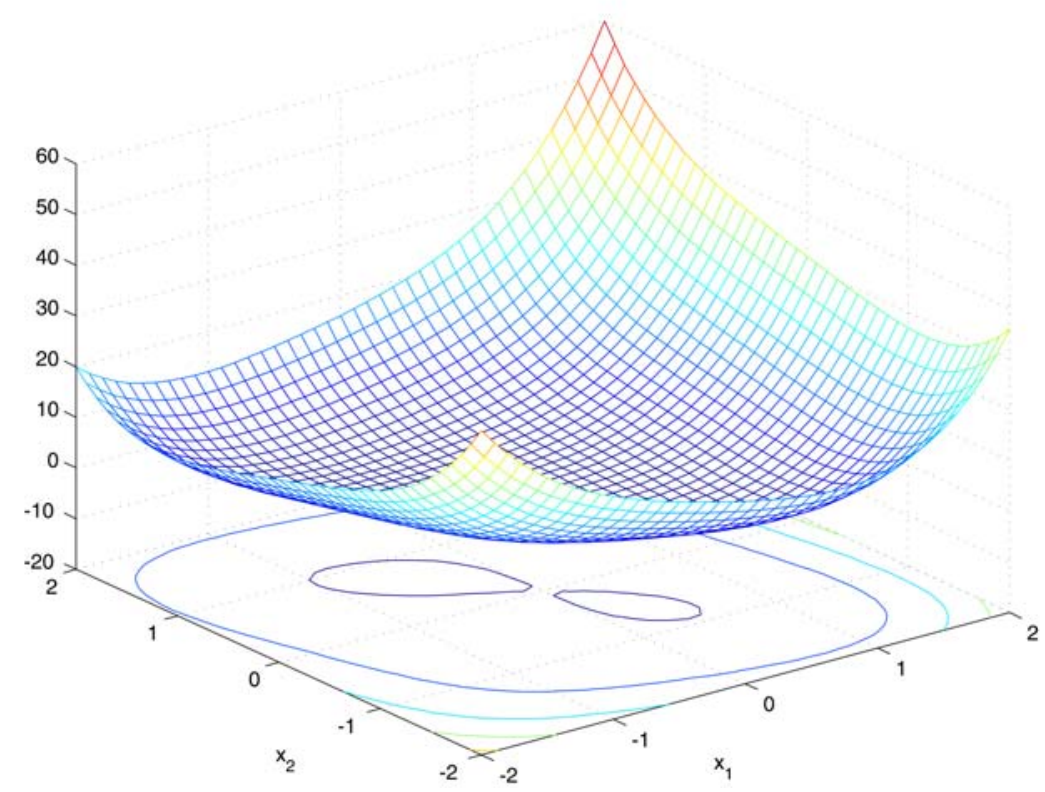

Figure 4.2: Example polynomial $p_{1}\left(x_{1}, x_{2}\right)$

The first-order conditions for minimality of the polynomial $p_{1}\left(x_{1}, x_{2}\right)$ are given by:

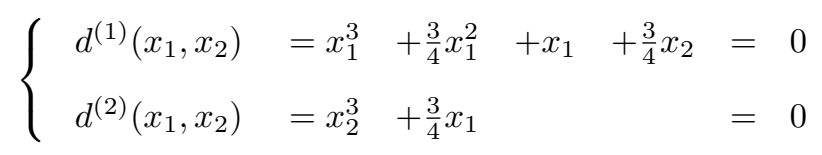

of which the real solutions constitute the stationary points of $p_{1}\left(x_{1}, x_{2}\right)$.

Note that this system of equations is indeed in Gröbner basis form already, with respect to a total degree monomial ordering, since for both of the variables $x_{1}$ and $x_{2}$ there is an index $i$ for which $L T\left(d^{(i)}\right)$ is a pure power of the variable: $\operatorname{LT}\left(d^{(1)}\right)=x_{1}^{3}$ and $\operatorname{LT}\left(d^{(2)}\right)=x_{2}^{3}$. The ideal $I$ is generated by $d^{(1)}\left(x_{1}, x_{2}\right)$ and $d^{(2)}\left(x_{1}, x_{2}\right)$ and the quotient space $\mathbb{R}\left[x_{1}, x_{2}\right] / I$ is of dimension $(2 d-1)^{n}=9$. A basis is given by the set of monomials $\left\{1, x_{1}, x_{1}^{2}, x_{2}, x_{1} x_{2}, x_{1}^{2} x_{2}, x_{2}^{2}, x_{1} x_{2}^{2}, x_{1}^{2} x_{2}^{2}\right\}$. In terms of this basis (which corresponds to a convenient permutation of the total degree reversed lexicographic monomial ordering) the matrices $A_{x_{1}}^{T}$ and $A_{x_{2}}^{T}$ are easily computed by 
the Stetter-Möller matrix method as:

$$
A_{x_{1}}^{T}=\left(\begin{array}{rrrrrrrrr}
0 & 1 & 0 & 0 & 0 & 0 & 0 & 0 & 0 \\
0 & 0 & 1 & 0 & 0 & 0 & 0 & 0 & 0 \\
0 & -1 & -\frac{3}{4} & -\frac{3}{4} & 0 & 0 & 0 & 0 & 0 \\
0 & 0 & 0 & 0 & 1 & 0 & 0 & 0 & 0 \\
0 & 0 & 0 & 0 & 0 & 1 & 0 & 0 & 0 \\
0 & 0 & 0 & 0 & -1 & -\frac{3}{4} & -\frac{3}{4} & 0 & 0 \\
0 & 0 & 0 & 0 & 0 & 0 & 0 & 1 & 0 \\
0 & 0 & 0 & 0 & 0 & 0 & 0 & 0 & 1 \\
0 & \frac{9}{16} & 0 & 0 & 0 & 0 & 0 & -1 & -\frac{3}{4}
\end{array}\right) \text { and }
$$

Of course, for such small dimensions computations are easy and it is not difficult to verify that $A_{x_{1}}^{T}$ and $A_{x_{2}}^{T}$ commute.

To locate the global minimum of $p_{1}\left(x_{1}, x_{2}\right)$, attention is first focused on the matrices $A_{x_{1}}^{T}$ and $A_{x_{2}}^{T}$. The eigenvalues of these matrices constitute the values of $x_{1}$ and $x_{2}$, respectively, at the stationary points of the system of equations (4.9). Using a direct eigenvalue solver, all the eigenvalues of the matrices $A_{x_{1}}^{T}$ and $A_{x_{2}}^{T}$ are computed. Both matrices have distinct eigenvalues, which can be paired by matching their common eigenspaces, yielding 9 solutions in $\mathbb{C}^{2}$. Restricting to real solutions, 3 stationary points are obtained: $(-0.631899,0.779656),(0.346826,-0.638348)$, and $(0,0)$, which can be classified, respectively, as a global minimizer, a local minimizer and a saddle point. The corresponding critical values of $p_{1}\left(x_{1}, x_{2}\right)$ are obtained by plugging the stationary points into $p_{1}\left(x_{1}, x_{2}\right)$, yielding the criterion values $-0.402778,-0.201376$, and 0 , respectively.

The extension of the Stetter-Möller matrix method mentioned in this Chapter, focuses attention on the matrix $A_{p_{1}\left(x_{1}, x_{2}\right)}^{T}$. The eigenvalues of the matrix $A_{p_{1}\left(x_{1}, x_{2}\right)}^{T}$ are the values of $p_{1}\left(x_{1}, x_{2}\right)$ at the stationary points. Therefore, the smallest real eigenvalue of this matrix is a main candidate to yield the global minimum of $p_{1}\left(x_{1}, x_{2}\right)$. Any non-real stationary point that corresponds to a real function value $p_{1}\left(x_{1}, x_{2}\right)$ is 
discarded because it is of no interest in this application.

The matrix $A_{p_{1}\left(x_{1}, x_{2}\right)}^{T}$ can be constructed using the same monomial basis $B$ and quotient space $\mathbb{R}\left[x_{1}, x_{2}\right] / I$ as introduced above. This yields the $9 \times 9$ matrix $A_{p_{1}\left(x_{1}, x_{2}\right)}^{T}$ :

$$
\left(\begin{array}{rrrrrrrrr}
0 & -\frac{1}{4} & \frac{13}{16} & -\frac{3}{16} & \frac{3}{2} & 0 & 0 & 0 & 0 \\
0 & -\frac{13}{16} & -\frac{55}{64} & -\frac{39}{64} & -\frac{3}{16} & \frac{3}{2} & 0 & 0 & 0 \\
0 & \frac{55}{64} & -\frac{43}{256} & \frac{165}{256} & -\frac{135}{64} & -\frac{21}{16} & -\frac{9}{8} & 0 & 0 \\
0 & 0 & 0 & 0 & -\frac{1}{4} & \frac{13}{16} & -\frac{3}{16} & \frac{3}{2} & 0 \\
0 & 0 & 0 & 0 & -\frac{13}{16} & -\frac{55}{64} & -\frac{39}{64} & -\frac{3}{16} & \frac{3}{2} \\
0 & \frac{27}{32} & 0 & 0 & \frac{55}{64} & -\frac{43}{256} & \frac{165}{256} & -\frac{135}{64} & -\frac{21}{16} \\
0 & \frac{9}{64} & -\frac{9}{8} & 0 & 0 & 0 & 0 & -\frac{1}{4} & \frac{13}{16} \\
0 & \frac{405}{256} & \frac{63}{64} & \frac{27}{32} & 0 & 0 & 0 & -\frac{13}{16} & -\frac{55}{64} \\
0 & -\frac{1503}{1024} & \frac{27}{32} & -\frac{189}{256} & \frac{27}{32} & 0 & 0 & \frac{55}{64} & -\frac{43}{256}
\end{array}\right)
$$

The real eigenvalues of the matrix $A_{p_{1}\left(x_{1}, x_{2}\right)}^{T}$ are computed as $-0.402778,-0.201376$, 0 . The smallest one of these is the global minimum and its location can be read off from the corresponding eigenvector. This (normalized) eigenvector is computed as $(1,-0.631899,0.399297,0.779656,-0.492664,0.311314,0.607864,-0.384108$, $0.242718)^{T}$ and has the same structure as the monomial basis $B$. The value of the $x_{1}$ coordinate can be read off from the eigenvector as it is the second element, -0.631899 , and the value of the $x_{2}$ coordinate can be read off as the fourth element, 0.779656 . This approach allows for computing the global minimum and its minimizer at once without explicitly constructing the matrices $A_{x_{1}}^{T}$ and $A_{x_{2}}^{T}$ and without performing eigenvalue/eigenvector computations on each of them.

As a final check, a local optimization technique, steepest descent, is applied to the polynomial $p_{1}\left(x_{1}, x_{2}\right)$ from the starting locations $(0.4,-0.6)$ and $(-0.6,0.8)$. The first starting location results in a minimum at $(0.3468,-0.6384)$ with criterion value -0.2014 . The second starting location results in a minimum at $(-0.6319,0.7797)$ with criterion value -0.4028 . The latter of these two values has the lowest criterion value and matches the location and value found by both eigenvalue approaches. 


\section{Chapter 5}

\section{An $n$ D-systems approach in polynomial optimization}

In Chapter 4 an extension of the Stetter-Möller matrix method is introduced for computing the global minimum of a multivariate Minkowski dominated polynomial $p_{\lambda}\left(x_{1}, \ldots, x_{n}\right)$. The Stetter-Möller matrix method can be applied to the class of Minkowski dominated polynomials because its system of first-order conditions is in Gröbner basis form and has a finite number of zeros due to its construction. This approach yields a matrix $A_{p_{\lambda}\left(x_{1}, \ldots, x_{n}\right)}^{T}$ whose real eigenvalues are the function values of the stationary points of the polynomial $p_{\lambda}\left(x_{1}, \ldots, x_{n}\right)$. The global minimum of $p_{\lambda}\left(x_{1}, \ldots, x_{n}\right)$ can therefore be computed by the smallest real eigenvalue of this matrix. The involved eigenvectors are structured and because of this structure the values of the minimizers $\left(x_{1}, \ldots, x_{n}\right)$ can be read off from the eigenvectors (see the previous chapter for details).

A serious bottleneck in this approach from a computational point of view is constituted by solving the eigenproblem of the matrix $A_{r}^{T}$ introduced in Chapter 4 . As a matter of fact, the $N \times N$ matrix $A_{r}^{T}$ quickly grows large, since $N=m^{n}$ where $m$ equals $2 d-1$ and $2 d$ denotes the total degree of the polynomial $p_{\lambda}\left(x_{1}, \ldots, x_{n}\right)$. On the other hand, the matrix $A_{r}^{T}$ may be highly sparse and structured. This holds in particular for the choices $r\left(x_{1}, \ldots, x_{n}\right)=x_{i}$.

Large and sparse eigenproblems are especially suited for a modern iterative eigenproblem solver (e.g., based on a Jacobi-Davidson [39], [94], or an Arnoldi method [77]). An advantage of such an iterative solver is that it has the ability to focus on certain eigenvalues first. In our application, this would be the smallest real eigenvalue. This allows to address the critical values of a polynomial $p_{\lambda}\left(x_{1}, \ldots, x_{n}\right)$ directly, as they appear among the (real) eigenvalues of the matrix $A_{p_{\lambda}}^{T}$, without determining all the stationary points of $p_{\lambda}\left(x_{1}, \ldots, x_{n}\right)$ first. Iterative eigenvalue solvers and the modifications of their implementations to improve their efficiency in this special application of polynomial optimization, are discussed in Chapter 6 . 
Another important advantage of iterative eigenproblem solvers is that they only require the action of the matrix $A_{r}^{T}$ on given vectors $v$, which avoids the need to build the large matrix $A_{r}^{T}$ explicitly. This matrix-free approach to compute the action of the matrix $A_{r}^{T}$ as the input for iterative eigenproblem solvers, is the main subject of this chapter.

To avoid building the large matrix $A_{r}^{T}$ one can associate the system of firstorder derivatives of $p_{\lambda}$ with an $n \mathrm{D}$-system of difference equations, by interpreting the variables in the polynomial equations as shift operators $\sigma_{1}, \ldots, \sigma_{n}$ working on a multidimensional time series $y_{t_{1}, t_{2}, \ldots, t_{n}}$. Then calculation of the action of $A_{r}^{T}$ on a given vector $v$ requires solving for $y_{t_{1}, t_{2}, \ldots, t_{n}}$ using the difference equations. The vector $v$ corresponds to an initial state of the associated $n \mathrm{D}$-system. See [5] and [40] for similar ideas in the 2D-case. This set-up is presented in the first section of this chapter.

The usage of the $n \mathrm{D}$-system in global polynomial optimization using the StetterMöller matrix method in combination with an iterative eigenproblem solver is described in Section 5.2.

The huge number of required iterations is the main reason why the action of a matrix $A_{r}^{T}$ has to be computed efficiently. This is studied in Section 5.3 and its subsections. One way to compute efficiently the action of $A_{r}^{T}$ on $v$ with an $n \mathrm{D}$-system is by first setting up a corresponding shortest path problem and to apply an algorithm, like Dijkstra's algorithm [32] or Floyd's algorithm [38], to solve it. A drawback is that the computation of an optimal shortest path along these lines can be quite expensive. On the other hand, the numerical complexity of the computation of the action of $A_{r}^{T}$ based on a shortest path solution can be shown to depend only linearly on the total degree of the polynomial $r$. Interestingly, suboptimal paths can easily be designed which also achieve a numerical complexity which depends linearly on the total degree of $r$. In the case of two-dimensional systems when there is no additional structure in the first-order derivatives of $p_{\lambda}$, the shortest path problem can be solved analytically. For three-dimensional systems the situation is more complicated but a number of partial results are available and presented in this chapter.

A numerical example is given in the first section of Chapter 7 where the approach described in this chapter is demonstrated by means of a worked example and compared to other approaches available in the literature for global optimization: SOSTOOLS, GloptiPoly and SYNAPS.

\subsection{The $n$ D-system}

In this section we pursue a state-space approach with respect to the computation of the action of the linear operation of multiplication by a polynomial $r$ within $\mathbb{R}\left[x_{1}, \ldots, x_{n}\right] / I$, i.e., the action of the matrix $A_{r}$. More precisely, we will be con- 
cerned with the action of $A_{r}^{T}$ rather than with the action of $A_{r}$, as this will allow for a meaningful system-theoretic interpretation.

To this end we set up in discrete time an autonomous multidimensional system, also called an $n \mathrm{D}$-system, associated with the set of polynomials $d^{(i)},(i=1, \ldots, n)$ representing the first-order conditions as in (4.2). With any monomial $x_{1}^{\alpha_{1}} x_{2}^{\alpha_{2}} \cdots x_{n}^{\alpha_{n}}$ we associate an $n \mathrm{D}$-shift operator $\sigma_{1}^{\alpha_{1}} \sigma_{2}^{\alpha_{2}} \cdots \sigma_{n}^{\alpha_{n}}$ which acts on any multidimensional time series $y_{t_{1}, t_{2}, \ldots, t_{n}}$ according to the rule:

$$
\sigma_{1}^{\alpha_{1}} \sigma_{2}^{\alpha_{2}} \cdots \sigma_{n}^{\alpha_{n}}: y_{t_{1}, t_{2}, \ldots, t_{n}} \mapsto y_{t_{1}+\alpha_{1}, t_{2}+\alpha_{2}, \ldots, t_{n}+\alpha_{n}} .
$$

Imposing the usual linearity properties, this allows one to associate a homogeneous multidimensional difference equation with an arbitrary polynomial $r\left(x_{1}, x_{2}, \ldots, x_{n}\right)$ as follows:

$$
r\left(\sigma_{1}, \sigma_{2}, \ldots, \sigma_{n}\right) y_{t_{1}, t_{2}, \ldots, t_{n}}=0 .
$$

Applying this set-up to the system of polynomial equations $d^{(i)}$ for $i=1, \ldots, n$ as in (4.2) a system of $n$ linear homogeneous multidimensional difference equations is obtained, which can be written in the form:

$$
y_{t_{1}, \ldots, t_{i-1}, t_{i}+m, t_{i+1}, \ldots, t_{n}}=f^{(i)}\left(\sigma_{1}, \ldots, \sigma_{n}\right) y_{t_{1}, t_{2}, \ldots, t_{n}}, \quad(i=1, \ldots, n)
$$

where $f^{(i)}=-\frac{1}{2 d \lambda} \frac{\partial}{\partial x_{i}} q\left(x_{1}, \ldots, x_{n}\right) \in \mathbb{R}\left[x_{1}, \ldots, x_{n}\right]$ of total degree strictly less than $m$ (in the same way as in Equation (4.4)).

Let for each multidimensional time instant $t=\left(t_{1}, \ldots, t_{n}\right)$ the 'total time' be denoted by $|t|:=t_{1}+\ldots+t_{n}$. Equation (5.3) expresses the fact that the value of $y_{\bar{t}_{1}, \bar{t}_{2}, \ldots, \bar{t}_{n}}$ at any multidimensional 'time instant' $\bar{t}=\left(\bar{t}_{1}, \bar{t}_{2}, \ldots, \bar{t}_{n}\right)$, such that $\max \left\{\bar{t}_{1}, \bar{t}_{2}, \ldots, \bar{t}_{n}\right\}$ is greater than or equal to $m$, can be obtained from the set of values of $y_{t_{1}, t_{2}, \ldots, t_{n}}$ for which the multidimensional time instants have a total time $|t|:=t_{1}+$ $t_{2}+\ldots+t_{n}$ strictly less than the total time $|\bar{t}|=\bar{t}_{1}+\bar{t}_{2}+\ldots+\bar{t}_{n}$. As a consequence, any multidimensional time series $y_{t_{1}, t_{2}, \ldots, t_{n}}$ satisfying this system of recursions is uniquely determined by the finite set of (total degree reversed lexicographically ordered) values:

$$
w_{0,0, \ldots, 0}:=\left\{y_{t_{1}, t_{2}, \ldots, t_{n}} \mid t_{1}, t_{2}, \ldots, t_{n} \in\{0,1, \ldots, m-1\}\right\} .
$$

Conversely, each choice for $w_{0,0, \ldots, 0}$ yields a corresponding solution for $y_{t_{1}, t_{2}, \ldots, t_{n}}$. In state-space terms, the set of values $w_{0,0, \ldots, 0}$ acts as an initial state for the autonomous homogeneous system of multidimensional difference equations (5.3). This point of view can be formalized by introducing the state vector $w_{t_{1}, t_{2}, \ldots, t_{n}}$ at the multidimensional time instant $\left(t_{1}, t_{2}, \ldots, t_{n}\right)$ as the set of values:

$$
w_{t_{1}, t_{2}, \ldots, t_{n}}:=\left\{y_{t_{1}+s_{1}, t_{2}+s_{2}, \ldots, t_{n}+s_{n}} \mid s_{1}, s_{2}, \ldots, s_{n} \in\{0,1, \ldots, m-1\}\right\} .
$$

The elements of the state vector $w_{t_{1}, t_{2}, \ldots, t_{n}}$ should be ordered again by the total degree reversed lexicographical ordering.

According to this definition, two state vectors $w_{t_{1}, t_{2}, \ldots, t_{n}}$ and $w_{t_{1}+\alpha_{1}, t_{2}+\alpha_{2}, \ldots, t_{n}+\alpha_{n}}$, with $\alpha_{i} \geq 0$ for all $i=1, \ldots, n$, are related by:

$$
w_{t_{1}+\alpha_{1}, t_{2}+\alpha_{2}, \ldots, t_{n}+\alpha_{n}}=\sigma_{1}^{\alpha_{1}} \sigma_{2}^{\alpha_{2}} \cdots \sigma_{n}^{\alpha_{n}} w_{t_{1}, t_{2}, \ldots, t_{n}}
$$


where the $n \mathrm{D}$-shift operates on such state vectors in an element-wise fashion. Since this operator is linear, the latter relation can also be cast in the usual matrix-vector form. This requires a choice of basis. If this choice is made to correspond to the basis $B$ for the quotient space $\mathbb{R}\left[x_{1}, \ldots, x_{n}\right] / I$ and the associated monomial ordering, it holds that:

$$
w_{t_{1}, \ldots, t_{i-1}, t_{i}+1, t_{i+1}, \ldots, t_{n}}=\sigma_{i} w_{t_{1}, t_{2}, \ldots, t_{n}}=A_{x_{i}}^{T} w_{t_{1}, t_{2}, \ldots, t_{n}}
$$

where the matrix $A_{x_{i}}$ again denotes the matrix associated with multiplication by the polynomial $x_{i}$ within the quotient space $\mathbb{R}\left[x_{1}, \ldots, x_{n}\right] / I$. Note that its transpose $A_{x_{i}}^{T}$ is involved in this relationship. As a consequence the following holds:

$$
w_{t_{1}+\alpha_{1}, t_{2}+\alpha_{2}, \ldots, t_{n}+\alpha_{n}}=\left(A_{x_{1}}^{T}\right)^{\alpha_{1}}\left(A_{x_{2}}^{T}\right)^{\alpha_{2}} \cdots\left(A_{x_{n}}^{T}\right)^{\alpha_{n}} w_{t_{1}, t_{2}, \ldots, t_{n}},
$$

which shows that the general solution to the autonomous multidimensional system with initial state $w_{0,0, \ldots, 0}$ is given by:

$$
w_{t_{1}, t_{2}, \ldots, t_{n}}=\left(A_{x_{1}}^{T}\right)^{t_{1}}\left(A_{x_{2}}^{T}\right)^{t_{2}} \cdots\left(A_{x_{n}}^{T}\right)^{t_{n}} w_{0,0, \ldots, 0} .
$$

More generally, for an arbitrary polynomial $r\left(x_{1}, \ldots, x_{n}\right)$ it holds that:

$$
r\left(\sigma_{1}, \ldots, \sigma_{n}\right) w_{t_{1}, \ldots, t_{n}}=A_{r\left(x_{1}, \ldots, x_{n}\right)}^{T} w_{t_{1}, \ldots, t_{n}} .
$$

Note that $y_{t_{1}, t_{2}, \ldots, t_{n}}$ can be read off from this as being an element of $w_{t_{1}, t_{2}, \ldots, t_{n}}$.

Now we intend to use the recursions in Equation (5.3) rather than Equation (5.9) to compute $w_{t_{1}, t_{2}, \ldots, t_{n}}$ (and $y_{t_{1}, t_{2}, \ldots, t_{n}}$ ). In this way the action of a matrix $A_{r\left(x_{1}, \ldots, x_{n}\right)}^{T}$ on a given vector $v$ can be computed without constructing this matrix explicitly: only the relations between the time series as represented by (5.3) are required. The values of the time series in the initial state $w_{0, \ldots, 0}$ are known as they are chosen as the values in the given vector $v$.

A more detailed formulation of the $n \mathrm{D}$-systems approach is described in Theorem 3.1 of [13].

Recall that our interest is in computing the eigenvalues of the matrix

$A_{r\left(x_{1}, \ldots, x_{n}\right)}$, which coincide with the eigenvalues of its transpose $A_{r\left(x_{1}, \ldots, x_{n}\right)}^{T}$ and which may conveniently be studied from the perspective of the autonomous $n \mathrm{D}$-system introduced above. Note that if $v$ is an eigenvector of $A_{x_{i}}^{T}$ with a corresponding eigenvalue $\xi_{i}$, then it holds that:

$$
A_{x_{i}}^{T} v=\xi_{i} v .
$$

In terms of the $n \mathrm{D}$-system this implies that the choice $w_{0, \ldots, 0}:=v$ for the initial state produces a scaled version for the state: $w_{0, \ldots, 0,1,0, \ldots, 0}=\xi_{i} v$, which relates to a shift in the multidimensional time space by 1 in the direction of the $i$-th time axis only. However, the vectors $w_{0, \ldots, 0}$ and $w_{0, \ldots, 0,1,0, \ldots, 0}$ have $m^{n}-m^{(n-1)}$ elements in common (in shifted positions), showing that the eigenvectors of $A_{x_{i}}^{T}$ exhibit a special structure. This corresponds to the Stetter vector structure discussed in Chapter 4. 
Example 5.1. Let us again consider the polynomial:

$$
p_{1}\left(x_{1}, x_{2}\right)=\left(x_{1}^{4}+x_{2}^{4}\right)+x_{1}^{3}+2 x_{1}^{2}+3 x_{1} x_{2}
$$

and the associated first-order conditions for minimality:

$$
\left\{\begin{array}{lllll}
d^{(1)}\left(x_{1}, x_{2}\right) & =x_{1}^{3}+\frac{3}{4} x_{1}^{2}+x_{1} & +\frac{3}{4} x_{2} & =0 \\
d^{(2)}\left(x_{1}, x_{2}\right) & =x_{2}^{3}+\frac{3}{4} x_{1} & & =0
\end{array}\right.
$$

as studied before in Example 4.3. The polynomials $f^{(1)}\left(x_{1}, x_{2}\right)$ and $f^{(2)}\left(x_{1}, x_{2}\right)$ in this example are:

$$
\left\{\begin{array}{l}
f^{(1)}\left(x_{1}, x_{2}\right)=-\frac{3}{4} x_{1}^{2} \quad-x_{1} \quad-\frac{3}{4} x_{2} \\
f^{(2)}\left(x_{1}, x_{2}\right)=-\frac{3}{4} x_{1}
\end{array}\right.
$$

Now we are not directly interested in computing the global minimum of $p_{1}\left(x_{1}, x_{2}\right)$ but rather in (i) computing the action of the matrices $A_{x_{1}}^{T}$ and $A_{x_{2}}^{T}$ and, more generally, (ii) computing the action of the matrix $A_{r\left(x_{1}, x_{2}\right)}^{T}$ on a given vector $v$ without constructing these matrices explicitly. Recall that the action on a given vector serves as the input for an iterative eigenproblem solver to compute some of the eigenvalues of the corresponding matrix.

(i) To focus attention on the computation of the actions of the matrices $A_{x_{1}}^{T}$ and $A_{x_{2}}^{T}$ directly from $f^{(1)}\left(x_{1}, x_{2}\right)$ and $f^{(2)}\left(x_{1}, x_{2}\right)$, without constructing these matrices explicitly, the following associated system of two-dimensional difference equations is considered:

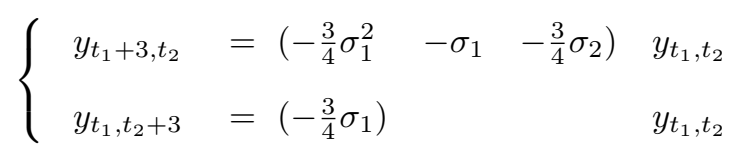

which is written in the following equivalent form:

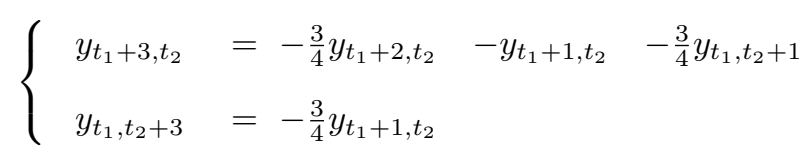

An initial state for this autonomous 2-dimensional system is constituted by $w_{0,0}=$ $\left(y_{0,0}, y_{1,0}, y_{2,0}, y_{0,1}, y_{1,1}, y_{2,1}, y_{0,2}, y_{1,2}, y_{2,2}\right)^{T}$ which corresponds to a $3 \times 3$ square array of values at integer time instants $\left(t_{1}, t_{2}\right)$ in the 2 -dimensional time plane. The relations between the various values of the two-dimensional time series in the equations in (5.16) for $t_{1}=t_{2}=0$ are displayed in Figure 5.1 by the arrows. The red square denotes the initial state $w_{0,0}$.

The action of the matrix $A_{x_{1}}^{T}$ on $w_{0,0}$ yields the vector $A_{x_{1}}^{T} w_{0,0}=w_{1,0}=\left(y_{1,0}\right.$, $\left.y_{2,0}, y_{3,0}, y_{1,1}, y_{2,1}, y_{3,1}, y_{1,2}, y_{2,2}, y_{3,2}\right)^{T}$, which also corresponds to a $3 \times 3$ square array of integer time instants $\left(t_{1}, t_{2}\right)$ in the 2-dimensional time plane. Compared to the location of the initial state $w_{0,0}$, this array is shifted by one unit along 


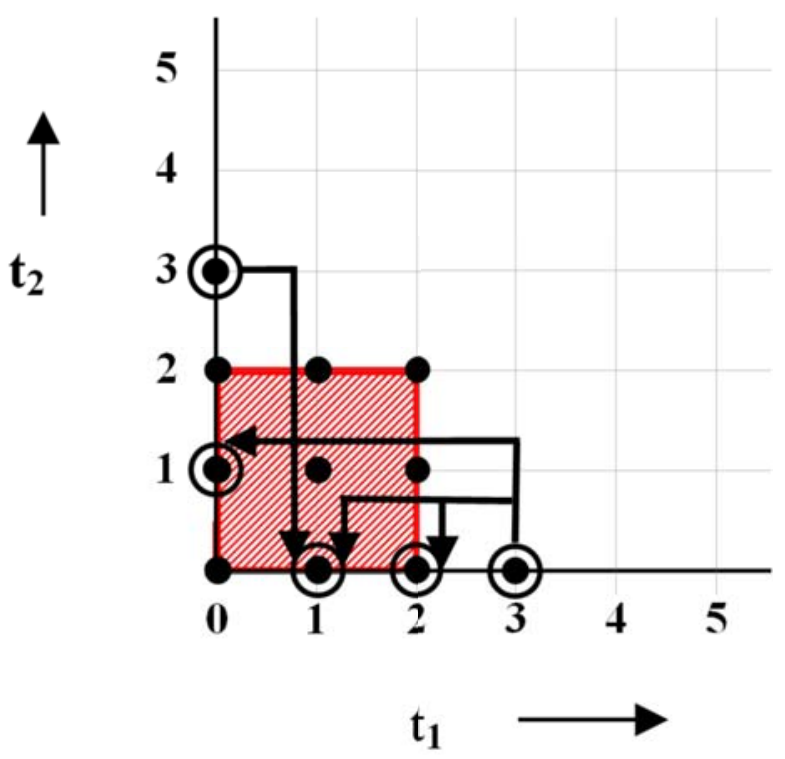

Figure 5.1: Relationship between time series in Equation (5.16) and the initial state $w_{0,0}$ (red square)

the $t_{1}$-axis. Thus there is an overlap of six two-dimensional time instants between the two arrays, which makes that only the additional computation of $y_{3,0}, y_{3,1}$ and $y_{3,2}$ is required. The values of $y_{3,0}$ and $y_{3,1}$ are readily obtained from the given values in the initial state through application of the first difference equation in (5.16). However, computation of $y_{3,2}$ requires prior computation of $y_{0,3}$. The latter can only be achieved through application of the second difference equation of (5.16). Similarly, the action of the matrix $A_{x_{2}}^{T}$ on $w_{0,0}$ yields the vector $A_{x_{2}}^{T} w_{0,0}=w_{0,1}=\left(y_{0,1}, y_{1,1}, y_{2,1}, y_{0,2}, y_{1,2}, y_{2,2}, y_{0,3}, y_{1,3}, y_{2,3}\right)^{T}$, which can be computed in a similar fashion.

(ii) More generally, the action of any matrix $r\left(A_{x_{1}}^{T}, A_{x_{2}}^{T}\right)$ on $w_{0,0}$, where $r\left(x_{1}, x_{2}\right)$ is some arbitrary polynomial, can be computed as a linear combination of the values of the two-dimensional time series $y_{t_{1}, t_{2}}$ at various $3 \times 3$ square arrays of two-dimensional time instants. For instance, the action of the matrix $A_{p_{1}\left(x_{1}, x_{2}\right)}^{T}=p_{1}\left(A_{x_{1}}^{T}, A_{x_{2}}^{T}\right)$ on $w_{0,0}$ can be obtained by a linear combination of the individual actions of the matrices $\left(A_{x_{1}}^{T}\right)^{4},\left(A_{x_{2}}^{T}\right)^{4},\left(A_{x_{1}}^{T}\right)^{3},\left(A_{x_{1}}^{T}\right)^{2}$ and $A_{x_{1}}^{T} A_{x_{2}}^{T}$ on $w_{0,0}$. In Figure 5.2 the locations of the time instants of these five actions on $w_{0,0}$ are visualized.

Here it is noted that the action of any matrix of the form $\left(A_{x_{1}}^{T}\right)^{\alpha_{1}}\left(A_{x_{2}}^{T}\right)^{\alpha_{2}}$ on $w_{0,0}$ yields the vector $\left(A_{x_{1}}^{T}\right)^{\alpha_{1}}\left(A_{x_{2}}^{T}\right)^{\alpha_{2}} w_{0,0}=\left(y_{\alpha_{1}, \alpha_{2}}, y_{\alpha_{1}+1, \alpha_{2}}, y_{\alpha_{1}+2, \alpha_{2}}, y_{\alpha_{1}, \alpha_{2}+1}\right.$, $\left.y_{\alpha_{1}+1, \alpha_{2}+1}, y_{\alpha_{1}+2, \alpha_{2}+1}, y_{\alpha_{1}, \alpha_{2}+2}, y_{\alpha_{1}+1, \alpha_{2}+2}, y_{\alpha_{1}+2, \alpha_{2}+2}\right)^{T}$. A systematic procedure to arrive at the value of $y_{t_{1}, t_{2}}$ at an arbitrary $2 \mathrm{D}$-time instant $\left(t_{1}, t_{2}\right)$, is to compute 


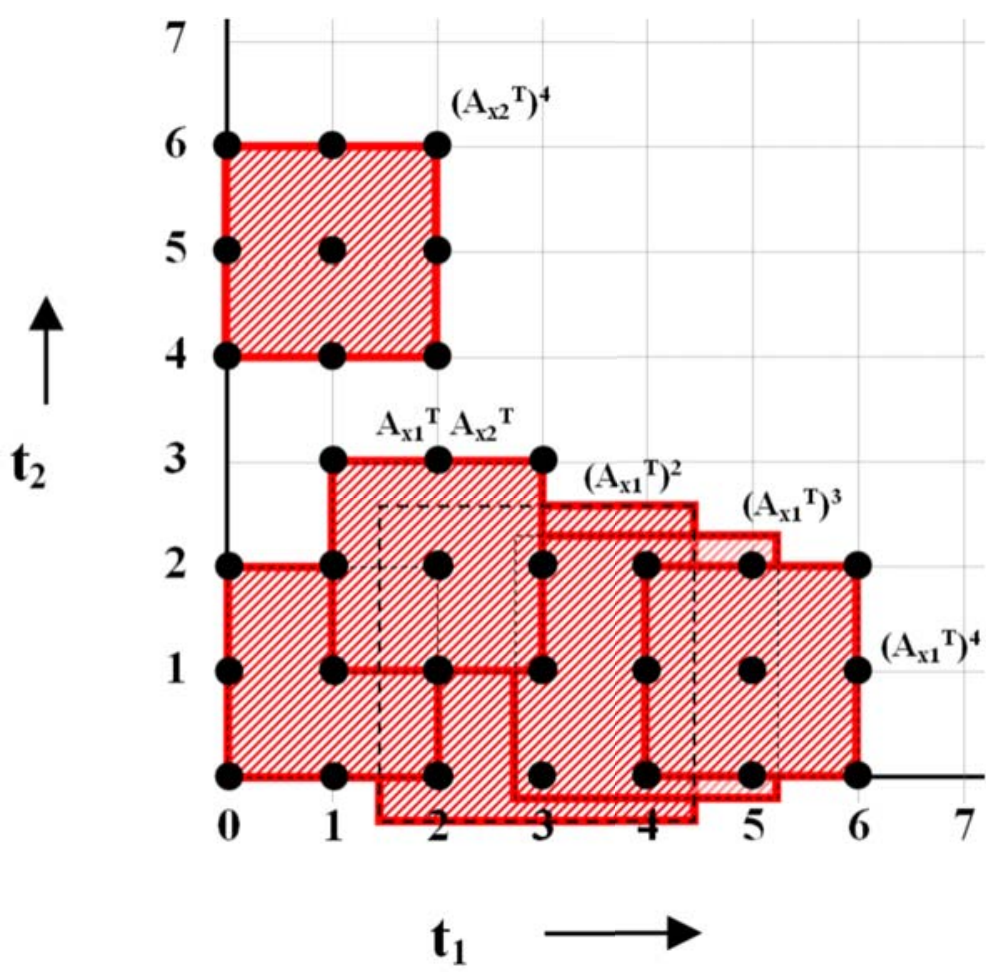

Figure 5.2: Time instants to compute the action of $A_{p_{1}}^{T}$

the values along the (finite) diagonals for which $t_{1}+t_{2}$ is constant, for increasing values of this constant.

One last remark concerns the computation of all the coordinates of a stationary point from the eigenvector of a single matrix such as $A_{x_{1}}^{T}$. Note that if $v$ denotes an eigenvector of the matrix $A_{x_{1}}^{T}$ corresponding to an eigenvalue $\xi_{1}$, then the vector $v=$ $\left(y_{0,0}, y_{1,0}, y_{2,0}, y_{0,1}, y_{1,1}, y_{2,1}, y_{0,2}, y_{1,2}, y_{2,2}\right)^{T}$, and the vector $A_{x_{1}}^{T} v=\left(y_{1,0}, y_{2,0}, y_{3,0}\right.$, $\left.y_{1,1}, y_{2,1}, y_{3,1}, y_{1,2}, y_{2,2}, y_{3,2}\right)^{T}$ differ by the scalar factor $\xi_{1}$. This implies that the columns of the matrix

$$
\left(\begin{array}{cccc}
y_{0,0} & y_{1,0} & y_{2,0} & y_{3,0} \\
y_{0,1} & y_{1,1} & y_{2,1} & y_{3,1} \\
y_{0,2} & y_{1,2} & y_{2,2} & y_{3,2}
\end{array}\right)
$$

are all dependent: each column is equal to $\xi_{1}$ times its preceding column. If the vector $v$ also happens to be an eigenvector of the matrix $A_{x_{2}}^{T}$ corresponding to an eigenvalue $\xi_{2}$ (the theory of the previous chapter makes clear that such vectors $v$ always exist and in case of distinct eigenvalues of $A_{x_{1}}$ will automatically have this property) then the rows of the matrix exhibit a similar pattern: each row is equal 
to $\xi_{2}$ times its preceding row. Obviously, in such a situation the value $y_{0,0}$ can not be zero and it therefore can be used to produce a normalized common eigenvector $\left(1, \xi_{1}, \xi_{1}^{2}, \xi_{2}, \xi_{1} \xi_{2}, \xi_{1}^{2} \xi_{2}, \xi_{2}^{2}, \xi_{1} \xi_{2}^{2}, \xi_{1}^{2} \xi_{2}^{2}\right)^{T}$ of the matrices $A_{x_{1}}^{T}$ and $A_{x_{2}}^{T}$ from which the multi-eigenvalue $\left(\xi_{1}, \xi_{2}\right)$ can be read off directly.

\subsection{Usage of the $n$ D-system in polynomial optimization}

Modern methods for the solution of large eigenvalue problems have the attractive feature that they do not operate on the matrix $A_{r}^{T}$ directly. Instead they iteratively perform the action of the linear operator at hand, for which it suffices to implement a computer routine that computes this action for any given vector. The $n$ D-systems approach supports this and it offers a framework to compute the action of $A_{r}^{T}$ on a vector $v$, by initializing the initial state as $w_{0, \ldots, 0}:=v$ and using the $n$ recursions (5.3) in combination with the relationship (5.10) to obtain the vector $r\left(\sigma_{1}, \ldots, \sigma_{n}\right) w_{0, \ldots, 0}=A_{r}^{T} w_{0, \ldots, 0}$. Such an approach entirely avoids an explicit construction of the matrix $A_{r}$. Note that $r\left(\sigma_{1}, \ldots, \sigma_{n}\right) w_{0, \ldots, 0}$ consists of a linear combination of state vectors $w_{t_{1}, \ldots, t_{n}}$; each monomial term $r_{\alpha_{1}, \ldots, \alpha_{n}} x_{1}^{\alpha_{1}} \cdots x_{n}^{\alpha_{n}}$ that occurs in $r\left(x_{1}, \ldots, x_{n}\right)$ corresponds to a weighted state vector $r_{\alpha_{1}, \ldots, \alpha_{n}} w_{\alpha_{1}, \ldots, \alpha_{n}}$. This makes clear that for any choice of polynomial $r$ the vector $r\left(\sigma_{1}, \ldots, \sigma_{n}\right) w_{0, \ldots, 0}$ can be constructed from the same multidimensional time series $y_{t_{1}, \ldots, t_{n}}$ which is completely determined by (and computable from) the $n$ difference equations (5.3) and the initial state $w_{0, \ldots, 0}$.

There are two possible ways to retrieve the minimal value and the global mini$\operatorname{mizer}(\mathrm{s})$ of the polynomial $p_{\lambda}\left(x_{1}, \ldots, x_{n}\right)$.

(i) If attention is focused on the computation of all the stationary points of the criterion $p_{\lambda}$ first, then the actions of the matrices $A_{x_{i}}^{T}$, for all $i=1, \ldots, n$, play a central role since their eigenvalues constitute the coordinates of these stationary points. Given an initial state $w_{0, \ldots, 0}$, which is composed of $m^{n}$ values of the time series $y_{t_{1}, \ldots, t_{n}}$ (those for which $t_{j}<m$ for all $j$ ), each of these actions requires the computation of only $m^{n-1}$ additional values of the time series $y_{t_{1}, \ldots, t_{n}}$ (namely those for which $t_{i}=m$ and $t_{j}<m$ if $j \neq i$ ). However, most of these additional values can not be obtained directly (by application of a single recursion from the set (5.3)) from the initial state $w_{0, \ldots, 0}$. They require the use of more than one recursion from the set (5.3) and they involve the computation of values of $y_{t_{1}, \ldots, t_{n}}$ at certain other multidimensional time instants as well.

(ii) If attention is focused on the computation of the critical values of the criterion $p_{\lambda}$ first, rather than on the stationary points, then the polynomial $r$ may be chosen as $p_{\lambda}$ which requires the computation of values of $y_{t_{1}, \ldots, t_{n}}$ at multidimensional time instants which are somewhat further away from the origin $(0, \ldots, 0)$ than in the previous approach. For the computation of the action of the matrix $A_{p_{\lambda}}^{T}$ the set of multidimensional time instants $\left(t_{1}, \ldots, t_{n}\right)$ at which the value of $y_{t_{1}, \ldots, t_{n}}$ needs to be determined is clearly larger than for each of the matrices $A_{x_{i}}^{T}$. But for $A_{p_{\lambda}}^{T}$ only the smallest real eigenvalue is required that corresponds to a real stationary point, 
whereas the eigenvalues of the matrices $A_{x_{i}}^{T}$ correspond to the $i$-th coordinate of the stationary points (for which it is usually far less clear in advance in which range the value at the global optimum will be located). Therefore, when dealing with the matrix $A_{p_{\lambda}}^{T}$ one may focus on the calculation of only a few eigenvalues, as one is interested in the smallest real eigenvalue that corresponds to a real solution point. Iterative methods allow the user to 'zoom in' on a few eigenvalues, whereas in case of $A_{x_{i}}^{T}$ all the real eigenvalues need to be computed, for each $i=1,2, \ldots, n$, and all resulting real critical points have to be substituted into the criterion function to find the global optimum.

In this thesis, approach (ii) of computing only the smallest real eigenvalue of the matrix $A_{p_{\lambda}}^{T}$ is investigated because only one eigenvalue is required when searching for the global minimum.

\subsection{Efficiency of the $n \mathbf{D}$-systems approach}

The following research questions are addressed in this section: (i) For a given multidimensional time instant $\left(t_{1}, \ldots, t_{n}\right)$, what is the most efficient way to compute the value of $y_{t_{1}, \ldots, t_{n}}$ from a given initial state $w_{0, \ldots, 0}$ using the $n$ difference equations (5.3)? And as a closely related question, what is the most efficient way to compute the whole state vector $w_{t_{1}, \ldots, t_{n}}$ ? (ii) Can we design a suboptimal heuristic procedure that computes the state vector $w_{t_{1}, \ldots, t_{n}}$ at acceptable computational costs?

\subsubsection{A linear complexity result for computing $y_{t_{1}, \ldots, t_{n}}$}

The first research question raised above is especially important when the efficiency of just one iteration of the iterative eigenvalue solver is under consideration. In this single iteration the state vector $w_{t_{1}, \ldots, t_{n}}$ has to be computed in an efficient way. This in contrast to the case where we study the efficiency of solving the eigenvalue problem as a whole.

The following result addresses the computational complexity that can be achieved by an optimal algorithm to compute $y_{t_{1}, \ldots, t_{n}}$ from $w_{0, \ldots, 0}$.

Theorem 5.1. Consider a set of $n$ multidimensional recursions of the form (5.3) and let an initial state $w_{0, \ldots, 0}$ be given. Then every algorithm that computes the value of $y_{t_{1}, \ldots, t_{n}}$, using only the recursions (5.3), has a computational complexity which increases at least linearly with the total time $|t|$.

Proof. Each recursion from the set (5.3) allows one to compute the value of $y_{t_{1}, \ldots, t_{n}}$ from a set of values for which the total times are all within the range $|t|-m,|t|-m+1, \ldots,|t|-1$. The largest total time among the entries of the initial state $w_{0, \ldots, 0}$ corresponds to $y_{m-1, \ldots, m-1}$ and is equal to $n(m-1)$. Therefore, to express $y_{t_{1}, \ldots, t_{n}}$ in terms of the quantities contained in the initial state requires at least $\lceil(|t|-n(m-1)) / m\rceil$ applications of a recursion from the set (5.3). Hence, the computational complexity of any algorithm along such lines increases at least linearly 
with $|t|$.

On the other hand, it is not difficult to design an algorithm which achieves a computational complexity that is indeed linear in $|t|$. This may proceed as follows: since $y_{t_{1}, \ldots, t_{n}}$ is contained in $w_{t_{1}, \ldots, t_{n}}=\left(A_{x_{1}}^{T}\right)^{t_{1}}\left(A_{x_{2}}^{T}\right)^{t_{2}} \cdots\left(A_{x_{n}}^{T}\right)^{t_{n}} w_{0,0, \ldots, 0}$, it can be computed by the joint action of $t_{1}+\ldots+t_{n}=|t|$ matrices of the form $A_{x_{i}}^{T}$. It is not difficult to compute a fixed uniform upper bound on the computational complexity involved in the action of each of the matrices $A_{x_{i}}^{T}$, because only the time instants that have a total time which does not exceed $n(m-1)$ can assist in this computation and their number is finite. In view of the previous theorem this shows that an optimal algorithm for the computation of $y_{t_{1}, \ldots, t_{n}}$ has a computational complexity that increases linearly with the total time $|t|$. Clearly, similar arguments and results also hold for the computation of a state vector $w_{t_{1}, \ldots, t_{n}}$.

\subsubsection{Formulation as a shortest path problem}

The problem of finding an optimal algorithm for the computation of $y_{t_{1}, \ldots, t_{n}}$ from $w_{0, \ldots, 0}$ using the recursions (5.3) can be cast into the form of a shortest path problem (SPP). As it turns out, this SPP will quickly become huge and difficult to solve. However, it is possible to set up a relaxation of the shortest path problem (RSPP) which is considerably smaller and easier to solve. In the 2D-case (with full recursions and uniform costs) the RSPP can be solved analytically and its solution happens to solve the SPP too. A central role in this approach is played by the notion of stable patterns, which are shifted along the $2 \mathrm{D}$-grid. The same approach leads to partial results in the $3 \mathrm{D}$-case (and in higher dimensions). It also underlies the design of the heuristic methods discussed in Section 5.3.3.

In general, a standard formulation of a shortest path problem requires the specification of a weighted directed graph $G=\left(V, E, W, v_{I}, v_{T}\right)$, consisting of a set $V$ of nodes, a set $E \subseteq V \times V$ of edges, a weight function $W: E \rightarrow \mathbb{R}$, a set of initial nodes $v_{I} \in V$ and a set of terminal nodes $v_{T} \in V$. To compute a shortest path from $v_{I}$ to $v_{T}$ with smallest total weight, one may apply any classical algorithm (e.g., those of Dijkstra or Floyd). The set $V$ should correspond to the various 'states' in which the computational procedure can be. It is natural to relate a node $v \in V$ in some way to a set of multidimensional time instants $\left(t_{1}, \ldots, t_{n}\right)$ for which the value of $y_{t_{1}, \ldots, t_{n}}$ is either already available or still requires computation. The edges $E$ represent 'state transitions' and they are naturally associated with the recursions in the set (5.3). The weight function $W$ is used to reflect the computational costs (e.g., the number of flops (floating point operations)) associated with these recursions.

In setting up a shortest path problem formulation, one may run into the problem that the number of elements in $V$ becomes infinite, since for $n \geq 2$ it may already happen that one can apply an infinite sequence of recursions without ever arriving at the specified multidimensional time instant $\left(t_{1}, \ldots, t_{n}\right)$. To avoid this, one may work backwards from $\left(t_{1}, \ldots, t_{n}\right)$, by figuring out sets of time instants with smaller total time which may assist in the computation of $y_{t_{1}, \ldots, t_{n}}$. Another feature of the problem 
is that many computations can be carried out in parallel since their exact order does not matter, so that many alternatives exist with equal performance. Already for small values of $n, m$ and $|t|$ this makes that the graph $G$ can become very large. Two helpful observations for constructing a useful shortest path formulation are: (i) any sequence of time instants which facilitates the computation of $y_{t_{1}, \ldots, t_{n}}$ from $w_{0, \ldots, 0}$ can always be reorganized such that the total time increases monotonically; (ii) the computation of values at time instants having the same total time can be carried out in any arbitrary order. Therefore, a node $v \in V$ can naturally be associated with a set of time instants all having the same total time, rather than with individual time instants. This is formalized in the following definition.

Definition 5.2. For $k=1,2, \ldots$, let $T_{k}$ be the set of all multidimensional time instants $t=\left(t_{1}, \ldots, t_{n}\right) \in \mathbb{N}_{0}^{n}$ for which $|t|=k$ and $\max \left\{t_{1}, \ldots, t_{n}\right\} \geq m$. Let $V_{k}$ be the power set of $T_{k}$ (i.e., the set of all its subsets). Let $V_{\star}$ be the set of time instants corresponding to $w_{0, \ldots, 0}$ (i.e., for which $\max \left\{t_{1}, \ldots, t_{n}\right\}<m$ ).

Given a specified time instant $t=\left(t_{1}, \ldots, t_{n}\right)$, define $V$ as the Cartesian product $V_{1} \times V_{2} \times \cdots \times V_{|t|}$. Define the set of initial nodes as $v_{I}=\{(\phi, \ldots, \phi)\}$ (where $\phi$ denotes the empty set) and the set of terminal nodes $v_{T}$ to consist of those tuples $v=\left(v_{1}, \ldots, v_{|t|}\right)$ for which $v_{|t|}=\{t\}$.

Define $E$ as the set of all the ordered pairs $(v, \tilde{v}) \in V \times V$ such that: $(i) \tilde{v}_{k}=v_{k}$ for precisely $|t|-1$ values of $k$ from the set $\{1, \ldots,|t|\}$; (ii) for the unique value of $k$ such that $\tilde{v}_{k} \neq v_{k}$ it holds that $v_{k}=v_{k+1}=\ldots=v_{|t|}=\phi$ and the set $\tilde{v}_{k}$ consists entirely of time instants $\left(\bar{t}_{1}, \ldots, \bar{t}_{n}\right)$ at which $y_{\bar{t}_{1}, \ldots, \bar{t}_{n}}$ can be computed from the values at the time instants contained in the union of sets $V_{\star} \cup v_{1} \cup v_{2} \cup \ldots \cup v_{k-1}$ through the application of a single recursion from the set (5.3).

Define $W: E \rightarrow \mathbb{R}$ to reflect the computational costs involved in the transitions from $v$ to $\tilde{v}$ contained in the set $E$. The computations for a time instant in the set $\tilde{v}_{k}$ which constitutes the difference between $v$ and $\tilde{v}$ through the application of a recursion from the set (5.3), require a certain number of flops as determined by the number of terms involved in that recursion. The computational costs of a transition from $v$ to $\tilde{v}$ are defined as the sum of all the (minimal) costs for the elements of that set $\tilde{v}_{k}$.

The weighted directed graph $G$ is defined as the tuple $G=\left(V, E, W, v_{I}, v_{T}\right)$. The shortest path problem (SPP) associated with $G$ models the optimal computation of $y_{t_{1}, \ldots, t_{n}}$ from $w_{0, \ldots, 0}$ using the recursions (5.3).

Note that the graph $G$ for the SPP has a tree structure rather than a network structure, which makes it possible to apply branch and bound techniques for tree searching. However, because $V=V_{1} \times V_{2} \times \cdots \times V_{|t|}$, the size of the SPP quickly becomes very large.

An interesting relaxation of the shortest path problem (RSPP) is obtained when condition (ii) in the definition of $E$ is replaced by the less restrictive condition that the set $\tilde{v}_{k}$ consists entirely of time instants $\left(t_{1}, \ldots, t_{n}\right)$ at which $y_{t_{1}, \ldots, t_{n}}$ can be computed from the values at the time instants contained in the union of sets $V_{\star} \cup T_{1} \cup \ldots \cup T_{k-2} \cup$ $v_{k-1}$ through the application of a single recursion from the set (5.3). In this case, each 
node $v=\left(v_{1}, \ldots, v_{|t|}\right) \in V$ can be restricted to the set $v_{k} \in V_{k}$ for which $k$ is as large as possible with $v_{k}$ non-empty. Then $V$ can be redefined as $V=V_{1} \cup \ldots \cup V_{|t|-1} \cup V_{|t|}$, the set of initial nodes can be replaced by a single initial node $v_{I}=\phi$ and the set of terminal nodes by a single terminal node $v_{T}=\{t\}$.

The number of nodes in $V$ is considerably smaller for the RSPP than for the SPP, and it becomes much easier to compute a solution. The optimal value of the RSPP provides a lower bound for the optimal value of the SPP. In case the recursions in (5.3) are not sparse, this lower bound is likely to be close to the optimal value of the SPP.

For the RSPP, each node in $V$ is a collection of time instants sharing the same total time, say $k$. Such a node is a subset of $T_{k}$, and the sets $T_{k}$ correspond to parallel hyperplanes in $\mathbb{N}_{0}^{n}$. Therefore, it is natural to compare the geometric patterns exhibited by nodes, by means of translations in $\mathbb{N}_{0}^{n}$. Such a translation $\tau_{s}$ involving a translation vector $s=\left(s_{1}, \ldots, s_{n}\right) \in \mathbb{Z}^{n}$, acts on a node $N$ by acting elementwise on each of the time instants $\left(t_{1}, \ldots, t_{n}\right)$ contained in $N$ according to the rule $\left(t_{1}, \ldots, t_{n}\right) \mapsto\left(t_{1}+s_{1}, \ldots, t_{n}+s_{n}\right)$. To describe the structure of an optimal solution to the RSPP, the concept of a stable pattern is useful.

Definition 5.3. In the graph $G=\left(V, E, W, v_{I}, v_{T}\right)$ corresponding to the $R S P P, a$ node $N_{b} \in V$ is said to exhibit a stable pattern if there exists a translation $\tau_{s}$ with the property that $N_{a}=\tau_{s}\left(N_{b}\right)$ is a node in $V$ for which $\left(N_{a}, N_{b}\right)$ is an edge in $E$.

Clearly, translation vectors $s$ associated with stable patterns have the property that $|s|=-1$ because of the condition that $\left(N_{a}, N_{b}\right)$ should be an edge in $E$. Note that a stable pattern may be associated with several different translation vectors. A situation of special interest occurs for translation vectors $s$ of the form $s=(0, \ldots, 0,-1,0, \ldots, 0)$ as they correspond to (backward) shifts along one of the $n$ different time axes.

When a node $N \in V_{k}$ exhibits a stable pattern for some associated translation $\tau_{s}$, then repeated application of $\tau_{s}$ produces a sequence of nodes in $V$ with decreasing total times $k, k-1, k-2, \ldots$, until the boundary of the non-negative orthant $\mathbb{N}_{0}^{n}$ is reached. Now the idea is to construct an optimal path which solves the RSPP in a backwards fashion as a composition of three parts: (i) a part which connects the terminal node $v_{T}=\{t\}$ to a node exhibiting a stable pattern, (ii) a part in which the stable pattern is repeatedly translated using an associated translation $\tau_{s}$ with $|s|=-1$, (iii) a part which connects it to the initial node $v_{I}=\phi$.

\section{The 2D-case}

In the 2D-case it is possible to solve the RSPP analytically, for the situation of 'full recursions' (i.e., the polynomials $f^{(i)}\left(x_{1}, x_{2}\right), i=1,2$, involve all the possible terms of total degree $\leq m-1$ with non-zero coefficients) when 'uniform costs' are applied (i.e., the costs associated with the application of a recursion to compute a value $y_{t_{1}, t_{2}}$ are always the same, for each recursion). 
Of course, if a stable pattern is to assist in the construction of a shortest path, the costs associated with its translation need to be as small as possible. For the case of full recursions and uniform costs, this implies that the size of a stable pattern needs to be as small as possible (i.e., the number of time instants involved). A stable pattern is called a minimal stable pattern if it does not contain a strict subset which is also a stable pattern.

In the $2 \mathrm{D}$-case, the nodes are subsets of the diagonals for which $t_{1}+t_{2}$ is constant. As an example, for $m=3$ a minimal stable pattern consists of a subset of 5 consecutive points on a diagonal. This is depicted in Figure 5.3, together with translations on several subdiagonals. For an arbitrary value of $m$ we have the following result.

Proposition 5.4. In the 2D-case with full recursions and uniform costs, a minimal stable pattern consists of $2 m-1$ consecutive points on a diagonal of constant total time. This minimal stable pattern can be associated both with a shift in the direction of the $t_{1}$-axis and with a shift in the direction of the $t_{2}$-axis.

Proof. To reach a point on a diagonal $T_{k}$ (with total time $k$ ) requires one of the two full recursions. Each such full recursion involves $m$ consecutive points on the first subdiagonal $T_{k-1}$. Therefore, any stable pattern involves at least a subset of $m$ consecutive points. To compute such a subset, each of its $m$ points also requires one of the two full recursions. Consider two consecutive points of this subset and suppose they are computed by employing two different recursions, then this requires either $2 m-1$ consecutive points on the next subdiagonal $T_{k-2}$, or it requires $2 m$ points (consisting of two disconnected groups of $m$ points) on $T_{k-2}$, which is less efficient. When all $m$ points involve the same recursion this also requires $2 m-1$ consecutive points on $t_{k-2}$. Hence any stable pattern involves at least a subset of $2 m-1$ consecutive points.

Now, a subset of $2 m-1$ consecutive points constitutes a stable pattern. To see this, one may require the $m-1$ points with largest $t_{1}$-values to be computed with the recursion involving $f^{(1)}$, and the $m-1$ points with largest $t_{2}$-values with the recursion involving $f^{(2)}$. The point in the middle (the $m$-th point of the stable pattern) may be computed either with the recursion involving $f^{(1)}$ or with the recursion involving $f^{(2)}$. In either case, this involves only a subset of $2 m-1$ consecutive points on the next subdiagonal. Hence we are dealing with a minimal stable pattern.

If the middle point of the stable pattern is computed with the recursion involving $f^{(1)}$, the translation involved is $s=(-1,0)$, which constitutes a (backward) shift in the $t_{1}$-direction. When the recursion involving $f^{(2)}$ is employed, the translation involves $s=(0,-1)$ which constitutes a (backward) shift in the $t_{2}$-direction. Hence the minimal stable pattern can be associated with shifts in both directions of the time axes.

The proof above makes clear that the minimal stable pattern of $2 m-1$ consecutive points can be used to construct the second part of an optimal solution path for the RSPP in this 2D-case, using a sequence of translations involving the vectors $(-1,0)$ and $(0,-1)$. It also is indicated how the first part of such a solution can be constructed 

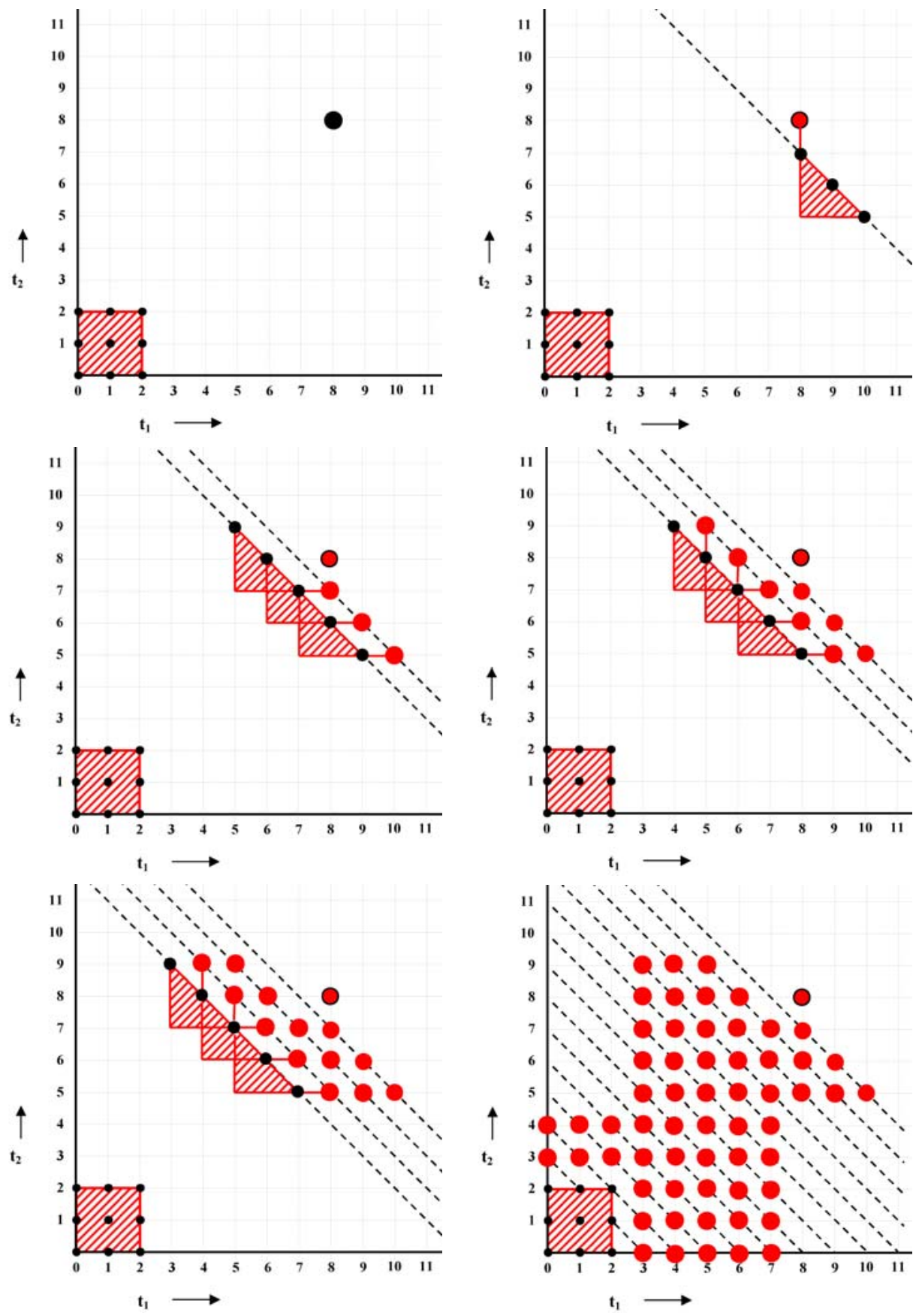

Figure 5.3: Constructing and translating a minimal stable pattern for $n=2, m=3$ 
which connects the terminal node to such a stable pattern. For the third part, note that the diagonal with total time $2 m-2$ consists of precisely $2 m-1$ consecutive points in the non-negative orthant. Its middle point, with coordinates $(m-1, m-1)$, corresponds to the right upper corner of the set of time-instants associated with $w_{0,0}$ and is therefore contained in $V_{\star}$. To compute $y_{t_{1}, t_{2}}$ at each of the other $2 m-2$ points requires the recursions $f^{(1)}$ and $f^{(2)}$ in an obvious way: for each points only one of the two recursions is feasible depending on which time-coordinate is $\geq m$. It should be clear that all points with total time $\leq 2 m-2$ not in $V_{\star}$ require computation. This is summarized in the following result, which is illustrated in Figure 5.4.

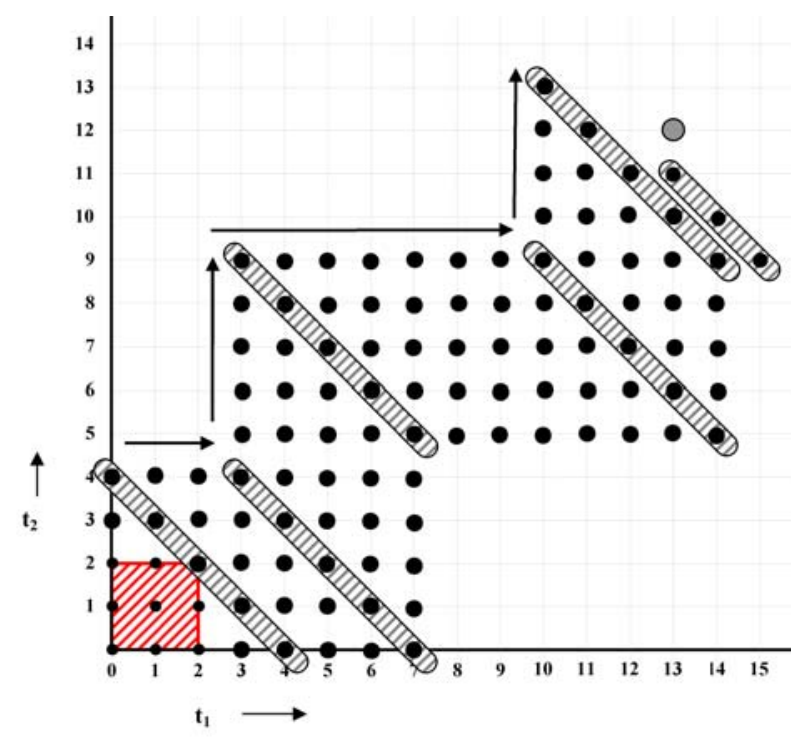

Figure 5.4: A shortest path to compute $y_{13,12}$

Proposition 5.5. In the 2D-case with full recursions and uniform costs, a solution to the RSPP has the following structure. (i) A part starting at $v_{I}=\phi$ followed by the nodes $v_{m}=T_{m}, v_{m+1}=T_{m+1}, \ldots, v_{2 m-2}=T_{2 m-2}$. (ii) A part involving the nodes $v_{2 m-1}, v_{2 m}, \ldots, v_{|t|-2}$ with the property that each of the nodes $v_{k}$ in this part involves a minimal stable pattern of $2 m-1$ consecutive points on the diagonal $T_{k}$ and that each two consecutive nodes are related by a translation $\tau_{s}$ with $s=(-1,0)$ or $s=(0,-1)$. (iii) A part involving the nodes $v_{|t|-1}$ and $v_{T}=\{t\}$. The node $v_{|t|-1}$ involves $m$ consecutive points on the diagonal $T_{|t|-1}$ such that there is an edge from $v_{|t|-2}$ to $v_{|t|-1}$ and an edge from $v_{|t|-1}$ to $v_{T}$.

Note that the above discussion is valid only when the terminal node $v_{T}=\{t\}$ satisfies $|t| \geq 2 m$. For a terminal node with total time $\leq 2 m-1$, no stable pattern occurs in the optimal path.

Interestingly, any solution to the RSPP in the 2D-case with full recursions and uniform costs is also a solution to the SPP itself. To see this, note that the RSPP 
has been obtained by considering only the time instants $\left(t_{1}, t_{2}\right)$ with $t_{1}+t_{2}=k-1$ and disregarding those with $t_{1}+t_{2}<k-1$ when the computation of a value $y_{t_{1}, t_{2}}$ at a time instant with $t_{1}+t_{2}=k$ is investigated. However, the relevant time-instants with $t_{1}+t_{2}<k-1$ are all in the triangular area of points that are both below and to the left of the $2 m-1$ points in the stable pattern on the diagonal with total time $k-1$. Hence, when the stable pattern is shifted along the time axes, this area is eventually entirely covered. See again Figure 5.3 and Figure 5.4.

Corollary 5.6. In the 2D-case with full recursions and uniform costs, a solution to the RSPP is also a solution to the SPP.

\section{The 3D-case}

In the 3D-case the situation is more complicated, also when restricting to the case of full recursions and uniform costs. Depending on the value of $m$, there may be several minimal stable patterns of different geometrical shape. Also, not every such stable pattern is associated with all of the three shifts along each of the time axes. On the other hand, it is possible to generalize the constructions in the 2D-case in a straightforward way to construct stable patterns which do have a symmetric geometrical shape and which are associated with all of the three shifts, although they are not minimal. Here we present partial results for the cases $m=2$ and $m=3$. These results also provide a rationale for the heuristic computational procedures developed and discussed in Section 5.3.3.

In the 3D-case with $m=2$, with full recursions and uniform costs, the initial state $w_{0,0,0}$ corresponds to values of $y_{t_{1}, t_{2}, t_{3}}$ at the time instants in $V_{\star}$, for which $t_{1}, t_{2}, t_{3} \in\{0,1\}$. Starting from the initial state, the three full recursions allow for the computation of $y_{t_{1}, t_{2}, t_{3}}$ at the time instants $(2,0,0),(0,2,0)$, and $(0,0,2)$, respectively, for which values are required at the time instants $(1,0,0),(0,1,0),(0,0,1)$, and $(0,0,0)$. This is depicted in Figures 5.5a and 5.5b. In Figure 5.6 a stable pattern is shown which allows for shifts in the directions of all the three time axes. This is a special instance of the following more general result for the $n \mathrm{D}$-case.

Proposition 5.7. In the $n D$-case a stable pattern is exhibited by translations of the set of time instants $N=\left\{\left(t_{1}, \ldots, t_{n}\right) \mid t_{1}+\ldots+t_{n}=n(m-1) ; t_{1} \geq 0, \ldots, t_{n} \geq 0\right\}$. This stable pattern allows for shifts along each of the $n$ time axes.

Proof. From the values of $y_{t_{1}, \ldots, t_{n}}$ at the time instants contained in $V_{\star}$, all its values in the non-negative orthant can be computed. This includes all the values at time instants for which the total time is $\leq n(m-1)$. Note that $(m-1, \ldots, m-1)$ is the time instant with the largest total time contained in $V_{\star}$. Now, the set $N$ describes all the time instants in the non-negative orthant with total time equal to $n(m-1)$. Together with all the time instants with a smaller total time, they allow for the (immediate) computation of $y_{t_{1}, \ldots, t_{n}}$ at all the time instants with total time $n(m-1)+1$. This includes all the translations of $N$ with translation vectors $s=(1,0, \ldots, 0), s=(0,1, \ldots, 0), \ldots, s=(0, \ldots, 0,1)$. This procedure can then be 


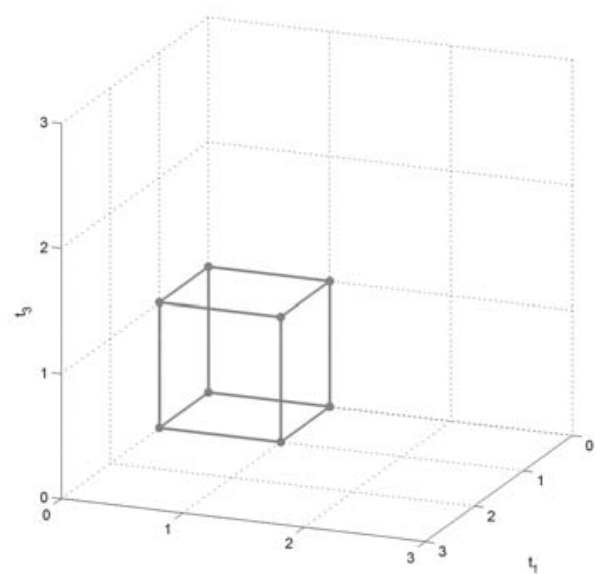

(a)

$$
\text { b }
$$

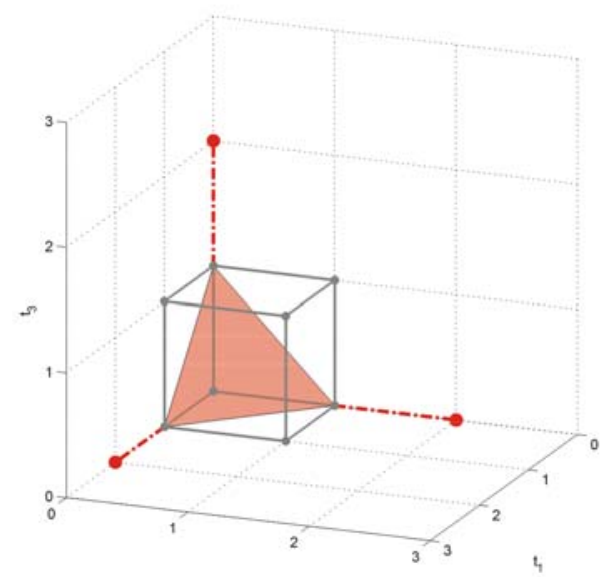

(b)

Figure 5.5: (a) The initial state $w_{0,0,0}$ (b) The initial state and the full recursions to compute $y_{2,0,0}, y_{0,2,0}, y_{0,0,2}$

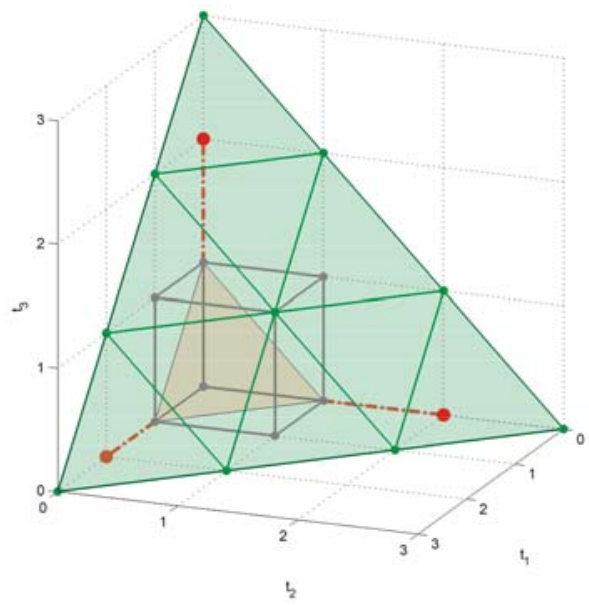

Figure 5.6: A stable pattern (green) for shifts in all three directions

repeated, yielding points with total time $n(m-1)+2$, etc., showing the pattern of points in $N$ to yield a stable pattern.

However, for $n>2$ the stable pattern $N$ in the above proposition is not minimal. To investigate minimality of stable patterns it is helpful to study the relationship 
between a point of total time $k$ and nearby points of total time $k-1$. (Alternatively, one may construct minimal stable patterns by deleting points from a stable pattern of the form $N$ until stability is lost.)

In Figure 5.7 it is shown for the case $n=3$ and $m=2$ how the points with total time 4 (blue layer, Figure a) are connected along the directions of the time axes (red lines) to nearby points of total time 3 (green layer, Figure b). In Figure 5.8b the points in these two consecutive layers are arranged in convenient triangulated patterns and shown from two different viewpoints (of which the last one will be used in the following).

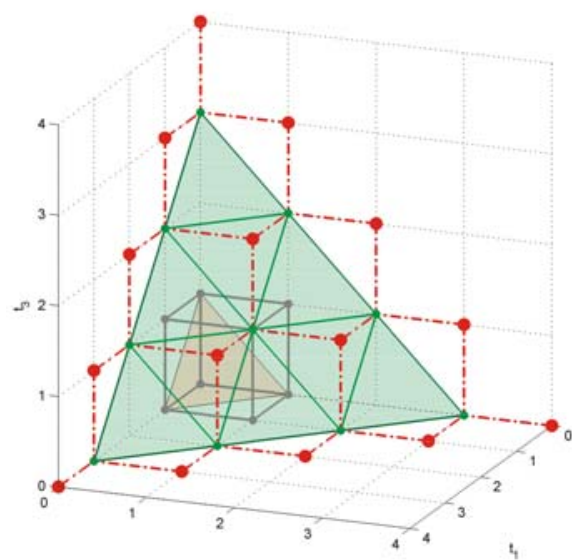

$(a)$

Figure 5.7: (a) Points with total time 4. (b) Connections of the points with total time 4 to nearby points of total time 3

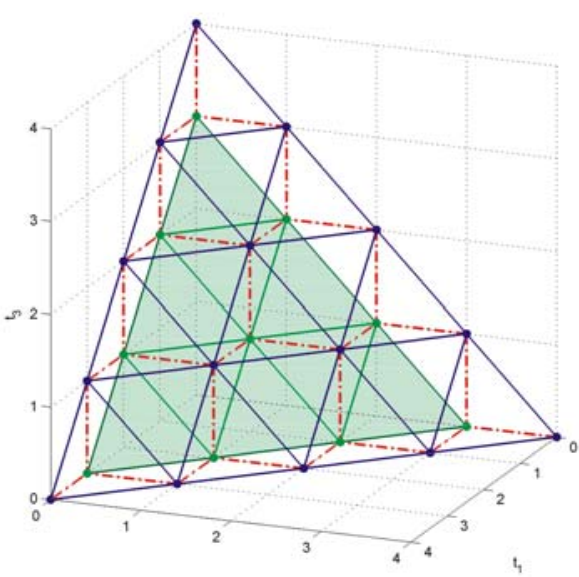

(b)

$\zeta$

In Figure 5.9 it is visualized how the three different full recursions associated with $f^{(1)}, f^{(2)}$ and $f^{(3)}$ may assist in the computation of a point with total time 4 , and also which points of total time 3 (red triangle) are required to achieve this.

For $n=3$ and $m=2$, the stable pattern $N$ in the previous proposition has 10 points. When two of its corner points are deleted, a minimal stable pattern of 8 points remains, which however allows only for a shift in just one of the directions of the time axes. But when only one corner point is deleted, a stable pattern results which allows for shifts in all three directions of the time axes.

As a consequence, a solution to the RSPP needs to take into account the coordinate values of the time instant $t=\left(t_{1}, t_{2}, t_{3}\right)$ associated with the terminal node $v_{T}$. First, a stable pattern containing 9 points can be used for shifting along the two axes corresponding to the two smallest values of $t_{1}, t_{2}$, and $t_{3}$. Then a cheaper minimal stable pattern containing 8 points can be used for shifting along the remaining third axis (this should be a subpattern of the previous pattern of 9 points). Note that such 

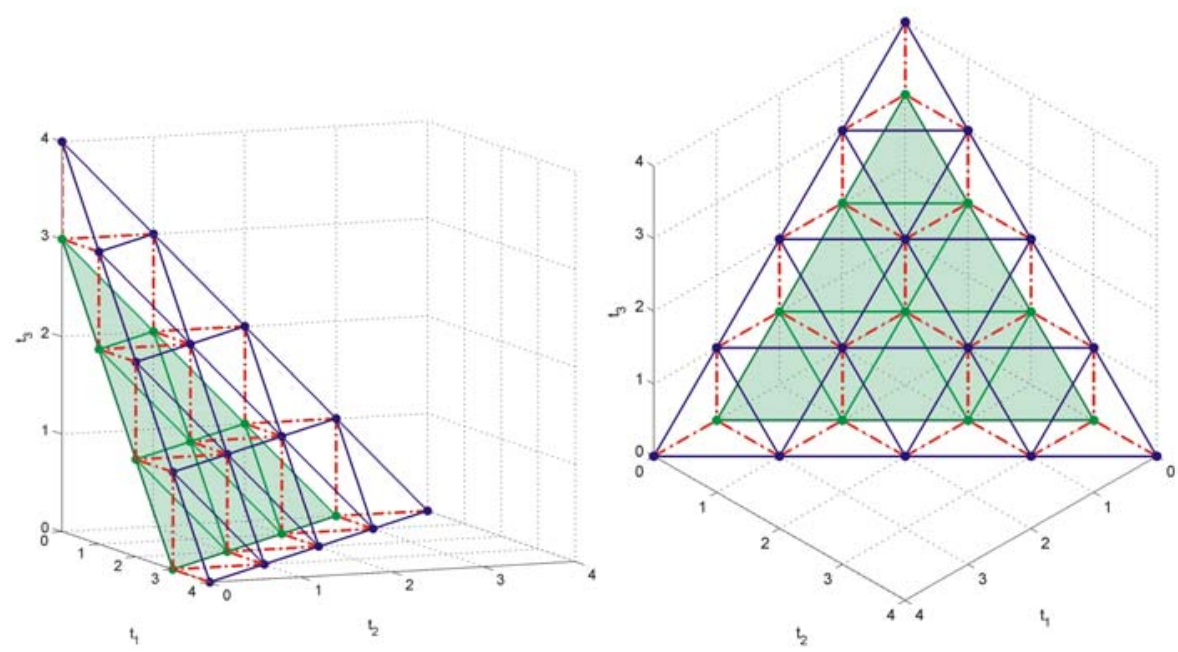

Figure 5.8: Two different viewpoints of Figure 5.7(b)

a solution also constitutes a solution to the SPP itself, because these stable patterns have convex configurations.

For the case $n=3$ and $m=3$, the stable pattern $N$ is depicted in Figure 5.10: all the points on the blue layer can be computed once all the points of the green layer are known. Here $N$ is a stable pattern which allows for shifts along each of the three time axes. However, $N$ is not minimal in this situation. Smaller stable patterns which allow for shifts in just one of the time axes are depicted in Figure 5.11: a green layer denotes the stable pattern from Figure 5.10 from which 4 points, denoted by the black dots, are removed. The patterns in the Figures $5.11 \mathrm{a}$, b, and c, respectively, allow for shifts along the $t_{1}, t_{2}$, and $t_{3}$ time axis. Although these three patterns are smaller than the non-minimal stable pattern $N$ in Figure 5.10, they are not minimal either.

When three (instead of four) points in one of the corners of $N$ in Figure 5.10 are deleted, a stable pattern remains which still allows for shifts in all three directions of the time axes. This demonstrates the complexity of the shortest path problem and its relaxation.

\subsubsection{Some heuristic procedures for the shortest path problem}

From the discussion in the previous section it is clear that the size and the complexity of the SPP increases rapidly with $n$. However, optimal solution of the SPP is not a goal by itself; it serves to facilitate the efficient computation of state vectors $w_{t_{1}, \ldots, t_{n}}$. 

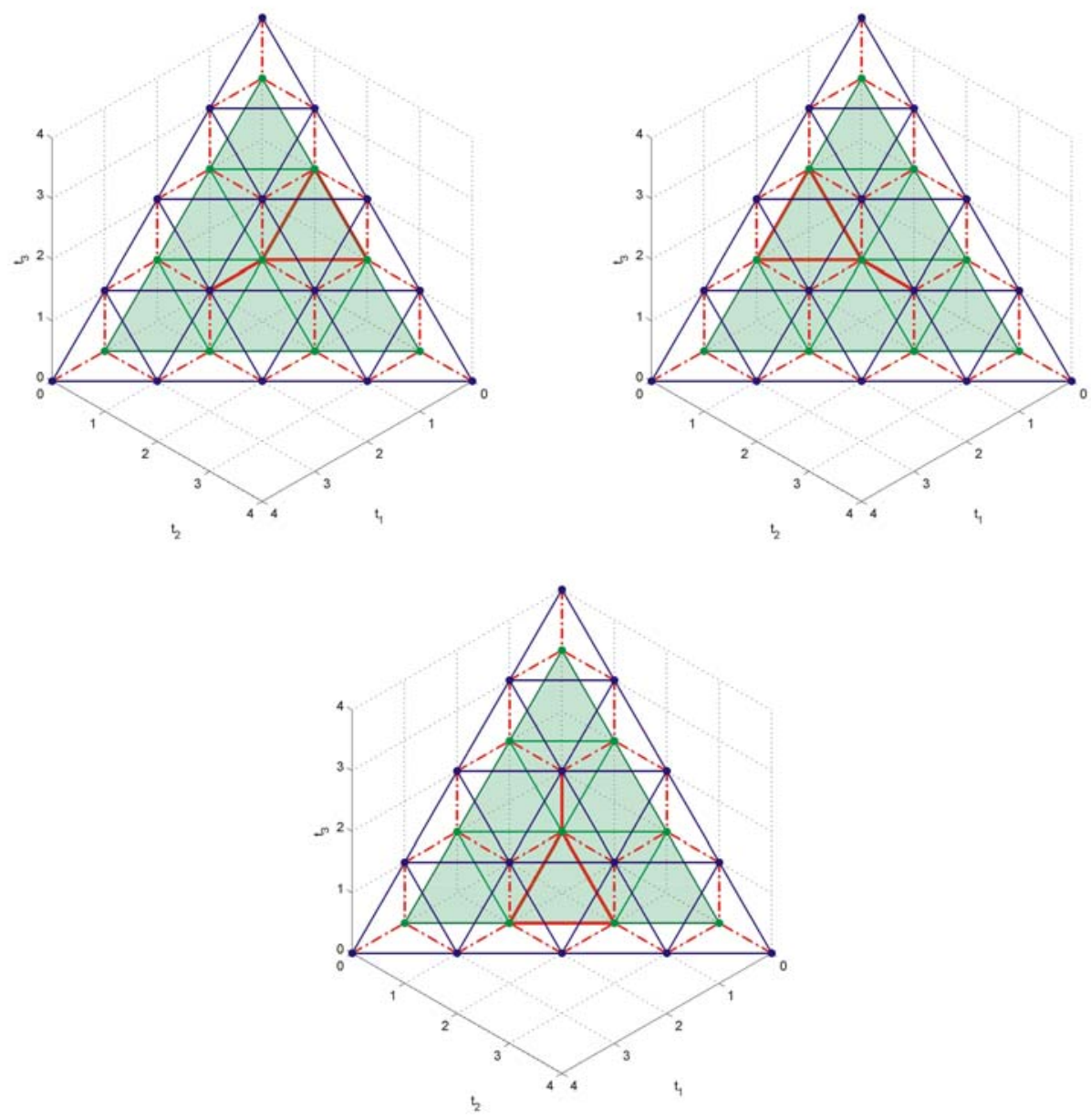

Figure 5.9: Computation of points with total time 4 using the three different recursions (associated with $f^{(1)}, f^{(2)}$ and $f^{(3)}$ ) involving points with total time 3

Note that in our application we need to solve the SPP only once (before the iterative eigenproblem solver is started), and that this solution can then be used in every iteration of the iterative eigenvalue solver (because the required path is the same in every iteration and already known). For the overall efficiency of the approach the number of iterations determines whether it is worthwhile to set up and solve the SPP, or if it is advisable to avoid this and to employ cheaper but suboptimal heuristic methods instead.

Using the insights from the previous section, five heuristic methods are developed to calculate the value $y_{t_{1}, \ldots, t_{n}}$ that appears in a state vector $w_{t_{1}, t_{2}, \ldots, t_{n}}$ with suboptimal but acceptable computational effort. The performance of these methods is 


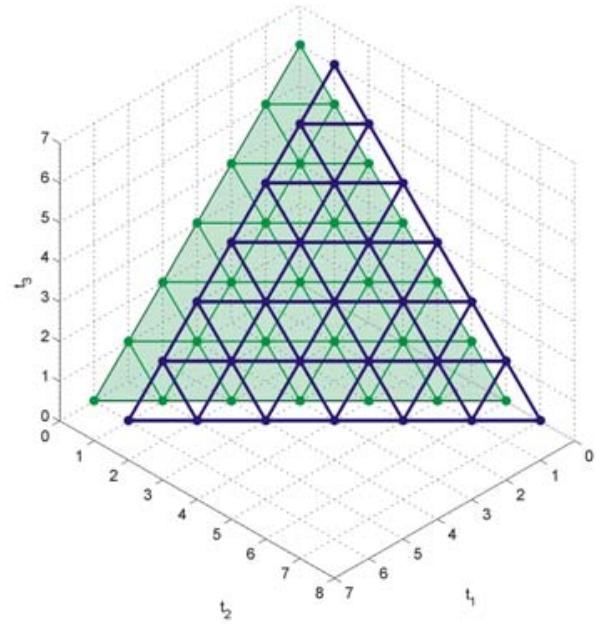

Figure 5.10: A non-minimal stable pattern for $n=3$ and $m=3$ allowing for shifts in three time directions

compared for a number of situations involving full recursions and uniform costs.

The heuristic methods under investigation are the following.

(i) The linear method starts by computing the values of $y_{t_{1}, \ldots, t_{n}}$ at all the points on and below the hyperplane which corresponds to the total time $n(m-1)$, thereby just covering the area (hypercube) of initial values. Then this stable pattern of values $N$ (see Proposition 5.7) is shifted step by step, with each step involving a single shift in one of the axis directions, until the requested location is reached. The sequence of shifts may depend on the multidimensional time instant at the requested location, but this does not affect the total amount of points encountered in this procedure.

(ii) The diagonal method proceeds by first computing $y_{t_{1}, \ldots, t_{n}}$ for all the time instants with constant total time $|t|=1$, then all those with total time $|t|=2$ and so on, until the requested location is reached.

(iii) The equalizing method proceeds by computing a value $y_{t_{1}, \ldots, t_{n}}$ by employing that recursion of the system (5.3) which reduces the largest possible coordinate of the time instant $y_{t_{1}, \ldots, t_{n}}$ (which is larger than $2 d-2$, to exclude negative time indices). It uses a lexicographic ordering in case there is more than one largest coordinate. The path between the initial state $w_{0, \ldots, 0}$ and the requested location is determined in a backwards fashion, i.e., starting from the requested location. This method has the effect that the calculated points are first directed towards the diagonal and then to the origin.

(iv) The axis method uses the same methodology as the equalizing method with the difference that it applies that recursion of the system (5.3) which reduces the smallest possible coordinate of the time instant $y_{t_{1}, \ldots, t_{n}}$ which is larger than $2 d-2$. This 
$(a)$

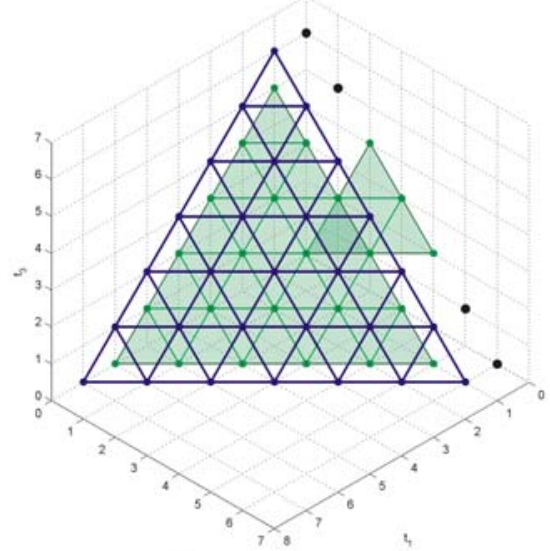

(b)

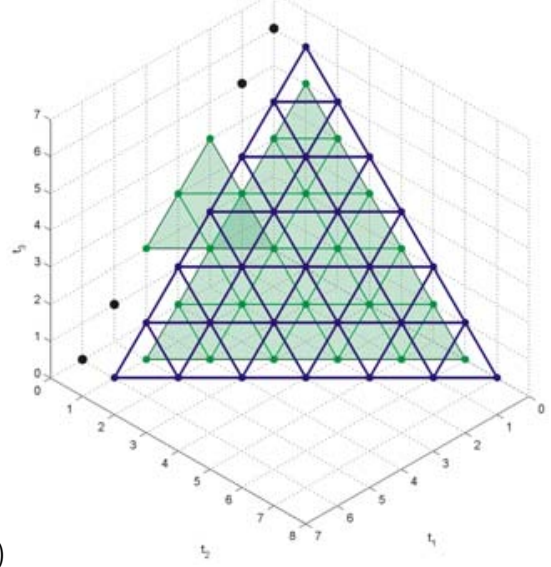

(c)

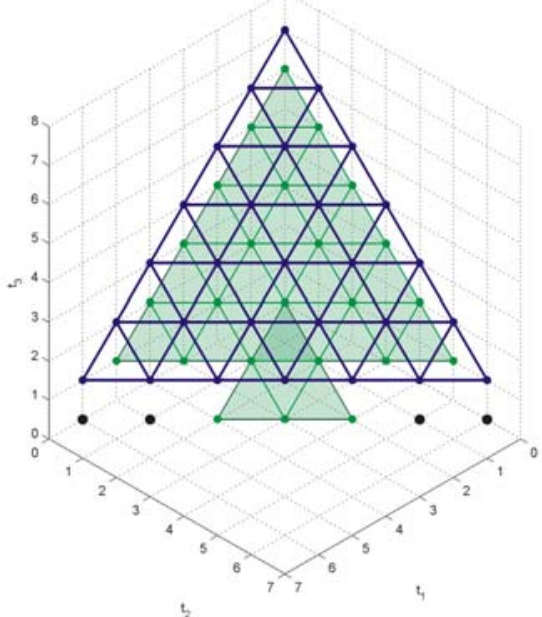

Figure 5.11: Non-minimal stable patterns (green layers) allowing for shifts along one time axis: (a) $t_{1}$ time axis, (b) $t_{2}$ time axis, (c) $t_{3}$ time axis

method has the effect that the calculated points are first directed towards the nearest axis and then to the origin.

(v) A slightly more sophisticated fifth method has been designed on the basis of insights gained from the test experiments with the first four methods introduced above. It is discussed later in this section and will be called the 'least-increments method'.

For the two-dimensional $(n=2)$ and three-dimensional $(n=3)$ cases the paths to a few particular points have been computed using the first four described methods. The results are collected in the Tables 5.1 and 5.2. For each method the total number 
of all stored points required for computing the requested point (i.e., the size of the path), the total number of floating point operations (flops) and the actual running time (in ms. obtained on a PC platform with an Intel Pentium PIV 2.8GHz processor and 512MB internal memory) are given for each requested point.

Table 5.1: Two-dimensional case $\left(2 \times 2\right.$ initial state $\left.w_{0,0}\right)$

\begin{tabular}{llrrrr}
\hline Requested point & & Linear & Diagonal & Equalizing & Axis \\
\hline$(0,10)$ & Stored points & 36 & 91 & 46 & 34 \\
& Flops & 100 & 265 & 167 & 118 \\
& Cpu time (ms) & 7 & 6 & 2 & 2 \\
$(0,50)$ & Stored points & 156 & 1431 & 546 & 154 \\
& Flops & 460 & 4285 & 2181 & 718 \\
$(0,100)$ & Cpu time (ms) & 32 & 23 & 25 & 8 \\
& Stored points & 306 & 5356 & 1921 & 304 \\
& Flops & 910 & 16060 & 7697 & 1468 \\
& Cpu time (ms) & 16 & 40 & 55 & 20 \\
\hline$(10,10)$ & Stored points & 66 & 276 & 64 & 143 \\
& Flops & 209 & 820 & 239 & 598 \\
$(50,50)$ & Cpu time (ms) & 30 & 20 & 1 & 20 \\
& Stored points & 306 & 5356 & 304 & 1723 \\
& Flops & 1009 & 16060 & 1199 & 7918 \\
$(100,100)$ & Cpu time (ms) & 40 & 40 & 10 & 60 \\
& Stored points & 606 & 20706 & 604 & 5948 \\
& Flops & 2009 & 62110 & 2399 & 27758 \\
& Cpu time (ms) & 20 & 120 & 50 & 220 \\
\hline
\end{tabular}

Table 5.2: Three-dimensional case $\left(2 \times 2 \times 2\right.$ initial state $\left.w_{0,0,0}\right)$

\begin{tabular}{llrrrr}
\hline Requested point & & Linear & Diagonal & Equalizing & Axis \\
\hline$(0,0,10)$ & Stored points & 120 & 560 & 159 & 88 \\
& Flops & 344 & 2216 & 832 & 399 \\
& Cpu time (ms) & 12 & 18 & 4 & 1 \\
$(0,0,50)$ & Stored points & 520 & 27720 & 4825 & 408 \\
& Flops & 1544 & 110856 & 32328 & 2199 \\
$(0,0,100)$ & Cpu time (ms) & 23 & 193 & 89 & 18 \\
& Stored points & 1020 & 192920 & 29808 & 808 \\
& Flops & 3044 & 771656 & 214696 & 4449 \\
$(10,10,10)$ & Cpu time (ms) & 32 & 1017 & 1875 & 33 \\
& Stored points & 320 & 7140 & 248 & 1868 \\
& Flops & 1040 & 28536 & 1379 & 10565 \\
$(50,50,50)$ & Cpu time (ms) & 20 & 40 & 1 & 20 \\
& Stored points & 1520 & 620620 & 1208 & 55348 \\
$(100,100,100)$ & Flops & 5040 & 2482456 & 7079 & 351861 \\
& Cpu time (ms) & 80 & 2774 & 60 & 2614 \\
& Stored points & 3020 & 4728720 & 2408 & 281948 \\
& Flops & 10040 & 18914856 & 14204 & 1892839 \\
& Cpu time (ms) & 50 & 265008 & 150 & 781646 \\
\hline
\end{tabular}

From this experiment we may conclude the following:

- The linear method indeed exhibits a linear complexity with respect to $|t|$. But in higher dimensions (e.g., $n>10$ ) the linear method may become inefficient because the simplex entirely covering the hypercube of initial values becomes very large. Although it constitutes a stable pattern associated with shifts in all 
$n$ time directions, it is non-minimal and especially for points near the initial hypercube more efficient paths can be constructed.

- The equalizing method performs best for points having (almost) equal coordinates (thus, points near the diagonal, for example the points $(10,10),(50,50)$ or $(100,100))$. For $n=2$ this method generates stable patterns which constitute an optimal solution to the shortest path problem for $y_{t_{1}, t_{2}}$ with $t_{1}=t_{2}$. For other points it is less efficient. Increasing the dimension $n$ for this method does not influence this behavior.

- The axis method performs well for points near the coordinate axes, (for example the points $(0,10),(0,50)$ or $(0,100))$. For $n=2$ this method generates stable patterns which constitute an optimal solution to the shortest path problem for $y_{t_{1}, t_{2}}$ with $t_{1}=0$ or $t_{2}=0$. Increasing the dimension $n$ for this method does not influence this behavior.

- The diagonal method does not exhibit a linear numerical complexity with respect to $|t|$ and is highly inefficient.

To further support and visualize these statements, some simulation experiments have been performed with $n=2$ and $m=3$, where the requested state vectors are further away from the origin: $w_{0,500}, w_{125,375}, w_{250,250}, w_{375,125}$ and $w_{500,0}$.

For the linear, diagonal, equalizing and axis methods, the points which are required for computing the state vectors $w_{0,500}, w_{250,250}$, and $w_{125,375}$, respectively, are displayed in three separate plots in the Figures 5.12, 5.13, 5.14 and 5.15.
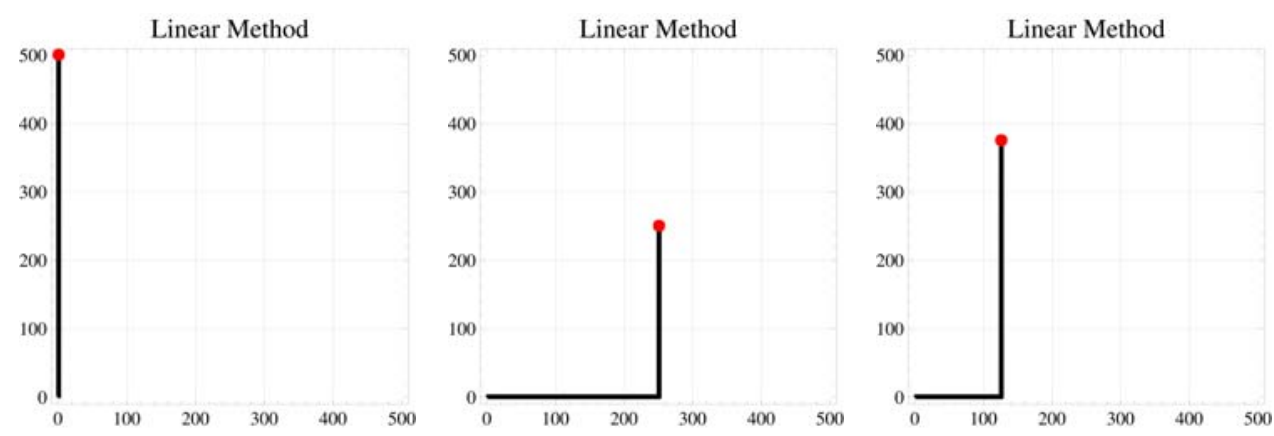

Figure 5.12: Points required by the Linear method for computing state vectors at time instants $(0,500),(250,250)$, and $(125,375)$ with a $3 \times 3$ initial state

From the Figures 5.12, 5.13, 5.14, and 5.15 one can see that the linear method has the best performance as it computes the fewest numbers of points in each situation and that the diagonal method has a highly inefficient performance for all locations of the requested state vectors. The equalizing and axis methods are only efficient in some specific situations: Figure 5.14 shows that the equalizing method is only efficient 

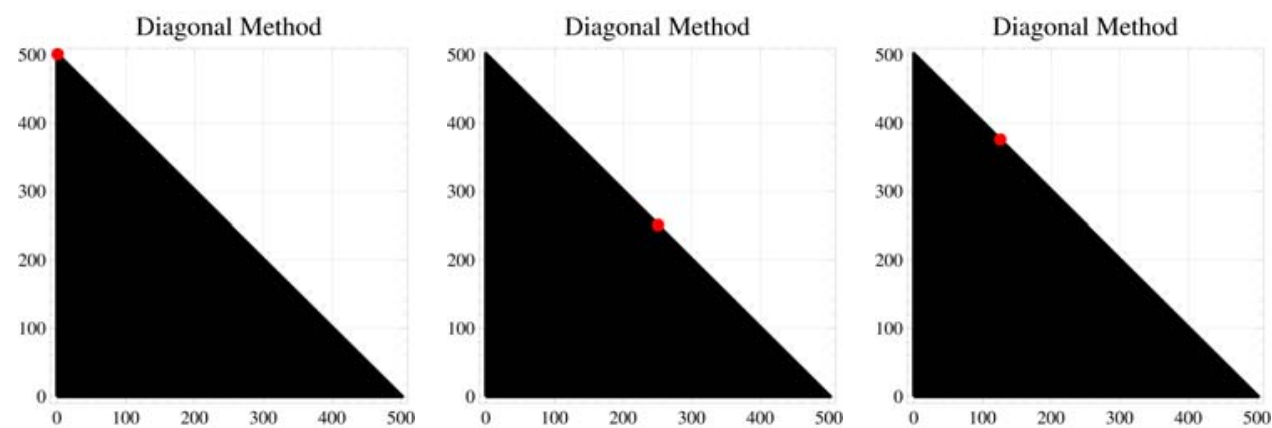

Figure 5.13: Points required by the Diagonal method for computing state vectors at time instants $(0,500),(250,250)$, and $(125,375)$ with a $3 \times 3$ initial state
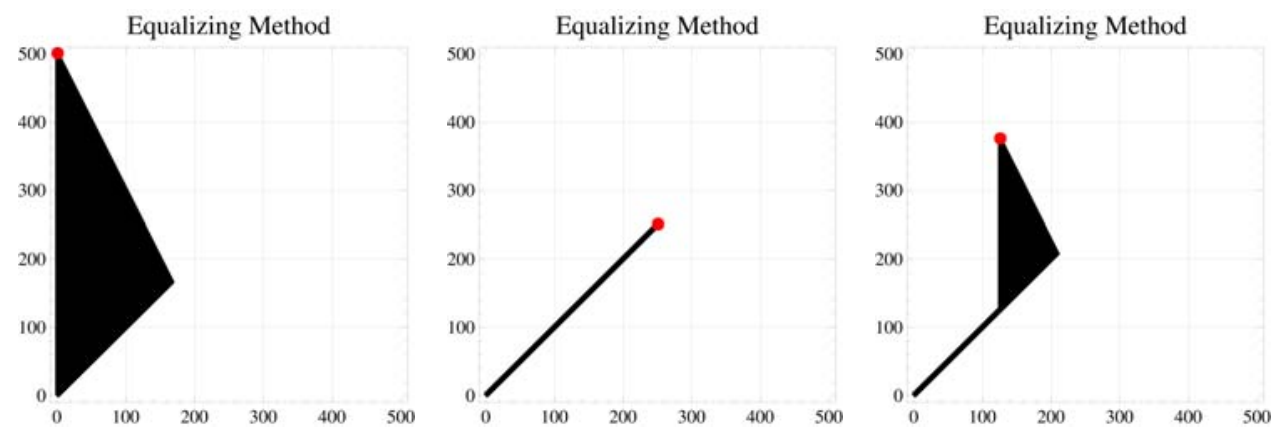

Figure 5.14: Points required by the Equalizing method for computing state vectors at time instants $(0,500),(250,250)$, and $(125,375)$ with a $3 \times 3$ initial state

for points located near the diagonal and Figure 5.15 shows that the axis method is only efficient for points located near the coordinate axes.

Performance worsens if one wants to compute a whole set of state vectors involving several different time instants at once, such as required, for example, for the operator $A_{r}^{T}$ when $r$ involves several terms. In Figure 5.16 the points are displayed which are computed by the linear, diagonal, equalizing, and axis method to facilitate the computation of five requested state vectors at the time instants $(0,500),(125,375)$, $(250,250),(375,125)$, and $(500,0)$ simultaneously. In the upper left corner the points computed by the linear method are plotted. This way of computing simultaneous paths to the requested time instants clearly is the most efficient (for this small example where $n=2$ ). Using the inefficient diagonal method, obviously too many points are computed. The bottom part of the plot displays the points required by the equalizing method and the axis method. The number of points evaluated by these four methods 

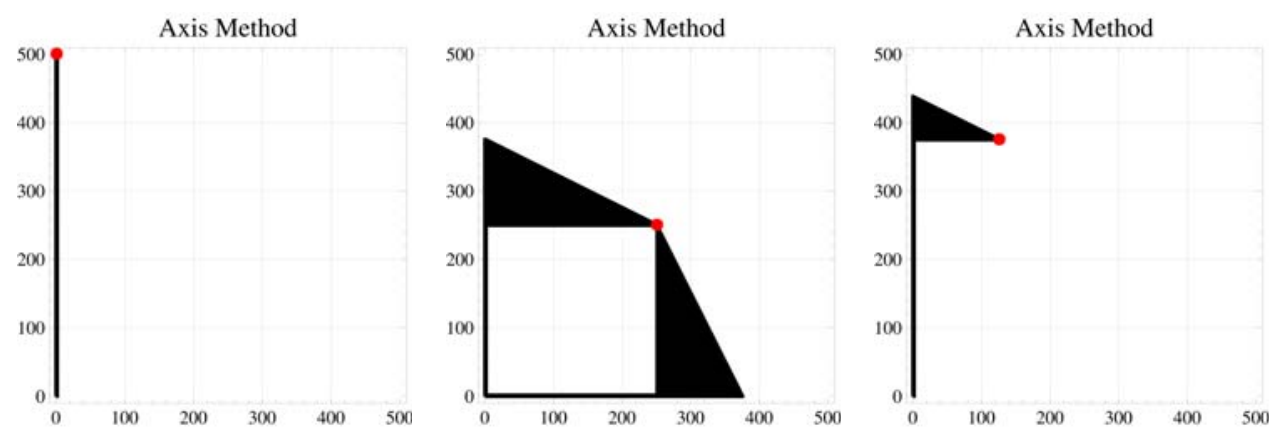

Figure 5.15: Points required by the Axis method for computing state vectors at time instants $(0,500),(250,250)$, and $(125,375)$ with a $3 \times 3$ initial state

are 7460, 127756, 113988 and 54597, respectively. Figure 5.17 shows the points of Figure 5.16 in the neighbourhood of the requested point $(250,250)$. From this figure, one can see that the thin paths showing up in the plots of the linear and equalizing methods in Figure 5.16 are built up from separate points located on diagonals of length $2 m-1=5$.

Because the diagonal, equalizing and axis methods are not efficient in every situation and because the linear method is only efficient for small values of $n$, but becomes less efficient when the dimension $n$ of the problem increases, a fifth method, the leastincrements method, is implemented. It is a combination of the equalizing and axis method and it applies that recurrence relation of the system (5.3) which requires a minimal number of new points to be calculated. It also proceeds in a backwards fashion (starting from a requested point and moving towards the initial state) but when constructing a path it takes the points into account that have already been included in the path. This method becomes more efficient than the linear method for larger values of $n$. Figure 5.18 shows a plot of all the evaluated points needed for the computation of the state vectors at the time instants $(0,500),(125,375),(250,250)$, $(375,125)$, and $(500,0)$ using this least-increments method. Obviously, more sophisticated methods can also be designed which take the global structure of the requested points into account.

To get an idea of the complexity of the first four heuristic methods additional computations are carried out using the values from Tables 5.1 and 5.2. The relative growth in the number of stored points needed for the computation of a requested point was investigated for two situations: when moving along the diagonal $t_{1}=t_{2}$ and when moving along a time axis. The increase in the number of stored points is displayed 

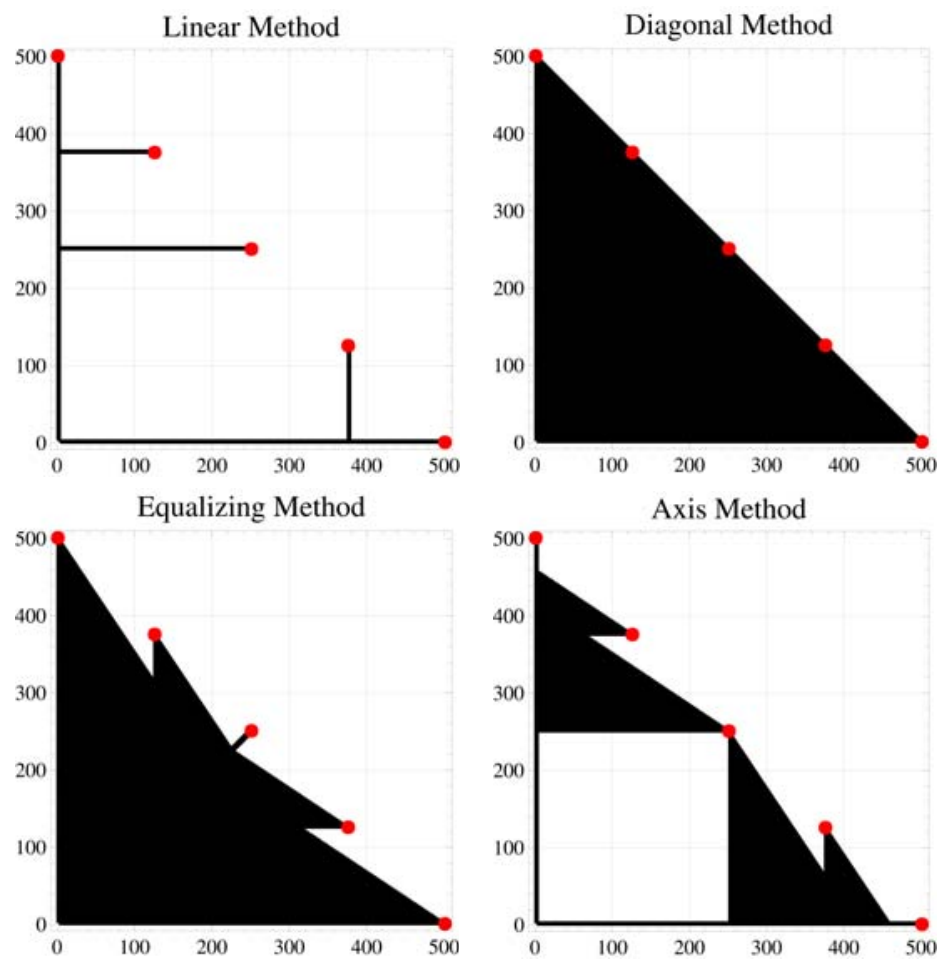

Figure 5.16: Points required by the linear, diagonal, equalizing and the axis method for computing state vectors at time instants $(0,500),(125,375),(250,250),(375,125)$, and $(500,0)$ with a $3 \times 3$ initial state

in Tables 5.3 and 5.4. An increase by a factor 2 in two dimensions indicates, for example, the increase of stored points when comparing the points $(0,50)$ and $(0,100)$ or the points $(50,50)$ and $(100,100)$. An increase factor of 5 in three dimensions concerns the increase of stored points when moving from $(10,10,10)$ to $(50,50,50)$, or from $(0,0,10)$ to $(0,0,50)$.

Table 5.3: Increase in the number of stored points along the diagonal

\begin{tabular}{lccc}
\hline Increase factor & $\begin{array}{c}\text { Diagonal method } \\
n=2 / n=3\end{array}$ & $\begin{array}{c}\text { Linear/Equalizing method } \\
n=2 \text { and } n=3\end{array}$ & $\begin{array}{c}\text { Axis method } \\
n=2 / n=3\end{array}$ \\
\hline 2 & $3.9 / 7.6$ & 2.0 & $3.5 / 5.1$ \\
5 & $19.4 / 86.9$ & 4.8 & $12.0 / 29.6$ \\
10 & $75.0 / 662.3$ & 9.4 & $41.6 / 150.9$ \\
\hline
\end{tabular}

From the Tables 5.3 and 5.4 it is concluded that for the equalizing method the number of points required for the computation of points with almost equal coordinates indeed grows linearly with the increase factor. For the axis method a similar statement 

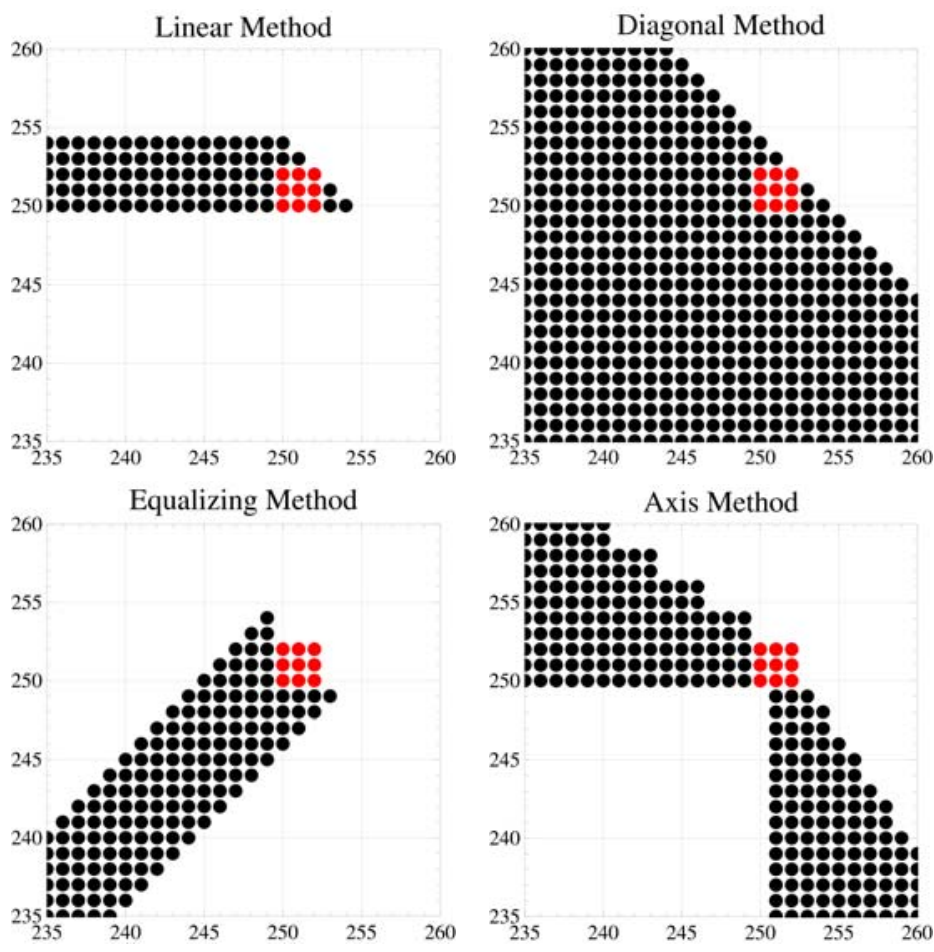

Figure 5.17: Points required by the linear, diagonal, equalizing and the axis method for computing state vectors at time instants $(0,500),(125,375),(250,250),(375,125)$, and $(500,0)$ with a $3 \times 3$ initial state zoomed in on a specific region

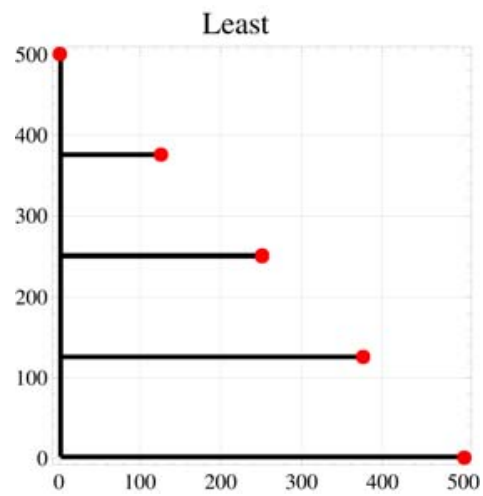

Figure 5.18: Points required by the least-increments method with a $3 \times 3$ initial state

holds for points along the time axes. 
Table 5.4: Increase in the number of stored points along the axis

\begin{tabular}{lccc}
\hline Increase factor & $\begin{array}{c}\text { Diagonal method } \\
n=2 / n=3\end{array}$ & $\begin{array}{c}\text { Linear/Axis method } \\
n=2 \text { and } n=3\end{array}$ & $\begin{array}{c}\text { Equalizing method } \\
n=2 / n=3\end{array}$ \\
\hline 2 & $3.7 / 7.0$ & 2.0 & $3.5 / 6.2$ \\
5 & $15.7 / 49.5$ & 4.5 & $11.9 / 30.3$ \\
10 & $58.9 / 344.5$ & 8.8 & $41.8 / 187.5$ \\
\hline
\end{tabular}

\subsubsection{Parallel computations in the $n$ D-systems approach}

Suppose one wants to compute the state vectors at the time instants $(0,500),(125,375)$, $(250,250),(375,125)$, and $(500,0)$. Figure 5.18 shows that the least-increments method determines five paths for this purpose. In every iteration of the eigenvalue solver the values of $w_{0,500}, w_{125,375}, w_{250,250}, w_{375,125}$, and $w_{500,0}$ have to be computed following these paths given the values of the initial state $w_{0,0}$. Because these five paths are well separated from each other (except in the very beginning near the initial state), one could think of determining and computing these paths of the least-increments method in parallel, i.e., each path is considered by one processor.

In such a situation a parallel approach is reasonable, but it has no effect when using an $n$ D-systems approach for global polynomial optimization. This is a conclusion from the parallel numerical examples described in Section 7.4. The same results are described in [53]. The reason that parallelization does not give any benefit in an $n$ D-systems approach for global polynomial optimization is the absence of long paths. Such long paths as displayed in Figure 5.18 can not occur: the smallest real eigenvalue of the operator $A_{p_{\lambda}\left(x_{1}, \ldots, x_{n}\right)}^{T}$ is of importance as it yields the value of the global minimum of $p_{\lambda}$. The total time of the state vectors $w_{t_{1}, \ldots, t_{n}}$, needed to compute the action of $A_{p_{\lambda}\left(x_{1}, \ldots, x_{n}\right)}^{T}$ is smaller than or equal to $2 d$, since the order of the polynomial $p_{\lambda}$ is $2 d$ (see the Equations (4.1) and (5.10)). The maximum total time of a time instant $y_{t_{1}, \ldots, t_{n}}$ in the initial state $w_{0, \ldots, 0}$ is $2 d-2$. As a result the occurring paths contain time instants with a total time increasing from $2 d-2$ to $2 d$ and therefore these paths are clearly shorter as the paths denoted in Figure 5.18.

The state vectors required to compute the action of the operator $A_{p_{\lambda}\left(x_{1}, \ldots, x_{n}\right)}^{T}$, among which also the initial state $w_{0, \ldots, 0}$, have the dimensions $(2 d-1) \times(2 d-1) \times$ $\cdots \times(2 d-1)$ and they all lie inside the region of points with maximum total time $2 d$, thereby forming a 'closed' region. These 'closed' regions are displayed for the 2dimensional and 3-dimensional case in the Figures 5.19(a) and 5.19(b), respectively, by a solid line.

The number of paths is obviously given by the number of monomial terms in the polynomial $p_{\lambda}$. Because of the fact that all these short paths start from the initial state $w_{0, \ldots, 0}$, there is much overlap especially near this initial state.

Remark 5.1. Note that even when the degree of $p_{\lambda}$ increases the paths remain short because only the initial state, containing the given values, becomes larger and still only a few steps in the $n \mathrm{D}$-system are required to follow one path. 


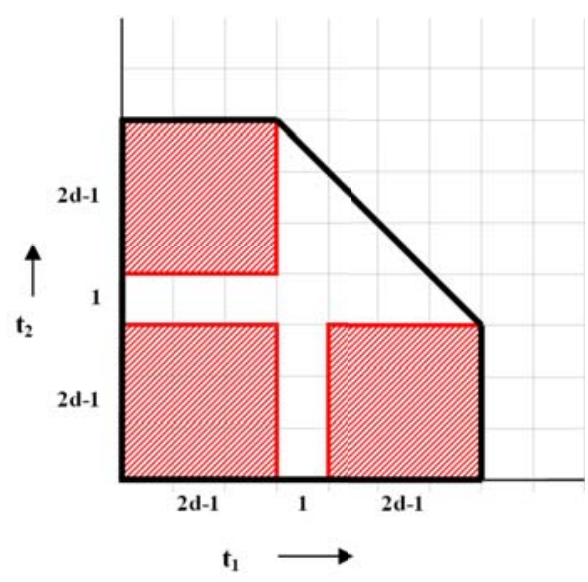

(a)

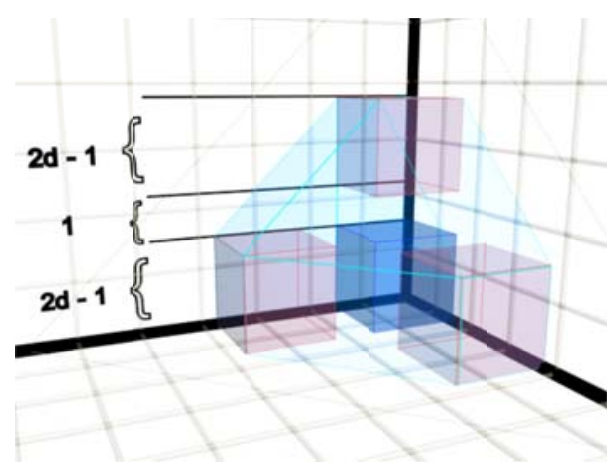

(b)

Figure 5.19: Region of points that occur in (a) 2-dimensional paths and (b) 3dimensional paths

Because the paths in the $n \mathrm{D}$-systems approach for polynomial optimization are short and have much overlap, the use of parallel computations is not advisable. The overhead of communication is too high in this case, especially for high-dimensional problems. In this particular case of polynomial optimization the difference between the performance of the linear method and the least-increments method will not be that high because of the shortness of the occurring paths.

As said before in the beginning of this section, in a more general case, parallelization can be an improvement of the efficiency of the $n \mathrm{D}$-system. One can imagine that in a certain application one wants to compute the action of a matrix $A_{r}$, where $r$ is a polynomial of high degree containing a lot of terms, without construction this matrix explicitly. The $n \mathrm{D}$-system in such a case contains long separate paths with little overlap and therefore every path can be considered by a single processor. Parallelization even becomes more suitable when the number of variables increases in such a situation, but this still requires further research. 


\section{Chapter 6}

\section{Iterative eigenvalue solvers in polynomial optimization}

Because only the smallest real eigenvalue of the large and sparse matrix $A_{p_{\lambda}}$ is needed for the polynomial optimization problem of a Minkowski dominated polynomial, it is a plausible choice to compute this eigenvalue with an iterative eigenvalue solver. Iterative eigenvalue solvers have the useful feature that they can focus on a certain subset of the eigenvalue spectrum. Moreover they can be implemented to operate in a matrix-free fashion: iterative solvers accept a device which computes the matrixvector product on a given vector as input. This avoids the explicit construction of the matrix under consideration and enables the use of the $n \mathrm{D}$-systems approach as described in Chapter 5. Iterative methods are based, for example, on an Arnoldi method or on a Jacobi-Davidson method. The Jacobi-Davidson (JD) method as presented by Sleijpen and van der Vorst (see [94]) is among the best iterative eigenvalue solvers. In the literature its implementation is called the JDQR method, for standard eigenvalue problems, and the JDQZ method, for generalized eigenvalue problems.

In the first two sections of this chapter a general introduction to iterative eigenvalue solvers is given, followed by a brief explanation of the techniques used in JacobiDavidson solvers (based on [58]). In the Sections 6.3 and 6.4 some of our own modifications of the Jacobi-Davidson method, which aim to improve its efficiency for polynomial optimization, are introduced. Among these improvements are the ability to focus on real eigenvalues only, which is non-standard among iterative solvers, and a procedure to project an approximate eigenvector to a close-by vector with Stetter structure. The development of a Jacobi-Davidson eigenvalue solver for a set of commuting matrices is described in Section 6.5 of this chapter. This Jacobi-Davidson method is called the JDCOMM method and has two distinctive properties: (i) it is able to target the smallest real eigenvalue of a large sparse matrix and moreover (ii) it computes the eigenvalues of the sparse matrix $A_{p_{\lambda}}$ while iterating with one of the 
much sparser commuting matrices $A_{x_{1}}, \ldots, A_{x_{n}}$ in the inner loop, causing a speed up in computation time and a decrease in required floating point operations. In this section also the pseudocode of the JDCOMM method is given. Numerical experiments using the approaches described in this chapter are given in Chapter 7 where the performance of the various approaches is compared to that of conventional iterative methods and of other methods for multivariate polynomial optimization.

\subsection{Iterative eigenvalue solvers}

In the previous chapters it was discussed how the global minimum of a Minkowski dominated polynomial $p_{\lambda}$ can be found by solving an eigenvalue problem. Unless the dimensions are small, this eigenvalue problem is preferably handled with iterative eigenvalue solvers because these allow for computations in a matrix-free fashion using an $n \mathrm{D}$-system as described in Chapter 5 . The matrix-free approach implements the action $A x$ of the matrix $A$ on a given vector $x$ without the explicit construction of the matrix $A$. This action is carried out repeatedly to approximate the eigenvectors and eigenvalues of the matrix $A$.

A well known iterative procedure for finding the largest eigenvalue / eigenvector pair is the power method [72]. This method operates as follows: let $v_{1}, v_{2}, \ldots, v_{N}$ be the $\mathrm{N}$ eigenvectors of an $N \times N$ matrix $A$, assuming that $N$ independent eigenvectors $v_{i}$ exist. Let $\lambda_{1}, \lambda_{2}, \ldots, \lambda_{N}$ be the corresponding eigenvalues such that $\left|\lambda_{1}\right|>$ $\left|\lambda_{2}\right| \geq \ldots \geq\left|\lambda_{N}\right|$. Then each $x \in \mathbb{R}^{N}$ can be written as a linear combination of the eigenvectors $v_{i}: x=\alpha_{1} v_{1}+\alpha_{2} v_{2}+\ldots+\alpha_{N} v_{N}$. The action of the matrix $A$ on the vector $x$ yields: $A x=\alpha_{1} \lambda_{1} v_{1}+\alpha_{1} \lambda_{2} v_{1}+\ldots+\alpha_{N} \lambda_{N} v_{N}$. More generally it holds that: $A^{k} x=\alpha_{1} \lambda_{1}^{k} v_{1}+\alpha_{2} \lambda_{2}^{k} v_{2}+\ldots+\alpha_{N} \lambda_{N}^{k} v_{N} \approx \alpha_{1} \lambda_{1}^{k} v_{1}$ when $k$ is large enough, because the eigenvalue $\lambda_{1}$ is dominant. Therefore, this method (extended with a renormalization procedure) allows for the iterative approximate calculation of the eigenvalue with the largest magnitude and its accompanying eigenvector.

The inverse power method is an iterative procedure which allows to compute the eigenvalue with the smallest magnitude [72]. This method operates with the matrix $A^{-1}$ rather than $A$. Note that the matrices $A$ and $A^{-1}$ have the same eigenvectors since $A^{-1} x=(1 / \lambda) x$ follows from $A x=\lambda x$. Since the eigenvalues of the matrix $A^{-1}$ are $1 / \lambda_{1}, \ldots, 1 / \lambda_{N}$, the inverse power method converges to the eigenvector $v_{n}$ associated with the eigenvalue $1 / \lambda_{N}$ : iteratively computing the action of $A^{-1}$ on a vector $x=\alpha_{1} v_{1}+\alpha_{2} v_{2}+\ldots+\alpha_{N} w_{N}$, where $v_{i}$ are the eigenvectors of the matrix, yields: $\left(A^{-1}\right)^{k} x=\alpha_{1}\left(1 / \lambda_{1}\right)^{k} v_{1}+\alpha_{2}\left(1 / \lambda_{2}\right)^{k} v_{2}+\ldots+\alpha_{N}\left(1 / \lambda_{N}\right)^{k} v_{N} \approx \alpha_{N}\left(1 / \lambda_{N}\right)^{k} v_{N}$ when $k$ is large enough.

Other, more advanced, iterative eigenvalue solvers are Arnoldi, Lanczos and Davidson methods [30] . Davidson's method is efficient for computing a few of the smallest eigenvalues of a real symmetric matrix. The methods by Lanczos [73] and Arnoldi $[4],[77]$ in their original form, tend to exhibit slow convergence and experience difficulties in finding the so-called interior eigenvalues. Interior eigenvalues are eigenvalues 
which remain in the inner part of the eigenvalue spectrum. A solution for this is to use the factorization of a shifted version of the matrix to solve linear systems of equations. However, such a factorization can be too expensive or even unfeasible, which make these methods unattractive. The Jacobi-Davidson method introduced and implemented by Sleijpen and van der Vorst [39],[94], called JDQR or JDQZ, avoids this problem by using an iterative approach to solve this system of equations. In this setting an approximate and therefore 'cheap' solution is accurate enough to have a fast convergence. Nowadays, the Jacobi-Davidson method has a well-understood convergence behavior and is considered one of the best eigenvalue solvers, especially for interior eigenvalues.

\subsection{The Jacobi-Davidson method}

Suppose a large and sparse $N \times N$ real or complex matrix $A$ is given. The JacobiDavidson method computes one or more relevant eigenvalues / eigenvector pairs $(\lambda, x)$ of the matrix $A$ which satisfy:

$$
A x=\lambda x .
$$

Here 'relevant' refers to the location of an eigenvalue in a specific part of the eigenvalue spectrum: for example the eigenvalue with the smallest magnitude, the largest real part or the eigenvalue close to some target value $\tau$. In the case of global polynomial optimization the most relevant eigenvalue would be the smallest real eigenvalue of the matrix $A_{p_{\lambda}}$. The deviation of an approximate eigenpair $(\theta, u)$ to a true eigenpair $(\lambda, x)$ is measured by the norm of the residual $r$, defined as:

$$
r=A u-\theta u .
$$

The Jacobi-Davidson method is a subspace method which means that approximate eigenvectors are sought in a subspace $\mathcal{U}$ [58]. Each iteration of such a method consist of two main phases: the subspace expansion phase and the subspace extraction phase. Both phases cooperate to obtain a fast and accurate convergence towards the relevant eigenvalues.

\subsubsection{Subspace expansion}

In the subspace expansion phase, we start from an approximate eigenpair $(\theta, u)$ of $(\lambda, x)$, where $u$ is an element of a low-dimensional subspace $\mathcal{U}$ and where $\theta$ is the Rayleigh quotient of $u$ :

$$
\theta=\frac{u^{*} A u}{u^{*} u}
$$

Note that this definition of $\theta$ implies that $r \perp u$ :

$$
u^{*} r=u^{*}(A u-\theta u)=u^{*} A u-\theta u^{*} u=0 .
$$

Obviously, one may require $u$ to be normalized to have norm 1 without loss of generality. 
The main issue of this phase is how to expand the current search space $\mathcal{U}$ with a new direction to get a better approximation in the next iteration. The key idea here is to look for an orthogonal direction $s \perp u$ such that $(u+s)$ satisfies $A(u+s)=\lambda(u+s)$, or in other words such that $(u+s)$ is proportional to the normalized eigenvector $x$. This implies that:

$$
A s-\theta s=(-A u+\theta u)+(\lambda u-\theta u)+(\lambda s-\theta s)
$$

which can be rewritten as:

$$
(A-\theta I) s=-r+(\lambda-\theta) u+(\lambda-\theta) s .
$$

Because $\|s\|$ and $(\lambda-\theta)$ both are expected to be small and much smaller than the other terms in this equation, the last term on the right-hand side of (6.6) is neglected. Furthermore, the term $(\lambda-\theta) u$ is projected out with the orthogonal projection matrix $\left(I-u u^{*}\right)$. Note that $\left(I-u u^{*}\right) u=0$ (for $\left.\|u\|=1\right),\left(I-u u^{*}\right) r=r$ (since $r \perp u$ ) and $\left(I-u u^{*}\right) s=s$ (upon requiring $s \perp u$ ). This gives rise to the Jacobi-Davidson correction equation:

$$
\left(I-u u^{*}\right)(A-\theta I)\left(I-u u^{*}\right) s=-r \quad \text { where } s \perp u .
$$

In a Jacobi-Davidson method the vector $s$ is solved approximately (with modest accuracy) from (6.7) in a matrix-free fashion using, for example, methods as GMRES of BiCGStab. A preconditioner can be used in this case to prevent for (possible) illconditioning and to (possibly) speed up the convergence. The vector $s$ is then used to expand the search space $\mathcal{U}$ : the vector $s$ is orthogonalized against all the vectors in $\mathcal{U}$ and added to it.

\subsubsection{Subspace extraction}

In the subspace extraction phase, the main goal is to compute an approximate eigenpair $(\theta, u)$ for the matrix $A$ with $u \in \mathcal{U}$. The extraction approach described here is the Rayleigh-Ritz approach (see [58]).

Let $U$ be an $N \times k$ matrix whose columns constitute an orthonormal basis of $\mathcal{U}$ and where $k \ll N$. Any vector $u \in \mathcal{U}$ can be written as $u=U c$, a linear combination of the columns of $U$ with $c$ a small $k$-dimensional vector. To compute an approximate eigenvector $u$, the Rayleigh-Ritz approach proceeds by computing $c$ by imposing the Ritz-Galerkin condition:

$$
r=A u-\theta u=A U c-\theta U c \perp \mathcal{U}
$$

which holds if and only if $(\theta, c)$ is an eigenpair of the $k$-dimensional projected eigenproblem:

$$
U^{*} A U c=\theta c
$$

(the orthonormality of $U$ is used here: $U^{*} U=I_{k}$ ). Once this low-dimensional eigenproblem is solved and an eigenpair $(\theta, c)$ is available, the approximate eigenvector 
for the original problem is computed as $u=U c$. The projected low-dimensional eigenproblem is usually solved by a direct method. The vector $u$ is called a Ritz vector. Moreover, because of (6.8) $r \perp u$ and $\theta$ is the Rayleigh quotient of $u$. Thus, $(\theta, U c)=(\theta, u)$ is the back-transformed eigenpair which acts as an approximation to $(\lambda, x)$ of the original problem (6.1).

An important property of the Jacobi-Davidson method is that it allows the user to focus on a subset of the eigenvalues via the specification of a target, as further discussed in Section 6.3 of this chapter.

In some cases the Rayleigh-Ritz extraction approach can give poor approximations. This issue is addressed by the Refined Rayleigh-Ritz extraction method. Given an approximate eigenpair $(\theta, c)$ of the small projected eigenproblem $(6.9)$, this refined approach solves:

$$
\hat{c}=\underset{c \in \mathbb{C}^{k},\|c\|=1}{\operatorname{argmin}}\|(A U-\theta U) c\|_{2}^{2} .
$$

The back-transformed eigenvector $\hat{u}=U \hat{c}$, called the refined Ritz vector, usually gives a much better approximation than the ordinary Ritz vector $u$ (see [94]). A new approximate eigenvalue $\hat{\theta}$ can be computed using the Rayleigh quotient (6.3) of $\hat{u}$.

Note that both the subspace expansion and the subspace extraction phase of the Jacobi-Davidson method can be performed in a matrix-free fashion as all the operations in which the matrix $A$ is involved are matrix-vector products, which can be computed without having the matrix $A$ explicitly at hand. This enables the use of the matrix-free $n \mathrm{D}$-systems approach discussed in Chapter 5 .

\subsubsection{Remaining important issues}

In the two previous subsections the main phases of the Jacobi-Davidson method, the subspace extension phase and the subspace extraction phase, are presented. In this subsection we discuss the important remaining issues of the Jacobi-Davidson method.

First, during the iterations the search space $\mathcal{U}$ grows. When this space reaches the maximum dimension $j_{\max }$ the method is restarted with a search space of dimension $j_{\text {min }}$ containing the $j_{\text {min }}$ most promising vectors. A 'thick' restart indicates that the method is restarted with the approximate eigenvectors from the previous $j_{\text {min }}$ iterations. The quantities $j_{\text {min }}$ and $j_{\max }$ are user specified parameters. Second, before the first iteration the search space $U$ is empty. One can decide to start with a Krylov search space of the matrix $A$ of dimension $j_{\min }$ to reach the wanted eigenvalue in less iteration steps. Finally, when one wants to compute more than one eigenvalue the so-called 'deflation step' becomes important. In this step the matrix $A$ is deflated to a matrix $\hat{A}$ such that it no longer contains the already computed eigenpair $(\lambda, x)$ : $\hat{A}=\left(I-x x^{*}\right) A\left(I-x x^{*}\right)$.

All the parameter options mentioned in this paragraph, and also the assumed tolerance and the maximum allowed number of iterations, have a considerable influence on the performance of the method. 


\subsubsection{Pseudocode of the Jacobi-Davidson method}

In this subsection, the pseudocode of the Jacobi-Davidson method, as presented in [58], is given in Algorithm 2. This method is denoted as the JD method. The JD method is used in Section 6.5 to compare with a modified implementation for commuting matrices. In this pseudocode the acronym RGS stands for a repeated Gram-Schmidt method to expand a given orthonormal basis with a vector $s$.

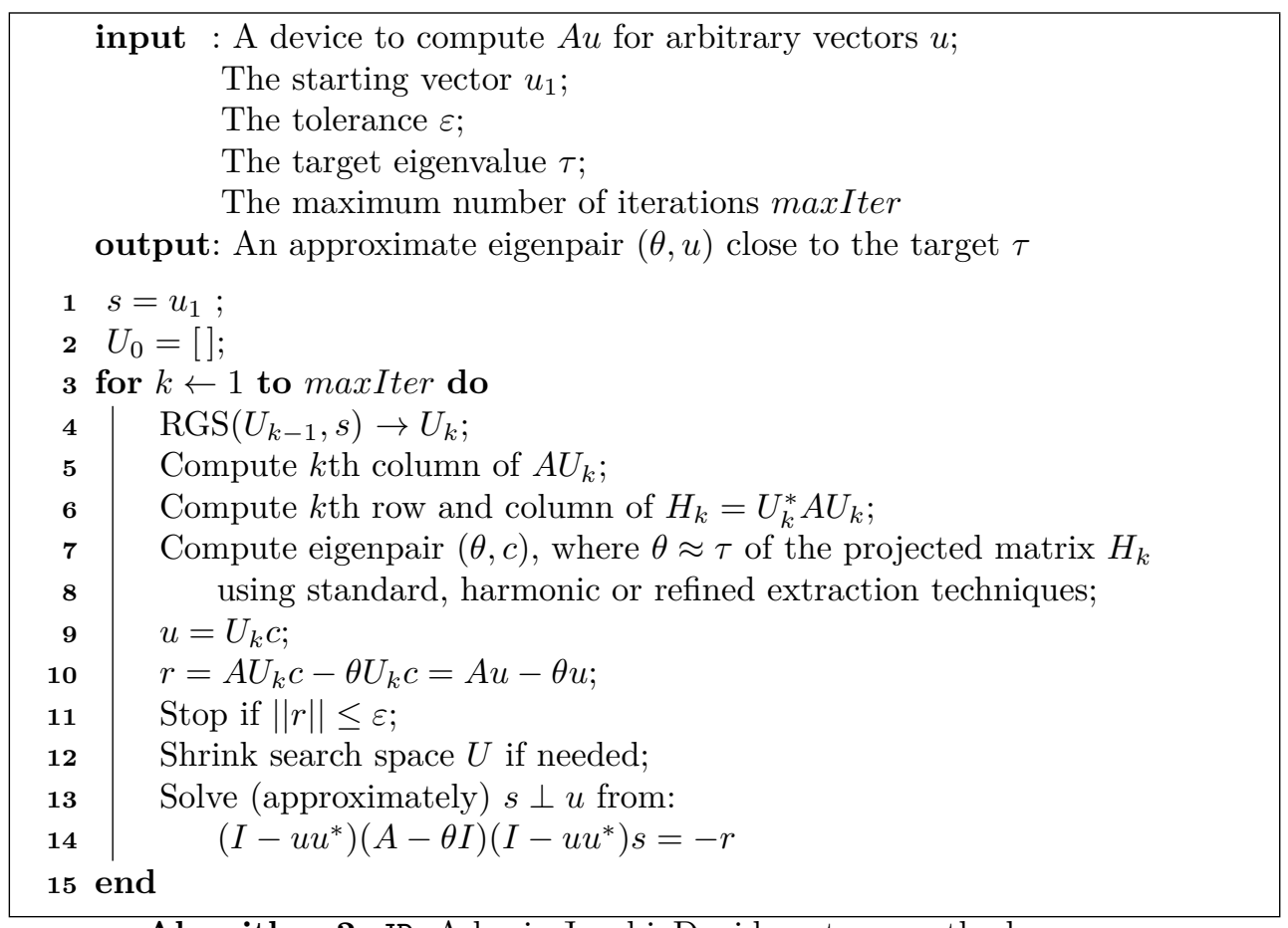

Algorithm 2: JD: A basic Jacobi-Davidson type method

Note that this algorithm has two nested loops as the correction equation in Line 14 is solved iteratively too. These loops are called the outer and inner loops. The convergence of the outer loop in Line 3 depends directly on the accuracy by which the correction equation (6.7) in Line 14 is solved.

\subsection{Target selection: the smallest real eigenvalue}

As mentioned before, it is one of the advantages of Jacobi-Davidson methods that they allow to focus on a certain subset of the eigenvalue spectrum. This is implemented through the specification of a target. This target can either be set to prefer the eigenvalue with the largest magnitude, the smallest magnitude, the largest real part or the smallest real part, resulting in a certain ordering of the eigenvalues of the small projected matrix $U^{*} A U$ of the eigenproblem in Equation (6.9). Because in our specific application only real eigenvalues are of interest, an additional sorting option 
is developed and added as an extension to the Jacobi-Davidson implementation. This sorting method first sorts the list of approximate complex eigenvalues of the matrix $U^{*} A U$ in increasing order with respect to the imaginary part. Then all entries with an imaginary part below a certain threshold are sorted in increasing order of the real part. With this sorting method the Jacobi-Davidson methods are made to focus on the desired eigenvalues for this application: the smallest real eigenvalues.

It should be stressed that the use of a threshold for the imaginary part is essential because of the iterative approach of the solvers. If no thresholding is used then the solver may not converge to a single eigenvalue. For example, suppose that the two smallest real eigenvalues are $\lambda_{1}=-117.6003$ and $\lambda_{2}=-93.4018$. However, because of the approximate approach of the eigenvalue solvers these values may arise as $\hat{\lambda}_{1}=-117.6006+2.316 \times 10^{-10} i$ and $\hat{\lambda}_{2}=-93.4012+4.794 \times 10^{-11} i$ resulting in the ordering $\left(\hat{\lambda}_{2}, \hat{\lambda}_{1}\right)^{T}$ if no thresholding is used. In a next iteration these small imaginary parts might be interchanged resulting in the opposite ordering $\left(\hat{\lambda}_{1}, \hat{\lambda}_{2}\right)^{T}$. Such a repeated change of target will have its effect on the convergence of the solver. With thresholding all imaginary parts below the threshold (i.e., $1 \times 10^{-10}$ ) are effectively treated as zero, thereby reducing this switching of the target.

Numerical experiments using this Jacobi-Davidson implementation are described in Chapter 7.

\subsection{Projection to Stetter-structure}

In the global polynomial optimization of a Minkowski dominated polynomial the eigenvectors of the matrices involved exhibit the Stetter structure as described in Section 3.3. If an approximate eigenpair is found by an iterative eigenvalue solver, the corresponding approximate eigenvector will in general not show the precise Stetter structure. The idea now is to project this vector to a nearby Stetter vector to accelerate the convergence to a desired accuracy. In this section two such projection methods are developed together with procedures to integrate this projection step into the Jacobi-Davidson algorithm.

\subsubsection{Projecting an approximate eigenvector}

An approximate eigenvector $u=\left(u_{1}, \ldots, u_{N}\right)$ of size $N=m^{n}$, where $m=2 d-$ 1, exhibits the Stetter structure if it is proportional to a vector of the form $v=$ $\left(v_{1}, \ldots, v_{N}\right)^{T}=\left(1, x_{1}, x_{1}^{2}, \ldots, x_{1}^{m-1}, x_{2}, x_{1} x_{2}, x_{1}^{2} x_{2}, \ldots, x_{1}^{m-1} x_{2}, x_{2}^{2}, x_{1} x_{2}^{2}, x_{1}^{2} x_{2}^{2}\right.$, $\left.\ldots, x_{1}^{m-1} x_{2}^{2}, \ldots, \ldots, x_{1}^{m-1} x_{2}^{m-1}, x_{3}, x_{1} x_{3}, \ldots, \ldots, x_{1}^{m-1} x_{2}^{m-1} \cdots x_{n}^{m-1}\right)^{T}$. See also Section 4.1. The idea of the projection method in this section is to find values in an approximate eigenvector $u$ for the quantities $x_{1}, x_{2}, \ldots, x_{n}$ such that the projected vector of $u$, the vector $\hat{u}$, is close to a nearby Stetter vector $v$.

However, particularly for polynomials with high total degree, the convergence can be dominated by higher powers of $x_{1}, x_{2}, \ldots, x_{n}$ if there is an $x_{i}$ with $\left|x_{i}\right|>1$. On the other hand, if all $x_{i}$ are of magnitude less than 1 , these high powers go to zero. This 
can potentially cause numerical difficulties. To overcome this problem the (natural) logarithmic transformation is applied to the entries of the eigenvector.

Example 6.1. For $n=2$ and $d=2$ (hence $m=3$ ) an eigenvector $v$ is structured as $\left(1, x_{1}, x_{1}^{2}, x_{2}, x_{1} x_{2}, x_{1}^{2} x_{2}, x_{2}^{2}, x_{1} x_{2}^{2}, x_{1}^{2} x_{2}^{2}\right)^{T}$. The logarithmic transformation applied to each of the entries of such a vector $v$ with Stetter structure results in:

$$
\log \left(\begin{array}{r}
1 \\
x_{1} \\
x_{1}^{2} \\
x_{2} \\
x_{1} x_{2} \\
x_{1}^{2} x_{2} \\
x_{2}^{2} \\
x_{1} x_{2}^{2} \\
x_{1}^{2} x_{2}^{2}
\end{array}\right)=\log \left(x_{1}\right)\left(\begin{array}{l}
0 \\
1 \\
2 \\
0 \\
1 \\
2 \\
0 \\
1 \\
2
\end{array}\right)+\log \left(x_{2}\right)\left(\begin{array}{l}
0 \\
0 \\
0 \\
1 \\
1 \\
1 \\
2 \\
2 \\
2
\end{array}\right) .
$$

In general a Stetter vector $v$ (where the first entry is normalized to 1) can be decomposed as follows using the same logarithmic transformation:

$$
\log (v)=\left(\begin{array}{c}
\log \left(v_{1}\right) \\
\vdots \\
\vdots \\
\log \left(v_{N}\right)
\end{array}\right)=\log \left(x_{1}\right) w_{1}+\log \left(x_{2}\right) w_{2}+\ldots+\log \left(x_{n}\right) w_{n}
$$

in which the vectors $w_{1}, \ldots, w_{n}$ are fixed vectors, containing the integer values 0 , $1, \ldots, m-1$ (each $m^{n-1}$ times) in a specific ordering depending on the monomial ordering that is used.

Remark 6.1. The right-hand side of Equation (6.12) can be extended without loss of generality with a term $\log \left(x_{0}\right) w_{0}$, where $w_{0}=(1, \ldots, 1)^{T}$, to be able to deal with eigenvectors in which the first entry is not normalized to 1.

The right-hand side of Equation (6.12) can be used to project a vector that has an approximate Stetter structure to the desired structure. Thus, we are looking for complex quantities $x_{1}, \ldots, x_{n}$ such that:

$$
\log (\hat{u})=\log \left(x_{1}\right) w_{1}+\log \left(x_{2}\right) w_{2}+\ldots+\log \left(x_{n}\right) w_{n}
$$

is a good approximation of $\log (u)$.

However, this logarithmic transformation is not straightforward when applying it to a complex or negative entry in the approximate eigenvector. To overcome this problem, all entries of a vector $u$, including complex and negative ones, are written in polar coordinates. This yields for a general vector $z$ :

$$
\log (z)=\log (|z|)+i \cdot \arg (z)
$$


so that the real part of $z$ is well determined for $z \neq 0$ whereas the imaginary part is determined only up to an integer multiple of $2 \pi$.

Writing the entries of the vectors $x=\left(x_{1}, \ldots, x_{n}\right)^{T}$ and $u$ as follows:

$$
x_{j}=r_{j} e^{i \cdot \phi_{j}} \quad j=1, \ldots, n
$$

and

$$
u_{j}=v_{j} e^{i \cdot \psi_{j}} \quad j=1, \ldots, N
$$

where $r_{j}=\left|x_{j}\right|, v_{j}=\left|u_{j}\right| \in \mathbb{R}^{+}$and $-\pi<\phi_{j} \leq \pi$ and $-\pi<\psi_{j} \leq \pi$, makes it possible to set up separate equations for the real and imaginary parts respectively:

$$
\log \left(v_{j}\right) \approx \log \left(r_{1}\right) w_{1 j}+\log \left(r_{2}\right) w_{2 j}+\ldots+\log \left(r_{n}\right) w_{n j} \quad j=1, \ldots, N
$$

and

$$
\psi_{j}+2 \pi k_{j} \approx \phi_{1} w_{1 j}+\phi_{2} w_{2 j}+\ldots+\phi_{n} w_{n j} \quad j=1, \ldots, N .
$$

Now Equation (6.17) can be written in matrix-vector form as follows:

$$
W\left(\begin{array}{c}
\log \left(r_{1}\right) \\
\log \left(r_{2}\right) \\
\vdots \\
\log \left(r_{n}\right)
\end{array}\right) \approx\left(\begin{array}{c}
\log \left(v_{1}\right) \\
\log \left(v_{2}\right) \\
\vdots \\
\log \left(v_{N}\right)
\end{array}\right)
$$

where the matrix $W$ of dimension $N \times n$ is constructed from the vectors $w_{1}, \ldots, w_{n}$ in Equation (6.12):

$$
W=\left(\begin{array}{l|l|l|l} 
& & & \\
w_{1} & w_{2} & \ldots & w_{n} \\
& & &
\end{array}\right)
$$

In Equation (6.19) we are looking for a non-negative vector $r=\left(r_{1}, \ldots, r_{n}\right)^{T}$ which can be solved approximately for the unknowns using a least-squares optimization approach. Here, the least-squares solution for $\log (r)$ equals:

$$
\log (r)=\left(W^{T} W\right)^{-1} W^{T} \log (v)
$$

so that the approximate left-hand side of $(6.19)$ is given by $\log (\hat{v})$ :

$$
\log (\hat{v})=W \log (r)=W\left(W^{T} W\right)^{-1} W^{T} \log (v)=P \log (v) .
$$

In Equation (6.22) the symmetric projection matrix $\left(W\left(W^{T} W\right)^{-1} W^{T}\right)$ is denoted by $P$ and is of dimension $N \times N$. 
Also Equation (6.18) can be written in matrix-vector form as:

$$
W\left(\begin{array}{c}
\phi_{1} \\
\phi_{2} \\
\vdots \\
\phi_{n}
\end{array}\right) \approx\left(\begin{array}{c}
\psi_{1} \\
\psi_{2} \\
\vdots \\
\psi_{N}
\end{array}\right)+2 \pi\left(\begin{array}{c}
k_{1} \\
k_{2} \\
\vdots \\
k_{N}
\end{array}\right)
$$

in which we need to solve for $\phi=\left(\phi_{1}, \phi_{2}, \ldots, \phi_{n}\right)^{T}$ and for an integer vector $k=$ $\left(k_{1}, k_{2}, \ldots, k_{N}\right)^{T}$. Note that $\phi$ satisfies: $-\pi<\phi \leq \pi$.

Then, for fixed values of $k$, the optimal least-squares solution for $\phi$ is described by:

$$
\phi^{L S}=\left(W^{T} W\right)^{-1} W^{T}(\psi+2 \pi k) .
$$

This yields the following approximate left-hand side $\hat{\psi}$ for Equation (6.23):

$$
\hat{\psi}+2 \pi k=W \phi^{L S}=W\left(W^{T} W\right)^{-1} W^{T}(\psi+2 \pi k)=P(\psi+2 \pi k),
$$

which can be rewritten as:

$$
\hat{\psi}=P(\psi+2 \pi k)-2 \pi k=P \psi+2 \pi(P-I) k .
$$

Here $\hat{\psi}$ denotes the approximate projected version of the vector $\psi$ for the chosen values of $k$. To find the true optimal value, we need to vary $k$ over the set of feasible integer values, which introduces a combinatorial complexity. In this case $\|(I-P)(\psi+2 \pi k)\|_{2}$ needs to be minimized over integer values of $k$, which need not be easy. Note here that the solution $\hat{k}=-\frac{1}{2 \pi} \psi$ is an exact, but non-integer, solution for $k$ and could be used as an initial guess for an optimization method.

When the (possibly) optimal value set for $k$ is determined, the vectors $\log (\hat{v})$ and $\hat{\psi}$ are combined and the result is transformed back, entry wise, into the non-logarithmic domain, to yield a new projected approximate eigenvector $\hat{u}$ with Stetter structure:

$$
\hat{u}=e^{\log (\hat{v})+i \cdot \hat{\psi}} .
$$

The corresponding (approximate) eigenvalue $\hat{\theta}$ is determined using the Rayleigh quotient of Equation (6.3), which yields a new eigenvalue/eigenvector pair $(\hat{\theta}, \hat{u})$.

Example 6.2. Suppose one wants to project an approximate eigenvector $u$ (where the first entry is normalized to 1$)$ of length $N=9(n=2, d=2$ and thus $m=3)$ to a close-by Stetter vector. Suppose that the vector $u$ is given by:

$$
u=\left(\begin{array}{rrrr}
1 & & \\
-4 & & \\
-5 & + & 29 i \\
-13 & - & 5 i \\
5 & + & 72 i \\
255 & - & 283 i \\
16 & + & 140 i \\
507 & - & 640 i \\
-4613 & + & 543 i
\end{array}\right) .
$$


This vector $u$ has much resemblance with a Stetter vector with a structure corresponding to $\left(1, x_{1}, x_{1}^{2}, x_{2}, x_{1} x_{2}, x_{1}^{2} x_{2}, x_{2}^{2}, x_{1} x_{2}^{2}, x_{1}^{2} x_{2}^{2}\right)^{T}$ where $x_{1}=-4-4 i$ and $x_{2}=-9-8 i$.

In polar coordinates the entries of the vector $u$ can be written as $u_{j}=v_{j} e^{i \cdot \psi_{j}}$ for $j=1, \ldots, N$. This yields in this example:

$$
v=\left(\begin{array}{r}
1.000 \\
4.000 \\
29.428 \\
13.928 \\
72.173 \\
380.938 \\
140.911 \\
816.486 \\
4644.849
\end{array}\right), \quad \psi=\left(\begin{array}{r}
0.000 \\
3.142 \\
1.742 \\
-2.774 \\
1.502 \\
-0.837 \\
1.457 \\
-0.901 \\
3.024
\end{array}\right)
$$

To project the vectors $v$ and $\psi$, the matrix $W$ and the projection matrix $P$ is required. To construct the matrix $W$, the logarithmic transformation is applied to the vector $\left(1, x_{1}, x_{1}^{2}, x_{2}, x_{1} x_{2}, x_{1}^{2} x_{2}, x_{2}^{2}, x_{1} x_{2}^{2}, x_{1}^{2} x_{2}^{2}\right)^{T}$. This yields:

$$
\log \left(\begin{array}{r}
1 \\
x_{1} \\
x_{1}^{2} \\
x_{2} \\
x_{1} x_{2} \\
x_{1}^{2} x_{2} \\
x_{2}^{2} \\
x_{1} x_{2}^{2} \\
x_{1}^{2} x_{2}^{2}
\end{array}\right)=\log \left(x_{1}\right) w_{1}+\log \left(x_{2}\right) w_{2}=\log \left(x_{1}\right)\left(\begin{array}{l}
0 \\
1 \\
2 \\
0 \\
1 \\
2 \\
0 \\
1 \\
2
\end{array}\right)+\log \left(x_{2}\right)\left(\begin{array}{l}
0 \\
0 \\
0 \\
1 \\
1 \\
1 \\
2 \\
2 \\
2
\end{array}\right) .
$$

The vectors $w_{1}$ and $w_{2}$ above constitute the matrix $W$ of dimensions $N \times n=9 \times 2$ :

$$
W=\left(\begin{array}{ll}
0 & 0 \\
1 & 0 \\
2 & 0 \\
0 & 1 \\
1 & 1 \\
2 & 1 \\
0 & 2 \\
1 & 2 \\
2 & 2
\end{array}\right) .
$$

The symmetric projection matrix $P$ of dimension $N \times N=9 \times 9$ is constructed using 
this matrix $W$ as follows:

$$
\begin{gathered}
\text { P }=W\left(W^{T} W\right)^{-1} W^{T}= \\
\left(\begin{array}{ccccccccc}
0 & 0 & 0 & 0 & 0 & 0 & 0 & 0 & 0 \\
0 & \frac{5}{48} & \frac{5}{24} & -\frac{1}{16} & \frac{1}{24} & \frac{7}{48} & -\frac{1}{8} & -\frac{1}{48} & \frac{1}{12} \\
0 & \frac{5}{24} & \frac{5}{12} & -\frac{1}{8} & \frac{1}{12} & \frac{7}{24} & -\frac{1}{4} & -\frac{1}{24} & \frac{1}{6} \\
0 & -\frac{1}{16} & -\frac{1}{8} & \frac{5}{48} & \frac{1}{24} & -\frac{1}{48} & \frac{5}{24} & \frac{7}{48} & \frac{1}{12} \\
0 & \frac{1}{24} & \frac{1}{12} & \frac{1}{24} & \frac{1}{12} & \frac{1}{8} & \frac{1}{12} & \frac{1}{8} & \frac{1}{6} \\
0 & \frac{7}{48} & \frac{7}{24} & -\frac{1}{48} & \frac{1}{8} & \frac{13}{48} & -\frac{1}{24} & \frac{5}{48} & \frac{1}{4} \\
0 & -\frac{1}{8} & -\frac{1}{4} & \frac{5}{24} & \frac{1}{12} & -\frac{1}{24} & \frac{5}{12} & \frac{7}{24} & \frac{1}{6} \\
0 & -\frac{1}{48} & -\frac{1}{24} & \frac{7}{48} & \frac{1}{8} & \frac{5}{48} & \frac{7}{24} & \frac{13}{48} & \frac{1}{4} \\
0 & \frac{1}{12} & \frac{1}{6} & \frac{1}{12} & \frac{1}{6} & \frac{1}{4} & \frac{1}{6} & \frac{1}{4} & \frac{1}{3}
\end{array}\right) .
\end{gathered}
$$

Note that the explicit matrix $P$ is constructed here for analytical purposes, but is avoided in numerical computations.

The vectors $\log (\hat{v})$ and $\hat{v}$ are computed as:

$$
\log (\hat{v})=P \cdot \log (v)=\left(\begin{array}{l}
0.000 \\
1.675 \\
3.349 \\
2.532 \\
4.207 \\
5.881 \\
5.064 \\
6.738 \\
8.413
\end{array}\right) \text { and } \hat{v}=e^{\log (\hat{v})}=\left(\begin{array}{r}
1.000 \\
5.337 \\
28.499 \\
12.576 \\
67.120 \\
358.234 \\
158.149 \\
844.083 \\
4505.076
\end{array}\right) .
$$

For a particular choice of $k=\left(k_{1}, \ldots, k_{N}\right)=(0,0,1,0,0,1,-1,0,0)$ the projected vector $\hat{\psi}$ is computed as:

$$
\hat{\psi}=P \psi+2 \pi(P-I) k=\left(\begin{array}{r}
0.000 \\
3.903 \\
1.523 \\
-2.424 \\
1.479 \\
-0.901 \\
1.435 \\
-0.945 \\
2.958
\end{array}\right)
$$

The entries in the projected vector $\log (\hat{v})$ satisfy the following relations in order to exhibit Stetter structure with respect to addition (because it is in the logarithmic 
domain):

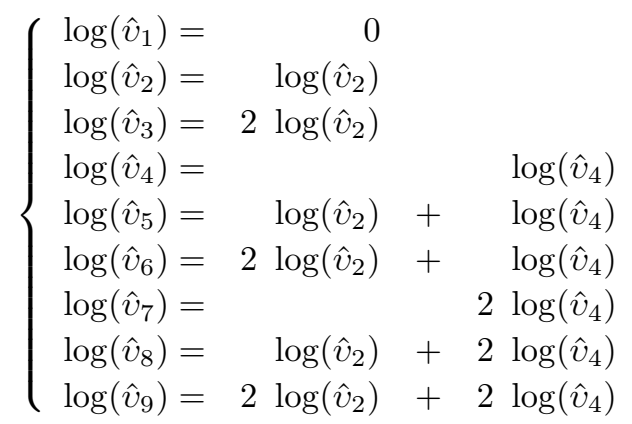

The entries in the vector $\hat{v}$ satisfy the following relations in order to exhibit Stetter structure with respect to multiplication:

$$
\begin{cases}\hat{v}_{1}=1 & \\ \hat{v}_{2}=\hat{v}_{2} & \\ \hat{v}_{3}=\hat{v}_{2}^{2} & \\ \hat{v}_{4}= & \hat{v}_{4} \\ \hat{v}_{5}=\hat{v}_{2} \cdot \hat{v}_{4} \\ \hat{v}_{6}=\hat{v}_{2}^{2} \cdot \hat{v}_{4} \\ \hat{v}_{7}= & \\ \hat{v}_{8}=\hat{v}_{2} \cdot \hat{v}_{4}^{2} \\ \hat{v}_{9}=\hat{v}_{2}^{2} \cdot \hat{v}_{4}^{2}\end{cases}
$$

Also the projected vector $\hat{\psi}$ exhibits Stetter structure with respect to addition (because this vector is also in the logarithmic domain). To show this, we use the leastsquares solution for $\phi$ in Equation (6.24) denoted by $\phi^{L S}$. We know from (6.25) that the following holds:

$$
\hat{\psi}=W \phi^{L S}-2 \pi k
$$

The vector $\phi^{L S}$ contains the two elements $\phi_{1}^{L S}$ and $\phi_{2}^{L S}$ which yields the following for $\hat{\psi}$ :

$$
\hat{\psi}=\left(\begin{array}{cc}
0 & 0 \\
1 & 0 \\
2 & 0 \\
0 & 1 \\
1 & 1 \\
2 & 1 \\
0 & 2 \\
1 & 2 \\
2 & 2
\end{array}\right)\left(\begin{array}{l}
\phi_{1}^{L S} \\
\phi_{2}^{L S}
\end{array}\right)-2 \pi\left(\begin{array}{c}
k_{1} \\
k_{2} \\
k_{3} \\
k_{4} \\
k_{5} \\
k_{6} \\
k_{7} \\
k_{8} \\
k_{9}
\end{array}\right)
$$

From the equation above we can derive the relations $\phi_{1}^{L S}=\hat{\psi}_{2}+2 \pi k_{2}$ and $\phi_{2}^{L S}=$ $\hat{\psi}_{4}+2 \pi k_{4}$. Substituting this back into the latter equation yields the structure in the 
vector $\hat{\psi}$ as follows:

$$
\hat{\psi}=\left(\begin{array}{cc}
0 & 0 \\
1 & 0 \\
2 & 0 \\
0 & 1 \\
1 & 1 \\
2 & 1 \\
0 & 2 \\
1 & 2 \\
2 & 2
\end{array}\right)\left(\hat{\psi}_{2} \hat{\psi}_{4}\right)+2 \pi\left(\begin{array}{cc}
0 & 0 \\
1 & 0 \\
2 & 0 \\
0 & 1 \\
1 & 1 \\
2 & 1 \\
0 & 2 \\
1 & 2 \\
2 & 2
\end{array}\right)\left(\begin{array}{c}
k_{2} \\
k_{4}
\end{array}\right)-2 \pi\left(\begin{array}{l}
k_{1} \\
k_{2} \\
k_{3} \\
k_{4} \\
k_{5} \\
k_{6} \\
k_{7} \\
k_{8} \\
k_{9}
\end{array}\right)
$$

This results in the following relations for the chosen values $k=(0,0,1,0,0,1,-1,0,0)$ :

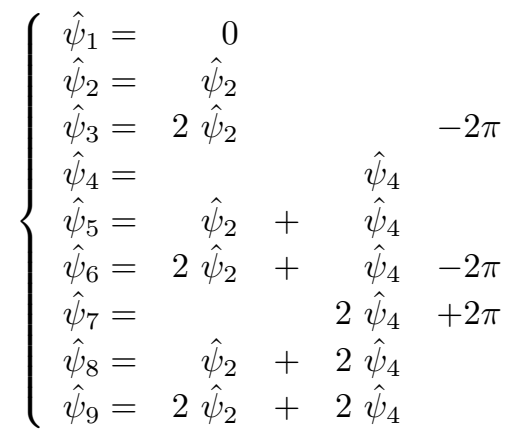

The projected vector is finally computed as $\log (\hat{v})+i \cdot \hat{\psi}$ and transformed back into the non-logarithmic domain to yield the vector $\hat{u}$ :

$$
\hat{u}=e^{\log (\hat{v})+i \cdot \hat{\psi}}=\left(\begin{array}{rrr}
1.000 & & \\
-3.862 & - & 3.684 i \\
1.350 & + & 28.454 i \\
-9.476 & - & 8.268 i \\
6.142 & + & 66.838 i \\
222.471 & - & 280.782 i \\
21.421 & + & 156.693 i \\
494.436 & - & 684.113 i \\
-4429.619 & + & 821.089 i
\end{array}\right) .
$$

The vector $\hat{u}$ exhibits the Stetter structure with respect to multiplication since it 
satisfies the following relations:

$$
\left\{\begin{array}{llll}
\hat{u}_{1}= & 1 & \\
\hat{u}_{2}= & \hat{u}_{2} & \\
\hat{u}_{3}= & \hat{u}_{2}^{2} & & \\
\hat{u}_{4}= & & & \hat{u}_{4} \\
\hat{u}_{5}= & \hat{u}_{2} & \cdot & \hat{u}_{4} \\
\hat{u}_{6}= & \hat{u}_{2}^{2} \cdot & \hat{u}_{4} \\
\hat{u}_{7}= & & & \hat{u}_{4}^{2} \\
\hat{u}_{8}= & \hat{u}_{2} \cdot & \hat{u}_{4}^{2} \\
\hat{u}_{9}= & \hat{u}_{2}^{2} \cdot & \hat{u}_{4}^{2}
\end{array}\right.
$$

Note that there are other possible choices for the values of $k=\left(k_{1}, \ldots, k_{9}\right)^{T}$ in Equation (6.34) which yield the same projected vector $\hat{u}$ as in (6.41). For example, the choices $k=(0,-2,-2,-1,-2,-1,-2,-1,0), k=(0,-3,-3,-1,-3,-3,-1,-2,-3)$ or $k=(0,-2,-1,0,-1,-1,-1,-2,-1)$ yield all the same vector $\hat{u}$ (this is because for these values the vectors $e^{i \cdot \hat{\psi}}$ in (6.41) are identical).

The 2-norm of the difference between the original vector $u$ and the projected vector $\hat{u}$ is 338.443 . This norm is larger than the 2-norm of the difference between the original vector $u$ and the Stetter vector corresponding to $\left(1, x_{1}, x_{1}^{2}, x_{2}, x_{1} x_{2}, x_{1}^{2} x_{2}, x_{2}^{2}, x_{1} x_{2}^{2}\right.$, $\left.x_{1}^{2} x_{2}^{2}\right)^{T}$, with given values $x_{1}=(-4-4 i)$ and $x_{2}=(-9-8 i)$, which is computed as 13.342. The large norm of $u-\hat{u}$ is caused by the errors in the last entries of the vector $u-\hat{u}$. These errors are relatively small but have a large influence on the computation of the norm. That the errors in the last entries of the vector $u-\hat{u}$ are larger than in the first entries is explained by the fact that this projection method operates in the logarithmic domain to regulate the influence of large dominating powers of the Stetter structure. When looking at the values of $x_{1}$ and $x_{2}$ before and after the projection we see that they are projected, respectively, from -4 and $(-13-5 i)$ in the vector $u$ to $(-3.862-3.684 i)$ and $(-9.476-8.268 i)$ in the projected vector $\hat{u}$. The latter values for $x_{1}$ and $x_{2}$ are close to the given values of $x_{1}=(-4-4 i)$ and $x_{2}=(-9-8 i)$.

A drawback of the above projection method is that the optimal values for $k$ in Equation (6.26) are not directly known: there exist various choices for $k$. Because of this, it is required to search for the optimal values for $k$ before this projection method can be applied. This amounts in determining the vector $k=\left(k_{1}, k_{2}, \ldots, k_{N}\right)^{T}$ such that the current estimate of the eigenpair improves. Currently, no efficient method to calculate $k$ is implemented other than a brute force search for a fixed maximum number of iterations. However, this requires a large amount of matrix-vector multiplications, which is contradictory to the initial goal of projection. To overcome this, the projection to Stetter structure discussed here is limited to the situation where the imaginary part of the approximate eigenvector is small enough to be discarded. This approach is used in the implementations of the Jacobi-Davidson methods and in the experiments of Chapter 7. 
A second approach for Stetter projection, which works for negative values as well as for complex values in the approximate eigenvector, is described next. The idea is to compare the structured eigenvector $v$ and the multiplication $\tilde{v}$ of the eigenvector with a variable $x_{i}: \quad \tilde{v}=x_{i} v$. Then the vectors $\tilde{v}$ and $v$ have entries in common which describe the relations between the separate entries in $v$ itself. If an approximate vector is indeed a 'true' eigenvector, then the values within the eigenvector should satisfy these relations too. The relations can be expressed in the form of $P\left(x_{1}, \ldots, x_{n}\right)^{T}-u=$ 0 for all the variables $x_{i}$. A linear least-squares method can compute the solution $\left(x_{1}, \ldots, x_{n}\right)^{T}$ with the smallest error $e=P\left(x_{1}, \ldots, x_{n}\right)^{T}-u$. This approach is illustrated with an example.

Example 6.3. As an example the situation with $n=2, d=2$ and $m=3$ is observed again where the eigenvector $v=\left(v_{1}, \ldots, v_{9}\right)$ is structured as $\left(1, x_{1}, x_{1}^{2}, x_{2}, x_{1} x_{2}, x_{1}^{2} x_{2}\right.$, $\left.x_{2}^{2}, x_{1} x_{2}^{2}, x_{1}^{2} x_{2}^{2}\right)^{T}$. When the vector $v$ is multiplied with the variable $x_{1}$ and when the vector $v$ is indeed an eigenvector, the following relations hold with respect to $x_{1}$ : $v_{1} x_{1}=v_{2}, v_{2} x_{1}=v_{3}, v_{4} x_{1}=v_{5}, v_{5} x_{1}=v_{6}, v_{7} x_{1}=v_{8}$ and $v_{8} x_{1}=v_{9}$. With respect to $x_{2}$ the following holds: $v_{1} x_{2}=v_{4}, v_{2} x_{2}=v_{5}, v_{3} x_{2}=v_{6}, v_{4} x_{2}=v_{7}, v_{5} x_{2}=v_{8}$, and $v_{6} x_{2}=v_{9}$. These relations can be expressed in matrix-vector form as:

$$
\left(\begin{array}{cc}
v_{1} & 0 \\
v_{2} & 0 \\
v_{4} & 0 \\
v_{5} & 0 \\
v_{7} & 0 \\
v_{8} & 0 \\
0 & v_{1} \\
0 & v_{2} \\
0 & v_{3} \\
0 & v_{4} \\
0 & v_{5} \\
0 & v_{6}
\end{array}\right)\left(\begin{array}{l}
x_{1} \\
x_{2}
\end{array}\right)-\left(\begin{array}{l}
v_{2} \\
v_{3} \\
v_{5} \\
v_{6} \\
v_{8} \\
v_{9} \\
v_{4} \\
v_{5} \\
v_{6} \\
v_{7} \\
v_{8} \\
v_{9}
\end{array}\right)=0
$$

By introducing a matrix $P$ and a vector $u$, Equation (6.43) can also be written as $P\left(x_{1}, x_{2}\right)^{T}-u=0$. Using a linear least-squares method the values for $x_{1}$ and $x_{2}$, which minimize the error $e=P\left(x_{1}, x_{2}\right)^{T}-u$, are computed.

Suppose for example the approximate (normalized) eigenvector is $u=\left(u_{1}, \ldots\right.$, $\left.u_{9}\right)=(1,4,6,2,18,43,27,76,224)^{T}$ (which has much resemblance with the Stetter vector $\left.(1,3,9,5,15,45,25,75,225)^{T}\right)$. A linear least-squares method yields the values $x_{1}=2.907$ and $x_{2}=5.106$ as the values which produce the smallest error $e$. When these values are substituted back into the symbolic structured eigenvector $v$, this yields the projected vector $\hat{u}=\left(\hat{u}_{1}, \ldots, \hat{u}_{9}\right)$ which exhibits Stetter structure: $\hat{u}=(1,2.91,8.45,5.11,14.84,43.15,26.07,75.79,220.33)^{T}$. To illustrate this Stetter structure in $\hat{u}$ the following relations hold for example: $\hat{u}_{5}=\hat{u}_{2} \hat{u}_{4}$ and $\hat{u}_{9}=\hat{u}_{2}^{2} \hat{u}_{4}^{2}$.

Remark 6.2. Note that the quantities $x_{1}$ and $x_{2}$ in Equation (6.43) can be computed independently from each other from the following: 


$$
\begin{gathered}
\min _{x_{1}}\left\|\left(\begin{array}{l}
v_{1} \\
v_{2} \\
v_{4} \\
v_{5} \\
v_{7} \\
v_{8}
\end{array}\right) x_{1}-\left(\begin{array}{l}
v_{2} \\
v_{3} \\
v_{5} \\
v_{6} \\
v_{8} \\
v_{9}
\end{array}\right)\right\|_{2}^{2} \text { and } \\
\min _{x_{2}}\left\|\left(\begin{array}{l}
v_{1} \\
v_{2} \\
v_{3} \\
v_{4} \\
v_{5} \\
v_{6}
\end{array}\right) x_{2}-\left(\begin{array}{l}
v_{4} \\
v_{5} \\
v_{6} \\
v_{7} \\
v_{8} \\
v_{9}
\end{array}\right)\right\|_{2}^{2} .
\end{gathered}
$$

When in (6.44) the involved vectors are denoted by $q_{1}$ and $q_{2}$, the linear leastsquares method yields $x_{i}=\frac{q_{1}^{T} q_{2}}{q_{1}^{T} q_{1}}$ as a solution for $\min _{x_{i}}\left\|q_{1} x_{i}-q_{2}\right\|_{2}^{2}$ for $i=1,2$. In this example this yields $x_{1}=\frac{9957}{3425}=2.907$ and $x_{2}=\frac{5693}{1115}=5.106$.

Example 6.2 (continued). Suppose one wants to project the vector $u$ from example 6.2 using the latter projection method. The vector $u$ is defined as $(1,-4,-5+29 i$, $-13-5 i, 5+72 i, 255-283 i, 16+140 i, 507-640 i,-4613+543 i)^{T}$. The projected vector $\hat{u}$ is computed as:

$$
\hat{u}=\left(\begin{array}{rrrr}
1.000 & & \\
-4.030 & - & 4.016 i \\
0.118 & + & 32.366 i \\
-9.152 & - & 8.053 i \\
4.548 & + & 69.202 i \\
259.551 & - & 297.153 i \\
18.911 & + & 147.389 i \\
515.626 & - & 669.935 i \\
-4768.156 & + & 629.428 i
\end{array}\right)
$$

and is structured like $\left(1, x_{1}, x_{1}^{2}, x_{2}, x_{1} x_{2}, x_{1}^{2} x_{2}, x_{2}^{2}, x_{1} x_{2}^{2}, x_{1}^{2} x_{2}^{2}\right)^{T}$ where $x_{1}$ and $x_{2}$ 
are given by:

$$
\begin{aligned}
& x_{1}=\frac{q_{1}^{T} q_{2}}{q_{1}^{T} q_{1}}=-4.030-4.016 i, \text { where } \\
& q_{1}=\left(\begin{array}{rrr}
1 & & \\
-4 & & \\
-13 & - & 5 i \\
5 & + & 72 i \\
16 & + & 140 i \\
507 & - & 640 i
\end{array}\right) \text { and } q_{2}=\left(\begin{array}{rrr}
-4 & & \\
-5 & + & 29 i \\
5 & + & 72 i \\
255 & - & 283 i \\
507 & - & 640 i \\
-4613 & + & 543 i
\end{array}\right) \text {, }
\end{aligned}
$$

and

$$
\begin{gathered}
x_{2}=\frac{q_{1}^{T} q_{2}}{q_{1}^{T} q_{1}}=-9.152-8.053 i \text {, where } \\
q_{1}=\left(\begin{array}{rrr}
1 & & \\
-4 & & \\
-5 & + & 29 i \\
-13 & - & 5 i \\
5 & + & 72 i \\
255 & - & 283 i
\end{array}\right) \text { and } q_{2}=\left(\begin{array}{rrr}
-13 & - & 5 i \\
5 & + & 72 i \\
255 & - & 283 i \\
16 & + & 140 i \\
507 & - & 640 i \\
-4613 & + & 543 i
\end{array}\right) .
\end{gathered}
$$

The 2-norm of the difference between $u$ and $\hat{u}$ is 181.349 , whereas it was 338.443 using the first projection method of this section. Moreover, the values of $x_{1}=-4$ and $x_{2}=(-13-5 i)$ in the vector $u$ are projected to $x_{1}=(-4.030-4.016 i)$ and $x_{2}=(-9.152-8.053 i)$ in the projected vector $\hat{u}$. These values are closer to the given values $x_{1}=(-4-4 i)$ and $x_{2}=(-9-8 i)$ than the values computed by the first projection method.

Although this projection method works for negative and complex entries of the approximate vectors and it works fast and without the need of additional matrixvector products with the matrix $A$, it is not yet implemented in combination with a Jacobi-Davidson method but it may be addressed in further research.

\subsubsection{Embedding Stetter projection in a Jacobi-Davidson method}

To embed the projection method of the previous section in a Jacobi-Davidson method, the Jacobi-Davidson implementation described in [58] is now modified as follows: let $\theta^{j}$ be the approximate smallest real eigenvalue of a matrix $A$ in iteration $j$ of the iterative eigenvalue solver corresponding to the approximate eigenvector $u^{j}$. Recall that the residual $r^{j}$ in iteration $j$ of this eigenvalue pair is defined as $r^{j}=A u^{j}-\theta^{j} u^{j}$. Now there are two possibilities: 
(i) If the norm of the residual $r^{j}$ is smaller than a specified tolerance threshold then the output of the eigenvalue solver is $\left\{u^{j}, \theta^{j}\right\}$ and $\theta^{j}$ is approximately the global minimum of the polynomial of interest.

(ii) If the norm of the residual $r$ is larger than or equal to the tolerance and $\max \left(\operatorname{Im}\left(u^{j}\right)\right)<1 \times 10^{-5}$ then the absolute values of $u^{j}$ are projected to the Stetter structure as described in the previous section yielding the vector $\hat{u}^{j}$. The corresponding eigenvalue $\hat{\theta}^{j}$ is computed using the Rayleigh quotient as in Equation (6.3). Finally, this new eigenvalue/eigenvector pair is accepted as a next improved pair if the norm of the residual decreases or is discarded otherwise:

$$
\left(u^{j+1}, \theta^{j+1}\right)= \begin{cases}\left(\hat{u}^{j}, \hat{\theta}^{j}\right) & \text { if }\left\|A \hat{u}^{j}-\hat{\theta}^{j} \hat{u}^{j}\right\|_{2}^{2}<r^{j} \\ \left(u^{j}, \theta^{j}\right) & \text { otherwise. }\end{cases}
$$

An implementation of the Jacobi-Davidson method using this method is applied to compute the global minimum of some polynomials. This approach only performs well in some particular cases when compared with the performance of the original Jacobi-Davidson implementation. This is described in detail in Section 7.3 of the next chapter.

Another method to embed the projection method of the previous section into the Jacobi-Davidson software is based on integrating the projected approximate eigenvector into the Refined Rayleigh-Ritz extraction phase of the method. This approach is described below.

The idea here is to perform a trade off between the residual and the amount of Stetter structure (as the amount of Stetter structure is maximal as the residual is zero). Here, the Refined Rayleigh-Ritz extraction as discussed in Section 6.2.2 is extended such that it also reflects the Stetter structure. This refined extraction is defined in Equation (6.10) where $A$ is the matrix of interest, $U$ is the $N \times k$ matrix that projects the problem to a $k$-dimensional problem and $\theta$ is an approximate eigenvalue for this problem. Then the approximate eigenvector is obtained by $U \hat{c}$. Now, the goal is to extend equation (6.10) such that the norm also reflects the Stetter structure:

$$
\hat{c}=\underset{c \in \mathbb{C}^{k},\|c\|=1}{\operatorname{argmin}}\|(A U-\theta U) c\|_{2}^{2}+\kappa
$$

where $\kappa$ is a function that penalizes deviation of $U c$ from the Stetter structure. Here $\kappa$ could for example be chosen as $\kappa=\alpha\|u-\hat{u}\|_{2}^{2}$, where $u=U c$ is the actual approximate eigenvector and where $\hat{u}$ is the projected Stetter vector of $u$. The positive quantity $\alpha$ is inversely proportional to $\|u-\hat{u}\|_{2}^{2}$ : it determines the amount of the difference between both vectors that is taken into account when computing the new vector $\hat{c}$. The value of $\alpha$ ranges between 0 and 1: $\alpha$ is zero at the beginning of the iterations and becomes closer to 1 when the convergence, and therefore also the amount of Stetter structure, increases. By extending the matrix $A U-\theta U$ as below, the original implementation based on a singular value decomposition on the matrix 
$A U-\theta U$ to solve Equation (6.49), can be used.

When minimizing the norm $\|(A U-\theta U) c\|_{2}^{2}$, the deviation of $u=U c$ from the Stetter structure can be taken into account too, as follows:

$$
\|(A U-\theta U) c\|_{2}^{2}+\alpha\|(U c-\hat{u})\|_{2}^{2}
$$

Note that the only unknown in this equation is $c$. Equation (6.50) can be written as:

$$
\begin{aligned}
& \|B c-v\|_{2}^{2} \quad \text { where } \\
& B=\left(\frac{A U-\theta U}{\alpha U}\right) \text { and } v=\left(\begin{array}{c}
0 \\
\alpha \hat{u}
\end{array}\right) \text {. }
\end{aligned}
$$

The remaining problem that has to be solved in this Refined Rayleigh-Ritz extraction method for projection, where the deviation from the Stetter structure is taken into account, is:

$$
\hat{c}=\underset{c \in \mathbb{C}^{k},\|c\|=1}{\operatorname{argmin}}\|B c-v\|_{2}^{2}
$$

involving the tall rectangular matrix $B$ and the vector $v$.

The problem of computing the minimum of $\|B c\|_{2}^{2}$ where $c \in \mathbb{C}^{k}$ and $\|c\|=1$ can be solved by using the Courant-Fischer Theorem as mentioned in [72]. Here the additional vector $-v$ makes the situation more difficult and as a result this theorem can not be applied directly here. A solution would be to extend this theorem or to use the method of Lagrange Multipliers [46] as shown below.

If no projection is used the matrix $B=A U-\theta U$ in Equation (6.52) is square since $\alpha=0$ and also $v=0$. Then a singular value decomposition of the matrix $(A U-\theta U)$ yields solutions for $\hat{c}$ as in the original implementation of the JacobiDavidson method.

When the involved matrix $B$ is not square, the problem (6.52) is solved using the method of Lagrange Multipliers. To apply such a technique the system of equations $B c-v$ has to be transformed first into a more convenient form. Applying a singular value decomposition to the (possibly) complex valued matrix $B$ yields $B=W D V^{*}$ with unitary matrices $W$ and $V$. The matrix $D$ is a rectangular matrix containing the singular values $d_{1}, \ldots, d_{k}$ of the matrix $B$ on its main diagonal. The problem 
(6.52) is rewritten as:

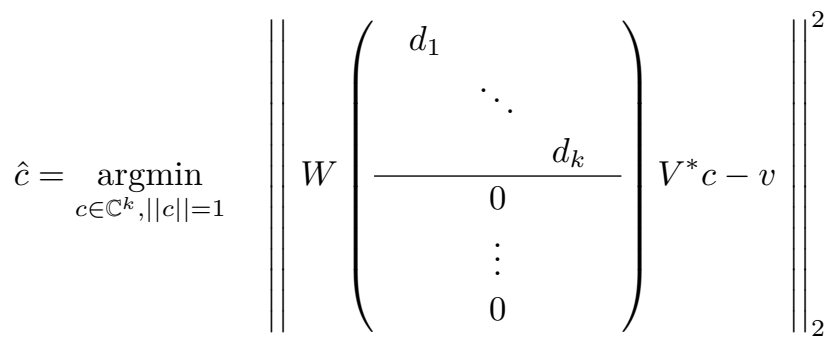

and

$$
\hat{c}=\underset{c \in \mathbb{C}^{k},\|c\|=1}{\operatorname{argmin}}\left\|\left(\begin{array}{ccc}
d_{1} & & \\
& \ddots & \\
& & d_{k} \\
0 & \vdots \\
& 0
\end{array}\right) V^{*} c-W^{*} v\right\|_{2}^{2} .
$$

Which can be simplified, using $\tilde{c}=V^{*} c$ and $\tilde{v}=W^{*} v$, to:

$$
\underset{\tilde{c} \in \mathbb{C}^{k},\|\tilde{c}\|=1}{\operatorname{argmin}}\left\|\left(\begin{array}{ccc}
d_{1} & & \\
& \ddots & \\
& & d_{k}
\end{array}\right) \tilde{c}-\tilde{v}\right\|_{2}^{2} .
$$

Note that the size of the vectors $\tilde{c}$ and $\tilde{v}$ is decreased such that the dimensions match to the matrix $\operatorname{diag}\left(\left(d_{1}, \ldots, d_{k}\right)^{T}\right)$. Note furthermore that $\|c\|=\|\tilde{c}\|$. Now the problem can be written as:

$$
\left\{\begin{array}{l}
\tilde{c}_{o p t}=\operatorname{argmin} f(\tilde{c}) \quad \text { where } f(\tilde{c})=\sum_{i=1}^{k}\left(d_{i} \tilde{c}_{i}-\tilde{v}_{i}\right)^{2} \\
\text { subject to } \\
g(\tilde{c})=\tilde{c}_{1}^{2}+\ldots+\tilde{c}_{k}^{2}-1=0
\end{array}\right.
$$

Now the theory of Lagrange multipliers can be applied because we are searching for the minimum $\tilde{c}_{o p t}$ of a multivariate function $f(\tilde{c})$ with continuous first derivatives subject to one constraint $g(\tilde{c})$. The theory of Lagrange multipliers is well-posed when the objective function and constraints are real-valued functions. In our case we are dealing with complex functions $f(\tilde{c})$ and $g(\tilde{c})$. Variational calculus can be easily extended to cope with this complication (see [52] and [93] for details). To prevent for complicated notation we will below present shortly how the Lagrange multiplier methods works for real-valued functions $f(\tilde{c})$ and $g(\tilde{c})$. 
The Lagrange function is constructed as:

$$
h(\tilde{c}, \lambda)=f(\tilde{c})+\lambda g(\tilde{c}) .
$$

If $\tilde{c}$ is a minimum for the constrained problem (6.56), then there exists a real $\lambda$ such that $(\tilde{c}, \lambda)$ is a stationary point for the Lagrange function (6.57). To compute the stationary points, the first-order conditions for optimality are considered: $\frac{\partial h}{\partial \lambda}$ and $\frac{\partial h}{\partial \tilde{c}_{1}}, \ldots, \frac{\partial h}{\partial \tilde{c}_{k}}$ are computed. The partial derivatives with respect to $\tilde{c}_{i}$ yield the equations:

$$
\tilde{c}_{i}=\frac{d_{i} \tilde{v}_{i}}{d_{i}^{2}+\lambda} \quad \text { for } i=1, \ldots, k
$$

These $\tilde{c}_{i}$, for $i=1, \ldots, k$, can be substituted into the partial derivative of $h(\tilde{c}, \lambda)$ with respect to $\lambda$, which is:

$$
\frac{\partial h}{\partial \lambda}=g(\tilde{c})=\tilde{c}_{1}^{2}+\ldots+\tilde{c}_{k}^{2}-1=0
$$

This yields a univariate polynomial equation in the only unknown $\lambda$ of degree $2 k$. From this univariate polynomial the real-valued value for the Lagrange multiplier $\lambda$ can be solved. Once $\lambda$ is known, the values for $\tilde{c}_{i}$ are computed by (6.58) and are backtransformed to $c_{i}$ by using the matrix $V$. In this way the solution $c=\left(c_{1}, \ldots, c_{k}\right)$ of problem (6.52) is computed. However, at this point this promising idea is not yet implemented in combination with a Jacobi-Davidson method but is subject for further research.

\subsection{JDCOMM: A Jacobi-Davidson type method for commuting matrices}

This section introduces a Jacobi-Davidson eigenvalue solver for commuting matrices to use in combination with the optimization method described in the previous sections and chapters. For the specific application of polynomial optimization, this solver is more efficient than other Jacobi-Davidson implementations. The optimization approach described in the previous chapter has some promising properties which are used in designing this efficient Jacobi-Davidson method: (i) because of the common eigenvectors, all the matrices $A_{p_{\lambda}}, A_{x_{1}}, \ldots, A_{x_{n}}$ commute, (ii) the matrices $A_{x_{1}}, \ldots, A_{x_{n}}$ are much sparser than the matrix $A_{p_{\lambda}}$, and finally, (iii) only the smallest real eigenvalue and its corresponding eigenvector of the matrix $A_{p_{\lambda}}$ are required to locate the (guaranteed) global optimum of $p_{\lambda}$, without addressing any (possible) local optimum $p_{\lambda}$ contains.

\subsubsection{The method JDCOMM}

Because the size of the commuting matrices mentioned in the previous section is usually very large, $N \times N$ with $N=m^{n}$ and $m=2 d-1$, it is not advisable to manipulate the full matrix in every iteration step of an algorithm and to address all 
the $N$ eigenvalues. Therefore it is a plausible choice to compute eigenvalues of the matrix $A_{p_{\lambda}}$ by means of a Jacobi-Davidson eigenvalue solver.

This section describes a Jacobi-Davidson eigenvalue solver called the JDCOMM method. The first important feature of this solver is that it targets on just one eigenvalue of the matrix $A_{p_{\lambda}}$ : it tries to compute the smallest real eigenvalue of the matrix $A_{p_{\lambda}}$ as it corresponds to the value of the global minimum of a polynomial $p_{\lambda}$. Available options of iterative eigenvalue solvers are to target on eigenvalues with the smallest or largest magnitudes or smallest or largest real parts. Computing the smallest real eigenvalue is our own developed option. The second important feature of the JDCOMM eigenvalue solver is that it takes advantage of the fact that the matrix $A_{p_{\lambda}}$ commutes with the other matrices $A_{x_{i}}, \ldots, A_{x_{n}}$ and the fact that the matrices $A_{x_{i}}, \ldots, A_{x_{n}}$ are much sparser than the matrix $A_{p_{\lambda}}$. This Jacobi-Davidson type method for commuting matrices, JDCOMM, is able to outperform a normal JacobiDavidson method by iterating with one of the very sparse matrices $A_{x_{i}}$ in the inner loop and only iterate with the less sparse matrix $A_{p_{\lambda}}$ in the outer loop. In general, in Jacobi-Davidson type methods, most of the work is done in the inner loops and therefore a speed up is expected.

\subsubsection{The JDCOMM expansion phase}

As a basis for the developed JDCOMM method we use the algorithm of the JD method described in [58]. The pseudocode of this JD method is given in Algorithm 2 of this thesis. To make it possible for the JDCOMM method to iterate with the sparse matrices $A_{x_{i}}$ in the inner loop and iterate with the less sparse matrix $A_{p_{\lambda}}$ in the outer loop, the expansion phase of the JD method, as described in Section 6.2.1, is extended and modified.

The Jacobi-Davidson expansion phase of the JDCOMM method works as follows. Suppose we have an approximate eigenpair $(\theta, v)$ for the matrix $A_{p_{\lambda}}$, where $v$ has unit norm and where $\theta$ is the Rayleigh quotient of $A_{p_{\lambda}}$ and $v$ defined as $\theta=v^{*} A_{p_{\lambda}} v$. The deviation of an approximate eigenpair $(\theta, v)$ to a true eigenpair of the matrix $A_{p_{\lambda}}$ is similarly as in Equation (6.2) measured by the norm of the residual $r$, defined here as:

$$
r=A_{p_{\lambda}} v-\theta v .
$$

We now look for an update $t \perp v$ such that the updated vector $v+t$ is a true eigenvector of the matrix $A_{p_{\lambda}}$ :

$$
A_{p_{\lambda}}(v+t)=\lambda(v+t)
$$

Rearranging the terms gives:

$$
\left(A_{p_{\lambda}}-\theta I\right) t=-r+(\lambda-\theta) v+(\lambda-\theta) t .
$$

Analogously as in Section 6.2.1, we derive the Jacobi-Davidson correction equation for the JDCOMM method: discarding the $(\lambda-\theta) t$ term, which is asymptotically secondorder (or third-order if $A_{p_{\lambda}}$ is symmetric), and projecting out the unknown quantity 
$(\lambda-\theta) v$ by the orthogonal projector $\left(I-v v^{*}\right)$ which also fixes $r$, gives rise to the Jacobi-Davidson correction equation:

$$
\left(I-v v^{*}\right)\left(A_{p_{\lambda}}-\theta I\right)\left(I-v v^{*}\right) t=-r \quad \text { where } t \perp v .
$$

In the Jacobi-Davidson method, the vast majority of the computational work is spent in solving the correction equation. Therefore, the simple but crucial idea is to make use of the sparser matrices $A_{x_{i}}, i=1, \ldots, n$, that have the same eigenvectors as $A_{p_{\lambda}}$.

Since $v$ is an approximate eigenvector for $A_{p_{\lambda}}$, it is likewise an approximate eigenvector for the matrix $A_{x_{i}}$. Let $\eta$ be the Rayleigh quotient of $A_{x_{i}}$ and $v: \eta=v^{*} A_{x_{i}} v$. Instead of Equation (6.61) we now want to update the vector $v$ such that we get an eigenvector for $A_{x_{i}}$ :

$$
A_{x_{i}}(v+t)=\mu(v+t)
$$

for a certain eigenvalue $\mu$ of $A_{x_{i}}$. Using the approximate value $\eta$ for $A_{x_{i}}$, this leads, similarly to Equation (6.62), to:

$$
\left(A_{x_{i}}-\eta I\right) t=-r+(\mu-\eta) v+(\mu-\eta) t,
$$

where the residual $r$ is defined here as:

$$
r=A_{x_{i}} v-\eta v
$$

By again neglecting the higher-order term $(\mu-\eta) t$ and projecting out the unknown term $(\mu-\eta) v$ by the orthogonal projector $\left(I-v v^{*}\right)$, we get the Jacobi-Davidson correction equation involving the matrix $A_{x_{i}}$ :

$$
\left(I-v v^{*}\right)\left(A_{x_{i}}-\eta I\right)\left(I-v v^{*}\right) t=-r \quad \text { where } t \perp v .
$$

The advantage of this correction equation compared to the correction equation in (6.63) is that the matrix-vector products with $A_{x_{i}}$ spent in approximately solving this equation are much cheaper than matrix-vector multiplication with $A_{p_{\lambda}}$. Typical practical examples indicate that the number of non-zeros of $A_{x_{i}}$ is often $10 \%$ or less than the number of non-zeros of $A_{p_{\lambda}}$.

\subsubsection{Pseudocode of the JDCOMM method}

In this subsection we give in Algorithm 3 the pseudocode of the JDCOMM method, the Jacobi-Davidson type method for commuting matrices. Note that in the subspace extraction phase of every (outer) iteration (Step 5 of the algorithm) we need to work with $A_{p_{\lambda}}$, since we should head for the leftmost real eigenvalue of $A_{p_{\lambda}}$.

We note that in Step 11, we may choose between two procedures. First, we may just compute $\eta$ by computing $A_{x_{i}} v$ and subsequently left-multiplying by $v^{*}$. This implies an extra cost of one matrix-vector product per iteration. Alternatively, we can store $A_{x_{i}} V$. 


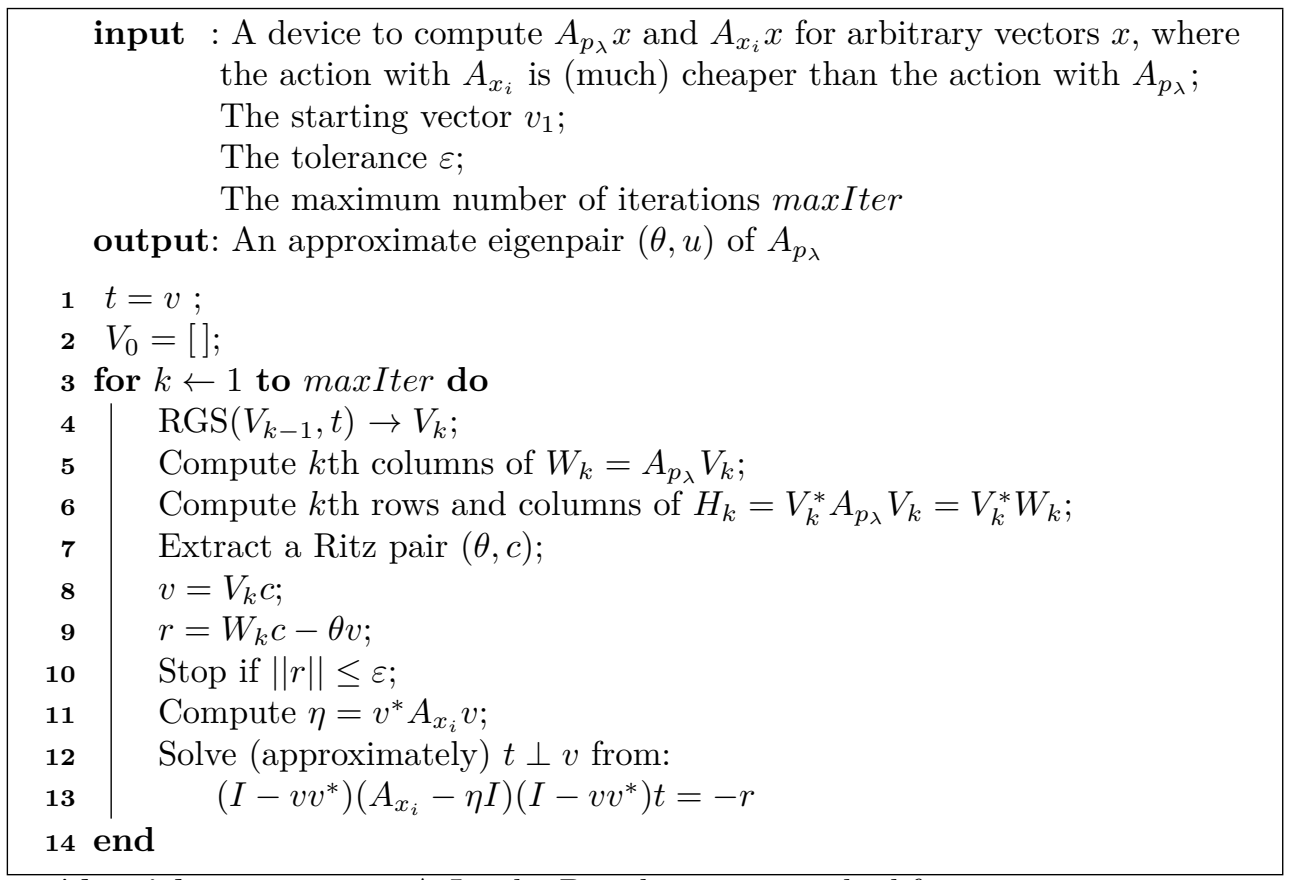

Algorithm 3: JDCOMM: A Jacobi-Davidson type method for commuting matrices

Remark 6.3. The JDCOMM method requires 'a device to compute $A_{p_{\lambda}} x$ and $A_{x_{i}} x$ for arbitrary vectors $x$ ' as some of the input variables (see Algorithm 3). Both devices can be implemented with an $n \mathrm{D}$-system as presented in Chapter 5 . This makes it possible to let the JDCOMM method operate in a matrix-free fashion too.

Section 7.5 of the next chapter describes some experiments in which the JDCOMM method is used to compute the global minima of some multivariate polynomials. 



\section{Chapter 7}

\section{Numerical experiments}

This chapter presents numerical experiments in which the global minima of various Minkowski dominated polynomials are computed using the approaches of the previous chapters. Section 7.1 describes the computations on a polynomial of order eight in four variables. To compute the global minimum of this polynomial conventional methods are used first. Then the $n \mathrm{D}$-systems approach is used in combination with the iterative eigenproblem solvers JDQR, JDQZ, and Eigs. Finally, the outcomes of these computations are compared with those of the software packages SOSTOOLS, GloptiPoly and SYNAPS. In Section 7.2 the $n$ D-systems approach is used to compute the global minima of 22 distinct polynomials. These 22 polynomials vary in the number of variables and in the total degree and therefore they also vary in the size of the involved linear operators and in their numerical conditioning. In this section the target selection, described in Section 6.3, is used to let the iterative solvers JD and JDQZ focus on the smallest real eigenvalues first. To put the performance of these methods into perspective it is compared with the performance of the SOSTOOLS package. The Sections 7.3 and 7.4 show the results of applying the projection method of Section 6.4 and the parallel approach of Section 5.3.4 on the same set of 22 polynomials. Finally, Section 7.5 describes four experiments in which the JDCOMM method is used to compute the global minima. The JDCOMM method is an extended version of the JD method for commuting matrices and is described in Section 6.5 of this thesis. The global minima in this section are also computed by the JD method and by the SOSTOOLS and GloptiPoly packages to be able to compare the performance and the efficiency of the various methods used. 


\subsection{An example of computing the global minimum of a polynomial of order 8}

In this section the Stetter-Möller approach, as described in the previous chapters, is used to compute the value and the location of the global minimum of a Minkowski dominated polynomial of order 8 . The polynomial under consideration here is the polynomial $p_{\lambda}$ with $d=4$ and $m=7$ containing four variables, $n=4$. Moreover, $\lambda$ equals 1 and the polynomial $q$ in Equation (4.1) is defined as $q\left(x_{1}, x_{2}, x_{3}, x_{4}\right)=$ $x_{1} x_{2} x_{3}^{2} x_{4}^{2}+3 x_{1} x_{2}+x_{2} x_{3}+x_{3} x_{4}^{2}+2 x_{3} x_{4}+x_{4}+8$ :

$$
\begin{gathered}
p_{1}\left(x_{1}, x_{2}, x_{3}, x_{4}\right)=\left(x_{1}^{8}+x_{2}^{8}+x_{3}^{8}+x_{4}^{8}\right)+ \\
x_{1} x_{2} x_{3}^{2} x_{4}^{2}+3 x_{1} x_{2}+x_{2} x_{3}+x_{3} x_{4}^{2}+2 x_{3} x_{4}+x_{4}+8 .
\end{gathered}
$$

The first-order conditions of this Minkowski dominated polynomial are given by:

$$
\left\{\begin{array}{lrllll}
d^{(1)}\left(x_{1}, x_{2}, x_{3}, x_{4}\right)=x_{1}^{7}+\frac{1}{8} x_{2} x_{3}^{2} x_{4}^{2}+\frac{3}{8} x_{2} & =0 \\
d^{(2)}\left(x_{1}, x_{2}, x_{3}, x_{4}\right)=x_{2}^{7}+\frac{1}{8} x_{1} x_{3}^{2} x_{4}^{2}+\frac{3}{8} x_{1}+\frac{1}{8} x_{3} & =0 \\
d^{(3)}\left(x_{1}, x_{2}, x_{3}, x_{4}\right) & =x_{3}^{7}+\frac{1}{4} x_{1} x_{2} x_{3} x_{4}^{2}+\frac{1}{8} x_{4}^{2}+\frac{1}{8} x_{2}+\frac{1}{4} x_{4}=0 \\
d^{(4)}\left(x_{1}, x_{2}, x_{3}, x_{4}\right)=x_{4}^{7}+\frac{1}{4} x_{1} x_{2} x_{3}^{2} x_{4}+\frac{1}{4} x_{3} x_{4}+\frac{1}{4} x_{3}+\frac{1}{8} & =0
\end{array}\right.
$$

Before we discuss the computation of the global minimum of this polynomial using the Stetter-Möller method with an $n \mathrm{D}$-system in combination with an iterative eigenvalue solver, we first follow two other, more 'conventional', approaches:

(i) the system of first-order partial derivatives which make up the first-order conditions is solved using standard software techniques. These solutions yield the stationary points of the polynomial $p_{1}$ which are used to compute the critical values.

(ii) the matrix $A_{p_{1}}$ is constructed explicitly and the global minimum is computed as the smallest real eigenvalue of this matrix. The eigenvalue computation is performed using a direct method.

For these two purposes standard routines from the software packages Mathematica and Matlab have been employed. For all the experiments throughout this section, use was made of Matlab 7.0.1 and Mathematica 5.2, running on an Intel Pentium PIV $2.8 \mathrm{GHz}$ platform with $512 \mathrm{MB}$ of internal memory.

(i) The real solutions of the system of equations (7.2) constitute the stationary points of $p_{1}\left(x_{1}, x_{2}, x_{3}, x_{4}\right)$. This system is solved using Mathematica with the NSolve routine. By substituting each real solution into the polynomial (7.1), we end up with 11 critical values: 8.003511, 7.329726, 6.742495, 6.723499, 6.045722, 5.866618, $5.731486,5.624409,5.491307,4.482528$, and 4.095165. The corresponding stationary points can be classified as a global minimizer, four local non-global minimizers, and six saddle points. The smallest one of these critical values yields the global minimum. The corresponding stationary point of this global minimum with value 4.095165 is 
given by:

$$
\begin{aligned}
& x_{1}=+0.876539 \\
& x_{2}=-0.903966 \\
& x_{3}=+0.862028 \\
& x_{4}=-0.835187
\end{aligned}
$$

(ii) In an attempt to obtain similar information in an independent fashion, the matrices $A_{x_{i}}$ for all $i=1, \ldots, 4$, and $A_{p_{1}}$ have been constructed explicitly using exact computation. The involved quotient space $\mathbb{R}\left[x_{1}, x_{2}, x_{3}, x_{4}\right] / I$ is of dimension $(2 d-1)^{n}=2401$. Therefore these matrices have 2401 eigenvalues. A property of these matrices is that they are highly sparse: the matrix $A_{x_{1}}$ contains only 4619 non-zero elements (percentage filled: $0.08 \%$ ), whereas the matrix $A_{p_{1}}$ contains 43178 non-zero elements (percentage filled: $0.75 \%$ ). See Figure 7.1 for a representation of the sparsity structure of the matrices $A_{x_{1}}$ and $A_{p_{1}}$.
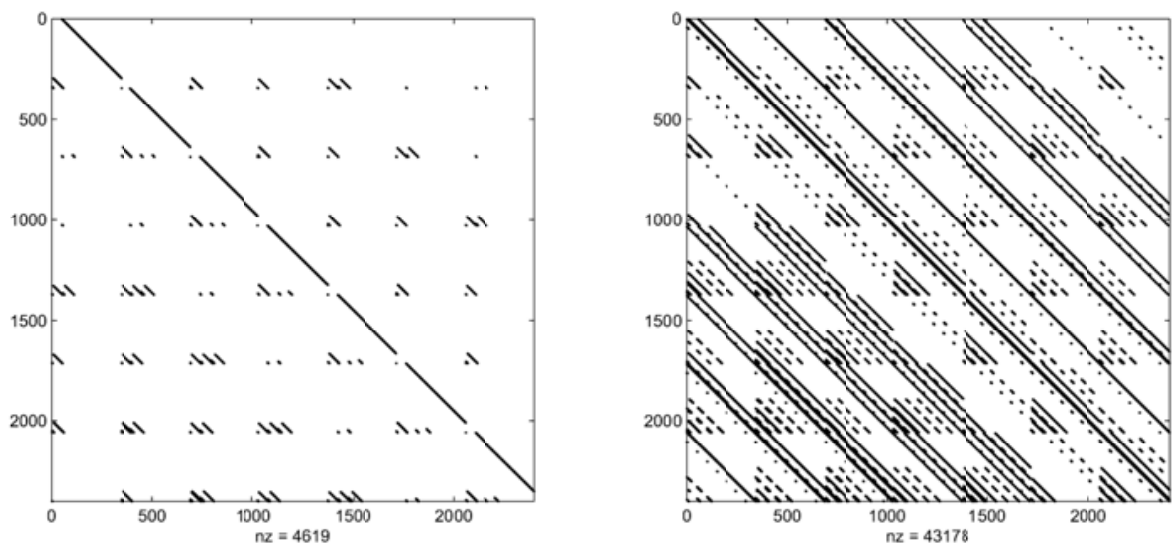

Figure 7.1: Sparsity structure of the matrices $A_{x_{1}}$ and $A_{p_{1}}$

Building the matrix $A_{p_{1}}$ took 465 seconds (whereas building the matrices $A_{x_{1}}$, $A_{x_{2}}, A_{x_{3}}$ and $A_{x_{4}}$ took 56,60,63, and 61 seconds, respectively). All the eigenvalues of the matrix $A_{p_{1}}$ are computed numerically in two ways: using the eigenvalue solver Eigensystem in Mathematica and the eigenvalue solver Eig in Matlab. In Table 7.1 the results of these computations are collected.

Table 7.1: Minimal real eigenvalue of the explicit matrix $A_{p_{1}}$

\begin{tabular}{llcl}
\hline Method & & Eigenvalue & Time (s) \\
\hline NSolve & solution of system $(7.2)$ & 4.09516474435915 & 744 \\
Eigensystem & construct $A_{p_{1}}$, compute all eigenvalues & 4.09516474435915 & $465+315$ \\
Eig & construct $A_{p_{1}}$, compute all eigenvalues & 4.09516474435924 & $465+176$ \\
\hline
\end{tabular}

The outcomes of these computations agree up to a limited number of decimal digits (the fact that the outcomes of the NSolve and the Eigenvalue function in Mathematica 
are exactly the same suggests that the NSolve function uses a matrix method too to solve the system of equations). To gain insight in the accuracy of the various methods, all the locations of the global minimum found with the above described methods have been used as the starting point for a local search method. Using a thirty digit working precision, which is a user option in the Mathematica/Maple software, the local search method obtains in all these cases the following coordinates for the global minimizer:

$$
\begin{aligned}
& x_{1}=+0.876539213106233894587289929758 \\
& x_{2}=-0.903966282304642050057296045914 \\
& x_{3}=+0.862027936174326572650513966373 \\
& x_{4}=-0.835187476756286528192781820247
\end{aligned}
$$

The corresponding criterion value of this point is computed as 4.095164744359157279770316678156 . These values have been used as the 'true' minimizer and global minimum of the polynomial $p_{1}\left(x_{1}, x_{2}, x_{3}, x_{4}\right)$ for the purpose of accuracy analysis of the numerical outcomes of the various computational approaches. The norm of the difference between this 'true' minimizer and the minimizer computed by the NSolve/Eigensystem and the Eig methods is $7.10543 \times 10^{-15}$ and $8.26006 \times 10^{-14}$, respectively.

Following the approach of the previous chapters, we then proceed to determine the global minimum of the polynomial (7.1) using the $n$ D-system implementation of the linear operator $A_{p_{1}}$ to compute only its smallest real eigenvalue with an iterative eigenvalue solver (instead of working with the explicit matrix $A_{p_{1}}$ ). The heuristic method used here is the least-increments method. The coordinates of the global minimum are computed from the corresponding eigenvector, employing the Stetter vector structure. For this purpose the iterative eigenvalue solvers JDQR, JDQZ, and Eigs have been used. JDQR is a normal - and JDQZ is a generalized iterative eigenvalue solver. Both methods employ Jacobi-Davidson methods coded in Matlab. The method Eigs is an iterative standard Matlab eigenproblem solver which uses (restarted) Arnoldimethods through ARPACK. The selection criterion of the iterative solvers used here is to focus on the eigenvalues with the smallest magnitude first, hoping to find the smallest real eigenvalue first.

For a fast convergence of these iterative eigenvalue methods, a balancing technique is used to balance the linear operator, see [27]. Balancing a linear operator or a matrix $M$ means finding a similarity transform $D^{-1} M D$, with $D$ a diagonal matrix, such that, for each $i$, row $i$ and column $i$ have (approximately) the same norm. The algorithms described in [27] help to reduce the norm of the matrix by using methods of Perron-Frobenius and the direct iterative method. Such a balancing algorithm is expected to be a good preconditioner for iterative eigenvalue solvers, especially when the matrix is not available explicitly.

In Table 7.2 the results of these computations are displayed. The columns denote the methods used, the minimal real eigenvalue computed, the error as the difference between this eigenvalue and the 'true' eigenvalue computed above, the number of 
required operator actions/matrix-vector products $(\mathrm{MVs})$, and the running time. For these computations the default parameters of the various methods are used.

Table 7.2: Minimal eigenvalue of the operator $A_{p_{1}}$ (one eigenvalue calculated)

\begin{tabular}{lccrr}
\hline Method & Eigenvalue & Error & \# MVs & Time (s) \\
\hline Eigs & 4.09516474405595 & $3.03 \times 10^{-10}$ & 3040 & 173 \\
JDQR & 4.09516474449668 & $1.38 \times 10^{-10}$ & 312 & 21 \\
JDQZ & 4.09516474427060 & $8.86 \times 10^{-11}$ & 302 & 21 \\
Eigs with balancing & 4.09516468968153 & $5.47 \times 10^{-8}$ & 2752 & 162 \\
JDQR with balancing & 4.09516473924322 & $5.12 \times 10^{-9}$ & 421 & 28 \\
JDQZ with balancing & 4.09516473593991 & $8.42 \times 10^{-9}$ & 389 & 28 \\
\hline
\end{tabular}

The global minimum computed by the JDQZ method produces the critical value that is the closest to the 'true' critical value computed by the local search method. For this setting, the $n \mathrm{D}$-systems approach uses the fewest operator actions. Using the corresponding eigenvector, the coordinates of the stationary point corresponding to this global minimum are computed as:

$$
\begin{aligned}
& x_{1}=+0.876539213107485 \\
& x_{2}=-0.903966282291641 \\
& x_{3}=+0.862027936168838 \\
& x_{4}=-0.835187476763094
\end{aligned}
$$

For the problem of finding the global minimum of a polynomial there are several other specialized software packages available, which employ different approaches. To put the performance of the $n \mathrm{D}$-systems approach into perspective, we will briefly discuss the outcomes of the computation of the global minimum of polynomial (7.1) when using the software packages SOSTOOLS, GloptiPoly and SYNAPS.

SOSTOOLS is a Matlab toolbox for formulating and solving sum of squares (SOS) problems (see [86], [90]). This toolbox uses the Matlab solver SeDuMi (see [96]) to solve the involved semi-definite programs (SDP). To compute the global minimum, SOSTOOLS searches for the largest possible $\gamma$ for which $p_{1}\left(x_{1}, x_{2}, x_{3}, x_{4}\right)-\gamma$ is still a sum of squares. This $\gamma$ may be the global minimum $p_{\star}$ we are looking for, depending on whether the polynomial $p_{1}\left(x_{1}, x_{2}, x_{3}, x_{4}\right)-p_{\star}$ can be written as a sum of squares of polynomials (see [87]). Note that the $n \mathrm{D}$-systems approach does not suffer from such a limitation.

GloptiPoly (see [54]) solves a multivariable polynomial optimization problem by building and solving convex linear matrix inequality (LMI) relaxations of the problem using the solver SeDuMi. It produces a series of lower bounds which converge to the global optimum we are looking for. The theory of moments and positive polynomials is used in the implementation of this software (see [74] and [75]).

SYNAPS [91] is a $\mathrm{C}++$ environment for symbolic and numeric computations. It provides a routine to search for the real solutions of a polynomial system of equations like system (7.2) within a given domain (based on a subdivision method). All the 
solutions of this system can be substituted into the polynomial (7.1) to find the global minimum.

The results of the computations using these software packages (using the default parameters) are collected in Table 7.3. Note that SOSTOOLS does not return the coordinates of the global minimum.

Table 7.3: Results of SOSTOOLS, GloptiPoly, and SYNAPS

\begin{tabular}{lcccc}
\hline Method & $x_{1}, x_{2}, x_{3}, x_{4}$ & Global Minimum & Error & Time $(\mathrm{s})$ \\
\hline SOSTOOLS & $(-,-,-,-)$ & 4.09516477401837 & $2.97 \times 10^{-8}$ & 10 \\
GloptiPoly & $(0.876535,-0.903963,0.862021,-0.835180)$ & 4.09516476247764 & $1.81 \times 10^{-8}$ & 11 \\
SYNAPS & $(0.876536,-0.903965,0.862026,-0.835184)$ & 4.09516474461324 & $2.50 \times 10^{-10}$ & 2 \\
\hline
\end{tabular}

When comparing the results in Table 7.2 and Table 7.3, we see that the methods SOSTOOLS, GloptiPoly and SYNAPS are faster than the methods using our $n$ D-systems approach. Moreover, the method Eigs performs poorly: it uses very many operator actions and needs a lot of running time. But where the methods in Table 7.3 appear to be faster, they are not as accurate as the iterative eigenvalue solvers in Table 7.2. This may be due to the default tolerance settings used in these software packages: a trade off is involved between computation time and accuracy/error. Although the methods in Table 7.3 outperform the $n$ D-systems approach in running time, the $n \mathrm{D}$ systems approach leaves us the possibility to increase its performance and accuracy by making use of the internal structure and the sparsity of the problem. The results of these attempts are described in the next sections.

\subsection{Computing the global minimum using target selection}

In [53] a set of 22 Minkowski dominated polynomials is generated. The number of variables in these polynomials ranges from 2 to 4 and the total degree ranges from 4 to 24. The explicit polynomials are given in Appendix B of this thesis and some characteristics are displayed in Table 7.4.

Table 7.4 shows the number $n$ of variables of the polynomials, the total degree $2 d$, the matrix size of the involved matrices $A_{p}^{T}$ which is computed as $(2 d-1)^{n}$, the value of the global minimum (also the smallest real eigenvalue $\lambda_{\min }$ ) and the number of terms of each of these polynomials.

The global minimum of all the polynomials in Table 7.4 is computed using the $n$ D-systems approach and an iterative solver. Each computation is repeated 20 times because of the randomly chosen first approximate eigenvector these solvers start with. The JD method, the implementation of the Jacobi-Davidson method by M. Hochstenbach in MATLAB (see [58]), is used for the research presented here. Also, the original Jacobi-Davidson method using QZ-decomposition, JDQZ [39], is used to compare the influence of different implementations of the iterative eigenvalue solver. Both implementations have various options that control their behavior. A preliminary analysis showed that some of these options should be adjusted to make sure that the algorithms are able to correctly converge to the smallest real eigenvalue. The set of parameters 
Table 7.4: Polynomial test cases of various complexities

\begin{tabular}{llrccc}
\hline$\#$ & $n$ & $2 d$ & Matrix size & Global minimum & Terms \\
\hline 1 & 2 & 8 & 49 & -7.259 & 11 \\
2 & 2 & 8 & 49 & -4.719 & 7 \\
3 & 2 & 10 & -20.71 & 19 \\
4 & 2 & 10 & 81 & -24.85 & 19 \\
5 & 4 & 4 & -24.45 & 13 \\
6 & 4 & 81 & -37.41 & 17 \\
7 & 2 & 121 & $-3.711 \times 10^{4}$ & 22 \\
8 & 2 & 12 & 121 & -52.77 & 23 \\
9 & 3 & 6 & 125 & -20.63 & 21 \\
10 & 3 & 6 & 125 & -142.9 & 19 \\
11 & 2 & 14 & 169 & -6116 & 36 \\
12 & 2 & 14 & 169 & -1115 & 33 \\
13 & 2 & 16 & 225 & $-2.927 \times 10^{6}$ & 46 \\
14 & 2 & 16 & 225 & -2258 & 40 \\
15 & 3 & 8 & 343 & -2398 & 34 \\
16 & 3 & 8 & 343 & -884.9 & 42 \\
17 & 2 & 24 & 529 & $-1.944 \times 10^{4}$ & 78 \\
18 & 2 & 24 & 529 & $-1.062 \times 10^{7}$ & 88 \\
19 & 3 & 10 & 729 & $-1.218 \times 10^{4}$ & 57 \\
20 & 3 & 10 & 729 & $-1.349 \times 10^{4}$ & 72 \\
21 & 3 & 12 & $-8.138 \times 10^{5}$ & 115 \\
22 & 3 & 12 & 1331 & $-1.166 \times 10^{6}$ & 112 \\
\hline
\end{tabular}

that is used is given in Table 7.5. The heuristic method used here to compute the matrix-vector products is the least-increments method and the target selection of the iterative solvers is chosen such that the smallest real eigenvalues are computed first.

Table 7.5: Settings for JD and JDQZ

\begin{tabular}{|c|c|c|c|c|c|}
\hline \multicolumn{3}{|c|}{ JD } & \multicolumn{3}{|c|}{ JDQZ } \\
\hline \# Eigenvalues & : & 1 & \# Eigenvalues & : & 1 \\
\hline Tolerance & : & $5 \times 10^{-8} \cdot(2 d-1)^{n}$ & Tolerance & : & $5 \times 10^{-8} \cdot(2 d-1)^{n}$ \\
\hline Imag. threshold & : & $1 \times 10^{-8} \cdot(2 d-1)^{n}$ & Imag. threshold & : & $1 \times 10^{-8} \cdot(2 d-1)^{n}$ \\
\hline Max \# iterations & : & 1000 & Max \# iterations & : & 1000 \\
\hline Min dimension & : & $0.5 \cdot(2 d-1)^{n}$ & Min dimension & : & $0.5 \cdot(2 d-1)^{n}$ \\
\hline Max dimension & : & $0.7 \cdot(2 d-1)^{n}$ & Max dimension & : & $0.7 \cdot(2 d-1)^{n}$ \\
\hline Start vector & : & Random & Start vector & : & Random \\
\hline Preconditioning & : & None & Preconditioning & : & None \\
\hline Extraction & : & Refined & $\begin{array}{l}\text { Schur Decomposi- } \\
\text { tion }\end{array}$ & : & No \\
\hline Expansion & : & JD & Conjugate pairs & : & No \\
\hline Linear Solver & : & GMRES & Linear solver & : & GMRES \\
\hline Fix target & : & 0.01 & Test space & : & 3 \\
\hline Krylov space & : & Yes & Lin solv tol & : & $0.7,0.49$ \\
\hline Max \# LS it. & : & 10 & Max \# LS it. & : & 15 \\
\hline Thick restart & : & 1 vector & Track & : & $1.0 \times 10^{-4}$ \\
\hline
\end{tabular}

The mean results of the 20 global minima computations of all the polynomials in the test set are given in Table 7.6. This table shows for JD and JDQZ the mean time and its standard deviation needed to compute the smallest real eigenvalue (denoted by mean \pm standard deviation), the mean required number of matrix-vector product and its standard deviation, and the error. This error is defined as the mean of the 
norm of the difference between the computed smallest real eigenvalue and the actual global minimum (computed by the Eig method on the explicit matrix $A_{p_{\lambda}}$ ).

Table 7.6: Results using JD and JDQZ with the least-increments method: time (mean \pm stdev), \# MVs (mean \pm stdev), and the error

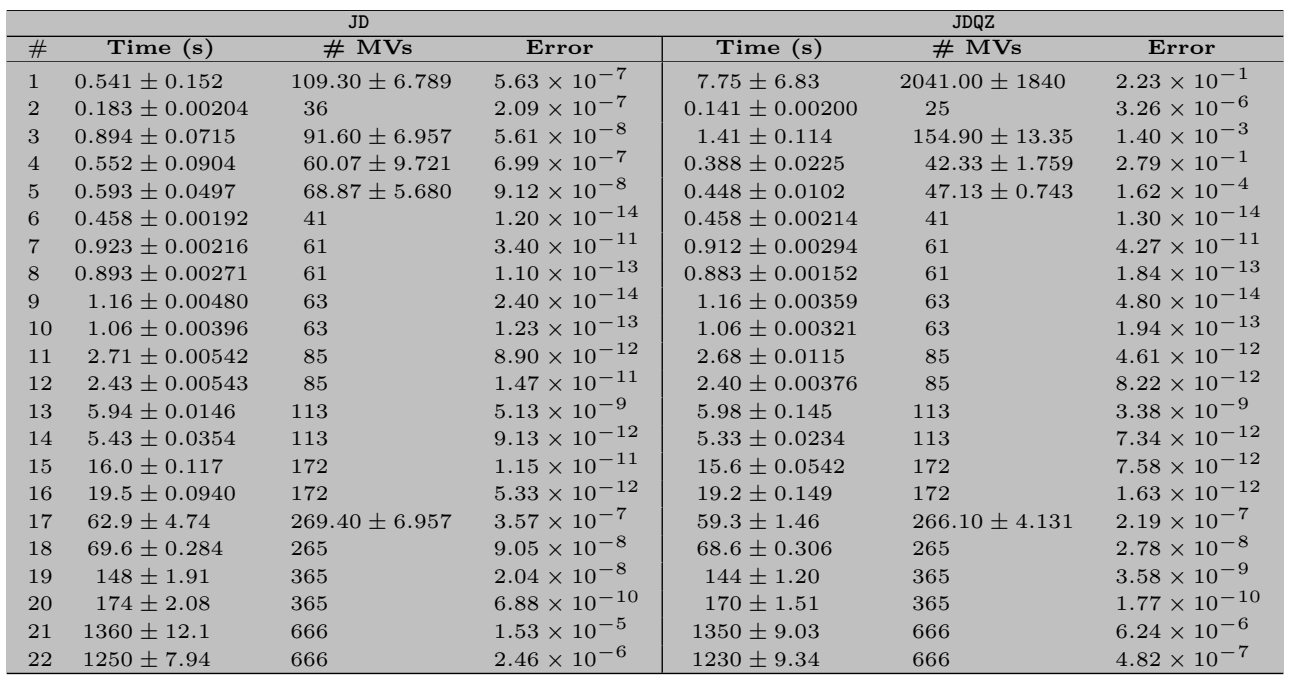

To place the performance results obtained above in the appropriate context, all cases are also tested with SOSTOOLS, the sum of squares toolbox for Matlab [90]. This software returns a certified lower bound for a polynomial $p$. Each test case from Table 7.4 is analyzed 20 times using SOSTOOLS with the default parameter settings. The mean processing time as well as the mean error, the mean of the norm of the difference between the computed smallest real eigenvalue of SOSTOOLS, and the actual global minimum, are recorded. The results are given in Table 7.7.

From this table it is clear that for the majority of the test cases the SOSTOOLS software approach is more efficient than the $n \mathrm{D}$-system implementation presented here. This is in accordance with the results described in [20]. In particular it appears that in the SOSTOOLS approach the processing time increases less rapidly with increasing complexity.

However, it should be noted that the error in the SOSTOOLS approach can be higher than the error in the $n \mathrm{D}$-systems implementation. In particular the test cases 17,18 , 21 and 22 can not accurately and reliably be solved by the SOSTOOLS software (at least not when using the default parameter settings of SOSTOOLS). These large errors can limit the actual application of this software on large problem instances.

Furthermore there are multivariate polynomials that are positive but can not be written as a sum of squares [92]. The global minimum of these polynomials can not be found directly using the SOSTOOLS approach. Such a limitation does not affect the Stetter-Möller matrix method. 
Table 7.7: Results using SOSTOOLS: time (mean \pm stdev) and the error

\begin{tabular}{lcc}
\hline$\#$ & Time $(\mathbf{s})$ & Error \\
\hline 1 & $1.00 \pm 1.58$ & $3.35 \times 10^{-9}$ \\
2 & $0.551 \pm 0.00617$ & $1.01 \times 10^{-9}$ \\
3 & $0.675 \pm 0.0150$ & $8.35 \times 10^{-9}$ \\
4 & $0.655 \pm 0.00650$ & $1.50 \times 10^{-8}$ \\
5 & $0.597 \pm 0.00662$ & $6.29 \times 10^{-9}$ \\
6 & $0.656 \pm 0.00302$ & $1.67 \times 10^{-8}$ \\
7 & $1.16 \pm 0.00980$ & $1.51 \times 10^{-4}$ \\
8 & $0.974 \pm 0.0169$ & $4.46 \times 10^{-8}$ \\
9 & $0.691 \pm 0.00570$ & $8.24 \times 10^{-8}$ \\
10 & $0.748 \pm 0.00607$ & $1.20 \times 10^{-7}$ \\
11 & $1.56 \pm 0.0254$ & $5.05 \times 10^{-5}$ \\
12 & $1.44 \pm 0.0143$ & $6.12 \times 10^{-6}$ \\
13 & $3.11 \pm 0.0185$ & $3.22 \times 10^{-2}$ \\
14 & $2.34 \pm 0.0163$ & $2.40 \times 10^{-6}$ \\
15 & $1.58 \pm 0.0153$ & $3.86 \times 10^{-6}$ \\
16 & $1.56 \pm 0.0176$ & $8.84 \times 10^{-7}$ \\
17 & $14.2 \pm 0.0206$ & $1.65 \times 10^{+4} \pm 3.77 \times 10^{-12}$ \\
19 & $24.1 \pm 0.0266$ & $4.50 \times 10^{+5}$ \\
20 & $4.48 \pm 0.0112$ & $8.58 \times 10^{-5}$ \\
21 & $4.77 \pm 0.00916$ & $3.47 \times 10^{-5}$ \\
22 & $19.4 \pm 0.0105$ & $1.77 \times 10^{-2}$ \\
\hline
\end{tabular}

\subsection{Projection to Stetter structure}

The effect of the projection of approximate eigenvectors to nearby approximate vectors with Stetter structure on the convergence of the JD software is analyzed using the first five polynomials of the set of polynomials mentioned in the previous section. The result of this experiment is that with the settings presented in Table 7.5 and the projection described in Section 6.4, the JD eigenvalue solver is no longer able to converge to the desired eigenvalue, as can be seen in Figure 7.2 which shows the residual $r^{j}$ before and after projection in the first test case for each iteration (see also [53]).

From this it is clear that the projection finds an improvement in each iteration. However, this improvement is discarded by the eigenvalue solver in the next iteration. A possible explanation for this may be the fact that the projected vector is no longer contained in the original search space and therefore the improvement is eliminated in the subsequent search space expansion and extraction of the new approximate eigenpair. A possible solution for this problem is also given in Section 6.4. Nonetheless these results strengthen the assumption that projection to Stetter structure can help to speed up the convergence.

Further experimental analysis shows that if the expansion type of the JD eigenvalue solver is changed from ' $\mathrm{jd}$ ' to 'rqi' then the eigenvalue solver can converge to the desired eigenvalue when projection is used. The Jacobi-Davidson method is an extension of the RQI (Rayleigh Quotient Iteration) where in the first part of the convergence process an inverse method is used to increase the convergence speed [58]. Table 7.8 shows the results of the first five test cases with the JD method with JD expansion, the JD method with RQI expansion without projection and the JD method 


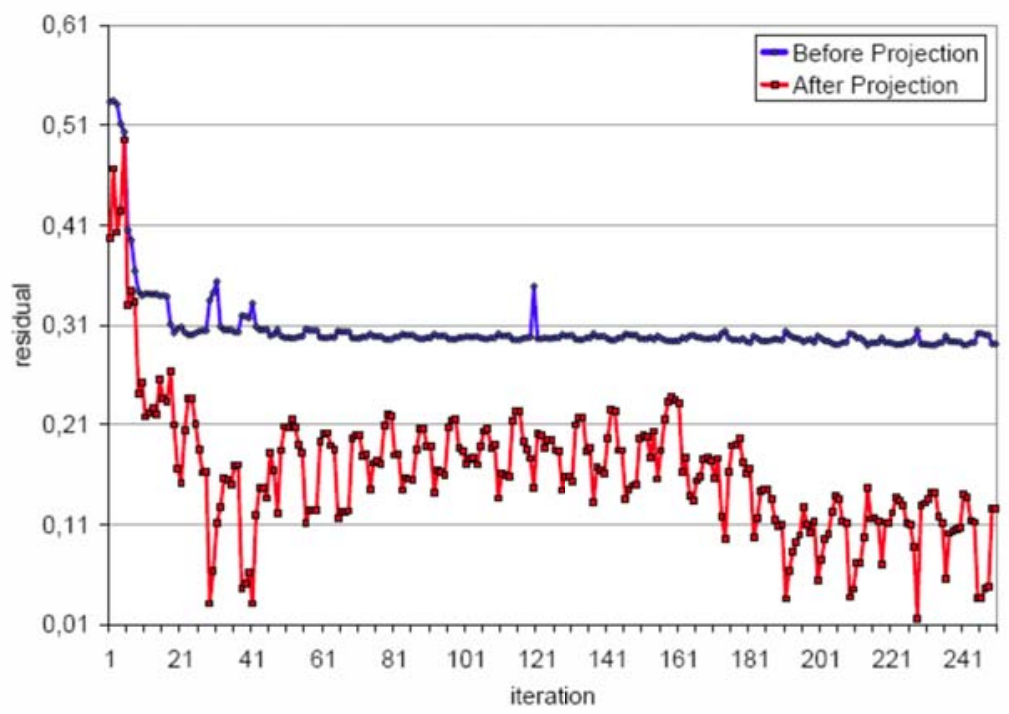

Figure 7.2: Residuals before and after projection during 250 iterations of the JD eigenvalue solver

with RQI expansion with projection.

Table 7.8: Results for the projection with JD: \# MVs (mean \pm stdev) and the error

\begin{tabular}{ccc|cc|cc}
\hline & \multicolumn{2}{c}{ JD } & \multicolumn{2}{c}{ RQI \& No projection } & \multicolumn{2}{c}{ RQI \& Projection } \\
\hline \# & \# MVs & Error & \# MVs & Error & \# MVs & Error \\
\hline 1 & $109.3 \pm 6.789$ & $5.63 \times 10^{-7}$ & $1661 \pm 1088$ & $4.45 \times 10^{-5}$ & $71.93 \pm 7.741$ & $4.69 \times 10^{-7}$ \\
2 & 36 & $2.09 \times 10^{-7}$ & $34.53 \pm 3.871$ & $1.23 \times 10^{-6}$ & 36 & $2.46 \times 10^{-7}$ \\
3 & $91.60 \pm 6.957$ & $5.61 \times 10^{-8}$ & $82.07 \pm 9.721$ & $2.92 \times 10^{-6}$ & $85.73 \pm 7.741$ & $5.26 \times 10^{-6}$ \\
4 & $60.07 \pm 9.721$ & $6.99 \times 10^{-7}$ & $57.87 \pm 7.039$ & $6.46 \times 10^{-7}$ & $57.13 \pm 7.039$ & $3.26 \times 10^{-7}$ \\
5 & $68.87 \pm 5.680$ & $9.12 \times 10^{-8}$ & $69.60 \pm 9.109$ & $2.73 \times 10^{-7}$ & $66.93 \pm 5.470$ & $6.46 \times 10^{-8}$ \\
\hline
\end{tabular}

This table does not show a difference between the three approaches except for the first test case. This first test case requires a relatively large number of operator actions with the original JD implementation. If the expansion method is changed to RQI then the number of operator actions increases more than tenfold to a level that is comparable with the JDQZ implementation. However, if the projection is applied with the RQI expansion then the required number of operator actions decreases below the number of operator actions required with the original implementation. This means that significantly less iterations of the eigenvalue solver are required because an iteration with projection requires two additional operator actions compared to a standard iteration: (i) an operator action for the Rayleigh quotient (6.3) to determine the new approximate eigenvalue and (ii) an operator action for the residual check in Equation (6.48).

Although no additional improvements can be seen with the presented settings, 
apart from the first test case, the results presented here can serve as an initial proof of concept that projection to Stetter structure can be used to increase the convergence speed of the eigenvalue solvers, at least if the initial estimate is accurate enough.

\subsection{Parallel computations in the $n$ D-systems approach}

Although a parallel implementation of the least-increments method is not very promising as explained in Section 5.3.4, we carried this out nevertheless. The results of computing the global minimum of the test set of 22 polynomials using iterative solvers and a parallel $n \mathrm{D}$-system implementation are given in Table 7.9 (see also [53]). The least-increments method is implemented here to compute each path in the $n \mathrm{D}$-system on a separate processor. The number of required paths are shown in the last column of Table 7.4 as the number of terms of each polynomial. The number of processors used here is 4 . Table 7.9 shows clearly that the computing time is longer but the required number of operator actions is roughly identical in this parallel environment in comparison with the results in Table 7.6. However, it is also clear to see that the increase in computation time for both the parallel JD and JDQZ method is not that high when the number of paths and the size of the involved matrix/operator increases: for example for the last 2 cases where the increase in computation time is roughly 2 for both the iterative methods.

Table 7.9: Results for the parallel implementation of the $n$ D-system for JD and JDQZ: time (mean \pm stdev), \# MVs (mean \pm stdev) and the error

\begin{tabular}{|c|c|c|c|c|c|c|}
\hline & \multicolumn{3}{|c|}{ JD } & \multicolumn{3}{|c|}{ JDQZ } \\
\hline$\#$ & Time (s) & \# MVs & Error & Time (s) & \# MVs & Error \\
\hline 2 & $26 \pm 0.131$ & 36 & $5.52 \times 10^{-7}$ & $27.2 \pm 0.557$ & 41 & $1.90 \times 10^{-6}$ \\
\hline 4 & $34.9 \pm 1.38$ & $59.33 \pm 6.351$ & $1.17 \times 10^{-7}$ & $31.6 \pm 0.737$ & $43 \pm 3.464$ & $4.65 \times 10^{-1}$ \\
\hline 5 & $70.3 \pm 1.39$ & $77.67 \pm 6.351$ & $5.48 \times 10^{-8}$ & $64.8 \pm 0.918$ & $47.67 \pm 0.577$ & $8.61 \times 10^{-5}$ \\
\hline 6 & $76.7 \pm 3.08$ & $52 \pm 11$ & $1.40 \times 10^{-14}$ & $73.6 \pm 3.29$ & $44.67 \pm 6.351$ & $1.40 \times 10^{-14}$ \\
\hline 8 & $47.5 \pm 2.01$ & $68.33 \pm 6.351$ & $9.10 \times 10^{-14}$ & $47.9 \pm 0.674$ & 71 & $7.90 \times 10^{-14}$ \\
\hline 9 & $67.8 \pm 1.89$ & $70.33 \pm 6.351$ & $7.00 \times 10^{-15}$ & $68.6 \pm 1.28$ & $66.67 \pm 3.512$ & $3.40 \times 10^{-14}$ \\
\hline 10 & $70.1 \pm 7$ & $74 \pm 11$ & $4.00 \times 10^{-14}$ & $68.5 \pm 4.28$ & $75 \pm 6.928$ & $1.55 \times 10^{-13}$ \\
\hline 11 & $78.3 \pm 9.35$ & $136.3 \pm 38.63$ & $9.10 \times 10^{-12}$ & $68.2 \pm 1.87$ & $95.67 \pm 9.238$ & $4.25 \times 10^{-12}$ \\
\hline 12 & $69.3 \pm 3.84$ & $99.67 \pm 16.80$ & $4.75 \times 10^{-12}$ & $67.3 \pm 0.601$ & $95.67 \pm 9.238$ & $7.21 \times 10^{-12}$ \\
\hline 13 & $118 \pm 13.8$ & $131.3 \pm 31.75$ & $6.98 \times 10^{-9}$ & $114 \pm 1.46$ & 129 & $1.86 \times 10^{-9}$ \\
\hline 18 & $307 \pm 1.40$ & 265 & $9.65 \times 10^{-8}$ & $304 \pm 2.56$ & 265 & $2.28 \times 10^{-8}$ \\
\hline 19 & $614 \pm 15.4$ & 365 & $9.38 \times 10^{-9}$ & $614 \pm 10.2$ & 365 & $4.35 \times 10^{-9}$ \\
\hline 20 & $615 \pm 10.1$ & 365 & $2.39 \times 10^{-9}$ & $613 \pm 10$ & 365 & $2.66 \times 10^{-10}$ \\
\hline 21 & $2580 \pm 636$ & $702.7 \pm 63.51$ & $2.10 \times 10^{-5}$ & $2160 \pm 29.5$ & 666 & $5.64 \times 10^{-6}$ \\
\hline 22 & $2150 \pm 44.3$ & 666 & $2.19 \times 10^{-6}$ & $2130 \pm 33.2$ & 666 & $4.96 \times 10^{-7}$ \\
\hline
\end{tabular}

\subsection{Numerical experiments with JDCOMM software}

In this section a Matlab implementation of the JDCOMM method, based on the pseudocode of Algorithm 3, is used to compute the global minimum of several polynomials. 
Table 7.10: Sparsity of the matrices $A_{p_{1}}$ and $A_{x_{1}}, A_{x_{2}}, A_{x_{3}}, A_{x_{4}}$

\begin{tabular}{lcc}
\hline Matrix of dimension $2401 \times 2401$ & Number of non-zeros & \% filled \\
\hline$A_{p_{1}}$ & 1326556 & 23.01 \\
$A_{x_{1}}$ & 63186 & 1.10 \\
$A_{x_{2}}$ & 52558 & 0.91 \\
$A_{x_{3}}$ & 52663 & 0.91 \\
$A_{x_{4}}$ & 54238 & 0.94 \\
\hline
\end{tabular}

To put the performance of this method into perspective, its performance is compared with the performance of the JD method. The JD method iterates with the relatively 'expensive' operator $A_{p_{\lambda}}$, whereas the JDCOMM method tries to outperform the JD method by targeting on the smallest real eigenvalue while iterating with a 'cheap' operator $A_{x_{i}}$ in the inner loop and with the operator $A_{p_{\lambda}}$ in the outer loop. Finally, the outcomes of both the methods JD and JDCOMM are compared to the outcomes of the SOSTOOLS and GloptiPoly software.

All the results throughout this section are obtained with Matlab version $R 2007 B$ running on an Intel Pentium PIV 2.8GHz platform with $1024 \mathrm{MB}$ of internal memory.

\subsubsection{Experiment 1}

Here we demonstrate the computation of the global minimum of a multivariate polynomial by using the JD and the JDCOMM method. For the first experiment a polynomial $p_{\lambda}$ with $n=4, d=4, m=7$, and $\lambda=1$ is considered:

$$
\begin{gathered}
p_{1}\left(x_{1}, x_{2}, x_{3}, x_{4}\right)=\left(x_{1}^{8}+x_{2}^{8}+x_{3}^{8}+x_{4}^{8}\right)+ \\
14 x_{1}^{4} x_{2} x_{3} x_{4}+6 x_{1} x_{2}^{4} x_{3} x_{4}-11 x_{2}^{3} x_{3}^{2} x_{4}^{2}+x_{1} x_{2} x_{3}^{2} x_{4}^{2}+8 x_{2} x_{3} x_{4}^{3}+ \\
+x_{3} x_{4}^{2}+3 x_{1} x_{2}+x_{2} x_{3}+2 x_{3} x_{4}+x_{4}+8 .
\end{gathered}
$$

Considering the quotient space $\mathbb{R}\left[x_{1}, x_{2}, x_{3}, x_{4}\right] / I$ of dimension $N=(2 d-1)^{n}=$ 2401 , the matrices $A_{p_{1}}, A_{x_{1}}, A_{x_{2}}, A_{x_{3}}$, and $A_{x_{4}}$ are constructed explicitly. From the 2401 eigenvalues of these matrices, we are interested in the smallest real eigenvalue and corresponding eigenvector of the matrix $A_{p_{1}}$. Table 7.10 shows the differences in the number of non-zero elements of all the involved matrices. The matrices $A_{x_{i}}, i=$ $1, \ldots, 4$, are much sparser than the matrix $A_{p_{1}}$. See also Figure 7.3 for a representation of the sparsity structure of these matrices.

One approach would be to compute all the eigenvalues of the matrix $A_{p_{1}}$ using a direct solver and to select the smallest real one as being the global optimum of polynomial $p_{1}\left(x_{1}, x_{2}, x_{3}, x_{4}\right)$. A similar approach would be to compute all the eigenvalues of the matrices $A_{x_{1}}, A_{x_{2}}, A_{x_{3}}$ or $A_{x_{4}}$ and to read off the coordinates of the stationary points from the corresponding eigenvectors. The global optimum of $p_{1}\left(x_{1}, x_{2}, x_{3}, x_{4}\right)$ can then be selected from all the stationary points by computing the associated function values and to pick out the smallest real one. Computing all the eigenvalues of the matrices $A_{p_{1}}, A_{x_{1}}, A_{x_{2}}, A_{x_{3}}$, and $A_{x_{4}}$ using a direct solver takes 57.9, 56.4, 54.5, 52.8, and 48.8 seconds respectively. The global minimizer we are looking for has the value 

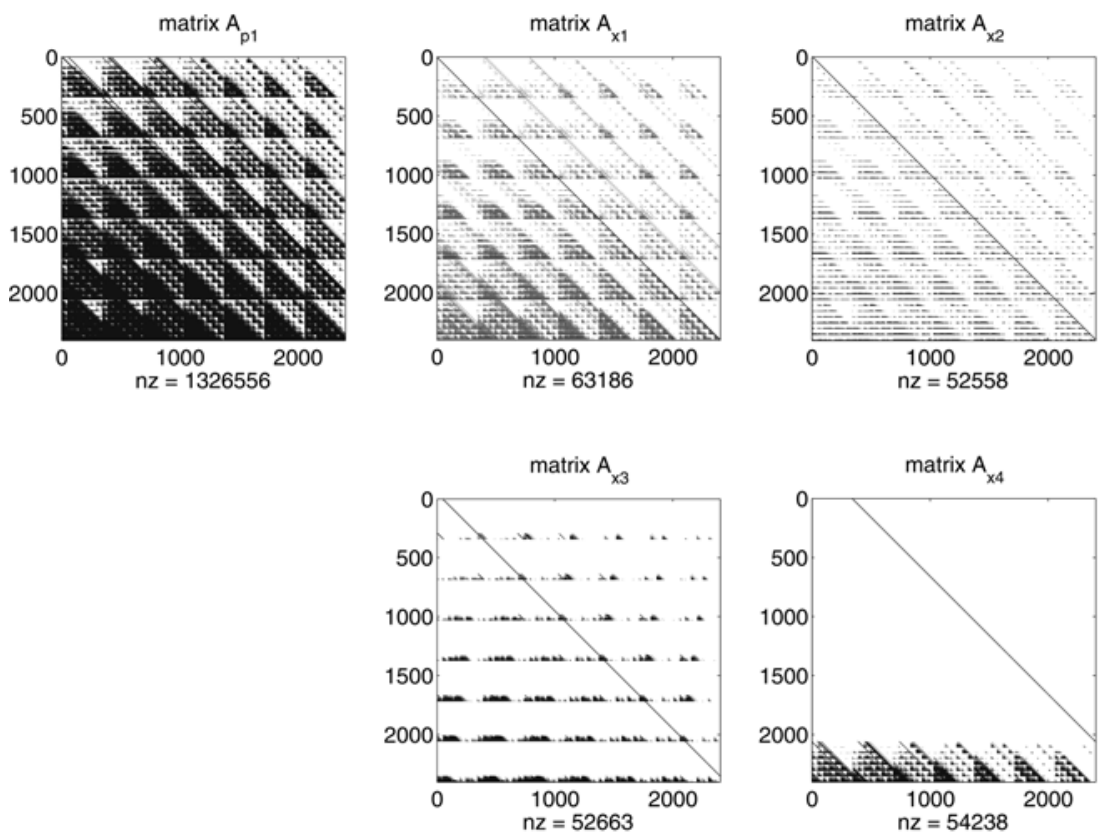

Figure 7.3: Sparsity structure of the matrices $A_{p_{1}}$ and $A_{x_{i}}, i=1, \ldots, 4$

Table 7.11: Comparison between performance of JD and JDCOMM

\begin{tabular}{lccccc}
\hline Method & MV $A_{p_{1}}$ & MV $A_{x_{i}}$ & Flops $\times 10^{8}$ & Time (s) & Global minimum \\
\hline JD & 844 & 0 & 11.20 & 50.0 & $-3.127 \times 10^{6}$ \\
JDCOMM $A_{x_{1}}$ & 69 & 459 & 1.21 & 5.8 & $-3.127 \times 10^{6}$ \\
JDCOMM $A_{x_{2}}$ & 87 & 657 & 1.50 & 7.5 & $-3.127 \times 10^{6}$ \\
JDCOMM $A_{x_{3}}$ & 89 & 679 & 1.54 & 7.6 & $-3.127 \times 10^{6}$ \\
JDCOMM $A_{x_{4}}$ & 66 & 426 & 1.11 & 4.9 & $-3.127 \times 10^{6}$ \\
\hline
\end{tabular}

$-3.127 \times 10^{6}$ and is attained at the point $x_{1}=7.085, x_{2}=7.186, x_{3}=-6.716$, and $x_{4}=6.722$. The location of these eigenvalues with respect to the whole eigenvalue spectrum of each matrix is depicted in Figure 7.4.

A more efficient way of computing the global optimum is by using a JacobiDavidson type eigenvalue solver for a set of commuting matrices and try to compute only the smallest real eigenvalue of our polynomial $p_{1}$. Table 7.11 shows the results of a conventional JD method and the JDCOMM method for computing the smallest real eigenvalue of the matrix $A_{p_{1}}$, as described in Section 6.5. The JD method used here is described in [58]. The settings used for both the JD and JDCOMM are: the tolerance on the residual norm for the convergence of the eigenpair is chosen as $10^{-6}$; the restart parameters for the minimal and maximal dimensions of the search spaces are chosen as 10 and 30 .

The first column of Table 7.11 denotes the method used and the last column shows the smallest real eigenvalue computed by the corresponding method. The second and 

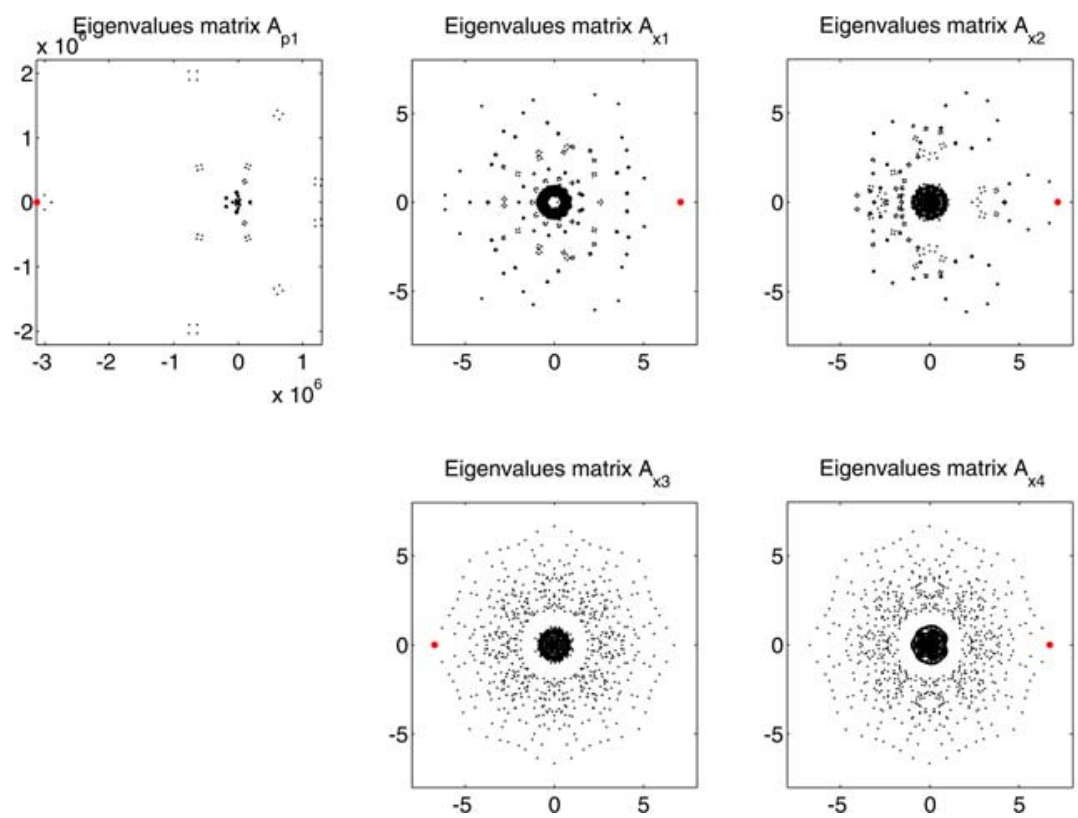

Figure 7.4: Eigenvalue spectra of the matrices $A_{p_{1}}$ and $A_{x_{i}}, i=1, \ldots, 4$

third columns contain the number of matrix-vector products of the method with the matrix $A_{p_{1}}$ and the matrices $A_{x_{i}}$ respectively. The fourth column shows the amount of floating point operations (flops) needed to perform all the matrix-vector products; the amount of flops is computed as the sum of the products of the number of multiplications with the number of non-zeros of the corresponding matrices: i.e., the JDCOMM method which iterates with the matrix $A_{x_{1}}$ in the inner loop and with the matrix $A_{p_{1}}$ in the outer loop requires 69 iterations with the matrix $A_{p_{1}}$ and 459 iterations with the matrix $A_{x_{1}}$. This costs $69 \times 1326556+459 \times 63186=120534738$ flops. Finally, column five shows the required computation time.

It is easy to see that all the methods compute the same leftmost real eigenvalue, and moreover, that the performance of the JDCOMM method is much better than the performance of the JD method, in terms of computation time as well as in required floating point operations.

Figure 7.5, shows the plot of the norms of the residuals in the eigenvalue equation (at each JD outer step), against the number of matrix-vector products.

To put the performance of the JD approach of this paper into perspective, we discuss briefly the outcomes of the computation of the global minimum of polynomial (7.6) by the software packages SOSTOOLS and GloptiPoly, which employ totally different approaches. Both, SOSTOOLS and GloptiPoly, compute the same global optimum and locations of polynomial $p_{1}$ as shown in Table 7.11 (using the default parameter settings). To determine these, SOSTOOLS uses 19.3 seconds and GloptiPoly uses 17.2 seconds. This shows that the JDCOMM method has the potential to outperform these 

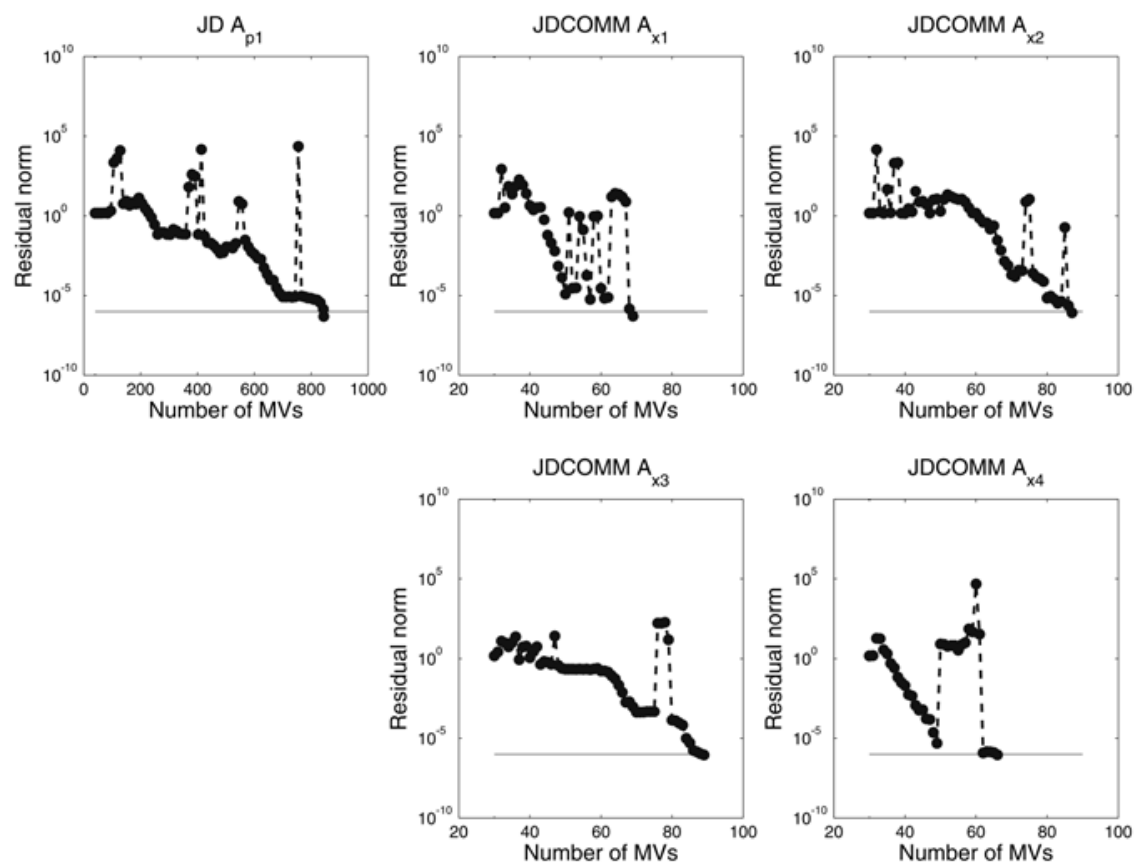

Figure 7.5: Residual norms against MVs with $A_{p_{1}}$ for the JD and JDCOMM methods

methods in this particular application.

\subsubsection{Experiment 2}

In a second experiment we consider a slightly larger problem of higher degree: a polynomial $p_{\lambda}$ with $n=4, d=5, m=9$, and $\lambda=1$ is considered:

$$
\begin{gathered}
p_{1}\left(x_{1}, x_{2}, x_{3}, x_{4}\right)=\left(x_{1}^{10}+x_{2}^{10}+x_{3}^{10}+x_{4}^{10}\right)+ \\
-7 x_{1}^{3} x_{2}^{4} x_{4}^{2}+3 x_{1} x_{2}^{3} x_{3}^{3} x_{4}^{2}+x_{2}^{3} x_{3}^{4} x_{4}^{2}+2 x_{1} x_{2} x_{3}^{6} x_{4}+ \\
-x_{1}^{4} x_{2} x_{3}^{3}+2 x_{2}^{3} x_{4}^{5}+3 .
\end{gathered}
$$

The quotient space $\mathbb{R}\left[x_{1}, x_{2}, x_{3}, x_{4}\right] / I$ has dimension $N=(2 d-1)^{n}=6561$, which yields matrices $A_{p_{1}}, A_{x_{1}}, A_{x_{2}}, A_{x_{3}}$, and $A_{x_{4}}$ of dimensions $6561 \times 6561$. Table 7.12 shows the differences in the number of non-zero elements of all the involved matrices. See also Figure 7.6.

Computing all the 6561 eigenvalues of the matrices using a direct method takes $548,345,358,339$, and 389 seconds respectively. The results of computing the smallest real eigenvalue of the matrix $A_{p_{1}}$ using the JD and JDCOMM methods, are displayed in Table 7.13. The parameters for the JD methods are slightly changed to $10^{-10}$ for 
Table 7.12: Sparsity of the matrices $A_{p_{1}}$ and $A_{x_{1}}, A_{x_{2}}, A_{x_{3}}, A_{x_{4}}$

\begin{tabular}{lcc}
\hline Matrix of dimension $6561 \times 6561$ & Number of non-zeros & \%filled \\
\hline$A_{p_{1}}$ & 9929906 & 23.07 \\
$A_{x_{1}}$ & 299594 & 0.70 \\
$A_{x_{2}}$ & 254523 & 0.59 \\
$A_{x_{3}}$ & 254845 & 0.59 \\
$A_{x_{4}}$ & 279887 & 0.65 \\
\hline
\end{tabular}
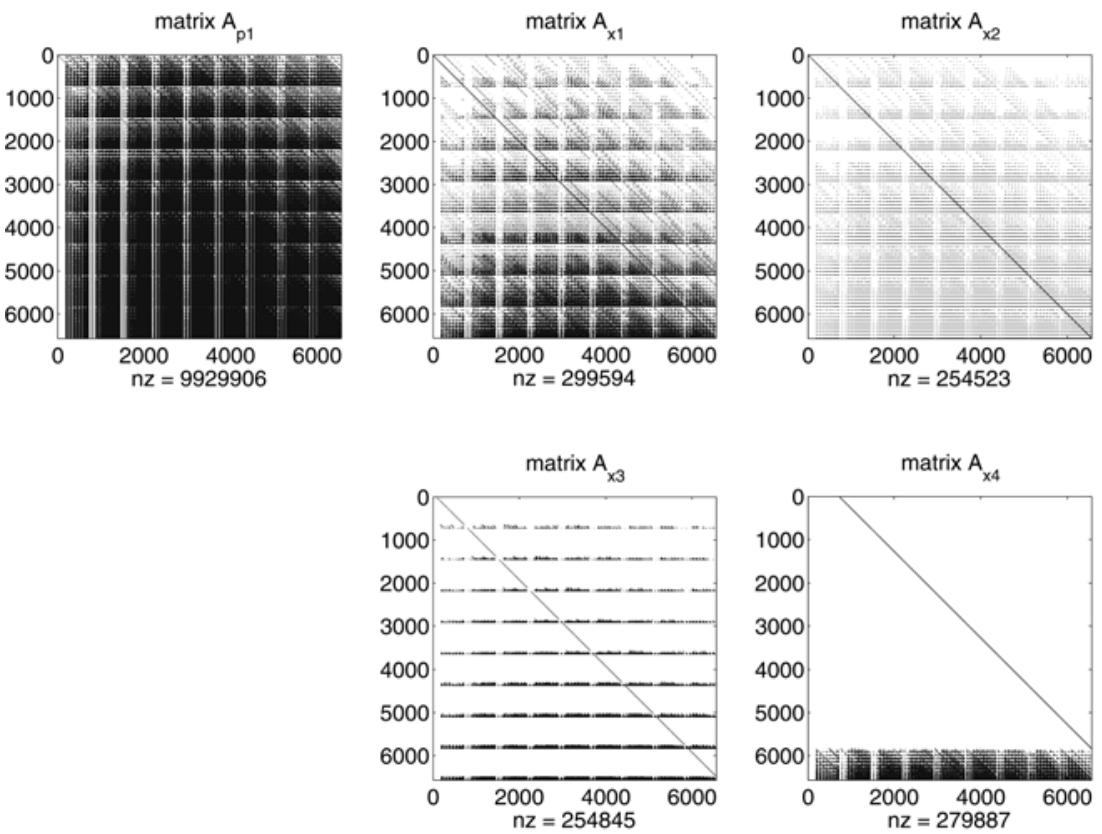

Figure 7.6: Sparsity structure of the matrices $A_{p_{1}}$ and $A_{x_{i}}, i=1, \ldots, 4$

Table 7.13: Comparison between performance of JD and JDCOMM

\begin{tabular}{lccccc}
\hline Method & MV $A_{p_{1}}$ & MV $A_{x_{i}}$ & Flops $\times 10^{8}$ & Time (s) & Global minimum \\
\hline JD & 180 & 0 & 17.9 & 69.9 & -36228.7 \\
JDCOMM $A_{x_{1}}$ & 63 & 543 & 7.9 & 32.8 & -36228.7 \\
JDCOMM $A_{x_{2}}$ & 58 & 488 & 7.0 & 28.3 & -36228.7 \\
JDCOMM $A_{x_{3}}$ & 44 & 334 & 5.2 & 19.8 & -36228.7 \\
JDCOMM $A_{x_{4}}$ & 72 & 642 & 8.9 & 37.6 & -36228.7 \\
\hline
\end{tabular}

the tolerance on the residual norm and to 10 and 20 for the restart parameters of the minimal and maximal dimensions of the search spaces.

A plot of the norms of the residuals in the eigenvalue equation (at each JD outer step) against the number of matrix-vector products is shown in Figure 7.7.

Again, all the methods compute the same global optimum of -36228.7 and this global optimum is attained at the stationary point with coordinates $x_{1}=3.044$, $x_{2}=-3.216, x_{3}=2.983$, and $x_{4}=3.064$. These eigenvalues are displayed jointly with the eigenvalue spectra of the matrices in Figure 7.8. In this case, the JDCOMM methods 

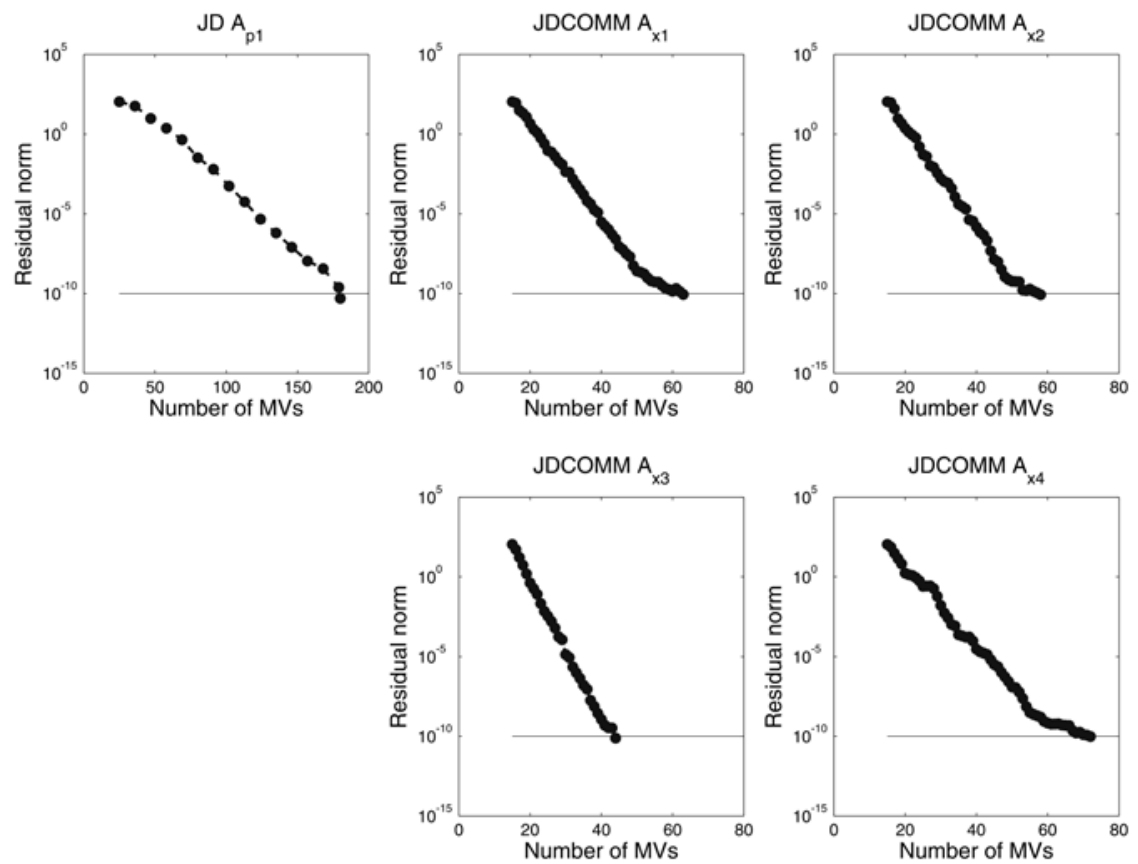

Figure 7.7: Residual norms against MVs with $A_{p_{1}}$ for the JD and JDCOMM methods

also perform better than the conventional JD method in terms of required flops and computation time. To compare this performance with SOSTOOLS and GloptiPoly: both methods compute the same global optimum and same location as found with the JD methods and require 114 and 122 seconds respectively.

\subsubsection{Experiment 3}

In the previous two experiments, we were looking for the smallest real eigenvalues of the matrix $A_{p_{1}}$ which were located on the outside of the eigenvalue spectrum. See Figures 7.4 and 7.8. Finding an eigenvalue at the outside of the spectrum is relatively easy for a Jacobi-Davidson type method. When the required eigenvalue lies more in the interior of the spectrum, computation becomes more difficult in the sense that the choice of parameters for the Jacobi-Davidson method plays a more important role as will be shown in this experiment. 

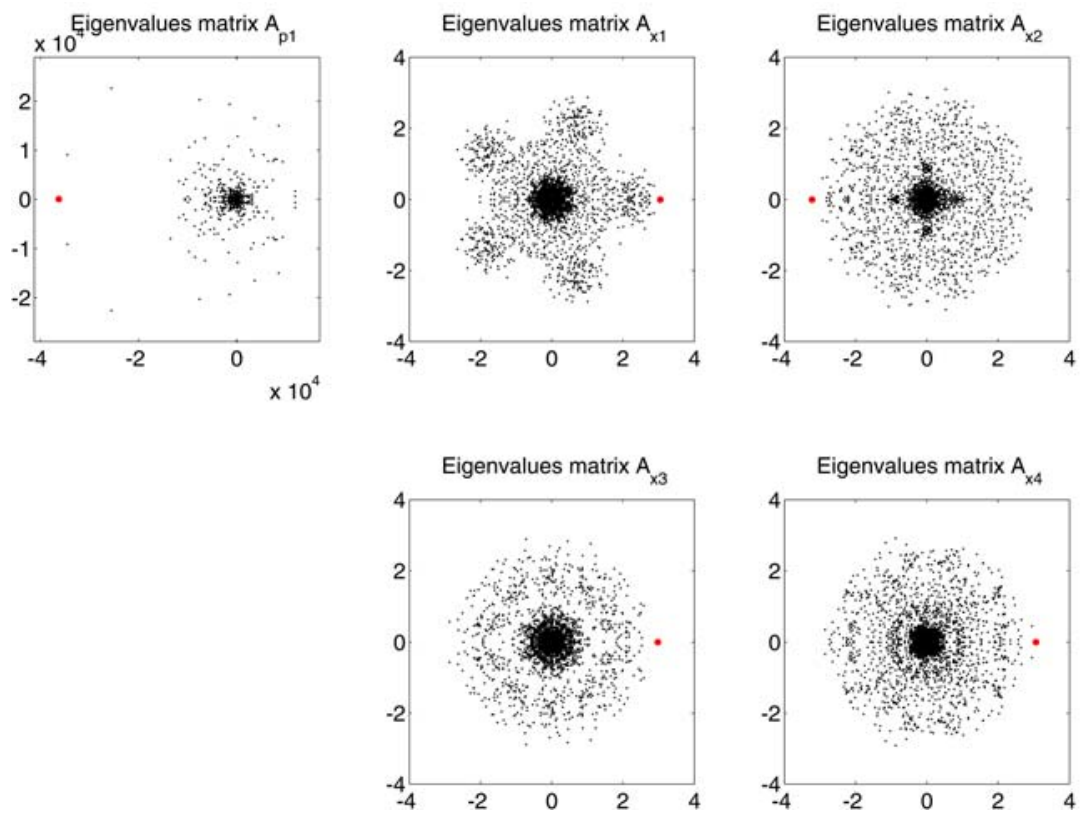

Figure 7.8: Eigenvalue spectra of the matrices $A_{p_{1}}$ and $A_{x_{i}}, i=1, \ldots, 4$

Table 7.14: Sparsity of the matrices $A_{p_{1}}$ and $A_{x_{1}}, \ldots, A_{x_{5}}$

\begin{tabular}{lcc}
\hline Matrix of dimension $3125 \times 3125$ & Number of non-zeros & \%filled \\
\hline$A_{p_{1}}$ & 2595635 & 26.58 \\
$A_{x_{1}}$ & 161729 & 1.66 \\
$A_{x_{2}}$ & 159527 & 1.63 \\
$A_{x_{3}}$ & 146546 & 1.50 \\
$A_{x_{4}}$ & 163614 & 1.68 \\
$A_{x_{5}}$ & 149176 & 1.53 \\
\hline
\end{tabular}

Consider a Minkowski dominated polynomial in 5 variables $(n=5)$, a total degree of $6(d=3, m=5)$, and $\lambda=1$ :

$$
\begin{gathered}
p_{1}\left(x_{1}, x_{2}, x_{3}, x_{4}\right)=\left(x_{1}^{6}+x_{2}^{6}+x_{3}^{6}+x_{4}^{6}+x_{5}^{6}\right)+ \\
2 x_{2}^{3} x_{5}^{2}-6 x_{3}^{2} x_{4} x_{5}^{2}-3 x_{2} x_{3} x_{5}^{3}-6 x_{1} x_{4}^{2} x_{5}^{2}+6 x_{1}^{3} x_{2}^{2}+3 x_{1}^{3} x_{2}+ \\
9 x_{1}^{2} x_{3} x_{5}+9 x_{1}^{2} x_{2} x_{4}-8 x_{1} x_{2}^{2} x_{4}-2 x_{3}^{3}-9 x_{3} x_{4} x_{5}-6 x_{2} x_{3} x_{5}+ \\
-8 x_{3}^{2}-2 x_{5}^{2}-5 .
\end{gathered}
$$

The corresponding quotient space $\mathbb{R}\left[x_{1}, x_{2}, x_{3}, x_{4}, x_{5}\right] / I$ has dimension $N=(2 d-$ $1)^{n}=3125$. Using this quotient space, the matrices $A_{p_{1}}, A_{x_{1}}, A_{x_{2}}, A_{x_{3}}, A_{x_{4}}$, and $A_{x_{5}}$ of dimensions $3125 \times 3125$ can be constructed. Table 7.14 shows the sparsity of all the involved matrices.

When trying to compute the smallest real eigenvalue of the matrix $A_{p_{1}}$ using the JD or JDCOMM method with $10^{-6}$ as the residual norm and 10 and 70 as the minimal and 
Table 7.15: Comparison between performance of JD and JDCOMM

\begin{tabular}{lrcccc}
\hline Method & MV $A_{p_{1}}$ & MV $A_{x_{i}}$ & Flops $\times 10^{8}$ & Time (s) & Global minimum \\
\hline JD & 499 & 0 & 13.0 & 48.5 & -2892.25 \\
JDCOMM $A_{x_{1}}$ & 99 & 389 & 3.2 & 10.2 & -2892.25 \\
JDCOMM $A_{x_{2}}$ & 109 & 499 & 3.6 & 12.7 & -2892.25 \\
JDCOMM $A_{x_{3}}$ & 83 & 213 & 2.5 & 6.0 & -2892.25 \\
JDCOMM $A_{x_{4}}$ & 85 & 235 & 2.6 & 6.6 & -2892.25 \\
JDCOMM $A_{x_{5}}$ & 81 & 191 & 2.4 & 5.6 & -2892.25 \\
\hline
\end{tabular}

maximal dimensions of the search spaces, this fails: the first computed eigenvalue is a complex eigenvalue. Only the fifth computed eigenvalue turns out to yield the smallest real eigenvalue of the matrix $A_{p_{1}}$. The five first eigenvalues computed by the JD and JDCOMM methods are: $-3346.2+651.314 i,-3346.2-651.314 i,-3146.58+661.606 i$, $-3146.58-661.606 i$ and finally -2892.25 . The coordinates of the global minimizer with value -2892.25 are $x_{1}=3.0922, x_{2}=-3.0680, x_{3}=3.6112, x_{4}=3.6562$, and $x_{5}=-4.0769$. The smallest real eigenvalue of the matrix $A_{p_{1}}$ lies slightly more into the inside of the spectrum of eigenvalues, enclosed between two complex conjugate pairs of eigenvalues, as can be seen in the top-left subfigure of Figure 7.9.
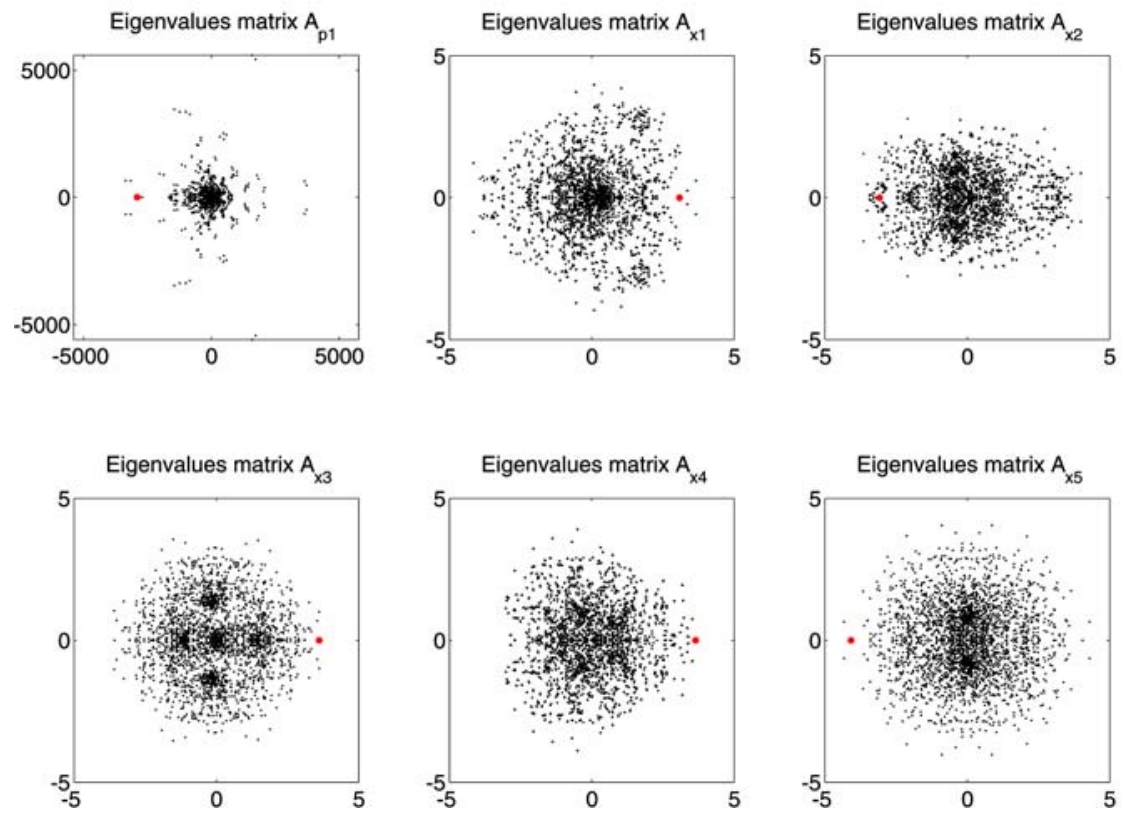

Figure 7.9: Eigenvalue spectra of the matrices $A_{p_{1}}$ and $A_{x_{i}}, i=1, \ldots, 5$

The computation time and the number of matrix-vector products needed by the JD and JDCOMM methods for the computation of the first five eigenvalues, are summarized in Table 7.15. Also in this situation, the JDCOMM methods with the matrices $A_{x_{i}}$ again need less computation time. When the maximal dimension of the search spaces is slightly increased from 70 to 80 the JD methods are capable of computing the smallest 
Table 7.16: Sparsity of the matrices $A_{p_{1}}$ and $A_{x_{1}}, A_{x_{2}}, A_{x_{3}}, A_{x_{4}}$

\begin{tabular}{lcc}
\hline Matrix of dimension $6561 \times 6561$ & Number of non-zeros & \% filled \\
\hline$A_{p_{1}}$ & 1255231 & 2.92 \\
$A_{x_{1}}$ & 45717 & 0.11 \\
$A_{x_{2}}$ & 59756 & 0.14 \\
$A_{x_{3}}$ & 41129 & 0.10 \\
$A_{x_{4}}$ & 41712 & 0.10 \\
\hline
\end{tabular}

real eigenvalue immediately, but then the JDCOMM methods become (almost) just as fast as the conventional JD method. Respectively, the JD and JDCOMM method with the matrices $A_{x_{1}}, A_{x_{2}}, A_{x_{3}}, A_{x_{4}}$, and $A_{x_{5}}$ need $3.5,3.7,3.5,3.8,3.7$, and 3.6 seconds and $2.1 \times 10^{8}, 2.2 \times 10^{8}, 2.2 \times 10^{8}, 2.2 \times 10^{8}, 2.2 \times 10^{8}$, and $2.2 \times 10^{8}$ flops. To compute the same global minimizer including its location, SOSTOOLS and GloptiPoly require as much as 9.2 and 8.7 seconds.

\subsubsection{Experiment 4}

In this final experiment we study an optimization problem of the same dimensions as the previous one: $n=4, d=5$, and $\beta=1$ with the following Minkowski-dominated polynomial:

$$
\begin{gathered}
p_{1}\left(x_{1}, x_{2}, x_{3}, x_{4}\right)=\left(x_{1}^{10}+x_{2}^{10}+x_{3}^{10}+x_{4}^{10}\right)+3 x_{1}^{5} x_{2} x_{3} x_{4}+ \\
+x_{1}^{5} x_{3} x_{4}^{2}+7 x_{1}^{5} x_{3} x_{4}-5 x_{1}^{3} x_{3}^{2} x_{4}+ \\
-7 x_{1}^{2} x_{2} x_{3}^{2} x_{4}^{2}-5 x_{1} x_{3}^{3} x_{4}^{3}-5
\end{gathered}
$$

The involved quotient space and thus also the matrices $A_{p_{1}}, A_{x_{1}}, A_{x_{2}}, A_{x_{3}}$, and $A_{x_{4}}$ are of dimension $6561 \times 6561$. Table 7.16 shows the differences in the number of nonzero elements between those matrices. See Figure 7.10 for a representation of the sparsity structure of these matrices.

The global minimizer, the smallest real eigenvalue of the matrix $A_{p_{1}}$, we are looking for has value -206.5 and is located at the point with coordinates $x_{1}=1.79$, $x_{2}=1.43, x_{3}=-1.54$, and $x_{4}=1.54$, which are the corresponding eigenvalues of the matrices $A_{x_{1}}, A_{x_{2}}, A_{x_{3}}$, and $A_{x_{4}}$. The location of these eigenvalues with respect to the entire eigenvalue spectrum of each matrix is depicted with red dots in Figure 7.11.

The smallest real eigenvalue of the matrix $A_{p_{1}}$ is not located very close to the exterior of the spectrum: there are 34 eigenvalues with a real part smaller than -206.5 . See the top-left subfigure of Figure 7.11.

The smallest real eigenvalue is computed with the JD and JDCOMM method using various search space parameters. The minimal (mindim) and maximal (maxdim) dimensions of the search space are varied between 5 and 100. See Table 7.17 for the results. Blanks in this table indicate that the corresponding method with these settings is unable to converge to the requested eigenvalue using a maximum of 1000 outer iterations and 10 inner iterations with GMRES. Note here that the standard JD 

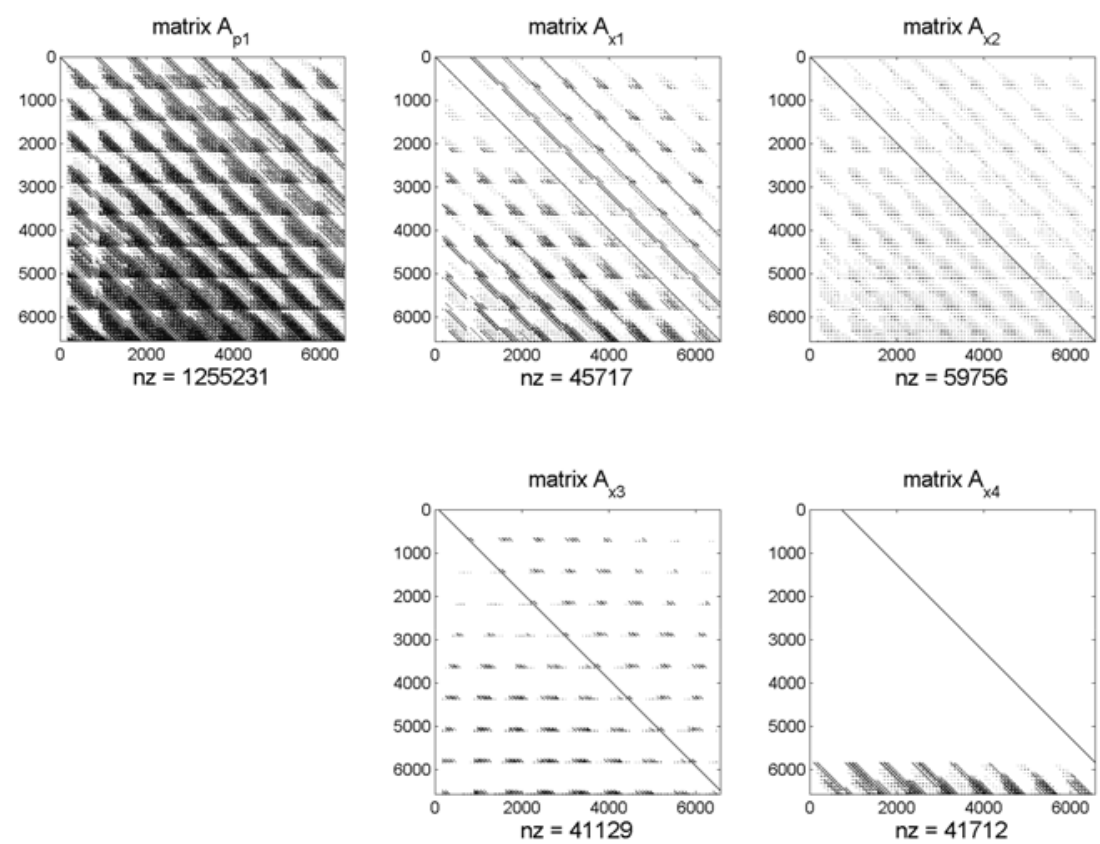

Figure 7.10: Sparsity structure of the matrices $A_{p_{1}}$ and $A_{x_{i}}, i=1, \ldots, 4$

Table 7.17: Comparison between performance of JD and JDCOMM using various search space dimensions

\begin{tabular}{|c|c|c|c|c|c|c|}
\hline \multirow{2}{*}{$\begin{array}{l}\text { Value } \\
\text { mindim }\end{array}$} & \multirow{2}{*}{$\begin{array}{l}\text { Value } \\
\text { maxdim }\end{array}$} & \multicolumn{5}{|c|}{ Time (s) } \\
\hline & & $A_{p_{1}}$ & $A_{x_{1}}$ & $A_{x_{2}}$ & $A_{x_{3}}$ & $A_{x_{4}}$ \\
\hline 5 & 30 & & & 11.6 & & \\
\hline 10 & 15 & & & 10.0 & & \\
\hline 10 & 25 & & & 15.4 & & \\
\hline 10 & 50 & & 10.7 & 6.1 & & \\
\hline 10 & 75 & & & 6.8 & 41.5 & \\
\hline 10 & 100 & 139.9 & 10.1 & 7.7 & 20.8 & 20.7 \\
\hline
\end{tabular}

method on the matrix $A_{p_{1}}$ computes the requested eigenvalue only in the case when the maximum search space dimension maxdim is 100, whereas the JDCOMM method using the matrix $A_{x_{2}}$ works in all cases with a superior performance. Note also that the increase of maxdim from 25 to 50 more than halves the computation time of the JDCOMM method on the matrix $A_{x_{2}}$. Moreover, this is the fastest computation shown in Table 7.17 .

In Table 7.18 we give the more detailed results (with respect to required matrixvector products and flops) of the situation where all the eigenvalue methods succeed 

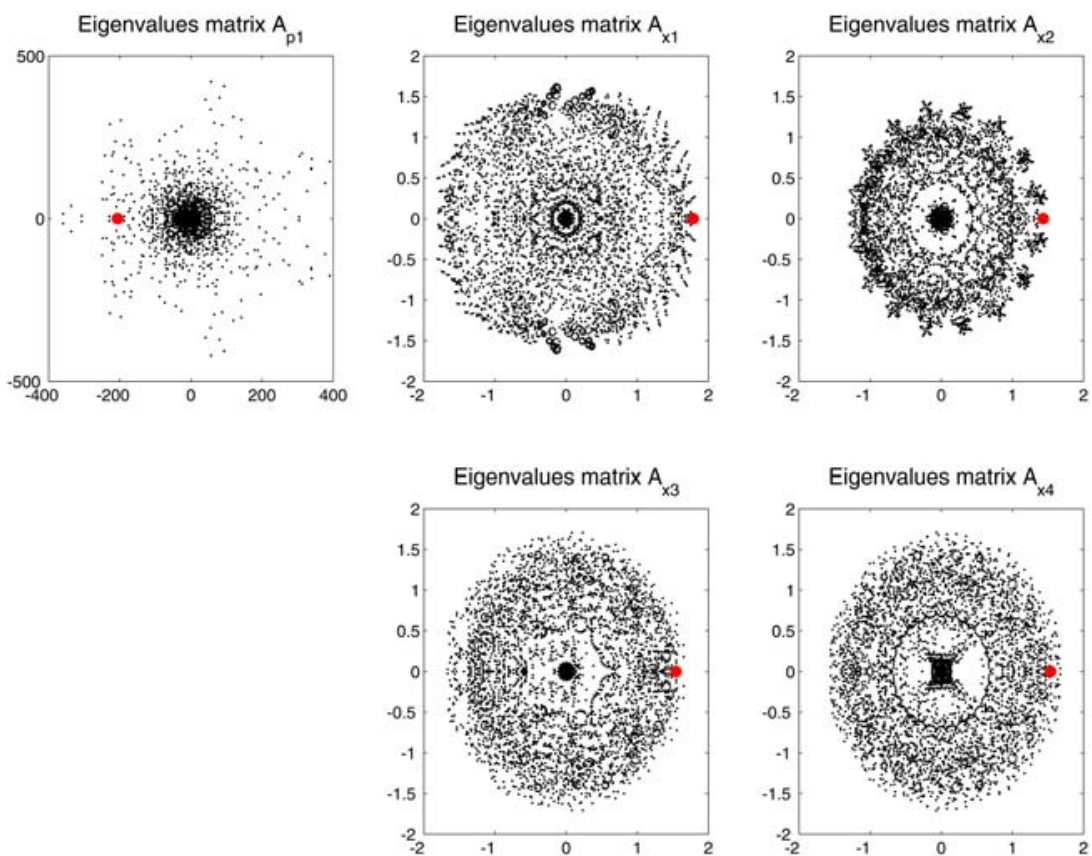

Figure 7.11: Eigenvalue spectra of the matrices $A_{p_{1}}$ and $A_{x_{i}}, i=1, \ldots, 4$

Table 7.18: Details of the performance of JD and JDCOMM with mindim $=10$ and maxdim $=100$

\begin{tabular}{lccccc}
\hline Method & MV $A_{p_{1}}$ & MV $A_{x_{i}}$ & Flops $\times 10^{9}$ & Time (s) & Global minimum \\
\hline JD & 5941 & 0 & 7.5 & 139.9 & -206.5 \\
JDCOMM $A_{x_{1}}$ & 237 & 1607 & 0.4 & 10.1 & -206.5 \\
JDCOMM $A_{x_{2}}$ & 302 & 2322 & 0.5 & 7.6 & -206.5 \\
JDCOMM $A_{x_{3}}$ & 725 & 6975 & 1.2 & 20.8 & -206.5 \\
JDCOMM $A_{x_{4}}$ & 636 & 5996 & 1.1 & 20.7 & -206.5 \\
\hline
\end{tabular}

to compute the requested eigenvalue; mindim is 10 and maxdim is 100 (last row of Table 7.17).

Figure 7.12 shows the plot of the norms of the residuals in the eigenvalue equation (at each JD outer step) against the number of matrix-vector products with $A_{p_{1}}$. All methods compute the same global minimum and the identical location.

To reveil the performance of a Krylov based eigenvalue solver, we also apply the Matlab eigs method, which is an implicitly restarted Arnoldi method [95], to this problem. This method computes the requested smallest real eigenvalue in 62 seconds as the 151th eigenvalue. We used no additional parameter settings here; 

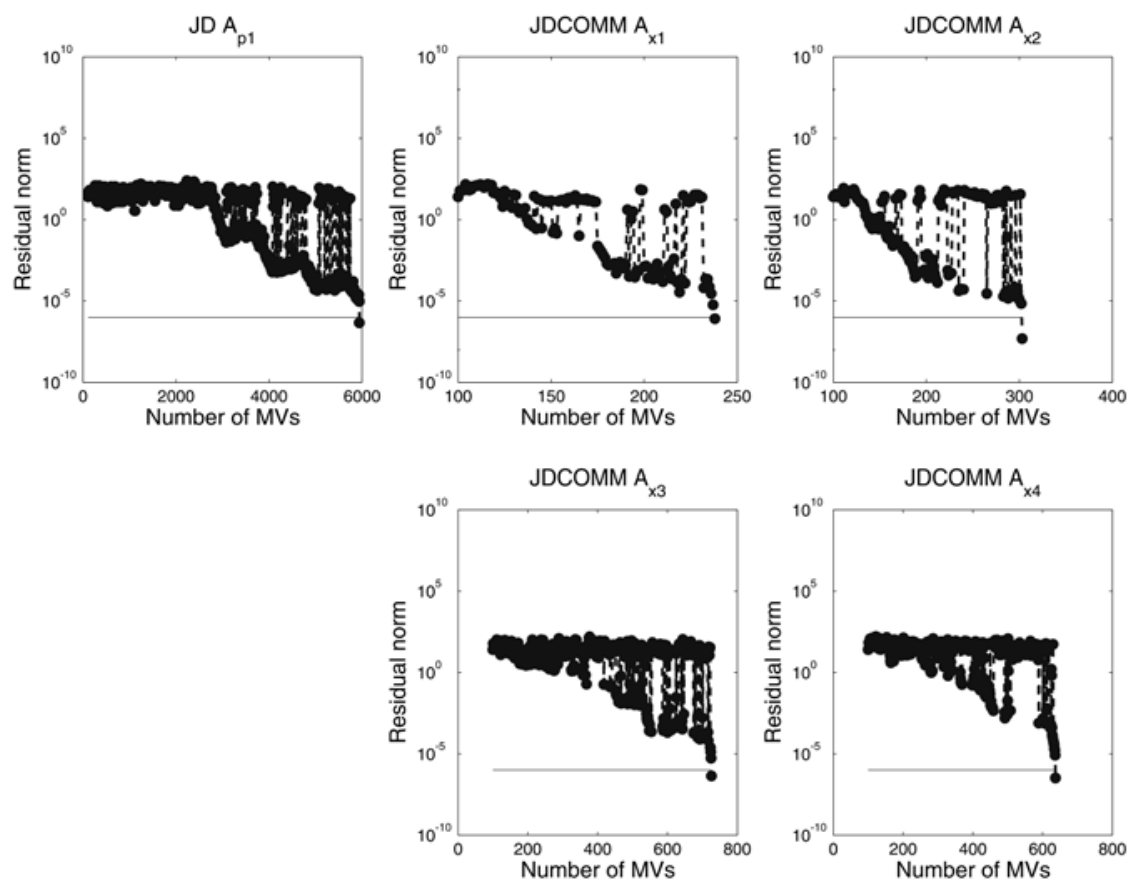

Figure 7.12: Residual norms against MVs with $A_{p_{1}}$ for the JD and JDCOMM methods

when using additional settings to target the required eigenvalue, eigs even requires more computation time.

Comparing the performance of the JDCOMM method in this example with SOSTOOLS and GloptiPoly, we find that both methods compute the same minimizer and require 42 and 39 seconds, respectively. 



\section{Part III}

\section{$H_{2}$ Model-order Reduction}





\section{Introduction to Part III}

In linear systems theory, an important class of input-output systems is that of strictly causal asymptotically stable LTI systems of finite order. From an input-output point of view such systems are characterized by their transfer functions, which are strictly proper rational functions having all their poles in the stability domain. This stability domain is either the open left-half plane in continuous time, or the open unit disk in discrete time.

When building a model from input-output data, when studying model behavior under special restrictive conditions, or when building a large model by interconnecting several parts, one is often confronted with the task to obtain a good quality approximation model of the lowest possible complexity.

To measure the quality of a candidate approximation model, one usually employs a criterion function or a distance measure to quantify the discrepancy between that candidate model and the model of high complexity.

To measure the complexity of a model from the class of systems described above, the order of the model, which is equal to the McMillan degree of its rational transfer function, is commonly used. Thus, the model approximation problem addresses the question of how to obtain a low-order model which fits well with an available more complicated model description. The model-order reduction problem focuses on the question of finding a low-order approximation to a given high-order model.

To quantify the difference between two given models, many different criteria and distance functions have been proposed and investigated in the literature of the past decades. Clearly, the intended model usage is of importance here. Sometimes it is of importance to minimize the worst case consequences of a simplified model description (e.g., for certain applications in robust control). In other cases it may be of importance to maximize average accuracy or expected performance, or to minimize prediction error variance. Criteria which measure the difference between two given models in a system theoretically meaningful way are generally preferred, as they allow one to assess the impact of a model-order reduction step more directly. However, they may be difficult to compute and hard to optimize, and they may exhibit many local non-global optima. It should be mentioned that other model-order reduction procedures, which are only partly criterion based and which share other qualities such as uniqueness of 
optima and ease of computation, are popular too; this includes balance-and-truncate type methods and many variants of Padé approximation.

While the $H_{\infty}$ and the Hankel norm are used very often as a criterion to measure the difference between two given models in a system theoretically meaningful way, here only the $\mathrm{H}_{2}$-norm criterion is discussed. The $\mathrm{H}_{2}$ model-order reduction problem has many applications and connections to other problems in systems and control theory. The $H_{2}$ model-order reduction problem is extensively studied in the literature, i.e., for example [2], [7], [41], [49] and [101] and the references therein. 


\section{Chapter 8}

\section{$H_{2}$ model-order reduction}

\subsection{Introduction}

The model-order reduction problem deals with finding an approximating system $G(s)$ of low order to a given system $H(s)$ of high order. We start from a linear timeinvariant (LTI) system of order $N$. The order $N$ corresponds to the McMillan degree of the transfer function, the number of states in a minimal state-space form. From an input-output point of view, such a system is fully characterized by its transfer function. In this thesis we focus on the single-input, single-output (SISO) case only but the various results can be generalized to the multiple-input, multiple-output (MIMO) case too.

In the SISO case, the transfer function is a real rational proper function of order $N$. Thus, when solving the model-order reduction problem one intends to find lower order approximations of order $n<N$, which are close to the given system of order $N$, disregarding any initial conditions. Working with an approximation of lower order is common in practical applications because of efficiency or performance reasons, especially when the order $N$ of the given system is high.

In this thesis the $\mathrm{H}_{2}$ model-order reduction problem is solved by algebraic methods and is approached as a rational optimization problem. The rational optimization problem is reformulated as the problem of finding solutions of a corresponding quadratic system of polynomial equations, possibly containing one or more additional parameters. This approach was first introduced in [50]. In this article an algebraic method is described for computing a globally optimal real approximation $G(s)$ of order $N-1$ to $H(s)$. The distance between $G(s)$ and $H(s)$ is measured by the $H_{2}$-norm. Stability is required for the $H_{2}$-norm to make sense, for the given system $H(s)$ and for the approximating system $G(s)$. The $H_{2}$-norm is important as it provides a system theoretically meaningful way to measure the distance between two systems. However, this norm is also notorious for admitting local non-global optima, and for being hard to optimize. 
In this thesis the approach of [50] is generalized and extended and, as a result, the $H_{2}$ model reduction problem from order $N$ to $N-1, N-2$, and $N-3$ is solved. For computing the real approximations of orders $N-1, N-2$, and $N-3$, the algebraic Stetter-Möller matrix method for solving systems of polynomial equations of Section 3.3 is used. This approach guarantees that the globally best approximation to the given system is found (while typically many local optima exist).

For compact notation we introduce the concept of a co-order: the case where the order of the given system of order $N$ is reduced to order $N-k$ is called the co-order $k$ case.

Remark 8.1. In the algebraic approach to global $H_{2}$-approximation of [50], it is convenient to impose the technical condition that all the $N$ zeros of $d(s)$, the poles of $H(s)$, are distinct for the co-order $k=1$ case. Extensions may be developed in the future to handle the case with poles of multiplicities larger than 1 too, but this will complicate notation and the numerical procedures. In the setup presented in this and in the next chapters we shall impose a similar constraint for ease of exposition for all co-orders $k$.

This chapter is structured as follows: in Section 8.2 the $H_{2}$ model-order reduction problem is introduced and formulated. In Section 8.3 a system of quadratic equations is constructed for the co-order $k$ case, by reparameterizing the $H_{2}$ model-order reduction problem introduced in Section 8.2. This is a generalization of the algebraic approach taken in [50]. The system of equations here contains the variables $x_{1}, \ldots, x_{N}$, and the additional real-valued parameters $\rho_{1}, \rho_{2}, \ldots, \rho_{k-1}$. It is in a particular Gröbner basis form from which it is immediately clear that it admits a finite number of solutions. In Section 8.4 a homogeneous polynomial of degree three is given for the co-order $k$ case which coincides with the $H_{2}$ model-order reduction criterion for the difference between $H(s)$ and $G(s)$ at the stationary points. This is again a generalization of the third order polynomial for the co-order $k=1$ case presented in [50]. Section 8.5 shows how the Stetter-Möller matrix method of Section 3.3 is used to solve the system of quadratic equations in the co-order $k$ case.

Applying the Stetter-Möller matrix method transforms the problem into an associated eigenvalue problem. From the solutions of this eigenvalue problem, the corresponding feasible solutions for the real approximation $G(s)$ of order $N-k$ can be selected. The $H_{2}$-criterion is used to select the globally best approximation: the smallest real criterion value yields the globally optimal approximation.

Following this approach for the co-order $k=1$ case, leads to a large conventional eigenvalue problem. The transformation into an eigenvalue problem and computing its solutions is the subject of Chapter 9. This largely builds on [50] but in Chapter 9 this technique is extended to a matrix-free version by using an $n \mathrm{D}$-system approach as presented in Chapter 5. In this way we are able to compute the globally optimal approximation $G(s)$ of order $N-1$ of a given system $H(s)$ of order $N$.

In the Chapters 10 and 11 the more difficult problems of computing a globally optimal real $H_{2}$-approximation $G(s)$ for the co-order $k=2$ and $k=3$ case are 
addressed. The practical relevance of the co-order two and three problem derives from the fact that the poles of a real transfer function are either real or they show up as complex conjugate pairs. These poles determine the characteristic modes of the system. Model-order reduction techniques often tend to remove unimportant modes, but in the co-order one case only one mode gets discarded. To remove a complex conjugate pair of poles requires a new technique other than provided by the co-order one case. Indeed, repeated application of the co-order one technique to achieve a larger reduction of the model order, can easily be shown to be non-optimal.

The co-order two and three problems are approached by generalizing and extending the ideas for the co-order $k=1$ case. Using again the reparameterization technique from Section 8.3 a quadratic system of equations is set up. In the co-order $k \geq 2$ case, the coefficients in the complex system of equations involve $k-1$ additional real variables. In addition, any feasible complex solution of this system must satisfy $k-1$ linear constraints. When taking the additional linear condition(s) in the co-order 2 and co-order 3 case into account, the Stetter-Möller matrix method approach yields eigenvalue problems other than the conventional eigenvalue problem in the co-order one case:

- Chapter 10 describes how the Stetter-Möller matrix method in the co-order $k=2$ case yields a polynomial eigenvalue problem. Such a polynomial eigenvalue problem can be rewritten as a generalized eigenvalue problem by using linearization techniques. This generalized eigenvalue problem is finally solved accurately by Kronecker canonical form techniques.

- In Chapter 11 the co-order $k=3$ case is studied in which application of the Stetter-Möller matrix method leads to a two-variable polynomial eigenvalue problem involving rectangular matrices. This problem is reducible to a polynomial eigenvalue problem in one variable involving a single square matrix. To perform this reduction a generalization of the Kronecker Canonical form technique for a one-variable polynomial matrix is developed and used.

From the eigenvalues and corresponding eigenvectors of these eigenvalue problems, the feasible solutions for the approximation problem can be selected and constructed. To perform the selection of the globally optimal solution $G(s)$, the $H_{2}$ model-order reduction criterion mentioned in Theorem 8.2 in Section 8.4 is evaluated for $k=2$ and $k=3$.

Each of the Chapters 9, 10, and 11 contain several worked examples.

\subsection{The $H_{2}$ model-order reduction problem}

When studying model-order reduction and system approximation from a geometric point of view, it is convenient to start from an enveloping inner product space which contains both the system to be approximated and the subset of systems in which an approximation is to be found. 
In the $H_{2}$-approximation problem in continuous time, this enveloping space is taken to be the Hardy space $\mathcal{H}_{2}\left(\Pi^{+}\right)$of complex functions that are square integrable on the boundary $i \mathbb{R}$ of $\Pi^{+}=\{s \in \mathbb{C} \mid \operatorname{Re}(s)>0\}$ and analytic on the closure of the right-half plane $\Pi^{+}$. Such functions are in one-to-one relationship with square integrable functions on the imaginary axis $\partial \Pi^{+}=i \mathbb{R}$, which occur as their limits for $s \rightarrow i \omega$ with $\omega \in \mathbb{R}$. The associated $H_{2}$-inner product of two functions $G(s)$ and $H(s)$ is given by:

$$
\langle G, H\rangle=\frac{1}{2 \pi i} \int_{i \mathbb{R}} G(s) H\left(-s^{*}\right)^{*} d s=\frac{1}{2 \pi} \int_{-\infty}^{\infty} G(i \omega) H(i \omega)^{*} d \omega,
$$

where the asterisk denotes complex conjugation. An important subset of $\mathcal{H}_{2}\left(\Pi^{+}\right)$is formed by $S$, the class of strictly proper rational functions which have all their poles in the open left-half plane $\Pi^{-}=\{s \in \mathbb{C} \mid \operatorname{Re}(s)<0\}$. Such functions correspond to transfer functions of continuous time stable and strictly causal LTI systems of finite order.

When a state-space realization $(A, B, C)$ is employed (thus $D=0)$ for $H(s)$ (i.e., it holds that $\left.H(s)=C(s I-A)^{-1} B\right)$, then it is well-known [102] that its squared $H_{2}$-norm can be computed as:

$$
\|H\|_{H_{2}}^{2}=C P C^{*}
$$

in which $P$ satisfies the continuous time Lyapunov equation:

$$
A P+P A^{*}=-B B^{*}
$$

In discrete time, an analogous framework is constituted by the enveloping Hardy space $\mathcal{H}_{2}(\mathbb{E})$ of complex functions which are square integrable on the boundary $\mathbb{T}=$ $\{z \in \mathbb{C}|z|=1\}$ of $\mathbb{E}=\{z \in \mathbb{C}|z|>1\}$ and analytic on the closure of $\mathbb{E}$. These functions are in one-to-one correspondence with square integrable functions on the unit circle $\mathbb{T}=\{z \in \mathbb{C}|| z \mid=1\}$, which occur as the radial limits of these functions in $\mathcal{H}_{2}(\mathbb{E})$. The associated $H_{2}$-inner product is now given by:

$$
\langle\hat{G}, \hat{H}\rangle=\frac{1}{2 \pi i} \oint_{\mathbb{T}} \hat{G}(z) \hat{H}\left(\frac{1}{z^{*}}\right)^{*} \frac{d z}{z}=\frac{1}{2 \pi} \int_{0}^{2 \pi} \hat{G}\left(e^{i \Omega}\right) \hat{H}\left(e^{i \Omega}\right)^{*} d \Omega .
$$

An important subset of $\mathcal{H}_{2}(\mathbb{E})$ is the class of strictly proper rational functions which have all their poles inside the unit disk $\mathbb{D}=\{z \in \mathbb{C}|| z \mid<1\}$. When represented as a Laurent series about infinity, a function $\hat{H}(z)$ in this class attains the form:

$$
\hat{H}(z)=\hat{H}_{0}+\hat{H}_{1} z^{-1}+\hat{H}_{2} z^{-2}+\ldots
$$

of which the corresponding $H_{2}$-norm satisfies:

$$
\|\hat{H}\|_{H_{2}}^{2}=\sum_{k=0}^{\infty}\left|\hat{H}_{k}\right|^{2}<\infty .
$$


When a state-space realization $(\hat{A}, \hat{B}, \hat{C}, \hat{D})$ is employed for $\hat{H}(z)$ (i.e., it holds that $\left.\hat{H}(z)=\hat{D}+\hat{C}(z I-\hat{A})^{-1} \hat{B}\right)$, then it is well-known [102] that its squared $H_{2}$-norm can be computed as:

$$
\|\hat{H}\|_{H_{2}}^{2}=\hat{C} \hat{P} \hat{C}^{*}+\hat{D} \hat{D}^{*},
$$

in which $\hat{P}$ satisfies the discrete time Lyapunov-Stein equation:

$$
\hat{P}-\hat{A} \hat{P} \hat{A}^{*}=\hat{B} \hat{B}^{*} .
$$

Remark 8.2. Note that the presence of a constant term $\hat{H}(\infty)=\hat{D}$ causes $\hat{H}(z)$ to be proper rather than strictly proper, which constitutes a slight difference between the discrete-time case and the continuous-time case (where $D=0$ ). However, in $\mathcal{H}_{2}$ model-order reduction the role played by $\hat{D}$ is simple and easily understood - it can be assigned an optimal value irrespective of $\hat{A}, \hat{B}$, and $\hat{C}$ and it does not affect the order. Therefore it is convenient to restrict again to strictly proper rational transfer functions also in the discrete-time case.

There exists an isometric isomorphism between the subspace of strictly proper rational transfer functions of $\mathcal{H}_{2}(\mathbb{E})$ in the discrete time case and the corresponding subspace $S$ of $\mathcal{H}_{2}\left(\Pi^{+}\right)$in the continuous time case which leaves the McMillan degree unchanged. Such an isometry is for example given by:

$$
\hat{H}(z) \mapsto H(s)=\frac{\sqrt{2}}{1-s} \hat{H}\left(\frac{1+s}{1-s}\right)
$$

of which the inverse mapping is given by:

$$
H(s) \mapsto \hat{H}(z)=\frac{\sqrt{2}}{z+1} H\left(\frac{z-1}{z+1}\right) .
$$

This mapping has the additional property that it transforms the associated discrete time Lyapunov-Stein equation (8.8) into the corresponding continuous time Lyapunov equation (8.3), leaving its solution $\hat{P}=P$ unchanged, when taking a state-space realization $(\hat{A}, \hat{B}, \hat{C})$ of $\hat{H}(z)$ into a corresponding realization $(A, B, C)=((\hat{A}-I)$ $\left.(\hat{A}+I)^{-1}, \sqrt{2}(\hat{A}+I)^{-1} \hat{B}, \hat{C}\right)$ of $H(s)$.

In discrete time, the sequence of coefficients $\left\{\hat{H}_{k}\right\}$ constitutes the impulse response of the system with transfer function $\hat{H}(z)$. Conversely, the transfer function $\hat{H}(z)$ is the $z$-transform of the impulse response. The different expressions for computing the $H_{2}$-norm either in the frequency domain or in the time domain reflect Parseval's identity. Similarly, in continuous time it holds that the transfer function $H(s)$ is the Laplace transform of the impulse response $C e^{A t} B$, of which the square of its $H_{2}$-norm is equivalently given by:

$$
\int_{0}^{\infty}\left|C e^{A t} B\right|^{2} d t
$$

These properties make that the $\mathrm{H}_{2}$-norm has a highly relevant system theoretic interpretation. 
Because of the order preserving isometry between the discrete time and continuous time cases, we can restrict to the continuous time case without loss of generality.

In this thesis we shall restrict to real transfer functions in continuous time, both for the given system $H(s)$ and for the candidate approximations $G(s)$. Following the approach and notation of [50], we start from a given function:

$$
H(s)=\frac{e(s)}{d(s)}
$$

of McMillan degree $N \geq 1$, in which $d(s)=s^{N}+d_{N-1} s^{N-1}+\ldots+d_{1} s+d_{0}$ is a monic real polynomial of degree $N$ that is Hurwitz (i.e., all its zeros are in $\Pi^{-}$) and $e(s)=e_{N-1} s^{N-1}+\ldots+e_{1} s+e_{0}$ is a real polynomial of degree $\leq N-1$ that is co-prime with $d(s)$. I.e., it does not identically vanish and it does not have any zeros in common with $d(s)$. For a given approximation of order $n<N$, the set $S_{n}$, the submanifold of $S$ of candidate approximations, consist of all the real functions $G(s)=\frac{b(s)}{a(s)}$ for which $a(s)=s^{n}+a_{n-1} s^{n-1}+\ldots+a_{1} s+a_{0}$ is a monic real polynomial of degree $n$ that is Hurwitz, and $b(s)=b_{n-1} s^{n-1}+\ldots+b_{1} s+b_{0}$ is a real polynomial of degree $\leq n-1$ that is co-prime with $a(s)$.

The subspace $S_{n}$ of $S$ of (strictly proper rational asymptotically stable) functions of McMillan degree $n$ constitutes a smoothly embedded submanifold, so that any critical point of the norm of $H(s)-G(s)$ is characterized by the property that $H(s)-G(s)$ is orthogonal to the tangent space of $S_{n}$ at $G(s)$.

Thus, using this notation the $\mathrm{H}_{2}$ model-order reduction problem consists of finding an approximation $G \in S_{n}$ to the given function $H$ of degree $N$, which minimizes the associated $\mathrm{H}_{2}$-criterion function:

$$
V_{H}(G)=\|H-G\|_{H_{2}}^{2}
$$

where $\|.\|_{H_{2}}^{2}$ denotes the squared $H_{2}$-norm, as induced by the $H_{2}$-inner product on $\mathcal{H}_{2}\left(\Pi^{+}\right)$.

To compute the $\mathrm{H}_{2}$-criterion later on in this chapter, the squared $\mathrm{H}_{2}$-norm of a rational stable transfer function $H(s)$ is required. This norm $\|H(s)\|_{H_{2}}^{2}$ equals $\langle H, H\rangle$. For the continuous time case where all the poles of the transfer function $H(s)$ are in the open left-half plane $\Pi^{-}$, this norm is defined using Equation (8.1) as follows:

$$
\begin{aligned}
\langle H, H\rangle=\|H(s)\|_{H_{2}}^{2} & =\frac{1}{2 \pi i} \int_{i \mathbb{R}} H(s) H\left(-s^{*}\right)^{*} d s \\
& =\frac{1}{2 \pi} \int_{-\infty}^{\infty} H(i \omega) H(i \omega)^{*} d \omega .
\end{aligned}
$$


Remark 8.3. Note that in the MIMO case, the corresponding $H_{2}$-inner product is obtained by applying the trace operator to the expressions given above for the SISO case. The state-space formulation needs to be adapted in a similar fashion, by including the trace operator.

\subsection{A reparameterization of the $H_{2}$ model-order reduction problem}

From an algebraic point of view, note that the squared $H_{2}$-norm of $H(s)-G(s)$ in (8.13) can be worked out to constitute a (rather complicated) rational expression in terms of the $2 n$ coefficients of $a(s)$ and $b(s)$, when

$$
G(s)=\frac{b(s)}{a(s)} .
$$

In Section 8.4 of this chapter we show how this criterion can be evaluated at some special points of interest by means of a third order homogeneous polynomial.

From a geometric point of view it is appropriate to regard the problem of minimizing the criterion function in (8.13) as a projection problem within the Hardy space $\mathcal{H}_{2}\left(\Pi^{+}\right)$of functions that are analytic on the open right-half plane $\Pi^{+}$and which satisfy the property that their $H_{2}$-norm attains a well-defined finite value.

Proceeding as in [50], it is well known that the space $S_{n}$ constitutes a smooth submanifold of $\mathcal{H}_{2}\left(\Pi^{+}\right)$. Therefore, if $b(s) / a(s)$ is an optimal approximation in $S_{n}$ to $e(s) / d(s)$ with respect to the $H_{2}$-norm, then $b(s) / a(s)$ is an orthogonal projection of $e(s) / d(s)$ onto $S_{n}$. The difference

$$
\frac{e(s)}{d(s)}-\frac{b(s)}{a(s)}
$$

is perpendicular to the tangent space to $S_{n}$ at the point $G(s)$. The tangent space to $S_{n}$ at $G(s)$ is easily computed as the set $\left\{t(s) / a(s)^{2} \mid t(s)\right.$ polynomial of degree $\left.\leq 2 n-1\right\}$. Now, from the theory of Hardy spaces it is known that the orthogonal complement of this tangent space in $\mathcal{H}_{2}\left(\Pi^{+}\right)$is obtained as the set $\left\{a(-s)^{2} R(s) \mid R(s) \in \mathcal{H}_{2}\left(\Pi^{+}\right)\right\}$. Consequently, $G(s)$ is a stationary point if and only if there exists a function $R(s) \in$ $\mathcal{H}_{2}\left(\Pi^{+}\right)$for which:

$$
\frac{e(s)}{d(s)}-\frac{b(s)}{a(s)}=a(-s)^{2} R(s)
$$

(see [8], [9], and [80]).

Clearly $R(s)$ needs to be a real and rational function and its poles are in the open left-half plane $\Pi^{-}$. Since the factor $a(-s)^{2}$ has its zeros in the open right-half plane $\Pi^{+}$none of its factors cancel and it follows upon multiplication by $a(s) d(s)$ that there exists a non-vanishing real polynomial $q(s)=a(s) d(s) R(s)$ such that:

$$
e(s) a(s)-b(s) d(s)=a(-s)^{2} q(s) .
$$

Note that $q(s) \not \equiv 0$ since $H(s)$ and $G(s)$ have different degrees. Note also that the degree of $q(s)$ is $\leq k-1$. The value $k=N-n \geq 1$ is the co-order of the approximation $G(s)$. 
One may now take the $n$ coefficients of the monic polynomial $a(s)$ together with the $n$ coefficients of $b(s)$ and the $N-n$ coefficients of $q(s)$ as the $N+n$ optimization parameters in conjunction with the $N+n$ equations associated with the terms in (8.18). This leads to a polynomial system of equations, which is structured in the sense that the coefficients of $b(s)$ show up in a linear fashion, but which may still be quite hard to solve algebraically because the system is not in Gröbner basis form. By means of a suitable reparameterization this structure can be reworked.

When the model-order is reduced by $k$ from order $N$ to order $n=N-k$, then the degree of $q(s)$ is at most $k-1$. We shall employ the notation:

$$
q(s)=q_{0}+q_{1} s+\ldots+q_{k-1} s^{k-1} \not \equiv 0 .
$$

A reparameterization is used which involves the $N$ zeros $\delta_{1}, \ldots, \delta_{N}$ of the given polynomial $d(s)$, which upon substitution into Equation (8.18) enables the elimination of the polynomial $b(s)$ from the problem. As stated before, we here require the technical assumption that the $N$ zeros $\delta_{1}, \ldots, \delta_{N}$ of $d(s)$ are all distinct.

We first focus on the generic situation where $q_{0} \neq 0$. For $i=0,1, \ldots, k-1$ we define $\rho_{i}=\frac{q_{i}}{q_{0}}$ and let the polynomial $\rho(s)$ be:

$$
\rho(s)=\frac{q(s)}{q_{0}}=1+\rho_{1} s+\rho_{2} s^{2}+\ldots+\rho_{k-1} s^{k-1} .
$$

Note that $\rho(s)$ is of degree $\leq k-1$ and that $\rho_{0}=\frac{q_{0}}{q_{0}}=1$.

Then Equation (8.18) can be manipulated as follows. Multiplying both sides by $q_{0}$ and introducing:

$$
\tilde{a}(s):=q_{0} a(-s)
$$

yields:

$$
e(s) \tilde{a}(-s)-q_{0} b(s) d(s)=\tilde{a}(s)^{2} \rho(s) .
$$

Note that $\tilde{a}(s)$ is not monic, in contrast to $a(s)$.

Evaluation of Equation (8.22) at the $N$ distinct zeros $\delta_{1}, \ldots, \delta_{N}$ of the polynomial $d(s)$ gives:

$$
e\left(\delta_{i}\right) \tilde{a}\left(-\delta_{i}\right)=\tilde{a}\left(\delta_{i}\right)^{2} \rho\left(\delta_{i}\right), \quad(i=1, \ldots, N) .
$$

Note that in Equation (8.23) it holds that $\tilde{a}\left(\delta_{i}\right) \neq 0$, and that the equations no longer involve the polynomial $b(s)$. This equation constitutes a system of $N$ equations in $N$ unknowns.

When studying the $\mathrm{H}_{2}$ model-order reduction problem for co-order $k$, the polynomial $\tilde{a}(s)$ is, for future computational convenience, treated as a polynomial of degree $\leq N-1$, while its degree is actually $N-k$. For $k=1$ we write:

$$
\tilde{a}(s)=\tilde{a}_{N-1} s^{N-1}+\tilde{a}_{N-2} s^{N-2}+\ldots+\tilde{a}_{1} s+\tilde{a}_{0} .
$$

For $k>1$ we use a similar notation, where we then have to add the additional constraints:

$$
\tilde{a}_{N-1}=\cdots=\tilde{a}_{N-k+1}=0 .
$$


This allows us to employ a joint framework in which to study the $H_{2}$ model-order reduction problem for various co-orders $k$.

In the co-order $k$ case equation (8.23) involves $N$ equations and $k-1$ additional constraints, in terms of $N+k-1$ unknowns (the $N$ coefficients of $\tilde{a}(s)$ and the $k-1$ unknown parameters $\rho_{1}, \ldots, \rho_{k-1}$ in $\left.\rho(s)\right)$.

We now reparameterize by introducing the $N$ quantities $x_{i}:=\tilde{a}\left(\delta_{i}\right)$, for $i=$ $1, \ldots, N$, to characterize the polynomial $\tilde{a}(s)$. Note that a polynomial of degree $N-1$ has $N$ degrees of freedom and is fully characterized by its values at $N$ distinct points. These values are related to the coefficients of the polynomial $\tilde{a}(s)$ in a linear way, by means of a Vandermonde matrix involving the interpolation points. To be explicit, let $V\left(\beta_{1}, \beta_{2}, \ldots, \beta_{N}\right)$ denote a Vandermonde matrix in the variables $\beta_{1}, \ldots, \beta_{N}$ :

$$
V\left(\beta_{1}, \beta_{2}, \ldots, \beta_{N}\right)=\left(\begin{array}{ccccc}
1 & \beta_{1} & \beta_{1}^{2} & \ldots & \beta_{1}^{N-1} \\
1 & \beta_{2} & \beta_{2}^{2} & \ldots & \beta_{2}^{N-1} \\
\vdots & \vdots & \vdots & & \vdots \\
1 & \beta_{N} & \beta_{N}^{2} & \ldots & \beta_{N}^{N-1}
\end{array}\right)
$$

As in [50], note that the quantities $x_{i}:=\tilde{a}\left(\delta_{i}\right)=\tilde{a}_{N-1} \delta_{i}^{N-1}+\ldots+\tilde{a}_{1} \delta_{i}+\tilde{a}_{0}$ (for $i=1, \ldots, N$ ) depend linearly on the coefficients $\tilde{a}_{0}, \ldots, \tilde{a}_{N-1}$ through the fixed Vandermonde matrix $V\left(\delta_{1}, \ldots, \delta_{N}\right)$ in the following way:

$$
\left(\begin{array}{c}
x_{1} \\
x_{2} \\
\vdots \\
x_{N}
\end{array}\right)=\left(\begin{array}{c}
\tilde{a}\left(\delta_{1}\right) \\
\tilde{a}\left(\delta_{2}\right) \\
\vdots \\
\tilde{a}\left(\delta_{N}\right)
\end{array}\right)=V\left(\delta_{1}, \ldots, \delta_{N}\right)\left(\begin{array}{c}
\tilde{a}_{0} \\
\tilde{a}_{1} \\
\vdots \\
\tilde{a}_{N-1}
\end{array}\right) .
$$

Likewise the quantities $\tilde{a}\left(-\delta_{i}\right)=\tilde{a}_{N-1}\left(-\delta_{i}\right)^{N-1}+\ldots+\tilde{a}_{1}\left(-\delta_{i}\right)+\tilde{a}_{0}$ in Equation (8.23) depend linearly on the coefficients of $\tilde{a}(s)$ too. This relation can be expressed, by using the matrix $V\left(-\delta_{1}, \ldots,-\delta_{N}\right)$, as follows:

$$
\left(\begin{array}{c}
\tilde{a}\left(-\delta_{1}\right) \\
\tilde{a}\left(-\delta_{2}\right) \\
\vdots \\
\tilde{a}\left(-\delta_{N}\right)
\end{array}\right)=V\left(-\delta_{1}, \ldots,-\delta_{N}\right)\left(\begin{array}{c}
\tilde{a}_{0} \\
\tilde{a}_{1} \\
\vdots \\
\tilde{a}_{N-1}
\end{array}\right) .
$$

In this expression existence of the inverse of the Vandermonde matrix $V\left(\delta_{1}, \ldots, \delta_{N}\right)$ is guaranteed by the poles $\delta_{1}, \delta_{2}, \ldots, \delta_{N}$ being all distinct, as its determinant is given by:

$$
\operatorname{det} V\left(\delta_{1}, \ldots, \delta_{N}\right)=\prod_{i>j}\left(\delta_{i}-\delta_{j}\right) .
$$

Combining Equations (8.27) and (8.28) allows to express the quantities $\tilde{a}\left(-\delta_{i}\right)$ in terms of $x_{1}, \ldots, x_{N}$ through fixed Vandermonde matrices involving the zeros $\delta_{1}, \ldots, \delta_{N}$ 
of $d(s)$ as follows:

$$
\left(\begin{array}{c}
\tilde{a}\left(-\delta_{1}\right) \\
\tilde{a}\left(-\delta_{2}\right) \\
\vdots \\
\tilde{a}\left(-\delta_{N}\right)
\end{array}\right)=V\left(-\delta_{1}, \ldots,-\delta_{N}\right) V\left(\delta_{1}, \ldots, \delta_{N}\right)^{-1}\left(\begin{array}{c}
x_{1} \\
x_{2} \\
\vdots \\
x_{N}
\end{array}\right)
$$

Hence, Equation (8.23) attains the following form:

$$
\left(\begin{array}{c}
\frac{\rho\left(\delta_{1}\right)}{e\left(\delta_{1}\right)} x_{1}^{2} \\
\frac{\rho\left(\delta_{2}\right)}{e\left(\delta_{2}\right)} x_{2}^{2} \\
\vdots \\
\frac{\rho\left(\delta_{N}\right)}{e\left(\delta_{N}\right)} x_{N}^{2}
\end{array}\right)=V\left(-\delta_{1}, \ldots,-\delta_{N}\right) V\left(\delta_{1}, \ldots, \delta_{N}\right)^{-1}\left(\begin{array}{c}
x_{1} \\
x_{2} \\
\vdots \\
x_{N}
\end{array}\right)
$$

or:

$$
\left(\begin{array}{c}
\frac{\rho\left(\delta_{1}\right)}{e\left(\delta_{1}\right)} x_{1}^{2} \\
\frac{\rho\left(\delta_{2}\right)}{e\left(\delta_{2}\right)} x_{2}^{2} \\
\vdots \\
\frac{\rho\left(\delta_{N}\right)}{e\left(\delta_{N}\right)} x_{N}^{2}
\end{array}\right)=M\left(\delta_{1}, \ldots, \delta_{N}\right)\left(\begin{array}{c}
x_{1} \\
x_{2} \\
\vdots \\
x_{N}
\end{array}\right)
$$

in which the matrix $M$ is given by $M\left(\delta_{1}, \ldots, \delta_{N}\right)=V\left(-\delta_{1}, \ldots,-\delta_{N}\right) V\left(\delta_{1}, \ldots\right.$, $\left.\delta_{N}\right)^{-1}$. Note that the quantities $e\left(\delta_{i}\right)$, for $i=1, \ldots, N$, are all non-zero because $e(s)$ and $d(s)$ are co-prime. The matrix $M$ is entirely available as a (numerical) matrix since the entries can easily be computed explicitly in terms of the poles $\delta_{1}, \ldots, \delta_{N}$ of $H(s)$ only. The coefficients in the equations in system (8.32) can be complex. This is the case when $H(s)$ has a complex conjugate pair of poles.

Theorem 8.1. The entry $m_{i, j}$ in row $i$ and column $j$ of the matrix $M\left(\delta_{1}, \ldots, \delta_{N}\right)$ is given by the non-zero quantity:

$$
m_{i, j}=-\frac{d\left(-\delta_{i}\right)}{\left(\delta_{i}+\delta_{j}\right) d^{\prime}\left(\delta_{j}\right)},
$$

where $d(s)=\left(s-\delta_{1}\right)\left(s-\delta_{2}\right) \cdots\left(s-\delta_{N}\right)$.

Proof. The entries in row $i$ of the matrix $M\left(\delta_{1}, \ldots, \delta_{N}\right)$ are obtained by solving the linear system of equations:

$$
\left(\begin{array}{llll}
m_{i 1} & m_{i 2} & \ldots & m_{i N}
\end{array}\right) V\left(\delta_{1}, \ldots, \delta_{N}\right)=\left(\begin{array}{llll}
1 & -\delta_{i} & \ldots & \left(-\delta_{i}\right)^{N-1}
\end{array}\right)
$$

According to Cramer's rule, the entry $m_{i, j}$ is given by:

$$
m_{i, j}=\frac{\operatorname{det} V\left(\delta_{1}, \delta_{2}, \ldots, \delta_{j-1},-\delta_{i}, \delta_{j+1}, \ldots, \delta_{N}\right)}{\operatorname{det} V\left(\delta_{1}, \delta_{2}, \ldots, \delta_{N}\right)} .
$$


This yields the expression:

$$
m_{i, j}=\frac{\prod_{k>l, k, l \neq j}\left(\delta_{k}-\delta_{l}\right) \prod_{k>j}\left(\delta_{k}+\delta_{i}\right) \prod_{j>l}\left(-\delta_{i}-\delta_{l}\right)}{\prod_{k>l}\left(\delta_{k}-\delta_{l}\right)} .
$$

Canceling common factors yields:

$$
\begin{aligned}
m_{i, j}= & \frac{\prod_{k>j}\left(\delta_{k}+\delta_{i}\right) \prod_{j>l}\left(-\delta_{i}-\delta_{l}\right)}{\prod_{k>j}\left(\delta_{k}-\delta_{j}\right) \prod_{j>l}\left(\delta_{j}-\delta_{l}\right)} \\
= & \frac{\left(-\delta_{i}-\delta_{1}\right) \cdots\left(-\delta_{i}-\delta_{j-1}\right)\left(-\delta_{i}-\delta_{j+1}\right) \cdots\left(-\delta_{i}-\delta_{N}\right)}{\left(\delta_{j}-\delta_{1}\right) \cdots\left(\delta_{j}-\delta_{j-1}\right)\left(\delta_{j}-\delta_{j+1}\right) \cdots\left(\delta_{j}-\delta_{N}\right)} .
\end{aligned}
$$

The denominator expression equals $d^{\prime}\left(\delta_{j}\right)$, while the numerator equals $-\frac{d\left(-\delta_{i}\right)}{\left(\delta_{i}+\delta_{j}\right)}$. The polynomial $d(s)$ has its zeros at $\delta_{1}, \delta_{2}, \ldots, \delta_{N}$ in the open left-half plane $\Pi^{-}$, which are all of multiplicity 1 . Therefore it holds that, $d\left(-\delta_{i}\right) \neq 0,\left(\delta_{i}+\delta_{j}\right) \neq 0$ and $d^{\prime}\left(\delta_{j}\right) \neq 0$ for all pairs $(i, j)$. Hence, the entries $m_{i, j}$ are non-zeros for all pairs $(i, j)$, which proves the theorem.

Note that Equation (8.32) can be regarded as a system of $N$ equations in the $N$ unknowns $x_{1}, \ldots, x_{N}$ with coefficients that depend on the parameters provided by $\rho\left(\delta_{i}\right)$. For the case of co-order $k=1$ the number of free parameters in $\rho\left(\delta_{i}\right)$ is $k-1=0$. This case was studied in [50]. As in that paper, we intend to proceed here by solving for the quantities $x_{1}, x_{2}, \ldots, x_{N}$, which then determine the polynomial $\tilde{a}(s)$, from which $q_{0}$ and $a(s)$ can be obtained. Subsequently, the polynomial $b(s)$ can be obtained as described in Section 8.6.

In the co-order $k$ case, where $k>1$, the solutions $x_{1}, x_{2}, \ldots, x_{N}$ should satisfy the additional constraints $\tilde{a}_{N-1}=\cdots=\tilde{a}_{N-k+1}=0$ too. These constraints can in view of Equation (8.27) be cast in the form of a linear constraint on the parameters $x_{1}, \ldots, x_{N}$ with coefficients that depend on $\delta_{1}, \ldots, \delta_{N}$ only. Moreover, note that for all values for $k \geq 1$ a feasible solution requires that $x_{i} \neq 0$ for all $i=1, \ldots, N$, so that the trivial solution to the system of equations (8.32) must be discarded.

Furthermore it is important here to note that for $k \geq 1$ the system of equations (8.32) for the quantities $x_{1}, x_{2}, \ldots, x_{N}$ is in Gröbner basis form with respect to any total degree monomial ordering for fixed values of $\rho_{0}, \rho_{1}, \ldots, \rho_{k-1}$. This observation is crucial as it provides a way to solve this system of equations by applying the StetterMöller matrix method mentioned in Section 3.3. This is studied in Section 8.5 of this chapter. 


\subsection{The $H_{2}$-approximation criterion}

In this section we present for the co-order $k$ case a way to evaluate the $H_{2}$-criterion of the difference $H(s)-G(s)$ directly in terms of a solution $\left(x_{1}, \ldots, x_{N}\right)$, and $\left(\rho_{1}, \ldots\right.$, $\left.\rho_{k-1}\right)$ of the system of quadratic equations (8.32) by means of a third order homogeneous polynomial that depends on the associated values of $\rho_{1}, \ldots, \rho_{k-1}$. This third order polynomial is described in Theorem 8.2 below and is a generalization of the polynomial in Theorem 5.2 in [50] which covers the co-order 1 case.

Theorem 8.2. (Criterion for co-order $k$ ) Let $H(s)=\frac{e(s)}{d(s)}$ and $G(s)=\frac{b(s)}{a(s)}$ be two strictly proper real rational $\mathrm{H}_{2}$ functions of McMillan degree $N$ and $n$, respectively, with $(d(s), e(s))$ and $(a(s), b(s))$ two co-prime pairs of real polynomials, such that $G(s)$ constitutes a stationary point of the $H_{2}$-approximation criterion $V_{H}(G)$ for $H$ on the submanifold $S_{n}$. Let $q(s)$ be the associated polynomial which satisfies e $(s) a(s)-$ $b(s) d(s)=a(-s)^{2} q(s)$. Then $V_{H}(G)$ equals:

$$
V_{H}(G)=\left\|\frac{e(s)}{d(s)}-\frac{b(s)}{a(s)}\right\|_{H_{2}}^{2}=\sum_{i=1}^{N} \frac{\rho\left(\delta_{i}\right)^{2} \rho\left(-\delta_{i}\right)}{e\left(\delta_{i}\right) d^{\prime}\left(\delta_{i}\right) d\left(-\delta_{i}\right)} x_{i}^{3},
$$

where $\delta_{1}, \delta_{2}, \ldots, \delta_{N}$ denote the $N$ distinct poles of $d(s)=\left(s-\delta_{1}\right)\left(s-\delta_{2}\right) \cdots\left(s-\delta_{N}\right)$, where $x_{i}=\tilde{a}\left(\delta_{i}\right)($ for $i=1,2, \ldots, N)$ with $\tilde{a}(s)=q_{0} a(-s), q_{0} \neq 0$ and $\rho(s)=\frac{q(s)}{q_{0}}$.

Proof. To prove this result, we proceed entirely analogous as in the proof of Theorem 5.2 in [50]. The $\mathrm{H}_{2}$-criterion function is defined as:

$$
V_{H}(G)=\left\|\frac{e(s)}{d(s)}-\frac{b(s)}{a(s)}\right\|_{H_{2}}^{2}=\left\|\frac{a(s) e(s)-b(s) d(s)}{a(s) d(s)}\right\|_{H_{2}}^{2} .
$$

Using Expression (8.18) the numerator of this expression is written as $a(-s)^{2} q(s)$. By using Equation (8.14) we introduce an integral to compute the criterion:

$$
V_{H}(G)=\left\|\frac{a(-s)^{2} q(s)}{a(s) d(s)}\right\|_{H_{2}}^{2}=\frac{1}{2 \pi} \int_{-\infty}^{\infty} \frac{a(-i \omega) a(i \omega) q(-i \omega) q(i \omega)}{d(i \omega) d(-i \omega)} d \omega .
$$

Application of Cauchy's Residue Theorem of complex analysis yields:

$$
V_{H}(G)=\sum_{i=1}^{N} \frac{a\left(-\delta_{i}\right) a\left(\delta_{i}\right) q\left(-\delta_{i}\right) q\left(\delta_{i}\right)}{d^{\prime}\left(\delta_{i}\right) d\left(-\delta_{i}\right)}=\sum_{i=1}^{N} \frac{\tilde{a}\left(-\delta_{i}\right) \tilde{a}\left(\delta_{i}\right) \rho\left(-\delta_{i}\right) \rho\left(\delta_{i}\right)}{d^{\prime}\left(\delta_{i}\right) d\left(-\delta_{i}\right)}
$$

since $\frac{q(s)}{q_{0}}=\rho(s)$. From Equation (8.23) we know that $\tilde{a}\left(-\delta_{i}\right)=\frac{\tilde{a}\left(\delta_{i}\right)^{2} \rho\left(\delta_{i}\right)}{e\left(\delta_{i}\right)}$ for $i=$ $1, \ldots, N$. Substituting this, it follows that:

$$
V_{H}(G)=\left\|\frac{e(s)}{d(s)}-\frac{b(s)}{a(s)}\right\|_{H_{2}}^{2}=\sum_{i=1}^{N} \frac{\tilde{a}\left(\delta_{i}\right)^{3} \rho\left(\delta_{i}\right)^{2} \rho\left(-\delta_{i}\right)}{e\left(\delta_{i}\right) d^{\prime}\left(\delta_{i}\right) d\left(-\delta_{i}\right)}
$$


which can also be denoted by $V_{H}\left(x_{1}, \ldots, x_{N}, \rho_{1}, \ldots, \rho_{k-1}\right)$ as:

$$
V_{H}\left(x_{1}, \ldots, x_{N}, \rho_{1}, \ldots, \rho_{k-1}\right)=\sum_{i=1}^{N} \frac{\rho\left(\delta_{i}\right)^{2} \rho\left(-\delta_{i}\right)}{e\left(\delta_{i}\right) d^{\prime}\left(\delta_{i}\right) d\left(-\delta_{i}\right)} x_{i}^{3} .
$$

This proves the theorem.

For $k=1$ and $\rho(s) \equiv 1$, Equation (8.43) specializes to the known results of Theorem 5.2 in [50].

Note that for fixed values of $\rho_{1}, \rho_{2}, \ldots, \rho_{k-1}$, the coefficients of this homogeneous polynomial in $x_{1}, x_{2}, \ldots, x_{N}$ of total degree 3 are all computable from the given transfer function $H(s)$ of order $N$.

\subsection{Stetter-Möller matrix method for $H_{2}$ model-order reduction}

In this section an approach is given to solve the system of equations (8.32) for the quantities $x_{1}, x_{2}, \ldots, x_{N}$ and $\rho_{1}, \ldots, \rho_{k-1}$ by applying the Stetter-Möller matrix method as described in Section 3.3. This method gives rise to an eigenvalue problem in terms of the $k-1$ coefficients of $\rho(s)$. For $k>1$, there will be $k-1$ additional constraints because the solutions for $x_{1}, x_{2}, \ldots, x_{N}$ are only feasible when the coefficients $\tilde{a}_{N-1}, \ldots, \tilde{a}_{N-k+1}$ of the polynomial $\tilde{a}(s)$ are all zero. In other words, we intend to find values for $\rho_{0}, \rho_{1}, \ldots, \rho_{k-1}$ for which the system of equations admits non-zero solutions for $x_{1}, x_{2}, \ldots, x_{N}$ which make all the coefficients $\tilde{a}_{N-1}, \ldots, \tilde{a}_{N-k+1}$ equal to zero. As said before, the coefficients $\tilde{a}_{N-1}, \ldots, \tilde{a}_{N-k+1}$, can be expressed linearly in terms of the quantities $x_{1}, x_{2}, \ldots, x_{N}$, with coefficients that constitute the last $k-1$ rows of the matrix $V\left(\delta_{1}, \delta_{2}, \ldots, \delta_{N}\right)^{-1}$.

Thus, by applying the Stetter-Möller matrix method as introduced in Chapter 3, we intend to transform the system of $N$ equations:

$$
\left(\begin{array}{c}
\frac{\rho\left(\delta_{1}\right)}{e\left(\delta_{1}\right)} x_{1}^{2} \\
\frac{\rho\left(\delta_{2}\right)}{e\left(\delta_{2}\right)} x_{2}^{2} \\
\vdots \\
\frac{\rho\left(\delta_{N}\right)}{e\left(\delta_{N}\right)} x_{N}^{2}
\end{array}\right)=M\left(\delta_{1}, \ldots, \delta_{N}\right)\left(\begin{array}{c}
x_{1} \\
x_{2} \\
\vdots \\
x_{N}
\end{array}\right)
$$

subject to the $k-1$ additional constraints:

$$
\left(\begin{array}{c}
\tilde{a}_{N-1} \\
\tilde{a}_{N-2} \\
\vdots \\
\tilde{a}_{N-k+1}
\end{array}\right)=\Gamma\left(\delta_{1}, \ldots, \delta_{N}\right)\left(\begin{array}{c}
x_{1} \\
x_{2} \\
\vdots \\
x_{N}
\end{array}\right)=\left(\begin{array}{c}
0 \\
0 \\
\vdots \\
0
\end{array}\right),
$$

into a structured polynomial eigenvalue problem of size $2^{N} \times 2^{N}$ involving the $k-1$ parameters in the polynomial $\rho(s)$. Here the matrix $\Gamma\left(\delta_{1}, \ldots, \delta_{N}\right)$ is defined to consist of the last $k-1$ rows of the matrix $V\left(\delta_{1}, \ldots, \delta_{N}\right)^{-1}$ in reversed order: 


$$
\Gamma\left(\delta_{1}, \ldots, \delta_{N}\right)=\left(\begin{array}{cccccc}
0 & \ldots & 0 & 0 & & 1 \\
\vdots & & \vdots & & . \cdot & \\
0 & \ldots & 0 & 1 & & 0
\end{array}\right) V\left(\delta_{1}, \ldots, \delta_{N}\right)^{-1} .
$$

This extends the approach of [50] to co-orders $k>1$ and from a conventional eigenvalue problem for $k=1$ to a multi- parameter polynomial eigenvalue problem formulation as worked out in the next chapters.

For fixed values of $\rho(s)$, the equations in system (8.44) are in Gröbner basis form with respect to any total degree monomial ordering. The polynomial expressions associated with these equations generate an ideal $I$. Now the idea is to choose a suitable polynomial $r\left(x_{1}, x_{2}, \ldots, x_{N}\right)$ and to address the linear operator $\mathcal{A}_{r}$ which performs multiplication by the polynomial $r$ modulo the ideal $I$ in the quotient space $\mathbb{C}$ $\left[x_{1}, x_{2}, \ldots, x_{N}\right] / I$. This quotient space is finite-dimensional and therefore constitutes a finite set of $2^{N}$ (possibly complex) solutions $\left(x_{1}, \ldots, x_{N}\right)$. For each solution a corresponding approximation $G(s)$ of order $N-k$ can be computed. Note that $G(s)$ is only a feasible solution if it is real and stable.

A monomial basis here is given by the following set $B$ :

$$
B=\left\{x_{1}^{\alpha_{1}} \cdots x_{N}^{\alpha_{N}} \mid \alpha_{1}, \ldots, \alpha_{N} \in\{0,1\}\right\} .
$$

With respect to $B$, the operator $\mathcal{A}_{r}$ can now be represented by a matrix $A_{r}^{T}$ of size $2^{N} \times 2^{N}$ as shown in Section 3.3.

Upon choosing $r\left(x_{1}, x_{2}, \ldots, x_{N}\right)$ as the polynomial $x_{i}$ the matrix $A_{x_{i}}^{T}$ can be constructed, for each $i=1,2, \ldots, N$. As discussed in Section 3.3, the tuple of matrices $\left(A_{x_{1}}^{T}, A_{x_{2}}^{T}, \ldots, A_{x_{N}}^{T}\right)$ constitutes a matrix solution to the system of equations $(8.44)$ we intend to solve. All the $2^{N}$ solutions of this system can be obtained by computing the $2^{N} N$-tuples of eigenvalues of the matrices $A_{x_{1}}^{T}, A_{x_{2}}^{T}, \ldots, A_{x_{N}}^{T}$.

The matrices $A_{r}^{T}$, for arbitrary polynomials $r$, all commute and thus they have common eigenvectors. The eigenvalues of the matrix $A_{r}^{T}$ correspond to the values of $r$ at the (possibly complex) solutions $\left(x_{1}, x_{2}, \ldots, x_{N}\right)$ to the system of equations (8.44). Similar properties obviously hold for the matrices $A_{r}$. If all the eigenvalues have multiplicity one, the eigenvectors of a matrix $A_{r}^{T}$ exhibit the Stetter vector structure and therefore the values $x_{1}, x_{2}, \ldots, x_{N}$ can be obtained from the entries of such a Stetter vector (see Section 3.3 again). Thus, an eigenpair computation on a single matrix $A_{r}^{T}$ for a well chosen polynomial $r$ can bring all the solutions to the system of equations (8.44). All the $2^{N}$ scalar solutions can be obtained in this way by working with one matrix $A_{r}^{T}$ only.

The approach above can be used, to set up a classical eigenvalue problem for the co-order $k=1$ case to solve the system of equations, since the additional variables $\rho_{i}$ do not show up here.

In the co-order $k>1$, the additional variables $\rho_{1}, \ldots, \rho_{k-1}$ show up in the quadratic system of equations (8.44). We now consider the linear operator which performs multiplication by the polynomial $r$ within the quotient space $\mathbb{C}\left(\rho_{1}, \ldots\right.$, 
$\left.\rho_{k-1}\right)\left[x_{1}, x_{2}, \ldots, x_{N}\right] / I\left(\rho_{1}, \ldots, \rho_{k-1}\right)$ where $I\left(\rho_{1}, \ldots, \rho_{k-1}\right)$ denotes the associated ideal generated by the polynomials in the system of equations (8.44). Applying the Stetter-Möller matrix method gives rise to matrices $A_{r}\left(\rho_{1}, \ldots, \rho_{k-1}\right)^{T}$ which are $r a-$ tional in $\rho_{1}, \ldots, \rho_{k-1}$.

Because we require for $k>1$ any feasible solution to satisfy all the constraints in (8.45), we consecutively choose $r$ as the polynomials involved in these $k-1$ additional constraints $\tilde{a}_{N-1}, \ldots, \tilde{a}_{N-k+1}$. This yields a polynomial eigenvalue problem involving $k-1$ matrices $A_{\tilde{a}_{N-1}}\left(\rho_{1}, \ldots, \rho_{k-1}\right)^{T}, \ldots, A_{\tilde{a}_{N-k+1}}\left(\rho_{1}, \ldots, \rho_{k-1}\right)^{T}$ which are rational in $\rho_{1}, \ldots, \rho_{k-1}$ but can be made polynomial in these parameters by getting rid of the (common) denominators.

In the Chapters 9, 10, and 11 we show procedures for the co-order $k=1, k=2$, and $k=3$ case, respectively, to solve the corresponding eigenvalue problems. These procedures lead to solutions $x_{1}, \ldots, x_{N}$, and $\rho_{1}, \ldots, \rho_{k-1}$ of the systems of equations (8.44) which satisfy the constraints in (8.45). Subsequently, these solutions yield approximations $G(s)$ as described in the next section.

\subsection{Computing the approximation $G(s)$}

Let $x_{1}, \ldots, x_{N}$ and $\rho_{1}, \ldots, \rho_{k-1}$ be a solution of the system of equations (8.44), which also satisfy the additional constraints $\tilde{a}_{N-1}=\cdots=\tilde{a}_{N-k+1}=0$ in (8.45). Using this solution an approximation of order $N-k$ can be computed as described in the next theorem.

Theorem 8.3. (Co-order $k)$ Let $H(s)=e(s) / d(s)$ be a given strictly stable proper rational transfer function of order $N$ which has $N$ distinct poles $\delta_{1}, \ldots, \delta_{N}$. In the coorder $k$ case, consider the associated linear system of equations (8.44). Let $x_{1}, \ldots, x_{N}$ and $\rho_{1}, \ldots, \rho_{k-1}$ constitute a solution to this set of equations, which satisfies the additional constraints $\tilde{a}_{N-1}=\cdots=\tilde{a}_{N-k+1}=0$ in (8.45). Then a feasible approximation $G(s)=b(s) / a(s)$ of order $N-k$ can be computed as follows, provided that it happens that $q_{0} \neq 0$ :

- From Equation (8.27) the polynomial $\tilde{a}(s)$ is constructed by computing its coefficients: once $x_{i}=\tilde{a}\left(\delta_{i}\right)$ for $i=1, \ldots, N$ are known, the coefficients $\tilde{a}_{0}, \ldots$, $\tilde{a}_{N-1}$ are computed by:

$$
\left(\begin{array}{c}
\tilde{a}_{0} \\
\vdots \\
\tilde{a}_{N-1}
\end{array}\right)=V\left(\delta_{1}, \ldots, \delta_{N}\right)^{-1}\left(\begin{array}{c}
x_{1} \\
\vdots \\
x_{N}
\end{array}\right) .
$$

- Feasibility requires that the 'leading' coefficients $\tilde{a}_{N-1}, \ldots, \tilde{a}_{N-k+1}$ of the polynomial $\tilde{a}(s)$ are zero. Subsequently, the parameter $q_{0}$ is read off from the coefficient $\tilde{a}_{N-k}$ by using: $q_{0}=(-1)^{N-k} \tilde{a}_{N-k}$. 
- Feasibility requires furthermore $q_{0}$ to be non-zero, in which case a $(-s)$ is determined as $\tilde{a}(s) / q_{0}$. The polynomial a(s) of degree $N-k$ should be Hurwitz. Note that only solutions $x_{1}, \ldots, x_{N}$ which yield a real-valued polynomial a $(s)$ are of importance, which requires the parameters $x_{i}$ to be either real or to occur as complex conjugate pairs. Note also that feasibility requires $\rho_{1}, \ldots, \rho_{k-1}$ to be real-valued.

- Finally, the polynomial b(s) is obtained from Equation (8.18) as follows:

$$
b(s)=\frac{\left.e(s) a(s)-q_{0} a(-s)^{2} \rho(s)\right)}{d(s)},
$$

where the polynomial $\rho(s)$ is of degree $\leq k-1$ as in (8.20).

These steps yield an approximation $G(s)=\frac{b(s)}{a(s)}$ of order $N-k$. The approximation which yields the smallest real value for the $H_{2}$-criterion $V_{H}\left(x_{1}, \ldots, x_{N}, \rho_{1}, \ldots, \rho_{k-1}\right)$, as described in (8.43), is the globally optimal approximation of order $N-k$ to the given system $H(s)$.

Proof. The proof is straightforward and therefore omitted. 


\section{Chapter 9}

\section{$\mathrm{H}_{2}$ Model-order reduction from order $\mathrm{N}$ to $\mathrm{N}-1$}

In the co-order $k=1$ case, the polynomial $\rho(s)$ in (8.44) is of degree 0 . Then it holds that $\rho(s) \equiv 1$ and also $\rho\left(\delta_{i}\right)=1$ for $i=1, \ldots, N$ in (8.44). Then the system of equations (8.44) yields the following $N$ polynomial equations in the $N$ unknowns $x_{1}, \ldots, x_{N}$ :

$$
\left(\begin{array}{c}
\frac{1}{e\left(\delta_{1}\right)} x_{1}^{2} \\
\frac{1}{e\left(\delta_{2}\right)} x_{2}^{2} \\
\vdots \\
\frac{1}{e\left(\delta_{N}\right)} x_{N}^{2}
\end{array}\right)-M\left(\delta_{1}, \ldots, \delta_{N}\right)\left(\begin{array}{c}
x_{1} \\
x_{2} \\
\vdots \\
x_{N}
\end{array}\right)=\left(\begin{array}{c}
0 \\
0 \\
\vdots \\
0
\end{array}\right)
$$

where the matrix $M$ is again $V\left(-\delta_{1}, \ldots,-\delta_{N}\right) V\left(\delta_{1}, \ldots, \delta_{N}\right)^{-1}$.

\subsection{Solving the system of quadratic equations}

Upon choosing the polynomial $r\left(x_{1}, x_{2}, \ldots, x_{N}\right)$ in Section (8.5) equal to each of the monomials $x_{i}$, the $2^{N} \times 2^{N}$ matrices $A_{x_{i}}^{T}$, for $i=1,2, \ldots, N$, are constructed by applying the Stetter-Möller matrix method. Here we consider the quotient space $\mathbb{C}\left[x_{1}, \ldots, x_{N}\right] / I$, where $I$ is the ideal generated by the polynomials involved in the system of equations (9.1).

The eigenvalues of the matrix $A_{x_{i}}^{T}$ are the values of $x_{i}$ at the solutions of the corresponding system of equations. Moreover, once one eigenpair of some matrix $A_{x_{i}}^{T}$ for some particular $i$ is known, the values of $x_{1}, \ldots, x_{N}$ can be read off from the eigenvector because this vector exhibits the Stetter-structure. Note that only real eigenvalues are of interest in our specific application.

Any (real) solution $\left(x_{1}, \ldots, x_{N}\right)$ to the system of equations (9.1) gives rise to a corresponding set of coefficients $\tilde{a}_{0}, \tilde{a}_{1}, \ldots, \tilde{a}_{N-1}$ from which $q_{0}=(-1)^{N-1} \tilde{a}_{N-1}$ and, 
subsequently, the coefficients $a_{1}, a_{2}, \ldots, a_{N-2}$ can be computed as follows: If $q_{0}=0$, an infeasible solution is obtained (this occurs for instance if $x_{1}=\cdots=x_{N}=0$ ). If $q_{0} \neq 0$, it holds that $a_{N-2}=(-1)^{N-2} \tilde{a}_{N-2} / q_{0}, \ldots, a_{1}=-\tilde{a}_{1} / q_{0}$ and $a_{0}=\tilde{a}_{0} / q_{0}$. The polynomial $a(s)=s^{N-1}+a_{N-2} s^{N-2}+\ldots+a_{1} s+a_{0}$ is required to be Hurwitz and real. Otherwise an infeasible solution is being considered, which should then be discarded. Once the polynomial $a(s)$ is known, $b(s)$ can be computed too which yields approximations $G(s)=\frac{b(s)}{a(s)}$ of order $N-1$. The method described so far in this section for $k=1$ coincides with the algebraic approach developed in [50].

In [50] all the matrices $A_{x_{i}}^{T} i=1, \ldots, N$ were constructed explicitly whereas applying the $n \mathrm{D}$-system technique of Chapter 5 makes it possible here to compute the solutions of (9.1) for $k=1$ in a matrix-free fashion by associating the polynomials that generate $I$ with a system of $N$ simultaneous recursions for an $N$-dimensional time-series (i.e., defined on an $N$-dimensional grid). The transposed matrix $A_{r}^{T}$, then serves as the dynamical matrix in a state-space description of a recursion associated with $r\left(x_{1}, x_{2}, \ldots, x_{N}\right)$ for the same $N$-dimensional time-series. This has the benefit that these matrices of dimensions $2^{N} \times 2^{N}$, do not have to be stored into memory. This leads to significant savings on required memory and CPU time.

Another improvement of the approach of [50] can be achieved as follows. Note that the method described so far allows one to compute all the minima, saddle points and maxima alike for the $\mathrm{H}_{2}$ model-order reduction criterion. It is then possible to select the globally optimal approximation by computing the $\mathrm{H}_{2}$-norm of the difference $H(s)-G(s)$ for every feasible solution $G(s)$ and selecting the one for which this criterion is minimal. To arrive at a global minimum directly, one somehow needs to evaluate the $\mathrm{H}_{2}$-criterion. For reasons of computational efficiency it is preferable to be able to do this in terms of the quantities $x_{1}, \ldots, x_{N}$.

The complex homogeneous third order polynomial given in Theorem 8.2 turns out to be helpful here. In the co-order $k=1$ case this polynomial attains the form:

$$
V_{H}\left(x_{1}, x_{2}, \ldots, x_{N}\right)=\left\|\frac{e(s)}{d(s)}-\frac{b(s)}{a(s)}\right\|_{H_{2}}^{2}=\sum_{i=1}^{N} \frac{1}{e\left(\delta_{i}\right) d^{\prime}\left(\delta_{i}\right) d\left(-\delta_{i}\right)} x_{i}^{3} .
$$

This polynomial coincides at the solutions $\left(x_{1}, \ldots, x_{N}\right)$ of the system of equations (9.1) with the $H_{2}$ model-order reduction criterion.

Upon choosing the polynomial $r\left(x_{1}, x_{2}, \ldots, x_{N}\right)$ in Section (8.5) as the polynomial equal to $V_{H}\left(x_{1}, x_{2}, \ldots, x_{N}\right)$, the $2^{N} \times 2^{N}$ matrix $A_{V_{H}}^{T}$ can be constructed through the Stetter-Möller matrix method by working modulo the ideal $I$. Here the ideal $I$ is generated by the polynomials on the left-hand side of the system of equations (9.1).

This makes it possible to compute the global optimum more directly and more efficiently which offers a computational advantage: the values of $V_{H}$ at the solutions of (9.1) show up as the eigenvalues of the matrix $A_{V_{H}}^{T}$. This enables the possibility to compute only the solution that corresponds to the smallest positive real eigenvalue $\lambda$ of the matrix $A_{V_{H}}^{T}$. The values $x_{1}, \ldots, x_{N}$, which are real or complex conjugate pairs, 
can be read off from the corresponding eigenvector. The solution $x_{1}, \ldots, x_{N}$ yields the approximation $G(s)$ of order $N-1$ with the smallest distance between all the real approximations $G(s)$ and the original system $H(s)$ of order $N$. If this solution happens to be feasible it constitutes the globally optimal approximation $G(s)$ of order $N-1$. If this is not the case, we discard this solution and proceed with the second smallest real eigenvalue $\lambda$ of the matrix $A_{V_{H}}^{T}$.

This approach avoids the computation of all the $2^{N}$ eigenvalues of the matrix $A_{V_{H}}^{T}$ or of the matrices $A_{x_{i}}^{T}$ for $i=1, \ldots, N$. Computing the smallest real eigenvalue(s) of a sparse matrix directly can be achieved by employing iterative eigenvalue solvers, as discussed in Chapter 6 . The goal here is to achieve a substantial gain in computational efficiency.

Remark 9.1. When computing the global minimum of a Minkowski dominated polynomial $p$, as discussed in Part II of this thesis, one may construct the matrix $A_{p}^{T}$ using the Stetter-Möller matrix method. The ideal $I$ which is used to construct this matrix is generated by the polynomials in the system of first-order conditions of the polynomial $p$. The smallest real eigenvalue of this matrix yields the value and the location of the global minimum of $p$. The polynomials which generate the ideal $I$ are directly related with the 'criterion' $p$ since they are the partial derivatives of $p$.

Here we are working with the matrix $A_{V_{H}}^{T}$ and an ideal $I$ which is generated by polynomials involved in the system of equations we want to solve. Note that the third order criterion $V_{H}$ is not related to these polynomials. Note furthermore that it is still valid to employ the $n \mathrm{D}$-systems approach in this case. We have similar structures in the $n \mathrm{D}$-system but the degrees of the structures equal $m=2$ instead of an odd degree $m=2 d-1$ (see Equations (4.2) and (5.3)).

\subsection{Computing the approximation $G(s)$}

Let $x_{1}, \ldots, x_{N}$ be a solution to the system of equations (9.1) which is read off from the Stetter eigenvector corresponding to the eigenvalue $\lambda_{\min }$. This eigenvalue $\lambda_{\text {min }}$ is the smallest real eigenvalue obtained by an eigenvalue computation on the matrix $A_{V_{H}}^{T}$ as in the previous section. Then the globally optimal approximation $G(s)$ of order $N-1$ is computed as described in Theorem 8.3 for $k=1$.

The method described in this chapter is an extension of the model order reduction approach developed by Hanzon, Maciejowski and Chou as described in [50]. In that paper approximations $G(s)$ of order $N-1$ are constructed by computing all the eigenvalues of all the matrices $A_{x_{i}}^{T}$ for $i=1, \ldots, N$. In this chapter this method is extended and improved by working with the single operator $A_{V_{H}}^{T}$ as discussed above. Working with this operator enables the ability to zoom in on the smallest real eigenvalue(s). Therefore iterative eigenvalue solvers as described in Chapter 6 are used, which furthermore make it possible to use the $n \mathrm{D}$-systems approach of Chapter 5. In this way one can compute only some eigenvalues of the operator in a matrix-free fashion, which increases the efficiency of the approach. Co-order one model reduction 
using the approach from this chapter is also discussed in [18]. The approach of this chapter is further extended to the co-order two and three cases in the Chapters 10 and 11 .

\subsection{Example}

In [50] the highest possible order of a system, for a co-order $k=1$ model reduction, was 9 . For larger values of the model-order severe memory problems occurred. In this section the potential of the reduction technique in combination with an $n \mathrm{D}$-system is demonstrated by an example which involves the reduction of a system of order 10 to a system of order 9 without explicitly constructing any matrices. All the calculations throughout this section are done on a Dual Xeon Pentium IV $3.2 \mathrm{GHz}$ with $4096 \mathrm{MB}$ internal memory using Mathematica 5.0.1 and Matlab 6.5.

The system $H(s)$ to be reduced has a $10^{\text {th }}$ order transfer function. This system is constructed to have Hankel singular values $10,9, \ldots, 1$ and is described by a statespace representation involving the following matrices $A, B$, and $C$ :

$$
A=\left(\begin{array}{cccccccccc}
\frac{-9}{80} & \frac{39}{380} & \frac{-11}{120} & \frac{-13}{20} & \frac{-3}{32} & \frac{-9}{25} & \frac{-9}{40} & \frac{3}{35} & \frac{-11}{80} & \frac{81}{220} \\
\frac{39}{380} & \frac{-169}{1800} & \frac{143}{1700} & \frac{169}{200} & \frac{13}{150} & \frac{39}{100} & \frac{21}{100} & \frac{-13}{150} & \frac{13}{100} & \frac{-351}{1000} \\
\frac{-11}{120} & \frac{143}{1700} & \frac{-121}{1600} & \frac{-143}{100} & \frac{-11}{140} & \frac{-11}{25} & \frac{-77}{400} & \frac{11}{125} & \frac{-121}{1000} & \frac{33}{100} \\
\frac{13}{20} & \frac{-169}{200} & \frac{143}{100} & \frac{-169}{1400} & \frac{-13}{10} & \frac{-13}{100} & \frac{-91}{100} & \frac{13}{250} & \frac{-143}{500} & \frac{117}{200} \\
\frac{-3}{32} & \frac{13}{150} & \frac{-11}{140} & \frac{13}{10} & \frac{-1}{12} & \frac{-6}{5} & \frac{-21}{100} & \frac{2}{15} & \frac{-11}{80} & \frac{27}{70} \\
\frac{9}{25} & \frac{-39}{100} & \frac{11}{25} & \frac{-13}{100} & \frac{6}{5} & \frac{-18}{125} & \frac{-63}{25} & \frac{3}{50} & \frac{-11}{25} & \frac{81}{100} \\
\frac{-9}{40} & \frac{21}{100} & \frac{-77}{400} & \frac{91}{100} & \frac{-21}{100} & \frac{63}{25} & \frac{-441}{800} & \frac{21}{25} & \frac{-77}{200} & \frac{567}{500} \\
\frac{-3}{35} & \frac{13}{150} & \frac{-11}{125} & \frac{13}{250} & \frac{-2}{15} & \frac{3}{50} & \frac{-21}{25} & \frac{-2}{75} & \frac{11}{25} & \frac{-27}{50} \\
\frac{-11}{80} & \frac{13}{100} & \frac{-121}{1000} & \frac{143}{500} & \frac{-11}{80} & \frac{11}{25} & \frac{-77}{200} & \frac{-11}{25} & \frac{-121}{400} & \frac{99}{100} \\
\frac{81}{220} & \frac{-351}{1000} & \frac{33}{100} & \frac{-117}{200} & \frac{27}{70} & \frac{-81}{100} & \frac{567}{500} & \frac{27}{50} & \frac{99}{100} & \frac{-729}{200}
\end{array}\right),
$$


which yields a transfer function $H(s)=C(s I-A)^{-1} B$, available in exact format but displayed in numerical format:

$$
\begin{aligned}
H(s)= & \frac{8.00769 \times 10^{-6}+1.71974 s+260.671 s^{2}+1254.63 s^{3}+899.401 s^{4}+\ldots}{1.60154 \times 10^{-7}+0.0541886 s+17.9691 s^{2}+147.766 s^{3}+138.541 s^{4}+\ldots} \\
& \frac{\ldots+1246.85 s^{5}+181.506 s^{6}+276.659 s^{7}-1.22269 s^{8}+15.77 s^{9}}{\cdots+252.726 s^{5}+99.6262 s^{6}+71.4313 s^{7}+19.6362 s^{8}+5.15548 s^{9}+s^{10}} .
\end{aligned}
$$

The poles of this transfer function are located at:

$$
\begin{array}{ll}
-4.0242 & -0.1807+0.8543 i \\
-0.2962+3.0209 i & -0.1807-0.8543 i \\
-0.2962-3.0209 i & -0.1313 \\
-0.0215+2.1720 i & -3.091 \times 10^{-3} \\
-0.0215-2.1720 i & -2.960 \times 10^{-6}
\end{array}
$$

and the zeros at:

$$
\begin{aligned}
0.5866+3.5000 i & -0.3625-1.0208 i \\
0.5866-3.5000 i & -0.2274 \\
-0.0683+2.1804 i & -6.815 \times 10^{-3} \\
-0.0683-2.1804 i & -4.660 \times 10^{-6} \\
-0.3625+1.0208 i &
\end{aligned}
$$

To compute an approximation $G(s)$ of order 9 , a system of 10 quadratic equations in $x_{1}, \ldots, x_{10}$ needs to be solved. This system has a finite number of complex solutions $\left(x_{1}, \ldots, x_{10}\right)$ and the associated complex homogeneous polynomial $V_{H}$ of degree 3 , which coincides with the $H_{2}$-criterion function at these solutions, is computed as:

$$
\begin{aligned}
V_{H}= & 3.83344 \times 10^{-20} x_{1}^{3}+\left(1.36467 \times 10^{-14}-1.00376 \times 10^{-15} i\right) x_{2}^{3}+ \\
& \left(1.36467 \times 10^{-14}+1.00376 \times 10^{-15} i\right) x_{3}^{3}+\left(3.58175 \times 10^{-7}+\right. \\
& \left.2.48189 \times 10^{-7} i\right) x_{4}^{3}+\left(3.58175 \times 10^{-7}-2.48189 \times 10^{-7} i\right) x_{5}^{3}+ \\
& 0.415865 x_{6}^{3}-\left(9.03372 \times 10^{-10}-1.13998 \times 10^{-9} i\right) x_{7}^{3}- \\
& \left(9.03372 \times 10^{-10}+1.13998 \times 10^{-9} i\right) x_{8}^{3}+1.93482 \times 10^{7} x_{9}^{3}+ \\
& 1.97345 \times 10^{13} x_{10}^{3} .
\end{aligned}
$$

Note that the polynomial $V_{H}$ is highly ill-conditioned since the values of the coefficients range between $10^{-20}$ and $10^{13}$, whereas an imaginary part of $10^{-7}$ seems to be significant.

To compare the results of the present approach with the approach of [50], we first construct likewise the $2^{10} \times 2^{10}=1024 \times 1024$ matrices $A_{x_{i}}^{T}, i=1, \ldots, 10$, explicitly with Mathematica. Then the built-in function Eigensystem is used to calculate all the eigenvalues and all the eigenvectors of these matrices. For each matrix $A_{x_{i}}^{T}$ this takes between 440 and 540 seconds together with the eigensystem computation (which takes 
between 70 and 100 seconds). To compute and store all these matrices an amount of $925 \mathrm{MB}$ of internal memory is required.

Following the approach of [50], all the 1024 tuples $x_{1}, \ldots, x_{10}$ are determined from the mutual eigenvectors of the matrices $A_{x_{1}}^{T}, \ldots, A_{x_{10}}^{T}$. Each such tuple yields an approximation of order $N-1$ which is either feasible or infeasible. By substituting these tuples into the polynomial $V_{H}$, we compute the $H_{2}$-criterion value for each feasible solution. The solution with the smallest real $H_{2}$-criterion value is used to compute the polynomials $b(s)$ and $a(s)$ which yield the feasible globally optimal stable approximation $G(s)=\frac{b(s)}{a(s)}$ of order 9 with an $H_{2}$-criterion value of 0.000489593 :

$$
\begin{aligned}
G(s)= & \frac{1.69597+260.566 s+1254.59 s^{2}+899.268 s^{3}+1246.84 s^{4}+\ldots}{0.0538714+17.9585 s+147.756 s^{2}+138.523 s^{3}+252.714 s^{4}+\ldots} \\
& \frac{\ldots+181.474 s^{5}+276.659 s^{6}-1.22433 s^{7}+15.7699 s^{8}}{\ldots+99.6209 s^{5}+71.4287 s^{6}+19.6358 s^{7}+5.15532 s^{8}+s^{9}} .
\end{aligned}
$$

The solution used to compute this approximation is:

$$
\begin{array}{ll}
x_{1}=78.6020 & x_{6}=0.000289407 \\
x_{2}=-0.180875-0.343180 i & x_{7}=-0.00193041+0.00371561 i \\
x_{3}=-0.180875+0.343180 i & x_{8}=-0.00193041-0.00371561 i \\
x_{4}=-0.00155184-0.00377204 i & x_{9}=5.99264 \times 10^{-6} \\
x_{5}=-0.00155184+0.00377204 i & x_{10}=2.91654 \times 10^{-6}
\end{array}
$$

The Hankel singular values of the approximation $G(s)$ are now changed to the values: $9.68,8,7, \ldots, 1$. Note that the coefficients of the approximation $G(s)$ are very similar to the coefficients of the original problem $H(s)$. Effectively, one pole/zero pair of the original transfer function at $s=0$ is removed by the model-order reduction algorithm and has left the other poles and zeros nearly unchanged.

Another computational approach is by working with the matrix $A_{V_{H}}^{T}$. First, we explicitly construct this matrix and compute all its eigenvalues. Second, we do not use the explicit matrix $A_{V_{H}}^{T}$ but we use the matrix-free approach for the associated operator of the matrix $A_{V_{H}}^{T}$ together with an iterative eigenvalue solver and an $n \mathrm{D}$ system, to compute only the smallest real eigenvalue of $A_{V_{H}}^{T}$ which immediately will lead to a feasible globally optimal approximation of order 9.

To construct the matrix $A_{V_{H}}^{T}$ explicitly and to compute all its eigenvalues and eigenvectors is computationally a hard job: it takes, respectively, 27164 and 82 seconds. Furthermore, it takes $275 \mathrm{MB}$ of internal memory to store all the data. The matrix $A_{V_{H}}^{T}$ has 45 real eigenvalues, including the zero eigenvalue. The smallest positive real eigenvalue $\lambda_{\min }$ is computed as 0.000489593 . This smallest real eigenvalue yields the solution $x_{1}, \ldots, x_{10}$ since it is read off from the corresponding eigenvector. The solution $x_{1}, \ldots, x_{10}$ computed here turns out to be the same solution as found before in (9.9). This leads to the same globally optimal approximation $G(s)$ of order 9 as in (9.8). The $H_{2}$-criterion is again 0.000489593 , which exactly equals the eigenvalue $\lambda_{\min }=0.000489593$. 
In Figure 9.1 all the eigenvalues of the matrix $A_{V_{H}}^{T}$, excluding the zero eigenvalue (as it will not lead to a feasible approximation), are plotted in the complex plane. The best stable approximation $G(s)$ corresponds to the real eigenvalue which is as small as possible. Figure 9.2 shows the eigenvalues of the matrix $A_{V_{H}}^{T}$ near the real axis around the origin.

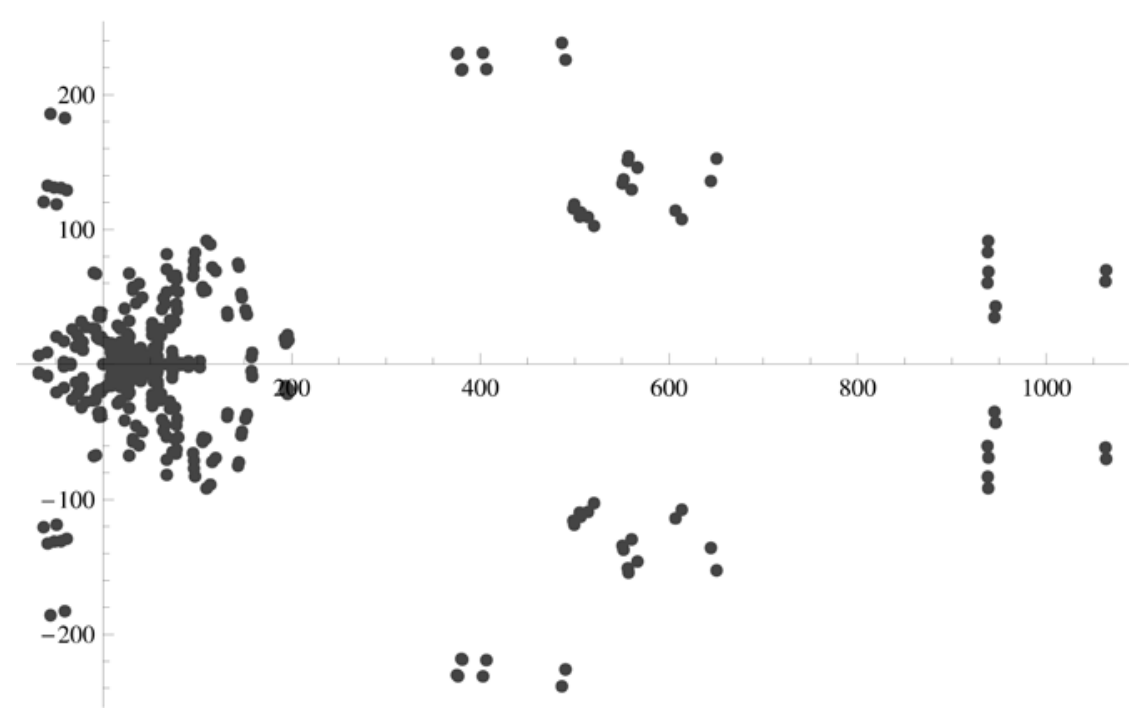

Figure 9.1: All eigenvalues of the matrix $A_{V_{H}}^{T}$

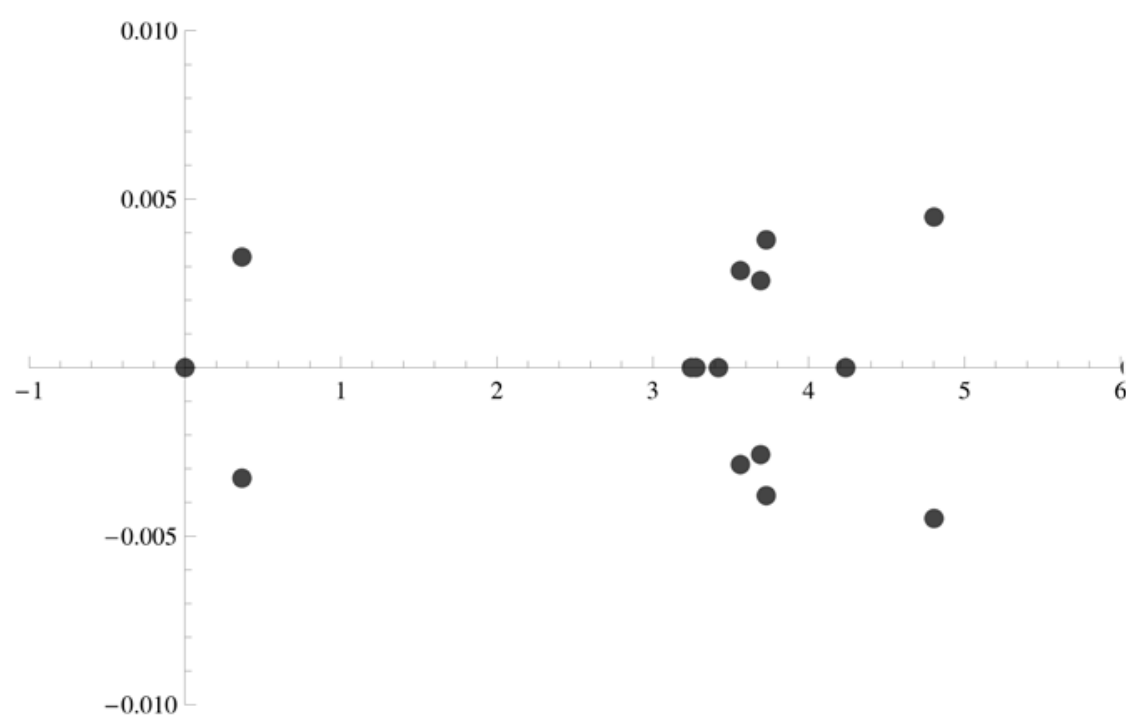

Figure 9.2: Zoomed in on the smallest real eigenvalues of the matrix $A_{V_{H}}^{T}$

The matrices involved in these computations are all sparse. All the matrices 
contain $1024^{2}=1048576$ elements of which 98415 are non-zero in the matrices $A_{x_{i}}^{T}$ (9.4\% filled), $i=1, \ldots, N$. The matrix $A_{V_{H}}^{T}$ contains 550100 non-zeros $(52.5 \%$ filled). See Figure 9.3 for a representation of the sparsity structure of the matrices $A_{x_{1}}^{T}, A_{x_{2}}^{T}$ and of the matrix $A_{V_{H}}^{T}$, respectively.
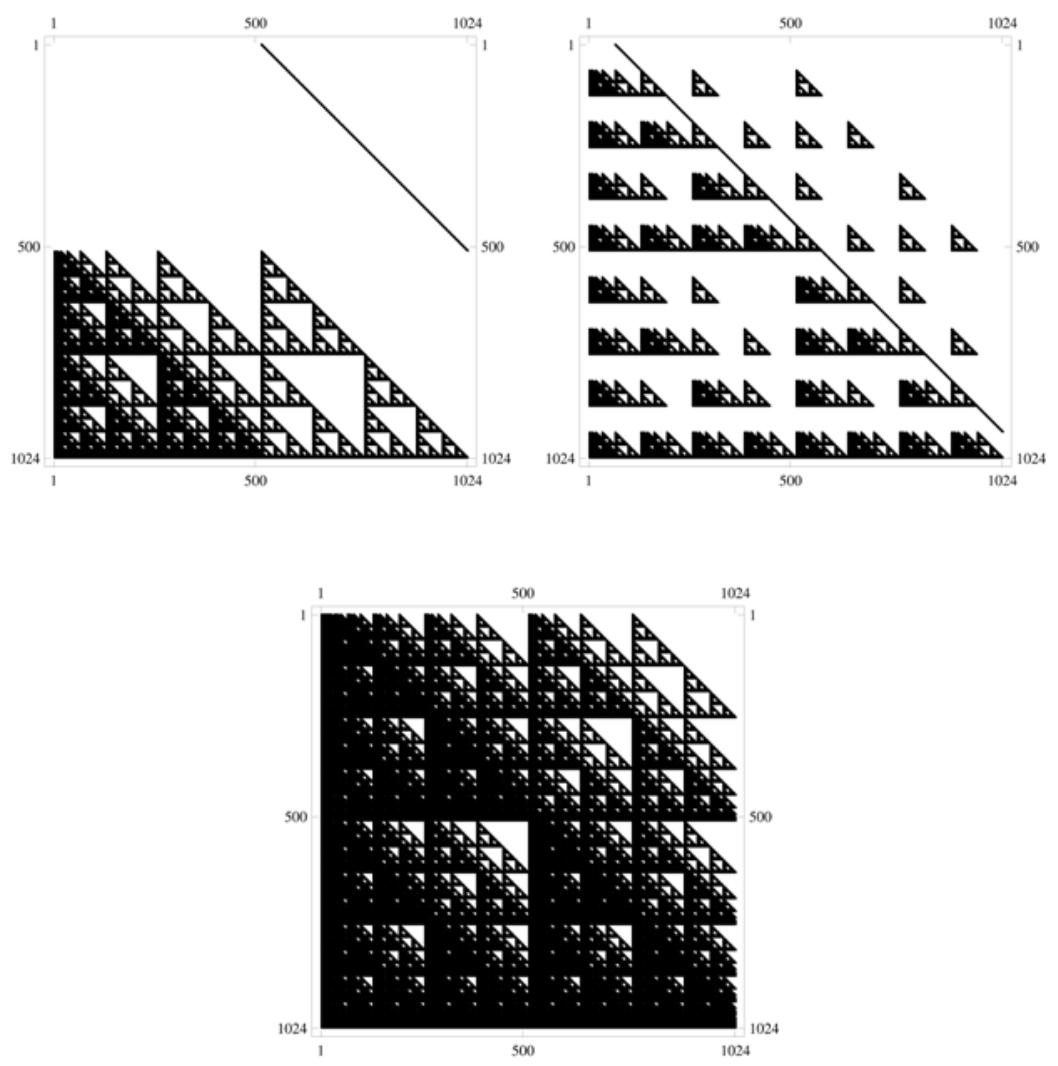

Figure 9.3: Sparsity representation of the matrices $A_{x_{1}}^{T}$ (9.4\% filled), $A_{x_{2}}^{T}(9.4 \%$ filled $)$ and $A_{V_{H}}^{T}(52.5 \%$ filled $)$

To compute only the smallest non-zero real eigenvalue/eigenvector of the operator $A_{V_{H}}^{T}$ using an $n$ D-systems approach, the iterative eigenvalue solvers JDQR, JDQZ and Eigs are used. These solvers are introduced and discussed in Chapter 6. Because the condition numbers of the operators involved are very large, a similar balancing technique is used as described in [27], and employed before in Section 7.1.

The smallest real non-zero eigenvalue computed matrix-free using the operator, is equal to the eigenvalue computed before, with the only exception that the computation only takes about 200 seconds and the memory requirements are minimal 
because the matrix is never constructed explicitly. The memory requirements for this matrix-free approach stay below $100 \mathrm{MB}$. Therefore, one may conclude that using an iterative eigenvalue solver in combination with an $n \mathrm{D}$-system is the most efficient approach to compute the smallest real eigenvalue of such a sparse matrix $A_{V_{H}}^{T}$.

In practice, the algebraic techniques presented here may be combined with numerical local search techniques to arrive at practical algorithms, for instance to save on hardware requirements, computation time, or to deal with numerical accuracy issues. How to deal with numerical ill-conditioning is an important research topic when one wants to solve problems that are of a somewhat larger size. Such implementation issues are still under investigation. 



\section{Chapter 10}

\section{$H_{2}$ Model-order reduction from order $\mathrm{N}$ to $\mathrm{N}-2$}

When reducing the model-order of a given system $H(s)$ from order $N$ to $N-2$, hence $k=2$, the degree of the polynomial $\rho(s)$ in Equation (8.20) is smaller than or equal to one:

$$
\rho(s)=1+\rho_{1} s \not \equiv 0 .
$$

Using this polynomial, (8.44) yields the following system of equations for the co-order $k=2$ case:

$$
\left(\begin{array}{c}
\frac{1+\rho_{1} \delta_{1}}{e\left(\delta_{1}\right)} x_{1}^{2} \\
\frac{1+\rho_{1} \delta_{2}}{e\left(\delta_{2}\right)} x_{2}^{2} \\
\vdots \\
\frac{1+\rho_{1} \delta_{N}}{e\left(\delta_{N}\right)} x_{N}^{2}
\end{array}\right)-M\left(\delta_{1}, \ldots, \delta_{N}\right)\left(\begin{array}{c}
x_{1} \\
x_{2} \\
\vdots \\
x_{N}
\end{array}\right)=\left(\begin{array}{c}
0 \\
0 \\
\vdots \\
0
\end{array}\right)
$$

where $M\left(\delta_{1}, \ldots, \delta_{N}\right)=V\left(-\delta_{1}, \ldots,-\delta_{N}\right) V\left(\delta_{1}, \ldots, \delta_{N}\right)^{-1}$. This system of equations is parameterized rationally by the parameter $\rho_{1}$.

As stated by Equation (8.45), there is one additional constraint on the coefficients of $\tilde{a}(s)$ involved in the co-order $k=2$ case. This constraint is defined by:

$$
\tilde{a}_{N-1}\left(x_{1}, x_{2}, \ldots, x_{N}\right)=\gamma_{1,1} x_{1}+\gamma_{1,2} x_{2}+\ldots+\gamma_{1, N} x_{N}=0 .
$$

Note that the quantities $\left.\gamma_{1,1}, \ldots, \gamma_{1, N}\right)$ in this constraint depend on the poles $\delta_{1}, \ldots, \delta_{N}$ and that it does not contain the parameter $\rho_{1}$. Note furthermore that the system of equations (10.2) yields $N$ equations in the quantities $x_{1}, \ldots, x_{N}$, and the parameter $\rho_{1}$. Together with the additional constraint $\tilde{a}_{N-1}\left(x_{1}, x_{2}, \ldots, x_{N}\right)=0$, there are in total $N+1$ equations in the $N+1$ unknowns.

To solve the $H_{2}$ model-order reduction problem for the co-order $k=2$ case, we need to compute the solutions $\left(x_{1}, x_{2}, \ldots, x_{N}, \rho_{1}\right)$ of the system of equations $(10.2)$ 
which satisfy the linear constraint in (10.3).

Till now we have treated the general case where $q_{0} \neq 0$ in (8.20). If $q_{0}=0$ we end up in a slightly different situation which can be treated as follows. If $q_{0}=0$, then the polynomial $q(s)$ in (8.19) equals $q_{1} s$. This allows one to rewrite Equation (8.18) into the form:

$$
e(s) \tilde{a}(-s)-q_{1} b(s) d(s)=\tilde{a}(s)^{2} s
$$

in which $\tilde{a}(s):=q_{1} a(-s)$ is again a polynomial of degree $N-2$ having all its zeros in the open right-half plane. Along similar lines it now follows that plugging in the values of the distinct zeros $\delta_{1}, \ldots, \delta_{N}$ of $d(s)$ and reparameterizing in terms of the quantities $x_{i}:=\tilde{a}\left(\delta_{i}\right)$, one arrives at a system of equations of the form:

$$
\left(\begin{array}{c}
\frac{\delta_{1}}{e\left(\delta_{1}\right)} x_{1}^{2} \\
\frac{\delta_{2}}{e\left(\delta_{2}\right)} x_{2}^{2} \\
\vdots \\
\frac{\delta_{N}}{e\left(\delta_{N}\right)} x_{N}^{2}
\end{array}\right)-M\left(\delta_{1}, \ldots, \delta_{N}\right)\left(\begin{array}{c}
x_{1} \\
x_{2} \\
\vdots \\
x_{N}
\end{array}\right)=\left(\begin{array}{c}
0 \\
0 \\
\vdots \\
0
\end{array}\right)
$$

with the matrix $M\left(\delta_{1}, \ldots, \delta_{N}\right)$ as before. Note that no additional parameter shows up in this system of equations. The problem of solving this system of equations can be cast into the form of an eigenvalue problem along similar lines as the approach for the co-order $k=1$ case of the previous chapter. That approach results in $2^{N}$ solutions. Since there are $N$ equations and an additional constraint in $N$ unknowns, it will often happen that none of the solutions will satisfy the additional constraint $\tilde{a}_{N-1}=0$, in which case no feasible solutions occur.

Combining the outcomes for the two situations $q_{0} \neq 0$ and $q_{0}=0$, we conclude that all the stationary points of the $\mathrm{H}_{2}$ model-order reduction criterion can be computed.

The idea now is to cast (10.2) and (10.3) into one single polynomial eigenvalue problem using the Stetter-Möller method. The solutions $\left(x_{1}, x_{2}, \ldots, x_{N}, \rho_{1}\right)$ of this eigenvalue problem are solutions of the system of equations (10.2) which also satisfy the constraint (10.3). This is the topic of Section 10.1. To solve such a polynomial eigenvalue problem a linearization method is applied in Section 10.2 such that it becomes equivalent with a linear but singular generalized eigenvalue problem. Because in general the eigenvalues of such a singular eigenvalue problem can not be computed directly, we have to compute the Kronecker canonical form of the singular pencil, as described in Section 10.3, to split off the unwanted eigenvalues. Section 10.4 describes how to compute a feasible approximation of order $N-2$ using the solutions obtained from the generalized eigenvalue problem. To put together all the techniques mentioned in this chapter, we give a single algorithm for the globally optimal $\mathrm{H}_{2}$ model-order reduction for the co-order $k=2$ case in Section 10.5. This algorithm is used in the three examples described in Section 10.6. 


\subsection{Solving the system of quadratic equations}

For the co-order $k=2$ case, a single parameter $\rho_{1}$ shows up in the system of equations (10.2). We want to use the Stetter-Möller matrix method here to solve this system of equations and we consider the linear operator which performs multiplication by a polynomial $r\left(x_{1}, \ldots, x_{N}\right)$ within the quotient space $\mathbb{C}\left(\rho_{1}\right)\left[x_{1}, x_{2}, \ldots, x_{N}\right] / I\left(\rho_{1}\right)$. Here the coefficients are from the field of complex rational functions in $\rho_{1}$ and $I\left(\rho_{1}\right)$ denotes the associated ideal generated by the polynomials in the system of equations (10.2), regarded to be rationally parameterized by $\rho_{1}$.

Multiplication by any polynomial $r\left(x_{1}, \ldots, x_{N}\right)$ within this quotient space is represented by the linear operator $\mathcal{A}_{r\left(x_{1}, \ldots, x_{N}\right)}$. With respect to an identical monomial basis as in (8.47), this linear operator is represented by the $2^{N} \times 2^{N}$ matrix $A_{r\left(x_{1}, \ldots, x_{N}\right)}\left(\rho_{1}\right)^{T}$ which depends rationally on $\rho_{1}$. The $2^{N}$ eigenvalues of this matrix are the values of the polynomial $r\left(x_{1}, \ldots, x_{N}\right)$ at the solutions of the system of equations (10.2).

A particularly interesting choice for $r\left(x_{1}, \ldots, x_{N}\right)$ is now provided by the polynomial $\tilde{a}_{N-1}$ in (10.3) to incorporate the requirement that any feasible solution of (10.2) satisfies $\tilde{a}_{N-1}=0$ :

$$
r\left(x_{1}, \ldots, x_{N}\right)=\tilde{a}_{N-1} .
$$

This gives rise to the linear operator $\mathcal{A}_{\tilde{a}_{N-1}}$ which is represented by the $2^{N} \times 2^{N}$ matrix $A_{\tilde{a}_{N-1}}\left(\rho_{1}\right)^{T}$. The eigenvalues of this matrix coincide with the values of the polynomial $\tilde{a}_{N-1}$ at the solutions of the system of equations (10.2). Because we require $\tilde{a}_{N-1}=0$, the eigenvalue $\lambda$ in $A_{\tilde{a}_{N-1}}\left(\rho_{1}\right)^{T} v=\lambda v$ is required to be zero too. Thus, we examine the remaining problem $A_{\tilde{a}_{N-1}}\left(\rho_{1}\right)^{T} v=0$, which can be regarded as a rational eigenvalue problem in the unknown $\rho_{1}$. Thus, we are looking for non-trivial solutions of:

$$
A_{\tilde{a}_{N-1}}\left(\rho_{1}\right)^{T} v=0 .
$$

The (real) values of $\rho_{1}$ that make the matrix $A_{\tilde{a}_{N-1}}\left(\rho_{1}\right)^{T}$ singular, are of importance now. For such $\rho_{1}$ any associated eigenvector $v$ in its kernel allows for the computation of a solution $\left(x_{1}, x_{2}, \ldots, x_{N}\right)$ by reading them off from the eigenvector which exhibits the Stetter structure.

The matrix $A_{\tilde{a}_{N-1}}\left(\rho_{1}\right)^{T}$ is rational in $\rho_{1}$ but can be made polynomial in $\rho_{1}$ by premultiplication of each row by the least common denominator (in terms of $\rho_{1}$ ) of the rational entries in $\rho_{1}$ in that row. This yields a polynomial eigenvalue problem. This premultiplication does not change the eigenvalues $\rho_{1}$ of the matrix. However, spurious eigenvalues/solutions can be introduced for values of $\rho_{1}$ where $\rho\left(\delta_{i}\right)=1+\rho_{1} \delta_{i}=0$ for some $i$. To avoid the occurrence of spurious solutions as much as possible, it is necessary not to multiply the rows of $A_{\tilde{a}_{N-1}}\left(\rho_{1}\right)^{T}$ by any polynomial factor which does not remove a pole for $\rho_{1}$. The following result specifies a convenient choice of multiplication factors to achieve this goal. 
Theorem 10.1. The least common denominator of the rational entries in $\rho_{1}$ in the row of the matrix $A_{\tilde{a}_{N-1}}\left(\rho_{1}\right)^{T}$ corresponding to the coefficients of the normal form of a product $x_{1}^{\alpha_{1}} x_{2}^{\alpha_{2}} \cdots x_{N}^{\alpha_{N}} \tilde{a}_{N-1}\left(x_{1}, x_{2}, \ldots, x_{N}\right)$ with $\alpha_{1}, \alpha_{2}, \ldots, \alpha_{N} \in\{0,1\}$, is generically given by:

$$
\rho\left(\delta_{1}\right)^{\alpha_{1}} \rho\left(\delta_{2}\right)^{\alpha_{2}} \cdots \rho\left(\delta_{N}\right)^{\alpha_{N}}
$$

Proof. Note that $\tilde{a}_{N-1}\left(x_{1}, x_{2}, \ldots, x_{N}\right)=\gamma_{1,1} x_{1}+\gamma_{1,2} x_{2}+\ldots+\gamma_{1, N} x_{N}$ is a homogeneous polynomial of total degree 1 . When multiplied by a monomial $x_{1}^{\alpha_{1}} x_{2}^{\alpha_{2}}$ $\cdots x_{N}^{\alpha_{N}}$ an expression of $N$ terms results, all of total degree $|\alpha|+1$. For each term it holds that at most one of the variables $x_{i}$ is of degree 2. Each reduction step, in which (for some index $i$ ) a quantity $x_{i}^{2}$ is replaced by the expression $\frac{e\left(\delta_{i}\right)}{\rho\left(\delta_{i}\right)}\left(m_{i, 1} x_{1}+m_{i, 2} x_{2}+\cdots+m_{i, N} x_{N}\right)$, produces new terms of lower degree. These terms are either reduced (this happens for the term involving $m_{i, i} x_{i}$ and for all the terms $m_{i, j} x_{j}$ with $j$ such that $\alpha_{j}=0$ ) or they contain another squared variable (this happens for the terms $m_{i, j} x_{j}$ with $j$ such that $\alpha_{j}=1$; then $x_{j}^{2}$ occurs). In the latter case, the variable $x_{i}$ is not present in such a term. Hence, any new term produced from it by a later reduction step with reintroduces the variable $x_{i}$, is then reduced. We conclude that a second replacement of the quantity $x_{i}^{2}$ does not occur: the reduction algorithm produces reduced terms by replacing quantities $x_{i}^{2}$ for each index $i$ at most once per term.

The variables $x_{i}$ for which the index $i$ satisfies $\alpha_{i}=0$ initially do not show up in the monomial. Therefore, by the same argument, the term generated from it do not require replacement of a quantity $x_{i}^{2}$ anywhere in the reduction process. In this way it follows that the reduction process for the product of $\tilde{a}_{N-1}$ and the monomial $x_{1}^{\alpha_{1}} x_{2}^{\alpha_{2}} \cdots x_{N}^{\alpha_{N}}$ involves replacements of the quantities $x_{i}^{2}$ for precisely those indices $\alpha_{i}=1$. This makes clear that the least common denominator of the entries in the corresponding row of $A_{\tilde{a}_{N-1}}\left(\rho_{1}\right)^{T}$ is always contained in the product $\rho\left(\delta_{1}\right)^{\alpha_{1}} \rho\left(\delta_{2}\right)^{\alpha_{2}} \cdots \rho\left(\delta_{N}\right)^{\alpha_{N}}$. It is easily verified by considering an example that generically this least common denominator is in fact equal to this expression.

Using the result of this theorem the rational matrix $A_{\tilde{a}_{N-1}}\left(\rho_{1}\right)^{T}$ is made polynomial in $\rho_{1}$ as follows:

$$
\tilde{A}_{\tilde{a}_{N-1}}\left(\rho_{1}\right)^{T}=
$$

$$
\left(\begin{array}{ccc}
\rho\left(\delta_{1}\right)^{\alpha_{1,1}} \cdots \rho\left(\delta_{N}\right)^{\alpha_{1, N}} & & \\
& \ddots & \\
& & \rho\left(\delta_{1}\right)^{\alpha_{2^{N}, 1} \cdots \rho\left(\delta_{N}\right)^{\alpha_{2^{N}, N}}}
\end{array}\right) A_{\tilde{a}_{N-1}}\left(\rho_{1}\right)^{T}
$$

where the $2^{N} \times 2^{N}$ matrix $\tilde{A}_{\tilde{a}_{N-1}}\left(\rho_{1}\right)^{T}$ is polynomial in $\rho_{1}$. Note that this implies that the coefficients in the rows of $\tilde{A}_{\tilde{a}_{N-1}}\left(\rho_{1}\right)^{T}$ correspond to the coefficients of the normal form of the expressions $\rho\left(\delta_{1}\right)^{\alpha_{1}} \rho\left(\delta_{2}\right)^{\alpha_{2}} \cdots \rho\left(\delta_{N}\right)^{\alpha_{N}} x_{1}^{\alpha_{1}} x_{2}^{\alpha_{2}} \cdots x_{N}^{\alpha_{N}}$ $\tilde{a}_{N-1}\left(x_{1}, x_{2}, \ldots, x_{N}\right)$. 
The values of $\rho_{1}$ that make the polynomial matrix $\tilde{A}_{\tilde{a}_{N-1}}\left(\rho_{1}\right)^{T}$ singular are called the polynomial eigenvalues of $\tilde{A}_{\tilde{a}_{N-1}}\left(\rho_{1}\right)^{T}$. Thus, the problem is transformed into the following polynomial eigenvalue problem:

$$
\tilde{A}_{\tilde{a}_{N-1}}\left(\rho_{1}\right)^{T} v=0
$$

where $\rho_{1}$ is the unknown parameter and $v$ the eigenvector.

As a corollary on the degree of the parameter $\rho_{1}$ in the involved matrix we have:

Corollary 10.2. The polynomial degree of the matrix $\tilde{A}_{\tilde{a}_{N-1}}\left(\rho_{1}\right)^{T}$ with respect to $\rho_{1}$ is equal to $N-1$.

Proof. When carrying out multiplication of a row of $A_{\tilde{a}_{N-1}}\left(\rho_{1}\right)^{T}$ by the associated polynomial factor $\rho\left(\delta_{1}\right)^{\alpha_{1}} \rho\left(\delta_{2}\right)^{\alpha_{2}} \cdots \rho\left(\delta_{N}\right)^{\alpha_{N}}$, the polynomial degree may increase to at most $N$. The latter may potentially only occur when all the $\alpha_{i}$ are equal to 1 ; i.e., when addressing the row of $A_{\tilde{a}_{N-1}}\left(\rho_{1}\right)^{T}$ containing the coefficients of the normal form of $\tilde{a}_{N-1}\left(x_{1}, x_{2}, \ldots, x_{N}\right) x_{1} x_{2} \cdots x_{N}$. However, in that case all the $N$ terms initially need reduction, so that all the terms have at least one factor $\rho\left(\delta_{i}\right)$ in their denominator. Multiplication by $\rho\left(\delta_{1}\right) \rho\left(\delta_{2}\right) \cdots \rho\left(\delta_{N}\right)$ therefore leads to some cancelation of factors for each term in the normal form, so that the resulting polynomial degree is at most $N-1$.

It is easily verified that the degree $N-1$ does in fact occur for the polynomial coefficient of $x_{1} x_{2} \cdot x_{N}$ in the normal form of each of the quantities $\rho\left(\delta_{1}\right) \cdots \rho\left(\delta_{i-1}\right)$ $\rho\left(\delta_{i+1}\right) \cdots \rho\left(\delta_{N}\right) \tilde{a}_{N-1}\left(x_{1}, x_{2}, \ldots, x_{N}\right) x_{1} \cdots x_{i-1} x_{i+1} \cdots x_{N}$. To see this, note that no reduction step for each term needs to be considered, because the total degree drops below $N$ as soon as a reduction step is applied. Therefore, the coefficient of $x_{1} x_{2} \cdot x_{N}$ in the normal form equals $\rho\left(\delta_{1}\right) \cdots \rho\left(\delta_{i-1}\right) \rho\left(\delta_{i+1}\right) \cdots \rho\left(\delta_{N}\right) \gamma_{i, i}$. Since $\gamma_{1, i}=\frac{1}{d^{\prime}\left(\delta_{i}\right)} \neq 0$ and since the expressions $\rho\left(\delta_{j}\right)=1+\delta_{j} \rho_{1}$ are of degree 1 ( $\delta_{j}$ is in $\Pi^{-}$, hence non-zero), the degree $N-1$ is indeed achieved.

To summarize: to find all the solutions of the system of equations (10.2), one needs to compute: (i) all the eigenvalues $\rho_{1}$ such that the polynomial matrix $\tilde{A}_{\tilde{a}_{N-1}}\left(\rho_{1}\right)^{T}$ becomes singular, and (ii) all the corresponding eigenvectors $v$, for which $\tilde{A}_{\tilde{a}_{N-1}}\left(\rho_{1}\right)^{T} v=$ 0 , from which to read off the values of $x_{1}, \ldots, x_{N}$. The $N$-tuples of values $\left(x_{1}, \ldots, x_{N}\right)$, thus obtained, together with the corresponding polynomial eigenvalues $\rho_{1}$, constitute all the solutions of the quadratic system of equations (10.2) which satisfy the constraint $\tilde{a}_{N-1}\left(x_{1}, \ldots, x_{N}\right)=0$ in $(10.3)$.

In the next section we show an approach to solve the polynomial eigenvalue problem $\tilde{A}_{\tilde{a}_{N-1}}\left(\rho_{1}\right)^{T} v=0$ in (10.10) by linearizing it into a generalized eigenvalue problem in order to obtain the eigenvalues $\rho_{1}$ and the eigenvectors $v$. 


\subsection{Linearizing a polynomial eigenvalue problem}

Polynomial eigenvalue problems have been well studied in the literature and numerical algorithms for its solution are available [55]. These problems occur for example in structural mechanics, molecular dynamics, gyroscopic systems and constrained leastsquares problems (see, e.g., [11] and [72]). Usually the problem is first linearized with respect to $\rho_{1}$, yielding a generalized eigenvalue problem with an associated matrix pencil. This linearization is the subject of this section.

The standard tool for the numerical solution of a generalized eigenvalue problem is the QZ-algorithm, which however is notoriously ill-conditioned if the matrix pencil has a singular part [42]. To remove any singular part that may be present, we may proceed algebraically as in the algorithm for the computation of the Kronecker canonical form of a matrix pencil (see [34] and [42]). This can be implemented with exact and symbolic arithmetic when required. Once the singular part and the associated 'indeterminate' eigenvalues are removed from the matrix pencil, the square regular part remains for which the (finite and infinite) eigenvalues can subsequently be computed by standard means. Computing the Kronecker canonical form of a singular matrix pencil and deflating the pencil to get rid of the unwanted eigenvalues, is the subject of Section 10.3.

First we introduce the generalized eigenvalue problem and some of its features. Let $B$ and $C$ be two $m_{1} \times m_{2}$ matrices with complex-valued entries. The associated matrix pencil is the one-parameter family of matrices $B+\rho_{1} C$, where $\rho_{1}$ ranges over all complex values. The generalized eigenvalue problem for $B+\rho_{1} C$ consists of finding values for $\rho_{1}$ and associated non-zero vectors $w \in \mathbb{C}^{m_{2}}$ such that:

$$
\left(B+\rho_{1} C\right) w=0 .
$$

Note that the conventional eigenvalue problem for a given square $m_{2} \times m_{2}$ matrix $A$ occurs when choosing $m_{1}=m_{2}, B=A$ and $C=-I_{m_{2}}$, producing the associated equation $\left(A-\rho_{1} I_{m_{2}}\right) w=0$. Because $B$ and $C$ in general are allowed to be rectangular matrices rather than square matrices, the generalized eigenvalue problem may exhibit a few features that do not occur for conventional eigenvalue problems. Another issue that may occur in the square case $m_{1}=m_{2}$, is that the matrix $C$ may be singular, so that the generalized eigenvalue problem can not be transformed directly into an equivalent conventional eigenvalue problem upon premultiplication by $C^{-1}$.

Obviously, a solution to the generalized eigenvalue problem $\left(B+\rho_{1} C\right) w=0$ requires a value of $\rho_{1}$, which makes $B+\rho_{1} C$ into an $m_{1} \times m_{2}$ matrix having a set of linearly dependent columns. When $m_{1}<m_{2}$, any value for $\rho_{1}$ will do, resulting in indeterminate eigenvalues. More precisely, indeterminate eigenvalues occur when the column rank of $B+\rho_{1} C$ is less than $m_{2}$ for all values of $\rho_{1}$. A zero eigenvalue occurs when $B w=0$ admits non-trivial solutions for $w$. This requires the column rank of $B$ to be less than $m_{2}$. An infinite eigenvalue refers to a situation in which the column rank of $C$ is less than $m_{2}$. Note that upon division by $\rho_{1}$, we may deal with the equation $\left(\frac{1}{\rho_{1}} B+C\right) w=0$ and $\rho_{1} \rightarrow \infty$ refers to $C w=0$. Instead of considering the (right) 
generalized eigenvalue problem $\left(B+\rho_{1} C\right) w=0$ one may also consider the left generalized eigenvalue problem $u^{*}\left(B+\rho_{1} C\right)=0^{T}$. Thus, linear dependence of the rows of $B+\rho_{1} C$ for various values of $\rho_{1}$ (including infinity) can be given similar meaning too.

As demonstrated in the previous section, the problem of finding solutions of the system of equations (10.2) subject to (10.3) can be cast in the form of a polynomial eigenvalue problem involving the square polynomial matrix $\tilde{A}_{\tilde{a}_{N-1}}\left(\rho_{1}\right)^{T}$. The problem of computing all the values $\rho_{1}$ and corresponding non-trivial vectors $v$ that satisfy $\tilde{A}_{\tilde{a}_{N-1}}\left(\rho_{1}\right)^{T} v=0$ can be approached by rewriting it as a generalized eigenvalue problem as follows.

First, expand the polynomial matrix $\tilde{A}_{\tilde{a}_{N-1}}\left(\rho_{1}\right)^{T}$ as a matrix polynomial in $\rho_{1}$ of degree $N-1$, by:

$$
\tilde{A}_{\tilde{a}_{N-1}}\left(\rho_{1}\right)^{T}=A_{0}+\rho_{1} A_{1}+\ldots+\rho_{1}^{N-1} A_{N-1} .
$$

Applying a linearization technique as mentioned, e.g., in [55], [56] or [72], makes the polynomial eigenvalue problem (10.10), where the involved matrix is written as (10.12), equivalent to a generalized eigenvalue problem:

$$
\left(A_{0}+\rho_{1} A_{1}+\ldots+\rho_{1}^{N-1} A_{N-1}\right) v=0 \quad \Longleftrightarrow \quad\left(B+\rho_{1} C\right) \tilde{v}=0
$$

where $\rho_{1}$ occurs linearly and where the matrices $B$ and $C$ are (large) block-partitioned square matrices with the following structure:

$$
\begin{gathered}
B=\left(\begin{array}{cccc}
0 & I & & 0 \\
\vdots & & \ddots & \\
0 & 0 & & I \\
A_{0} & A_{1} & \ldots & A_{N-2}
\end{array}\right), \\
C=\left(\begin{array}{cccc}
-I & & 0 & 0 \\
& \ddots & & \vdots \\
0 & & -I & 0 \\
0 & \ldots & 0 & A_{N-1}
\end{array}\right) .
\end{gathered}
$$

The eigenvectors $\tilde{v}$ of the generalized eigenvalue problem $\left(B+\rho_{1} C\right) \tilde{v}=0$ in $(10.13)$ have a built-in structure, which is given by:

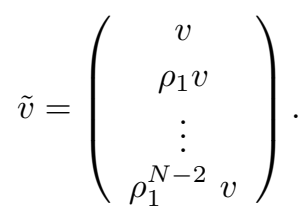

The block vectors $v$ inside such an eigenvector $\tilde{v}$ are eigenvectors of the original polynomial eigenvalue problem (10.10). Note that the eigenvectors $\tilde{v}$ have additional 
structure, because the vectors $v$ are structured too as being Stetter vectors (with a structure corresponding to the chosen monomial basis).

The matrices $B$ and $C$ in this set-up have dimensions $(N-1) 2^{N} \times(N-1) 2^{N}$. This gives rise to $(N-1) 2^{N}$ generalized eigenvalues. Because spurious eigenvalues can be introduced by making the matrix $A_{\tilde{a}_{N-1}}\left(\rho_{1}\right)^{T}$ polynomial in $\rho_{1}$, one has to check which eigenvalues (which can be numerical values, indeterminate or infinite) are eigenvalues of the problem (10.10) too. Recall that the roots $-1 / \delta_{i}$ for all $i=1, \ldots, N$ of the terms $\left(1+\rho_{1} \delta_{1}\right),\left(1+\rho_{1} \delta_{2}\right), \ldots,\left(1+\rho_{1} \delta_{N}\right)$ are the quantities that may be introduced as spurious eigenvalues when constructing the polynomial matrix $\tilde{A}_{\tilde{a}_{N-1}}\left(\rho_{1}\right)^{T}$.

The equivalence between the polynomial eigenvalue problem and the generalized eigenvalue problem, is expressed by the fact that if one has computed a solution of the generalized eigenvalue problem (10.13), then this yields a solution of the polynomial eigenvalue problem (10.10) too, and vice versa. Once the eigenvalues $\rho_{1}$ and eigenvectors $\tilde{v}$ of generalized eigenvalue problem (10.13) are found and the appropriate ones have been selected, the values $x_{1}, \ldots, x_{N}$ can be read off from the vectors $\tilde{v}$. This provides a method to find all the solutions of the polynomial eigenvalue problem (10.10) and, therefore, to find all the solutions of the system of equations (10.2) that satisfy the constraint $\tilde{a}_{N-1}=0$ in (10.3).

\subsection{The Kronecker canonical form of a matrix pencil}

A matrix pencil $B+\rho_{1} C$ of dimensions $m \times n$ is regular when the involved matrices are square $(m=n)$ and the determinant is not identically zero. When a pencil is singular, then $m \neq n$, or $m=n$ and the determinant of the matrix is $\equiv 0$. Therefore, when a pencil is rectangular it is singular by definition.

The matrix pencil $B+\rho_{1} C$ of size $(N-1) 2^{N} \times(N-1) 2^{N}$ in (10.13) of the previous section will in general be singular, although it involves square matrices. Singular generalized eigenvalue problems may have indeterminate, infinite, zero and non-zero finite eigenvalues and are well-known to be numerically highly ill-conditioned. The eigenvalues which cause this singularity of the eigenvalue problem are unwanted as they do not have any meaning for our $\mathrm{H}_{2}$ model-order reduction problem. A solution here is to decompose, using exact arithmetic, the matrix pencil $B+\rho_{1} C$ into a singular part, which corresponds to indeterminate eigenvalues, and a regular part, which corresponds to infinite and finite eigenvalues, and to split off the unwanted eigenvalues. This can be achieved by using the computation of the Kronecker canonical form (see [6], [34] and [42]).

In the conventional square case, the matrix pencil $A-\rho_{1} I_{n}$ can be conveniently characterized by means of its Jordan canonical form. The sizes of the Jordan blocks for the various eigenvalues of the matrix $A$ provide geometrical information on the associated eigenspaces, both for right eigenvectors and for left eigenvectors. The Kronecker canonical form is a generalization of the Jordan canonical form to the possible rectangular and singular case, and applies to arbitrary $m \times n$ matrix pencils $B+\rho_{1} C$. 
By computing such a decomposition into the Kronecker canonical form it is possible to split off the unwanted eigenvalues of the generalized eigenvalue problem.

In the procedure to compute the Kronecker Canonical form of a pencil $B+\rho_{1} C$ of dimension $m \times n$, one transforms this pencil into another convenient pencil $D+\rho_{1} E$ with an additional block structure, as stated in the theorem below.

Theorem 10.3. Let $B+\rho_{1} C$ be an $m \times n$ complex-valued matrix pencil. Then there exists an $m \times m$ invertible matrix $W$ and an $n \times n$ invertible matrix $V$ for which the matrix pencil $D+\rho_{1} E$ obtained as:

$$
W^{-1}\left(B+\rho_{1} C\right) V=D+\rho_{1} E
$$

attains the form:

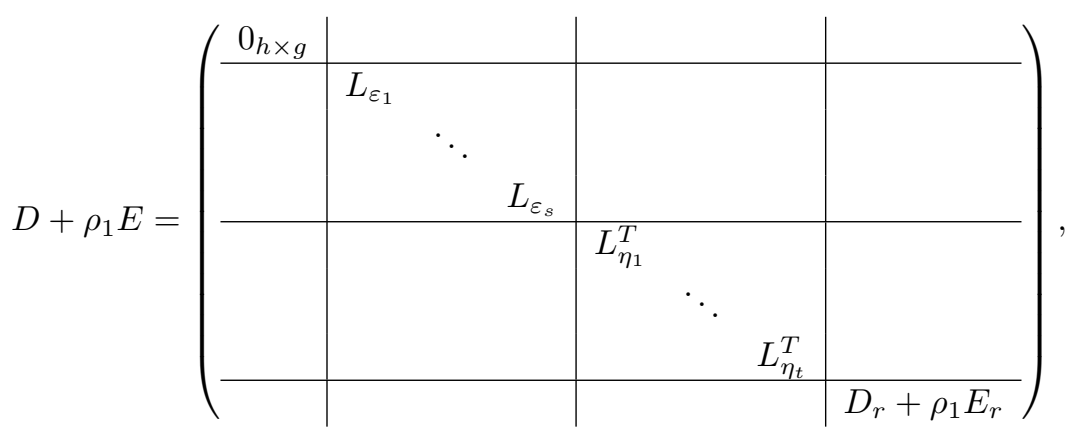

where the blocks $0_{h \times g}, L_{\varepsilon_{1}}, \ldots, L_{\varepsilon_{s}}$ and $L_{\eta_{1}}^{T}, \ldots, L_{\eta_{t}}^{T}$ correspond to indeterminate eigenvalues. It holds that $g=\operatorname{dim}\left(\operatorname{ker}\left(\begin{array}{c}B \\ C\end{array}\right)\right), h=\operatorname{dim}\left(\operatorname{ker}\left(\begin{array}{c}B^{T} \\ C^{T}\end{array}\right)\right), 0<\varepsilon_{1} \leq$ $\cdots \leq \varepsilon_{s}$ and $0<\eta_{1} \leq \cdots \leq \eta_{t}$. Furthermore, $D_{r}+\rho_{1} E_{r}$ is a square $n_{r} \times n_{r}$ regular matrix pencil, i.e., $\operatorname{det}\left(D_{r}+\rho_{1} E_{r}\right)$ does not vanish identically.

Any block of the form $L_{\varepsilon}$ is given by:

$$
L_{\varepsilon}=\left(\begin{array}{ccccc}
\rho_{1} & 1 & 0 & \ldots & 0 \\
0 & \rho_{1} & 1 & & \vdots \\
\vdots & \vdots & \ddots & \ddots & \vdots \\
0 & 0 & \ldots & \rho_{1} & 1
\end{array}\right) \text { of dimension } \varepsilon \times(\varepsilon+1)
$$

and $L_{\eta}^{T}$ is given by:

$$
L_{\eta}^{T}=\left(\begin{array}{cccc}
\rho_{1} & 0 & \ldots & 0 \\
1 & \rho_{1} & & \vdots \\
0 & 1 & \ddots & 0 \\
\vdots & & \ddots & \rho_{1} \\
0 & \ldots & 0 & 1
\end{array}\right) \text { of dimension }(\eta+1) \times \eta
$$


For any $W$ and $V$ which bring $B+\rho_{1} C$ into such a form, the dimensions $h, g, \varepsilon_{1}$, $\ldots, \varepsilon_{s}$ (when ordered), $\eta_{1}, \ldots, \eta_{t}$ (when ordered) and $n_{r}$ are uniquely determined by the matrices $B+\rho_{1} C$ and so is the Jordan form of the regular part $D_{r}+\rho_{1} E_{r}$. This Jordan form is given by:

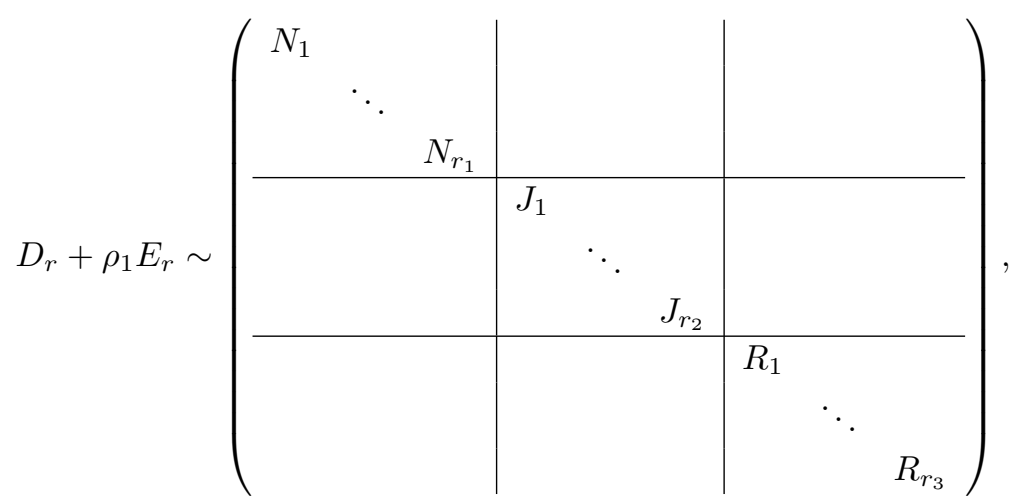

with:

$$
\begin{aligned}
& N_{j}=\left(\begin{array}{cccc}
1 & \rho_{1} & & 0 \\
& \ddots & \ddots & \\
& & & \rho_{1} \\
0 & & & 1
\end{array}\right) \text { of dimension } k_{j} \times k_{j}\left(1 \leq k_{1} \leq \cdots \leq k_{r_{1}}\right), \\
& J_{j}=\left(\begin{array}{cccc}
\rho_{1} & 1 & & 0 \\
& \ddots & \ddots & \\
& & & 1 \\
0 & & & \rho_{1}
\end{array}\right) \text { of dimension } l_{j} \times l_{j}\left(1 \leq l_{1} \leq \cdots \leq l_{r_{2}}\right),
\end{aligned}
$$

and

$$
R_{j}=\left(\begin{array}{cccc}
\rho_{1}-\alpha_{j} & 1 & & 0 \\
& \ddots & \ddots & \\
& & & 1 \\
0 & & & \rho_{1}-\alpha_{j}
\end{array}\right) \text { of dimension } m_{j} \times m_{j}\left(1 \leq m_{1} \leq \cdots \leq m_{r_{3}}\right) \text {. }
$$


Example 10.1. As an illustrative example, the Kronecker canonical form of a pencil $B+\rho_{1} C$ with $h=2, g=1, \varepsilon_{1}=2, \varepsilon_{2}=3, \eta_{1}=1$ and $\eta_{2}=2$ is shown below:

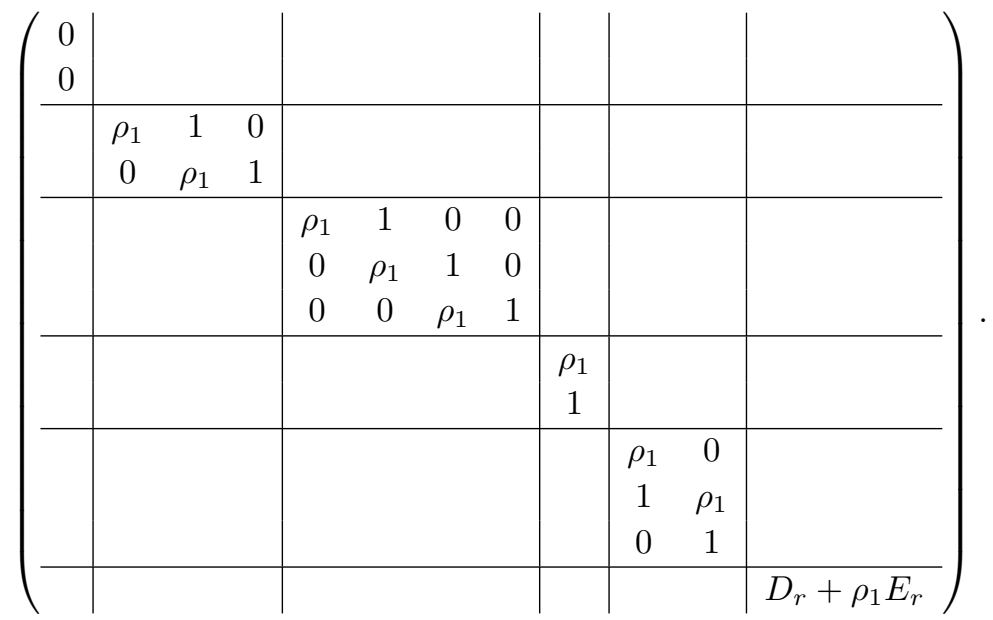

The empty blocks correspond to zero blocks of appropriate dimensions. The pencil $D_{r}+\rho_{1} E_{r}$ is the regular part of the pencil $B+\rho_{1} C$, which can also be brought into a canonical form as in (10.20).

Matrix pencils $B+\rho_{1} C$ and $D+\rho_{1} E$, of dimension $m \times n$, related by Equation (10.16), where $W$ and $V$ are square non-singular matrices independent of $\rho_{1}$, are called strictly equivalent. The eigenvector $\tilde{v}$ in the eigenvalue problem $\left(B+\rho_{1} C\right) \tilde{v}=0$ is transformed conformably to $w=V^{-1} \tilde{v}$ such that the following equation holds:

$$
W^{-1}\left(B+\rho_{1} C\right) V\left(V^{-1} \tilde{v}\right)=\left(D+\rho_{1} E\right) w=0 .
$$

As shown in Theorem 10.3, the Kronecker Canonical form allows for a decomposition of a singular matrix pencil into a regular and a singular part. The singular part relates to all the so-called indeterminate eigenvalues. The regular part is what we are interested in since it relates to infinite and finite eigenvalues. For our purposes, the indeterminate, the infinite and the zero eigenvalues will not yield feasible solutions for our model-order reduction problem and should therefore all be excluded. The associated Jordan block structure for these eigenvalues can be obtained with exact arithmetic (if one starts from an exact matrix pencil $B+\rho_{1} C$ ). In this way it is possible to arrive algebraically at a smaller sized exact regular matrix pencil in which both matrices are invertible. Its eigenvalues can then be computed reliably by standard numerical methods.

In the next subsections the decomposition of a singular matrix pencil into a singular and a regular part and the deflation of a matrix pencil is discussed. Actually, the parts corresponding to indeterminate, zero and infinite eigenvalues are split off using exact arithmetic and the finite eigenvalues of the remaining (regular) part are computed using numerical arithmetic. 
Although this procedure is not new in the literature (see, e.g., [34] and [42])), we go through the details here. The reason for this is that a generalized version of this procedure will be exploited for the co-order $k=3$ case in Chapter 11 to solve a polynomial eigenvalue problem in two variables.

\subsubsection{The singular part of a pencil}

Consider a singular matrix pencil $B+\rho_{1} C$ of dimension $m \times n$. We denote by $r$ the (algebraic) rank of the pencil, i.e., the largest of the orders of minors that do not vanish identically. Because of the singularity it follows that at least one of the inequalities $r<n$ and $r<m$ holds. Assume for now that $r<n$.

Then, there may exist a dependency between the rows and/or the columns of the matrix pencil with constant coefficients. If $g$ and $h$ are the maximal number of constant independent solutions of the equations:

$$
\left(B+\rho_{1} C\right) z=0 \quad \text { and } \quad\left(B^{T}+\rho_{1} C^{T}\right) \hat{z}=0,
$$

respectively, then the first $g$ columns and the first $h$ rows of $\left(B+\rho_{1} C\right)$ can be transformed into zeros. This yields the zero block $0_{h \times g}$ of dimension $h \times g$ in (10.17). If this block $0_{h \times g}$ is split off, the remaining pencil contains no longer any linear dependence with constant coefficients among the rows or the columns.

Furthermore, a linear dependency between the columns of $B+\rho_{1} C$ may exist, such that:

$$
\left(B+\rho_{1} C\right) z\left(\rho_{1}\right)=0
$$

has a non-zero solution $z$ depending polynomially on $\rho_{1}$ : $z\left(\rho_{1}\right)$. Every non-zero solution $z\left(\rho_{1}\right)$ determines some dependence among the columns of this matrix pencil. We restrict ourselves to solutions $z\left(\rho_{1}\right)$ of least possible degree $\varepsilon$ for $\rho_{1}$ :

$$
z\left(\rho_{1}\right)=z_{0}-\rho_{1} z_{1}+\rho_{1}^{2} z_{2}-\ldots+(-1)^{\varepsilon} \rho_{1}^{\varepsilon} z_{\varepsilon}
$$

When we substitute this solution $z\left(\rho_{1}\right)$ into the original problem (10.27), this yields:

$$
\begin{array}{ccc}
\left(B+\rho_{1} C\right)\left(z_{0}-\rho_{1} z_{1}+\rho_{1}^{2} z_{2}-\ldots+(-1)^{\varepsilon} \rho_{1}^{\varepsilon} z_{\varepsilon}\right)= \\
1 & \cdot\left(+B z_{0}\right) & + \\
\rho_{1} & \cdot\left(-B z_{1}+C z_{0}\right) & + \\
\rho_{1}^{2} & \cdot\left(+B z_{2}-C z_{1}\right) & + \\
\vdots & \vdots & \vdots \\
\rho_{1}^{\varepsilon} & \cdot\left((-1)^{\varepsilon} B z_{\varepsilon}+(-1)^{\varepsilon-1} C z_{\varepsilon-1}\right) & + \\
\rho_{1}^{\varepsilon+1} & \cdot\left((-1)^{\varepsilon} C z_{\varepsilon}\right) & =0 .
\end{array}
$$


Working out the relationships for the coefficients of the powers of $\rho_{1}$ in (10.29), yields the following in matrix-vector format:

$$
\left(\begin{array}{ccccc}
B & 0 & \ldots & \ldots & 0 \\
C & B & & & \vdots \\
0 & C & \ddots & & \vdots \\
0 & 0 & \ddots & \ddots & \vdots \\
\vdots & \vdots & & \ddots & B \\
0 & 0 & \ldots & \ldots & C
\end{array}\right)\left(\begin{array}{c} 
\\
z_{0} \\
-z_{1} \\
z_{2} \\
\vdots \\
(-1)^{\varepsilon} z_{\varepsilon}
\end{array}\right)=M_{\varepsilon}\left(\begin{array}{c}
z_{0} \\
-z_{1} \\
z_{2} \\
\vdots \\
(-1)^{\varepsilon} z_{\varepsilon}
\end{array}\right)=0
$$

where the block matrix $M_{\varepsilon}$ has dimensions $(\varepsilon+2) m \times(\varepsilon+1) n$ and has rank < $(\varepsilon+1) n$. Note that any non-zero vector in the kernel of the matrix $M_{\varepsilon}$ in $(10.30)$ yields a corresponding solution $z\left(\rho_{1}\right)$ of degree $\varepsilon$ in (10.27). Note furthermore that the matrices $M_{\varepsilon}$ only depend on the known matrices $B$ and $C$.

Now let the (algebraic) rank of a matrix $M_{i}$ be denoted by $r_{i}=\operatorname{rank}\left(M_{i}\right)$. Because $\varepsilon$ was chosen as the least possible degree of $z\left(\rho_{1}\right)$ for which a solution exists, it holds for the matrices:

$$
M_{0}=\left(\begin{array}{l}
B \\
C
\end{array}\right), M_{1}=\left(\begin{array}{cc}
B & 0 \\
C & B \\
0 & C
\end{array}\right), \ldots, M_{\varepsilon-1}=\left(\begin{array}{cccc}
B & 0 & \ldots & 0 \\
C & B & & \vdots \\
0 & C & \ddots & \vdots \\
\vdots & \vdots & \ddots & B \\
0 & 0 & \ldots & C
\end{array}\right),
$$

that $r_{0}=n, r_{1}=2 n, \ldots, r_{\varepsilon-1}=\varepsilon n$.

Theorem 4 in Chapter 12 of [42] shows that if (10.27) has a solution $z\left(\rho_{1}\right)$ of minimal degree $\varepsilon$ and $\varepsilon>0$, then $\left(B+\rho_{1} C\right)$ is strictly equivalent to:

$$
\left(\begin{array}{cc}
L_{\varepsilon} & 0 \\
0 & D+\rho_{1} E
\end{array}\right)
$$

where the submatrix $L_{\varepsilon}$ of dimension $\varepsilon \times(\varepsilon+1)$ exhibits the structure given in (10.18). Furthermore, it holds that $\left(D+\rho_{1} E\right) z\left(\rho_{1}\right)=0$ has no solution $z\left(\rho_{1}\right)$ of degree less then $\varepsilon$.

The eigenvalue problem $\left(B+\rho_{1} C\right) \tilde{v}=0$ is transformed using transformation matrices $V$ and $W$ as follows:

$$
W^{-1}\left(B+\rho_{1} C\right) V\left(V^{-1} \tilde{v}\right)=\left(\begin{array}{cc}
L_{\varepsilon} & 0 \\
0 & D+\rho_{1} E
\end{array}\right) w=\left(\begin{array}{l}
0 \\
0
\end{array}\right) .
$$


By partitioning the new eigenvector $w$ as $w=\left(\begin{array}{c}w_{1} \\ w_{2}\end{array}\right)$, we get the following two equations:

$$
\begin{cases}L_{\varepsilon} w_{1} & =0 \\ \left(D+\rho_{1} E\right) w_{2} & =0\end{cases}
$$

Note that also holds: $\tilde{v}=V\left(\begin{array}{c}w_{1} \\ w_{2}\end{array}\right)$. The first equation in (10.34) yields:

$$
\left\{\begin{array}{cccc}
\rho_{1} w_{1,1} & + & w_{1,2} & =0 \\
\rho_{1} w_{1,2} & + & w_{1,3} & =0 \\
& \vdots & & \\
\rho_{1} w_{1, \varepsilon}+ & w_{1, \varepsilon+1} & =0
\end{array}\right.
$$

When the first element $w_{1,1}$ of the vector $w_{1}$ is chosen equal to one, then the only solution of $(10.35)$ has the structure $\left(1,-\rho_{1}, \rho_{1}^{2}, \ldots,\left(-\rho_{1}\right)^{\varepsilon}\right)^{T}$. Therefore, any choice of $\rho_{1}$ provides a non-trivial eigenvector $w_{1}$ depending on $\rho_{1}$ : it generates an eigenvector $z$ for $B+\rho_{1} C$ through $z=V\left(\begin{array}{l}w_{1} \\ w_{2}\end{array}\right)$, by choosing $w_{2}=0$, or by choosing a solution pair $\left(\rho_{1}, w_{2}\right)$ to the second equation $\left(D+\rho_{1} E\right) w_{2}=0$ and fixing $\rho_{1}$ to the same value to generate $w_{1}$. Therefore, this eigenvalue is recorded as an indeterminate eigenvalue with multiplicity $\varepsilon$.

In [42] it is shown how to obtain the quasi block diagonal form (10.32) by choosing appropriate transformation matrices $W$ and $V$. Consider the pencil $B+\rho_{1} C$ as a family of operators mapping $\mathbb{C}^{n}$ to $\mathbb{C}^{m}$. With a suitable choice of bases the matrix $B+\rho_{1} C$ takes up the form of (10.32). Such a suitable choice corresponds to taking the vectors $z_{0}, \ldots, z_{\varepsilon}$, involved in a solution $z\left(\rho_{1}\right)$ in Equation (10.27), as part of the basis for $\mathbb{C}^{n}$. Subsequently, the vectors $B z_{1}, \ldots, B z_{\varepsilon}$ are taken as part of the basis for $\mathbb{C}^{m}$. This is allowed since $\left\{z_{0}, \ldots, z_{\varepsilon}\right\}$ and $\left\{B z_{1}, \ldots, B z_{\varepsilon}\right\}$ are linearly independent sets (see [42]). Choosing the vectors $z_{0}, \ldots, z_{\varepsilon}$ and $B z_{1}, \ldots, B z_{\varepsilon}$ as the first columns for the new bases in $\mathbb{C}^{n}$ and $\mathbb{C}^{m}$, respectively, yields transformation matrices $V$ and 
$W$ as follows:

$$
\begin{aligned}
& W=\left(\begin{array}{l|l|l|l|l|l} 
& & & & & \\
B z_{1} & \ldots & B z_{\varepsilon} & p_{\varepsilon+1} & \ldots & p_{m} \\
& & & & &
\end{array}\right), \\
& V=\left(\begin{array}{c|c|c|c|c|c} 
& & & & & \\
z_{0} & \ldots & z_{\varepsilon} & q_{\varepsilon+1} & \ldots & q_{n-1} \\
& & & & &
\end{array}\right)
\end{aligned}
$$

where $p_{\varepsilon+1}, \ldots, p_{m}$ and $q_{\varepsilon+1}, \ldots, q_{n-1}$ are basis column vectors to complete the matrices up to square and invertible matrices.

Then it can easily be shown by using the relationships in (10.30) (i.e., $B z_{0}=0$, $B z_{i+1}=C z_{i}$, for $i=0, \ldots, \varepsilon-1$, and $\left.(-1)^{\varepsilon} C z_{\varepsilon}=0\right)$, that the pencil $W^{-1}\left(B+\rho_{1} C\right) V$ is equivalent with:

$$
\left(\begin{array}{ccccc|c}
0 & 1 & 0 & \ldots & 0 \\
0 & 0 & 1 & \ldots & 0 & * \\
\vdots & \vdots & & \ddots & & \\
0 & 0 & 0 & \ldots & 1 & \\
\hline 0 & 0 & 0 & \ldots & 0 & \\
\vdots & \vdots & \vdots & & \vdots & * \\
0 & 0 & 0 & \ldots & 0
\end{array}\right)+\rho_{1}\left(\begin{array}{ccccc|c}
1 & 0 & \ldots & 0 & 0 & \\
0 & 1 & \ldots & 0 & 0 & * \\
\vdots & \vdots & \ddots & & \vdots & \\
0 & 0 & \ldots & 1 & 0 & \\
\hline 0 & 0 & \ldots & 0 & 0 & \\
\vdots & \vdots & & \vdots & \vdots & * \\
0 & 0 & \ldots & 0 & 0 & *
\end{array}\right)
$$

Now we conclude that this pencil has its upper left block equal to $L_{\varepsilon}$ and its lower left block equal to 0, similar as in Equation (10.32).

Remark 10.1. The upper right block of the transformed matrix pencil (10.37) does not equal a zero block as in (10.32) at this point. To make also this block zero, one more transformation is required. We do not address this transformation here and assume the upper right block of the pencil (10.37) to be zero without performing this transformation. This is possible because the quasi diagonal blocks in Equation (10.32) are not affected by this transformation. Moreover, when we continue splitting off singular parts, we split off the upper right (non-zero) block, the bottom left (zero) block and the structured block $L_{\varepsilon}$ to get rid of the unwanted eigenvalue(s). For details see the third part of the proof of Theorem 4 in [42]. In fact, this amounts to choosing $p_{\varepsilon+1}, \ldots, p_{m}$ and $q_{\varepsilon+1}, \ldots, q_{n-1}$ in a special way. 
In the latter we have shown how to transform a pencil $B+\rho_{1} C$ into a form $B_{1}+\rho_{1} C_{1}$ containing one block $L_{\varepsilon_{1}}$, once a solution $z\left(\rho_{1}\right)$ for $\left(B+\rho_{1} C\right) z\left(\rho_{1}\right)=0$ of minimal degree $\varepsilon_{1}$ for $\rho_{1}$ is available. Subsequently, if $B_{1}+\rho_{1} C_{1}$ has a nonzero solution of minimal degree $\varepsilon_{2}$, where $\varepsilon_{2} \geq \varepsilon_{1}$, then it is possible to do one more transformation. Repeating until no more solutions $z\left(\rho_{1}\right)$ are found, leads to a transformed pencil:

$$
\left(\begin{array}{c|cccc|c}
0_{h \times g} & & & & \\
\hline & L_{\varepsilon_{1}} & & & & \\
& & L_{\varepsilon_{2}} & & & \\
& & & \ddots & & \\
& & & & L_{\varepsilon_{s}} & \\
\hline & & & & & D_{s}+\rho_{1} E_{s}
\end{array}\right)
$$

with $0<\varepsilon_{1} \leq \varepsilon_{2} \leq \ldots \leq \varepsilon_{s}$. Then the block $D_{s}+\rho_{1} E_{s}$ has full column rank and, thus, all its columns are linearly independent.

Remark 10.2. Suppose one has computed more than one non-zero solution $z\left(\rho_{1}\right)$ satisfying Equation (10.27), which, e.g., may happen when the kernel of the matrix $M_{\varepsilon}$ contains more than one non-zero vector. Let us denote two of these vectors by $\tilde{z}\left(\rho_{1}\right)$ and $\hat{z}\left(\rho_{1}\right)$. Then the transformation $W^{-1}\left(B+\rho_{1} C\right) V$ can be performed using the vectors $\tilde{z}\left(\rho_{1}\right)$ and $\hat{z}\left(\rho_{1}\right)$ simultaneously to speed up the computation. Then the matrices $W$ and $V$ are structured as in (10.36) but contain as first columns $B \tilde{z}_{1}, \ldots, B \tilde{z}_{\varepsilon}, B \hat{z}_{1}, \ldots, B \hat{z}_{\varepsilon}$, and $\tilde{z}_{0}, \ldots, \tilde{z}_{\varepsilon}, \hat{z}_{0}, \ldots, \hat{z}_{\varepsilon}$, respectively. Note that this simultaneous transformation is only possible if all the columns in $W$ and $V$ are linearly independent. If this is the case, this transformation results in an equivalent pencil structured as:

$$
\left(\begin{array}{cc|c}
L_{\varepsilon} & 0 & \\
0 & L_{\varepsilon} & \\
\hline & &
\end{array}\right),
$$

where both the submatrices $L_{\varepsilon}$ exhibit the structure as in (10.18).

When (10.38) has been achieved with $D_{s}+\rho_{1} E_{s}$ of full column rank, one can restart this procedure with the transposed pencil:

$$
D_{s}^{T}+\rho_{1} E_{s}^{T}
$$

to make all the rows of the pencil linearly independent and bring it into the form (10.17) with $0<\eta_{1} \leq \eta_{2} \leq \ldots \leq \eta_{t}$, and $0<\varepsilon_{1} \leq \varepsilon_{2} \leq \ldots \leq \varepsilon_{s}$. The blocks $L_{\eta}^{T}$ are of dimensions $(\eta+1) \times \eta$ and are structured as in (10.19). 
Note that the corresponding equations of $L_{\eta}^{T} w_{1}=0$ are:

$$
\left\{\begin{array}{rll} 
& \rho_{1} w_{1,1} & =0 \\
w_{1,1}+ & \rho_{1} w_{1,2} & =0 \\
\vdots & & \\
w_{1, \varepsilon-1}+\rho_{1} w_{1, \varepsilon} & =0 \\
w_{1, \varepsilon} & & =0
\end{array}\right.
$$

It is easy to see from (10.41) that only the trivial solution $w_{1}=0$ exists. The value of $\rho_{1}$ does not play a role in (10.41). The blocks $L_{\eta}^{T}$ are said to generate indeterminate eigenvalues $\rho_{1}$ too.

The quantities $\varepsilon_{i}$ and $\eta_{i}$ are called the minimal indices for, respectively, the columns and the rows of a pencil. One property of strictly equivalent pencils is that they have exactly the same minimal indices.

During the transformation process, some special situations can occur: (i) if $\varepsilon_{1}+$ $\varepsilon_{2}+\ldots+\varepsilon_{s}=m$, then the dimension of the pencil $D_{s}+\rho_{1} E_{s}$ is $0 \times 0$, (ii) if $r=n$, then there are no dependent columns and therefore the blocks $L_{\varepsilon}$ will be absent, (iii) if $r=m$, then there are no dependent rows and therefore the blocks $L_{\eta}^{T}$ will be absent.

Finally the pencil $B+\rho_{1} C$ is transformed into a quasi block diagonal matrix as in (10.17) containing a regular (hence square) block $D_{r}+\rho_{1} E_{r}$.

\subsubsection{The regular part of a pencil}

A regular pencil $D_{r}+\rho_{1} E_{r}$ is square, say of dimension $n_{r} \times n_{r}$, such that its determinant does not vanish identically. In general, if a pencil is regular and the characteristic polynomial of degree $k \leq n_{r}$ is equal to $p_{k}\left(\rho_{1}\right)=\operatorname{det}\left(D_{r}+\rho_{1} E_{r}\right)$, then the pencil has $k$ finite eigenvalues (counting multiplicities) which are the zeros of $p_{k}\left(\rho_{1}\right)$ and $n_{r}-k$ infinite eigenvalues.

The regular part $D_{r}+\rho_{1} E_{r}$ in $(10.17)$ can be transformed into a Jordan canonical form as in (10.20), containing the Jordan blocks $N_{1}, \ldots, N_{r_{1}}, J_{1}, \ldots, J_{r_{2}}$ and $R_{1}, \ldots, R_{r_{3}}$ (see [34] and [42]). These blocks correspond with infinite, zero and nonzero finite eigenvalues, respectively. The sizes of the blocks $N_{i}, J_{i}$, and $R_{i}$, the associated structure and the eigenvalues are all uniquely determined by the matrices $B$ and $C$ (but the order in which the blocks appear may vary).

In the application to $\mathrm{H}_{2}$ model-order reduction we do not compute all these regular blocks. Once the pencil is brought into the form (10.17), we remove the infinite and zero eigenvalues of the regular part by exact arithmetic and proceed with the remaining regular pencil, containing only finite non-zero eigenvalues, using conventional numerical methods. We will show in this subsection the canonical form of a regular matrix pencil, containing the Jordan blocks of infinite, zero and non-zero finite eigenvalues. 
Formally, when the regular pencil $D_{r}+\rho_{1} E_{r}$ in (10.17) attains the canonical form (10.20), then the Kronecker canonical form of the pencil $B+\rho_{1} C$ is obtained.

The Jordan blocks $N_{j}$ in (10.20) are square blocks of possibly different dimensions $k_{j} \times k_{j}$ with a structure as in (10.21). The equations resulting from $N_{j} w_{1}=0$, where $w_{1}$ is the corresponding part of the eigenvector $w$, are:

$$
\left\{\begin{array}{llll}
w_{1,1} & + & \rho_{1} w_{1,2} & =0 \\
w_{1,2} & + & \rho_{1} w_{1,3} & =0 \\
& \vdots & & \\
w_{1, k_{j}-1}+\rho_{1} w_{1, k_{j}} & =0 \\
w_{1, k_{j}} & & & =0
\end{array}\right.
$$

These equations only admit the trivial solution $w_{1}=0$. Therefore, $\rho_{1}=\infty$ does not play a role in our problem. The blocks $N_{j}$ are said to generate infinite eigenvalues. The number of infinite eigenvalues amounts to the sum of the associated dimensions $k_{j}$ for these blocks.

The Jordan blocks $J_{j}$ in (10.20) are square blocks of possibly different dimensions $l_{j} \times l_{j}$ with a structure as in (10.22). The corresponding equations following from $J_{j} w_{1}=0$, are:

$$
\left\{\begin{array}{llll}
\rho_{1} w_{1,1} & + & w_{1,2} & =0 \\
\rho_{1} w_{1,2} & + & w_{1,3} & =0 \\
& \vdots & & \\
\rho_{1} w_{1, l_{j}-1}+w_{1, l_{j}} & =0 \\
\rho_{1} w_{1, l_{j}} & & & =0
\end{array}\right.
$$

In this case there are two possible situations: if $\rho_{1} \neq 0$, then $J_{j} w_{1}$ only admits the trivial solution. If $\rho_{1}=0$ it admits the non-trivial solution $w_{1}=(1,0, \ldots, 0)^{T}$, or any vector proportional to it. This block corresponds to a zero eigenvalue with algebraic multiplicity $l_{j}$.

The Jordan blocks corresponding to non-zero finite eigenvalues are denoted by the $R_{j}$ blocks of dimension $m_{j} \times m_{j}$ in Equation (10.20). They exhibit the structure as in (10.23). The finite eigenvalues have the values $\rho_{1}=\alpha_{j}$ with multiplicity equal to the sum of the associated dimensions $m_{j}$, for each value of $\alpha_{j}$. The corresponding equations resulting from $R_{j} w_{1}=0$ are:

$$
\left\{\begin{array}{lll}
\left(\rho_{1}-\alpha_{j}\right) w_{1,1} & +w_{1,2}=0 \\
\left(\rho_{1}-\alpha_{j}\right) w_{1,2} & +w_{1,3}=0 \\
& \vdots & \\
\left(\rho_{1}-\alpha_{j}\right) w_{1, m_{j}-1}+w_{1, m_{j}} & =0 \\
\left(\rho_{1}-\alpha_{j}\right) w_{1, m_{j}} & & =0
\end{array}\right.
$$


They only admit the trivial solution $w_{1}=0$, if $\rho_{1} \neq \alpha_{j}$, and for $\rho_{1}=\alpha_{j}$ we get non-trivial solutions in the span of $(1,0, \ldots, 0)^{T}$.

\subsubsection{Deflation of a singular pencil}

As shown in the previous two subsections, the singular pencil $B+\rho_{1} C$ in (10.13) can be transformed into the Kronecker canonical form. For the application at hand it is sufficient to split off the singular part. If desired, infinite and zero eigenvalues of the regular part of the pencil may also be removed by exact arithmetic as they do not have any meaning for this application either.

Then the remaining pencil is regular and only contains non-zero finite eigenvalues. Moreover, the pencil is generally much better conditioned than the original pencil. Let us denote the regular pencil $D_{r}+\rho_{1} E_{r}$, with its infinite and zero eigenvalues removed, as the pencil $F-\rho_{1} G$. Then the matrices $F$ and $G$ are square and invertible matrices and therefore this generalized eigenvalue problem $\left(F-\rho_{1} G\right) w=0$ can be treated as a conventional eigenvalue problem by looking at $\left(G^{-1} F-\rho_{1} I\right) w=0$. The matrix involved here is a smaller, but still exact, matrix $G^{-1} F$ containing only non-zero finite eigenvalues which can be computed in a reliable way by numerical methods. The eigenvalues $\rho_{1}$ and corresponding eigenvectors of the matrix $G^{-1} F$, lead to solutions $\left(\rho_{1}, x_{1}, \ldots, x_{N}\right)$ of the system of quadratic equations (10.2) which satisfy the additional constraint (10.3).

\subsection{Computing the approximation $G(s)$}

Let $x_{1}, \ldots, x_{N}$ and $\rho_{1}$ be a solution of the system of equations (10.2), which satisfies the additional constraint $\tilde{a}_{N-1}=0$ in (10.3). Using this solution an approximation $G(s)$ of order $N-2$ can be computed as described in Theorem 8.3 for $k=2$.

From the approximations obtained with the approach given in Theorem 8.3 for $k=2$, it is straightforward to select those that are feasible, i.e., which give rise to real stable approximations $G(s)$ of order $N-2$. It is then possible to select the globally optimal approximation by computing the $H_{2}$-norm $V_{H}$ of the difference $H(s)-G(s)$ for every feasible solution $G(s)$ and selecting the one for which this criterion value is minimal.

The third order polynomial, given in Theorem 8.2, which represents the $H_{2}$ criterion of $H(s)-G(s)$, attains for the co-order $k=2$ case the form:

$$
\begin{aligned}
V_{H}\left(x_{1}, x_{2}, \ldots, x_{N}, \rho_{1}\right) & =\left\|\frac{e(s)}{d(s)}-\frac{b(s)}{a(s)}\right\|_{H_{2}}^{2} \\
& =\sum_{i=1}^{N} \frac{\left(1+\rho_{1} \delta_{i}\right)^{2}\left(1-\rho_{1} \delta_{i}\right)}{e\left(\delta_{i}\right) d^{\prime}\left(\delta_{i}\right) d\left(-\delta_{i}\right)} x_{i}^{3} .
\end{aligned}
$$


Remark 10.3. A disadvantage of this approach, from the perspective of computational efficiency, is that in this way one can only decide about global optimality of a solution when all the solutions of the polynomial eigenvalue problem have been computed and further analyzed.

In the co-order one case, the third order polynomial $V_{H}\left(x_{1}, x_{2}, \ldots, x_{N}\right)$ coincides at the solutions $\left(x_{1}, \ldots, x_{N}\right)$ of the system of equations with the $H_{2}$-criterion. In that case, the third order polynomial offers an additional computational advantage, as seen before in Chapter 9: if $V_{H}\left(x_{1}, x_{2}, \ldots, x_{N}\right)$ denotes the third order polynomial, then the globally optimal approximation of order $N-1$ can be computed by computing the smallest real eigenvalue of the matrix $A_{V_{H}}^{T}$ which avoids the computation of all the eigenvalues of the matrix $A_{V_{H}}^{T}$. This is achieved by using iterative eigenproblem solvers. In the co-order $k=2$ case the situation is more difficult: the eigenvalues of the matrix $\tilde{A}_{\tilde{a}_{N-1}}\left(\rho_{1}\right)^{T}$ do not correspond to $H_{2}$-criterion values directly.

Currently it is investigated whether the third order polynomial specified in Equation (10.42) can still be employed by iterative polynomial eigenproblem solvers to limit the number of eigenvalues and solutions that require computation. Possibly, one could modify the available Jacobi-Davidson methods for a polynomial eigenvalue problem, see [61], [62], in such a way that it iterates with the matrix $\tilde{A}_{\tilde{a}_{N-1}}\left(\rho_{1}\right)^{T}$ but targets on small positive real values of the polynomial criterion function $V_{H}\left(x_{1}, x_{2}, \ldots, x_{N}, \rho_{1}\right)$ first (in the same spirit as the JDCOMM method developed in Section 6.5 for conventional eigenvalue problems with commuting matrices). This approach is currently under investigation.

\subsection{Algorithm for $H_{2}$ model-order reduction for the co-order $k=2$ case}

For implementation purposes, the techniques discussed in the previous sections are collected into a single algorithm to perform globally optimal $\mathrm{H}_{2}$ model-order reduction for the co-order $k=2$ case.

1. For the given transfer function $H(s)$ of order $N$ to be reduced, construct the system of quadratic equations (10.2).

2. Construct the matrix $A_{\tilde{a}_{N-1}}\left(\rho_{1}\right)^{T}$ by applying the Stetter-Möller matrix method using the ideal $I\left(\rho_{1}\right)$ generated by the polynomials in (10.2). This matrix is rational in $\rho_{1}$. Construct the polynomial matrix $\tilde{A}_{\tilde{a}_{N-1}}\left(\rho_{1}\right)^{T}$ by multiplication of the rows with suitable scalar expressions as given by Theorem 10.1.

3. Use a linearization technique which transforms the polynomial eigenvalue problem $\tilde{A}_{\tilde{a}_{N-1}}\left(\rho_{1}\right)^{T} v=0$ into the generalized eigenvalue problem $\left(B+\rho_{1} C\right) z=0$.

4. Partly compute the Kronecker canonical form of the matrix pencil $\left(B+\rho_{1} C\right)$ and deflate it to the regular pencil $\left(F+\rho_{1} G\right)$ by splitting off the blocks corresponding to the indeterminate, infinite and zero eigenvalues. 
5. Compute the non-zero finite eigenvalues $\rho_{1}$ and the corresponding eigenvectors $w$ of the regular generalized eigenvalue problem $\left(F+\rho_{1} G\right) w=0$.

6. Determine the corresponding solutions $\left(x_{1}, \ldots, x_{N}\right)$ by computing solutions $v$ to the polynomial eigenvalue problem $\tilde{A}_{\tilde{a}_{N-1}}\left(\rho_{1}\right)^{T} v=0$ and by reading off the values of $x_{1}, \ldots, x_{N}$ from the entries of the eigenvectors $v$ at the specified positions. These solutions $\left(x_{1}, \ldots, x_{N}\right)$ are constructed to satisfy the constraint $\tilde{a}_{N-1}=0$ in (10.3).

7. Extend the already computed solution set (for $q_{0} \neq 0$ ) with any solutions to (10.5), for $q_{0}=0$, which also satisfy the linear constraint (10.3).

8. Compute all the corresponding $H_{2}$-criterion values, i.e., by substituting $\left(x_{1}\right.$, $\left.\ldots, x_{N}\right)$ and the corresponding $\rho_{1}$ into the third order polynomial specified in Equation (10.42).

9. Select the feasible solution $\left(\hat{x}_{1}, \ldots, \hat{x}_{N}\right)$ and $\hat{\rho}_{1}$ that produces the smallest (positive) real criterion value.

10. Use the selected solution $\left(\hat{x}_{1}, \ldots, \hat{x}_{N}\right)$ and $\hat{\rho}_{1}$ to compute the real stable polynomial $\tilde{a}(s)$ from Equation (8.27). This is a polynomial of order $N-2$ since the coefficient $\tilde{a}_{N-1}$ is zero. Furthermore, read off $q_{0}$ from the coefficient $\tilde{a}_{N-2}$ as $q_{0}=(-1)^{N-2} \tilde{a}_{N-2}$.

11. Compute $a(s)$ from $\tilde{a}(s)$ and $q_{0}$.

12. Compute $b(s)$ using Equation (8.49).

13. The globally optimal approximation $G(s)$ of order $N-2$ is given by $G(s)=$ $b(s) / a(s)$.

\subsection{Examples}

In this section three worked examples are presented to illustrate the co-order $k=2$ techniques discussed in this chapter.

In Subsection 10.6.1 a system of order 6 is reduced to order 4. The approximation of order 4 is computed by applying the co-order $k=1$ technique of Chapter 9 twice and the co-order $k=2$ technique of the present chapter once. The co-order $k=2$ technique is applied here without computing the Kronecker canonical form. Instead, a balancing method is used to improve the ill-conditioning of the singular matrix pencil. The results for both approaches are very similar.

Subsection 10.6.2 describes the reduction of a system of order 7 to a globally optimal $\mathrm{H}_{2}$ approximation of order 5. Computing the Kronecker canonical form is necessary in this example, because otherwise all the eigenvalues of the singular matrix pencil are incorrectly specified to be 0 , indeterminate or infinite by all the numerical methods we have available, due to ill-conditioning of the singular pencil. 
Subsection 10.6.3 describes an example of a globally optimal $H_{2}$ model-order reduction of order 4 to order 2. The co-order $k=1$ and $k=2$ techniques are applied again and for this example the results turn out to be quite different. The co-order $k=2$ technique turns out to exhibit the best performance in terms of the $H_{2^{-}}$ approximation criterion in comparison with the co-order $k=1$ technique.

\subsubsection{Example 1}

The transfer function $H(s)$ of a continuous time system studied in this example is of order 6 . We shall compare two different ways to arrive at an approximation $G(s)$ of order 4: (i) by applying the co-order two technique once, and (ii) by applying the co-order one technique, described in Chapter 9, twice consecutively.

The transfer function $H(s)$ is given in an exact format by:

$$
H(s)=\frac{-48-184 s+216 s^{2}-124 s^{3}+16 s^{4}+24 s^{5}}{\frac{445}{2916}+\frac{27383}{21870} s+\frac{30001}{7290} s^{2}+\frac{3503}{486} s^{3}+\frac{11773}{1620} s^{4}+\frac{182}{45} s^{5}+s^{6}} .
$$

The poles of $H(s)$ are $\delta_{1}=-\frac{8}{9}-\frac{5}{9} i, \delta_{2}=-\frac{8}{9}+\frac{5}{9} i, \delta_{3}=-\frac{2}{3}-\frac{1}{2} i, \delta_{4}=-\frac{2}{3}+\frac{1}{2} i$, $\delta_{5}=-\frac{3}{5}, \delta_{6}=-\frac{1}{3}$, which are all in the open left-half plane $\Pi^{-}$. The zeros of $H(s)$ are located at $-3.27927,-0.205398,0.666667-1.24722 i, 0.666667+1.24722 i$ and 1.48466. The impulse response and the Bode diagrams of this system are shown as plots in blue in Figures 10.5 and 10.6.

(i) We first apply the co-order two technique and construct the corresponding system of quadratic equations (10.2). This system has 6 equations that each involve the variables $x_{1}, x_{2}, x_{3}, x_{4}, x_{5}, x_{6}$, and the parameter $\rho_{1}$. To use the Stetter-Möller matrix method, a quotient space $\mathbb{C}\left(\rho_{1}\right)\left[x_{1}, \ldots, x_{6}\right] / I\left(\rho_{1}\right)$ is needed with an appropriate basis. The dimension of this quotient space $\mathbb{C}\left(\rho_{1}\right)\left[x_{1}, \ldots, x_{6}\right] / I\left(\rho_{1}\right)$ is $2^{6}=64$. The basis $B$ contains 64 elements: $\left(1, x_{1}, x_{2}, x_{1} x_{2}, x_{3}, x_{1} x_{3}, x_{2} x_{3}, x_{1} x_{2} x_{3}, \ldots, x_{1} x_{2} x_{3} x_{4} x_{5} x_{6}\right)$. The linear constraint $\tilde{a}_{N-1}\left(x_{1}, x_{2}, x_{3}, x_{4}, x_{5}, x_{6}\right)=0$, is computed using the elements of the last row of the inverted Vandermonde matrix as defined in (8.45). It attains the following exact form:

$$
\begin{aligned}
\tilde{a}_{N-1}= & \left(\frac{31709313}{12721865}+\frac{88691598}{12721865} i\right) x_{1} & +\left(\frac{31709313}{12721865}-\frac{88691598}{12721865} i\right) x_{2} & + \\
& -\left(\frac{18895680}{1467661}+\frac{5190480}{1467661} i\right) x_{3} & -\left(\frac{18895680}{1467661}-\frac{5190480}{1467661} i\right) x_{4} & + \\
& \frac{6834375}{181826} x_{5} & -\frac{2187}{130} x_{6} & =0 .
\end{aligned}
$$

The next step is to construct the matrix $A_{\tilde{a}_{N-1}}\left(\rho_{1}\right)^{T}$, which represents multiplication by the polynomial $\tilde{a}_{N-1}\left(x_{1}, \ldots, x_{6}\right)$ in the quotient space $\mathbb{C}\left(\rho_{1}\right)\left[x_{1}, \ldots, x_{6}\right] /$ $I\left(\rho_{1}\right)$. Here $I\left(\rho_{1}\right)$ denotes the ideal generated by the 6 polynomials of the quadratic system of equations. The rows of this matrix are then multiplied elementwise with suitable scalar expressions given in Theorem 10.1 to arrive at the polynomial matrix $\tilde{A}_{\tilde{a}_{N-1}}\left(\rho_{1}\right)^{T}$. The dimension of the polynomial matrix is $64 \times 64$ and it is sparse and structured: only 2123 of the 4096 entries are non-zero. See Figure 10.1 for a representation of the sparsity and the structure of the matrix $\tilde{A}_{\tilde{a}_{N-1}}\left(\rho_{1}\right)^{T}$. 


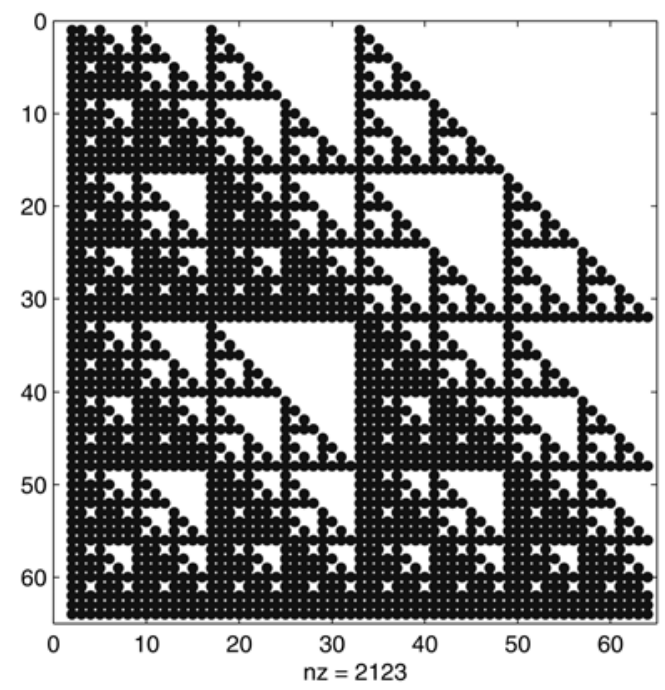

Figure 10.1: Sparsity and structure of the matrix $\tilde{A}_{\tilde{a}_{N-1}}\left(\rho_{1}\right)^{T}$

To find the polynomial eigenvalues $\rho_{1}$ of this matrix, we rewrite the polynomial eigenvalue problem as a generalized eigenvalue problem $\left(B+\rho_{1} C\right) z=0$ which is linear in $\rho_{1}$ and with matrices $B$ and $C$ obtained from $\tilde{A}_{\tilde{a}_{N-1}}\left(\rho_{1}\right)^{T}$ according to the Equations $(10.12)-(10.14)$. The matrices $B$ and $C$ are of dimension $(N-1) 2^{N} \times$ $(N-1) 2^{N}=320 \times 320$. See Figure 10.2 for a representation of the sparsity structure of the matrices $B$ and $C$.
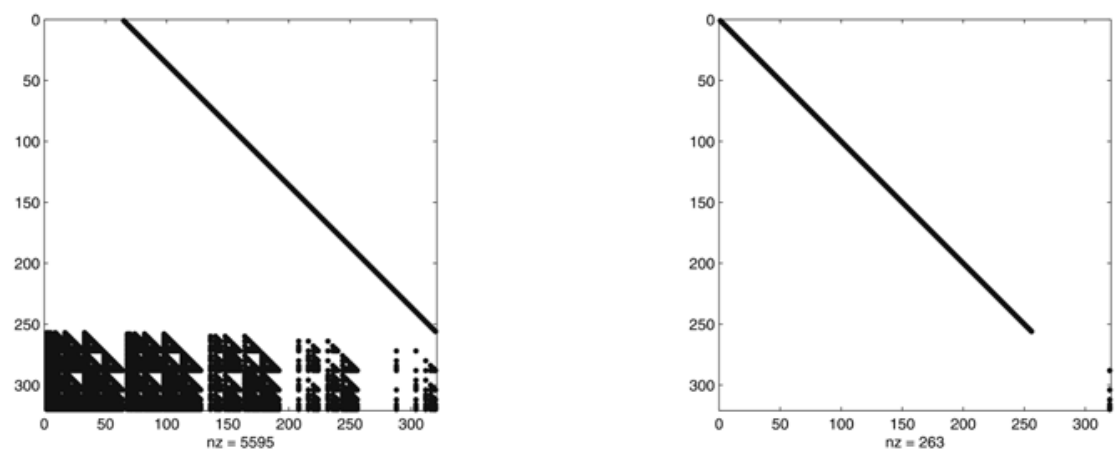

Figure 10.2: Sparsity structure of the matrices $B$ and $C$

Although the matrix pencil $B+\rho_{1} C$ is singular and highly ill-conditioned, we can in this case avoid to compute the Kronecker canonical form using a numerical 
balancing technique to compute the generalized eigenvalues of the singular matrix pencil $B+\rho_{1} C$ in an accurate and fast manner. Such a balancing method for matrix pencils is described in, e.g., [78] and [100]. This technique makes it possible to apply a standard QZ-algorithm to a singular generalized eigenvalue problem by reducing the condition number of the matrices $B$ and $C$ (using exact arithmetic). With this technique, the condition number of the matrix $B$ is reduced from $1.96 \times 10^{30}$ to 3.84 and the condition number of the matrix $C$ from 8.84 to 1 .

The third order polynomial $V_{H}$ in the variables $x_{1}, \ldots, x_{6}$ and $\rho_{1}$ that is used to evaluate the $H_{2}$-criterion at the solutions to the system of equations (10.2), is (numerically) given by:

$$
\begin{aligned}
& V_{H}= \\
& (0.000183786+0.000592891 i)\left(1-\left(\frac{8}{9}+\frac{5}{9} i\right) \rho_{1}\right)^{2}\left(1+\left(\frac{8}{9}+\frac{5}{9} i\right) \rho_{1}\right) x_{1}^{3}+ \\
& (0.000183786-0.000592891 i)\left(1-\left(\frac{8}{9}-\frac{5}{9} i\right) \rho_{1}\right)^{2}\left(1+\left(\frac{8}{9}-\frac{5}{9} i\right) \rho_{1}\right) x_{2}^{3}+ \\
& (-0.00416733-0.000388594 i)\left(1-\left(\frac{2}{3}+\frac{1}{2} i\right) \rho_{1}\right)^{2}\left(1+\left(\frac{2}{3}+\frac{1}{2} i\right) \rho_{1}\right) x_{3}^{3}+ \\
& (-0.00416733+0.000388594 i)\left(1-\left(\frac{2}{3}-\frac{1}{2} i\right) \rho_{1}\right)^{2}\left(1+\left(\frac{2}{3}-\frac{1}{2} i\right) \rho_{1}\right) x_{4}^{3}+ \\
& -0.0428712\left(1-\frac{3}{5} \rho_{1}\right)^{2}\left(1+\frac{3}{5} \rho_{1}\right) x_{5}^{3}+0.285547\left(1-\frac{1}{3} \rho_{1}\right)^{2}\left(1+\frac{1}{3} \rho_{1}\right) x_{6}^{3} .
\end{aligned}
$$

Out of the 320 polynomial eigenvalues, the eigenvalue $\rho_{1}=-0.737003$ gives the smallest feasible real criterion value of 3.739. The values of $x_{1}, \ldots, x_{6}$ are read off from the corresponding eigenvector $z$, which yields the corresponding solution:

$$
\begin{aligned}
& x_{1}=6.32076+30.81856 i \\
& x_{2}=6.32076-30.81856 i \\
& x_{3}=3.02124+17.02393 i \\
& x_{4}=3.02124-17.02393 i \\
& x_{5}=11.7087 \\
& x_{6}=5.02394
\end{aligned}
$$

This solution also satisfies the constraint $\tilde{a}_{N-1}=0$ in Equation (10.44). From this solution, the polynomials $a(s)$ and $b(s)$ and the approximating system $G(s)=\frac{b(s)}{a(s)}$ of order 4 are computed as:

$$
G(s)=\frac{-84.3988+113.051 s-79.9198 s^{2}+28.344 s^{3}}{0.260026+0.909555 s+1.95544 s^{2}+1.60726 s^{3}+s^{4}} .
$$

The poles of the system $G(s)$ are located at $-0.500535-0.930033 i,-0.500535$ $+0.930033 i,-0.303095-0.375816 i,-0.303095+0.375816 i$, and the zeros at 0.667803 $-1.24920 i, 0.667803+1.24920 i, 1.48403$. When comparing the poles and zeros of $H(s)$ and $G(s)$ one may interpret the outcome $G(s)$ such that two real zeros and two real poles are removed from $H(s)$ and the remaining complex conjugate pairs of poles and zeros are adapted. Figure 10.3 shows a plot of the locations of the poles of $H(s)$ and the poles of its approximation $G(s)$ in the complex plane. 


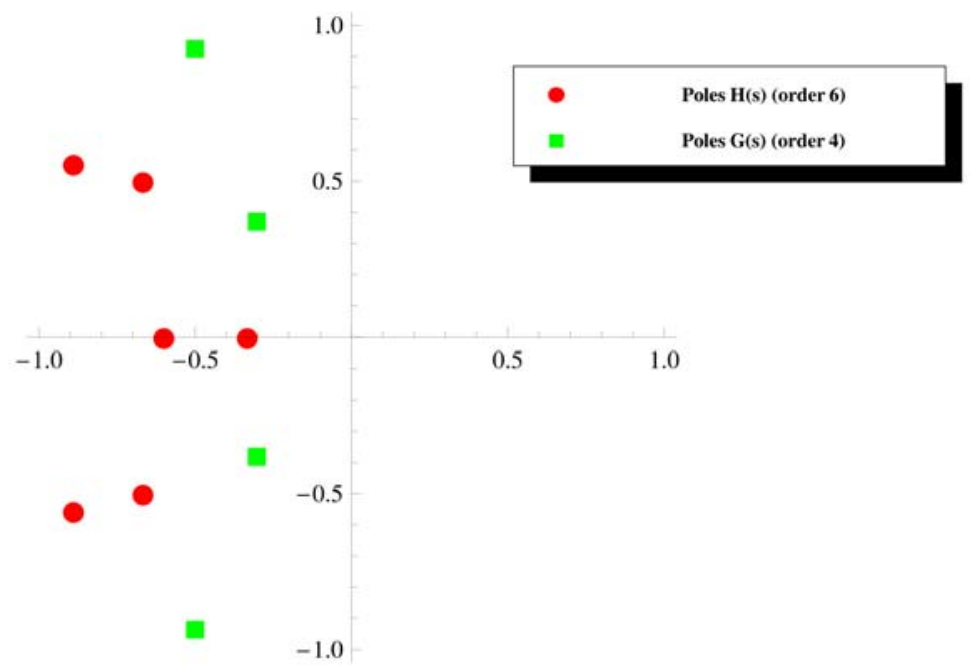

Figure 10.3: The poles of $H(s)$ (red circles) and of $G(s)$ (green squares)

(ii) The co-order $k=1$ technique as described in Chapter 9 is now applied twice to $H(s)$ to arrive at an approximation of order 4 in another way and to compare the results with the globally optimal approximation found by the co-order $k=2$ technique above. Using the co-order one technique, first an approximation $G_{1}(s)$ of order 5 is computed:

$$
\begin{gathered}
G_{1}(s)= \\
\frac{-10.2783-73.8312 s+107.911 s^{2}-79.6939 s^{3}+29.515 s^{4}}{0.0328014+0.374281 s+1.20205 s^{2}+2.1838 s^{3}+1.82557 s^{4}+s^{5}} .
\end{gathered}
$$

The poles of $G_{1}(s)$ are located at $-0.508403-0.877968 i,-0.508403+0.877968 i$, $-0.338916-0.358517 i,-0.338916+0.358517 i$, and -0.130928 , and its $H_{2}$-criterion value, $\|H(s)-G(s)\|_{H_{2}}^{2}$, is 2.488 .

Applying the co-order one technique once again to the transfer function $G_{1}(s)$ to find an approximation $G_{2}(s)$ of order 4 gives:

$$
G_{2}(s)=\frac{-83.8768+112.212 s-79.2856 s^{2}+28.0997 s^{3}}{0.258479+0.903876 s+1.94628 s^{2}+1.59856 s^{3}+s^{4}} .
$$

The poles of $G_{2}(s)$ have slightly different locations as the poles of $G(s)$ : they are located at $-0.496926-0.930667 i,-0.496926+0.930667 i,-0.302353-0.375237 i$, and $-0.302353+0.375237 i$. The zeros of $G_{2}(s)$ are located at $0.667769-1.25010 i$, $0.667769+1.25010 i$, and 1.48604 . In comparison to the poles of $G_{1}(s)$ only the slowest mode at 0.130928 is discarded, whereas the other poles are slightly adapted. Figure 10.4 shows a plot of the poles of $H(s), G_{1}(s)$, and $G_{2}(s)$ in the complex plane. 
The $H_{2}$ model-order reduction error between the original system $H(s)$ and the approximation $G_{2}(s)$ of order 4 is 3.742 , whereas the model-order reduction error of the approximation $G(s)$ computed by the co-order $k=2$ technique was 3.739 .

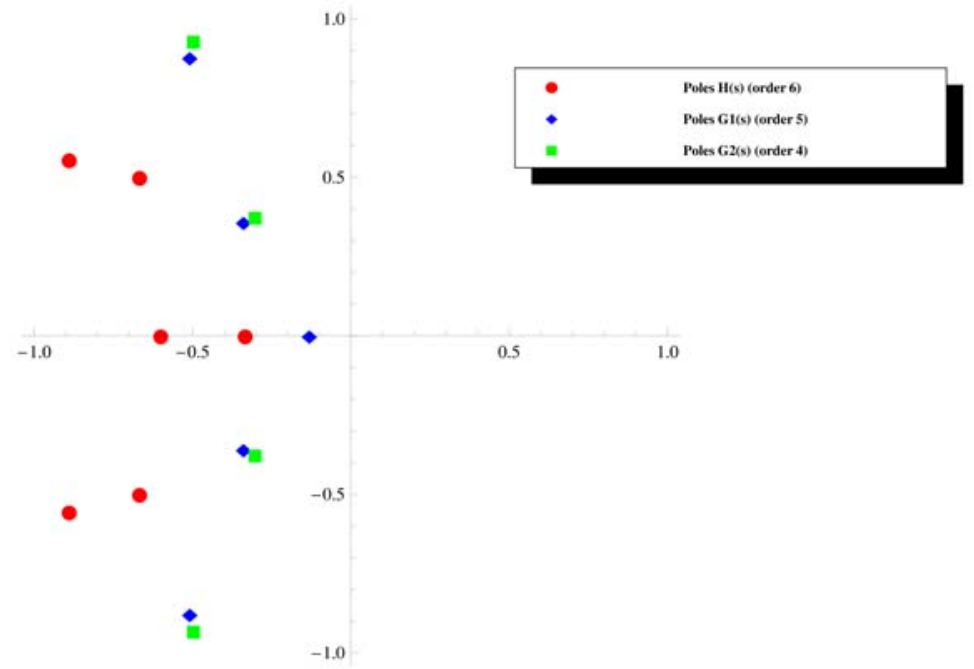

Figure 10.4: The poles of $H(s)$ (red circles), $G_{1}(s)$ (blue diamonds), and $G_{2}(s)$ (green squares)

The Figures 10.5 and 10.6 show the impulse responses and the Bode diagrams of the systems $H(s)$ (blue), $G(s)$ (green), and $G_{2}(s)$ (red). The plots of $G(s)$ and $G_{2}(s)$ virtually overlap in both figures (only $G_{2}(s)$ is visible as it is plotted last) and differ only slightly from $H(s)$. This shows the approximations of order 4 to be of high-quality.

\subsubsection{Example 2}

In this example the system to be reduced is chosen to be a system of order $N=7$, given by the following transfer function:

$$
\begin{gathered}
H(s)= \\
\frac{2+3 s-3 s^{2}+s^{3}-2 s^{4}-12 s^{5}+s^{6}}{\left(s+\frac{1}{2}\right)\left(s+\frac{1}{3}\right)\left(s+\frac{1}{8}\right)\left(s+\frac{1}{2}+\frac{i}{2}\right)\left(s+\frac{1}{2}-\frac{i}{2}\right)\left(s+\frac{1}{3}+\frac{i}{3}\right)\left(s+\frac{1}{3}-\frac{i}{3}\right)} .
\end{gathered}
$$

We now want to compute its globally optimal stable real approximation of order 5. Note that the poles of $H(s)$ are $\delta_{1}=-\frac{1}{2}, \delta_{2}=-\frac{1}{2}-\frac{i}{2}, \delta_{3}=-\frac{1}{2}+\frac{i}{2}, \delta_{4}=-\frac{1}{3}$, $\delta_{5}=-\frac{1}{3}-\frac{i}{3}, \delta_{6}=-\frac{1}{3}+\frac{i}{3}$, and $\delta_{7}=-\frac{1}{8}$. Its zeros are $-0.745632,-0.472807$, $0.162586-0.780510 i, 0.162586+0.780510 i, 0.734023$, and 12.1592 . 


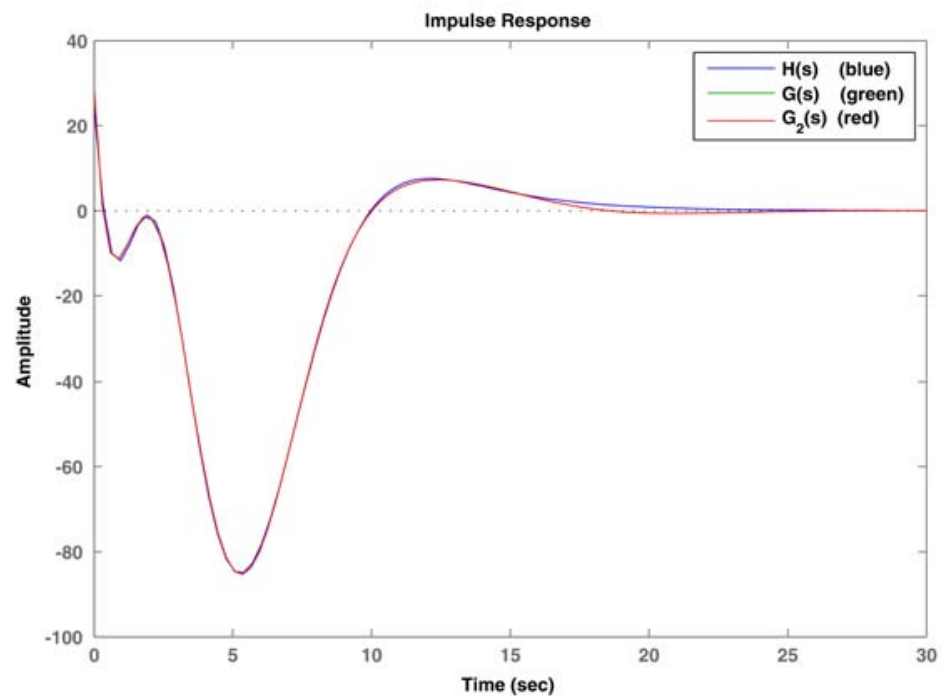

Figure 10.5: Impulse responses of $H(s), G(s)$, and $G_{2}(s)$

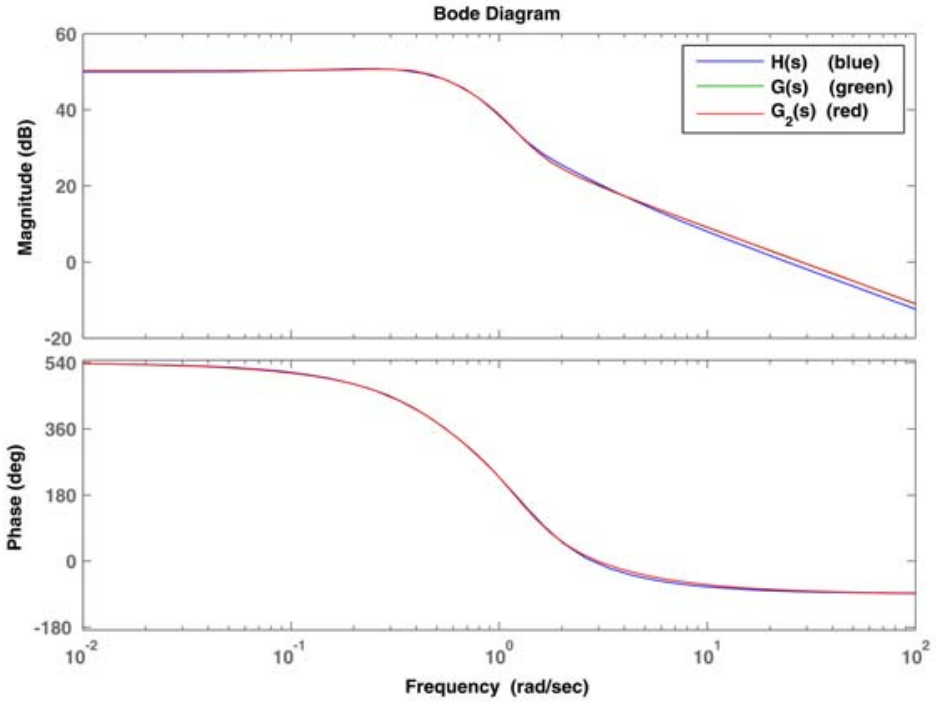

Figure 10.6: Bode diagrams of $H(s), G(s)$, and $G_{2}(s)$ 
The system of corresponding quadratic equations in $x_{1}, \ldots, x_{7}$ and $\rho_{1}$ is:

$$
\left(\begin{array}{c}
\frac{1+\rho_{1} \delta_{1}}{e\left(\delta_{1}\right)} x_{1}^{2} \\
\frac{1+\rho_{1} \delta_{2}}{e\left(\delta_{2}\right)} x_{2}^{2} \\
\frac{1+\rho_{1} \delta_{3}}{e\left(\delta_{3}\right)} x_{3}^{2} \\
\frac{1+\rho_{1} \delta_{4}}{e\left(\delta_{4}\right)} x_{4}^{2} \\
\frac{1+\rho_{1} \delta_{5}}{e\left(\delta_{5}\right)} x_{5}^{2} \\
\frac{1+\rho_{1} \delta_{6}}{e\left(\delta_{6}\right)} x_{6}^{2} \\
\frac{1+\rho_{1} \delta_{7}}{e\left(\delta_{7}\right)} x_{7}^{2}
\end{array}\right)-M\left(\delta_{1}, \ldots, \delta_{7}\right)\left(\begin{array}{c}
x_{1} \\
x_{2} \\
x_{3} \\
x_{4} \\
x_{5} \\
x_{6} \\
x_{7}
\end{array}\right)=\left(\begin{array}{l}
0 \\
0 \\
0 \\
0 \\
0 \\
0 \\
0
\end{array}\right),
$$

where $M\left(\delta_{1}, \ldots, \delta_{7}\right)$ is the complex matrix given by $V\left(-\delta_{1}, \ldots,-\delta_{7}\right) V\left(\delta_{1}, \ldots, \delta_{7}\right)^{-1}$.

The linear constraint, defined by Equation (10.3), is given by:

$$
\begin{aligned}
\tilde{a}_{N-1}= & \frac{2304}{5} x_{1}+\left(\frac{18144}{1625}-\frac{45792}{1625} i\right) x_{2}+\left(\frac{18144}{1625}+\frac{45792}{1625} i\right) x_{3} \\
& -\frac{23328}{25} x_{4}+\left(\frac{688176}{5785}+\frac{559872}{5785} i\right) x_{5}+\left(\frac{688176}{5785}-\frac{559872}{5785} i\right) x_{6} \\
& +\frac{2359296}{11125} x_{7}=0 .
\end{aligned}
$$

It is used to construct the matrix $A_{\tilde{a}_{N-1}}\left(\rho_{1}\right)^{T}$. This matrix is rational in $\rho_{1}$, and after diagonal rescaling (according to Theorem 10.1) to make it polynomial in $\rho_{1}$, a matrix $\tilde{A}_{\tilde{a}_{N-1}}\left(\rho_{1}\right)^{T}$ is obtained. The total degree of $\rho_{1}$ in this matrix is $N-1=6$ (see Corollary 10.2). The size of the matrix $\tilde{A}_{\tilde{a}_{N-1}}\left(\rho_{1}\right)^{T}$ is $2^{N} \times 2^{N}=128 \times 128$. The sparsity structure of this matrix is shown in Figure 10.7.

To find the solutions of the quadratic system of polynomial equations (10.51), which also satisfy the linear constraint (10.52), one has to find the solutions of the polynomial eigenvalue problem $\tilde{A}_{\tilde{a}_{N-1}}\left(\rho_{1}\right)^{T} v=0$. This is done by writing the polynomial matrix $\tilde{A}_{\tilde{a}_{N-1}}\left(\rho_{1}\right)^{T}$ as $A_{0}+\rho_{1} A_{1}+\ldots+\rho_{1}^{6} A_{6}$ and by using a linearization technique. This yields the generalized eigenvalue problem $\left(B+\rho_{1} C\right) \tilde{v}=0$, with the matrices $B$ and $C$ defined as:

$$
\begin{aligned}
B & =\left(\begin{array}{cccccc}
0 & I & 0 & 0 & 0 & 0 \\
0 & 0 & I & 0 & 0 & 0 \\
0 & 0 & 0 & I & 0 & 0 \\
0 & 0 & 0 & 0 & I & 0 \\
0 & 0 & 0 & 0 & 0 & I \\
A_{0} & A_{1} & A_{2} & A_{3} & A_{4} & A_{5}
\end{array}\right), \\
C & =\left(\begin{array}{cccccc}
-I & 0 & 0 & 0 & 0 & 0 \\
0 & -I & 0 & 0 & 0 & 0 \\
0 & 0 & -I & 0 & 0 & 0 \\
0 & 0 & 0 & -I & 0 & 0 \\
0 & 0 & 0 & 0 & -I & 0 \\
0 & 0 & 0 & 0 & 0 & A_{6}
\end{array}\right) .
\end{aligned}
$$




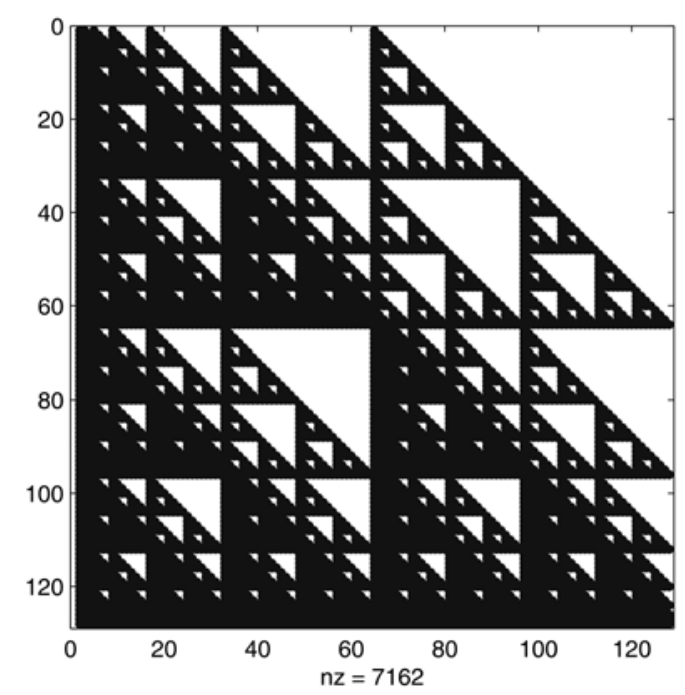

Figure 10.7: Structure of the matrix $\tilde{A}_{\tilde{a}_{N-1}}\left(\rho_{1}\right)^{T}$

The eigenvector $\tilde{v}$ in the generalized eigenvalue problem $\left(B-\rho_{1} C\right) \tilde{v}=0$ is structured as $\left(v^{T}, \rho_{1} v^{T}, \rho_{1}^{2} v^{T}, \rho_{1}^{3} v^{T}, \rho_{1}^{4} v^{T}, \rho_{1}^{5} v^{T}\right)^{T}$ and the matrices $B$ and $C$ are of dimensions $(N-1) 2^{N} \times(N-1) 2^{N}=768 \times 768$.

The matrix pencil $B+\rho_{1} C$ is singular and computing the eigenvalues of this pencil using standard numerical methods fails: all the 768 computed eigenvalues are incorrectly specified to be 0 , indeterminate and infinite, due to ill-conditioning of the singular pencil. In this case, a balancing technique as used in the previous example does not work either. Therefore we resorted to compute the Kronecker canonical form of this pencil using exact arithmetic.

Once the Kronecker canonical form is (partially) obtained the blocks corresponding to the unwanted indeterminate, infinite and zero eigenvalues, which cause the ill-conditioning, are split off. In this way we first split off six indeterminate eigenvalues, which reduces the size of the pencil to $762 \times 762$. This $762 \times 762$ pencil is the regular part of the matrix pencil $B+\rho_{1} C$ which contains zero, infinite and finite non-zero eigenvalues. Furthermore, a total number of 441 zero and infinite eigenvalues are determined by computing the associated Jordan blocks with exact arithmetic. Also these values are split off, because they also have no meaning for the model-order reduction problem. This yields a pencil of size $321 \times 321$ which only contains nonzero finite eigenvalues. These are the eigenvalues which are of importance for the $\mathrm{H}_{2}$ model-order reduction problem. This pencil is denoted by $F+\rho_{1} G$.

The regular generalized eigenvalue problem $\left(F+\rho_{1} G\right) w=0$ can now easily be solved by a numerical method, since it is no longer ill-conditioned and does not include any unwanted eigenvalues which hamper the computation. 
Of the 321 eigenvalues $\rho_{1}$, only 26 are real-valued. The values for $x_{1}, \ldots, x_{7}$ are read off from the corresponding eigenvectors. The 26 tuples $\left(x_{1}, \ldots, x_{7}, \rho_{1}\right)$ are substituted into the available third order polynomial $V_{H}\left(x_{1}, \ldots, x_{7}, \rho_{1}\right)$, which coincides with the $\mathrm{H}_{2}$-criterion at the solutions of the system of equations. It turns out that there is only one solution which yields a real and feasible approximation $G(s)$ of order five: $\rho_{1}=2.03804, x_{1}=-0.0583908, x_{2}=0.0960792-0.0601039 i$, $x_{3}=0.0960792+0.0601039 i, x_{4}=-0.0207708, x_{5}=0.0179519-0.0274311 i$, $x_{6}=0.0179519+0.0274311 i, x_{7}=-0.00383800$ with an $H_{2}$-criterion value of 0.228778 . This system $G(s)$ is the $H_{2}$ globally optimal approximation of order 5 of the original system $H(s)$ of order 7 and is computed as:

$$
G(s)=\frac{6.109-12.02 s+14.93 s^{2}-14.41 s^{3}+1.257 s^{4}}{0.007068+0.10286 s+0.4956 s^{2}+1.210 s^{3}+1.573 s^{4}+s^{5}} .
$$

The system $G(s)$ has its poles at $-0.366841 \pm 0.192807 i,-0.358033 \pm 0.454934 i$, and -0.122789 and its zeros at $0.161729 \pm 0.781139 i, 0.734038$, and 10.4062 .

Computing the globally optimal approximation of order 5 of the original system $H(s)$ of order 7 by applying the co-order one technique twice, results in virtually the same (numerical) approximation $G(s)$.

When comparing the poles of the system $H(s)$ and $G(s)$ one finds that effectively two real poles and two real zeros are removed and the remaining poles and zeros are only adapted slightly. Figure 10.8 shows the poles of the transfer function $H(s)$ and its approximation $G(s)$ obtained by the co-order $k=2$ technique. Figure 10.9 shows the poles of the transfer function $H(s)$ and the poles of its approximations $G_{1}(s)$ of order 6 and $G_{2}(s)$ of order 5 obtained by co-order $k=1$ techniques.

For completeness the impulse responses and Bode diagrams of the systems $H(s)$ (blue) and $G(s)$ (green) are given in the Figures 10.10 and 10.11, which show highquality approximations.

\subsubsection{Example 3}

In this example a system $H(s)$ of order 4 is given which has four complex poles. The globally optimal approximations of order 2 are computed by applying the co-order one technique twice, which results in the systems $G_{1}(s)$, and $G_{2}(s)$, and the co-order two technique once, which results in the system $G(s)$. The results are given in Table 10.1 which presents the locations of the poles and zeros, and the $H_{2}$-criterion values $\left(V_{H}=\|H(s)-G(s)\|_{H_{2}}^{2}\right)$ of the systems $H(s), G_{1}(s), G_{2}(s)$ and $G(s)$.

Applying the co-order one technique twice, yields a significantly worse performance than applying the co-order two technique once: the $H_{2}$-error of $G(s)$ is 9.612 , whereas the $H_{2}$-error of $G_{2}(s)$ is 10.92 . Moreover, the system $G_{2}(s)$ has a completely different behavior from the system $G(s)$, as shown in Figures 10.13 and 10.14, which show the impulse responses and the Bode diagrams of the systems $H(s)$ (blue), $G_{2}(s)$ (green), and $G(s)$ (red). 


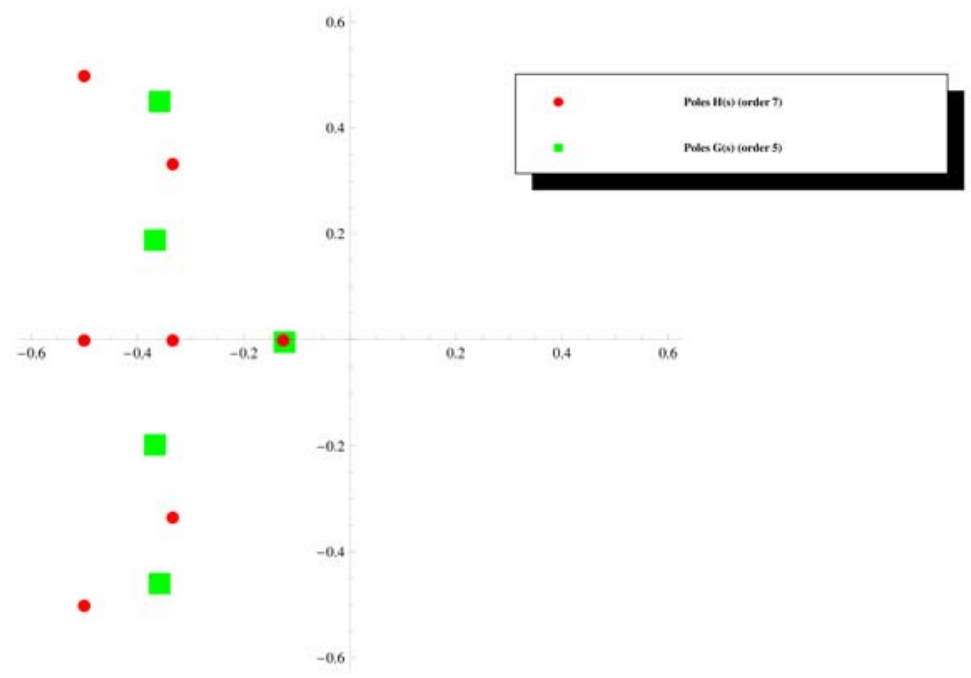

Figure 10.8: Poles of $H(s)$ and its approximation $G(s)$ computed by the co-order two technique

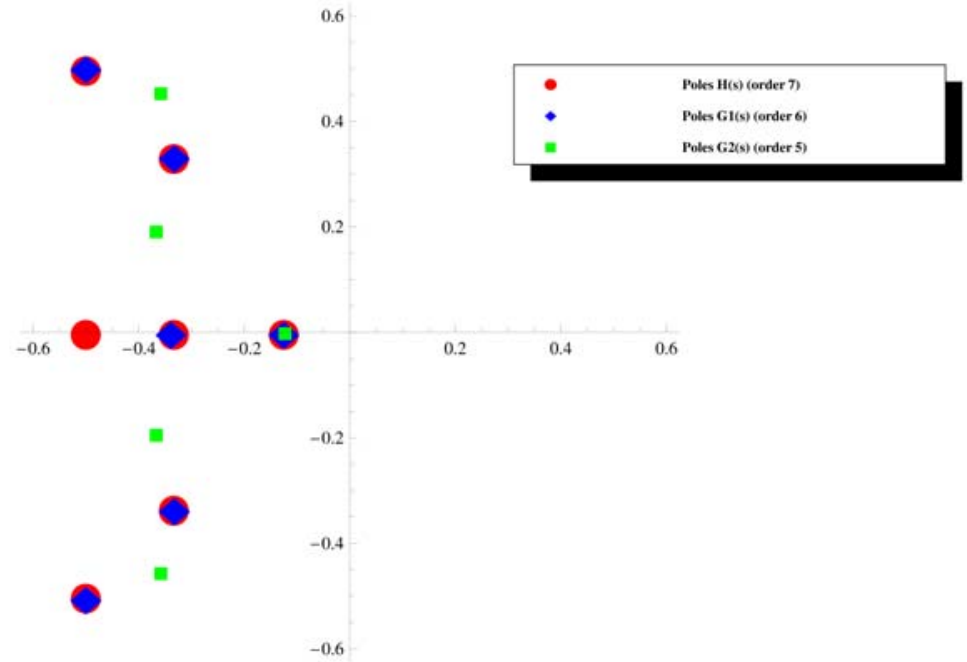

Figure 10.9: Poles of $H(s)$ and its approximations $G_{1}(s)$ and $G_{2}(s)$ computed by repeated application of the co-order one technique 


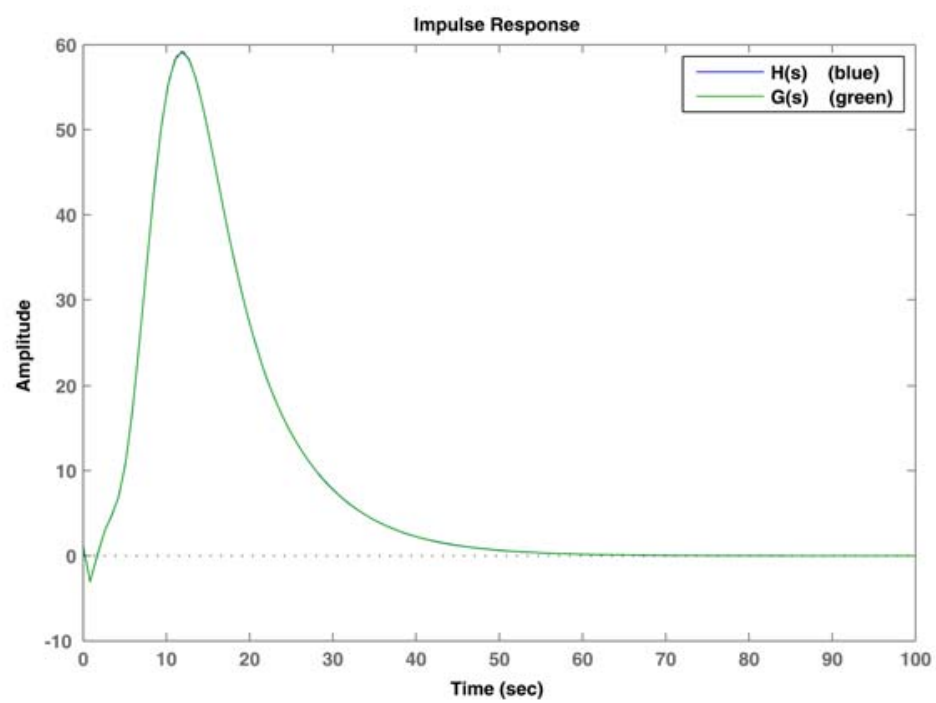

Figure 10.10: Impulse responses of $H(s)$ and $G(s)$

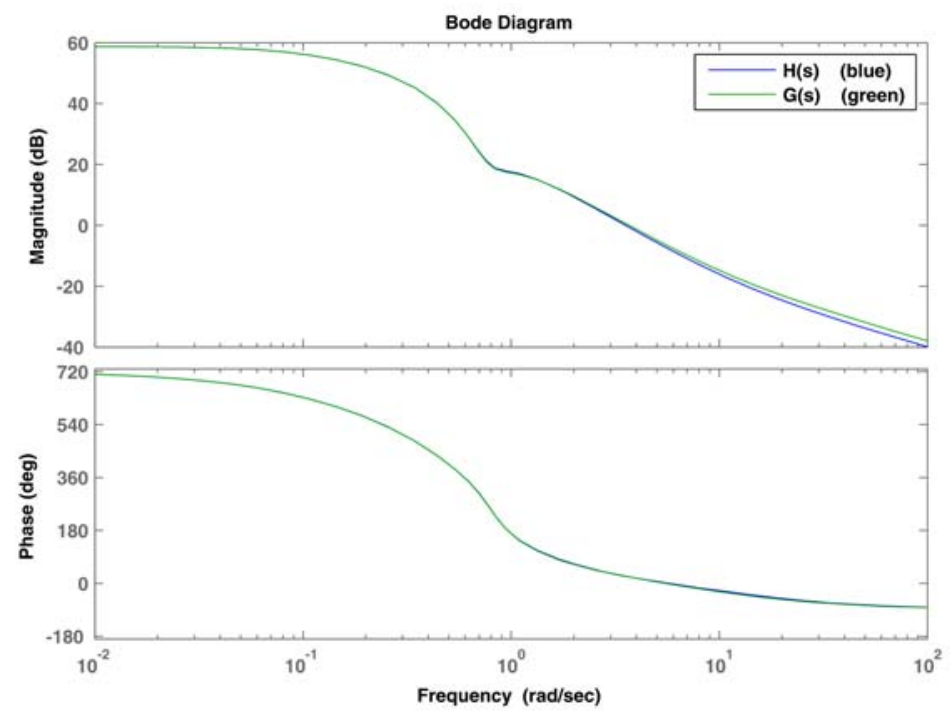

Figure 10.11: Bode diagrams of $H(s)$ and $G(s)$ 
Table 10.1: Reducing the model-order of a system of order 4

\begin{tabular}{clll}
\hline Transfer function & \multicolumn{1}{c}{ Poles } & \multicolumn{1}{c}{ Zeros } & $V_{H}$ \\
\hline$H(s)=$ & $-0.7692-0.8065 i$ & $0.01962-0.1794 i$ & 0 \\
$\frac{-0.9429+1.169 s-29 s^{2}+s^{3}}{0.01634+0.1977 s+1.475 s^{2}+1.681 s^{3}+s^{4}}$ & $-0.7692+0.8065 i$ & $0.01962+0.1794 i$ & \\
& $-0.07143-0.08974 i$ & 28.96 & \\
& $-0.07143+0.08974 i$ & & \\
\hline$G_{1}(s)=$ & $-0.1666-0.1894 i$ & $0.02391-0.1867 i$ & 7.044 \\
$\frac{-0.3929+0.5303 s-11.09 s^{2}}{0.006300+0.096601 s+0.4322 s^{2}+s^{3}}$ & $-0.1666+0.1894 i$ & $0.02391+0.1867 i$ & \\
\hline$G_{2}(s)=$ & -0.09905 & & \\
\hline$-2.183-12.77 s$ & -1.034 & -0.1709 & 10.92 \\
$0.03092+1.064 s+s^{2}$ & -0.02989 & & \\
\hline$G(s)=$ & & & \\
\hline$-22.23-0.7325 s$ & $-0.5660-1.043 i$ & -30.34 & \\
\hline $1.408+1.132 s+s^{2}$ & $-0.5660+1.043 i$ & & \\
\hline
\end{tabular}

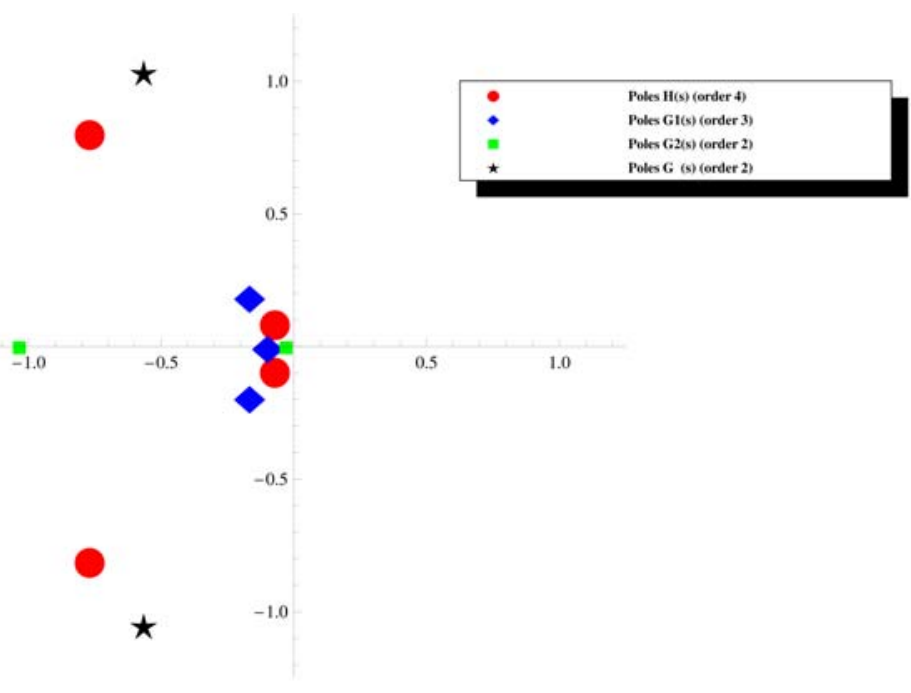

Figure 10.12: Poles of $H(s), G_{1}(s), G_{2}(s)$, and $G(s)$ 


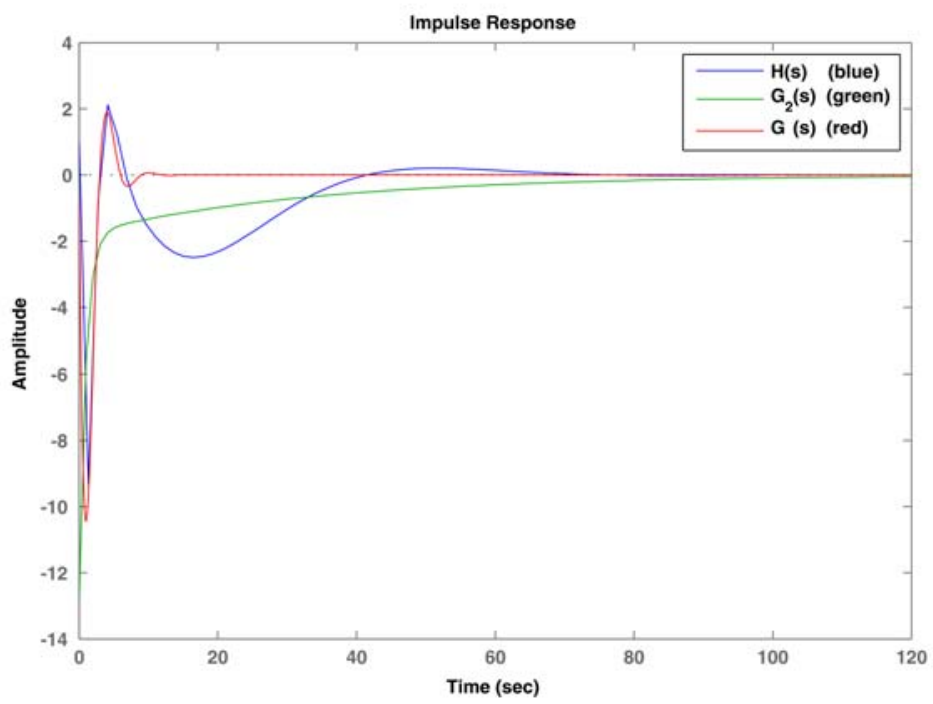

Figure 10.13: Impulse responses of $H(s), G_{2}(s)$, and $G(s)$

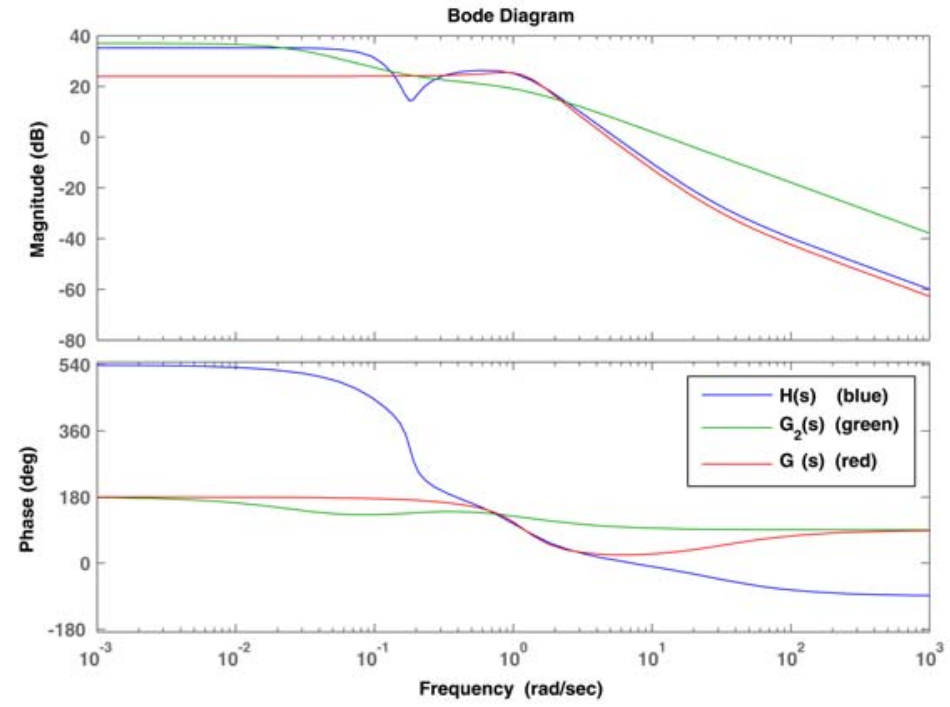

Figure 10.14: Bode diagrams of $H(s), G_{2}(s)$, and $G(s)$ 


\section{Chapter 11}

\section{$H_{2}$ Model-order reduction from order $\mathrm{N}$ to $\mathrm{N}-3$}

When the order of the model is reduced from order $N$ to $N-3$, the co-order $k=3$ case, the degree of the polynomial $q(s)$ in Equation (8.20) is smaller than or equal to $k-1=2: q(s)=q_{0}+q_{1} s+q_{2} s^{2} \not \equiv 0$. For $q_{0} \neq 0$, the polynomial $\rho(s)$ in Equation (8.20) becomes: $\rho(s)=\frac{q(s)}{q_{0}}=1+\rho_{1} s+\rho_{2} s^{2} \not \equiv 0$, where $\rho_{1}=\frac{q_{1}}{q_{0}}$ and $\rho_{2}=\frac{q_{2}}{q_{0}}$. Then the polynomial $\rho\left(\delta_{i}\right)$ becomes $1+\rho_{1} \delta_{i}+\rho_{2} \delta_{i}^{2}$ for $i=1, \ldots, N$ in the system of equations (8.44). This yields a system of quadratic equations containing the two unknown parameters $\rho_{1}$ and $\rho_{2}$ with the following structure:

$$
\left(\begin{array}{cc}
\frac{\left(1+\rho_{1} \delta_{1}+\rho_{2} \delta_{1}^{2}\right)}{e\left(\delta_{1}\right)} x_{1}^{2} \\
\frac{\left(1+\rho_{1} \delta_{2}+\rho_{2} \delta_{2}^{2}\right)}{e\left(\delta_{2}\right)} x_{2}^{2} \\
\vdots \\
\frac{\left(1+\rho_{1} \delta_{N}+\rho_{2} \delta_{N}^{2}\right)}{e\left(\delta_{N}\right)} x_{N}^{2}
\end{array}\right)-M\left(\delta_{1}, \ldots, \delta_{N}\right)\left(\begin{array}{c}
x_{1} \\
x_{2} \\
\vdots \\
x_{N}
\end{array}\right)=\left(\begin{array}{c}
0 \\
0 \\
\vdots \\
0
\end{array}\right)
$$

where $M\left(\delta_{1}, \ldots, \delta_{N}\right)=V\left(-\delta_{1}, \ldots,-\delta_{N}\right) V\left(\delta_{1}, \ldots, \delta_{N}\right)^{-1}$.

As discussed in Section 8.5, there are two constraints on the coefficients of the polynomial $\tilde{a}(s)$ in the co-order $k=3$ case which have to be satisfied in order to find feasible approximations $G(s)$ of order $N-3$. Using Equation (8.45) for $k=3$, these constraints are denoted by:

$$
\left\{\begin{array}{l}
\tilde{a}_{N-1}\left(x_{1}, x_{2}, \ldots, x_{N}\right)=\gamma_{1,1} x_{1}+\gamma_{1,2} x_{2}+\ldots+\gamma_{1, N} x_{N}=0 \\
\tilde{a}_{N-2}\left(x_{1}, x_{2}, \ldots, x_{N}\right)=\gamma_{2,1} x_{1}+\gamma_{2,2} x_{2}+\ldots+\gamma_{2, N} x_{N}=0
\end{array}\right.
$$

Note that the quantities $\gamma_{i, 1}, \ldots, \gamma_{i, N}$ (for $i=1,2$ ) in these constraints depend on $\delta_{1}, \ldots, \delta_{N}$. 
The system of equations (11.1) and the two additional constraints in (11.2) represent $N+2$ equations in $N+2$ unknowns. The $N+2$ unknowns show up as the $N$ quantities $x_{1}, \ldots, x_{N}$ together with the unknown parameters $\rho_{1}$ and $\rho_{2}$ in (11.1).

When a solution $\left(x_{1}, \ldots, x_{N}, \rho_{1}, \rho_{2}\right)$ of the system of equations (11.1) is known, and this solution also satisfies the additional constraints $\tilde{a}_{N-1}=0$ and $\tilde{a}_{N-2}=0$ in (11.2), then an approximation $G(s)$ of order $N-3$ can be computed. To obtain the globally optimal approximation $G(s)$, all the solutions $\left(x_{1}, \ldots, x_{N}, \rho_{1}, \rho_{2}\right)$ are required to select the best one. Section 11.1 describes how solutions of (11.1), which satisfy both the additional constraints (11.2), can be obtained by using the StetterMöller matrix method. This approach leads to a two-parameter eigenvalue problem involving two matrices and one common eigenvector. Linearizing and solving such a two parameter polynomial eigenvalue problem are the subjects of the Sections 11.2 and 11.3. Section 11.4 shows how to arrive at an approximation $G(s)$ once these solutions of the system of equations (11.1) and the constraints (11.2) are known. As a proof of principle, an example is worked out in Section 11.5.

\subsection{Solving the system of quadratic equations}

Suppose a system of the form (11.1) together with the constraints (11.2) is given. The idea now is to use the Stetter-Möller method in the same way as in the previous chapters to find solutions $x_{1}, \ldots, x_{N}$, and $\rho_{1}$ and $\rho_{2}$ which lead to approximations $G(s)$ of order $N-3$.

Using the polynomials in (11.2) we apply the Stetter-Möller matrix method and consider the linear multiplication operators $\mathcal{A}_{\tilde{a}_{N-1}}\left(\rho_{1}, \rho_{2}\right)$ and $\mathcal{A}_{\tilde{a}_{N-2}}\left(\rho_{1}, \rho_{2}\right)$ within the quotient space $\mathbb{C}\left(\rho_{1}, \rho_{2}\right)\left[x_{1}, \ldots, x_{N}\right] / I\left(\rho_{1}, \rho_{2}\right)$. Here $I\left(\rho_{1}, \rho_{2}\right)$ denotes the ideal generated by the parameterized set of polynomials of the quadratic system of equations (11.1). With respect to an identical monomial basis $B$ as in (8.47), these linear operators are represented by the matrices $A_{\tilde{a}_{N-1}}\left(\rho_{1}, \rho_{2}\right)^{T}$ and $A_{\tilde{a}_{N-2}}\left(\rho_{1}, \rho_{2}\right)^{T}$, which denote multiplication by $\tilde{a}_{N-1}$ and $\tilde{a}_{N-2}$.

We know from Section 3.3 that the eigenvalues $\lambda$ in the eigenvalue problem $A_{\tilde{a}_{N-i}}$ $\left(\rho_{1}, \rho_{2}\right)^{T} v=\lambda v$, coincide with the values of the polynomial $\tilde{a}_{N-i}$ at the solutions of the system of equations (11.1), for $i=1,2$. Because the values of $\tilde{a}_{N-1}$ and $\tilde{a}_{N-2}$ are required to be zero, the resulting problem is: $A_{\tilde{a}_{N-1}}\left(\rho_{1}, \rho_{2}\right)^{T} v=0$ and $A_{\tilde{a}_{N-2}}\left(\rho_{1}, \rho_{2}\right)^{T} v=0$. Thus, actually we are searching here for values of $\rho_{1}$ and $\rho_{2}$ which make the matrices $A_{\tilde{a}_{N-1}}\left(\rho_{1}, \rho_{2}\right)^{T}$ and $A_{\tilde{a}_{N-2}}\left(\rho_{1}, \rho_{2}\right)^{T}$ simultaneously singular.

Both the matrices $A_{\tilde{a}_{N-1}}\left(\rho_{1}, \rho_{2}\right)^{T}$ and $A_{\tilde{a}_{N-2}}\left(\rho_{1}, \rho_{2}\right)^{T}$ are rational in $\rho_{1}$ and $\rho_{2}$ and can be made polynomial in $\rho_{1}$ and $\rho_{2}$. Using Theorem 10.1 the matrices $A_{\tilde{a}_{N-1}}\left(\rho_{1}, \rho_{2}\right)^{T}$ and $A_{\tilde{a}_{N-2}}\left(\rho_{1}, \rho_{2}\right)^{T}$ are made polynomial in $\rho_{1}$ and $\rho_{2}$ by multiplying elementwise the rows of the matrices with well chosen quantities. The polynomial matrices in $\rho_{1}$ and $\rho_{2}$ are denoted by the matrices $\tilde{A}_{\tilde{a}_{N-1}}\left(\rho_{1}, \rho_{2}\right)^{T}$ and $\tilde{A}_{\tilde{a}_{N-2}}\left(\rho_{1}, \rho_{2}\right)^{T}$. These matrices have dimensions $2^{N} \times 2^{N}$ and the total degree of $\rho_{1}$ and $\rho_{2}$ occurring in the polynomial matrices is $N-1$ (analogous to the result in Corollary 10.2). 
To find all the solutions of the system of quadratic equations (11.1) subject to (11.2) one needs to compute:

(i) all the pairs $\left(\rho_{1}, \rho_{2}\right)$ such that the matrices $\tilde{A}_{\tilde{a}_{N-1}}\left(\rho_{1}, \rho_{2}\right)^{T}$ and $\tilde{A}_{\tilde{a}_{N-2}}\left(\rho_{1}, \rho_{2}\right)^{T}$ simultaneously become singular. Thus, the problem can be written as a polynomial two-parameter eigenvalue problem involving both the matrices:

$$
\left\{\begin{array}{l}
\tilde{A}_{\tilde{a}_{N-1}}\left(\rho_{1}, \rho_{2}\right)^{T} v=0 \\
\tilde{A}_{\tilde{a}_{N-2}}\left(\rho_{1}, \rho_{2}\right)^{T} v=0
\end{array}\right.
$$

Note that here a common eigenvector $v$ is present in both the equations.

(ii) Furthermore, all the corresponding eigenvectors $v$ are required. These eigenvectors exhibit the Stetter structure and therefore the values of $x_{1}, \ldots, x_{N}$ can be read off from the eigenvectors. The $N$-tuples of values $x_{1}, \ldots, x_{N}$, thus obtained, together with the corresponding eigenvalues $\rho_{1}$ and $\rho_{2}$, constitute all the solutions of the system of equations (11.1) which simultaneously satisfy the constraints (11.2). From these solutions approximations $G(s)$ of order $N-3$ can be computed, as shown in Section 11.4.

A problem as in Equation (11.3) is highly structured and because of the known background of the problem we are allowed to suppose that it will admit a finite number of solutions. In general however such a problem will have no solutions. It turns out that techniques from linearizing a polynomial matrix and the Kronecker canonical form computation of a singular matrix pencil with one parameter, introduced in Section 10.3, are useful to reliably compute the solutions of this eigenvalue problem in two parameters. This is the subject of the Sections 11.2 and 11.3.

\subsection{Linearizing the two-parameter eigenvalue problem}

A polynomial two-parameter eigenvalue problem (11.3), which involves two polynomial matrices and one common eigenvector, is, to the best of our knowledge, new in the literature. A solution method does not readily exist so far. Note that the problem (11.3) has special additional structure: we know from the algebraic techniques, used to construct the eigenvalue problem, that the matrices $\tilde{A}_{\tilde{a}_{N-1}}\left(\rho_{1}, \rho_{2}\right)^{T}$ and $\tilde{A}_{\tilde{a}_{N-2}}\left(\rho_{1}, \rho_{2}\right)^{T}$ commute and that there will be a finite number of solutions. At first sight, however, without such additional structure, one would generically expect the problem (11.3) not to have any solutions, as it would be overdetermined: there are $2^{N+1}$ equations and only $2^{N}+1$ degrees of freedom (the $2^{N}-1$ free entries in the normalized eigenvector $v$ and the 2 parameters $\rho_{1}$ and $\rho_{2}$ ). Also, when an eigenvector $v$ and eigenvalues $\rho_{1}$ and $\rho_{2}$ are found which satisfy $\tilde{A}_{\tilde{a}_{N-1}}\left(\rho_{1}, \rho_{2}\right)^{T} v=0$, it is in general unlikely that this same solution will also satisfy $\tilde{A}_{\tilde{a}_{N-2}}\left(\rho_{1}, \rho_{2}\right)^{T} v=0$.

A first step to solve the two-parameter polynomial eigenvalue problem (11.3), is to construct an equivalent eigenvalue problem which is linear in $\rho_{1}$ and $\rho_{2}$. This can be 
achieved by applying the linearization technique of Section 10.2 twice on each of the matrices $\tilde{A}_{\tilde{a}_{N-1}}\left(\rho_{1}, \rho_{2}\right)^{T}$ and $\tilde{A}_{\tilde{a}_{N-2}}\left(\rho_{1}, \rho_{2}\right)^{T}$. Such an approach is given in Appendix A including an example to demonstrate the procedure.

By applying the technique of Appendix A to linearize (11.3), we arrive at the equivalent linear two-parameter eigenvalue problem:

$$
\left\{\begin{array}{l}
\left(B_{1}+\rho_{1} C_{1}+\rho_{2} D_{1}\right) w=0 \\
\left(B_{2}+\rho_{1} C_{2}+\rho_{2} D_{2}\right) w=0
\end{array}\right.
$$

where $B_{i}, C_{i}, D_{i}(i=1,2)$ are large structured matrices of dimensions $(N-1)^{2} 2^{N} \times$ $(N-1)^{2} 2^{N}$. The eigenvector $w$ in (11.4) is built up from products of the original eigenvector $v$ and powers of $\rho_{1}$ and $\rho_{2}$.

In the literature on solving two-parameter linear eigenvalue problems (see, e.g., [57], [59], [60]) commonly the problem with distinct eigenvectors is addressed:

$$
\left\{\begin{array}{l}
\left(X_{1}+\rho_{1} Y_{1}+\rho_{2} Z_{1}\right) w_{1}=0 \\
\left(X_{2}+\rho_{1} Y_{2}+\rho_{2} Z_{2}\right) w_{2}=0
\end{array}\right.
$$

Therefore this theory can not be applied directly to our problem. Moreover and more importantly, most of this literature deals with iterative solvers to compute only some eigenvalues, whereas we need all the eigenvalues of the two-parameter eigenvalue problem (11.3). No literature is available on linear two-parameter eigenvalue problems involving a common eigenvector $w$.

\subsection{The Kronecker canonical form of a two-parameter matrix pen- cil}

Due to the linearization technique, the first $(N-1)^{2}-1$ block rows capture the structure imposed on the eigenvector $w$ and the last block row represents the equations of the original non-linear two-parameter eigenvalue problem. Therefore, the first $\left((N-1)^{2}-1\right) 2^{N}$ equations of $\left(B_{1}+\rho_{1} C_{1}+\rho_{2} D_{1}\right) w=0$, are identical to the first $\left((N-1)^{2}-1\right) 2^{N}$ equations represented by $\left(B_{2}+\rho_{1} C_{2}+\rho_{2} D_{2}\right) w=0$.

When all the equations represented by (11.4) are written as one matrix-vector product with the eigenvector $w$, this yields:

$$
\left[\begin{array}{c}
B_{1}+\rho_{1} C_{1}+\rho_{2} D_{2} \\
B_{2}+\rho_{1} C_{2}+\rho_{2} D_{2}
\end{array}\right] w=0 .
$$

where the involved matrix contains $\left((N-1)^{2}-1\right) 2^{N}$ duplicate rows. When all duplicates are removed and only the unique rows are maintained, an equivalent linear non-square eigenvalue problem arises:

$$
\left(B+\rho_{1} C+\rho_{2} D\right) w=0
$$


where the matrices $B, C$, and $D$ have at most $\left((N-1)^{2}+1\right) 2^{N}$ unique rows and exactly $(N-1)^{2} 2^{N}$ columns. Let us denote the dimensions of these matrices by $m \times n$.

The equivalence of (11.7) and (11.3) implies that (11.7) has a finite number of solutions too.

Let us now consider the rectangular eigenvalue problem (11.7) as a one-parameter eigenvalue problem in $\rho_{1}$ with matrices containing entries from the field $\mathbb{C}\left(\rho_{2}\right)$. Then (11.7) can be rewritten as the equivalent homogeneous system of equations:

$$
\left(P\left(\rho_{2}\right)+\rho_{1} Q\left(\rho_{2}\right)\right) w=0
$$

where $P\left(\rho_{2}\right)=B+\rho_{2} D$ and $Q\left(\rho_{2}\right)=C$, both of dimensions $m \times n$.

Because the one-parameter pencil $P+\rho_{1} Q$ in problem (11.8) is rectangular it is singular by definition. We now could proceed as in Section 10.3: by computing the Kronecker Canonical form of this one-parameter matrix pencil and by splitting off its singular parts, one can try to determine the regular part of the pencil. Then one expects to compute solutions $\rho_{1}$ and $\rho_{2}$ of the original problem (11.3) from this regular part.

The next subsections describe the transformation into the Kronecker canonical form, the structure of the involved transformed matrices and three methods to determine these transformation matrices to split off singular parts using exact arithmetic. We use here in fact a generalization of the techniques of the Kronecker canonical form computations for singular one-parameter pencils in $\mathbb{C}$ presented in Section 10.3 of the previous chapter.

\subsubsection{Transformation into the Kronecker canonical form}

When varying the value of $\rho_{1}$ in Equation (11.8) it may happen that solutions exist for all values of $\rho_{1}$, but other solutions may exist only for special values of $\rho_{1}$. To emphasize the dependence of the solution set on the value of $\rho_{1}$, we shall write $w\left(\rho_{1}\right)$.

The matrix pencil $P+\rho_{1} Q$ can be brought into Kronecker canonical form. This involves a transformation into the form:

$$
W^{-1}\left(P+\rho_{1} Q\right) V=D+\rho_{1} E
$$

where $V$ (of size $n \times n)$ and $W$ (of size $m \times m$ ) are invertible transformation matrices with entries in $\mathbb{C}\left(\rho_{2}\right)$. The solutions $\tilde{w}\left(\rho_{1}\right)$ to the set of equations:

$$
\left(D+\rho_{1} E\right) \tilde{w}=0
$$

are related to those of the original problem $\left(P+\rho_{1} Q\right) w=0$ according to:

$$
w\left(\rho_{1}\right)=V \tilde{w}\left(\rho_{1}\right)
$$

Note that the systems of equations are equivalent when working over $\mathbb{C}\left(\rho_{2}\right)$, i.e., every solution $\tilde{w}\left(\rho_{1}\right)$ corresponds to a unique solution $w\left(\rho_{1}\right)$ and vice versa, since $V$ is invertible. 
Also, invertibility of $W$ is required for equivalence of the two systems. However, when values for $\rho_{2}$ are plugged in, $V$ and $W$ may fail to be invertible. Because $V$ and $W$ are invertible over $\mathbb{C}\left(\rho_{2}\right)$, their determinants are non-zero rational expressions, so that there is only a finite set of values for $\rho_{2}$ which may compromise invertibility.

Therefore, we may proceed with the new set of equations $\left(D+\rho_{1} E\right) \tilde{w}=0$ in (11.10) and we should complement the solutions $w=V \tilde{w}$ with an additional analysis for the finite set of values for $\rho_{2}$ which destroy invertibility of $V$ or $W$.

As in Chapter 10, the pencil $D+\rho_{1} E$ is in Kronecker canonical form, if it has the quasi block diagonal structure:

$$
D+\rho_{1} E=
$$

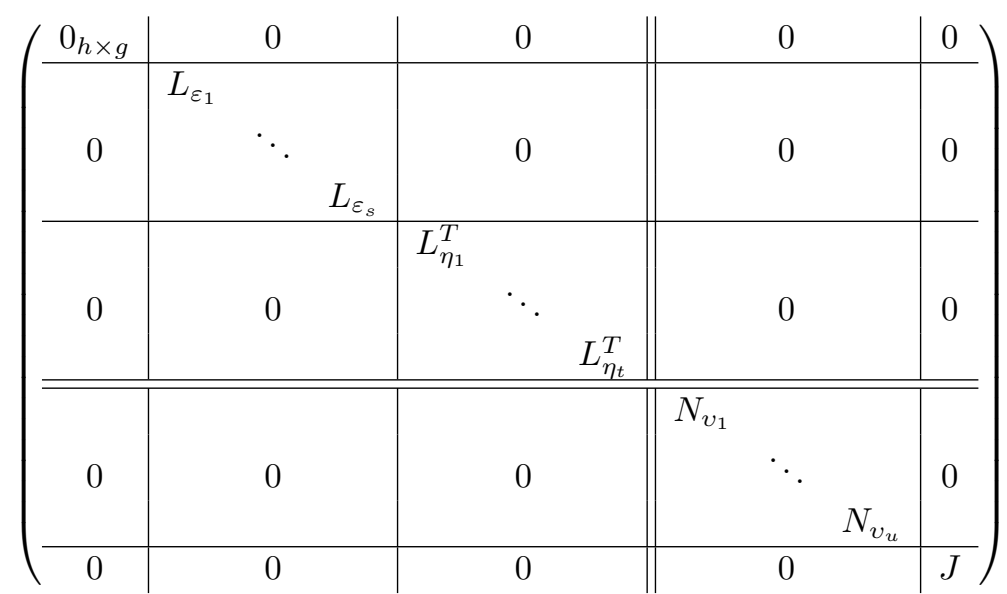

where

$$
\begin{aligned}
L_{\varepsilon} & =\left(\begin{array}{ccccc}
\rho_{1} & 1 & 0 & \ldots & 0 \\
0 & \rho_{1} & 1 & & \vdots \\
\vdots & \vdots & \ddots & \ddots & \vdots \\
0 & 0 & \ldots & \rho_{1} & 1
\end{array}\right) \text { is of size } \varepsilon \times(\varepsilon+1), \\
L_{\eta}^{T} & =\left(\begin{array}{cccc}
\rho_{1} & 0 & \ldots & 0 \\
1 & \rho_{1} & & \vdots \\
0 & 1 & \ddots & 0 \\
\vdots & & \ddots & \rho_{1} \\
0 & \ldots & 0 & 1
\end{array}\right) \text { is of size }(\eta+1) \times \eta,
\end{aligned}
$$

and

$$
N_{v}=\left(\begin{array}{cccc}
1 & \rho_{1} & & 0 \\
& \ddots & \ddots & \\
& & & \rho_{1} \\
0 & & & 1
\end{array}\right) \text { is of size } v \times v
$$


and where $J$ is the Jordan form of a $\kappa \times \kappa$ square matrix pencil which is regular and only has finite eigenvalues.

As seen before in the previous chapter, every matrix pencil $P+\rho_{1} Q$ has a unique Kronecker canonical form, provided that the dimensions $\varepsilon_{1} \leq \varepsilon_{2} \leq \cdots \leq \varepsilon_{s}$, $\eta_{1} \leq \eta_{2} \leq \cdots \leq \eta_{t}, v_{1} \leq v_{2} \leq \cdots \leq v_{u}$ and the Jordan blocks $J$ are ordered in some prespecified way.

A solution to the problem $\left(D+\rho_{1} E\right) \tilde{w}=0$ involves a partitioned eigenvector $\tilde{w}$ structured as:

$$
\tilde{w}=\left(\begin{array}{c}
\tilde{w}_{0} \\
\tilde{w}_{1} \\
\vdots \\
\tilde{w}_{s} \\
\hline \tilde{w}_{s+1} \\
\vdots \\
\tilde{w}_{s+t} \\
\tilde{w}_{s+t+1} \\
\vdots \\
\tilde{w}_{s+t+u} \\
\tilde{w}_{s+t+u+1}
\end{array}\right) .
$$

Each of the parts in $\tilde{w}$ may yield a solution, to be combined with solutions to other parts. Each part will always admit the trivial solution. Furthermore, it holds that:

- $\tilde{w}_{0}$ is always free to choose, irrespective of $\rho_{1}$.

- $\tilde{w}_{i}, i=1, \ldots, s$ needs to satisfy $L_{\varepsilon_{i}} \tilde{w}_{i}=0$. Hence,

$$
\tilde{w}_{i}=\alpha_{i}\left(\begin{array}{c}
1 \\
-\rho_{1} \\
\rho_{1}^{2} \\
\vdots \\
\left(-\rho_{1}\right)^{\varepsilon_{i}}
\end{array}\right),
$$

for arbitrary $\alpha_{i} \in \mathbb{C}\left(\rho_{2}\right)$, and $\rho_{1}$ is free to choose.

- $\tilde{w}_{s+i}, i=1, \ldots, t$, needs to satisfy $L_{\eta_{i}}^{T} \tilde{w}_{s+i}=0$, which only admits the trivial solution $\tilde{w}_{s+i}=0$.

- $\tilde{w}_{s+t+i}, i=1, \ldots, u$, needs to satisfy $N_{v_{i}} \tilde{w}_{s+t+i}=0$, which only admits the trivial solution $\tilde{w}_{s+t+i}=0$.

- $\tilde{w}_{s+t+u+1}$ needs to satisfy $J \tilde{w}_{s+t+u+1}=0$. For given $\rho_{2}$, only a finite number of values for $\rho_{1}$ will do, i.e., the eigenvalues which are finite. 
We can conclude from this that non-trivial solutions only exist for a finite set of values for $\rho_{1}$ if and only if $g=0$ and $s=0$ in the Kronecker canonical form (11.12) of the singular matrix pencil $P+\rho_{1} Q$ in (11.9) (thus no blocks of the form $L_{\varepsilon}$ ).

The dimension of the regular part involving the block $J$ is $\kappa \times \kappa$. This means that there are $\kappa$ equations in $\kappa-1$ unknowns in the (normalized) eigenvector $\tilde{w}_{s+t+u+1}$ plus the two unknowns $\rho_{1}$ and $\rho_{2}$ in the eigenvalue problem $J\left(\rho_{1}, \rho_{2}\right) \tilde{w}_{s+t+u+1}$. This means that we have created an underdetermined system of equations. Such a system allows an infinite number of additional solutions. This is in contradiction of what we know when we apply the Stetter-Möller matrix method to the problem at hand: the commuting operators $\tilde{A}_{\tilde{a}_{N-1}}\left(\rho_{1}, \rho_{2}\right)^{T}$ and $\tilde{A}_{\tilde{a}_{N-2}}\left(\rho_{1}, \rho_{2}\right)^{T}$ will only allow for a finite number of solutions. Therefore one may conclude that generically there will exist no regular part in this pencil, thus $\kappa=0$.

Therefore, a finite number of solutions for $\rho_{1}$ and $\rho_{2}$ may only exist, if and only if, $g=0, s=0$, and $\kappa=0$ and we conclude the following:

Proposition 11.1. The pencil $P+\rho_{1} Q$ with $P, Q \in \mathbb{C}\left(\rho_{2}\right)^{m \times n}$ has a finite set of pairs $\left(\rho_{1}, \rho_{2}\right)$ at which the two-parameter eigenvalue problem $\left(P+\rho_{1} Q\right) w=0$ admits a non-trivial solution if and only if its Kronecker canonical form over $\mathbb{C}\left(\rho_{2}\right)$ only contains singular blocks:

$$
L_{\eta}^{T}=\left(\begin{array}{cccc}
\rho_{1} & 0 & \ldots & 0 \\
1 & \rho_{1} & & \vdots \\
0 & 1 & \ddots & 0 \\
\vdots & & \ddots & \rho_{1} \\
0 & \ldots & 0 & 1
\end{array}\right)
$$

(possibly of size $1 \times 0$ ) which correspond to indeterminate eigenvalues and blocks of the form:

$$
N_{v}=\left(\begin{array}{cccc}
1 & \rho_{1} & & 0 \\
& \ddots & \ddots & \\
& & & \rho_{1} \\
0 & & & 1
\end{array}\right)
$$

which correspond to infinite eigenvalues.

As a consequence, the generic solution set for $D+\rho_{1} E$, when considered over the field $\mathbb{C}\left(\rho_{2}\right)$, is just the trivial set $\tilde{w}\left(\rho_{1}\right)=0$. Hence, we have the following result:

Proposition 11.2. The finite solutions for $\left(\rho_{1}, \rho_{2}\right)$ correspond to values for $\rho_{2}$ which make the transformation matrices $V$ and $W$ singular (as numerical values). Hence, we arrive at the one-parameter problem to investigate the existence of non-trivial solutions to:

$$
\begin{cases}V\left(\rho_{2}\right) z_{v}=0 & \text { of size } m \times m \\ W\left(\rho_{2}\right) z_{w}=0 & \text { of size } n \times n .\end{cases}
$$


Now problem (11.8) is reshaped into the form of two one-parameter eigenvalue problems, which can be solved by using the techniques presented in Chapter 10. The main goal now is to determine the transformation matrices $V\left(\rho_{2}\right)$ and $W\left(\rho_{2}\right)$ which bring the singular pencil $P+\rho_{1} Q$ into the Kronecker canonical form. This is the main subject of the next three subsections where three methods are described to determine these matrices $V\left(\rho_{2}\right)$ and $W\left(\rho_{2}\right)$.

Remark 11.1. The only singular blocks that may be split off from the singular pencil $P+\rho_{1} Q$, are the blocks $L_{\eta}^{T}$ of dimension $(\eta+1) \times \eta$, see Proposition 11.1. Such blocks can be obtained by looking at transformations:

$$
W\left(\rho_{2}\right)^{-1}\left(P^{T}+\rho_{1} Q^{T}\right) V\left(\rho_{2}\right),
$$

which means that we continue to work with the transposed versions of the matrices $P$ and $Q$. A consequence of this is that we find as singular blocks the transposes of the blocks $L_{\eta}^{T}$ which then are of dimensions $\eta \times(\eta+1)$.

\subsubsection{Computing the transformation matrices for a linear two-parameter matrix pencil}

We consider the transposed version of the pencil in Equation (11.8). The dimensions of the matrices $P\left(\rho_{2}\right)^{T}$ and $Q\left(\rho_{2}\right)^{T}$ are $m \times n$. Because the rectangular one-parameter pencil $P\left(\rho_{2}\right)^{T}+\rho_{1} Q\left(\rho_{2}\right)^{T}$ is singular, the rank $r$ will be $r<m, r<n$ or both. We assume for now that $r<n$. This means that there is dependence between the columns of this pencil and that:

$$
\left(P\left(\rho_{2}\right)^{T}+\rho_{1} Q\left(\rho_{2}\right)^{T}\right) z\left(\rho_{1}, \rho_{2}\right)=0
$$

has a non-zero solution $z\left(\rho_{1}, \rho_{2}\right)$ depending polynomially on $\rho_{1}$ and $\rho_{2}$. Take such a solution $z$ in which the degree of $\rho_{1}$ is $\eta_{1}$, the degree of $\rho_{2}$ is $\eta_{2}$ and where $\eta_{1}$ is as small as possible. Once $\eta_{1}$ is chosen, then subsequently $\eta_{2}$ should be as small as possible too. Then:

$$
z\left(\rho_{1}, \rho_{2}\right)=z_{0}\left(\rho_{2}\right)-\rho_{1} z_{1}\left(\rho_{2}\right)+\rho_{1}^{2} z_{2}\left(\rho_{2}\right)+\ldots+(-1)^{\eta_{1}} \rho_{1}^{\eta_{1}} z_{\eta_{1}}\left(\rho_{2}\right)
$$


with $z_{0}\left(\rho_{2}\right), z_{1}\left(\rho_{2}\right), \ldots, z_{\eta_{1}}\left(\rho_{2}\right)$ all polynomial in $\rho_{2}$ with a maximal degree of $\eta_{2}$. The idea is to substitute this expression for $z\left(\rho_{1}, \rho_{2}\right)$ into (11.22), which leads to:

$$
\begin{aligned}
& \left(P\left(\rho_{2}\right)^{T}+\rho_{1} Q\left(\rho_{2}\right)^{T}\right)\left(z_{0}\left(\rho_{2}\right)-\rho_{1} z_{1}\left(\rho_{2}\right)+\rho_{1}^{2} z_{2}\left(\rho_{2}\right)+\ldots+(-1)^{\eta_{1}} \rho_{1}^{\eta_{1}} z_{\eta_{1}}\left(\rho_{2}\right)\right)= \\
& \left.\begin{array}{lll}
1 & \cdot\left(\begin{array}{ll}
+P\left(\rho_{2}\right)^{T} z_{0}\left(\rho_{2}\right) & \\
\rho_{1} & \cdot \\
\rho_{1}^{2} & -P\left(\rho_{2}\right)^{T} z_{1}\left(\rho_{2}\right)
\end{array}\right. \\
+P\left(\rho_{2}\right)^{T} z_{2}\left(\rho_{2}\right) & +Q\left(\rho_{2}\right)^{T} z_{0}\left(\rho_{2}\right)
\end{array}\right)\left\{\begin{array}{l}
+ \\
+ \\
+
\end{array}\right.
\end{aligned}
$$

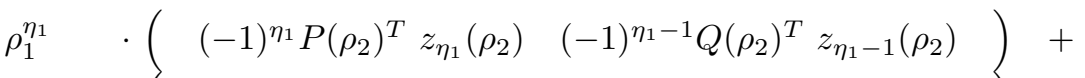

$$
\begin{aligned}
& \rho_{1}^{\eta_{1}+1} \cdot\left(\begin{array}{lll}
(-1)^{\eta_{1}} & Q\left(\rho_{2}\right)^{T} & z_{\eta_{1}}\left(\rho_{2}\right)
\end{array}\right)=0
\end{aligned}
$$

When the relationships for the coefficients are worked out, this generates a system of equations which can be expressed in matrix-vector form as follows:

$$
\left.\begin{array}{ccccc}
P\left(\rho_{2}\right)^{T} & 0 & \ldots & \ldots & 0 \\
Q\left(\rho_{2}\right)^{T} & P\left(\rho_{2}\right)^{T} & & & \vdots \\
0 & Q\left(\rho_{2}\right)^{T} & \ddots & & \vdots \\
0 & 0 & \ddots & \ddots & \vdots \\
\vdots & \vdots & & \ddots & P\left(\rho_{2}\right)^{T} \\
0 & 0 & \ldots & \ldots & Q\left(\rho_{2}\right)^{T}
\end{array}\right)\left(\begin{array}{c} 
\\
z_{0}\left(\rho_{2}\right) \\
-z_{1}\left(\rho_{2}\right) \\
z_{2}\left(\rho_{2}\right) \\
\vdots \\
(-1)^{\eta_{1}} z_{\eta_{1}}\left(\rho_{2}\right)
\end{array}\right)=
$$

where the block matrix $M_{\eta_{1}, \eta_{2}}\left(\rho_{2}\right)$ has dimensions $\left(\eta_{1}+2\right) m \times\left(\eta_{1}+1\right) n$. The rank of the matrix $M_{\eta_{1}, \eta_{2}}\left(\rho_{2}\right)$ is denoted by $r_{\eta_{1}, \eta_{2}}$ and it holds that $r_{\eta_{1}, \eta_{2}}<\left(\eta_{1}+1\right) n$.

Since the variable $\eta_{2}$ denotes the degree of $\rho_{2}$ in $z\left(\rho_{1}, \rho_{2}\right), z_{i}\left(\rho_{2}\right)$ can be written as:

$$
z_{i}\left(\rho_{2}\right)=z_{i, 0}+\rho_{2} z_{i, 1}+\rho_{2}^{2} z_{i, 2}+\ldots+\rho_{2}^{\eta_{2}} z_{i, \eta_{2}}
$$

for $i=0, \ldots, \eta_{1}$.

When (11.26) is substituted into the equations of (11.25) and the relationships for the coefficients are worked out, a system of equations can be set up which only involves constant coefficients. When $P\left(\rho_{2}\right)^{T}=B^{T}+\rho_{2} D^{T}$ and $Q\left(\rho_{2}\right)^{T}=C^{T}$ are plugged into the expressions, the resulting system of equations can be written in 
matrix-vector form as follows:

$$
M_{\eta_{1}, \eta_{2}}\left(\begin{array}{c}
z_{0,0} \\
\vdots \\
\frac{z_{0, \eta_{2}}}{\vdots} \\
\hline z_{\eta_{1}, 0} \\
\vdots \\
z_{\eta_{1}, \eta_{2}}
\end{array}\right)=0
$$

where the matrix $M_{\eta_{1}, \eta_{2}}$ is a block matrix containing the coefficient matrices $B^{T}$, $C^{T}$, and $D^{T}$ from Equation (11.7). The matrix $M_{\eta_{1}, \eta_{2}}$ is independent of $\rho_{1}$ and $\rho_{2}$ and has a dimension of $\left(\eta_{1}+2\right)\left(\eta_{2}+2\right) m \times\left(\eta_{1}+1\right)\left(\eta_{2}+1\right) n$.

Any non-zero vector in the kernel of the matrix $M_{\eta_{1}, \eta_{2}}$ in (11.27) yields a corresponding solution $z\left(\rho_{1}, \rho_{2}\right)$ of degree at most $\eta_{1}$ and $\eta_{2}$ for $\rho_{1}$ and $\rho_{2}$, respectively. Therefore, a computation of the kernel of the matrix $M_{\eta_{1}, \eta_{2}}$ suffices to compute a vector $z\left(\rho_{1}, \rho_{2}\right)$ in $(11.22)$.

Example 11.1. To illustrate the technique described above, a small example is given. Note that this example only serves the purpose of making clear how to construct the block matrix $M_{\eta_{1}, \eta_{2}}$ in (11.27).

Suppose the two-parameter linear matrix pencil $B^{T}+\rho_{1} C^{T}+\rho_{2} D^{T}$ (with matrices of dimensions $m \times n)$ is given and one wants to check whether there is a solution $z\left(\rho_{1}, \rho_{2}\right)$ of $\left(B^{T}+\rho_{1} C^{T}+\rho_{2} D^{T}\right) z\left(\rho_{1}, \rho_{2}\right)=0$ where the degree of $\rho_{1}$ is $\eta_{1}=1$ and the degree of $\rho_{2}$ is $\eta_{2}=2$.

First, the expression $\left(B^{T}+\rho_{1} C^{T}+\rho_{2} D^{T}\right) z\left(\rho_{1}, \rho_{2}\right)=0$ is written as $\left(P\left(\rho_{2}\right)^{T}+\right.$ $\left.\rho_{1} Q\left(\rho_{2}\right)^{T}\right) z\left(\rho_{1}, \rho_{2}\right)=0$ where $P\left(\rho_{2}\right)^{T}=B^{T}+\rho_{2} D^{T}$ and $Q\left(\rho_{2}\right)^{T}=C^{T}$.

We know that every solution $z\left(\rho_{1}, \rho_{2}\right)$ should take the form $z_{0}\left(\rho_{2}\right)-\rho_{1} z_{1}\left(\rho_{2}\right)$, because $\eta_{1}=1$. If such a solution is substituted into $\left(P\left(\rho_{2}\right)^{T}+\rho_{1} Q\left(\rho_{2}\right)^{T}\right) z\left(\rho_{1}, \rho_{2}\right)=$ 0 , and the relationships for the coefficients of the powers of $\rho_{1}$ are worked out, it can be written as:

$$
\left(\begin{array}{cc}
P\left(\rho_{2}\right)^{T} & 0 \\
Q\left(\rho_{2}\right)^{T} & P\left(\rho_{2}\right)^{T} \\
0 & Q\left(\rho_{2}\right)^{T}
\end{array}\right)\left(\begin{array}{r}
z_{0}\left(\rho_{2}\right) \\
-z_{1}\left(\rho_{2}\right)
\end{array}\right)=M_{1,2}\left(\rho_{1}, \rho_{2}\right)\left(\begin{array}{r}
z_{0}\left(\rho_{2}\right) \\
-z_{1}\left(\rho_{2}\right)
\end{array}\right)=0
$$

where the dimension of the block matrix $M_{1,2}\left(\rho_{1}, \rho_{2}\right)$ is $\left(\eta_{1}+2\right) m \times\left(\eta_{1}+1\right) n=$ $3 m \times 2 n$.

The degree of $\rho_{2}$ is chosen here as 2 and therefore $z_{i}\left(\rho_{2}\right)$ can be written as $z_{i}\left(\rho_{2}\right)=z_{i, 0}+\rho_{2} z_{i, 1}+\rho_{2}^{2} z_{i, 2}$ for $i=0,1$. This can be substituted into (11.28). When the relationships for the coefficients are worked out and $P\left(\rho_{2}\right)^{T}=B^{T}+\rho_{2} D^{T}$ 
and $Q\left(\rho_{2}\right)^{T}=C^{T}$ are plugged in too, the following system of equations arises:

$$
M_{1,2}\left(\begin{array}{c}
z_{0,0} \\
z_{0,1} \\
z_{0,2} \\
\hline z_{1,0} \\
z_{1,1} \\
z_{1,2}
\end{array}\right)=0
$$

where the dimension of $M_{1,2}$ is $\left(\eta_{1}+2\right)\left(\eta_{2}+2\right) m \times\left(\eta_{1}+1\right)\left(\eta_{2}+1\right) n=12 m \times 6 n$ and where $M_{1,2}$ is structured as:

$$
M_{1,2}=\left(\begin{array}{ccc|ccc}
B^{T} & 0 & 0 & 0 & 0 & 0 \\
D^{T} & B^{T} & 0 & 0 & 0 & 0 \\
0 & D^{T} & B^{T} & 0 & 0 & 0 \\
0 & 0 & D^{T} & 0 & 0 & 0 \\
\hline C^{T} & 0 & 0 & B^{T} & 0 & 0 \\
0 & C^{T} & 0 & D^{T} & B^{T} & 0 \\
0 & 0 & C^{T} & 0 & D^{T} & B^{T} \\
0 & 0 & 0 & 0 & 0 & D^{T} \\
\hline 0 & 0 & 0 & C^{T} & 0 & 0 \\
0 & 0 & 0 & 0 & C^{T} & 0 \\
0 & 0 & 0 & 0 & 0 & C^{T} \\
0 & 0 & 0 & 0 & 0 & 0
\end{array}\right) .
$$

If the rank of this matrix $M_{1,2}$ is smaller than $6 n$, one can compute solutions $z\left(\rho_{1}, \rho_{2}\right)$ of $\left(B^{T}+\rho_{1} C^{T}+\rho_{2} D^{T}\right) z\left(\rho_{1}, \rho_{2}\right)=0$, by computing non-zero vectors in the kernel of this matrix: a non-zero vector in this kernel is structured as $\left[z_{0,0}^{T}, z_{0,1}^{T}, z_{0,2}^{T}\right.$, $\left.z_{1,0}^{T}, z_{1,1}^{T}, z_{1,2}^{T}\right]^{T}$ and from these (sub)vectors, one can construct the solution $z\left(\rho_{1}, \rho_{2}\right)$ as $z\left(\rho_{1}, \rho_{2}\right)=\left(z_{0,0}+\rho_{2} z_{0,1}+\rho_{2}^{2} z_{0,2}\right)-\rho_{1}\left(z_{1,0}+\rho_{2} z_{1,1}+\rho_{2}^{2} z_{1,2}\right)$.

Once a solution $z\left(\rho_{1}, \rho_{2}\right)=z_{0}\left(\rho_{2}\right)-\rho_{1} z_{1}\left(\rho_{2}\right)+\ldots+(-1)^{\eta_{1}} \rho_{1}^{\eta_{1}} z_{\eta_{1}}\left(\rho_{2}\right)$ of $(11.22)$ has become available, it can be used to split off one or more singular parts. As stated in Proposition 11.1, the only singular parts that occur in such a pencil are $L_{\eta}^{T}(11.14)$ of dimension $\left(\eta_{1}+1\right) \times \eta_{1}$. Because we are working with the transposes of the matrices $P\left(\rho_{2}\right)$ and $Q\left(\rho_{2}\right)$, we find the transposed version of the singular blocks $L_{\eta}^{T}$. Singular blocks (11.13) of dimension $\varepsilon_{1} \times\left(\varepsilon_{1}+1\right)$ can not occur here because they would allow for an infinite number of solutions to the original problem.

If there exists a solution $z\left(\rho_{1}, \rho_{2}\right)$, where the degree of $\rho_{1}$ is $\eta_{1}$, the degree of $\rho_{2}$ is $\eta_{2}$ and $\eta_{1}$ is as small as possible, then the matrix $P\left(\rho_{2}\right)+\rho_{1} Q\left(\rho_{2}\right)$ is equivalent to a matrix of the form:

$$
\left(\begin{array}{cc}
L_{\eta_{1}}^{T} & 0 \\
0 & \tilde{P}\left(\rho_{2}\right)+\rho_{1} \tilde{Q}\left(\rho_{2}\right)
\end{array}\right) .
$$

The singular block $L_{\eta_{1}}^{T}$ has dimension $\left(\eta_{1}+1\right) \times \eta_{1}$ and it corresponds to unwanted indeterminate eigenvalues. Therefore it can legally be split off from the pencil 
resulting in a smaller sized pencil $\tilde{P}\left(\rho_{2}\right)+\rho_{1} \tilde{Q}\left(\rho_{2}\right)$, still containing all the relevant (meaningful) solutions. If there are more than one solution $z\left(\rho_{1}, \rho_{2}\right)$ of degrees $\eta_{1}$ and $\eta_{2}$, let us say $g$ solutions, then $g$ singular blocks of dimensions $\left(\eta_{1}+1\right) \times \eta_{1}$ can be split off at once, provided that independence for the associated basis holds.

The transformation of $P\left(\rho_{2}\right)+\rho_{1} Q\left(\rho_{2}\right)$ into a quasi diagonal pencil (11.31) can be done using a generalization of the transformation technique mentioned in Section 10.3.1 which is based on choosing appropriate transformation matrices $V$ and $W$. Consider the pencil $P\left(\rho_{2}\right)^{T}+\rho_{1} Q\left(\rho_{2}\right)^{T}$ of dimension $m \times n$ as a family of operators mapping $\mathbb{C}^{n}\left(\rho_{2}\right)$ to $\mathbb{C}^{m}\left(\rho_{2}\right)$. With a suitable choice of bases in the spaces the matrix corresponding to the operator $P\left(\rho_{2}\right)+\rho_{1} Q\left(\rho_{2}\right)$ takes the form (11.31).

Such a suitable choice corresponds to taking the vectors $z_{0}\left(\rho_{2}\right), \ldots, z_{\eta_{1}}\left(\rho_{2}\right)$ of a solution $z\left(\rho_{1}, \rho_{2}\right)$ of $(11.22)$ as a part of the basis for $\mathbb{C}^{n}\left(\rho_{2}\right)$ and $P\left(\rho_{2}\right)^{T} z_{1}\left(\rho_{2}\right), \ldots$, $P\left(\rho_{2}\right)^{T} z_{\eta_{1}}\left(\rho_{2}\right)$ as a part of the basis for $\mathbb{C}^{m}\left(\rho_{2}\right)$. This is allowed since $\left\{z_{0}\left(\rho_{2}\right), \ldots\right.$, $\left.z_{\eta_{1}}\left(\rho_{2}\right)\right\}$ and $\left\{P\left(\rho_{2}\right)^{T} z_{1}\left(\rho_{2}\right), \ldots, P\left(\rho_{2}\right)^{T} z_{\eta_{1}}\left(\rho_{2}\right)\right\}$ are linearly independent sets. If the bases for $\mathbb{C}^{m}\left(\rho_{2}\right)$ and $\mathbb{C}^{n}\left(\rho_{2}\right)$ are completed with standard basis column vectors $w_{\eta_{1}+1}, \ldots, w_{m}$ and $v_{\eta_{1}+2}, \ldots, v_{n}$ to make $W$ and $V$ square and invertible, then the transformation:

$$
W^{-1}\left(\rho_{2}\right)\left(P\left(\rho_{2}\right)^{T}+\rho_{1} Q\left(\rho_{2}\right)^{T}\right) V\left(\rho_{2}\right)
$$

yields the equivalent pencil (11.31), up to the upper right zero block. To make the upper right block also zero, the columns $w_{\eta_{1}+1}, \ldots, w_{m}$ and $v_{\eta_{1}+2}, \ldots, v_{n}$ should be chosen in a special way.

In order to get rid of all the singular parts $L_{\eta_{i}}^{T}$ of the matrix pencil, one has to search for solutions $z\left(\rho_{1}, \rho_{2}\right)$ for different values of $\eta_{1}$ and $\eta_{2}$. We have previously required $\eta_{1}$ to be minimal first, and (given $\eta_{1}$ ) $\eta_{2}$ to be minimal next. Once one or more such solutions $z\left(\rho_{1}, \rho_{2}\right)$ are found, one has to construct the transformation matrices $W\left(\rho_{2}\right)$ and $V\left(\rho_{2}\right)$ and to apply these as in (11.32). This can be repeated iteratively until no more singular parts exist. Note that the parts that are split off increase in size in every step which makes it possible to put limitations on the dimensions of the next singular blocks that can be found. E.g., if $m \times n$ is currently reduced to $14 \times 12$ and $\eta_{1}=4$ is exhausted, we know that (i) $14-12=2$ more blocks will be found and (ii) these are of dimension $6 \times 5$ and $8 \times 7$ or two blocks of dimension $7 \times 6$, since all other options are no longer available.

Example 11.1 (continued). In Example 11.1 the value of $\eta_{1}$ is chosen as 1 and an approach is given to compute the vectors $z_{0}\left(\rho_{2}\right)$ and $z_{1}\left(\rho_{2}\right)$ which satisfy the equations in (11.28). To transform the pencil $P\left(\rho_{2}\right)^{T}+\rho_{1} Q\left(\rho_{2}\right)^{T}$ into the equivalent form 
(11.31), the transformation matrices $V\left(\rho_{2}\right)$ and $W\left(\rho_{2}\right)$ are constructed as follows:

$$
\begin{aligned}
& W\left(\rho_{2}\right)=\left(\begin{array}{l|l|l|l} 
& & & \\
P\left(\rho_{2}\right)^{T} z_{1}\left(\rho_{2}\right) & w_{2} & \ldots & w_{m} \\
& & &
\end{array}\right), \\
& V\left(\rho_{2}\right)=\left(\begin{array}{l|l|l|l|l}
z_{0}\left(\rho_{2}\right) & z_{1}\left(\rho_{2}\right) & v_{3} & \ldots & v_{n} \\
& & & &
\end{array}\right)
\end{aligned}
$$

where $P\left(\rho_{2}\right)^{T} z_{1}\left(\rho_{2}\right), z_{0}\left(\rho_{2}\right)$ and $z_{1}\left(\rho_{2}\right)$ are the first columns of the matrices $W\left(\rho_{2}\right)$ and $V\left(\rho_{2}\right)$ and where $w_{2}, \ldots, w_{m}$ and $v_{3}, \ldots, v_{n}$ are basis column vectors to complete these matrices up to square invertible matrices.

We know from the equations in (11.25) that the following holds: $P\left(\rho_{2}\right)^{T} z_{0}\left(\rho_{2}\right)$ $=0,(-1)^{\eta_{1}} Q\left(\rho_{2}\right)^{T} z_{\eta_{1}}\left(\rho_{2}\right)=0$ and $P\left(\rho_{2}\right)^{T} z_{i}\left(\rho_{2}\right)=Q\left(\rho_{2}\right)^{T} z_{i-1}\left(\rho_{2}\right)$ for $i=1, \ldots, \eta_{1}$. In this example this means the following:

$$
\left\{\begin{array}{l}
P\left(\rho_{2}\right)^{T} z_{0}\left(\rho_{2}\right)=0 \\
Q\left(\rho_{2}\right)^{T} z_{1}\left(\rho_{2}\right)=0 \\
P\left(\rho_{2}\right)^{T} z_{1}\left(\rho_{2}\right)=Q\left(\rho_{2}\right)^{T} z_{0}\left(\rho_{2}\right)
\end{array}\right.
$$

The required transformation here is:

$$
W\left(\rho_{2}\right)^{-1}\left(P\left(\rho_{2}\right)^{T}+\rho_{1} Q\left(\rho_{2}\right)^{T}\right) V\left(\rho_{2}\right) .
$$

This yields for $W\left(\rho_{2}\right)^{-1}\left(P\left(\rho_{2}\right)^{T} V\left(\rho_{2}\right)+\rho_{1} Q\left(\rho_{2}\right)^{T} V\left(\rho_{2}\right)\right)$ the following:

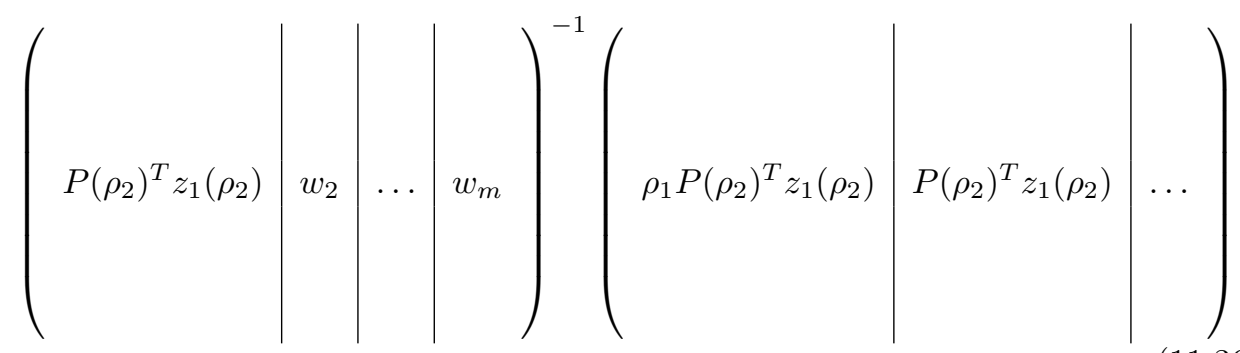


which is equivalent with (11.31) and where the upper left block is the transposed of $L_{\eta_{1}}^{T}$ and is of dimension $\eta_{1} \times\left(\eta_{1}+1\right)=1 \times 2$ :

$$
L_{\eta_{1}}=\left(\begin{array}{ll}
\rho_{1} & 1
\end{array}\right)^{T}
$$

Finally, this singular $L_{\eta_{1}}^{T}$ part can be split off from the matrix pencil.

Once all the singular parts of the pencil $P\left(\rho_{2}\right)^{T}+\rho_{1} Q\left(\rho_{2}\right)^{T}$ are split off, all the transformation matrices $V\left(\rho_{2}\right)$ and $W\left(\rho_{2}\right)$ are available. Using the result in Proposition 11.2, one has compute the values which make each of the transformation matrices $V\left(\rho_{2}\right)$ and $W\left(\rho_{2}\right)$ singular as in (11.20). When the solutions $\rho_{2}$ of $(11.20)$ are known, they can be substituted into the original problem (11.3), which yields the square polynomial eigenvalue problems in the only parameter $\rho_{1}$ :

$$
\left\{\begin{array}{l}
\tilde{A}_{\tilde{a}_{N-1}}\left(\rho_{1}\right)^{T} v=0 \\
\tilde{A}_{\tilde{a}_{N-2}}\left(\rho_{1}\right)^{T} v=0
\end{array}\right.
$$

From an eigenvector $v$ of (11.38), the values for $x_{1}, \ldots, x_{N}$ can be read off, since these vectors exhibit the Stetter structure. All the tuples of solutions $\left(\rho_{1}, \rho_{2}, x_{1}, \ldots\right.$, $x_{N}$ ) found in this way are the solutions of the quadratic system of equations (11.1) which satisfy both the constraints (11.2). The solutions of which the values for $\rho_{1}$ and $\rho_{2}$ are real, may give feasible approximations $G(s)$ of order $N-3$. The real and stable approximation $G(s)$ with the smallest $H_{2}$-criterion value is the globally optimal approximation of order $N-3$.

Remark 11.2. Using this approach, it is possible to split off singular parts of a matrix pencil which is linear in $\rho_{1}$ and $\rho_{2}$. After applying a transformation $W^{-1}\left(\rho_{2}\right)\left(P\left(\rho_{2}\right)^{T}+\right.$ $\left.\rho_{1} Q\left(\rho_{2}\right)^{T}\right) V\left(\rho_{2}\right)$, the lower right block $\tilde{P}\left(\rho_{2}\right)+\rho_{1} \tilde{Q}\left(\rho_{2}\right)$ of the equivalent pencil in (11.31) is not necessarily linear in $\rho_{2}$ anymore. Therefore it is possible that an extra linearization step (as described in Section 11.2) is needed after splitting off a singular part. With such a linearization the matrix dimensions may grow rapidly.

\subsubsection{Computing the transformation matrices for a non-linear two-parameter matrix pencil}

In Equation (11.22) and in the steps thereafter, it does not matter whether the matrices $P\left(\rho_{2}\right)$ and $Q\left(\rho_{2}\right)$ are linear in $\rho_{2}$ or not. Moreover, even the variable $\rho_{1}$ is allowed to show up in a non-linear fashion when following a different approach than in the previous section. Without the linearization step, which brings (11.3) into the form of (11.4), it is possible to work with smaller sized matrices. This is the subject of this subsection. 
The two-parameter polynomial eigenvalue problem (11.3) involving the two matrices $\tilde{A}_{\tilde{a}_{N-1}}\left(\rho_{1}, \rho_{2}\right)^{T}$ and $\tilde{A}_{\tilde{a}_{N-2}}\left(\rho_{1}, \rho_{2}\right)^{T}$ can be jointly written as:

$$
\left[\begin{array}{c}
\tilde{A}_{\tilde{a}_{N-1}}\left(\rho_{1}, \rho_{2}\right)^{T} \\
\tilde{A}_{\tilde{a}_{N-2}}\left(\rho_{1}, \rho_{2}\right)^{T}
\end{array}\right] v=A\left(\rho_{1}, \rho_{2}\right) v=0
$$

where the matrix $A\left(\rho_{1}, \rho_{2}\right)$ is rectangular and polynomial in both $\rho_{1}$ and $\rho_{2}$. This matrix has $2^{N}$ columns and at most $2^{N+1}$ (unique) rows. The total degree of the terms in $\rho_{1}$ and $\rho_{2}$ in the matrix $A\left(\rho_{1}, \rho_{2}\right)$ is denoted by $\tilde{N}$ and, as stated in Corollary 10.2, is equal to $N-1$ (where $N$ is the order of the original model $H(s)$ to reduce). Because we are searching for blocks $L_{\eta_{1}}^{T}$ to split off, we continue working with the transposed version of the matrix $A\left(\rho_{1}, \rho_{2}\right)$. Thus, the matrix $A\left(\rho_{1}, \rho_{2}\right)^{T}$ can be written as:

$$
A\left(\rho_{1}, \rho_{2}\right)^{T}=\left(M_{0}\left(\rho_{2}\right)+\rho_{1} M_{1}\left(\rho_{2}\right)+\ldots+\rho_{1}^{\tilde{N}} M_{\tilde{N}}\left(\rho_{2}\right)\right)
$$

where the matrices $M_{i}\left(\rho_{2}\right)$ are expanded as:

$$
M_{i}\left(\rho_{2}\right)=M_{i, 0}+\rho_{2} M_{i, 1}+\rho_{2}^{2} M_{i, 2}+\ldots+\rho_{2}^{\tilde{N}-i} M_{i, \tilde{N}-i}
$$

for $i=0, \ldots, \tilde{N}$.

The matrix $A\left(\rho_{1}, \rho_{2}\right)^{T}$ is rectangular of dimension $m \times n$. Note that the dimensions of the matrix $A\left(\rho_{1}, \rho_{2}\right)^{T}$ are much smaller than the dimensions of the (linearized) matrices $P\left(\rho_{2}\right)^{T}$ and $Q\left(\rho_{2}\right)^{T}$ in the previous subsection. Therefore $m$ and $n$ denote different, smaller values here. Also in this non-linear case one should be able to split off singular parts. Assuming that there is dependency between the columns of the matrix $A\left(\rho_{1}, \rho_{2}\right)^{T}$ means that:

$$
A\left(\rho_{1}, \rho_{2}\right)^{T} z\left(\rho_{1}, \rho_{2}\right)=0
$$

has a non-zero solution $z\left(\rho_{1}, \rho_{2}\right)$ depending on $\rho_{1}$ and $\rho_{2}$. Take such a solution $z$ in which the degree of $\rho_{1}$ is $\eta_{1}$, the degree of $\rho_{2}$ is $\eta_{2}$ and where $\eta_{1}$ is as small as possible. Then $z\left(\rho_{1}, \rho_{2}\right)$ can be written as:

$$
z\left(\rho_{1}, \rho_{2}\right)=z_{0}\left(\rho_{2}\right)-\rho_{1} z_{1}\left(\rho_{2}\right)+\rho_{1}^{2} z_{2}\left(\rho_{2}\right)+\ldots+(-1)^{\eta_{1}} \rho_{1}^{\eta_{1}} z_{\eta_{1}}\left(\rho_{2}\right)
$$

where $z_{0}\left(\rho_{2}\right), z_{1}\left(\rho_{2}\right), \ldots, z_{\eta_{1}}\left(\rho_{2}\right)$ all polynomial in $\rho_{2}$.

Substituting such a solution $z\left(\rho_{1}, \rho_{2}\right)$ into (11.42) where the matrix $A\left(\rho_{1}, \rho_{2}\right)^{T}$ is written as in (11.40), yields the following when the relationships for the coefficients of the powers of $\rho_{1}$ are worked out and the resulting expressions are written in matrix- 
vector form:

$$
\left(\begin{array}{cccc}
M_{0}\left(\rho_{2}\right) & & & 0 \\
M_{1}\left(\rho_{2}\right) & M_{0}\left(\rho_{2}\right) & & \\
M_{2}\left(\rho_{2}\right) & M_{1}\left(\rho_{2}\right) & \ddots & \\
\vdots & M_{2}\left(\rho_{2}\right) & \ddots & M_{0}\left(\rho_{2}\right) \\
M_{\tilde{N}}\left(\rho_{2}\right) & \vdots & & M_{1}\left(\rho_{2}\right) \\
& M_{\tilde{N}}\left(\rho_{2}\right) & & M_{2}\left(\rho_{2}\right) \\
& & \ddots & \vdots \\
0 & & & M_{\tilde{N}}\left(\rho_{2}\right)
\end{array}\right)\left(\begin{array}{c}
z_{0}\left(\rho_{2}\right) \\
-z_{1}\left(\rho_{2}\right) \\
\vdots \\
(-1)^{\eta_{1}} z_{\eta_{1}}\left(\rho_{2}\right)
\end{array}\right)=
$$

where the block matrix $M_{\eta_{1}, \eta_{2}}\left(\rho_{2}\right)$ has dimension $\left(\eta_{1}+\tilde{N}+1\right) m \times\left(\eta_{1}+1\right) n$. Furthermore it holds that the rank $r_{\eta_{1}, \eta_{2}}<\left(\eta_{1}+1\right) n$.

Because $\eta_{2}$ denotes the degree of $z\left(\rho_{1}, \rho_{2}\right)$ with respect to $\rho_{2}$, we can write:

$$
z_{i}\left(\rho_{2}\right)=z_{i, 0}+\rho_{2} z_{i, 1}+\rho_{2}^{2} z_{i, 2}+\ldots+\rho_{2}^{\eta_{2}} z_{i, \eta_{2}}
$$

for $i=0, \ldots, \eta_{1}$. When (11.45) is substituted into the equations of (11.44) and the matrices $M_{i, j}$ in Equation (11.41) are used, the following system of equations in matrix-vector form is constructed:

$$
M_{\eta_{1}, \eta_{2}}\left(\begin{array}{c}
z_{0,0} \\
\vdots \\
\frac{z_{0, \eta_{2}}}{\vdots} \\
\frac{z_{\eta_{1}, 0}}{\vdots} \\
z_{\eta_{1}, \eta_{2}}
\end{array}\right)=0
$$

where the matrix $M_{\eta_{1}, \eta_{2}}$ is a matrix of $\left(\eta_{2}+\tilde{N}+1\right)\left(\eta_{1}+\tilde{N}+1\right) m$ rows and $\left(\eta_{1}+\right.$ 1) $\left(\eta_{2}+1\right) n$ columns containing the $m \times n$ coefficient matrices $M_{i, j}$ from Equation (11.41).

Example 11.1 (continued). To illustrate the construction of the matrix $M_{\eta_{1}, \eta_{2}}$, using this approach, the example of the previous subsection is continued.

Suppose that the two-parameter matrix pencil $A\left(\rho_{1}, \rho_{2}\right)^{T}$ of dimension $m \times n$ is given where the total degree of the terms is $\tilde{N}=3$ and one wants to check whether 
there is a polynomial solution $z\left(\rho_{1}, \rho_{2}\right)$ of $A\left(\rho_{1}, \rho_{2}\right)^{T} z=0$ where the degree of $\rho_{1}$ is $\eta_{1}=1$ and the degree of $\rho_{2}$ is $\eta_{2}=2$.

Using (11.40), the expression $A\left(\rho_{1}, \rho_{2}\right)^{T} z\left(\rho_{1}, \rho_{2}\right)=0$ can be written as:

$$
\left(M_{0}\left(\rho_{2}\right)+\rho_{1} M_{1}\left(\rho_{2}\right)+\rho_{1}^{2} M_{2}\left(\rho_{2}\right)+\rho_{1}^{3} M_{3}\left(\rho_{2}\right)\right) z\left(\rho_{1}, \rho_{2}\right)=0
$$

where:

$$
\begin{aligned}
& M_{0}\left(\rho_{2}\right)=M_{0,0}+\rho_{2} M_{0,1}+\rho_{2}^{2} M_{0,2}+\rho_{2}^{3} M_{0,3} \\
& M_{1}\left(\rho_{2}\right)=M_{1,0}+\rho_{2} M_{1,1}+\rho_{2}^{2} M_{1,2} \\
& M_{2}\left(\rho_{2}\right)=M_{2,0}+\rho_{2} M_{2,1} \\
& M_{3}\left(\rho_{2}\right)=M_{3,0},
\end{aligned}
$$

and where every matrix $M_{i, j}$ is of dimension $m \times n$. Recall again that the dimensions $m$ and $n$ are smaller than in the previous subsection because no linearization step is involved here.

Every solution $z\left(\rho_{1}, \rho_{2}\right)$ should take the form $z_{0}\left(\rho_{2}\right)-\rho_{1} z_{1}\left(\rho_{2}\right)$ because $\eta_{1}=1$. If such a solution is substituted into (11.47), and the relationships for the coefficients of the powers of $\rho_{1}$ are worked out, we find:

$$
\left(\begin{array}{cc}
M_{0}\left(\rho_{2}\right) & 0 \\
M_{1}\left(\rho_{2}\right) & M_{0}\left(\rho_{2}\right) \\
M_{2}\left(\rho_{2}\right) & M_{1}\left(\rho_{2}\right) \\
M_{3}\left(\rho_{2}\right) & M_{2}\left(\rho_{2}\right) \\
0 & M_{3}\left(\rho_{2}\right)
\end{array}\right)\left(\begin{array}{r}
z_{0}\left(\rho_{2}\right) \\
-z_{1}\left(\rho_{2}\right)
\end{array}\right)=M_{1,2}\left(\rho_{2}\right)\left(\begin{array}{r}
z_{0}\left(\rho_{2}\right) \\
-z_{1}\left(\rho_{2}\right)
\end{array}\right)=0
$$

where the dimension of the block matrix $M_{1,2}\left(\rho_{2}\right)$ is $\left(\eta_{1}+\tilde{N}+1\right) m \times\left(\eta_{1}+1\right) n=$ $5 m \times 2 n$.

The degree of $\rho_{2}$ is $\eta_{2}=2$ and therefore $z_{i}\left(\rho_{2}\right)$ can be written as $z_{i}\left(\rho_{2}\right)=$ $z_{i, 0}+\rho_{2} z_{i, 1}+\rho_{2}^{2} z_{i, 2}$ for $i=0,1$. This can be substituted into (11.49). When the relationships for the coefficients of the powers of $\rho_{2}$ are worked out again, the following system of equations in matrix-vector form occurs:

$$
M_{1,2}\left(\begin{array}{c}
z_{0,0} \\
z_{0,1} \\
z_{0,2} \\
\hline z_{1,0} \\
z_{1,1} \\
z_{1,2}
\end{array}\right)=0
$$

where the dimension of the matrix $M_{1,2}$ is $\left(\eta_{2}+\tilde{N}+1\right)\left(\eta_{1}+\tilde{N}+1\right) m \times\left(\eta_{1}+1\right)\left(\eta_{2}+\right.$ 
1) $n=30 m \times 6 n:$

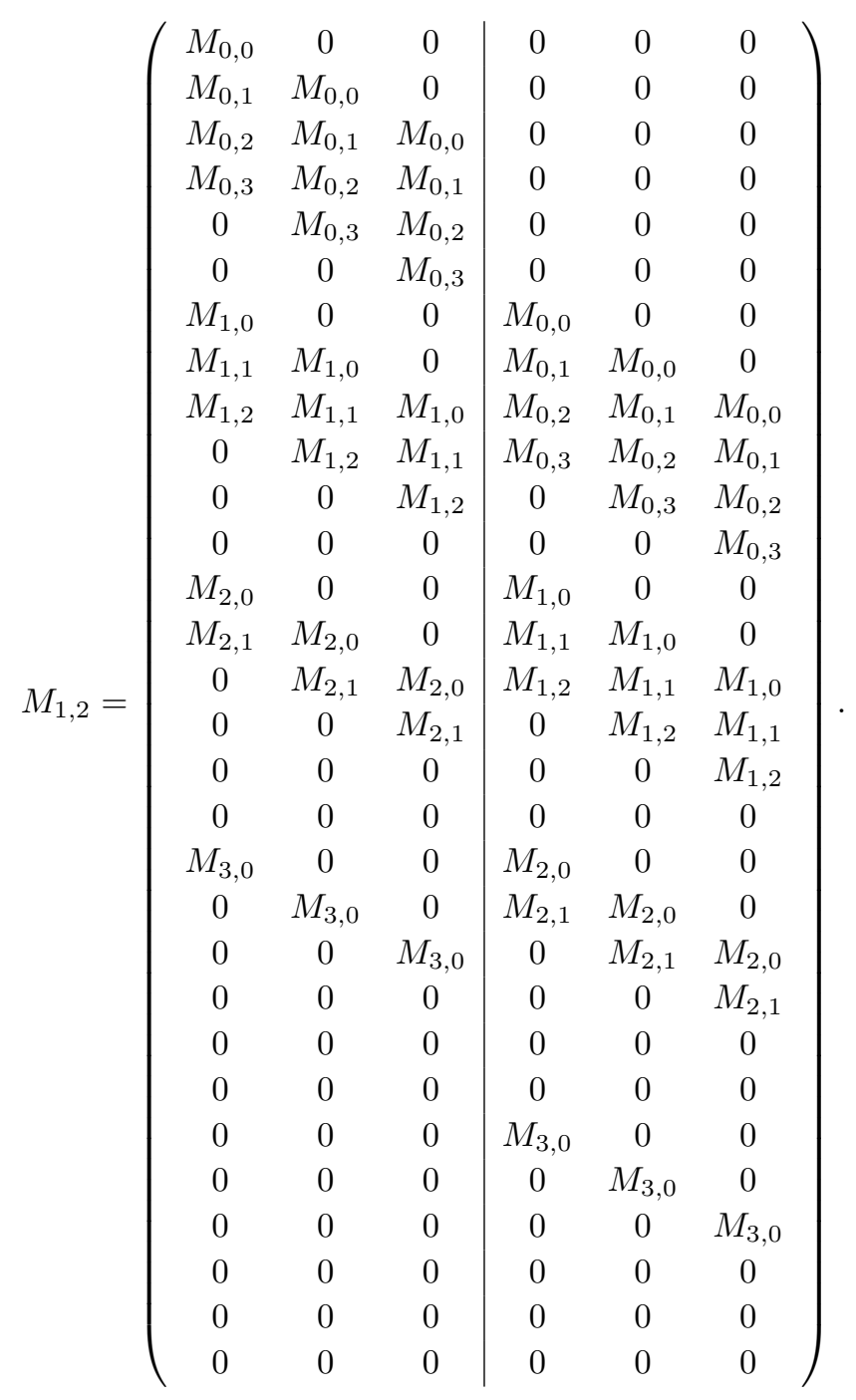

Note that the zero rows are redundant here and may be removed from the matrix $M_{1,2}$.

If the rank of this matrix $M_{1,2}$ is smaller than $6 n$, one can compute all solutions $z\left(\rho_{1}, \rho_{2}\right)$ of $A\left(\rho_{1}, \rho_{2}\right)^{T} z=0$ : every non-zero vector in the kernel of this matrix yields a solution $z\left(\rho_{1}, \rho_{2}\right)$. Every vector in the kernel has the structure $\left[z_{0,0}^{T}, z_{0,1}^{T}, z_{0,2}^{T}, z_{1,0}^{T}, z_{1,1}^{T}, z_{1,2}^{T}\right]^{T}$ and one can construct the solution $z\left(\rho_{1}, \rho_{2}\right)$ from it as: $z\left(\rho_{1}, \rho_{2}\right)=\left(z_{0,0}+\rho_{2} z_{0,1}+\rho_{2}^{2} z_{0,2}\right)-\rho_{1}\left(z_{1,0}+\rho_{2} z_{1,1}+\rho_{2}^{2} z_{1,2}\right)$.

In this approach, the structure of the matrix $V\left(\rho_{2}\right)$ is unchanged: the first columns of $V\left(\rho_{2}\right)$ are chosen (as before) as the vectors $z_{0}\left(\rho_{2}\right), \ldots, z_{\eta_{1}}\left(\rho_{2}\right)$. However, the first columns of the transformation matrix $W$ are chosen in a different way. The $\eta_{1}$ first columns of $W\left(\rho_{1}, \rho_{2}\right)$ are denoted by $w_{0}, \ldots, w_{\eta_{1}-1}$ and are given by the following 
expression:

$$
w_{c}=M_{0}\left(\rho_{2}\right) z_{c+1}\left(\rho_{2}\right)-\sum_{i=2}^{\tilde{N}} \rho_{1}^{(i-1)} \sum_{j=i}^{\min (i+c, \tilde{N})} M_{j}\left(\rho_{2}\right) z_{i+c-j}\left(\rho_{2}\right),
$$

for $c=0, \ldots, \eta_{1}-1$. Both the matrices $V\left(\rho_{2}\right)$ and $W\left(\rho_{1}, \rho_{2}\right)$ are completed with standard basis vectors to make them square and invertible.

The transformation:

$$
W\left(\rho_{1}, \rho_{2}\right)^{-1}\left(M_{0}\left(\rho_{2}\right)+\rho_{1} M_{1}\left(\rho_{2}\right)+\ldots+\rho_{1}^{\tilde{N}} M_{\tilde{N}}\left(\rho_{2}\right)\right) V\left(\rho_{2}\right)
$$

yields an equivalent pencil which has the same structure, up to the upper right zero block, as the pencil in Equation (11.31) and where the block $L_{\eta_{1}}^{T}$ has the same structure and dimensions of $\left(\eta_{1}+1\right) \times \eta_{1}$ as the block in (11.14). To make the upper right block also zero, the standard basis vectors of the matrices $W$ and $V$ should be chosen in a special way.

Using this technique one is able to split off singular parts of a non-linear matrix pencil in two parameters: search for solutions $z\left(\rho_{1}, \rho_{2}\right)$ of minimal but increasing degrees $\eta_{1}$ for $\rho_{1}$ and degrees $\eta_{2}$ for $\rho_{2}$ (which are as small as possible for the given degree $\left.\eta_{1}\right)$ and split off the singular parts of dimensions $\left(\eta_{1}+1\right) \times \eta_{1}$ until no more singular parts exist or until the matrix size has become $0 \times 0$. Because the existence of a regular part is impossible (because of the same reasons mentioned in the previous section), the solutions $\rho_{1}$ and $\rho_{2}$ of (11.39) are the values which make the transformation matrices $V\left(\rho_{2}\right)$ and $W\left(\rho_{1}, \rho_{2}\right)$ singular.

Remark 11.3. In the approach of Subsection 11.3.2, it is possible that the pencil is non-linear after splitting off a singular part. Therefore an additional linearization step is required. In the approach presented in this subsection, the pencil remains non-linear after splitting off a singular part. Therefore the dimensions of the matrices involved in this case are always smaller than in the linear case of Subsection 11.3.2.

Remark 11.4. A drawback of this approach is that the solutions $\rho_{1}$ and $\rho_{2}$ may remain among the values which make the matrix $W\left(\rho_{1}, \rho_{2}\right)$ singular. In that case one has to solve a square polynomial eigenvalue problem in two variables, which can be difficult, especially for large matrices or high powers of $\rho_{1}$ and $\rho_{2}$. Moreover, computing the inverse of $W\left(\rho_{1}, \rho_{2}\right)$ to perform the transformation of the matrix pencil, can be computationally challenging when using exact arithmetic because of the existence of two variables with possibly high degrees.

Remark 11.5. The value of $\tilde{N}$ can change (increase or decrease), during the process of splitting off singular parts of the matrix pencil. This has a direct effect on the decomposition of the matrix pencil in (11.40) and (11.41). 
Example 11.1 (continued). The transformation matrices for the running example using this approach are constructed as follows. The matrix $V$ is structured as:

$$
V\left(\rho_{2}\right)=\left(\begin{array}{l|l|l|l|l} 
& & & & \\
z_{0}\left(\rho_{2}\right) & z_{1}\left(\rho_{2}\right) & v_{3} & \ldots & v_{n} \\
& & & &
\end{array}\right) .
$$

The transformation matrix $W$ contains only one prescribed column. Equation (11.52) provides for $c=0$ an expression for the first column of this matrix:

$$
M_{0}\left(\rho_{2}\right) z_{1}\left(\rho_{2}\right)-\rho_{1} M_{2}\left(\rho_{2}\right) z_{0}\left(\rho_{2}\right)-\rho_{1}^{2} M_{3}\left(\rho_{2}\right) z_{0}\left(\rho_{2}\right) .
$$

By following the Equations in (11.49), it turns out that this is equal to:

$$
\left(M_{0}\left(\rho_{2}\right)-\rho_{1} M_{1}\left(\rho_{2}\right)-\rho_{1}^{2} M_{2}\left(\rho_{2}\right)\right) z_{1}\left(\rho_{2}\right),
$$

which results in the following matrix $W\left(\rho_{2}\right)$ :

$$
W\left(\rho_{2}\right)=\left(\begin{array}{ll|l|l|l}
\left(M_{0}\left(\rho_{2}\right)-\rho_{1} M_{1}\left(\rho_{2}\right)-\rho_{1}^{2} M_{2}\left(\rho_{2}\right)\right) & z_{1}\left(\rho_{2}\right) & w_{2} & \ldots & w_{m} \\
& & &
\end{array}\right) .
$$

The columns $v_{3}, \ldots, v_{n}$, and $w_{2}, \ldots, w_{m}$ denote standard basis vectors to make the matrices $V$ and $W$ square and invertible.

When the transformation:

$$
W\left(\rho_{1}, \rho_{2}\right)^{-1}\left(M_{0}\left(\rho_{2}\right)+\rho_{1} M_{1}\left(\rho_{2}\right)+\rho_{1}^{2} M_{2}\left(\rho_{2}\right)+\rho_{1}^{3} M_{3}\left(\rho_{2}\right)\right) V\left(\rho_{2}\right)
$$

is applied, an equivalent pencil emerges and a similar transposed version of the singular part $L_{\eta_{1}}^{T}$ of dimension $1 \times 2$ as in (11.37) can be split off from the pencil.

\subsubsection{A second approach to compute the transformation matrices for a non-linear two-parameter matrix pencil}

This section presents a second approach to split off singular parts of a polynomial/ non-linear matrix pencil. This approach differs from the approach described in the previous section in the way the expansion of the involved polynomial matrices is performed. Although this approach will not work in general, it exhibits some useful 
properties. First, the transformation matrix $W$ in this approach contains only one variable $\rho_{2}$, whereas it contained both the variables $\rho_{1}$ and $\rho_{2}$ in the previous subsection. Because the matrix $W$ only contains one variable, it is (i) less challenging in a computational point of view to compute the inverse of $W$ and (ii) much easier to compute the values which make the matrix $W$ singular.

Consider the transpose of the matrix $A\left(\rho_{1}, \rho_{2}\right)$ in Equation (11.39) and let us denote its dimensions by $m \times n$. The variable $\tilde{N}$ is the total degree of $\rho_{1}$ and $\rho_{2}$ in the polynomial matrix $A\left(\rho_{1}, \rho_{2}\right)^{T}$. In the first step, $\tilde{N}$ is therefore equal to $N-1$ where $N$ is the order of the original system $H(s)$. Let us start by expanding the polynomial matrix as follows:

$$
A\left(\rho_{1}, \rho_{2}\right)^{T}=\left(B\left(\rho_{2}\right)+\rho_{1} C\left(\rho_{1}, \rho_{2}\right)\right)
$$

where the matrix $B\left(\rho_{2}\right)$ is:

$$
B\left(\rho_{2}\right)=B_{0}+\rho_{2} B_{1}+\rho_{2}^{2} B_{2}+\ldots+\rho_{2}^{\tilde{N}} B_{\tilde{N}}
$$

and where $C\left(\rho_{1}, \rho_{2}\right)$ is:

$$
\begin{aligned}
& C\left(\rho_{1}, \rho_{2}\right)=
\end{aligned}
$$

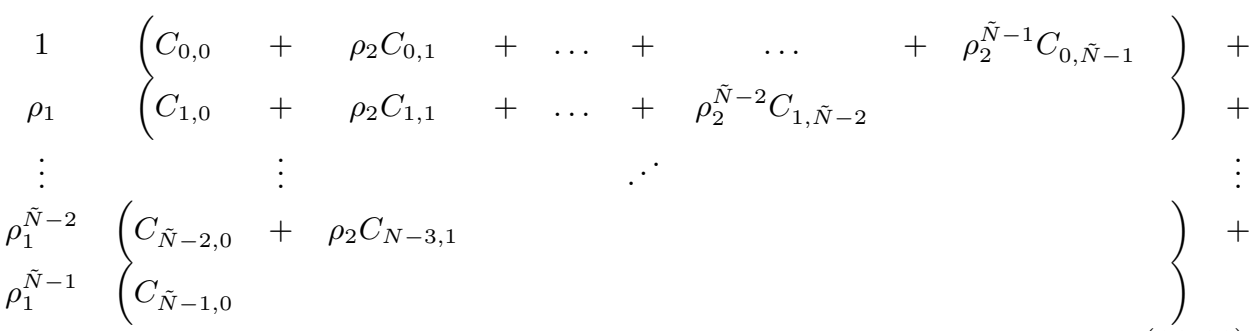

The rank $r$ of the matrix $A\left(\rho_{1}, \rho_{2}\right)^{T}$ will be $r<m$ or $r<n$. Assuming that the rank $r<n$ means that there is dependency between the columns of the matrix $A\left(\rho_{1}, \rho_{2}\right)^{T}$ and that:

$$
A\left(\rho_{1}, \rho_{2}\right)^{T} z\left(\rho_{1}, \rho_{2}\right)=0
$$

has a non-zero solution $z\left(\rho_{1}, \rho_{2}\right)$ depending on $\rho_{1}$ and $\rho_{2}$. Take such a solution $z$ in which the degree of $\rho_{1}$ is $\eta_{1}$, the degree of $\rho_{2}$ is $\eta_{2}$ and $\eta_{1}$ is as small as possible. Then $z\left(\rho_{1}, \rho_{2}\right)$ can be written as:

$$
z_{0}\left(\rho_{2}\right)-\rho_{1} z_{1}\left(\rho_{2}\right)+\rho_{1}^{2} z_{2}\left(\rho_{2}\right)+\ldots+(-1)^{\eta_{1}} \rho_{1}^{\eta_{1}} z_{\eta_{1}}\left(\rho_{2}\right)
$$

where $z_{0}\left(\rho_{2}\right), z_{1}\left(\rho_{2}\right), \ldots, z_{\eta_{1}}\left(\rho_{2}\right)$ are all polynomial in $\rho_{2}$. Substituting such a solution 
$z\left(\rho_{1}, \rho_{2}\right)$ into (11.62) where the matrix $A\left(\rho_{1}, \rho_{2}\right)^{T}$ is expanded as in (11.59) leads to:

$$
\begin{aligned}
& \left(B\left(\rho_{2}\right)+\rho_{1} C\left(\rho_{1}, \rho_{2}\right)\right)\left(z_{0}\left(\rho_{2}\right)-\rho_{1} z_{1}\left(\rho_{2}\right)+\rho_{1}^{2} z_{2}\left(\rho_{2}\right)+\ldots+(-1)^{\eta_{1}} \rho_{1}^{\eta_{1}} z_{\eta_{1}}\left(\rho_{2}\right)\right)= \\
& \left.\begin{array}{ll}
1 & \cdot\left(\begin{array}{ll}
+B\left(\rho_{2}\right) z_{0}\left(\rho_{2}\right) & \\
\rho_{1} & \cdot \\
\rho_{1}^{2} & -B\left(\rho_{2}\right) z_{1}\left(\rho_{2}\right)
\end{array}\right. \\
+B\left(\rho_{2}\right) z_{2}\left(\rho_{2}\right) & +C\left(\rho_{1}, \rho_{2}\right) z_{0}\left(\rho_{2}\right)
\end{array}\right)\left\{\begin{array}{l}
+ \\
+ \\
+
\end{array}\right.
\end{aligned}
$$

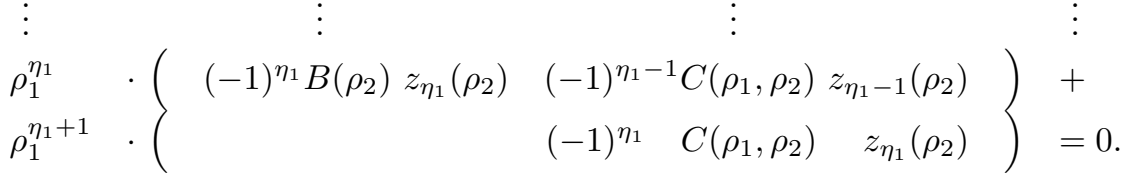

One can now work out the relationships for the coefficients of the powers of $\rho_{1}$ and write all the resulting expressions in matrix-vector form, as follows:

$$
\left.\begin{array}{ccccc}
B\left(\rho_{2}\right) & 0 & \ldots & \ldots & 0 \\
C\left(\rho_{1}, \rho_{2}\right) & B\left(\rho_{2}\right) & & & \vdots \\
0 & C\left(\rho_{1}, \rho_{2}\right) & \ddots & & \vdots \\
0 & 0 & \ddots & \ddots & \vdots \\
\vdots & \vdots & & \ddots & B\left(\rho_{2}\right) \\
0 & 0 & \ldots & \ldots & C\left(\rho_{1}, \rho_{2}\right)
\end{array}\right)\left(\begin{array}{c} 
\\
z_{0} \\
-z_{1} \\
z_{2} \\
\vdots \\
(-1)^{\eta_{1}} z_{\eta_{1}}
\end{array}\right)=
$$

Here the block matrix $M_{\eta_{1}, \eta_{2}}\left(\rho_{1}, \rho_{2}\right)$ has dimension $\left(\eta_{1}+2\right) m \times\left(\eta_{1}+1\right) n$. The rank of the matrix $M_{\eta_{1}, \eta_{2}}\left(\rho_{1}, \rho_{2}\right)$ is $r_{\eta_{1}, \eta_{2}}<\left(\eta_{1}+1\right) n$.

Because $\eta_{2}$ is the degree of $\rho_{2}$ in $z\left(\rho_{1}, \rho_{2}\right)$, we can write for $z_{i}\left(\rho_{2}\right)$ :

$$
z_{i}\left(\rho_{2}\right)=z_{i, 0}+\rho_{2} z_{i, 1}+\rho_{2}^{2} z_{i, 2}+\ldots+\rho_{2}^{\eta_{2}} z_{i, \eta_{2}}
$$

for $i=0, \ldots, \eta_{1}$.

When (11.66) is substituted into (11.65), the matrices $B\left(\rho_{2}\right)$ and $C\left(\rho_{1}, \rho_{2}\right)$ are written as in (11.60) and (11.61) and the relationships for the coefficients of the 
terms in $\rho_{1}$ and $\rho_{2}$ are worked out again, the following system of equations emerges:

$$
M_{\eta_{1}, \eta_{2}}\left(\begin{array}{c}
z_{0,0} \\
\vdots \\
z_{0, \eta_{2}} \\
\frac{\vdots}{z_{\eta_{1}, 0}} \\
\vdots \\
z_{\eta_{1}, \eta_{2}}
\end{array}\right)=0
$$

where the matrix $M_{\eta_{1}, \eta_{2}}$ is a block matrix containing the coefficient matrices $B_{0}, \ldots$, $B_{\tilde{N}}$, and $C_{0,0}, \ldots, C_{\tilde{N}-1,0}$ from the Equations (11.60) and (11.61). The dimension of this matrix is $\frac{1}{2}\left(\eta_{1}+2\right)\left(\eta_{2}+\tilde{N}+1\right)\left(\eta_{2}+\tilde{N}+2\right) m \times\left(\eta_{1}+1\right)\left(\eta_{2}+1\right) n$. Note that this matrix is independent of $\rho_{1}$ and $\rho_{2}$. Every non-zero vector in the kernel of the matrix $M_{\eta_{1}, \eta_{2}}$ provides a vector $z\left(\rho_{1}, \rho_{2}\right)$ which is a solution of (11.59).

Remark 11.6. The system of equations (11.67) in general is too restrictive in comparison with the approach of the previous section. This is due to the fact that the powers of $\rho_{1}$ and $\rho_{2}$ play a simultaneous role in the equations in (11.67). This is the reason why this approach does not work in general. However, this approach is useful because when the system of equations (11.65) admits a solution, we have a solution for the system of equations (11.44) of the previous subsection too. In this way we can still split off a singular block of the matrix pencil. Note that the system of equations (11.44) may have solutions which are not of this more 'restrictive' form.

Example 11.1 (continued). Using this approach, $A\left(\rho_{1}, \rho_{2}\right)^{T} z\left(\rho_{1}, \rho_{2}\right)=0$ is written as:

$$
\left(B\left(\rho_{2}\right)+\rho_{1} C\left(\rho_{1}, \rho_{2}\right)\right) z\left(\rho_{1}, \rho_{2}\right)=0
$$

where

$$
B\left(\rho_{2}\right)=B_{0}+\rho_{2} B_{1}+\rho_{2}^{2} B_{2}+\rho_{2}^{3} B_{3}
$$

and

$$
C\left(\rho_{1}, \rho_{2}\right)=\left(C_{0,0}+\rho_{2} C_{0,1}+\rho_{2}^{2} C_{0,2}\right)+\rho_{1}\left(C_{1,0}+\rho_{2} C_{1,1}\right)+\rho_{1}^{2}\left(C_{2,0}\right) .
$$

We know that every solution $z\left(\rho_{1}, \rho_{2}\right)$ should take the form $z_{0}\left(\rho_{2}\right)-\rho_{1} z_{1}\left(\rho_{2}\right)$ because $\eta_{1}$ is chosen as 1 . If such a solution is substituted into (11.68), and the relationships of the coefficients of the powers of $\rho_{1}$ are equated to zero, it can be written as:

$$
\left(\begin{array}{cc}
B\left(\rho_{2}\right) & 0 \\
C\left(\rho_{1}, \rho_{2}\right) & B\left(\rho_{2}\right) \\
0 & C\left(\rho_{1}, \rho_{2}\right)
\end{array}\right)\left(\begin{array}{r}
z_{0}\left(\rho_{2}\right) \\
-z_{1}\left(\rho_{2}\right)
\end{array}\right)=M_{1,2}\left(\rho_{1}, \rho_{2}\right)\left(\begin{array}{r}
z_{0}\left(\rho_{2}\right) \\
-z_{1}\left(\rho_{2}\right)
\end{array}\right)=0
$$

where the dimension of the block matrix $M_{1,2}\left(\rho_{1}, \rho_{2}\right)$ is $\left(\eta_{1}+2\right) m \times\left(\eta_{1}+1\right) n=$ $3 m \times 2 n$. 
The degree of $\rho_{2}$ is $\eta_{2}=2$ and therefore $z_{i}\left(\rho_{2}\right)$ can be written as $z_{i}\left(\rho_{2}\right)=z_{i, 0}+$ $\rho_{2} z_{i, 1}+\rho_{2}^{2} z_{i, 2}$ for $i=0,1$. This can be substituted into (11.71). When the notations (11.69) and (11.70) are used for the matrices $B\left(\rho_{2}\right)$ and $C\left(\rho_{1}, \rho_{2}\right)$ and the relationships for the coefficients of the terms in $\rho_{1}$ and $\rho_{2}$ are worked out again, the following system of equations occurs:

$$
M_{1,2}\left(\begin{array}{c}
z_{0,0} \\
z_{0,1} \\
z_{0,2} \\
\hline z_{1,0} \\
z_{1,1} \\
z_{1,2}
\end{array}\right)=0
$$

where the dimension of the matrix $M_{1,2}$ is $\left(\frac{1}{2} \cdot 3 \cdot 6 \cdot 7\right) m \times(2 \cdot 3) n=63 m \times 6 n$. When the zero block rows of the matrix $M_{1,2}$ are discarded, the dimension becomes 
$31 m \times 6 n:$

$$
M_{1,2}=\left(\begin{array}{ccc|ccc}
B_{0} & 0 & 0 & 0 & 0 & 0 \\
B_{1} & B_{0} & 0 & 0 & 0 & 0 \\
B_{2} & B_{1} & B_{0} & 0 & 0 & 0 \\
B_{3} & B_{2} & B_{1} & 0 & 0 & 0 \\
0 & B_{3} & B_{2} & 0 & 0 & 0 \\
0 & 0 & B_{3} & 0 & 0 & 0 \\
C_{1,0} & 0 & 0 & B_{0} & 0 & 0 \\
C_{2,0} & 0 & 0 & 0 & 0 & 0 \\
C_{1,1} & C_{1,0} & 0 & B_{1} & B_{0} & 0 \\
C_{3,0} & 0 & 0 & 0 & 0 & 0 \\
C_{2,1} & C_{2,0} & 0 & 0 & 0 & 0 \\
C_{1,2} & C_{1,1} & C_{1,0} & B_{2} & B_{1} & B_{0} \\
0 & C_{3,0} & 0 & 0 & 0 & 0 \\
0 & C_{2,1} & C_{2,0} & 0 & 0 & 0 \\
0 & C_{1,2} & C_{1,1} & B_{3} & B_{2} & B_{1} \\
0 & 0 & C_{3,0} & 0 & 0 & 0 \\
0 & 0 & C_{2,1} & 0 & 0 & 0 \\
0 & 0 & C_{1,2} & 0 & B_{3} & B_{2} \\
0 & 0 & 0 & 0 & 0 & B_{3} \\
0 & 0 & 0 & C_{1,0} & 0 & 0 \\
0 & 0 & 0 & C_{2,0} & 0 & 0 \\
0 & 0 & 0 & C_{1,1} & C_{1,0} & 0 \\
0 & 0 & 0 & C_{3,0} & 0 & 0 \\
0 & 0 & 0 & C_{2,1} & C_{2,0} & 0 \\
0 & 0 & 0 & C_{1,2} & C_{1,1} & C_{1,0} \\
0 & 0 & 0 & 0 & C_{3,0} & 0 \\
0 & 0 & 0 & 0 & C_{2,1} & C_{2,0} \\
0 & 0 & 0 & 0 & C_{1,2} & C_{1,1} \\
0 & 0 & 0 & 0 & 0 & C_{3,0} \\
0 & 0 & 0 & 0 & 0 & C_{2,1} \\
0 & 0 & 0 & 0 & 0 & C_{1,2}
\end{array}\right) .
$$

If the rank of this matrix $M_{1,2}$ is smaller than $6 n$, one can compute solutions $z\left(\rho_{1}, \rho_{2}\right)$ of $A\left(\rho_{1}, \rho_{2}\right)^{T} z\left(\rho_{1}, \rho_{2}\right)=0$, by computing non-zero vectors in the kernel of this matrix. From the vectors $z_{0,0}, z_{0,1}, z_{0,2}, z_{1,0}, z_{1,1}, z_{1,2}$, which are parts of a vector in the kernel of $M_{1,2}$, one can construct the solution $z$ as $z\left(\rho_{1}, \rho_{2}\right)=\left(z_{0,0}+\rho_{2} z_{0,1}+\right.$ $\left.\rho_{2}^{2} z_{0,2}\right)-\rho_{1}\left(z_{1,0}+\rho_{2} z_{1,1}+\rho_{2}^{2} z_{1,2}\right)$.

The vectors $z\left(\rho_{1}, \rho_{2}\right)$ are used to split off singular parts of the singular matrix pencil $A\left(\rho_{1}, \rho_{2}\right)^{T}=B\left(\rho_{2}\right)+\rho_{1} C\left(\rho_{1}, \rho_{2}\right)$ : first construct transformation matrices $V\left(\rho_{2}\right)$ and $W\left(\rho_{2}\right)$, second, perform the transformation $W\left(\rho_{2}\right)^{-1}\left(B\left(\rho_{2}\right)+\rho_{1} C\left(\rho_{1}, \rho_{2}\right) V\left(\rho_{2}\right)\right.$ and finally split off one or more singular parts of dimensions $\left(\eta_{1}+1\right) \times \eta_{1}$ with the same structure as in (11.14). 
The difference here with respect to the previous approach lies in the construction of the transformation matrices $V$ and $W$. The first columns of the matrices $V$ are chosen, as usual, as the $\eta_{1}+1$ vectors $z_{0}\left(\rho_{2}\right), \ldots, z_{\eta_{1}}\left(\rho_{2}\right)$ which makes this a polynomial matrix in $\rho_{2}$. The first columns of the matrix $W$ are chosen as the $\eta_{1}$ vectors $B\left(\rho_{2}\right) z_{1}\left(\rho_{2}\right), \ldots, B\left(\rho_{2}\right) z_{\eta_{1}}\left(\rho_{2}\right)$. In this approach both the transformation matrices are polynomial in the variable $\rho_{2}$, whereas in the previous approach the matrix $W$ was polynomial in both $\rho_{1}$ and $\rho_{2}$. This makes it easier to compute the inverse of the transformation matrix $W$.

After all the singular parts of the matrix pencil $B\left(\rho_{2}\right)+\rho_{1} C\left(\rho_{1}, \rho_{2}\right)$ are split off, there should not exist a regular part which admits other solutions than the trivial solution. Because the existence of a regular part is impossible (for the same reasons mentioned in Section 11.3.1), the solutions for $\rho_{1}$ and $\rho_{2}$ correspond to the values which make the transformation matrices $V\left(\rho_{2}\right)$ and $W\left(\rho_{2}\right)$ singular as in (11.20) of Proposition 11.2.

Remark 11.7. The value of $\tilde{N}$ can change (increase or decrease), during the process of splitting off singular parts of the matrix pencil. This has a direct effect on the decomposition of the matrix pencil (11.59) in (11.60) and (11.61).

\subsection{Computing the approximation $G(s)$}

Let $x_{1}, \ldots, x_{N}$ and $\rho_{1}$ and $\rho_{2}$ be a solution of the system of equations (11.1) which also satisfies the additional constraints $\tilde{a}_{N-1}=0$ and $\tilde{a}_{N-2}=0$ in (11.2). Using such a solution an approximation of order $N-3$ can be computed as described in Theorem 8.3 for $k=3$.

From the set of solutions to the quadratic system of equations (11.1) that satisfy the constraints $\tilde{a}_{N-1}=0$ and $\tilde{a}_{N-2}=0$ in (11.2), it is straightforward to select those that are feasible, i.e., which give rise to a real stable approximation $G(s)$ of order $N-3$. It is then possible to select the globally optimal approximation by computing the $H_{2}$-norm of the difference $H(s)-G(s)$ for every feasible solution $G(s)$ and selecting the one for which this criterion is minimal. This $H_{2}$-norm of $H(s)-G(s)$ is, according to Theorem 8.2 for $k=3$, given by:

$$
\begin{aligned}
V_{H}\left(x_{1}, x_{2}, \ldots, x_{N}, \rho_{1}, \rho_{2}\right) & =\left\|\frac{e(s)}{d(s)}-\frac{b(s)}{a(s)}\right\|_{H_{2}}^{2} \\
& =\sum_{i=1}^{N} \frac{\left(1+\rho_{1} \delta_{i}+\rho_{2} \delta_{i}^{2}\right)^{2}\left(1-\rho_{1} \delta_{i}+\rho_{2} \delta_{i}^{2}\right)}{e\left(\delta_{i}\right) d^{\prime}\left(\delta_{i}\right) d\left(-\delta_{i}\right)} x_{i}^{3} .
\end{aligned}
$$




\subsection{Example}

\subsubsection{Model-order reduction from 4 to 1}

The system $H(s)$ to be reduced is a system of order 4 and is described in state space form with the matrices $A, B$, and $C$ :

$$
A=\left(\begin{array}{cccc}
-\frac{34}{15} & 1 & 0 & 0 \\
-\frac{77}{36} & 0 & 1 & 0 \\
-\frac{247}{270} & 0 & 0 & 1 \\
-\frac{5}{36} & 0 & 0 & 0
\end{array}\right), B=\left(\begin{array}{r}
2 \\
4 \\
-9 \\
-2
\end{array}\right), C=\left(\begin{array}{llll}
1 & 0 & 0 & 0
\end{array}\right) .
$$

This yields the fourth-order transfer function $H(s)=C(s I-A)^{-1} B$ :

$$
H(s)=\frac{-2-9 s+4 s^{2}+2 s^{3}}{\frac{5}{36}+\frac{247}{270} s+\frac{77}{36} s^{2}+\frac{34}{15} s^{3}+s^{4}} .
$$

The poles of the system $H(s)$ are $\delta_{1}=-\frac{2}{3}+\frac{1}{2} i, \delta_{2}=-\frac{2}{3}-\frac{1}{2} i, \delta_{3}=-\frac{3}{5}$ and $\delta_{4}=-\frac{1}{3}$. Because the reduction is from order 4 to 1 , it is easily possible to compute the optimal approximation $G(s)$ of order one, by using other numerical methods, see [89]. The globally optimal $\mathrm{H}_{2}$-approximation of order one obtained in that way, is:

$$
G(s)=\frac{-3.1894}{0.174884+s}
$$

which has one pole located at -0.174884 .

This system $G(s)$ is the approximation one also wants to compute with the approach described in this chapter using the Stetter-Möller matrix method and the Kronecker canonical form techniques for two-parameter pencils.

The system of quadratic equations (11.1) corresponding to the given transfer function $H(s)$ of order four, is given by:

$$
\left(\begin{array}{c}
\frac{1+\delta_{1} \rho_{1}+\delta_{1}^{2} \rho_{2}}{e\left(\delta_{1}\right)} x_{1}^{2} \\
\frac{1+\delta_{2} \rho_{1}+\delta_{2}^{2} \rho_{2}}{e\left(\delta_{2}\right)} x_{2}^{2} \\
\frac{1+\delta_{3} \rho_{1}+\delta_{3}^{2} \rho_{2}}{e\left(\delta_{3}\right)} x_{3}^{2} \\
\frac{1+\delta_{4} \rho_{1}+\delta_{4}^{2} \rho_{2}}{e\left(\delta_{4}\right)} x_{4}^{2}
\end{array}\right)-M\left(\delta_{1}, \delta_{2}, \delta_{3}, \delta_{4}\right)\left(\begin{array}{l}
x_{1} \\
x_{2} \\
x_{3} \\
x_{4}
\end{array}\right)=\left(\begin{array}{l}
0 \\
0 \\
0 \\
0
\end{array}\right)
$$

with the matrix $M\left(\delta_{1}, \delta_{2}, \delta_{3}, \delta_{4}\right)$ as before. The constraints $\tilde{a}_{N-1}$ and $\tilde{a}_{N-2}$, as defined in (11.2), take the form:

$$
\left\{\begin{array}{l}
\tilde{a}_{N-1}=\left(-\frac{6480}{2977}+\frac{7380}{2977} i\right) x_{1}-\left(\frac{6480}{2977}+\frac{7380}{2977} i\right) x_{2}+\frac{3375}{229} x_{3}-\frac{135}{13} x_{4}=0 \\
\tilde{a}_{N-2}=\left(\frac{6678}{2977}-\frac{15048}{2977} i\right) x_{1}+\left(\frac{6678}{2977}+\frac{15048}{2977} i\right) x_{2}-\frac{5625}{229} x_{3}+\frac{261}{13} x_{4}=0 .
\end{array}\right.
$$

In order to find the solutions of the quadratic system of equations (11.78), which also satisfy both the linear constraints $(11.79)$, the matrices $A_{\tilde{a}_{N-1}}\left(\rho_{1}, \rho_{2}\right)^{T}$ and 
$A_{\tilde{a}_{N-2}}\left(\rho_{1}, \rho_{2}\right)^{T}$ are constructed. The monomial basis $B$ used for constructing these matrices is:

$$
\begin{gathered}
B=\left\{1, x_{1}, x_{2}, x_{1} x_{2}, x_{3}, x_{1} x_{3}, x_{2} x_{3}, x_{1} x_{2} x_{3}, x_{4}, x_{1} x_{4}, x_{2} x_{4},\right. \\
\left.x_{1} x_{2} x_{4}, x_{3} x_{4}, x_{1} x_{3} x_{4}, x_{2} x_{3} x_{4}, x_{1} x_{2} x_{3} x_{4}\right\} .
\end{gathered}
$$

After making the matrices $A_{\tilde{a}_{N-1}}\left(\rho_{1}, \rho_{2}\right)^{T}$ and $A_{\tilde{a}_{N-2}}\left(\rho_{1}, \rho_{2}\right)^{T}$ polynomial in $\left(\rho_{1}, \rho_{2}\right)$ (see Theorem 10.1), they are denoted by $\tilde{A}_{\tilde{a}_{N-1}}\left(\rho_{1}, \rho_{2}\right)^{T}$ and $\tilde{A}_{\tilde{a}_{N-2}}\left(\rho_{1}, \rho_{2}\right)^{T}$. The dimensions of these matrices are $2^{4} \times 2^{4}=16 \times 16$ and the total degree of all the terms is $N-1=3$ (analogous to the result in Corollary 10.2). The trivial/zero solution can be split off from the matrices immediately by removing the first column, which is a zero column, and the first row of both the matrices. This brings the dimensions of both the matrices to $15 \times 15$. The sparsity structure of the matrices $\tilde{A}_{\tilde{a}_{N-1}}\left(\rho_{1}, \rho_{2}\right)^{T}$ and $\tilde{A}_{\tilde{a}_{N-2}}\left(\rho_{1}, \rho_{2}\right)^{T}$ is given in Figure 11.1.

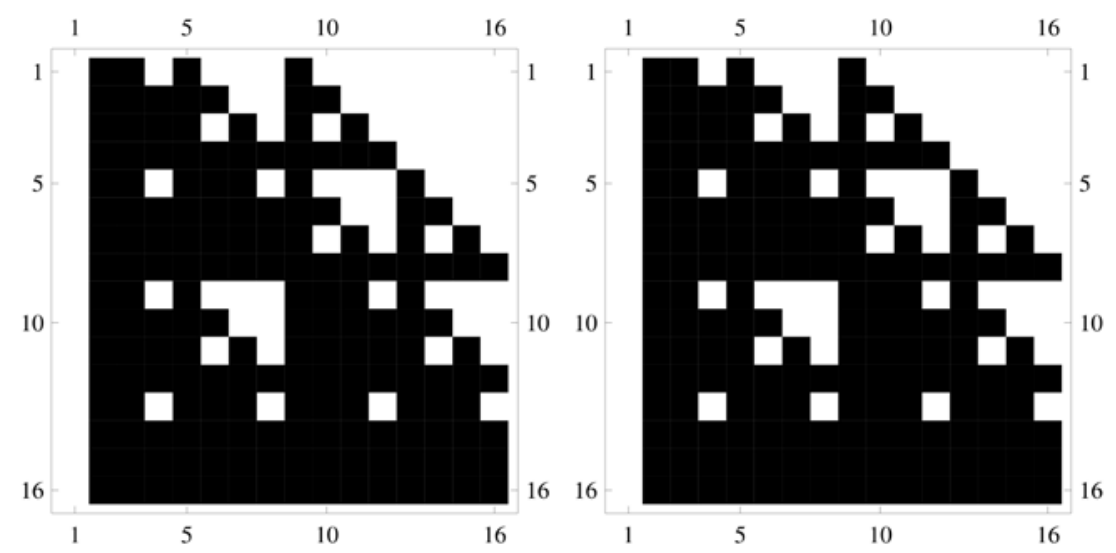

Figure 11.1: Sparsity structure of the matrices $\tilde{A}_{\tilde{a}_{N-1}}\left(\rho_{1}, \rho_{2}\right)^{T}$ and $\tilde{A}_{\tilde{a}_{N-2}}\left(\rho_{1}, \rho_{2}\right)^{T}$

The solutions of the quadratic system of equations (11.78), can be computed from the polynomial eigenvalues of the polynomial matrices $\tilde{A}_{\tilde{a}_{N-1}}\left(\rho_{1}, \rho_{2}\right)^{T}$ and $\tilde{A}_{\tilde{a}_{N-2}}\left(\rho_{1}, \rho_{2}\right)^{T}$. Thus the remaining problem is the following:

$$
\left\{\begin{aligned}
\tilde{A}_{\tilde{a}_{N-1}}\left(\rho_{1}, \rho_{2}\right)^{T} v & =0 \\
\tilde{A}_{\tilde{a}_{N-2}}\left(\rho_{1}, \rho_{2}\right)^{T} v & =0
\end{aligned}\right.
$$

In the previous section, three techniques are presented to compute the eigenvalue pairs $\left(\rho_{1}, \rho_{2}, v\right)$. The linear approach of Subsection 11.3.2 first linearizes both the matrices with respect to $\rho_{1}$ and $\rho_{2}$ and then joins the rows together in one matrix, removing duplicate rows. This yields a rectangular and therefore singular matrix of dimension $\left((N-1)^{2}+1\right) 2^{N} \times(N-1)^{2} 2^{N}=150 \times 135$.

Both the approaches of the Subsections 11.3.3 and 11.3.4 admit to work with the non-linearized polynomial matrices of dimensions $15 \times 15$. These approaches 
are preferred because of computational efficiency. These approaches stack both the matrices on top of each other to generate the rectangular and singular matrix

$$
\left[\begin{array}{c}
\tilde{A}_{\tilde{a}_{N-1}}\left(\rho_{1}, \rho_{2}\right)^{T} \\
\tilde{A}_{\tilde{a}_{N-2}}\left(\rho_{1}, \rho_{2}\right)^{T}
\end{array}\right]=A_{1}\left(\rho_{1}, \rho_{2}\right)
$$

of dimension $30 \times 15$. The quantities $\rho_{1}$ and $\rho_{2}$ are the polynomial eigenvalues of this matrix and the values for $x_{1}, x_{2}, x_{3}$, and $x_{4}$ can be read off from the corresponding (Stetter) eigenvectors $v$.

Before splitting off singular parts of the rectangular matrix $A_{1}\left(\rho_{1}, \rho_{2}\right)^{T}$, we first try to get an impression of the real-valued eigenvalue pairs $\rho_{1}$ and $\rho_{2}$ of the matrices $\tilde{A}_{\tilde{a}_{N-1}}\left(\rho_{1}, \rho_{2}\right)^{T}$ and $\tilde{A}_{\tilde{a}_{N-2}}\left(\rho_{1}, \rho_{2}\right)^{T}$ as follows: a range of real values for $\rho_{1}$ is substituted into both the matrices. Both matrices then only contain one variable: $\rho_{2}$. The eigenvalues $\rho_{2}$ of the matrices can then be computed by solving the corresponding polynomial eigenvalue problem (in Section 10.2 it is shown how the eigenvalues of a polynomial matrix in one variable can be computed). The chosen real values for $\rho_{1}$ are plotted against the computed real values $\rho_{2}$ in a $\left(\rho_{1}, \rho_{2}\right)$-plot for both matrices. This plot is displayed in Figure 11.2.

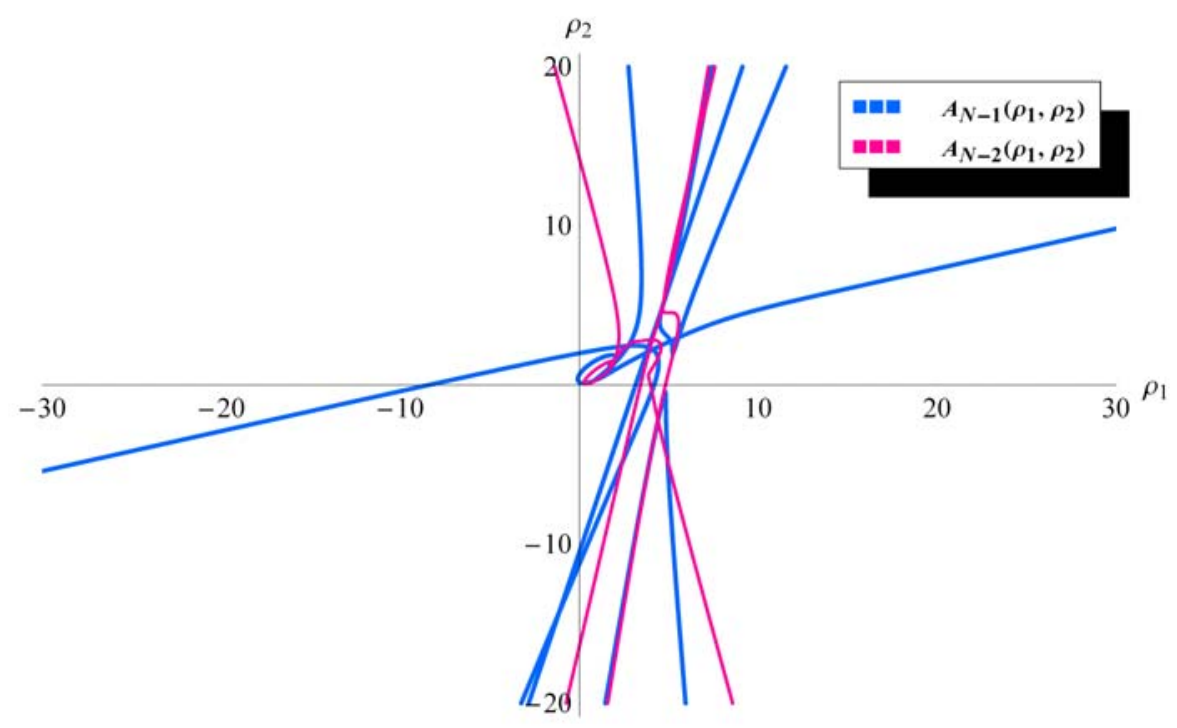

Figure 11.2: Real eigenvalue pairs $\left(\rho_{1}, \rho_{2}\right)$ of the matrices $\tilde{A}_{\tilde{a}_{N-1}}\left(\rho_{1}, \rho_{2}\right)^{T}$ and $\tilde{A}_{\tilde{a}_{N-2}}\left(\rho_{1}, \rho_{2}\right)^{T}$

We require $\rho_{1}$ and $\rho_{2}$ to be real eigenvalues of the matrices $\tilde{A}_{\tilde{a}_{N-1}}\left(\rho_{1}, \rho_{2}\right)^{T}$ and $\tilde{A}_{\tilde{a}_{N-2}}\left(\rho_{1}, \rho_{2}\right)^{T}$ at the same time. Therefore the interesting locations $\left(\rho_{1}, \rho_{2}\right)$ in Figure 11.2 are located on the intersection of the red and blue curves in the plot. In this way we obtain five interesting real valued non-trivial pairs $\left(\rho_{1}, \rho_{2}\right)$ which are eigenvalues 
of both matrices simultaneously. The other intersections of both curves correspond to the trivial solutions $x_{1}=0, \ldots, x_{N}=0$. Figure 11.3 shows a zoomed version of Figure 11.2 near these 5 real points which are marked with green dots.

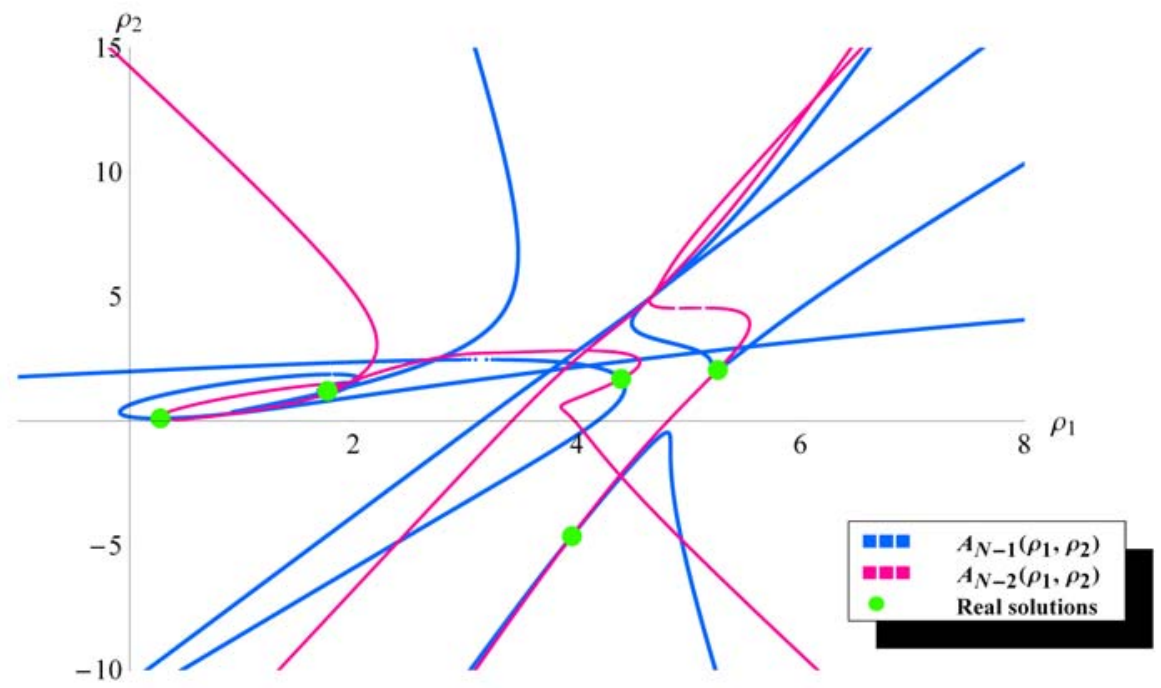

Figure 11.3: Five important real eigenvalue pairs $\left(\rho_{1}, \rho_{2}\right)$ of the matrices $\tilde{A}_{\tilde{a}_{N-1}}\left(\rho_{1}, \rho_{2}\right)^{T}$ and $\tilde{A}_{\tilde{a}_{N-2}}\left(\rho_{1}, \rho_{2}\right)^{T}$

Splitting off singular blocks from the matrix $A_{1}\left(\rho_{1}, \rho_{2}\right)^{T}$ is done by performing transformations $W^{-1} A_{1}\left(\rho_{1}, \rho_{2}\right)^{T} V$ with suitable square and invertible matrices $V$ and $W$. When performing these transformations iteratively to the matrix $A_{1}\left(\rho_{1}, \rho_{2}\right)^{T}$, one can split off one or more singular parts in every iteration.

Finally, one has a set of transformation matrices at hand. All the values $\rho_{2}$ (and possibly $\rho_{1}$ ) which make the matrices $V$ and $W$ singular are the (partial) solutions of the system of equations (11.78). The results of these reduction processes are given in the Tables 11.1 and 11.2 for the approaches of Subsections 11.3.3 and 11.3.4, respectively. The tables show the dimensions of the matrices, the degrees $\eta_{1}$ and $\eta_{2}$ of the solution $z\left(\rho_{1}, \rho_{2}\right)$, the dimensions of the transposed version of the singular parts $L_{\eta_{1}}^{T}$ which are split off and the variables involved in the matrices.

Table 11.1: Results of reduction process of two-parameter pencil $A_{1}\left(\rho_{1}, \rho_{2}\right)^{T}$ using the approach of Subsection 11.3.3.

\begin{tabular}{lccccc}
\hline Matrix & Dimensions & $\eta_{1}$ & $\eta_{2}$ & Dimensions of $L_{\eta_{1}}$ & Variables \\
\hline$A_{1}\left(\rho_{1}, \rho_{2}\right)^{T}$ & $15 \times 30$ & 0 & 0 & $4(0 \times 1)$ & $\rho_{1}, \rho_{2}$ \\
$A_{2}\left(\rho_{1}, \rho_{2}\right)^{T}$ & $15 \times 26$ & 1 & 1 & $6(1 \times 2)$ & $\rho_{1}, \rho_{2}$ \\
$A_{3}\left(\rho_{1}, \rho_{2}\right)^{T}$ & $9 \times 14$ & 5 & 4 & $5 \times 6$ & $\rho_{1}, \rho_{2}$ \\
$A_{4}\left(\rho_{1}, \rho_{2}\right)^{T}$ & $4 \times 8$ & 4 & 7 & $4 \times 5$ & $\rho_{1}, \rho_{2}$ \\
$A_{5}\left(\rho_{1}, \rho_{2}\right)^{T}$ & $0 \times 3$ & - & - & - & - \\
\hline
\end{tabular}


Table 11.2: Results of reduction process of two-parameter pencil $A_{1}\left(\rho_{1}, \rho_{2}\right)^{T}$ using the approach of Subsection 11.3.4.

\begin{tabular}{lccccc}
\hline Matrix & Dimensions & $\eta_{1}$ & $\eta_{2}$ & Dimensions of $L_{\eta_{1}}$ & Variables \\
\hline$A_{1}\left(\rho_{1}, \rho_{2}\right)^{T}$ & $15 \times 30$ & 0 & 0 & $4(0 \times 1)$ & $\rho_{1}, \rho_{2}$ \\
$A_{2}\left(\rho_{1}, \rho_{2}\right)^{T}$ & $15 \times 26$ & 1 & 2 & $1 \times 2$ & $\rho_{1}, \rho_{2}$ \\
$A_{3}\left(\rho_{1}, \rho_{2}\right)^{T}$ & $14 \times 24$ & 3 & 3 & $3 \times 4$ & $\rho_{1}, \rho_{2}$ \\
$A_{4}\left(\rho_{1}, \rho_{2}\right)^{T}$ & $11 \times 20$ & 1 & 8 & $1 \times 2$ & $\rho_{1}, \rho_{2}$ \\
$A_{5}\left(\rho_{1}, \rho_{2}\right)^{T}$ & $10 \times 18$ & 3 & 15 & $2(3 \times 4)$ & $\rho_{1}, \rho_{2}$ \\
$A_{6}\left(\rho_{1}, \rho_{2}\right)^{T}$ & $4 \times 10$ & 0 & 0 & $0 \times 3$ & $\rho_{2}$ \\
$A_{7}\left(\rho_{1}, \rho_{2}\right)^{T}$ & $4 \times 7$ & 0 & 3 & $3(0 \times 1)$ & $\rho_{2}$ \\
$A_{8}\left(\rho_{1}, \rho_{2}\right)^{T}$ & $4 \times 4$ & - & - & - & - \\
\hline
\end{tabular}

When the non-linear approach of Subsection 11.3.4 is used, the eigenvalues $\rho_{2}$ of the pencil $A_{1}\left(\rho_{1}, \rho_{2}\right)^{T}$ are among the values of $\rho_{2}$ which make the transformation matrices $V\left(\rho_{2}\right)$ and $W\left(\rho_{2}\right)$ singular. For this small example it was possible to compute the values of $\rho_{2}$ exactly as the zeros of the determinants of the transformation matrices $V$ and $W$. Once the values for $\rho_{2}$ are known, these can be substituted into the matrices $\tilde{A}_{\tilde{a}_{N-1}}\left(\rho_{1}, \rho_{2}\right)^{T}$ and $\tilde{A}_{\tilde{a}_{N-2}}\left(\rho_{1}, \rho_{2}\right)^{T}$. Then the corresponding values for $\rho_{1}$ are the values which make the matrices $\tilde{A}_{\tilde{a}_{N-1}}\left(\rho_{1}, \rho_{2}\right)^{T}$ and $\tilde{A}_{\tilde{a}_{N-2}}\left(\rho_{1}, \rho_{2}\right)^{T}$ simultaneously singular for this known value of $\rho_{2}$. These values for $\rho_{1}$ can also be determined by computing the zeros of the determinants of $\tilde{A}_{\tilde{a}_{N-1}}\left(\rho_{1}, \rho_{2}\right)^{T}$ and $\tilde{A}_{\tilde{a}_{N-2}}\left(\rho_{1}, \rho_{2}\right)^{T}$.

Only real values of $\rho_{1}$ and $\rho_{2}$ yield solutions which lead to feasible approximations of order 1. After determining all the pairs $\left(\rho_{1}, \rho_{2}\right)$ which make the matrices $V\left(\rho_{2}\right)$, $W\left(\rho_{2}\right), \tilde{A}_{\tilde{a}_{N-1}}\left(\rho_{1}, \rho_{2}\right)^{T}$ and $\tilde{A}_{\tilde{a}_{N-2}}\left(\rho_{1}, \rho_{2}\right)^{T}$ simultaneously singular, it turned out that the matrices $V\left(\rho_{2}\right)$ and $W\left(\rho_{2}\right)$ used for the reduction of dimension $10 \times 18$ to $4 \times 10$ (see Table 11.2) contained five real-valued pairs $\left(\rho_{1}, \rho_{2}\right)$. These values are computed as zeros from some factor of the determinants. The five real values of $\rho_{1}$ are the real zeros of:

$$
\begin{aligned}
& -73724881053644800000+394401417758222784000 \rho_{1}- \\
& 543650121764883196800 \rho_{1}^{2}+343281065062589182600 \rho_{1}^{3}- \\
& 117176950555078573308 \rho_{1}^{4}+22474612752967348983 \rho_{1}^{5}- \\
& 2288807613803985228 \rho_{1}^{6}+96606717088729788 \rho_{1}^{7},
\end{aligned}
$$

and the five real values of $\rho_{2}$ are the real zeros of:

$$
\begin{aligned}
& 55748192717662688-608378684643388064 \rho_{2}+ \\
& 1093069815842928726 \rho_{2}^{2}-737547208075861557 \rho_{2}^{3}+ \\
& 188328490866932812 \rho_{2}^{4}+2970655440662889 \rho_{2}^{5}- \\
& 10165163659763936 \rho_{2}^{6}+1789013279420922 \rho_{2}^{7} .
\end{aligned}
$$

The numerical values of these five pairs $\left(\rho_{1}, \rho_{2}\right)$ are: $(3.95361,-4.61399),(0.274237$, $0.112808),(1.76776,1.21776),(5.25849,2.05994)$, and $(4.39521,1.70287)$.

Once the values for $\rho_{1}$ and $\rho_{2}$ are available, the values for $x_{1}, x_{2}, x_{3}, x_{4}$ can be read off from the corresponding (normalized) Stetter eigenvectors. Using these five tuples 
of solutions $\left(\rho_{1}, \rho_{2}, x_{1}, x_{2}, x_{3}, x_{4}\right)$, the polynomials $a(s)$ and $b(s)$ can be determined. This yields five corresponding transfer functions of order one:

$$
\begin{aligned}
G(s) & =\frac{3.37127}{-6.48665+s}, \\
G(s) & =\frac{3.87128}{-0.129578+s}, \\
G(s) & =\frac{396.044}{-0.409222+s}, \\
G(s) & =\frac{2.97122}{4.43913+s}, \\
G(s) & =\frac{-3.1894}{0.174884+s},
\end{aligned}
$$

with corresponding $H_{2}$-criterion values $8.24643,11.1784,437.85,8.13223$, and 6.16803 , respectively.

The $H_{2}$-criterion values are computed by substituting the values of $\rho_{1}, \rho_{2}$, and $x_{1}, \ldots, x_{4}$ into the third order polynomial:

$$
\begin{aligned}
V_{H}= & (-0.121789+0.00691205 i)\left(1-(0.666667+0.5 i) \rho_{1}+\right. \\
& \left.(0.194444+0.666667 i) \rho_{2}\right)^{2}\left(1+(0.666667+0.5 i) \rho_{1}+\right. \\
& \left.(0.194444+0.666667 i) \rho_{2}\right) x_{1}^{3}-(0.121789+0.00691205 i) \\
& \left(1-(0.666667-0.5 i) \rho_{1}+(0.194444-0.666667 i) \rho_{2}\right)^{2} \\
& \left(1+(0.666667-0.5 i) \rho_{1}+(0.194444-0.666667 i) \rho_{2}\right) x_{2}^{3}- \\
& 1.60977\left(1-0.6 \rho_{1}+0.36 \rho_{2}\right)^{2}\left(1+0.6 \rho_{1}+0.36 \rho_{2}\right) x_{3}^{3}+ \\
& 9.74309\left(1-0.333333 \rho_{1}+0.111111 \rho_{2}\right)^{2} \\
& \left(1+0.333333 \rho_{1}+0.111111 \rho_{2}\right) x_{4}^{3} .
\end{aligned}
$$

It is easy to see that only the last two approximations $G(s)$, are feasible because only those two have a pole in the open left half plane $\Pi^{-}$. Since the last approximation has the smallest real criterion value, this approximation $G(s)$ is the globally optimal $H_{2}$-approximation of order one. This is the same transfer function as computed before in Equation (11.77):

$$
G(s)=\frac{-3.1894}{0.174884+s} .
$$

The corresponding values of the solution of the system of equations (11.78) and the two additional constraints in (11.79) are:

$$
\begin{aligned}
& \rho_{1}=4.39521 \\
& \rho_{2}=1.70287 \\
& x_{1}=2.56458+1.52372 i \\
& x_{2}=2.56458-1.52372 i \\
& x_{3}=2.36141 \\
& x_{4}=1.54876 .
\end{aligned}
$$




\subsubsection{Comparing the co-order three technique with the co-order one and two technique}

Finally, the performance of the co-order one, co-order two and co-order three techniques is compared with each other. The same system $H(s)$ as in Subsection 11.5.1 of order four is reduced to order one by applying the co-order three reduction once, by applying a co-order one and co-order two reduction consecutively and by applying the co-order one technique three times.

Table 11.3 shows the outcomes of these computations ordered by an increasing $H_{2}$-criterion value $V_{H}=\|H(s)-G(s)\|_{H_{2}}^{2}$. The co-order 3 technique performs the best, whereas the other techniques show only a slightly worse performance in terms of the $H_{2}$-criterion value $V_{H}$.

Table 11.3: Reducing the model-order of a system of order 4

\begin{tabular}{lccc}
\hline Method & G(s) of order 1 & Pole of G(s) & $V_{H}$ \\
\hline Co-order 3 & $\frac{-3.1894}{0.17488+s}$ & -0.174884 & 6.16803 \\
Co-order 2, co-order 1 & $\frac{-3.1584}{0.17266+s}$ & -0.172657 & 6.16826 \\
Co-order 1, co-order 1, co-order 1 & $\frac{-3.1523}{0.17287+s}$ & -0.172869 & 6.16828 \\
Co-order 1, co-order 2 & $\frac{-3.1716}{0.16854+s}$ & -0.168542 & 6.17014 \\
\hline
\end{tabular}




\section{Chapter 12}

\section{Conclusions \& directions for further research}

\subsection{Conclusions}

In Chapter 4 of this thesis a global optimization method is worked out to compute the global optimum of a Minkowski dominated polynomial. This method is based on the Stetter-Möller matrix method which transforms the problem of finding the solutions of the system of first-order conditions into an eigenvalue problem involving a large and sparse matrix. This method is described in [81] and a similar approach in [50] and [48]. A drawback of this approach is that the involved matrices are usually very large. The efficiency of this method is improved in this thesis by using a matrix-free implementation of the matrix-vector products and by using an iterative eigenvalue solver instead of a direct solver.

In Chapter 5 we have developed a routine that computes the action of the large and sparse matrix, provided by the Stetter-Möller matrix method, on a given vector without having this matrix explicitly at hand. To avoid building the large matrix the system of first-order conditions are associated with an $n \mathrm{D}$-system of difference equations. Such an $n$ D-system yields a matrix-free routine and such a routine is all that is needed for modern iterative eigenproblem solvers as input. It will turn out that this works perfectly together which proves the computational feasibility of this approach.

Section 5.3 focuses on improving the efficiency of computing the action of such a matrix on a vector. One way to compute the action of the matrix by an $n \mathrm{D}$-system efficiently, is by setting up a corresponding shortest path problem (SPP) and solve it as described in Section 5.3.2. A drawback here is that this SPP will quickly become huge and difficult to solve. However, it it is possible to set up a relaxation of the shortest path problem (RSPP) which is considerably smaller and easier to solve. In the 2D-case the RSPP is solved analytically and its solution happens to solve the 
SPP too. A central role in this approach is played by the notion of stable patterns, which are shifted along the $2 \mathrm{D}$-grid. The same approach leads to partial results in the 3D-case (and higher dimensions).

The results of Section 5.3.2 also underlie the design of the heuristic methods discussed in Section 5.3.3. These heuristic procedures are developed to arrive cheaply at suboptimal paths with acceptable performance. We have implemented five of these heuristics and compared their performance.

Another way to improve the efficiency of an $n \mathrm{D}$-system is to apply parallel computing techniques as described in Section 5.3.4. Here the 'least-increments' method is implemented in such a way that it computes each path in the $n \mathrm{D}$-system on a separate processor. The parallel $n \mathrm{D}$-system is applied in combination with the JDQZ and the JD methods. However, it turns out that parallelization is not very useful in this application of the $n \mathrm{D}$-system.

The use of iterative eigenvalue solvers is described in Chapter 6. The iterative eigenvalue solver implementations we used in this thesis are JDQR, JDQZ and JD. An advantage of such an iterative eigenvalue solver, is that it is able to focus on a subset of eigenvalues of the involved matrix. Here the most interesting eigenvalue is the smallest real eigenvalue. Focusing on the smallest real eigenvalue of a matrix is not a usual parameter setting for an iterative eigenvalue solver. Therefore this option is developed and implemented as described in Section 6.3: the selection criterion is adapted such that it is no longer necessary to compute all the eigenvalues of the matrix but that it becomes possible to focus on the smallest real eigenvalues.

An important observation is that the eigenvectors of the matrix at hand exhibit a large amount of internal structure, called the Stetter structure. In Section 6.4 some procedures are studied to project an approximate eigenvector to a close-by vector with Stetter structure to speed up the convergence process of the iterative solver. The main bottleneck was the existence of negative and complex entries in the approximate eigenvectors in combination with the use of a logarithmic transformation. Subsequently, two methods are given to embed such a projection method in the Jacobi-Davidson implementation.

The development of a Jacobi-Davidson eigenvalue solver for commuting matrices is described in Section 6.5. This Jacobi-Davidson method is called the JDCOMM method and is an extended version of the JD method. Its most important newly implemented feature is that it computes the eigenvalues of the matrix $A_{p}$ in the outer loop while iterating with a much sparser (but commuting) matrix $A_{x_{i}}$ in the inner loop. Most of the computation time is spent in this inner loop, while the outer loop is only used occasionally, which results in a speed up in computation time and a decrease in the amount of required floating point operations.

Chapter 7 presents the results of the numerical experiments in which the global minima of various Minkowski dominated polynomials are computed using the approaches and techniques mentioned in Part II of this thesis.

Section 7.1 describes the computations on a polynomial of order eight in four variables. To compute the global minimum of this polynomial conventional methods are 
used first. Then the $n \mathrm{D}$-systems approach is used in combination with the iterative eigenproblem solvers JDQR, JDQZ, and Eigs. Finally, the outcomes of these computations are compared with those of the software packages SOSTOOLS, GloptiPoly and SYNAPS. The JDQZ method computes the global minimum and its minimizer faster than the JDQR and Eigs method. However, the methods SOSTOOLS, GloptiPoly and SYNAPS are faster than the methods using our $n$ D-systems approach. But where these methods appear to be faster, they are not as accurate as the iterative eigenvalue solvers. This may be due to the default tolerance settings used in these software packages.

In Section 7.2 the $n \mathrm{D}$-systems approach is used to compute the global minima of 22 distinct polynomials. These 22 polynomials vary in the number of variables and in the total degree and therefore they also vary in the size of the involved linear operators and in their numerical conditioning. Here the target selection as described in Section 6.3 is used to let the iterative solvers JD and JDQZ focus on the smallest real eigenvalues first. To put the performance of these methods into perspective they are compared with the performance of the SOSTOOLS package. In the majority of the test cases the SOSTOOLS software approach is more efficient than the $n \mathrm{D}$-system implementation presented here. In particular it appears that in the SOSTOOLS approach the processing time increases less rapidly with increasing complexity. However, it should be noted that the error in the SOSTOOLS approach is higher in some cases than the error in the $n \mathrm{D}$ systems implementation. Some test cases can not accurately and reliably be solved by the SOSTOOLS software. These large errors can limit the actual application of this software on large problem instances. Furthermore there are multivariate polynomials that are positive but can not be written as a sum of squares [92]. The global minimum of such polynomials can not be found directly using the SOSTOOLS approach. Such a limitation does not affect the Stetter-Möller matrix method.

Section 7.3 shows the result of applying the projection method of Section 6.4. Projection to Stetter structure using a logarithmic transformation can be performed if the effects on complex and negative numbers are taken into account. The JD eigenvalue solver with JD expansion no longer converges to the desired eigenvector if projection of the absolute values is used. When the expansion is changed to Rayleigh Quotient Iteration (RQI) and the projection is applied, the number of matrix-vector computations is comparable to the numbers required in the original JD implementation in most cases and lower for some particular cases. This indicates that projection can indeed be used to increase the convergence speed of the iterative eigenvalue solvers at least for certain examples.

Section 7.4 shows the results of applying the parallel approach of Section 5.3.4 on the set of 22 polynomials. The number of processors used in parallel here is 4 . It turns out that the computing time is longer but the required number of matrixvector operations is roughly identical in this parallel approach in comparison with a sequential approach. Therefore, we conclude that parallelization is not appropriate for this type of problems. This is caused by the high density of short paths that, for an efficient calculation, require communication between the different path computations. 
Furthermore, for lower complexities the required overhead for multiple processors dominates the actual computational costs and therefore a parallel approach requires more processing time than a single processor implementation.

Finally, Section 7.5 describes three experiments in which the newly developed and implemented JDCOMM method is used to compute the global minima. The global minima in this section are also computed by the JD method and by the SOSTOOLS and GloptiPoly packages to be able to compare the performance and the efficiency of the various methods. The result here is that the JDCOMM method has a superior performance over the JD method and the SOSTOOLS and GloptiPoly methods for all the three experiments. The JDCOMM method uses fewer matrix-vector operations and therefore also needs less computation time.

The performance of the iterative eigenvalue solvers is only efficient when the parameter settings are well chosen. They are important to ensure the convergence to the desired eigenvalue. We have obtained additional insight on these settings. In particular, it is required to threshold the imaginary part of the target to prevent constant switching of target due to the approximations.

In Part III of this thesis the approach of [50] plays an important role. In that article the $\mathrm{H}_{2}$ model-order reduction problem from order $N$ tot $N-1$ is solved by algebraic methods. By approaching the problem as a rational optimization problem it is reformulated as the problem of finding solutions of a quadratic system of polynomial equations. In Chapter 8 this approach is generalized and extended which results in a joint framework in which to study the $\mathrm{H}_{2}$ model-order reduction problem for various co-orders $k \geq 1$. This makes it possible to solve the $H_{2}$ model-order reduction problem from order $N$ to $N-1, N-2$, and $N-3$.

For co-orders $k \geq 1$ we also end up with a system of equations in $k-1$ additional real parameters. Furthermore, any feasible solution of this system of equations should satisfy $k-1$ additional linear constraints. For computing the real approximations of order $N-k$, the algebraic Stetter-Möller matrix method for solving systems of polynomial equations of Section 3.3 is used. This approach casts the problem into a particular eigenvalue problem involving one or more matrices in the $k-1$ parameters. This approach guarantees also in this application that the globally best approximation to the given system is found (while typically many local optima exist). To select the global best solution the new and useful result of Theorem 8.2 is used where we present for the co-order $k$ case the homogeneous polynomial of degree three which coincides with the $\mathrm{H}_{2}$ model-order reduction criterion for the difference between $H(s)$ and $G(s)$ at the stationary points of the system of equations. This is a generalization of the third order polynomial for the co-order $k=1$ case presented in [50].

In Chapter 9 this approach for $k=1$ leads to a large conventional eigenvalue problem. The solutions of this eigenvalue problem lead to approximations of order $N-1$ as described in [50]. However, we improve the efficiency of this method by a matrix-free implementation which is possible by using the $n \mathrm{D}$-systems approach as presented in Chapter 5 in combination with an iterative Jacobi-Davidson eigenvalue 
solver as described in Chapter 6. The third order polynomial $V_{H}$ given in Section 9.1 offers an additional computational advantage. The globally optimal approximation of order $N-1$ is obtained by computing the smallest real eigenvalue of the matrix $A_{V_{H}}^{T}$ which avoids the computation of all the eigenvalues. This is achieved by using an iterative Jacobi-Davidson solver. In this way it is furthermore possible to work in a matrix-free fashion.

In [50] the highest possible order for a co-order $k=1$ model reduction was 9 . In Section 9.3 the potential of the reduction technique in combination with an $n \mathrm{D}$ system and an iterative solver on the matrix $A_{V_{H}}$ is demonstrated by an example which involves the reduction of a system of order 10 to a system of order 9 without explicitly constructing any matrices.

In the Chapters 10 and 11 the more difficult problems of computing a globally optimal real $H_{2}$-approximation $G(s)$ for the co-order $k=2$, and $k=3$ case are addressed. As shown in this thesis, repeated application of the co-order one technique to achieve a larger reduction of the model-order is non-optimal, which shows the practical relevance of the co-order two and three problem.

When taking the additional linear condition in the co-order $k=2$ case in Chapter 10 into account, the Stetter-Möller matrix method yields a rational matrix in $\rho$. Using the result of Theorem 10.1 in Section 10.1, this matrix is transformed into a polynomial matrix in $\rho$, which yields a polynomial eigenvalue problem. The important observation that the polynomial degree of $\rho$ in this matrix is equal to $N-1$ is given in Corollary 10.2. To solve this polynomial eigenvalue problem it is cast into an equivalent singular generalized eigenvalue problem in Section 10.2. To accurately compute the meaningful eigenvalues of the singular matrix pencil, the singular part and infinite eigenvalues are split off by computing the Kronecker canonical form, as described in Section 10.3. In Section 10.5 all these techniques are combined in one algorithm for the co-order $k=2$ case.

In Section 10.6 three examples are given where the algorithm is successfully applied to obtain a globally optimal approximation of order $N-2$. In the second example in Subsection 10.6.2 all the eigenvalues of the involved singular matrix pencil were incorrectly specified to be 0 , indeterminate or infinite by all the numerical methods we have available, due to ill-conditioning of the singular pencil. By computing the Kronecker canonical form the singular parts and the infinite eigenvalues are split off which enables to reliably compute the eigenvalues by a numerical method. Subsection 10.6.3 of this chapter describes an example of a globally optimal $H_{2}$ model-order reduction of order 4 to order 2 . The co-order $k=1$ and $k=2$ techniques are applied and their performance is compared which each other. The results for this example turn out to be quite different. The co-order $k=2$ technique turns out to exhibit the best performance in terms of the $H_{2}$-approximation criterion.

In Chapter 11 we show how the Stetter-Möller matrix method yields two rationally parameterized matrices in $\rho_{1}$ and $\rho_{2}$ when taking the two additional linear condition in the co-order $k=3$ case into account. Using the result in Theorem 10.1 again, both matrices are made polynomial in $\rho_{1}$ and $\rho_{2}$, which yields a two-parameter polynomial 
eigenvalue problem involving two matrices and one common eigenvector in Section 11.1. Both these matrices are joined together in one rectangular and singular matrix. Now the ideas of Chapter 10 regarding the Kronecker canonical form computations are useful to compute the values $\rho_{1}$ and $\rho_{2}$ which make these matrices simultaneously singular. In Section 11.3 three methods are given to split off singular parts of the involved matrix pencil, which make it possible to compute the solutions of the system of equations. The advantage of the second and third method is that the matrix dimensions stay as small as possible.

An important result is given by Proposition 11.1: the only singular blocks that occur in the singular part of the Kronecker canonical form in the co-order $k=3$ case, in order to admit a non-trivial solution, are the blocks $L_{\eta}^{T}$. This leads to another important result given in Proposition 11.2: the values $\rho_{1}$ and $\rho_{2}$ we are looking for are computed from the values which make the transformation matrices $V$ and $W$ singular. Using these values the globally optimal approximation $G(s)$ of order $N-3$ is computed An example is worked out in Section 11.5. The co-order $k=3$ technique exhibits a better performance on this example than the co-order $k=1$ and $k=2$ techniques.

\subsection{Directions for further research}

The approach taken in this thesis to compute the global minimum of a dominated polynomial can be extended in several ways. One immediate extension involves the dominating term $\lambda\left(x_{1}^{2 d}+\ldots+x_{n}^{2 d}\right)$ with $\lambda>0$, which may obviously be replaced by a dominating term of the form $\lambda_{1} x_{1}^{2 d}+\ldots+\lambda_{n} x_{n}^{2 d}$ with $\lambda_{i}>0$ for all $i \in\{1,2, \ldots, n\}$. Depending on the monomials which are allowed to feature in the polynomial $q\left(x_{1}, \ldots, x_{n}\right)$ and depending on the chosen monomial ordering, one may also extend the approach by using a dominating term of the form $\lambda_{1} x_{1}^{2 d_{1}}+\ldots+\lambda_{n} x_{n}^{2 d_{n}}$ with $\lambda_{i}>0$ and possibly different (but well-chosen) positive integers $d_{i}$. Also, one may consider generalizations which involve weighted total degree monomial orderings.

As we have argued in Chapter 4, the range of applicability of the presented method extends beyond that of dominated polynomials. In [48] it is shown how the infimum of an arbitrary multivariate real polynomial $q\left(x_{1}, \ldots, x_{n}\right)$ can be found as the limit for $\lambda \downarrow 0$ of the global minima of the dominated polynomials $q\left(x_{1}, \ldots, x_{n}\right)+$ $\lambda\left\|\left(x_{1}, \ldots, x_{n}\right)\right\|_{2 d}^{2 d}$. There it is also shown that if $\lambda>0$ decreases below a certain threshold value, no more bifurcations with respect to the set of stationary points will take place. Since the dominating term $\lambda\left\|\left(x_{1}, \ldots, x_{n}\right)\right\|_{2 d}^{2 d}$ can be regarded as a penalty term which has been added to the polynomial function $q\left(x_{1}, \ldots, x_{n}\right)$, it also allows one to compute bounds on the achievable global minimum of $q\left(x_{1}, \ldots, x_{n}\right)$ from the outcomes for values of $\lambda>0$, especially once the location of a global minimizer is known to be confined to some compact subset of $\mathbb{R}^{n}$. However, if $\lambda>0$ is taken too small, numerical problems are likely to arise.

A second way to deal with an arbitrary real polynomial $q\left(x_{1}, \ldots, x_{n}\right)$ applies if the first-order conditions generate an ideal with zero-dimensional variety and if it is known 
that the polynomial has a global minimum. In that case the first-order conditions can be brought in Gröbner basis form with respect to a total degree ordering (using the Buchberger algorithm, for example). Then the Stetter-Möller matrix approach is applicable to find the critical points and critical values of the polynomial.

The optimization method presented here can also be employed to find the global minimum of a real multivariate rational function in an iterative way by solving a sequence of associated global minimization problems involving real polynomials, see [64]. In this case, there are good opportunities for speeding up the iteration process by combining this algebraic approach with conventional numerical local search methods, which allow one to compute upper bounds on the global minimum. The general setup of an iteration step of such combined methods consists of the computation of a candidate value for the global minimum (e.g., by local search) and a test of global minimality of this candidate value by the algebraic construction of an eigenvalue problem as in the approach of this thesis. In case the global minimality test fails, the method will yield a new starting point for a local search method which is guaranteed to lead to an improved candidate value for the global minimum.

The fact that other software tools, such as, e.g., SOSTOOLS, are more efficient in some cases, indicates that further efficiency improvements can be obtained. First, the parameter settings for the iterative eigenvalue solvers can be further optimized. The main calibration in this research was aimed at ensuring the correct functioning of the software. Parameters for the maximum and minimum search space dimension can be optimized, which (possibly) results in a decrease in the required amount of matrix-vector operations.

Second, the projection to Stetter structure requires further analysis. Combining the projection with a Jacobi-Davidson method and tuning it such that a balance between 'increasing Stetter structure' and 'reducing residuals' is achieved, is expected to result in a decrease of the required amount of matrix-vector operations because both aspects work well together (in the optimum there is maximal Stetter structure and no residual). Then it is of interest to extend the refined extraction procedure of JD, such that it also takes into account the amount of Stetter structure. Such an extension is given in Subsection 6.4.2. The problem with the projection method is that a logarithmic transformation is used which is difficult when applied to negative or complex entries in the approximate eigenvector. In Section 6.4.1 a projection method is given which does not use the logarithmic transformation. Another aspect which should be analyzed further is the question of when to apply this Stetter projection. One can imagine that a bad approximate eigenvector is projected onto the wrong Stetter vector. The solution for this in the implementations for this research is that projection is only applied when the estimate of the eigenvector has become accurate enough.

The research described in Part III of this thesis, leaves us with a number of open research questions which require attention.

In the algebraic approach to global $H_{2}$-approximation of [50] for the co-order 
$k=1$ case, it is convenient to impose the technical condition that all the poles of the original given system $H(s)$ are distinct. We have imposed a similar constraint for ease of exposition for all co-orders $k$ in this thesis. Extensions may be developed in the future to handle the case with poles of multiplicities larger than 1 too.

Moreover, the algebraic techniques presented in this part of the thesis may be combined with numerical local search techniques to arrive at practical algorithms, for instance to save on hardware requirements, computation time, or to deal with numerical accuracy issues. To deal with numerical conditioning issues is an important research topic, to be able to deal with problems that are of a somewhat larger size.

A disadvantage of the co-order $k=2$ and $k=3$ case, from the perspective of computational efficiency, is that one can only decide about global optimality of a solution $G(s)$ when all the solutions of the polynomial eigenvalue problem have been computed and further analyzed. Currently it is investigated for the co-order $k=2$ case whether the third order polynomial $V_{H}$ can be employed by iterative polynomial eigenproblem solvers to limit the number of eigenvalues that require computation. Possibly, one could modify the available Jacobi-Davidson methods for a polynomial eigenvalue problem in such a way that it iterates with the involved matrix but targets on small positive real values of the criterion function $V_{H}$ first (in the same spirit as the JDCOMM method).

When we compute the Kronecker canonical forms of the singular matrix pencils in the co-order $k=2$ and $k=3$ case, we use exact arithmetic to split off the singular blocks of these pencils. This is only possible when the given transfer function $H(s)$ is given in exact format. Exact computations are computationally hard and, moreover, they become useless when the transfer function $H(s)$ is given in numerical format. To overcome this computational limitation it is advisable to use numerical algorithms to compute the Kronecker canonical forms of the singular matrix pencils as described for example in [34].

Finally, $\mathrm{H}_{2}$ model-order reduction of the multi-input, multi-output case along similar lines as the approach followed here will lead to further generalizations too. Furthermore, the discrete time case can be handled too when using the isometric isomorphism mentioned in Remark 8.2 which transforms the continuous time case into the discrete time case. 


\section{Bibliography}

[1] W. Adams and P. Loustaunau. An Introduction to Gröbner Bases, Graduate Studies in Mathematics, volume 3. American Mathematical Society, 1994. [cited at p. 11]

[2] P.R. Aigrain and E.M. Williams. Synthesis of n-reactance networks for desired transient response. Journal of Applied Physics, 20:597-600, 1949. [cited at p. 134]

[3] E.L. Allgower and K. Georg. Numerical Continuation Methods: An Introduction. Springer, 1990. [cited at p. 30]

[4] W.E. Arnoldi. The principle of minimized iteration in the solution of the matrix eigenvalue problem. Quarterly of Applied Mathematics, 9:17-29, 1951. [cited at p. 82]

[5] S. Attasi. Modeling and recursive estimation for double indexed sequences. In R.K. Mehra and D.G. Lainiotis, editors, System Identification: Advances and Case Studies, pages 289-348, New York, 1976. Academic Press. [cited at p. 52]

[6] Z. Bai, J. Demmel, J. Dongarra, A. Ruhe, H. van der Vorst, and editors. Templates for the solution of Algebraic Eigenvalue Problems: A Practical Guide. SIAM, 2000. [cited at p. 168]

[7] L. Baratchart. Existence and generic properties of $\mathrm{L}_{2}$ approximants for linear systems. IMA Journal of Mathematical Control and Information, 3:89-101, 1986. [cited at p. 134]

[8] L. Baratchart, M. Cardelli, and M. Olivi. Identification and rational $\mathrm{L}_{2}$ approximation: A gradient algorithm. Automatica, 27(2):413-417, 1991. [cited at p. 141]

[9] L. Baratchart and M. Olivi. Index of critical points in rational $\mathrm{L}_{2}$-approximation. Systems and Control Letters, 10(3):167-174, 1988. [cited at p. 141]

[10] T. Becker, V. Weispfenning, and H. Kredel. Gröbner Bases: A Computational Approach to Commutative Algebra, volume 141. Springer, 1993. [cited at p. 11, 23]

[11] M. Berhanu. The polynomial eigenvalue problem. Ph.D. Thesis, University of Manchester, Manchester, England, 2005. [cited at p. 166]

[12] A.W.J. Bierfert. Polynomial optimization via recursions and parallel computing. Master Thesis, Maastricht University, MICC (Department Knowledge Engineering), 2007. [cited at p. 43] 
[13] I.W.M. Bleylevens, B. Hanzon, and R.L.M. Peeters. A multidimensional systems approach to polynomial optimization. Proceedings of the MTNS, Leuven, Belgium, 2004. [cited at p. 4, 26, 54]

[14] I.W.M. Bleylevens, M.E. Hochstenbach, and R.L.M. Peeters. Polynomial optimization and a Jacobi-Davidson type method for commuting matrices. In preparation, 2009. [cited at p. 4]

[15] I.W.M. Bleylevens, R.L.M. Peeters, and B. Hanzon. A multidimensional systems approach to algebraic optimization. Proceedings of the HPOPT - the 8th International Workshop on High Performance Optimization Techniques: Optimization and Polynomials, Amsterdam, pages 12-13, 2004. [cited at p. 4, 5]

[16] I.W.M. Bleylevens, R.L.M. Peeters, and B. Hanzon. Efficiency improvement in an $\mathrm{nD}$-systems approach to polynomial optimization. Proceedings of the MEGA 2005: Effective Methods in Algebraic Geometry Computing in and with algebraic geometry: Theory, Algorithms, Implementations, Applications, Alghero, Sardinia, Italy, 2005. [cited at p. 4, 26]

[17] I.W.M. Bleylevens, R.L.M. Peeters, and B. Hanzon. Efficient polynomial optimization with an nD-systems approach. Proceedings of the 24th Benelux Meeting on Systems and Control, Houffalize, Belgium, page 46, 2005. [cited at p. 4]

[18] I.W.M. Bleylevens, R.L.M. Peeters, and B. Hanzon. An nD-systems approach to global polynomial optimization with an application to $\mathrm{H}_{2}$ model-order reduction. Proceedings of the 44th IEEE CDC and the ECC, Seville, Spain, pages 5107-5112, 2005. [cited at p. 4, 5, 154]

[19] I.W.M. Bleylevens, R.L.M. Peeters, and B. Hanzon. Optimal $\mathrm{H}_{2}$ model reduction from order N to N-2. Proceedings of the 13th ILAS Conference, Amsterdam, pages 76-77, 2006. [cited at p. 5]

[20] I.W.M. Bleylevens, R.L.M. Peeters, and B. Hanzon. Efficiency improvement in an $\mathrm{nD}$-systems approach to polynomial optimization. Journal of Symbolic Computation, 42(1-2):30-53, 2007. [cited at p. 4, 26, 35, 36, 46, 114]

[21] I.W.M. Bleylevens, R.L.M. Peeters, and B. Hanzon. Optimal $\mathrm{H}_{2}$ model reduction from order N to N-2. Internal Report M07-01, Department of Mathematics, Maastricht University, 2007. [cited at p. 5]

[22] I.W.M. Bleylevens, R.L.M. Peeters, and B. Hanzon. A generalized eigenvalue approach to $\mathrm{H}_{2}$ model-order reduction. Proceedings of the 27th Benelux Meeting on Systems and Control, Heeze, page 98, 2008. [cited at p. 5]

[23] B. Buchberger. Gröbner bases: An algorithmic method in polynomial ideal theory. In N.K. Bose, editor, Multidimensional Systems Theory, Mathematics and Its Applications, pages 184-232. D. Reidel Publishing Company, 1985. [cited at p. 11, 19, 34]

[24] J. Canny and I. Emiris. Efficient incremental algorithms for the sparse resultant and the mixed volume. Journal of Symbolic Computation, 20:117 Ü-149, 1995. [cited at p. 28]

[25] J. Canny, E. Kaltofen, and Y. Lakshman. Solving systems of non-linear polynomial equations faster. In Proceedings of the ACM-SIGSAM 1989 international symposium on Symbolic and algebraic computation 1989, pages 121-128, New York, 1989. ACM Press. [cited at p. 29] 
[26] A. Cayley. On the theory of elimination. Cambridge and Dublin Mathematical Journal, 3:210-270, 1865. [cited at p. 29]

[27] T.Y. Chen and J. Demmel. Balancing sparse matrices for computing eigenvalues. Linear Algebra and Its Applications, 309(1-3):261-287, 2000. [cited at p. 110, 158]

[28] D. Cox, J. Little, and D. O'Shea. Ideals, Varieties, and Algorithms. An Introduction to Computational Algebraic Geometry and Commutative Algebra. Springer, 2nd edition, 1997. [cited at p. 11, 17, 18, 19, 20, 23, 34]

[29] D. Cox, J. Little, and D. O'Shea. Using algebraic geometry. Springer, 1998. [cited at p. 35]

[30] E.R. Davidson. The iterative calculation of a few of the lowest eigenvalues and corresponding eigenvectors of large real-symmetric matrices. Journal of Computational Physics, 17:87Ü-94, 1975. [cited at p. 82]

[31] B.L. Van der Waerden. Algebra: Volume I. Springer-Verlag, 6th edition, 1964. [cited at p. 26]

[32] E.W. Dijkstra. A note on two problems in connexion with graphs. Numerische Mathematik, 1:269-271, 1959. [cited at p. 52]

[33] A.L. Dixon. The elimination of three quantics in two independent variables. In Proceedings of London Mathematical Society, volume 6, pages 468-478, 1908. [cited at p. 29]

[34] P. Van Dooren. The computation of Kronecker's canonical form of a singular pencil. Linear Algebra and Its Applications, 27:103-140, 1979. [cited at p. 166, 168, 172, 177, 236]

[35] D. Eisenbud, D. Grayson, M. Stillman, and B. Sturmfels. Mathematical Computations with Macaulay 2 (http://www.math.uiuc.edu/Macaulay2), volume 8. Springer Verlag, 2001. [cited at p. 25]

[36] I. Emiris and J. Canny. A practical method for the sparse resultant. In M. Bronstein, editor, Proceedings of the 1993 international symposium on Symbolic and algebraic computation ISSAC '93, pages 183-192, New York, 1993. ACM Press. [cited at p. 29]

[37] G. Farin. Curves and surfaces for computer aided geometric design, A practical guide. Academic Press, 1990. [cited at p. 32]

[38] R.W. Floyd. Algorithm 97: Shortest Path. Communications of the ACM, 5(6):345, 1962. [cited at p. 52]

[39] D.R. Fokkema, G.L.G. Sleijpen, and H.A. van der Vorst. Jacobi-Davidson style QR and QZ algorithms for the reduction of matrix pencils. SIAM Journal on Scientific Computing, 20(1):94-125, 1998. [cited at p. 51, 83, 112]

[40] E. Fornasini, P. Rocha, and S. Zampieri. State space realization of 2-D finitedimensional behaviours. SIAM Journal on Control and Optimization, 31(6):1502-1517, 1993. [cited at p. 52]

[41] P. Fulcheri and M. Olivi. Matrix rational $\mathrm{H}_{2}$ approximation: a gradient algorithm based on Schur analysis. SIAM Journal on Control and Optimization, 36(6):21032127, 1998. [cited at p. 134] 
[42] F.R. Gantmacher. The Theory of Matrices, volume 2. Chelsea Publishing Company, 1959. [cited at p. $166,168,172,173,174,175,177$ ]

[43] I.M. Gelfand, M.M. Kapranov, and A.V. Zelevinsky. Discriminants, Resultants and Multidimensional Determinants. Birkhäuser Verlag, 1994. [cited at p. 29]

[44] A. Giovini, T. Mora, G. Niesi, L. Robbiano, and C. Traverso. "One sugar cube, please", or Selection strategies in the Buchberger Algorithm. In S. Watt, editor, Proceedings ISSAC'91, pages 49-54, New York, 1991. ACM Press. [cited at p. 23]

[45] G. Greuel and G. Pfister. A SINGULAR Introduction to Commutative Algebra (http://www.singular.uni-kl.de). Springer Verlag, 2002. [cited at p. 25]

[46] G. Hadley. Nonlinear and dynamic programming. Addison-Wesley, 1964. [cited at p. 100]

[47] B. Hanzon and M. Hazewinkel. Constructive Algebra and Systems Theory. Royal Netherlands Academy of Arts and Sciences, Amsterdam, 2006. [cited at p. 26, 36]

[48] B. Hanzon and D. Jibetean. Global minimization of a multivariate polynomial using matrix methods. Journal of Global Optimization, 27:1-23, 2003. [cited at p. 3, 26, 35, $36,44,45,229,234,257,261]$

[49] B. Hanzon and J.M. Maciejowski. Constructive algebra methods for the $\mathrm{L}_{2}$ problem for stable linear systems. Automatica, 32(12):1645-1657, 1996. [cited at p. 134]

[50] B. Hanzon, J.M. Maciejowski, and C.T. Chou. Optimal $\mathrm{H}_{2}$ order-one reduction by solving eigenproblems for polynomial equations. ArXiv e-prints, 706, june 2007. [cited at p. 3, 5, 7, 26, 36, 135, 136, 140, 141, 143, 145, 146, 147, 148, 152, 153, 154, 155, 156, $229,232,233,235,257,258,259,261,263]$

[51] B. Hassett. Introduction to Algebraic Geometry. Cambridge University Press, 2007. [cited at p. 11]

[52] S. Haykin. Adaptive filter theory. Prentice Hall, 3th edition, 1991. [cited at p. 101]

[53] J. Heijman. A parallel approach to polynomial optimization. Master Thesis, Maastricht University, MICC (Department Knowledge Engineering), 2007. [cited at p. 4, 79, $112,115,117]$

[54] D. Henrion and J.B. Lasserre. GloptiPoly: Global optimization over polynomials with Matlab and SeDuMi. ACM Transactions on Mathematical Software (TOMS), 29(2):165-194, 2003. [cited at p. 111]

[55] N.J. Higham, R.C. Li, and F. Tisseur. Backward error of polynomial eigenproblems solved by linearization. SIAM Journal on Matrix Analysis and Applications, 29(4):1218-1241, 2007. [cited at p. 166, 167]

[56] N.J. Higham, D.S. Mackey, N. Mackey, and F. Tisseur. Symmetric linearizations for matrix polynomials. SIAM Journal on Matrix Analysis and Applications, 29(1):143159, 2006. [cited at p. 167]

[57] M.E. Hochstenbach, T. Kosir, and B. Plestenjak. A Jacobi-Davidson type method for the two-parameter eigenvalue problem. SIAM Journal on Matrix Analysis and Applications, 26(2):477-497, 2005. [cited at p. 198] 
[58] M.E. Hochstenbach and Y. Notay. The Jacobi-Davidson method. GAMMMitteilungen, 29(2):368-382, 2006. [cited at p. 81, 83, 84, 86, 98, 103, 112, 115, 119]

[59] M.E. Hochstenbach and B. Plestenjak. Backward error, condition numbers, and pseudospectrum for the multiparameter eigenvalue problem. Linear Algebra and Applications, 375:63-81, 2003. [cited at p. 198]

[60] M.E. Hochstenbach and B. Plestenjak. Harmonic Rayleigh-Ritz for the multiparameter eigenvalue problem. CASA report, Technische Universiteit Eindhoven, 06(35):1-16, 2006. [cited at p. 198]

[61] M.E. Hochstenbach and G.L.G. Sleijpen. Harmonic and refined Rayleigh-Ritz for the polynomial eigenvalue problem. Numerical Linear Algebra with Applications, 15:35-54, 2008. [cited at p. 180]

[62] E. Jarlebring and M.E. Hochstenbach. Polynomial two-parameter eigenvalue problems and matrix pencil methods for stability of delay-differential equations. Linear Algebra and Its Applications, 431:369-380, 2009. [cited at p. 180]

[63] D. Jibetean. Global optimization of rational multivariate functions. Technical Report PNA-R0120, CWI, Amsterdam, 2001. [cited at p. 4, 43]

[64] D. Jibetean. Algebraic Optimization with Applications to System Theory. Ph.D. Thesis, Vrije Universiteit, Amsterdam, 2003. [cited at p. 4, 44, 235]

[65] D. Kapur and Y.N. Lakshman. Elimination methods: an introduction. In B. Donald, D. Kapur, and J. Mundy, editors, Symbolic and Numerical Computation for Artificial Intelligence, pages 45-89. Academic Press, 1992. [cited at p. 28, 29]

[66] D. Kapur and T. Saxena. Comparison of various multivariate resultant formulations. In A.H.M. Levelt, editor, Proceedings of the 1995 international symposium on Symbolic and algebraic computation ISSAC '95, pages 187-195, New York, 1995. ACM Press. [cited at p. 29]

[67] D. Kapur, T. Saxena, and L. Yang. Algebraic and geometric reasoning using Dixon resultants. In J. von zur Gathen and M. Giesbrecht, editors, Proceedings of the International Symposium on Symbolic and Algebraic Computation ISSAC 1994, pages 99-107, New York, 1994. ACM Press. [cited at p. 28]

[68] V.B. Khazanov. On spectral properties of multiparameter polynomial matrices. Journal of Mathematical Sciences, 89(6):1775-1800, 1998. [cited at p. 247]

[69] V.B. Khazanov. To solving spectral problems for multiparameter polynomial matrices. Journal of Mathematical Sciences, 141(6):1690-1700, 2007. [cited at p. 247]

[70] D.E. Knuth. The Art of Computer Programming, Volume 2: Seminumerical Algorithms. Addison Wesley, 1981. [cited at p. 28]

[71] M. Kreuzer and L. Robbiano. Computational Commutative Algebra. 1 (http://cocoa.dima.unige.it). Springer Verlag, 2000. [cited at p. 25]

[72] P. Lancaster and M. Tismenetsky. Theory of matrices with applications. Academic Press, 2nd edition, 1985. [cited at p. 82, 100, 166, 167] 
[73] C. Lanczos. An iteration method for the solution of the eigenvalue problem of linear differential and integral operators. J. Research of the National Bureau of Standards, 45:255Ü-282, 1950. [cited at p. 82]

[74] J.B. Lasserre. Global optimization with polynomials and the problem of moments. SIAM Journal on Optimization, 11(3):796-817, 2001. [cited at p. 111]

[75] J.B. Lasserre. An explicit equivalent positive semidefinite program for nonlinear 0-1 programs. SIAM Journal on Optimization, 12(3):756-769, 2002. [cited at p. 111]

[76] D. Lazard. Resolution des systemes d'equation algebriques. Theoretical Computer Science, 15:77-110, 1981. [cited at p. 29]

[77] R.B. Lehoucq, D.C. Sorensen, and C. Yang. ARPACK Users' Guide: Solution of Large-Scale Eigenvalue Problems with Implicitly Restarted Arnoldi Methods. SIAM Publications, 1998. [cited at p. 51, 82]

[78] D. Lemonnier and P. van Dooren. Balancing regular matrix pencils. Journal on Matrix Analysis and Applications, 28(1):253-263, 2006. [cited at p. 184]

[79] F.S. Macaulay. Algebraic theory of modular systems. Cambridge Tracts in Mathematics and Mathematical physics, no. 19, 1916. [cited at p. 28, 29]

[80] L. Meier and D. Luenberger. Approximation of linear constant systems. IEEE Transactions on Automatic Control, 12(5):585-588, 1967. [cited at p. 141]

[81] H.M. Möller and H.J. Stetter. Multivariate polynomial equations with multiple zeros solved by matrix eigenproblems. Numerische Mathematik, 70:311-329, 1995. [cited at p. 3, 26, 35, 36, 229, 257, 261]

[82] H.M. Möller and R. Tenberg. Multivariate polynomial system solving using intersections of eigenspaces. Journal of Symbolic Computation, 32:513-531, 2001. [cited at p. 35]

[83] A.P. Morgan. Solving Polynomial Systems Using Continuation for Engineering and Scientific Problems. Prentice Hall, 1987. [cited at p. 30]

[84] B. Mourrain. Symbolic-numeric methods for solving polynomial equations and applications. In A. Dickenstein, editor, Lecture notes of the 1st Latin American School on Polynomial Systems, CIMPA, 2003. [cited at p. 28, 37]

[85] B. Mourrain and J.P. Pavone. Subdivision methods for solving polynomial equations. Journal of Symbolic Computation, 44:292-306, 2009. [cited at p. 32]

[86] P.A. Parrilo. Semidefinite programming relaxations for semialgebraic problems. Mathematical Programming, 96(2):293-320, 2003. [cited at p. 111]

[87] P.A. Parrilo and B. Sturmfels. Minimizing Polynomial Functions. Algorithmic and quantitative real algebraic geometry, DIMACS Series in Discrete Mathematics and Theoretical Computer Science, 60:83-100, 2003. [cited at p. 111]

[88] R.L.M. Peeters, I.W.M. Bleylevens, and B. Hanzon. A mixed algebraic-numerical global optimization approach to $\mathrm{H}_{2}$ model reduction. Proceedings of the 22th Benelux Meeting on Systems and Control, Lommel, Belgium, 2003. [cited at p. 4]

[89] R.L.M. Peeters, B. Hanzon, and D. Jibetean. Optimal $\mathrm{H}_{2}$ model reduction in statespace: a case study. Proceedings of the European Control Conference ECC 2003, Cambridge, UK, 2003. [cited at p. 222] 
[90] S. Prajna, A. Papachristodoulou, P. Seiler, and P.A. Parrilo. SOSTOOLS: Sum of squares optimization toolbox for MATLAB (http://www.mit.edu/ parrilo/sostools), 2004. [cited at p. $25,111,114]$

[91] G. Reis, B. Mourrain, R. Rouillier, and Ph. Trébuchet. An environment for Symbolic and Numeric Computation (http://www-sop.inria.fr/galaad/software/synaps). In Proceedings of the International Conference on Mathematical Software, pages 239249, 2002. [cited at p. 32, 111]

[92] T. Roh, B. Dumistrescu, and L. Vandenberghe. Interior-point algorithms for sum-ofsquares optimization of multidimensional trigonometric polynomials. In International Conference on Acoustics Speech and Signal Processing 200\%, pages 905-908, 2007. [cited at p. 114, 231]

[93] J. Shyu, S. Pei, and K. Fu. Complex Lagrange multiplier approach to the design of arbitrary complex coefficient FIR digital filters. Signal Processing, 35:117-130, 1994. [cited at p. 101]

[94] G.L.G. Sleijpen and H.A. van der Vorst. A Jacobi-Davidson iteration method for linear eigenvalue problems. SIAM Journal on Matrix Analysis and Applications, 17(2):401425, 1996. [cited at p. 51, 81, 83, 85]

[95] D.C. Sorensen. Implicit application of polynomial filters in a $k$-step Arnoldi method. SIAM Journal on Matrix Analysis and Applications, 1992. [cited at p. 128]

[96] J.F. Sturm. Using SeDuMi 1.02, a MATLAB toolbox for optimization over symmetric cones (http://sedumi.mcmaster.ca). Optimization Methods and Software, 11:625-653, 1999. [cited at p. 111]

[97] B. Sturmfels. Sparse elimination theory. In D. Eisenbud and L. Robbiano, editors, Proceedings Computational Algebraic Geometry and Commutative Algebra 1993, pages 264-298. Cambridge University Press, 1993. [cited at p. 29]

[98] B. Sturmfels. Solving systems of polynomial equations. American Mathematical Society, CBMS Regional Conferences Series, No 97, 2002. [cited at p. 26, 35, 37, 38]

[99] B. Sturmfels and A. Zelevinsky. Multigraded resultants of the Sylvester type. Journal of Algebra, 163(1):115-127, 1994. [cited at p. 29]

[100] R.C. Ward. Balancing the generalized eigenvalue problem. SIAM Journal on Scientific and Statistical Computing, 2(2):141-152, 1981. [cited at p. 184]

[101] D.A. Wilson. Model reduction for multivariable systems. International Journal of Control, 20(1):57-64, 1974. [cited at p. 134]

[102] K. Zhou, J.C. Doyle, and K. Glover. Robust and optimal control. Prentice Hall, 1996. [cited at p. 138, 139] 



\section{Appendices}

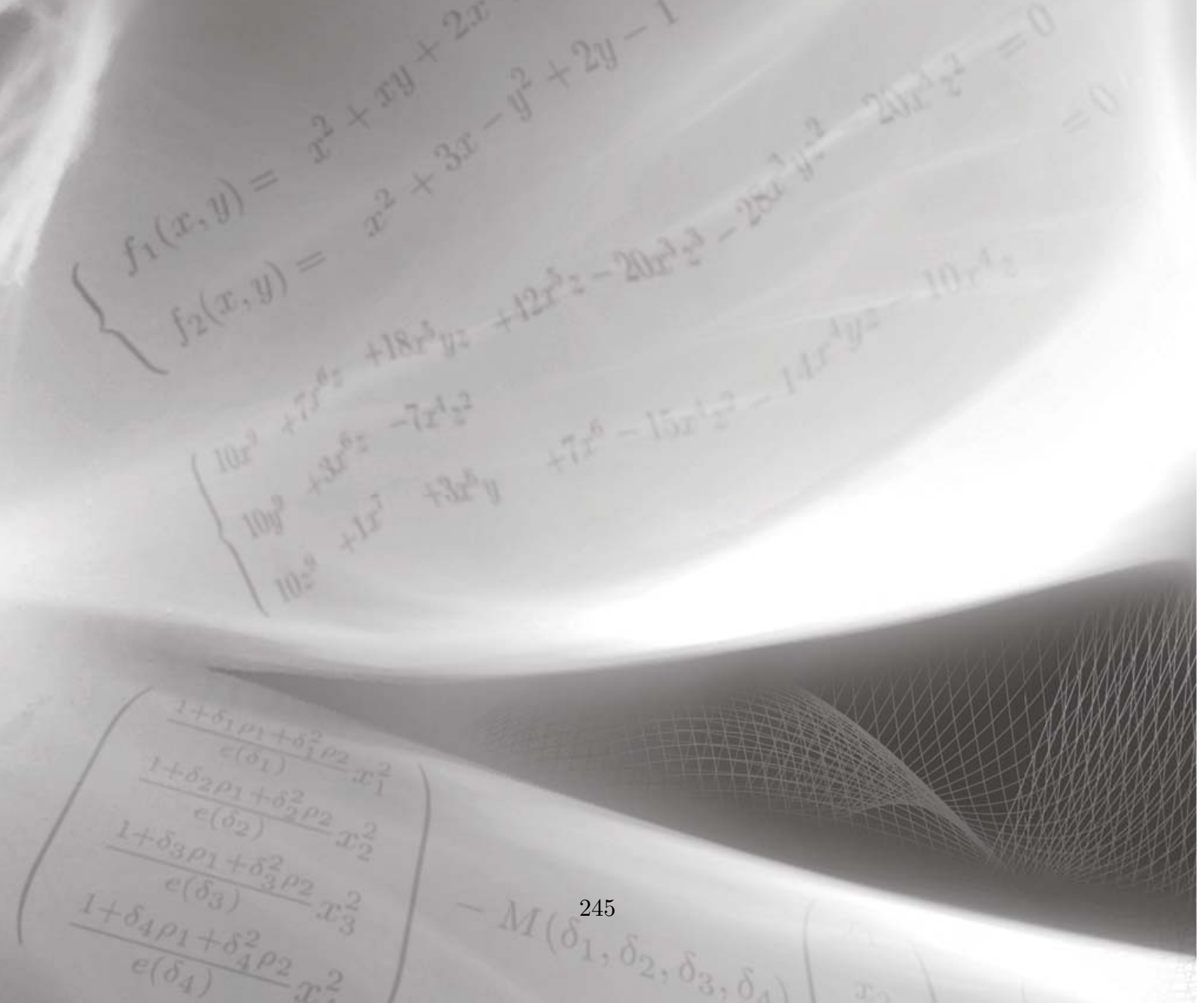





\section{Appendix A}

\section{A linearization technique for a two-parameter eigenvalue problem}

Let the polynomial eigenvalue problem:

$$
M(\mu, \lambda) v=0
$$

be given, where the $n \times n$ matrix $M$ is polynomial in $\mu$ and $\lambda$. The total degree of $\mu$ and $\lambda$ in the entries of $M$ is denoted by $m$. Using linearization techniques, one can construct an equivalent, but larger, eigenvalue problem which is linear in both $\lambda$ and $\mu$. One linearization technique is discussed in this appendix but various other approaches are possible. Other techniques to linearize a two parameter polynomial matrix can be found in the literature, see e.g., [68] and [69].

The linearization approach discussed in this appendix, first linearizes the problem with respect to $\mu$ and, subsequently, with respect to $\lambda$.

\section{A.1 Linearization with respect to $\mu$}

Let us first expand the polynomial matrix $M(\mu, \lambda)$ in Equation (A.1) as a polynomial in $\mu$ with coefficients that depend on $\lambda$ :

$$
M(\mu, \lambda)=M_{0}(\lambda)+M_{1}(\lambda) \mu+M_{2}(\lambda) \mu^{2}+\ldots+M_{m}(\lambda) \mu^{m} .
$$

Now a linearization technique can be applied with respect to $\mu$ only. The eigenvector $z$ for the eigenvalue problem, which is linear in $\mu$, is chosen as:

$$
z=\left(\begin{array}{c}
v \\
\mu v \\
\mu^{2} v \\
\vdots \\
\mu^{m-1} v
\end{array}\right) .
$$


Then the following holds:

$$
\begin{gathered}
M(\mu, \lambda) v= \\
\left(M_{0}(\lambda), M_{1}(\lambda), M_{2}(\lambda), \ldots, M_{m-1}(\lambda)\right) z+\mu\left(0,0,0, \ldots, M_{m}(\lambda)\right) z=0
\end{gathered}
$$

while the structure of $z$ is captured by:

$$
\left(\begin{array}{cccc}
0 & I & & 0 \\
& \ddots & \ddots & \\
0 & & 0 & I
\end{array}\right) z=\mu\left(\begin{array}{cccc}
I & 0 & & 0 \\
& \ddots & \ddots & \\
0 & & I & 0
\end{array}\right) z .
$$

Hence, problem (A.1) becomes equivalent to:

$$
\begin{gathered}
\left(\left(\begin{array}{cccc}
0 & I & & 0 \\
& \ddots & \ddots & \\
0 & & 0 & I \\
\hline M_{0}(\lambda) & M_{1}(\lambda) & \ldots & M_{m-1}(\lambda)
\end{array}\right)+\right. \\
\left.\mu\left(\begin{array}{cccc}
-I & 0 & & 0 \\
0 & \ddots & \ddots & \\
\hline 0 & \ldots & 0 & M_{m}(\lambda)
\end{array}\right)\right) z=\left(\begin{array}{c}
0 \\
\vdots \\
0 \\
0
\end{array}\right) .
\end{gathered}
$$

Denoting the involved matrices in the latter equations by the matrices $K(\lambda)$ and $L(\lambda)$, yields:

$$
(K(\lambda)+\mu L(\lambda)) z=0 .
$$

This eigenvalue problem is linear in $\mu$ but still polynomial in $\lambda$. The dimensions of the matrices $K(\lambda)$ and $L(\lambda)$ is $(m n) \times(m n)$. In a second step this problem is linearized with respect to $\lambda$.

Note that Equation (A.6) can also be rewritten as follows:

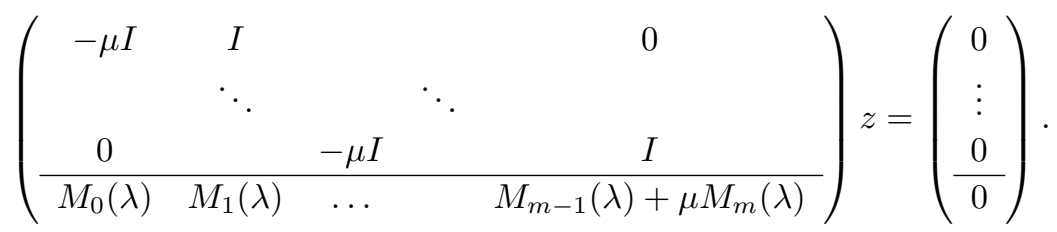

\section{A.2 Linearization with respect to $\lambda$}

Let us expand the problem $(K(\lambda)+\mu L(\lambda)) z=0$ in (A.7), which is linear in $\mu$ and polynomial in $\lambda$, by writing the involved matrix as a polynomial in $\lambda$ : 


$$
\begin{gathered}
K(\lambda)+\mu L(\lambda)= \\
\left(K_{0}+K_{1} \lambda+K_{2} \lambda^{2}+\ldots+K_{m} \lambda^{m}\right)+\mu\left(L_{0}+L_{1} \lambda+L_{2} \lambda^{2}+\ldots+L_{m} \lambda^{m}\right) .
\end{gathered}
$$

Now let us apply the same linearization technique with respect to $\lambda$ and introduce the eigenvector $w$ for the linearized problem:

$$
w=\left(\begin{array}{c}
z \\
\lambda z \\
\lambda^{2} z \\
\vdots \\
\lambda^{m-1} z
\end{array}\right)
$$

for which holds:

$$
\left(\begin{array}{cccc}
0 & I & & 0 \\
& \ddots & \ddots & \\
0 & & 0 & I
\end{array}\right) w=\lambda\left(\begin{array}{cccc}
I & 0 & & 0 \\
& \ddots & \ddots & \\
0 & & I & 0
\end{array}\right) w .
$$

Using this eigenvector $w$, the eigenvalue problem (A.7) becomes equivalent with:

$$
\begin{gathered}
\left(K_{0}+\mu L_{0}, K_{1}+\mu L_{1}, \ldots, K_{m-1}+\mu L_{m-1}\right) w+ \\
\lambda\left(0, \ldots, 0, K_{m}+\mu L_{m}\right) w=0 .
\end{gathered}
$$

Thus, we obtain:

$$
\begin{gathered}
\left(K_{0}, K_{1}, \ldots, K_{m-1}\right) w+\mu\left(L_{0}, L_{1}, \ldots, L_{m-1}\right) w+ \\
\lambda\left(0, \ldots, 0, K_{m}\right) w+\mu \lambda\left(0, \ldots, 0, L_{m}\right) w=0 .
\end{gathered}
$$

However, the total degree of $\mu$ and $\lambda$ in (A.1) does not exceed $m$, so that the matrix $M_{m}(\lambda)$ in (A.6) is in fact a constant matrix. This yields that the matrix $L(\lambda)$ in (A.7) and in (A.9) is also a constant matrix and therefore $L_{1}=L_{2}=\cdots=L_{m}=0$.

Therefore, we end up with the following linear problem:

$$
\begin{aligned}
& \left(\begin{array}{cccc}
\cdots & & & \cdots \\
\cdots & & & \cdots \\
\cdots & & & \cdots \\
\hline K_{0} & K_{1} & \ldots & K_{m-1}
\end{array}\right) w+
\end{aligned}
$$

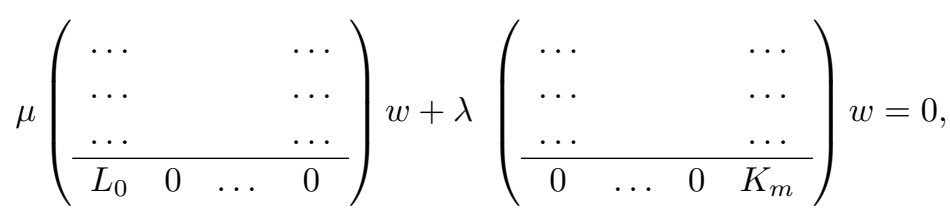


which is written as:

$$
\left(P_{0}+\mu P_{1}+\lambda P_{2}\right) w=0
$$

which is to be enhanced by suitable block rows to fix the particular structure of the eigenvector $w$. To achieve this goal, various ways exist. Now the problem is linear in both $\mu$ and $\lambda$ and the dimensions of the matrices $P_{0}, P_{1}$, and $P_{2}$ are $\left(m^{2} n\right) \times\left(m^{2} n\right)$. Note that the eigenvector $w$ is highly structured, since the vectors from which it is constructed, the vectors $z$, are structured too: $w=\left(z^{T}, \lambda z^{T}, \lambda^{2} z^{T}, \ldots, \lambda^{m-1} z^{T}\right)^{T}$ where $z=\left(v^{T}, \mu v^{T}, \mu^{2} v^{T}, \ldots, \mu^{m-1} v^{T}\right)^{T}$.

The eigenvalues of the original problem (A.1) can be found among the eigenvalues of (A.15) because the eigenvalue spectrum is unchanged. Moreover, the possible multiplicities among the eigenvalues in (A.1) are preserved when linearizing the polynomial problem. The eigenvectors of (A.1) can be recovered from the eigenvectors of (A.15) by making use of the internal structure of the eigenvectors $w$.

Remark A.1. Note that the matrix $M(\mu, \lambda)$ in (A.1) does not necessarily has to be square. Suppose that this matrix $M$ is not square but rectangular. Then the linearization technique of this section can still be applied if the dimensions of the block matrices $I$ and 0 are adapted conformably.

\section{A.3 Example}

Suppose a two-parameter eigenvalue problem is given involving a $n \times n$ matrix $M$ polynomial in $\mu$ and $\lambda$. The total degree of the entries in the parameters $\mu$ and $\lambda$ in the matrix $M$ is three: $m=3$. We can write the matrix $M(\mu, \lambda)$ as:

$$
\begin{gathered}
M(\mu, \lambda)=N_{0}+\mu N_{1}+\lambda N_{2}+\mu^{2} N_{3}+\mu \lambda N_{5}+ \\
\lambda^{2} N_{4}+\mu^{3} N_{6}+\lambda^{2} \mu N_{7}+\lambda \mu^{2} N_{8}+\lambda^{3} N_{9} .
\end{gathered}
$$

Using this, the polynomial matrix $M(\mu, \lambda)$ can be written as:

$$
\begin{aligned}
& M(\mu, \lambda)=\left(\left(N_{0}+\lambda N_{2}+\lambda^{2} N_{4}+\lambda^{3} N_{9}\right)+\right. \\
& \mu\left(N_{1}+\lambda N_{5}+\lambda^{2} N_{7}\right)+ \\
& \mu^{2}\left(N_{3}+\lambda N_{8}\right)+ \\
& \left.\mu^{3}\left(N_{6}\right)\right) \text {. }
\end{aligned}
$$

When the matrices $\left(N_{0}+\lambda N_{2}+\lambda^{2} N_{4}+\lambda^{3} N_{9}\right),\left(N_{1}+\lambda N_{5}+\lambda^{2} N_{7}\right),\left(N_{3}+\lambda N_{8}\right)$ and $\left(N_{6}\right)$ are denoted by the matrices $M_{0}(\lambda), M_{1}(\lambda), M_{2}(\lambda), M_{3}(\lambda)$, respectively, the following formulation is equivalent:

$$
M(\mu, \lambda)=M_{0}(\lambda)+\mu M_{1}(\lambda)+\mu^{2} M_{2}(\lambda)+\mu^{3} M_{3}(\lambda) .
$$


The first step of the linearization technique, transforms the problem $M(\mu, \lambda) v=0$ into an equivalent problem where the eigenvector $z$ is structured as:

$$
z=\left(\begin{array}{c}
v \\
\mu v \\
\mu^{2} v
\end{array}\right)
$$

and where the involved matrices are linear in $\mu$ :

$$
\left(\left(\begin{array}{ccc}
0 & I & 0 \\
0 & 0 & I \\
\hline M_{0}(\lambda) & M_{1}(\lambda) & M_{2}(\lambda)
\end{array}\right)+\mu\left(\begin{array}{ccc}
-I & 0 & 0 \\
0 & -I & 0 \\
\hline 0 & 0 & M_{3}(\lambda)
\end{array}\right)\right) z=\left(\begin{array}{l}
0 \\
0 \\
\hline 0
\end{array}\right) .
$$

This equals:

$$
\begin{gathered}
\left(\left(\begin{array}{ccc}
0 & I & 0 \\
0 & 0 & I \\
\hline\left(N_{0}+\lambda N_{2}+\lambda^{2} N_{4}+\lambda^{3} N_{9}\right) & \left(N_{1}+\lambda N_{5}+\lambda^{2} N_{7}\right) & \left(N_{3}+\lambda N_{8}\right)
\end{array}\right)+\right. \\
\left.\mu\left(\begin{array}{ccc}
-I & 0 & 0 \\
0 & -I & 0 \\
\hline 0 & 0 & N_{6}
\end{array}\right)\right) z=\left(\begin{array}{l}
0 \\
0 \\
\hline 0
\end{array}\right)
\end{gathered}
$$

Furthermore, the blocks $I$ and 0 denote identity blocks and zero blocks, respectively, of dimensions $n \times n$. The dimension of the matrices involved in the problems (A.20) and (A.21) is therefore $3 n \times 3 n$.

In the second step, the linearization with respect to $\lambda$ is carried out. First the matrix involved in Equation (A.21) is written as:

$$
\left(K_{0}+K_{1} \lambda+K_{2} \lambda^{2}+K_{3} \lambda^{3}\right)+\mu\left(L_{0}+L_{1} \lambda+L_{2} \lambda^{2}+L_{3} \lambda^{3}\right)
$$

where

$$
\begin{aligned}
K_{0}=\left(\begin{array}{ccc}
0 & I & 0 \\
0 & 0 & I \\
N_{0} & N_{1} & N_{3}
\end{array}\right), & K_{1}=\left(\begin{array}{ccc}
0 & 0 & 0 \\
0 & 0 & 0 \\
N_{2} & N_{5} & N_{8}
\end{array}\right), \\
K_{2}=\left(\begin{array}{ccc}
0 & 0 & 0 \\
0 & 0 & 0 \\
N_{4} & N_{7} & 0
\end{array}\right), & K_{3}=\left(\begin{array}{ccc}
0 & 0 & 0 \\
0 & 0 & 0 \\
N_{9} & 0 & 0
\end{array}\right), \\
L_{0}=\left(\begin{array}{ccc}
-I & 0 & 0 \\
0 & -I & 0 \\
0 & 0 & N_{6}
\end{array}\right), & \text { and } L_{1}=L_{2}=L_{3}=\left(\begin{array}{lll}
0 & 0 & 0 \\
0 & 0 & 0 \\
0 & 0 & 0
\end{array}\right) .
\end{aligned}
$$

Here the blocks $I$ and 0 denote again identity and zero blocks of dimensions $n \times n$. 
Using the eigenvector $w$ of size $9 n \times 1$ with a structure:

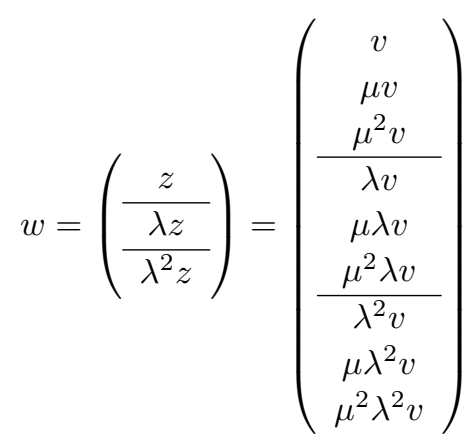

yields the following eigenvalue problem which is linear in $\mu$ and $\lambda$ :

$$
\begin{gathered}
\left(P_{0}+\mu P_{1}+\lambda P_{2}\right) w= \\
\left(\left(\begin{array}{ccc}
0 & I & 0 \\
0 & 0 & I \\
\hline K_{0} & K_{1} & K_{2}
\end{array}\right)+\mu\left(\begin{array}{ccc}
0 & 0 & 0 \\
0 & 0 & 0 \\
\hline L_{0} & 0 & 0
\end{array}\right)+\right. \\
\left.\lambda\left(\begin{array}{ccc}
-I & 0 & 0 \\
0 & -I & 0 \\
\hline 0 & 0 & K_{3}
\end{array}\right)\right) w=\left(\begin{array}{l}
0 \\
0 \\
0
\end{array}\right) .
\end{gathered}
$$

where every block $I$ and 0 has dimensions $3 n \times 3 n$. The dimensions of the matrices $P_{0}, P_{1}$, and $P_{2}$ are $\left(m^{2} n\right) \times\left(m^{2} n\right)=9 n \times 9 n$. Note that the last equation represented by (A.25) satisfies Equation (A.22) times the eigenvector $w$ and that the other rows are required to capture the structure of the eigenvector $w$.

Remark A.2. The existence of another possible approach to linearize a matrix is demonstrated below. The matrix $M(\mu, \lambda)$ in this example with polynomial coefficients in $\mu$ and $\lambda$ with a total degree 3 , can also be linearized in one step as follows:

$$
\begin{gathered}
\left(\begin{array}{cccccc}
0 & I & 0 & 0 & 0 & 0 \\
0 & 0 & I & 0 & 0 & 0 \\
0 & 0 & 0 & I & 0 & 0 \\
0 & 0 & 0 & 0 & I & 0 \\
0 & 0 & 0 & 0 & 0 & I \\
\hline N_{0} & N_{2} & N_{1} & N_{4} & N_{5} & N_{3}
\end{array}\right)+\mu\left(\begin{array}{ccccccc}
0 & 0 & 0 & 0 & 0 & 0 \\
-I & 0 & 0 & 0 & 0 & 0 \\
0 & 0 & 0 & 0 & 0 & 0 \\
0 & -I & 0 & 0 & 0 & 0 \\
0 & 0 & -I & 0 & 0 & 0 \\
\hline 0 & 0 & 0 & 0 & N_{8} & N_{6}
\end{array}\right)+ \\
\left.\lambda\left(\begin{array}{cccccc}
-I & 0 & 0 & 0 & 0 & 0 \\
0 & 0 & 0 & 0 & 0 & 0 \\
0 & -I & 0 & 0 & 0 & 0 \\
0 & 0 & 0 & 0 & 0 & 0 \\
0 & 0 & 0 & 0 & 0 & 0 \\
0 & 0 & 0 & N_{9} & N_{7} & 0
\end{array}\right)\right) \tilde{w}=\left(\begin{array}{c}
0 \\
0 \\
0 \\
0 \\
0 \\
0
\end{array}\right)
\end{gathered}
$$


where the eigenvector $\tilde{w}$ is structured as:

$$
\tilde{w}=\left(\begin{array}{c}
v \\
\lambda v \\
\mu v \\
\lambda^{2} v \\
\lambda \mu v \\
\mu^{2} v
\end{array}\right) .
$$

This problem is of dimension $6 n \times 6 n$ and the eigenvector $\tilde{w}$ only incorporates powers of $\lambda$ and $\mu$ up till a total degree of $m-1=2$. Note that the last equation represented by (A.26) equals $M(\mu, \lambda) v=0$ with the matrix $M(\mu, \lambda)$ as in Equation (A.16). 



\section{Appendix B}

\section{Polynomial test set}

\begin{tabular}{|c|c|}
\hline \# & Polynomial \\
\hline 1 & $\left(5 x^{8}+5 y^{8}\right)-5.0 x y^{6}+8.8 y^{6}+1.2 x y^{5}+5.4 y^{5}+9.2 x^{4} y^{3}-4.3 x^{4} y^{2}+3.5 x y^{2}-2.4 x^{5}+7.5 x$ \\
\hline 2 & $\left(5 x^{8}+5 y^{8}\right)+2 x^{2} y^{5}+3.8 x^{4} y^{2}-3.3 x^{6} y-1.9 y-7 x^{2}$ \\
\hline 3 & $\begin{array}{l}\left(5 x^{10}+5 y^{10}\right)+8.9 x y^{8}-8.8 x^{2} y^{7}+3.6 y^{7}-1.8 x^{2} y^{6}+6.5 y^{5}+1.0 x y^{4}+9.4 x^{4} y^{3}-2.9 x^{3} y^{3}+8.1 y^{2}+ \\
5.7 x^{6} y+8.7 x^{4} y+8.5 x^{3} y-2.5 x^{2} y-8.7 x^{5}+6.1 x^{4}+1.5 x^{2}-5.1\end{array}$ \\
\hline 4 & $\begin{array}{l}\left(5 x^{10}+5 y^{10}\right)+10 y^{8}-6 y^{6}+7 x^{4} y^{5}+9 y^{5}-5 x^{4} y^{4}+2 x y^{4}-4 y^{4}-1 x^{3} y^{3}+7 y^{3}+10 x^{6} y^{2}-3 x^{3} y^{2}+ \\
-2 x y^{2}-2 x^{5} y+3 x^{3} y+5 x^{2} y+7 x^{7}-7\end{array}$ \\
\hline 5 & $\left(5 x^{4}+5 y^{4}+5 z^{4}+5 w^{4}\right)+7.1 w^{2}-8.4 y^{2} w+6.8 x^{2} w+5.5 w-4.0 x y z+8.4 x z-4.8 z-9.5 x^{2}-8.7 x$ \\
\hline 6 & $\begin{array}{l}\left(5 x^{4}+5 y^{4}+5 z^{4}+5 w^{4}\right)-6.1 x w^{2}+9.4 z^{2} w+9.1 x y w-8.8 y w+2.1 w-4.0 y z^{2}-7.9 x z^{2}+7.1 z^{2}+ \\
-4.7 x y z-5.7 y z-5.8 x^{2} z+3.3 z+4.9 x\end{array}$ \\
\hline 7 & $\begin{array}{l}\left(5 x^{12}+5 y^{12}\right)+7.8 x y^{10}-9.3 y^{10}-1.1 x y^{9}+5.2 x y^{8}-7.7 x^{4} y^{7}+8.6 x^{3} y^{7}+3.6 x^{6} y^{5}-8.7 x^{4} y^{4}+ \\
3.1 x^{6} y^{3}+4.6 x^{5} y^{3}+2.5 x^{4} y^{3}+6.5 x^{3} y^{3}+4.6 x^{5} y^{2}-5.5 x^{4} y^{2}+2.5 x^{2} y^{2}+9.3 x^{4} y+2.7 x^{2} y-9.0 y+ \\
6.2 x^{3}-7.1 x^{2}\end{array}$ \\
\hline 8 & $\begin{array}{l}\left(5 x^{12}+5 y^{12}\right)-8.0 y^{10}+3.3 x^{4} y^{7}+4.5 x^{3} y^{7}-2.8 x^{3} y^{5}+1.8 y^{5}-6.1 x^{7} y^{4}+3.3 x^{3} y^{4}+4.0 x^{2} y^{4}+ \\
-3.2 x^{8} y^{3}+6.0 x^{5} y^{3}+3.1 x^{9} y^{2}-4.3 x^{6} y^{2}-4.4 x^{3} y^{2}+4.8 x^{7} y+9.7 x^{10}+7.8 x^{7}-7.7 x^{5}+7.2 x^{4}+ \\
1.0 x^{2}-3.8 x-6.3\end{array}$ \\
\hline 9 & $\begin{array}{l}\left(5 x^{6}+5 y^{6}+5 z^{6}\right)+1.8 y z^{4}+5.8 x z^{4}-8.5 x y z^{3}+4.6 y z^{3}-7.3 y^{3} z^{2}+3.3 x y^{2} z^{2}+6.1 x^{2} y z^{2}-5.8 z^{2}+ \\
-9.3 x y^{2} z+3.4 x^{3} y z+9.0 x^{3} z+4.3 x y^{4}-3.0 x^{3} y^{2}+8.1 x^{2} y^{2}+0.1 x y+4.7 x^{4}-7.3 x+9.8\end{array}$ \\
\hline 10 & $\begin{array}{l}\left(5 x^{6}+5 y^{6}+5 z^{6}\right)-3.8 y z^{4}+9.7 x y z^{3}+8.9 y z^{3}+8.4 x^{3} z^{2}-9.3 x^{2} y^{2} z-4.7 x^{3} y z+3.2 x^{2} y z-7.9 x y z+ \\
6.0 x^{2} z+8.0 x z+6.2 x^{2} y^{3}-6.4 x y^{3}+x^{3} y+7.0 x^{2} y+5.5 x-6.4\end{array}$ \\
\hline 11 & $\begin{array}{l}\left(5 x^{14}+5 y^{14}\right)-4.5 x^{2} y^{11}+5.6 y^{10}-3.1 x^{3} y^{9}+5.1 y^{9}-3.3 x^{2} y^{8}+9.0 x y^{8}+8.7 x^{3} y^{7}-3.3 x^{2} y^{7}+ \\
8.5 x^{5} y^{6}+5.7 x^{2} y^{6}-5.4 y^{6}-5.3 x^{6} y^{5}-1.9 x^{3} y^{5}-4.8 x^{2} y^{5}+0.1 y^{5}+2.1 x^{9} y^{4}+2.6 x^{7} y^{4}+2.6 x^{6} y^{4}+ \\
6.3 x^{5} y^{4}-2.9 x^{10} y^{3}+0.1 x^{7} y^{3}-3.1 x y^{3}-2.9 x^{4} y^{2}+4.3 x^{3} y^{2}+9.7 x^{11} y-7.3 x^{10} y-2.0 x^{9} y+8.8 x^{8} y+ \\
9.8 x^{6} y+x^{5} y-4.9 x y-3.9 x^{9}-5.4 x^{7}+6.9 x^{6}\end{array}$ \\
\hline 12 & $\begin{array}{l}\left(5 x^{14}+5 y^{14}\right)+3.1 x y^{11}-3.8 y^{11}+8.7 x^{2} y^{10}+8.4 y^{10}+9.2 x^{4} y^{7}-7.1 x y^{7}+5.1 x^{7} y^{6}+ \\
-4.0 x^{6} y^{6}+5.1 x^{3} y^{6}+4.7 x^{2} y^{6}-3.5 x^{5} y^{5}-4.7 x^{9} y^{4}+5.1 x^{6} y^{4}-7.1 x y^{4}-1.3 y^{4}+2.6 x^{6} y^{3}-8.6 x^{4} y^{3}+ \\
-4.9 x^{2} y^{3}+2.1 x y^{3}-9.1 y^{3}+7.8 x^{11} y^{2}+8.6 x^{8} y^{2}+5.7 x^{4} y^{2}+3.9 x y^{2}+0.1 x^{8} y+9.3 x y-9.4 x^{12}+ \\
3.0 x^{11}-8.8 x^{4}+0.5 x^{2}-7.1\end{array}$ \\
\hline 13 & $\begin{array}{l}\left(5 x^{16}+5 y^{16}\right)+0.4 y^{13}-4.0 x^{3} y^{12}-8.2 x y^{12}-2.2 y^{12}+7.1 x^{3} y^{11}-9.5 x^{5} y^{10}+ \\
-6.7 x^{3} y^{10}+8.4 x y^{9}+7.9 y^{9}-9.9 x^{7} y^{8}+9.1 x^{3} y^{8}-0.6 y^{8}+1.7 x^{8} y^{7}+1.7 x^{6} y^{7}-7.1 x^{5} y^{7}-6.4 x^{3} y^{7}+ \\
-3.8 x^{6} y^{6}+4.6 x^{5} y^{6}+0.1 x^{4} y^{6}-4.7 x^{2} y^{6}-9.7 x^{9} y^{5}-1.1 x^{6} y^{5}+9.8 x^{10} y^{4}+6.7 x^{9} y^{4}-3.1 x y^{4}+ \\
1.3 x^{12} y^{3}+4.5 x^{7} y^{3}+4.0 x y^{3}-3.6 y^{3}-3.8 x^{13} y^{2}+4.0 x^{10} y^{2}-9.1 x^{7} y^{2}-7.3 x^{6} y^{2}-0.7 x^{5} y^{2}+ \\
6.0 x^{4} y^{2}+6.5 x^{3} y^{2}-3.2 x^{11} y+4.3 x^{10}-0.3 x^{9}+7.3 x^{8}-2.0 x^{7}-10.0 x^{3}+1.1 x^{2}-4.4 x\end{array}$ \\
\hline 14 & $\begin{array}{l}\left(5 x^{16}+5 y^{16}\right)-4.5 x y^{13}-2.8 x^{2} y^{12}-0.9 y^{12}-4.8 x y^{10}+3.9 x^{4} y^{9}+6.3 x^{6} y^{8}+x^{2} y^{8}+ \\
3.4 x y^{8}+4.0 x^{7} y^{7}+0.3 x^{6} y^{7}+9.3 x^{8} y^{6}-4.4 x^{6} y^{6}+x^{5} y^{6}+8.3 x^{4} y^{6}-9.7 x^{3} y^{6}-3.9 y^{6}+5.7 x^{7} y^{5}+ \\
8.2 x^{5} y^{5}-3.3 y^{5}-9.1 x^{10} y^{4}+8.8 x^{7} y^{4}-6.9 x y^{4}+8.9 x^{12} y^{3}-9.5 x^{10} y^{3}+0.1 x^{5} y^{3}-1.5 x^{3} y^{3}+ \\
-0.5 x^{2} y^{3}+3.8 x^{11} y^{2}-9.1 x^{9} y^{2}+2.8 x^{8} y^{2}-0.6 x^{4} y^{2}+8.3 x^{2} y^{2}+9.4 x^{8} y+9.9 x^{6} y-6.4 x^{2} y-9.8 x^{13}+ \\
-6.8 x^{12}-5.5 x\end{array}$ \\
\hline
\end{tabular}




\section{Polynomial}

$15\left(5 x^{8}+5 y^{8}\right)+5 z^{8}+1.6 y z^{6}-9.4 x y^{2} z^{4}-0.5 x^{2} y z^{4}-9.9 x^{3} z^{4}-4.9 x^{2} z^{4}-3.6 z^{4}-4.5 x^{2} y^{2} z^{3}+$ $-0.4 x y^{2} z^{3}-2.5 y^{2} z^{3}-7.5 x y z^{3}+6.6 y^{3} z^{2}+3.6 x^{3} y z^{2}-9.0 y z^{2}+7.4 x^{2} z^{2}+7.7 x z^{2}-6.9 z^{2}+$ $-8.9 x^{2} y^{4} z+7.8 y^{4} z-4.1 y^{3} z-5.4 x^{4} y^{2} z+9.1 x^{3} y^{2} z+10.0 x^{6} z+9.5 x z+8.6 y^{6}+4.2 x^{4} y^{3}+6.8 x^{3} y^{3}+$ $2.7 x^{3} y^{2}+2.5 x y^{2}+5.4 x^{6} y-5.4 y+7.9 x^{4}$

$16\left(5 x^{8}+5 y^{8}\right)+5.0 z^{8}-3.4 x y z^{5}-1.4 y z^{5}-5.9 x z^{5}+2.1 y^{3} z^{4}-1.1 x y^{3} z^{3}-3.4 x^{2} y^{2} z^{3}-9.5 x y^{2} z^{3}+$ $5.2 y^{2} z^{3}+8.4 x y z^{3}+1.2 x^{4} z^{3}-1.1 z^{3}+8.6 y^{4} z^{2}+9.7 x^{2} y^{3} z^{2}-9.4 x y^{2} z^{2}+1.3 x^{2} y z^{2}-8.4 x y z^{2}-6.8 y^{6} z+$ $2.8 x y^{4} z+6.2 x^{3} y^{3} z-0.6 y^{3} z-5.2 x^{3} y^{2} z+3.8 x^{5} y z-1.3 x^{4} y z+5.3 x^{6} z-2.3 x^{3} z-6.9 x z+1.3 z-7.4 y^{6}+$ $2.8 x y^{4} z+6.2 x^{3} y^{3} z-0.6 y^{3} z-5.2 x^{3} y^{2} z+3.8 x^{5} y z-1.3 x^{4} y z+5.3 x^{6} z-2.3 x^{3} z-6.9 x z+1.3 z-7.4 y$
$5.7 x y^{5}+2.1 x^{3} y^{4}+2.8 x^{2} y^{4}+8.1 y^{4}-8.2 x^{3} y^{3}+1.4 x^{2} y^{3}+9.4 x^{3} y^{2}-10.0 x y^{2}-4.6 x^{3} y-2.7 x^{6}+7.7 x^{2}$

$17\left(5 x^{24}+5 y^{24}\right)-0.7 x y^{22}+1.2 y^{22}+8.2 x y^{20}+8.5 x^{2} y^{19}+6.4 y^{19}-2.1 x y^{17}-3.9 x^{5} y^{16}+$ $-4.2 x^{4} y^{16}-9.5 x^{2} y^{16}+7.9 x^{6} y^{15}-5.1 x^{5} y^{15}-4.0 x^{3} y^{15}-10.0 x^{2} y^{15}-4.6 x^{4} y^{14}-4.1 y^{14}+$
$-6.2 x^{10} y^{13}+1.8 x^{8} y^{13}+5.7 x^{5} y^{13}+7.8 x^{10} y^{12}+2.4 x^{8} y^{12}+8.3 x^{7} y^{12}+4.6 x^{4} y^{12}+$

$-0.6 y^{12}-7.4 x^{11} y^{11}-0.6 x^{9} y^{11}+0.9 x^{4} y^{11}+4.1 x^{9} y^{10}+1.6 x^{5} y^{10}+8.8 x^{3} y^{10}+0.7 x^{11} y^{9}+$ $-4.4 x^{9} y^{9}+9.1 x^{8} y^{9}-3.1 x^{15} y^{8}+8.6 x^{13} y^{8}+6.3 x^{9} y^{8}+9.7 x^{4} y^{8}-3.5 x^{16} y^{7}+0.8 x^{8} y^{7}+8.2 x^{5} y^{7}+$ $9.9 x^{2} y^{7}+7.0 x^{17} y^{6}+4.2 x^{7} y^{6}+9.6 x^{16} y^{5}+3.8 x^{9} y^{5}+4.2 x^{12} y^{4}-7.1 x^{11} y^{4}+9.3 x^{5} y^{4}+6.7 x^{4} y^{4}+$ $2.5 x^{19} y^{3}-2.2 x^{16} y^{3}+7.1 x^{14} y^{3}-6.9 x^{12} y^{3}+0.6 x^{11} y^{3}-5.2 x^{10} y^{3}-4.7 x y^{3}-2.8 x^{18} y^{2}+$ $2.5 x x^{16} y^{2}+5.3 x^{10} y^{2}+2.6 x^{5} y^{2}-4.7 x^{4} y^{2}-2.1 y^{2}-1.5 x^{21} y-2.3 x^{20} y+2.8 x^{15} y-5.1 x^{14} y+$ $6.6 x^{8} y+1.6 x^{7} y-9.0 x^{21}+5.4 x^{17}+8.4 x^{16}+4.8 x^{12}+0.3 x^{9}+9.1 x^{7}+8.4 x^{5}+1.9 x^{2}+1.5 x$
$\left(5 x^{24}+5 y^{24}\right)+2.2 y^{21}-4.3 x y^{20}+5.1 y^{20}-8.4 y^{18}+6.2 x^{6} y^{17}-1.6 x^{3} y^{17}-8.1 x^{4} y^{16}+$ $8.6 x y^{16}+6.9 x^{4} y^{15}-6.7 x^{3} y^{15}+5.8 x^{2} y^{15}-3.6 x^{7} y^{14}-8.1 x^{6} y^{14}+5.8 x^{5} y^{13}-2.7 x y^{13}+$ $-7.2 y^{13}+2.6 x^{10} y^{12}+8.9 x^{5} y^{12}-9.4 x^{11} y^{11}+0.1 x^{10} y^{11}+2.1 x^{4} y^{11}+4.5 x^{2} y^{11}+$ $6.7 y^{11}+8.3 x^{11} y^{10}+9.7 x^{10} y^{10}+8.6 x^{8} y^{10}+8.9 x^{4} y^{10}-2.4 x^{3} y^{10}-3.5 x^{12} y^{9}+2.2 x y^{9}+$ $6.7 x^{15} y^{8}+5.6 x^{13} y^{8}+3.2 x^{10} y^{8}+5.2 x^{8} y^{8}-9.1 x^{5} y^{8}+5.4 y^{8}-3.1 x^{7} y^{7}+4.8 x^{6} y^{7}+3.3 x y^{7}+$ $-2.2 x^{17} y^{6}-1.4 x^{16} y^{6}-0.5 x^{13} y^{6}+3.7 x^{12} y^{6}+6.7 x^{10} y^{6}-3.6 x^{9} y^{6}+3.9 x^{6} y^{6}-6.9 y^{6}+$ $-9.3 x^{11} y^{5}+7.7 x^{6} y^{5}-0.4 x^{2} y^{5}-4.1 x^{17} y^{4}-2.7 x^{15} y^{4}-8.7 x^{13} y^{4}-6.0 x^{11} y^{4}+3.2 x^{10} y^{4}+$ $-8.5 x^{9} y^{4}+4.1 x^{8} y^{4}-0.5 x^{6} y^{4}+7.7 x^{18} y^{3}+7.1 x^{16} y^{3}-8.1 x^{15} y^{3}-5.4 x^{13} y^{3}-4.6 x^{12} y^{3}+$
$8.1 x^{11} y^{3}+6.6 x^{10} y^{3}+0.2 x^{8} y^{3}+2.4 x^{7} y^{3}+5.8 x^{5} y^{3}-7.4 x^{4} y^{3}+9.0 x^{2} y^{3}+6.0 y^{3}-10.0 x^{18} y^{2}+$ $8.7 x^{14} y^{2}+9.3 x^{12} y^{2}-6.4 x^{11} y^{2}+8.7 x^{7} y^{2}-3.3 x y^{2}-7.7 y^{2}+9.6 x^{20} y-0.3 x^{10} y-8.6 x^{4} y-6.0 x^{2} y+$ $4.8 x^{20}-2.0 x^{13}+8.1 x^{10}+1.6 x^{4}$

$19\left(5 x^{10}+5 y^{10}+5 z^{10}\right)+7.6 x y z^{7}+2.3 y^{3} z^{6}+8.3 x y^{2} z^{6}-8.1 x^{3} z^{6}+0.0 x^{2} z^{6}+9.4 x z^{6}+9.0 z^{6}+$ $8.6 x^{2} y^{2} z^{5}-7.2 y z^{5}+6.5 x^{3} z^{5}+1.3 x^{2} z^{5}-8.6 x y^{3} z^{4}+5.7 x^{4} y z^{4}-7.8 x^{2} y z^{4}-1.3 y^{5} z^{3}-9.0 x^{2} y^{4} z^{3}+$ $1.5 y^{4} z^{3}-3.9 y^{3} z^{3}+6.8 x y^{2} z^{3}+x^{3} z^{3}+9.8 z^{3}+1.8 x^{2} y^{5} z^{2}+6.5 y^{4} z^{2}+9.2 x^{5} y^{2} z^{2}+0.1 x^{4} y z^{2}+$ $-7.3 x y z^{2}+9.9 x^{7} z^{2}-6.9 x^{5} z^{2}-1.9 x^{4} z^{2}-5.8 x^{3} z^{2}+7.2 x^{2} z^{2}+6.3 x y^{7} z+3.9 y^{7} z-3.8 x^{2} y^{6} z+$ $-2.3 x^{4} y^{4} z-5.0 y^{3} z-5.8 x^{5} y^{2} z-3.9 x^{4} y^{2} z+9.7 x^{2} y^{2} z+6.4 x^{3} y z+3.1 y z-7.9 x^{6} z-1.5 x^{5} z+4.6 x^{4} z+$ $-0.7 y^{7}-2.7 x^{3} y^{6}+6.6 x^{4} y^{5}-0.5 x y^{5}-4.6 y^{5}-9.6 x^{5} y^{4}-6.4 x^{4} y^{4}-1.9 x^{3} y^{2}-5.8 x^{3} y-5.8 x^{3}$

$20 \quad\left(5 x^{10}+5 y^{10}+5 z^{10}\right)-9.8 z^{6}-6.6 x^{2} y z^{5}+8.6 x^{2} z^{5}+3.8 x z^{5}-6.7 z^{5}+6.1 y^{4} z^{4}+6.3 x^{2} y^{3} z^{4}+$ $8.6 x y^{3} z^{4}+3.5 y^{2} z^{4}+9.3 x^{4} y z^{4}+7.7 x y z^{4}-0.7 y z^{4}+4.0 x^{3} z^{4}-1.4 x z^{4}+2.0 y^{6} z^{3}-6.1 x y^{5} z^{3}-9.9 y^{5} z^{3}+$ $8.6 x^{2} y^{4} z^{3}-2.7 x^{2} y^{3} z^{3}+0.7 x^{4} y^{2} z^{3}+2.0 x^{2} y^{2} z^{3}+7.1 x y^{2} z^{3}+7.1 x^{5} y z^{3}+9.4 x^{2} y z^{3}-3.1 y z^{3}+7.0 x^{3} z^{3}+$ $-2.7 x z^{3}+2.5 y^{7} z^{2}-1.8 x y^{6} z^{2}+2.8 x y^{5} z^{2}-2.2 x y^{4} z^{2}+9.1 y^{4} z^{2}-5.9 x^{2} y^{3} z^{2}+0.9 y^{3} z^{2}+9.5 x^{3} y^{2} z^{2}+$ $-2.5 x^{2} y^{2} z^{2}+6.9 y^{2} z^{2}-9.9 x^{5} y z^{2}+4.7 x^{3} y z^{2}+8.0 x^{2} y z^{2}+1.5 x^{5} z^{2}+5.2 x^{4} z^{2}-6.1 x^{3} z^{2}-7.7 x z^{2}+$ $6.9 y^{7} z-2.2 x^{2} y^{6} z+3.9 y^{5} z-8.7 x y^{4} z+8.2 x^{4} y^{3} z-9.2 x^{2} y^{3} z+2.1 x^{5} y^{2} z+3.3 x y^{2} z-1.9 x^{6} y z+$

$-9.6 x^{5} y z-1.4 x^{3} y z-4.1 x^{2} y z-3.1 y z+2.3 x^{5} z-5.0 x^{2} z+7.1 x z-5.4 x y^{8}-0.1 x^{2} y^{6}-2.8 x y^{5}-5.1 y^{5}+$ $-9.4 x y^{4}+0.7 x^{3} y^{2}+x y-1.8 x^{8}-4.3 x^{4}$ $21 \quad\left(5 x^{12}+5 y^{12}+5 z^{12}\right)+7.6 y z^{10}+8.4 y^{2} z^{9}+4.1 x^{2} z^{9}-6.8 x^{2} y z^{8}-4.8 x^{3} z^{8}+6.8 x z^{8}-2.1 y^{4} z^{7}+$
$-6.8 y^{3} z^{7}+1.7 x^{3} y z^{7}+4.9 x^{2} y z^{7}-0.3 y z^{7}-0.4 x^{3} z^{7}-7.1 y^{5} z^{6}+4.1 y^{4} z^{6}-1.3 x^{2} y^{3} z^{6}-3.1 x^{3} y^{2} z^{6}+$ $-8.8 x^{2} y^{2} z^{6}-6.8 x^{4} y z^{6}+5.9 y z^{6}-2.5 x^{4} z^{6}+2.2 y^{6} z^{5}+5.2 x^{2} y^{4} z^{5}+4.1 x^{3} y^{3} z^{5}+10.0 x y^{3} z^{5}+$ $-0.7 x^{3} y^{2} z^{5}+9.3 x^{2} y^{2} z^{5}-2.5 x y z^{5}-6.7 x^{6} z^{5}+4.7 x^{5} z^{5}+4.3 x y^{3} z^{4}+3.3 y^{3} z^{4}-9.1 x^{2} y^{2} z^{4}+7.3 y^{2} z^{4}+$ $-5.2 x^{6} y z^{4}-0.3 x^{4} y z^{4}-9.3 x^{6} z^{4}+0.0 x^{2} z^{4}-1.9 x^{2} y^{6} z^{3}-6.9 x y^{6} z^{3}-2.8 x y^{5} z^{3}-5.0 x^{4} y^{4} z^{3}+$ $-0.5 x^{3} y^{4} z^{3}-5.5 x^{5} y^{3} z^{3}+6.9 x y^{3} z^{3}-1.2 x^{4} y^{2} z^{3}+7.5 x^{7} y z^{3}-0.9 x^{6} y z^{3}+3.1 x^{2} y z^{3}+8.7 x^{3} z^{3}+$ $5.0 z^{3}-2.0 y^{8} z^{2}+3.2 y^{7} z^{2}+8.8 x^{2} y^{6} z^{2}-0.1 y^{6} z^{2}-1.9 x^{3} y^{5} z^{2}-7.4 y^{5} z^{2}+1.2 x^{3} y^{4} z^{2}+8.1 y^{4} z^{2}+$ $-6.7 x^{6} y^{3} z^{2}+8.1 x^{5} y^{3} z^{2}+6.9 x^{2} y^{3} z^{2}+4.7 x^{6} y^{2} z^{2}+8.2 x^{5} y^{2} z^{2}-0.9 x y z^{2}+4.4 y z^{2}+9.6 x^{9} z^{2}+$ $8.6 x^{6} z^{2}+x y^{9} z-4.5 y^{8} z-2.1 x^{3} y^{7} z-5.1 x^{2} y^{7} z-6.9 x^{4} y^{6} z-1.9 x^{3} y^{6} z+5.1 x y^{6} z+0.0 x^{3} y^{5} z+$ $+2.2 x^{2} y^{5} z+6.8 x y^{5} z+1.2 y^{5} z+9.5 x^{4} y^{4} z+1.1 x^{6} y^{3} z+3.3 x^{3} y^{3} z+6.1 x^{2} y^{3} z+9.9 x^{8} y^{2} z+6.9 x^{6} y^{2} z+$ $-2.1 y^{2} z+6.4 x^{7} y z+8.4 x^{2} y z+8.7 x^{5} z+9.8 x^{4} z-1.1 x^{2} z-4.6 x y^{9}+7.6 y^{9}-4.6 x y^{8}-5.5 x^{4} y^{7}+$ $1.9 x^{2} y^{7}+6.5 y^{7}-1.6 x^{5} y^{6}-0.8 x y^{6}-3.8 x^{5} y^{5}+9.7 x^{2} y^{5}-8.0 y^{5}+3.8 x^{3} y^{4}+1.7 x^{4} y^{2}+$ $8.4 x^{2} y^{2}-2.2 y^{2}+3.6 x^{10} y-3.3 x^{5} y-6.8 x y+2.6 y-8.9 x^{10}+8.4 x^{9}-9.8 x^{4}$

$22\left(5 x^{12}+5 y^{12}+5 z^{12}\right)+8.3 z^{10}+0.1 y^{2} z^{9}-5.7 x y z^{9}-0.4 x z^{9}+1.9 z^{9}+7.5 x^{2} y z^{8}-2.2 x z^{8}+$ $2.5 y^{4} z^{7}-6.7 y^{3} z^{7}-0.1 x^{3} y z^{7}+1.9 z^{7}-3.3 y^{5} z^{6}+1.1 x y^{3} z^{6}-0.7 x^{3} y^{2} z^{6}+4.4 y^{2} z^{6}+7.0 x^{4} y z^{6}+$ $-3.8 x^{5} z^{6}+4.0 x^{2} z^{6}+4.9 x z^{6}+2.0 x y^{4} z^{5}-2.0 y^{4} z^{5}+5.5 x^{4} y z^{5}+8.4 x y z^{5}-8.8 y z^{5}+9.9 y^{5} z^{4}+$ $6.6 x^{2} y^{4} z^{4}-7.2 x y^{4} z^{4}+6.5 x^{3} y^{3} z^{4}+6.1 x y^{3} z^{4}+5.4 x^{2} y^{2} z^{4}+3.6 x y^{2} z^{4}+9.4 y^{2} z^{4}-0.4 x^{4} y z^{4}+$ $6.8 x^{3} y z^{4}-4.9 x y z^{4}-6.2 y z^{4}+2.2 x^{6} z^{4}-4.9 x^{5} z^{4}-9.7 x^{4} z^{4}+6.8 z^{4}+3.7 x^{3} y^{4} z^{3}-7.3 x y^{4} z^{3}+$ $-1.8 y^{4} z^{3} 6.2 x^{3} y^{2} z^{3}-6.2 x^{4} y z^{3}+0.3 y z^{3}+6.7 x^{8} z^{3}-6.4 x^{4} z^{3}+4.0 x^{3} z^{3}-5.1 z^{3}+9.4 x y^{7} z^{2}+9.2 y^{6} z^{2}+$ $-7.6 x^{3} y^{5} z^{2}-9.0 x^{5} y^{4} z^{2}+7.4 x^{4} y^{4} z^{2}-7.1 x y^{4} z^{2}-7.5 x^{6} y^{3} z^{2}-9.2 x^{5} y^{3} z^{2}+1.4 x y^{3} z^{2}+0.8 y^{3} z^{2}+$ $0.6 x^{3} y^{2} z^{2}-7.1 x y^{2} z^{2}-4.3 y^{2} z^{2}+8.2 x^{7} y z^{2}+6.1 x y z^{2}+7.0 y z^{2}+8.7 x^{9} z^{2}+9.9 x^{8} z^{2}+5.7 x^{6} z^{2}+$ $-3.3 x^{4} y^{6} z-2.3 x^{3} y^{6} z+5.5 y^{6} z+4.2 x^{4} y^{5} z+2.4 x y^{5} z+1.1 x^{5} y^{4} z-6.7 x^{7} y^{3} z+3.0 x^{6} y^{3} z+6.7 x^{4} y^{3} z+$ $-5.4 y^{3} z-6.3 x^{5} y^{2} z-1.9 x y^{2} z-0.3 x^{8} y z-8.9 x^{5} y z+3.8 x^{5} z+1.1 x^{4} z+5.0 z+3.2 x^{2} y^{8}-3.3 x y^{7}+$ $7.7 x y^{6}+9.4 x^{6} y^{5}+1.4 x^{5} y^{5}-4.5 x^{2} y^{5}-5.2 x^{7} y^{4}+3.9 x^{6} y^{4}-6.4 x^{5} y^{4}+7.0 x^{8} y^{3}-5.6 x^{7} y^{3}-6.0 x^{5} y^{3}+$ $-2.1 x^{2} y^{3}+9.5 x^{9} y^{2}+9.3 x^{2} y^{2}-0.3 x y^{2}+5.3 x^{10} y-9.9 x^{9} y+2.7 x^{7} y+1.7 x^{3} y-2.9 y+2.7 x^{7}-4.0 x^{5}$ 


\section{Summary}

The problem of computing the solutions of a system of multivariate polynomial equations can be approached by the Stetter-Möller matrix method which casts the problem into a large eigenvalue problem. This Stetter-Möller matrix method forms the starting point for the development of computational procedures for the two main applications addressed in this thesis:

- The global optimization of a multivariate polynomial, described in Part II of this thesis, and

- the $H_{2}$ model-order reduction problem, described in Part III of this thesis.

Part I of this thesis provides an introduction in the background of algebraic geometry and an overview of various methods to solve systems of polynomial equations.

In Chapter 4 a global optimization method is worked out which computes the global minimum of a Minkowski dominated polynomial. The Stetter-Möller matrix method transforms the problem of finding solutions to the system of first-order conditions of this polynomial into an eigenvalue problem. This method is described in [48], [50] and [81]. A drawback of this approach is that the matrices involved in this eigenvalue problem are usually very large.

The research question which plays a central role in Part II of this thesis is formulated as follows: How to improve the efficiency of the Stetter-Möller matrix method, applied to the global optimization of a multivariate polynomial, by means of an $n D-$ systems approach? The efficiency of this method is improved in this thesis by using a matrix-free implementation of the matrix-vector products and by using an iterative eigenvalue solver instead of a direct eigenvalue solver.

The matrix-free implementation is achieved by the development and implementation of an $n \mathrm{D}$-system of difference equations as described in Chapter 5 . This yields a routine that computes the action of the involved large and sparse matrix on a given 
vector. This routine is used as input for the iterative eigenproblem solvers to compute the matrix-vector products in a matrix-free fashion.

To study the efficiency of such an $n \mathrm{D}$-system we have set up a corresponding shortest path problem. It turns out that this will quickly become huge and difficult to solve. However, it is possible to set up a relaxation of the shortest path problem that is easier to solve. This is described in the Sections 5.3.1 and 5.3.2. The conclusions about these shortest paths problems lead to some heuristic methods, as discussed in Section 5.3.3, to arrive cheaply at suboptimal paths with acceptable performance.

Another way to improve the efficiency of an $n \mathrm{D}$-system is to apply parallel computing techniques as described in Section 5.3.4. However, it turns out that parallelization is not very useful in this application of the $n \mathrm{D}$-system.

Iterative eigenvalue solvers are described in Chapter 6. An advantage of such an iterative eigenvalue solver is that it is compatible with an $n \mathrm{D}$-systems approach and that it is able to focus on a subset of eigenvalues such that it is no longer necessary to compute all the eigenvalues of the matrix. For global polynomial optimization the most interesting eigenvalue is the smallest real eigenvalue. Therefore this feature is developed and implemented in a Jacobi-Davidson eigenproblem solver, as described in Section 6.3. In Section 6.4 some procedures are studied to project an approximate eigenvector to a close-by vector with Stetter structure to speed up the convergence process of the iterative solver.

The development of the JDCOMM method, a Jacobi-Davidson eigenvalue solver for commuting matrices, is described in Section 6.5. The most important new implemented features of this matrix-free solver are that: (i) it computes the eigenvalues of the matrix of interest while iterating with a another, much sparser (but commuting), matrix which results in a speed up in computation time and a decrease in the required amount of floating point operations, and, (ii) it focuses on the smallest real eigenvalues first.

In Chapter 7 we present the results of the numerical experiments in which the global minima of various Minkowski dominated polynomials are computed using the approaches and techniques mentioned in Part II of this thesis. In the majority of the test cases the SOSTOOLS software approach is more efficient than the $n \mathrm{D}$-systems approach in combination with an iterative eigenvalue solver. Some test cases however can not accurately be solved by the SOSTOOLS software since they produce large errors. These large errors can limit the actual application of this software. In general our approach tends to give more accurate results. In Section 7.5 four experiments are described in which the newly developed JDCOMM method is used to compute the global minima. The result here is that the JDCOMM method has a superior performance over the other methods: the JDCOMM method requires fewer matrix-vector operations and therefore also uses less computation time.

The $\mathrm{H}_{2}$ model-order reduction problem, described in Part III of this thesis, deals with finding an approximating system of reduced order $N-k$ to a given system of order $N$. This is called the co-order $k$ case. In [50] an approach is introduced 
where the $\mathrm{H}_{2}$ global model-order reduction problem for the co-order $k=1$ case is reformulated as the problem of finding solutions of a quadratic system of polynomial equations.

The research question answered in Part III, is: How to find the global optimum to the $\mathrm{H}_{2}$ model-order reduction problem for a reduction of order $N$ to order $N-k$, using the techniques of the Stetter-Möller matrix method in combination with an nD-system and an iterative eigenvalue solver? Furthermore, we give answers to the following questions: How to improve the performance for co-order $k=1$ reduction?, and How to develop new techniques or extensions for co-order $k=2$ and $k=3$ reduction?

The approach introduced in [50] for the co-order $k=1$ case is generalized and extended in Chapter 8 which results in a joint framework in which to study the $\mathrm{H}_{2}$ model-order reduction problem for various co-orders $k \geq 1$. In the co-order $k \geq 1$ case the problem is reformulated as finding the solutions to the system of quadratic equations which contains $k-1$ additional parameters and whose solutions should satisfy $k-1$ additional linear constraints. The Stetter-Möller method is used to transform the problem of finding solutions of this system of quadratic equations into an eigenvalue problem containing one or more parameters. From the solutions of such an eigenvalue problem the corresponding feasible solutions for the real approximation $G(s)$ of order $N-k$ can be selected. A generalized version of the $H_{2}$-criterion is used to select the globally best approximation.

In Chapter 9 this approach leads in the co-order $k=1$ case to a large conventional eigenvalue problem, as described in [50]. We improve the efficiency of this method by a matrix-free implementation by using an $n \mathrm{D}$-system in combination with an iterative eigenvalue solver which targets the smallest real eigenvalue. In Section 9.3 the potential of this approach is demonstrated by an example which involves the efficient reduction of a system of order 10 to a system of order 9 .

In Chapter 10 we describe how the Stetter-Möller matrix method in the co-order $k=2$ case, when taking the additional linear constraint into account, yields a rational matrix in one parameter. We can set up a polynomial eigenvalue problem and to solve this problem it is rewritten as a generalized and singular eigenvalue problem. To accurately compute the eigenvalues of this singular matrix, the singular parts are split off by computing the Kronecker canonical form. In Section 10.6 three examples are given where this co-order $k=2$ approach is successfully applied to obtain a globally optimal approximation of order $N-2$.

Applying the Stetter-Möller matrix method for the co-order $k=3$ case in Chapter 11 yields two rational matrices in two parameters. This is cast into a two-parameter polynomial eigenvalue problem involving two matrices and one common eigenvector. Both matrices are joined together into one rectangular and singular matrix. Now the ideas of Chapter 10 regarding the Kronecker canonical form computations are useful to compute the values $\rho_{1}$ and $\rho_{2}$ which make these matrices simultaneously singular. An important result is described in this chapter: the solutions to the system of equations are determined by the values which make the transformation matrices, used in the Kronecker canonical form computations, singular. With these values the 
globally optimal approximation $G(s)$ of order $N-3$ is computed. An example is worked out at the end of this chapter where the co-order $k=3$ technique exhibits a better performance on this example than the co-order $k=1$ and $k=2$ techniques.

Chapter 12 provides concluding remarks and directions for further research of the results described in the Parts II and III of this thesis. 


\section{Samenvatting}

Het probleem van het berekenen van de oplossingen van een stelsel van polynomiale vergelijkingen kan worden benaderd met behulp van de Stetter-Möller matrix methode die het probleem omvormt tot een groot eigenwaarde probleem. Deze StetterMöller matrix methode vormt het uitgangspunt voor de ontwikkeling van computationele procedures voor de twee toepassingen die in dit proefschrift aan bod komen:

- De globale optimalisatie van een polynomiale functie in meerdere variabelen, zoals beschreven in deel II van deze thesis, en

- het $H_{2}$ model-orde reductie probleem, beschreven in deel III van deze thesis.

Deel I van deze thesis verstrekt een inleiding in de achtergrond van de algebraïsche meetkunde en een overzicht van diverse methodes om een stelsel van polynomiale vergelijkingen op te lossen.

In hoofdstuk 4 wordt een globale optimalisatiemethode uitgewerkt die het globale minimum van een Minkowski gedomineerde polynomiale functie berekent. De StetterMöller matrix methode transformeert het probleem van het vinden van oplossingen van het stelsel eerste-orde afgeleiden van deze polynomiale functie naar een eigenwaarde probleem. Deze aanpak is eerder beschreven in [48], [50] en [81]. Een nadeel van deze aanpak is dat de matrices in dit eigenwaarde probleem gewoonlijk zeer groot zijn.

De onderzoeksvraag die een centrale rol speelt in deel II van dit proefschrift is als volgt geformuleerd: Hoe kan de efficiëntie van de Stetter-Möller matrix methode, toegepast op de globale optimalisatie van een polynomiale functie, verbeterd worden door middel van een nD-systeem aanpak? De efficiëntie van deze methode is in dit proefschrift verbeterd door middel van een 'matrix-vrije' implementatie van de matrix-vector producten en door het gebruik van een iteratieve eigenwaarde solver in plaats van een directe eigenwaarde solver. 
De matrix-vrije implementatie is bereikt door de ontwikkeling en de implementatie van een $n \mathrm{D}$-systeem van differentie vergelijkingen zoals beschreven in hoofdstuk 5 . Dit resulteert in een routine die de actie berekent van de grote en ijle matrix op een gegeven vector. Deze routine wordt gebruikt als input voor de iteratieve eigenwaarde solvers om de matrix-vector producten te berekenen op een matrix-vrije manier.

Om de efficiëntie van een dergelijke $n \mathrm{D}$-systeem aanpak te bestuderen hebben we een overeenkomstig kortste pad probleem geformuleerd. Het blijkt dat dit kortste pad probleem al snel groot en moeilijk op te lossen is. Het is echter mogelijk om een relaxatie van het kortste pad probleem te formuleren die makkelijker op te lossen is. Dit is beschreven in de Secties 5.3.1 en 5.3.2. De conclusies met betrekking tot de kortste pad aanpak leiden tot een aantal heuristische methodes, zoals besproken in Sectie 5.3.3, om goedkoop suboptimale paden te berekenen met een aanvaardbare prestatie.

Een andere manier om de efficiëntie van de $n \mathrm{D}$-systeem aanpak te verbeteren is het toepassen van parallelle rekentechnieken zoals die in Sectie 5.3.4 worden beschreven. Het blijkt echter dat deze parallellisatie niet erg nuttig is om toe te passen bij de $n \mathrm{D}$-systeem aanpak.

Iteratieve eigenwaarde solvers worden beschreven in Hoofdstuk 6. Een voordeel van een dergelijke iteratieve eigenwaarde solver is dat het compatibel is met de $n \mathrm{D}$ systeem aanpak en dat deze in staat is te focussen op een subset van eigenwaardes zodat het niet langer nodig is om alle eigenwaardes van de matrix te berekenen. De meest interessante eigenwaarde voor de globale optimalisatie van een polynomiale functie is de kleinste reële eigenwaarde. Daarom is deze functie ontwikkeld en geïmplementeerd in een Jacobi-Davidson eigenwaarde solver zoals beschreven in Sectie 6.3. In Sectie 6.4 worden enkele procedures bestudeerd om een benaderende eigenvector te projecteren op een nabije eigenvector met Stetter structuur om zodoende het convergentieproces van de iteratieve solver te versnellen.

De ontwikkeling van de JDCOMM methode, een Jacobi-Davidson eigenwaarde solver voor een set van commuterende matrices, wordt beschreven in Sectie 6.5. De belangrijkste nieuwe eigenschappen geïmplementeerd in deze matrix-vrije solver zijn de volgende: (i) de methode berekent de eigenwaardes van de matrix, terwijl deze itereert met een andere, veel ijlere (maar commuterende) matrix, wat resulteert in versnelling van de rekentijd en een afname in het vereiste aantal floating-point operaties, en (ii) de methode richt zich enkel op het berekenen van de kleinste reële eigenwaarde.

In Hoofdstuk 7 worden de resultaten gepresenteerd van de numerieke experimenten waarin de globale minima van verschillende Minkowski gedomineerde polynomen worden berekend met behulp van de aanpak en de ontwikkelde technieken zoals beschreven in deel II van dit proefschrift. In de meerderheid van de experimenten is de SOSTOOLS software efficiënter dan de $n \mathrm{D}$-systeem aanpak in combinatie met een iteratieve eigenwaarde solver. Sommige experimenten kunnen echter niet nauwkeurig worden opgelost door de SOSTOOLS software omdat in deze gevallen grote afwijkingen ontstaan, wat de daadwerkelijke toepassing van deze software beperkt. In het algemeen leidt onze aanpak tot nauwkeurigere resultaten. In Sectie 7.5 worden vier ex- 
perimenten beschreven waarin de nieuw ontwikkelde JDCOMM methode wordt gebruikt om de globale minima te berekenen. Het resultaat hiervan is dat de JDCOMM methode superieure prestaties heeft ten opzichte van andere methodes: de JDCOMM methode vereist minder matrix-vector operaties en benodigt daarom ook minder rekentijd.

Het $H_{2}$ model-orde reductie probleem, beschreven in deel III van dit proefschrift, behandelt het vinden van een benaderend systeem van gereduceerde orde $N-k$, gegeven een systeem van orde $N$. Dit geval zal het co-orde $k$ geval genoemd worden. In [50] is een aanpak geïntroduceerd waar het globale $H_{2}$ model-orde reductie probleem voor het co-orde $k=1$ geval geherformuleerd is als het probleem van het vinden van oplossingen van een kwadratisch stelsel van polynomiale vergelijkingen.

De onderzoeksvraag beantwoord in deel III van dit proefschrift luidt als volgt: Hoe kan het globale optimum van het $\mathrm{H}_{2}$ model-orde reductie probleem van orde $\mathrm{N}$ naar orde $N-k$ bepaald worden met behulp van de technieken van de Stetter-Möller matrix methode in combinatie met een $n D$-systeem en een iteratieve eigenwaarde solver? Voorts worden antwoorden gegeven op de volgende vragen: Hoe kan de performance voor co-orde $k=1$ verbeterd worden? en Hoe kunnen nieuwe technieken of uitbreidingen voor co-orde $k=2$ en $k=3$ ontwikkeld worden?

De aanpak geïntroduceerd in [50] voor het co-orde $k=1$ geval hebben we gegeneraliseerd en uitgebreid, zoals beschreven in Hoofdstuk 8, wat resulteert in een gezamenlijk kader waarin het $\mathrm{H}_{2}$ model-orde reductie probleem bestudeerd kan worden voor diverse co-ordes $k \geq 1$. In het co-orde $k \geq 1$ geval is het probleem geherformuleerd als het vinden van de oplossingen van een stelsel van kwadratische vergelijkingen dat $k-1$ additionele parameters bevat en waarvan de oplossingen moeten voldoen aan $k-1$ additionele lineaire constraints. De Stetter-Möller matrix methode is gebruikt om het probleem van het vinden van oplossingen van dit stelsel van kwadratische vergelijkingen te transformeren in een eigenwaardeprobleem dat één of meer parameters bevat. Uit de oplossingen van een dergelijk eigenwaardeprobleem kunnen de bijbehorende feasible oplossingen voor de reële approximatie $G(s)$ van orde $N-k$ worden geselecteerd. Een gegeneraliseerde versie van het $\mathrm{H}_{2}$-criterium is gebruikt om de globaal beste approximatie te selecteren.

In Hoofdstuk 9 leidt deze aanpak in het co-orde $k=1$ geval tot een groot conventioneel eigenwaardeprobleem zoals beschreven in [50]. Wij verbeteren de efficiëntie van deze methode met behulp van een matrix-vrije implementatie gebruik makend van een $n \mathrm{D}$-systeem in combinatie met een iteratieve eigenwaarde solver die de kleinste reële eigenwaarde berekent. In Sectie 9.3 wordt het potentieel van deze benadering gedemonstreerd door een voorbeeld dat de efficiënte reductie van een systeem van orde 10 naar een systeem van orde 9 behandeld.

In Hoofdstuk 10 wordt beschreven hoe de Stetter-Möller matrix methode in het co-orde $k=2$ geval een rationale matrix in één parameter oplevert wanneer er rekening wordt gehouden met de additionele lineaire constraint. Op basis van deze rationale matrix kunnen we een polynomiaal eigenwaarde probleem formuleren. Om dit probleem op te lossen wordt het herschreven als een gegeneraliseerd en singulier 
eigenwaarde probleem. Om de eigenwaardes van deze singuliere matrix nauwkeurig te kunnen berekenen, worden de singuliere delen afgesplitst door de Kronecker canonieke vorm te berekenen. In Sectie 10.6 worden drie voorbeelden gegeven waar deze co-orde $k=2$ aanpak met succes is toegepast om een globaal optimale benadering van orde $N-2$ te verkrijgen.

Het toepassen van de Stetter-Möller matrix methode voor het co-orde $k=3$ geval in Hoofdstuk 11 leidt tot twee rationale matrices in twee parameters. Dit wordt herschreven als een twee-parameter polynomiaal eigenwaarde probleem dat twee matrices en één gemeenschappelijke eigenvector bevat. Beide matrices worden samengevoegd tot één rechthoekige en singuliere matrix. Hier zijn de ideeën van Hoofdstuk 10 met betrekking tot de Kronecker canonieke vorm berekeningen nuttig om de waardes van $\rho_{1}$ en $\rho_{2}$, die beide matrices gelijktijdig singulier maken, te berekenen. Een belangrijk resultaat is beschreven in dit hoofdstuk: de oplossingen van het stelsel vergelijkingen worden bepaald door de waardes die de transformatie matrices, die voor de verkrijging van de Kronecker canonieke vorm gebruikt worden, singulier maken. Met behulp van deze waardes wordt de globaal optimale benadering $G(s)$ van orde $N-3$ berekend. Aan het einde van dit hoofdstuk wordt een voorbeeld uitgewerkt waarbij de co-orde $k=3$ techniek een betere performance laat zien dan de co-orde $k=1$ en $k=2$ technieken.

Hoofdstuk 12 biedt concluderende opmerkingen en aanwijzingen voor verder onderzoek met betrekking tot de resultaten beschreven in de delen II en III van dit proefschrift. 


\section{Curriculum Vitae}

1979

1991-1997

1997-1998

1998-2003

2001-2002

2001-2003

2003-2004

2004-2010

2008-2009

2010-present
Born on March 12th 1979, Heerlen, the Netherlands.

Pre-university Education, Gymnasium Rolduc, Kerkrade.

Propaedeutic Chemical Technology, Hogeschool Limburg, Heerlen.

Master of Science in Knowledge Engineering, Math Major. Joint programme of the Maastricht University (the Netherlands) and the Limburgs Universitair Centrum (Belgium).

Master thesis at the Department of Mathematics of the Maastricht University with the title 'Polynomial optimization using algebraic techniques with applications to system theory'.

Part-time position as software developer at MateUM B.V., Maastricht.

Part-time position as developer of e-SAPAS, an electronic self- and peer-assessment system, at Faculty of General Sciences and Faculty of Law, Maastricht University, Maastricht.

Developer of e-SAPAS at Faculty of General Sciences, Maastricht University, Maastricht.

PhD Student, Faculty of Humanities and Sciences, MICC, Department of Mathematics, Maastricht University, Maastricht.

Software Developer at IDEE (Instrument Development Engineering \& Evaluation), Maastricht University, Maastricht.

Post-doctoral researcher at the BioMathematics and BioInformatics (BMI) group, Department of Knowledge Engineering, Maastricht University, Maastricht. 



\section{List of Publications}

[1] I.W.M. Bleylevens, M.E. Hochstenbach, and R.L.M. Peeters. Polynomial optimization and a Jacobi-Davidson type method for commuting matrices. Submitted, 2010.

[2] I.W.M. Bleylevens, R.L.M. Peeters, and B. Hanzon. A generalized eigenvalue approach to $\mathrm{H}_{2}$ model-order reduction. Proceedings of the 27 th Benelux Meeting on Systems and Control, Heeze, 2008.

[3] I.W.M. Bleylevens, R.L.M. Peeters, and B. Hanzon. Efficiency improvement in an $n \mathrm{D}$-systems approach to polynomial optimization. Journal of Symbolic Computation, 42(1-2): 30-53, 2007.

[4] I.W.M. Bleylevens, R.L.M. Peeters, and B. Hanzon. Optimal $\mathrm{H}_{2}$ model reduction from order $N$ to $N-2$. Internal Report M0\%-01, Department of Mathematics, Maastricht University, 2007.

[5] I.W.M. Bleylevens, R.L.M. Peeters, and B. Hanzon. Optimal $H_{2}$ model reduction from order $N$ to $N-2$. Proceedings of the 13th ILAS Conference, Amsterdam, 2006.

[6] I.W.M. Bleylevens, R.L.M. Peeters, and B. Hanzon. An $n$ D-systems approach to global polynomial optimization with an application to $\mathrm{H}_{2}$ model-order reduction. Proceedings of the 44th IEEE CDC and the ECC, Seville, Spain, 2005.

[7] I.W.M. Bleylevens, R.L.M. Peeters, and B. Hanzon. Efficiency improvement in an $n \mathrm{D}$-systems approach to polynomial optimization. Proceedings of the MEGA 2005: Effective Methods in Algebraic Geometry Computing in and with algebraic geometry: Theory, Algorithms, Implementations, Applications, Alghero, Sardinia, Italy, 2005.

[8] I.W.M. Bleylevens, R.L.M. Peeters, and B. Hanzon. Efficient polynomial optimization with an $n \mathrm{D}$-systems approach. Proceedings of the 24 th Benelux Meeting on Systems and Control, Houffalize, Belgium, 2005. 
[9] I.W.M. Bleylevens, B. Hanzon, and R.L.M. Peeters. A multidimensional systems approach to polynomial optimization. Proceedings of the MTNS, Leuven, Belgium, 2004.

[10] I.W.M. Bleylevens, R.L.M. Peeters, and B. Hanzon. A multidimensional systems approach to algebraic optimization. Proceedings of the HPOPT - the 8 th International Workshop on High Performance Optimization Techniques: Optimization and Polynomials, Amsterdam, 2004.

[11] R.L.M. Peeters, I.W.M. Bleylevens, and B. Hanzon. A mixed algebraic-numerical global optimization approach to $\mathrm{H}_{2}$ model reduction. Proceedings of the 22th Benelux Meeting on Systems and Control, Lommel, Belgium, 2003.

[12] I.W.M. Bleylevens. De berekening van de globale minima van een polynomiaal criterium. Master Thesis (in Dutch), Department of Mathematics, Maastricht University, 2003. 


\section{List of Symbols and Abbreviations}

\begin{tabular}{lll}
\hline Abbreviation & \multicolumn{1}{c}{ Description } & Definition \\
\hline ARPACK & Arnoldi algorithm & page 51 \\
Eig & Direct eigenvalue solver in Matlab & page 109 \\
Eigensystem & Eigensystem solver in Mathematica & page 109 \\
Eigs & Iterative eigenvalue solver in Matlab & page 110 \\
Flops & Floating point operations & page 60 \\
GloptiPoly & Multivariable polynomial optimization problem solver & page 52 \\
& in Matlab & \\
JD & Jacobi-Davidson method by M. Hochstenbach & page 81 \\
JDCOMM & Jacobi-Davidson method for commuting matrices & page 81 \\
JDQR & Jacobi-Davidson QR-method & page 81 \\
JDQZ & Jacobi-Davidson QZ-method & page 81 \\
LMI & Linear matrix inequality & page 111 \\
MIMO & Multiple-input, multiple-output & page 135 \\
NSolve & Solver for polynomial systems of equations in Mathe- & page 108 \\
& matica & \\
RGS & Repeated Gram-Schmidt & page 86 \\
RSPP & Relaxation of the shortest path problem & page 60 \\
SDP & Semi Definite Program & page 111 \\
SeDuMi & Self-dual-minimization / optimization package & page 111 \\
SISO & Single-input, single-output & page 135 \\
SOS & Sum of squares & page 111 \\
SOSTOOLS & Matlab toolbox for formulating and solving sum of & page 25 \\
SPP & square problems & \\
SYNAPS & Shortest path problem & page 60 \\
\hline & C++ solver for polynomial systems & page 52 \\
\hline
\end{tabular}




\section{List of Figures}

3.1 (a) $x$-values (b) $y$-values of solution paths of $H(x, y, t)=0(0 \leq t \leq 1)$. . 31

4.1 Example of a polynomial with multiple locations of the optimum . . . . . 44

4.2 Example polynomial $p_{1}\left(x_{1}, x_{2}\right) \ldots \ldots \ldots \ldots$. . . . . . . . 48

5.1 Relationship between time series in (5.16) and the initial state $w_{0,0} \ldots \ldots$

5.2 Time instants to compute the action of $A_{p_{1}}^{T} \ldots \ldots \ldots \ldots$

5.3 Constructing and translating a minimal stable pattern . . . . . . . . . . 64

5.4 A shortest path to compute $y_{13,12} \ldots \ldots \ldots \ldots$. . . . . . . . 65

5.5 (a) The initial state $w_{0,0,0}$ (b) The initial state and the full recursions . . 67

5.6 A stable pattern (green) for shifts in all three directions . . . . . . . . . . 67

5.7 (a) Points with total time 4. (b) Connections of the points . . . . . . 68

5.8 Two different viewpoints of Figure 5.7(b) . . . . . . . . . . . . . . 69

5.9 Computation of points with total time $4 \ldots \ldots \ldots$

5.10 A non-minimal stable pattern allowing shifts in three time directions . . . 71

5.11 Non-minimal stable patterns allowing for shifts along one time axis . . . . 72

5.12 Points required by the Linear method . . . . . . . . . . . . . . 74

5.13 Points required by the Diagonal method . . . . . . . . . . . . . . 75

5.14 Points required by the Equalizing method . . . . . . . . . . . . . 75

5.15 Points required by the Axis method . . . . . . . . . . . . . . 76

5.16 Points required by the linear, diagonal, equalizing and axis method . . . . 77

5.17 Points required by the linear, diagonal, equalizing and axis method . . . . 78

5.18 Points required by the least-increments method . . . . . . . . . . . 78

5.19 Region of points that occur in (a) 2-D paths and (b) 3-D paths . . . . . 80

7.1 Sparsity structure of the matrices $A_{x_{1}}$ and $A_{p_{1}} \ldots \ldots \ldots$. . . . . 109

7.2 Residuals before and after projection . . . . . . . . . . . . . 116

7.3 Sparsity structure of the matrices $A_{p_{1}}$ and $A_{x_{i}}, i=1, \ldots, 4 \ldots \ldots$. . . 119

7.4 Eigenvalue spectra of the matrices $A_{p_{1}}$ and $A_{x_{i}}, i=1, \ldots, 4 \ldots \ldots$

7.5 Residual norms against MVs with $A_{p_{1}}$ for the JD and JDCOMM methods . . 121

7.6 Sparsity structure of the matrices $A_{p_{1}}$ and $A_{x_{i}}, i=1, \ldots, 4 \ldots \ldots$. . . 122 
7.7 Residual norms against MVs with $A_{p_{1}}$ for the JD and JDCOMM methods . . 123

7.8 Eigenvalue spectra of the matrices $A_{p_{1}}$ and $A_{x_{i}}, i=1, \ldots, 4 \ldots \ldots \ldots$

7.9 Eigenvalue spectra of the matrices $A_{p_{1}}$ and $A_{x_{i}}, i=1, \ldots, 5 \ldots \ldots$

7.10 Sparsity structure of the matrices $A_{p_{1}}$ and $A_{x_{i}}, i=1, \ldots, 4 \ldots \ldots$. . . 127

7.11 Eigenvalue spectra of the matrices $A_{p_{1}}$ and $A_{x_{i}}, i=1, \ldots, 4 \ldots \ldots$. . 128

7.12 Residual norms against MVs with $A_{p_{1}}$ for the JD and JDCOMM methods . . 129

9.1 All eigenvalues of the matrix $A_{V_{H}}^{T} \ldots \ldots \ldots \ldots$. . . . . . . . 157

9.2 Zoomed in on the smallest real eigenvalues of the matrix $A_{V_{H}}^{T} \ldots \ldots . .157$

9.3 Sparsity representation of the matrices $A_{x_{1}}^{T}, A_{x_{2}}^{T}$ and $A_{V_{H}}^{T} \ldots \ldots \ldots$

10.1 Sparsity and structure of the matrix $\tilde{A}_{\tilde{a}_{N-1}}\left(\rho_{1}\right)^{T} \ldots \ldots \ldots$. . . . . . . 183

10.2 Sparsity structure of the matrices $B$ and $C \ldots \ldots$. . . . . . . . . . . . . . . . . .

10.3 The poles of $H(s)$ and $G(s) \ldots \ldots \ldots \ldots$. . . . . . . . . . . . . . . . . . . . . . . . . . . . . . .

10.4 The poles of $H(s), G_{1}(s)$, and $G_{2}(s) \ldots \ldots \ldots$. . . . . . . . . . . . . . . . . . . . 186

10.5 Impulse responses of $H(s), G(s)$, and $G_{2}(s) \ldots \ldots \ldots$

10.6 Bode diagrams of $H(s), G(s)$, and $G_{2}(s) \ldots \ldots \ldots \ldots$

10.7 Structure of the matrix $\tilde{A}_{\tilde{a}_{N-1}}\left(\rho_{1}\right)^{T} \ldots \ldots \ldots \ldots$. . . . . . . . . . . . . . . . . . . . . . . . . . . .

10.8 Poles of $H(s)$ and approximation $G(s) \ldots \ldots \ldots$. . . . . . . . . . . . . . . . . . . . . .

10.9 Poles of $H(s)$ and approximations $G_{1}(s)$ and $G_{2}(s) \ldots \ldots$. . . . 191

10.10Impulse responses of $H(s)$ and $G(s) \ldots \ldots \ldots 2$

10.11Bode diagrams of $H(s)$ and $G(s) \ldots \ldots \ldots \ldots$. . . . . . . . . . . . . . . . . . . . . . . . . . . .

10.12Poles of $H(s), G_{1}(s), G_{2}(s)$, and $G(s) \ldots \ldots \ldots$. . . . . . . . . . . . . . . . . . . .

10.13Impulse responses of $H(s), G_{2}(s)$, and $G(s) \ldots \ldots \ldots$. . . . . . . . . . . . . . . . . . 194

10.14Bode diagrams of $H(s), G_{2}(s)$, and $G(s) \ldots \ldots \ldots \ldots$

11.1 Sparsity structure of the matrices $\tilde{A}_{\tilde{a}_{N-1}}\left(\rho_{1}, \rho_{2}\right)^{T}$ and $\tilde{A}_{\tilde{a}_{N-2}}\left(\rho_{1}, \rho_{2}\right)^{T}$. . 223

11.2 Real eigenvalue pairs $\left(\rho_{1}, \rho_{2}\right)$ of the matrices . . . . . . . . . . 224

11.3 Five important real eigenvalue pairs $\left(\rho_{1}, \rho_{2}\right)$ of the matrices . . . . . 225 


\section{List of Tables}

2.1 Monomial orderings of the terms of $f(x, y, z) \ldots \ldots \ldots$

5.1 Two-dimensional case $\left(2 \times 2\right.$ initial state $\left.w_{0,0}\right) \ldots \ldots \ldots$. . . . . . . 73

5.2 Three-dimensional case $\left(2 \times 2 \times 2\right.$ initial state $\left.w_{0,0,0}\right) \ldots \ldots \ldots$

5.3 Increase in the number of stored points along the diagonal . . . . . . . . . 77

5.4 Increase in the number of stored points along the axis . . . . . . . . . 79

7.1 Minimal real eigenvalue of the explicit matrix $A_{p_{1}} \ldots \ldots$. . . . . 109

7.2 Minimal eigenvalue of the operator $A_{p_{1}}$ (one eigenvalue calculated) . . . 111

7.3 Results of SOSTOOLS, GloptiPoly, and SYNAPS . . . . . . . . . . . 112

7.4 Polynomial test cases of various complexities . . . . . . . . . . . . . . . . . . . . . . . . . . . .

7.5 Settings for JD and JDQZ . . . . . . . . . . . . . . . . 113

7.6 Results using JD and JDQZ with the least-increments method . . . . . . . 114

7.7 Results using SOSTOOLS . . . . . . . . . . . . . . . . . . 115

7.8 Results for the projection with JD . . . . . . . . . . . 116

7.9 Results for the parallel implementation of the $n$ D-system . . . . . . . . 117

7.10 Sparsity of the matrices $A_{p_{1}}$ and $A_{x_{1}}, A_{x_{2}}, A_{x_{3}}, A_{x_{4}} \ldots \ldots \ldots$. . . . . 118

7.11 Comparison between performance of JD and JDCOMM . . . . . . . . . . 119

7.12 Sparsity of the matrices $A_{p_{1}}$ and $A_{x_{1}}, A_{x_{2}}, A_{x_{3}}, A_{x_{4}} \ldots \ldots \ldots$. . . . . . . . . . . .

7.13 Comparison between performance of JD and JDCOMM . . . . . . . . . 122

7.14 Sparsity of the matrices $A_{p_{1}}$ and $A_{x_{1}}, \ldots, A_{x_{5}} \ldots \ldots \ldots \ldots$

7.15 Comparison between performance of JD and JDCOMM . . . . . . . . . . 125

7.16 Sparsity of the matrices $A_{p_{1}}$ and $A_{x_{1}}, A_{x_{2}}, A_{x_{3}}, A_{x_{4}} \ldots \ldots \ldots$

7.17 Comparison between performance using various search space dimensions . 127

7.18 Performance of JD and JDCOMM $($ mindim $=10$, maxdim $=100) \ldots$. . 128

10.1 Reducing the model-order of a system of order $4 \ldots \ldots 3$

11.1 Results of reduction process of two-parameter pencil $A_{1}\left(\rho_{1}, \rho_{2}\right)^{T}$. . . 225

11.2 Results of reduction process of two-parameter pencil $A_{1}\left(\rho_{1}, \rho_{2}\right)^{T} \ldots$. . 226

11.3 Reducing the model-order of a system of order $4 \ldots \ldots$. . . . . . . 228 


\section{Index}

$H_{2}$ model-order reduction problem, 140

$\mathrm{H}_{2}$-criterion, 152

$n$ D-shift, 54

$n$ D-system, 53

$z$-transform, 139

2D-case, 62

3D-case, 66

Arnoldi, 51, 82, 110

ARPACK, 110

autonomous, 53

axis method, 71

balance-and-truncate, 134

balancing, 110, 184

BiCGStab, 84

branch and bound, 61

Cartesian product, 61

Cauchy's Residue Theorem, 146

co-order, 141

combinatorial complexity, 90

commuting matrix, 46

continuous time, 138

conventional eigenvalue problem, 166

correction equation, 84, 103

Cramer's rule, 144

Davidson method, 82

deflation step, 85

diagonal method, 71

difference equation, 53

Dijkstra's algorithm, 52, 60

directed graph, 60

discrete time, 138

dominated polynomial, 44

Eig, 109

eigenspace, 49

Eigensystem, 109

Eigs, 110, 129 equalizing method, 71

flops, 60, 73

Floyd's algorithm, 52, 60

full recursion, 62,68

Galois Theory, 26

generalized eigenvalue problem, 166

generalized iterative eigenvalue solver, 110

geometric multiplicity, 47

geometric pattern, 62

global minimizer, 45, 49

GloptiPoly, 52, 111

GMRES, 84

Gröbner basis, 45

Gram-Schmidt, 86

Hankel norm, 134

Hardy space, 138

heuristic, 59, 60, 66, 70

homogeneous system, 53

hypercube, 71

hyperplane, 62,71

impulse response, 139

indeterminate eigenvalue, 166

infimum, 44

infinite eigenvalue, 166

initial node, 60

initial state, 53

interior eigenvalues, 82

inverse power method, 82

iterative eigenproblem solver, 51

Jacobi-Davidson, 51, 110

Jdqr, 110

Jdqz, 110

Jordan canonical form, 168

Kronecker canonical form, 168

Krylov search space, 85 
Lagrange Multiplier, 100

Lanczos method, 82

Laplace transform, 139

Laurent series, 138

layer, 68

least-increments method, 76

left generalized eigenvalue problem, 167

left-half plane, 133

linear complexity, 73

linear least-squares method, 96

linear matrix inequality, 111

linear method, 71

linear operator, 46

LMI, 111

local minimum, 49, 152

local search method, 110

Lyapunov equation, 138

Lyapunov-Stein equation, 139

matrix pencil, 166

matrix solution, 46, 148

McMillan degree, 133

MIMO, 135

minimal index, 177

minimal stable pattern, $63,67,74$

Minkowski, 44

model approximation problem, 133

model-order reduction problem, 133

monomial basis, 45

monomial ordering, 45

multidimensional system, 53

multidimensional time instant, 53

multidimensional time series, 53

nodes, 60

one-parameter problem, 202

orthant, 62,66

Padé approximation, 134

parallel, 61

Parseval's identity, 139

Perron-Frobenius, 110

polar coordinates, 88

polynomial eigenvalue, 137, 165

polynomial eigenvalue problem, 137, 165

power method, 82

power set, 61

preconditioner, 84, 110

quotient space, 45,54

QZ-algorithm, 166

Rayleigh-Ritz approach, 84

recursion, 58 refined Ritz vector, 85

regular matrix pencil, 168

relaxation of shortest path problem, 60,61

reversed lexicographic, 45

RGS, 86

Ritz vector, 85

RSPP, 60, 61

saddle point, 49, 152

SDP, 111

SeDuMi, 111

semi-definite program, 111

shift operator, 52,53

shortest path problem, 52, 60

similarity transform, 110

simplex, 73

singular matrix pencil, 168

SISO, 135

SOS, 111

SOSTOOLS, 52, 111

sparse, 51, 62

SPP, 60

stable pattern, $60,62,66,67,73$

state transition, 60

state vector, 53

Stetter vector, 110

strictly equivalent, 171

suboptimal, 70

subspace expansion, 83

subspace extraction, 84

sum of squares, 111

SYNAPS, 52, 111

terminal node, 60

theory of moments, 111

time instant, 53

total degree, 44,45

trace operator, 141

translation, 62, 66

tree, 61

unit disk, 133

upper bound, 60

variety, 45

Weak Hilbert Nullstellensatz, 34

weight function, 60 